\title{
Inwertsetzung regionaler traditioneller Produkte am Beispiel des neuen EU-Mitgliedsstaats Malta
}

\author{
Eine Untersuchung in den Bereichen \\ Kultur- und Sozialgeographie, Agrargeographie \\ und Tourismusgeographie \\ Dissertation \\ zur Erlangung des Doktorgrades \\ der Mathematisch-Naturwissenschaftlichen Fakultäten \\ der Georg-August-Universität zu Göttingen \\ vorgelegt von \\ Rainer Aschemeier \\ aus \\ Helmarshausen
}

Göttingen 2005 
D7

Referent: Prof. Dr. Bernd Cyffka

Korreferent: Prof. Dr. Werner Kreisel

Tag der mündlichen Prüfung: 03.11.2005 


\section{Inhalt}

Karten-, Tabellen- \& Abbildungsverzeichnis

S. IV

Abkürzungsverzeichnis

Hinweis zu mündlichen Zitaten \& Hinweis zur Rechtschreibung

S. VI

Danksagung

S. VI

1 - Forschungsfrage und Zielsetzung

S. VII

1.1 - Forschungsfrage

S. 1

1.2 - Zielsetzung

S. 1

S. 2

2 - Stand der Forschung

2.1 - Tourismus

S. 3

2.2 - Regionale Produkte Maltas

S. 3

2.3 - Landwirtschaft

S. 3

2.4 - Geschichte der Maltesischen Inseln

S. 4

2.5 - Verfügbare Länderkunden

S. 4

2.6 - Soziologie

S. 4

S. 5

3 - Die Maltesischen Inseln - Topographie und Naturraum

S. 6

3.1 - Topographie

3.2 - Anmerkung zur Darstellung der naturräumlichen Gegebenheiten

S. 6

3.3 - Geologie und Pedologie

3.3.1 - Terra Soils

3.3.2 - Xerorendzina

3.3.3 - Carbonat-Rohböden

3.4 - Klima

S. 7

S. 8

S. 8

S. 8

S. 9

S. 9

3.5 - Wasservorkommen

S. 10

3.6 - Vegetation

S. 10

3.7 - Die Fauna und ihre Stellung in Alltags- und Landeskultur

S. 12

4 - Entwicklungsgeschichte der Maltesischen Inseln

S. 14

5 - Malta und die Europäische Union

5.1 - Die Periode 1964 bis zum Ende der 1980er-Jahre

S. 20

5.2 - Maltas Europa-Politik von 1987-2003

S. 21

S. 25

5.3 - Protest der Malta Labour Party gegen den EU-Beitritt

S. 27

5.4 - Ausblick

S. 27

6 - Allgemeine Wirtschaftssituation der Republik Malta zum Zeitpunkt der EU-Erweiterung 2004 - Eine Kurzdarstellung

6.1 - Wirtschaftliche Grunddaten Maltas im Sommer 2004

S. 30

S. 30

6.2 - Ausblick und Prognose für Maltas Wirtschaftsentwicklung nach dem Beitritt zur EU und die Konsequenzen für die Vermarktung regionaler traditioneller Produkte

6.3 - Wettbewerbsstärken und -schwächen

7 - Auswertung einer touristischen Befragung zum Thema „regionale maltesische Produkte"

S. 38

7.1 - Fazit

S. 44

8 - Kultur und Folklore Maltas

S. 46

8.1 - Äußere Einflüsse und indigene Volkskultur

S. 46

8.2 - Zur Vergleichbarkeit des Untersuchungsraums mit anderen Regionen in Europa

S. 47

8.3 - Lokale Erzeugnisse als Bestandteil von Kultur und Folklore Maltas

S. 47

8.4 - Der Begriff „Folklore“ in der wissenschaftlichen Forschung

S. 48

9 - Die Debatte um Authentizität und Identität im Bereich der regionalen Produkte

S. 50

10 - Regionale traditionelle Produkte - Definition und Erläuterung

S. 52

10.1 - Definition

10.2 - Einschränkungen

S. 52

10.3 - Zusammenfassung

S. 54

S. 54

11 - Maltesische Produkte in den Augen der einheimischen Bevölkerung

S. 55

11.1 - Die zustimmende Haltung

S. 55

11.2 - Die ablehnende Haltung

S. 56 
11.3 - Effekte des EU-Beitritts auf die Haltung der Bevölkerung zu maltesischen Produkten

11.4 - Fazit und Ausblick

12.1 - Abriss der historischen Landwirtschaftsentwicklung und Diskussion der historischen Ursachen der heutigen sekundären Position des Agrarsektors in Maltas Volkswirtschaft

12.1.1 - Maltas Landwirtschaftsentwicklung von der Steinzeit bis zur Ritterherrschaft

12.1.2 - Unstimmigkeiten in der Darstellung der Landwirtschaft zu Zeiten der Johanniterherrschaft

S. 62

12.1.3 - Die Landwirtschaft zwischen französischer und britischer Kolonialzeit bis zum Zweiten Weltkrieg

12.1.4 - Maltas Agrarwirtschaft in den Nachkriegsjahren

12.1.5 - Entwicklungen der letzten Jahrzehnte und Auswirkungen von Tourismus und Erbteilung

S. 65

12.1.6 - Maltas Landwirtschaft nach dem EU-Beitritt

13.1 - Begründung für die folgende Nutzpflanzenauflistung im Rahmen der Abhandlung

13.2 - Auflistung der Anbaupflanzen

S. 73

13.3 - Obst

S. 73

13.4 - Gemüse

S. 79

13.5 - Leguminosen

S. 82

13.6 - Hackfrüchte

S. 83

13.7 - Getreide

S. 84

13.8 - Nüsse

S. 85

13.9 - Sonstige Anbaupflanzen

S. 86

13.10 - Nutzpflanzen, die keinem Anbau unterliegen

S. 88

14 - Regionale traditionelle Produkte Maltas und Gozos

S. 93

14.1 - Lebensmittel

14.1.1 - Backwaren

S. 95

14.1.2 - Gerichte

S. 105

14.1.3 - Getränke

S. 107

14.2 - Produkte des Kunsthandwerks

14.2.1 - Kunsthandwerk allgemein

14.2.2 - Kunsthandwerk Maltas und Gozos

S. 108

S. 111

S. 111

S. 111

15 - Streitfälle und nicht-regional-traditionelle Produkte

S. 123

15.1 - Moderne Produkte mit traditionellen Ausgangsstoffen

S. 125

16 - Agrargeographische Landnutzungsanalysen

S. 128

16.1 - Die Bedeutung der Erhebungen im Rahmen der Hauptthematik

S. 128

16.2 - Zur Methodik

16.3 - Zur Auswahl der Teilerhebungen

S. 129

16.4 - Zur Legende

S. 130

S. 133

17 - Die Ergebnisse der Landnutzungsanalysen im Einzelnen

S. 135

17.1 - Ergebnisse - Analyse 1: II-Miżieb

S. 135

17.2 - Ergebnisse - Analyse 2: II-Hofra

S. 139

17.3 - Ergebnisse - Analyse 3: Fort Binġemma

S. 142

17.4 - Ergebnisse - Analyse 4: Ǵnien Busewdien

S. 144

18 - Maltas Produzenten regionaler traditioneller Produkte

S. 148

18.1 - Einteilung nach Herstellergruppen

18.1.1 - Gruppe 1: Einzelpersonen oder kleinste Familienbetriebe

S. 149

18.1.2 - Gruppe 2: Einzelpersonen oder Familienbetriebe, die im Verband organisiert sind

S. 150

18.1.3 - Gruppe 3: Betriebe mit 10 Angestellten und mehr

S. 151

18.1.4 - Gruppe 4: „Alternative Hersteller“ mit eigenem Geschäftskonzept und Künstler

S. 154

S. 157

19 - Innovative Konzepte rund um regionale traditionelle maltesische Produkte

S. 161

19.1 - Verwertung der Kaktusfeige

S. 161

19.2 - „Farmhouse Cooking“

S. 162

20 - Ausgewählte Produkte im Hinblick auf ihre Entwicklungsfähigkeit in der Zukunft 20.1 - Zur Produktpsychologie bestimmter maltesischer Erzeugnisse

S. 164

20.2 - Artischocken

S. 164

S. 165

S. 167

20.2.1 - Wirtschaftliche Erfolgsaussichten für den Artischockenanbau in Malta

S. 168 
20.2.3 - Stärken-Schwächen-Analyse für maltesische Artischocken im Hinblick auf ihre Vermarktungsfähigkeit als regionales traditionelles Produkt

S. 169

20.3 - Olivenöl

20.3.1 - Einleitender Hinweis

20.3.2 - Diskussion der historischen Stellung der Olivenölproduktion in Malta und ihrer weit reichenden Ignorierung in wissenschaftlichen Arbeiten des 20. Jh.

20.3.3 - Olivenöl in der Vermarktung als regionales traditionelles Produkt

20.3.4 - Konsequenz

20.3.5 - Einschätzung der nationalen und internationalen Konkurrenz

20.3.6 - Stärken-Schwächen-Analyse für maltesisches Olivenöl im Hinblick auf seine Vermarktungsfähigkeit als regionales traditionelles Produkt

21 - Die Handwerksdörfer Ta'Qali und Ta'Dbieǵi

21.1 - Geschäftskartierungen in Ta'Qali

S. 179

21.2 - Kartierung Nr. 1

21.2.1 - Zur Methodik

21.2.2 - Auswertung

21.3 - Kartierung Nr. 2

S. 182

S. 183

S. 184

S. 184

S. 185

S. 188

S. 188

S. 189

S. 190

S. 190

S. 191

S. 192

S. 192

S. 192

S. 193

S. 193

22.9 - U.U. Subventionierung durch Regierung und
22.10 - Ausnutzung von Online-Business-Lösungen

S. 194

S. 200

S. 201 


\section{Karten-, Tabellen- \& Abbildungsverzeichnis}

\section{Fotos}

(Soweit nicht anders angegeben, alle Fotos: ASCHEMEIER)

Foto 1 - Ziegenherde bei Qrendi

S. 13

Foto 2 - Wahlfeier im Jahr 2003 auf der Republic Street in Valletta

S. 26

Foto 3 - Detailaufnahme eines Feigenkaktus der Sorte aћmar ingliż bei Bidnija

S. 88

Foto 4 - Detailaufnahme einer Kapernpflanze bei Xemxija.

S. 91

Foto 5 - Verpackte Ġbejniet (frisch).

Foto 6 - Werbung für Ǵbejna auf Milchpackung des Herstellers Benna.

S. 97

Foto 7 - Typischer Maltesischer Nougatverkaufsstand.

S. 98

Foto 8 - Qaghaq ta'l-ghasel in der Auslage eines Spezialitätengeschäfts in Mdina.

S. 103

Foto 9 - Traditioneller gozitanischer Salztopf in der Auslage der "Gozo Pottery Barn“

S. 106

Foto 10 - In den Geschäftsräumen von „Peter The Potter“, Ta'Qali

S. 116

Foto 11 - Die Geschäftsräume von „Bristow Pottery“, Ta'Qali

S. 119

Foto 12 - Silver Filigree im Juweliergeschäft „Denis Jewellery“, Ta'Qali

S. 119

Foto 13 - Ganutell-Dekoration im Dom von Mosta. Foto: MENHENNET

S. 120

Foto 14 - Schäden des Unwetters vom Oktober 2003 am Rand der Ortschaft Burmarrad

S. 122

Foto 15 - Erosionsrinnen auf einer Hangterrasse in der Nähe des Ǵnien Busewdien nach dem Unwetter im Oktober 2003

S. 145

Foto 16 - „Lace Lady“ bei der Arbeit

S. 146

Foto 17 - Das Label des Malta Crafts Council als Aufkleber auf der Verpackung von SchafsKäse

Foto 18 - Luftbildaufnahme des Meridiana Wine Estates in Ta'Qali. Foto: Meridiana

S. 152

Foto 19 - Übergroßes Werbebanner für den Softdrink Kinnie

S. 155

S. 155

Foto 20 - Bildhauer Joe Xuereb bei der Arbeit in seinem Ateiler in Ghajnsielem

S. 158

Foto 21 - Olivenölhersteller Sam Cremona

S. 159

Foto 22 - Die Präsentation im Rahmen der Veranstaltung „II Kćina I-Antika“ im Restaurant „Ir-Razzett L'Antik“ bei Qormi. Foto: Ir-Razzett L'Antik

Foto 22 - Artischocken im kleinbäuerlichen Anbau in der Region II-Hofra bei Mellieћa Foto: KORNITZKY

Foto 23 - Teilaufnahme der Ruinen der Villa Rustica von San Pawl Milqi

Foto 24 - Ölbaumanpflanzung Bajda Ridge

S. 172

Foto 25 - Überreste von römischen und ägyptischen Bleiankern aus der Region Burmarrad

S. 174

Foto 26 - Crafts Village Ta'Dbieǵi auf Gozo

S. 181

Foto 27 - Die typische Bauweise der Shops und Werkstätten in Ta'Qali

S. 182

Foto 28 - Die Beschriftung der Kunserva-Dose der Marke „Vera“

S. 190

\section{Karten}

Karte 1 - Die Maltesischen Inseln und Ihre Lage im Mittelmeer. EVANS (1963), S. 12 (geändert) S. 6

Karte 2 - Maltas Verträge mit dem Ausland in der Zeit von 1963-1982. Entwurf: AsCHEMEIER, Grafik: HÖFER

Karte 3 - NSO Viticulture Survey 1999/2000. NSO (2002a), S. 31

Karte 4 - Die landwirtschaftlichen Anbaugebiete der Insel Malta. Entwurf \& Grafik: AsCHEMEIER In Anlehnung an BOWEN JONES, DEWDNEY \& FISHER (1961), S. 236

Karte 5 - Landbewirtschaftung in II-Miżieb. Entwurf \& Grafik: ASCHEMEIER

S. 137

Karte 6 - Landbewirtschaftung in II-Hofra. Entwurf \& Grafik: ASCHEMEIER

S. 141

Karte 7 - Landbewirtschaftung im Bereich der Santi-Bingemma Uplands, westlich von Fort Binġemma. Entwurf \& Grafik: AsCHEMEIER

Karte 8 - Landbewirtschaftung im Ǵnien Busewdien. Entwurf \& Grafik: AsCHEMEIER

Karte 9 - Geschäftskartierung von Ta'Qali nach Angebotsstruktur und Preisniveau. Entwurf \& Grafik: ASCHEMEIER

Karte 10 - Geschäftskartierung von Ta'Qali nach Angebotsart. Entwurf \& Grafik: AsCHEMEIER

Karte I - Anbaukarte II-Miżieb

S. 187

Karte II - Anbauzone II-Hofra

Anhang

Anhang

Karte III - Anbauzone Santi-Binġemma Uplands

Anhang

Karte IV - Anbauzone Ġnien Busewdien 


\section{Tabellen}

(Für sämtliche Tabellen: Entwurf und Darstellung: ASCHEMEIER)

Tabelle 1 - Auflistung der Nutzpflanzen, die in Kap. 13 behandelt werden

S. 73

Tabelle 2 - Auflistung der Handwerksprodukte, die in Kap. 14.2.2 behandelt werden

S. 112

Tabelle 3 - Erhebungsschlüssel zur Kartierung der Produktkategorien in Ta'Qali

S. 185

Tabelle 4 - Die Geschäfte in Ta'Qali und inr Produktsortiment

\section{Abbildungen}

Abb. 1 - Klimadiagramm Valletta/Luqa aus dem Jahr 2000.

Quelle: https://www.ilexikon.com/Malta.html. Stand: 08.09.2005, überarbeitet: ASCHEMEIER

Abb. 2 - Zeittafel zur Geschichte der Maltesischen Inseln. Entwurf \& Grafik: AsCHEMEIER

Abb. 3 - Statistische Auswertung der Frage: „Fühlen Sie sich ausreichend über maltesische Produkte informiert?" Entwurf \& Grafik: AsCHEMEIER

Abb. 4 - Statistische Auswertung der Frage: „Kennen Sie typisch maltesische Produkte?“ Entwurf \& Grafik: AsCHEMEIER

Abb. 5 - Statistische Auswertung der Frage: „In welchen dieser Produktkategorien würden Sie am ehesten typisch maltesische Produkte vermuten?" Entwurf \& Grafik: ASCHEMEIER

Abb. 6 - Statistische Auswertung der Frage: „Fänden Sie es gut, wenn Sie maltesische Produkte auch in ihrem Heimatland kaufen könnten?" nach geschlechterbezogenem Antwortverhalten. Entwurf \& Grafik: ASCHEMEIER

Abb. 7 - Statistische Auswertung der Verknüpfung von Alterszusammensetzung der Befragten und der Auswertung der Frage „Fänden Sie es gut, wenn Sie maltesische Produkte auch in Ihrem Heimatland kaufen könnten?"

Abb. 8 - Statistische Auswertung des Sachverhalts wie in Abb. 7, hier als Kurve dargestellt. Entwurf \& Grafik: ASCHEMEIER

Abb 9 - Größenverteilung der landwirtschaftlichen Nutzflächen auf den Maltesischen Inseln Daten: NSO (2003a). Entwurf \& Grafik: AsCHEMEIER

Abb. 10 - Beschäftigte in der Landwirtschaft nach Altersklassen. ASCHEMEIER \& CYFFKA (2004b), S. 109

Abb. 11 - Maltas Ausgaben für den Import von Weintrauben in Lm. Daten: NSO (individuell zusammengestellt). Entwurf \& Grafik: AsCHEMEIER

Abb. 12 - Schwankungen beim Verkauf von Zwiebeln in Malta. Daten: NSO (individuell zusammengestellt). Entwurf \& Grafik: AsCHEMEIER

Abb. 13 - Die fünf Erzeugerzweige für regionale traditionelle Produkte auf den Maltesischen Inseln. Entwurf \& Grafik: AsCHEMEIER

S. 94

Abb. 14 - Werbeanzeige „Kinnie“

S. 108

Abb. 15 - „Lace-Making“ in Malta im 19. Jh. ZARB (1998), Bildteil

S. 113

Abb. 16 - Ganzseitige Zeitschriftenwerbung für Ċisk Lager

S. 124

Abb. 17 - IKONOS-Satellitenbildszene (28.03.2004) vom nordwestlichen Teil Maltas $\quad$ S. 129

Abb. 18 - Anbauzone II-Miżieb im IKONOS-Satellitenbild, Channel 3, Infrarot 136

Abb. 19 - Das Label des Malta Crafts Council

S. 152

Abb. 20 - Buchcover des bisher einzigen in Druckform publizierten Handwerkerverzeichnisses des Malta Crafts Councils

Abb. 21 - Vewertungswege der Kaktusfeige. Entwurf \& Grafik: AsCHEMEIER

Abb. 22 - Gesamtmenge verkaufter Artischocken auf den Maltesischen Inseln pro Jahr von 2002-2004. Daten: NSO (individuell zusammengestellt). Entwurf \& Grafik: ASCHEMEIER

Abb. 23 - Die Wappen der gozitanischen Ortschaften Zebbug̉ und Ghasri. BEZZINA (1988), S. 20 \& S. 42

Abb. 24 - Der Bereich Ta'Qali auf Malta. Entwurf \& Umsetzung: AscHEMEIER

S. 180

Abb. 25 - Vernetzungsmöglichkeiten der Folkoremuseen Gozos mit dem Crafts Village Ta'Dbieġi. Entwurf und Grafik: AsCHEMEIER 


\section{Abkürzungsverzeichnis}

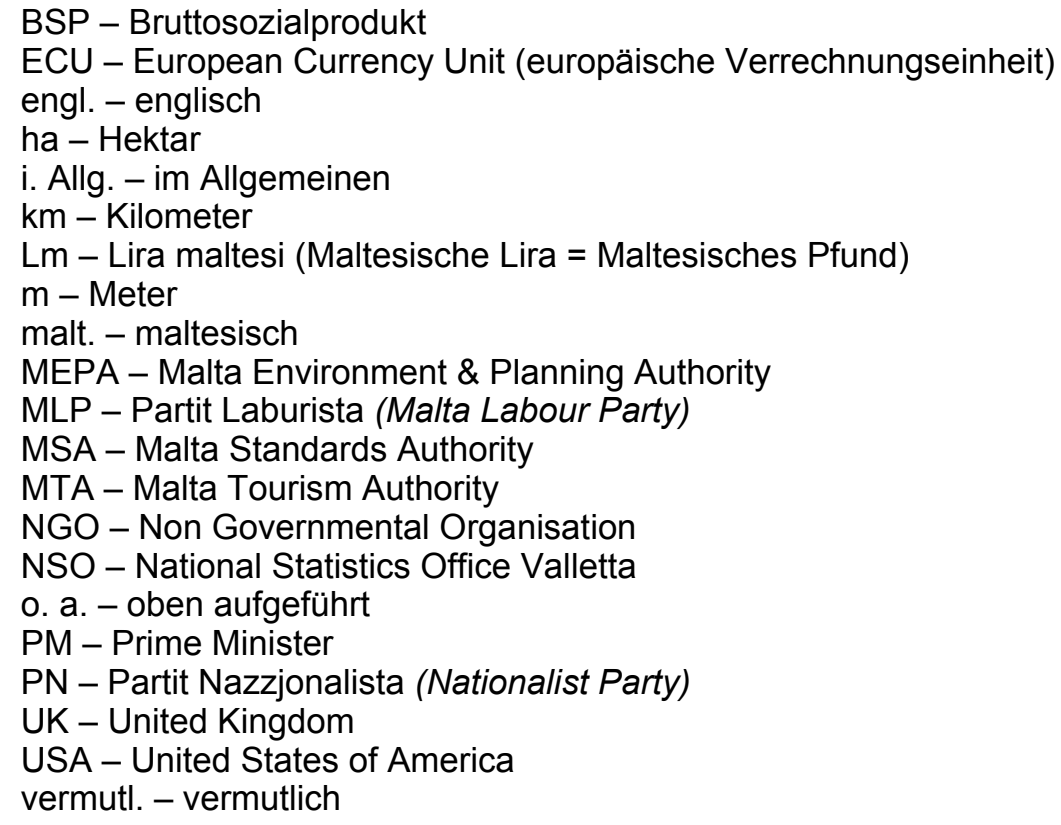

Des Weiteren gelten die offiziellen Abkürzungszeichen aus dem amtlichen Regelwerk der deutschen Rechtschreibung.

\section{Hinweis zu mündlichen Zitaten}

Mündliche Zitate werden vom Verfasser als wortwörtliches Transkript (vgl. PFAFFENBACH \& REUBER (2005)) wiedergegeben. Es ist zu bedenken, dass Englisch nicht die Muttersprache der befragten Malteser ist. Es kommen daher mündliche Zitate vor, die nicht in allen Belangen die korrekte Verwendung der englischen Sprache aufweisen. Aus Gründen der Lesbarkeit wurde aber auf diesbezügliche Hinweise (z.B. [sic]) in mündlichen Zitaten verzichtet.

\section{Hinweis zur Rechtschreibung}

Der Verfasser orientierte sich im Rahmen der vorliegenden Abhandlung an den Richtlinien der aktuellen Rechtschreibreform, wie sie im amtlichen Regelwerk der deutschen Rechtschreibung des Jahres 2005 dargelegt sind. 


\section{Danksagung}

Ich möchte den folgenden Personen sowie den Mitarbeitern der genannten Einrichtungen und Firmen danken, die durch ihre Auskunftsbereitschaft in den Experteninterviews, ihre Bereitschaft zur Kontaktvermittlung oder durch ihre unmittelbare Hilfeleistung zum Gelingen der vorliegenden Dissertation beigetragen haben:

Prof. Charles Agius

Reno Agius

Prof. Dr. Lino Briguglio (Institute for Small States \& Small Island States Studies, Uni Malta)

Denise Buckwell (Parliamentary Secretary - Ministry for Rural Affairs and the Environment)

Keith Buttig̉ieg

Godfrey Camillieri (Agricultural Research and Development Centre, Ghammieri)

Romina Cilia

Sam Cremona (Ghajn Razul Company, Wardija)

Matty Cremona (Schriftstellerin, Journalistin und TV-Köchin, Wardija)

Lina Deguara (Lehrerin, Mġarr)

Denis Jewellery (Ta'Qali)

Victor Farrugia (Plant Biotechnology Centre Malta, Lija)

Marica Gatt (Malta Beekeeper's Association)

Anthony Gellel (Registrar of the University of Malta)

Dr. Gigquel (Master Wine Group, Naxxar)

Lawrence Giordemaina (Meteorological Office - Malta International Airport)

Gozo Pottery Barn (Ta'Dbiegi)

Brian Grima (Bristow Pottery, Ta'Qali)

Anthony Mangion (Director Library Services - University of Malta, Msida)

Marsovin Winery (Marsa)

Meridiana Wine Estate (Ta'Qali)

Georg Merten (Deutscher Botschafter in Malta)

Hans-Werner Morawetz (Deutschland-Importeur maltesischer Produkte)

Pins \& Needles (Ta'Dbiegi)

Olivia Said (Mdina Glass, Ta'Qali)

Charles M. Saliba (Institute for Cellular Pharmacology, Mosta)

Frans Scerri (Xemxija Heritage/St. Paul's Bay Local Council)

John Schembri (University of Malta, Department of Human Geography)

Simon Sultana (Malta International Airport)

Charles J. Vassallo (Malta Crafts Council, Valletta)

Kristian Vella

Maudie Vella (Weaving \& Lace, Rabat)

Miriam Vella \& Familie (Frans Vella Company \& Sons, Qormi)

Joe Xuereb (Stone \& Arts Gallery, Ghajnsielem)

Chris Zahra (Association for Producers of Maltese Cheeselets (Ǵbejniet), Buskett)

Deutsch-Maltesische Gesellschaft (Adenau, Deutschland)

Department of Information of the Government of Malta

The Embassy of Germany in Malta

Institute for Cellular Pharmacology

Library of the University of Malta

MEPA - Malta Environment and Planning Authority

MIA - Malta International Airport

MIC - Malta-EU-Information Centre

Ministry of Rural Affairs and the Environment of the Government of Malta

MTA - Malta Tourism Authority

NSO - National Statistics Office

The University of Malta

Darüber hinaus möchte ich meinen Helferlnnen bei den in dieser Dissertation vorgestellten Landnutzungskartierungen danken: Sebastian Kornitzky, Katrin Kranz, Angela Sohst und Kerstin Thoelke. 
Besonderer Dank gebührt Herrn Carmelo Galea für seine unschätzbare Unterstützung bei meiner Forschungsarbeit und bei der Übersetzung maltesischer Texte, seine hilfreichen Tipps und Kontaktvermittlungen, seine Freundlichkeit und Gastfreundschaft und seine wertvolle Hilfe bei der Bewältigung der kleinen Hürden des maltesischen Alltags.

Ebenfalls besonders danken möchte ich Herrn Oberstudienrat Hans Barth, der mir durch seinen engagierten und motivierten Erdkundeunterricht während meiner Schulzeit die Liebe zur Geographie vermittelt hat und mir zudem ein geschätzter Kritiker bei der Fertigstellung der vorliegenden Schrift war.

Die GIS-Auswertungen der vorliegenden Dissertation wurden mit freundlicher Unterstützung der Firma MapInfo ermöglicht, die ihre Software leihweise zur Verfügung stellte. Der Autor bedankt sich sehr herzlich!

Vor allem möchte ich mich aber bei meinem Referenten, Herrn Prof. Dr. Bernd Cyffka und meinem Korreferenten Herrn Prof. Dr. Werner Kreisel für ihre persönliche Betreuung bedanken.

Auch den Herren PD Dr. Heiko Faust, Dr. Michael Waibel und Dr. Tobias Reeh möchte ich Danke sagen, weil sie mit mit ihrer offenen und unkomplizierten Art im persönlichen Gespräch viele gute Ratschläge geben konnten.

Gewidmet ist die Arbeit meinen Eltern sowie meiner verstorbenen Großmutter, die mich in meinem Studium und in allen anderen Lebensbereichen in außergewöhnlicher Art und Weise gefördert und unterstützt haben. 


\section{Forschungsfrage und Zielsetzung}

\subsection{Forschungsfrage}

Die Maltesischen Inseln waren jahrhundertelang Kolonie fremder Machthaber, bevor im Jahr 1964 die Unabhängigkeit von der britischen Krone erreicht werden konnte. Nach einer Verfassungsänderung im Jahr 1974 wurde Malta zur Republik mit einem - politisch lange Zeit nicht unproblematischen - Neutralitätsstatus. 30 Jahre später, im Mai 2004, erfolgte eine weitere Veränderung mit bedeutsamen Konsequenzen für den Inselstaat: Malta wurde EU-Mitglied.

Als kleinster EU-Staat mit vielen Subventionen, Sonderregelungen und Fördermitteln bedacht, bricht nun in Malta eine neue Phase der gesellschaftlichen und wirtschaftlichen Ausrichtung an. Die maltesische Wirtschaft steht jedoch nicht nur unter dem Einfluss von EU-Fördermitteln, sondern mit dem Beitritt setzt sich Malta auch völlig neuen Konkurrenzsituationen aus und muss sich im freien Handel des EU-Binnenmarkts behaupten.

Insbesondere die zahlreichen regionaltypischen Produkte der Inselgruppe sind von dieser Entwicklung betroffen. Sie blicken oft auf eine lange Tradition zurück, die im Einzelfall bis ins Jahr 200 v. Chr. zurückreicht. Gegenwärtig sind insbesondere diese traditionellen einheimischen Produkte auf dem „Prüfstand“. Werden sie dem Ansturm europäischer Importwaren standhalten können, der bereits wenige Wochen nach dem EU-Beitritt spürbar wurde? Können ausländische Imitate maltesischer Spezialitäten ausgeschlossen werden? Ist das Image der maltesischen Traditionsprodukte noch zeitgemäß, und wie kann es u.U. weiterentwickelt werden?

Die Anforderungen an die maltesischen Hersteller sind hoch. Zudem stellt sich im Rahmen von Maltas EUBeitritt die Frage, was ist überhaupt „typisch maltesisch“? Und was ist „regionaltypisch" oder „traditionell“? Insbesondere für den Begriff des „regionalen Produkts“ existieren unterschiedliche, häufig nicht zufrieden stellende Definitionen in der wissenschaftlichen Literatur.

Auch die nationale Identität der Bevölkerung befindet sich derzeit in einem Verschmelzungsprozess. Einerseits kann ein starkes Nationalgefühl der Malteser nicht verleugnet werden, andererseits existiert auch eine Affinität zu ausländischen Waren und Werten, die durch ihre Herkunft von außerhalb einer „Flucht aus dem Altag“ der engen Inselwelt psychologisch Raum geben.

Der „Zwergstaat Malta“ trifft auf den „Riesen Europa“. Welche Auswirkungen und Prozesse im Bereich der regionaltypischen Produkte kann das haben?

Aus der Literatur ist bekannt, dass der Megatrend Globalisierung einen zweiten Megatrend geschaffen hat: Die Regionalisierung ${ }^{1}$. Die Regionalisierung erwies sich gegenüber der Globalisierung als „realitätsnäherer Weg zur Rückgewinnung politischer Handlungsfreiheit" (MüLLER (2002), S. 150). Regionen und kleine Staaten, die im Rahmen der Globalisierungswelle ökonomisch „überrollt" zu werden drohten, konnten im Rahmen eines gegensteuernden Regionalisierungsprozesses oft erfolgreich in größere multilaterale Staatenbünde integriert werden. In der EU gibt es dafür viele Beispiele, so z. B. auch die von der EU beschlossene euro-mediterrane Freihandelszone ${ }^{2}$, die wiederum mittelbar Malta beeinflusst.

1 Vgl. z. B. MüLLER (2002), S. 150 ff. Der Begriff Regionalisierung ist in diesem Fall im ökonomisch-politischen Kontext zu verstehen.

2 Vgl. BMZ (2001) 


\subsection{Zielsetzung}

Die Dissertation untersucht Anzahl und Art sowie Verbreitung und Vermarktung regionaler traditioneller Produkte der Maltesischen Inseln.

Seit der einflussreichen Arbeit von BOWEN JONES, DEWDNEY \& FISHER (1961) gab es keine konkrete Bestandserhebung und Beschreibung maltesischer Agrarprodukte und Anbaufrüchte mehr. Eine Untersuchung der maltesischen Lebensmittel- und Kunsthandwerksprodukte gab es in wissenschaftlicher Form bisher noch gar nicht. Der Autor versucht mit der vorliegenden Abhandlung eine Datengrundlage zu schaffen und bestimmte Bereiche der Untersuchung von BOWEN JONES et al. fortzuschreiben.

Der Verfasser strebt des Weiteren im Rahmen seiner Dissertationsschrift folgende Aspekte an, die hiermit als Zielsetzung der Arbeit definiert werden.

- Bestandserhebung und Beschreibung der Agrarprodukte und Nutzpflanzen der Maltesischen Inseln. Hierbei: Fortschreibung eines Teils der Untersuchung von BOWEN JONES, DEWDNEY \& FISHER (1961), S. $195-273$

- Bestandserhebung und Beschreibung maltesischer Kunsthandwerksprodukte und Lebensmittel

- Geschäftskartierung des wichtigen Vermarktungszentrums Ta'Qali auf Malta

- Einführung und Definition des neuen geographischen Terminus technicus „regionales traditionelles Produkt"

- Untersuchung sozialgeographischer und psychologischer Verknüpfungen zwischen Maltas regionalen traditionellen Produkten und einheimischer sowie touristischer Kundschaft

- Analyse ausgewählter Landnutzungszonen dahin gehend, inwieweit sie noch als Quellgebiete für regionale traditionelle Agrarprodukte dienen bzw. inwieweit die Modernisierung der Agrarwirtschaft Auswirkungen auf den Anbau und die Produktion regionaler traditioneller Agrarprodukte hatte

- Abschätzung der ökonomischen Chancen für regionale traditionelle Produkte Maltas und Gozos für die inländische Vermarktung sowie für die Vermarktung auf dem EU-Binnenmarkt

- Abschätzung der Entwicklungsfähigkeit ausgewählter regionaler traditioneller Produkte der Maltesischen Inseln

- Erstellung eines schematischen Marketingkonzepts für maltesische Produkte

Als Endergebnis wird eine umfassende Kompilation der historischen und aktuellen Entwicklungen sowie der soziologischen und ökonomischen Strukturierung des Forschungsbereichs der regionalen traditionellen Produkte der Maltesischen Inseln angestrebt. 


\section{Stand der Forschung}

Die Malta-Forschung ist eine auffallend interdisziplinäre Forschungsrichtung. Während die Arbeiten aus dem Ausland vor allem aus den Bereichen Soziologie, Geographie und Geologie stammen, sind die Beiträge maltesischer Autoren in vielen Fällen keiner Forschungsrichtung eindeutig zuzuordnen.

Dieser Sachverhalt liegt möglicherweise auch an der Lehrstruktur der University of Malta, bei der viele Autoren wissenschaftlicher Veröffentlichungen jüngeren Datums als Dozenten beschäftigt sind. Dort gibt es vor allem im Bereich der Naturwissenschaften häufig keine unabhängigen Institute, sondern ein „Mediterranean Institute“, das von vornherein auf interdisziplinäre Forschung ausgelegt ist. Dieser Ansatz spiegelt sich in den Fachbeiträgen wider und sorgt dort für eine auffallende Vermischung der fachlichen Ausrichtung (vgl. z. B. CASSAR PULLICINO (1992), BusutTIL (1993), FRENDO (1994), ZARB (1998) oder FARRUGIA (2001)).

Eine Einordnung der wissenschaftlichen Arbeiten nach Disziplinen ist demnach oft schwierig.

\subsection{Tourismus}

Ein Großteil der wissenschaftlichen Arbeiten über Malta aus dem In- und Ausland beschäftigt sich mit der Ausbildung des Massentourismus auf den Maltesischen Inseln seit den 1960er Jahren und seinen wirtschaftlichen, ökologischen und soziologischen Effekten. Wichtige Beiträge aus Malta sind u. a. BRIGUGLIO (1996), SANT CASSIA (1999) und POLLACCO, J. (2003). Auffällig hierbei ist vor allem der Ansatz, eine aus maltesischer Sicht nachhaltige Tourismusstrategie zu entwickeln, den die genannten Autoren verfolgen.

Arbeiten aus dem Ausland stammen vorwiegend aus Großbritannien, so z. B. BLACK (1996), BOISSEVAIN (1996), MARKWICK (1999, 2001a \& 2001b), BLAKE, SINCLAIR \& SUGIYARTO (2003) und BRAMWELL (2003). Viele dieser Beiträge stehen in der Tradition der historisch-analytischen Forschung und untersuchen eher wie es zum derzeitigen Stand der Dinge in puncto Tourismus auf Malta kam, anstatt wie sich dieser in Zukunft entwickeln könnte. Dabei weist vor allem BRAMWELL (2003) darauf hin, dass viel Literatur der internationalen Malta-Forschung gar nicht oder nur unzureichend ausgewertet werde, was die mögliche Modernisierung des Tourismusmarketings auf den Inseln unnötig verlangsame und erschwere.

Einen wichtigen deutschsprachigen Beitrag zur maltesischen Tourismusforschung stellt die umfassende Dissertation von BESENTHAL (1994) dar, die auf internationaler Ebene die ausführlichste Darstellung der maltesischen Tourismusgeschichte sein dürfte.

Der Autor der vorliegenden Abhandlung stellte 2005 erstmals eine wissenschaftliche Verknüpfung zwischen regionalen traditionellen Produkten und dem Tourismus auf Malta her (ASCHEMEIER (2005)).

\subsection{Regionale Produkte Maltas}

Dies führt uns zum nächsten thematischen Aspekt, der im Rahmen der vorliegenden Abhandlung von zentraler Bedeutung ist: den regionalen Produkten der Maltesischen Inseln. Hierzu wurde bisher auffallend wenig veröffentlicht. Wissenschaftliche Publikationen zu diesem Themenkomplex gibt es streng genommen bisher nicht.

In Malta erschienen jedoch zahlreiche Arbeiten, die sich mit landestypischen Traditionen und Berufen beschäftigen, so z. B. CASSAR PULLICINO (1992 \& 1994), ZARB (1998), BORG, P. P. (2000 \& 2001), LANFRANCO (2002 \& 2003), MIFSUD-CHIRCOP (2003a \& 2003b) und CREMONA (2003).

Von den zuvor genannten Publikationen sind hinsichtlich eines thematischen Bezugs zu regionalen Produkten vor allem LANFRANCO (2002 \& 2003) und CREMONA (2003) hervorzuheben. AsCHEMEIER (2005) stellte, wie bereits erwähnt, erstmals eine wissenschaftliche Verknüpfung zwischen Tourismus und regionalen traditionellen Produkten auf Malta her. 


\subsection{Landwirtschaft}

Viele regionale traditionelle Produkte der Maltesischen Inseln haben ihren Ursprung in der Landwirtschaft. Aus diesem Grund sind wissenschaftliche Abhandlungen über die Agrarwirtschaftsstruktur der Inselgruppe von Bedeutung für die vorliegende Arbeit.

Die Vielfalt an veröffentlichten Schriften in diesem Bereich ist groß, wenngleich vor allem bei maltesischen Arbeiten der eingangs erläuterte interdisziplinäre Charakter innerhalb der Beiträge z. T. zu unausgewogenen Ergebnissen führt. Im weiteren Sinne agrargeographisch/wirtschaftsgeographisch orientierte Arbeiten finden sich z. B. bei BOWEN JONES (1955), BOWEN JONES, DEWDNEY \& FISHER (1961), KRETSCHMER (1980), Charlton \& Beeley (1987 \& 1993), Mizzi (1993), SHort (1997), Pollacco, C. (2003), Aschemeier \& CyffKA (2004b) sowie bei CYFFKA (2005).

CYFFKA (2005) untersuchte Aspekte der Degradierung ehemaliger agrarischer Nutzflächen in Abhängigkeit von der Änderung der Landbewirtschaftung. Beiträge aus einer ökologisch-biologisch motivierten Perspektive stammen u. a. von BusUtTIL (1993), MELI (1993), BORG, G. (1993), FARRUGIA (2001), und GALEA DE GIOVANNI (ohne Jahresangabe). Des Weiteren äußerte sich die Europäische Kommission in einem Kurzbericht über Maltas Landwirtschaft (KOMMISSION DER EUROPÄISCHEN GEMEINSCHAFTEN (2002)).

Von dieser Fülle an Material ist insbesondere die Arbeit von BOWEN JONES, DEWDNEY \& FISHER (1961) herauszustellen, die bis zum heutigen Tag immer wieder Einzug in agrargeographisch geprägte Forschungsprojekte findet. Aus jüngerer Zeit müssen die Beiträge von CHARLTON \& BEELEY (1987 \& 1993), SHORT (1997), FARRUGIA (2001) und CYFFKA (2005) besonders hervorgehoben werden.

Eine Einordnung der maltesischen Agrarstruktur in den Gesamtzusammenhang der mediterranen Landwirtschaft erfolgte unter Berücksichtigung der Arbeiten von ROTHER (1984 \& 1993) und SICK (1983).

\subsection{Geschichte der Maltesischen Inseln}

Zur geschichtlichen Entwicklung der Maltesischen Inselgruppe existiert eine fast unüberschaubare Vielzahl von Veröffentlichungen. Sie reicht von der trivialen Reiseliteratur bis hin zu mehrbändigen wissenschaftlichen Abhandlungen. Ein Literaturschwerpunkt liegt zweifellos auf der Zeit der Johanniterherrschaft, welche als glanzvollste und ereignisreichste Episode der maltesischen Historie immer wieder das Interesse von Einheimischen, Reisenden und Forschern weckt.

Arbeiten, die in der vorliegenden Abhandlung Berücksichtigung fanden sind EVANS (1963), NEHRING (1966), LUKE (1968), HUGHES (1972), MiNISTRY OF YOUTH AND THE ARTS (1994), FRENDO (1994), CASSAR (2000), LOCATELLI (2001), ATTARD (2002) und BLOUET (1987 \& 2004). Eine Einordnung der maltesischen Geschichte in den Kontext der Historie des Mittelmeerraums erfolgte anhand des Kompendiums von BRADFORD (1971).

BLOUET (1987 \& 2004) legte im geschichtlichen Bereich die einzige fachgeographische Arbeit vor, der aus diesem Grund in der vorliegenden Abhandlung besondere Aufmerksamkeit gewidmet wurde.

\subsection{Verfügbare Länderkunden}

Die noch immer einzige deutschsprachige Länderkunde über die Maltesischen Inseln stammt von NEHRING (1966). Daneben existiert eine länderkundliche Arbeit in italienischer Sprache von BIAGINI (1974) und das bereits erwähnte, im weiteren Sinne als länderkundlich zu bezeichnende Werk von BOWEN JONES, DEWDNEY \& FISHER (1961).

Die Arbeit von NeHRING ist umfassend und deckt im Wesentlichen alle wichtigen Bereiche der Geographie der Maltesischen Inselgruppe ab. Wie jedoch im Verlauf der Datenerhebung zur vorliegenden Abhandlung ersichtlich wurde, ist NEHRINGs Dissertation in einigen Punkten nicht eindeutig belegbar. 


\subsection{Soziologie}

Verschiedene Arbeiten aus der soziologischen Forschung beschäftigen sich mit den Maltesischen Inseln als Untersuchungsraum. In Augsburg wurde von der dortigen Universität in den 1980er Jahren die „Forschungsgruppe Malta“ gegründet, die unter der Leitung von HELGA und HORST REIMANN wertvolle Beiträge zur soziologischen Malta-Forschung geleistet hat. Der wichtigste Soziologe, der über Jahrzehnte in Malta arbeitete, ist gewiss JEREMY BoISSEVAIN, der rund dreißig Beiträge zum Thema vorgelegt hat.

Arbeiten jüngeren Datums beschäftigen sich vor allem mit der Situation der maltesischen Bevölkerung unter dem Einfluss des Massentourismus sowie mit der ambivalenten Position der Malteser zum EU-Beitritt inres Landes.

Folgende im engeren oder weiteren Sinne soziologisch motivierte Schriften von REIMANN (1979 \& 1991), CAssar Pullicino (1992), Besenthal (1994), BLACK (1996), BoIsseVAin (1996), ZARB (1998), MitcheLL (2002) und BRAMWELL (2003) wurden im Rahmen der vorliegenden Dissertation berücksichtigt.

\section{Hinweis}

Literatur, die zusätzlich zu der im Rahmen dieser Aufstellung zum Forschungsstand aufgelisteten verwendet wurde, wurde durchgängig in den Fußnoten kenntlich gemacht. 


\section{Die Maltesischen Inseln - Topographie und Naturraum}

\subsection{Topographie}

Die Maltesischen Inseln sind eine Gruppe kleiner flacher Inseln im zentralen Mittelmeerraum. Neben den bewohnten Hauptinseln Malta, Gozo (malt.: Gћawdex) und Comino (malt.: Kemmuna) gehören auch die unbesiedelten Inseln Filfla (auch: Filfola), die St. Paul's Islands ${ }^{3}$ (auch: Selmunett Islands, malt.: il-Gżejjer ta' San Pawl), Fungus Rock (malt.: il-Gebla tal-Ġeneral) und einige unbedeutende einzelne Felsen dazu (vgl. Karte 1). Sie bilden zusammen eine Gesamtfläche von $315,6 \mathrm{~km}^{24}$.

Die geographischen Koordinaten der Maltesischen Inselgruppe ${ }^{5}$ lauten:

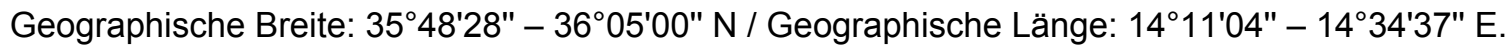

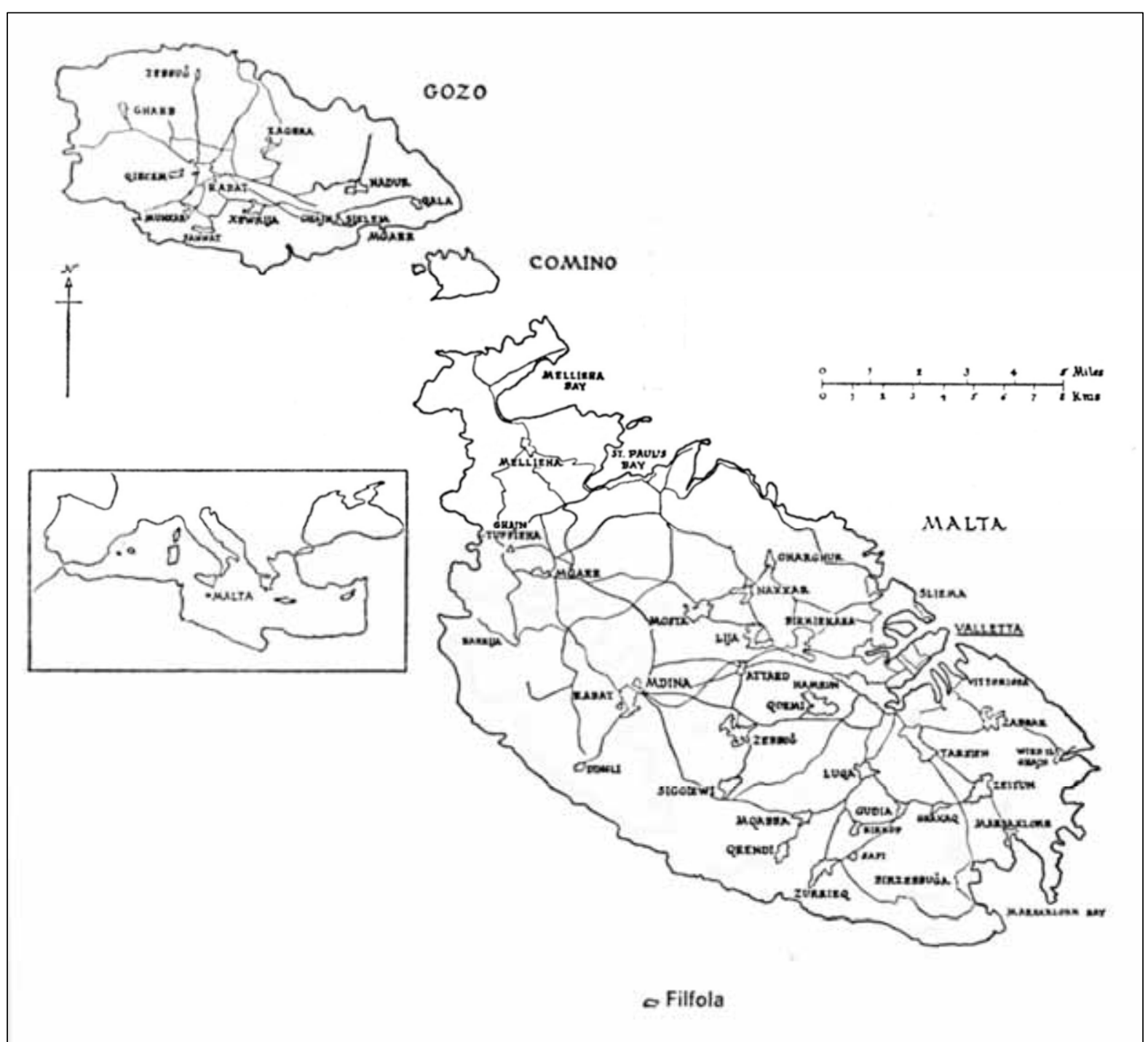

Karte 1: Die Maltesischen Inseln und ihre Lage im Mittelmeer. Quelle: EvANS (1963), S. 12 (verändert).

3 Faktisch handelt es sich dabei um nur eine Insel mit einer Halbinsel, die aber mit einer so schmalen Landbrücke verbunden sind, dass der allgemeine Eindruck entsteht, es handele sich um zwei voneinander getrennte Inseln (vgl. BRADFORD (1972)). Die Namensgebung in verschiedenen Sprachen geht aber in allen Fällen von der Mehrzahl aus.

4 Vgl. AZZOPARDI (2002), S. 22

5 Nach SCHEMBRI (1994), S. 106 
Die Maltesischen Inseln erstrecken sich 93 km südlich von Sizilien und 288 km nordöstlich von Tunesien ${ }^{6}$ in NW-SE-Ausrichtung am äußeren Rand der Afrikanischen Kontinentalplatte ${ }^{7}$. Ihnen vorgelagert ist der flache Malta-Ragusa-Schelf. Dieser ist Bestandteil eines submarinen Höhenzugs, der von der Halbinsel Ragusa auf Sizilien bis hin zu den Küsten Libyens und Tunesiens führt ${ }^{8}$. Er stellt den Überrest einer ehemaligen Landverbindung zwischen Südeuropa und Nordafrika dar, die noch im Pleistozän existierte ${ }^{9}$. Durch die Eisschmelze nach der letzten Eiszeit hob sich der Meeresspiegel, und die Maltesischen Inseln kamen zu ihrer isolierten Lage im Zentrum des Mittelmeers. Die Meerestiefen rund um die Maltesischen Inseln sind aufgrund der beschriebenen Situation relativ gering und betragen nur zwischen $<90-200 \mathrm{~m}$.

Der höchste Punkt der Hauptinsel Malta wird mit 251 m NN im Bereich der Kliffküste im Süden und Südwesten der Insel erreicht. Diese Region wird nach einem nahe gelegenen Ort Dingli Cliffs genannt. Von diesen Kliffs ausgehend fällt das Relief nach Nordosten ab, bis es im Bereich der Hauptsiedlungszone, der sog. Valletta Conurbation (vgl. Karte 4, S. 131), die flachste Ausprägung zeigt.

Trotz der topographischen Nähe zu Malta weist das Relief der kleineren Nachbarinsel Gozo erkennbare morphologische Unterschiede auf. Augenfällig ist zunächst die deutlich steilere Küstenlinie, die nur im Osten Gozos flach reliefierte Zonen zulässt. Bei Ta'Ćenć im Südwesten Gozos erreichen die schroffen Klippen Höhen von 135 m NN.

\subsection{Anmerkung zur Darstellung der naturräumlichen Gegebenheiten}

Als Basis des primären Sektors, und daher als natürlicher „Ausgangspunkt“ zahlreicher ursprünglicher maltesischer Produkte, soll im Folgenden die naturräumliche Ausstattung der Inseln - für eine anthropogeographische Arbeit verhältnismäßig ausführlich - dargestellt werden.

Dies ist zum einen aufgrund der Vielfältigkeit der agrarischen Produkte, deren Anbaumöglichkeiten in Malta stark den naturräumlichen Bedingungen unterliegen, angebracht. Zum anderen sei aber auch darauf hingewiesen, dass die lückenhafte Literatursituation im Rahmen der deutschsprachigen Malta-Forschung nach Meinung des Verfassers eine relativ ausführliche Darstellung des Naturraums rechtfertigt. In den letzten 50 Jahren legten lediglich NEHRING (1966), KRETSCHMER (1980) und BESENTHAL (1994) umfassende geographische Grundinformationen über den Untersuchungsraum in deutscher Sprache vor. Die Werke dieser Autoren sind nicht mehr im Buchhandel erhältlich und darüber hinaus nur selten in deutschen Universitätsbibliotheken zu finden.

\footnotetext{
6 Daten nach FARRUGIA (2001), S. 108

7 Vgl. ROTHER (1993), S. 20

8 Vgl. SCHEMBRI (1994), S. 106

9 Vgl. FARRUGIA (2001), S. 105
} 


\subsection{Geologie und Pedologie}

Die Geologie der Maltesischen Inseln besteht aus einer Abfolge von fünf Gesteinsformationen, die durch marine Sedimentationsprozesse entstanden sind.

Die fünf geologischen Schichten der Maltesischen $\operatorname{Inseln}^{10}$ sind:

- Lower Coralline Limestone (unterer Korallenkalk; bis 140m Mächtigkeit)

- Globigerina Limestone (Globigerinenkalk; 23m-207m Mächtigkeit)

- Blue Clay (Blauer Ton; bis 65m Mächtigkeit)

- Greensand (Grünlicher Sand; wenige Zentimeter bis $12 \mathrm{~m}$ Mächtigkeit)

- Upper Coralline Limestone (oberer Korallenkalk; bis 162m Mächtigkeit)

Oberer Korallenkalk und Globigerinenkalk werden als Baumaterial genutzt. Der obere Korallenkalk ist zunächst weich und kann einfach abgebaut werden. An der Luft härtet der Stein aus und erweist sich als relativ verwitterungsresistent.

Die schroff verwitternden Kalksteinhorizonte bilden über weite Flächen eine Karstlandschaft aus, werden aber auch oftmals vom Blue Clay überlagert, der für eine „weiche“ Überformung der Hänge sorgt und für die Agrarwirtschaft bedeutsam ist. Der Greensand spielt aufgrund seiner meist geringen Mächtigkeit kaum eine Rolle im Landschaftsbild.

Es gibt auf der Inselgruppe nur wenig Oberflächenwasser, das zudem nur periodisch auftritt (vgl. Kap. 3.5). Die Böden der Inseln sind von diesen Ausgangsbedingungen geprägt. Sie sind relativ jung und flachgründig. Die Ursache hierfür ist vor allem klimatisch bedingt, da mediterrane Klimate mit einer Trockenzeit und einer Regenzeit den Bodenbildungsprozess stark verlangsamen ${ }^{11}$. Die vorkommenden Böden gehören überwiegend zu den Chromic Cambisolen $(\mathrm{CM})^{12}$. Sie können in drei Gruppen eingeteilt werden, die im Folgenden näher vorgestellt werden. Die Bodentypen sind Terra Soils, Xerorendzinen sowie einige Carbonat-Rohböden auf Blue Clay ${ }^{13}$. Alle vorkommenden Böden werden landwirtschaftlich genutzt und sind durch anthropogen bedingte Bodenverlagerung teilweise stark verändert bzw. gestört.

\subsubsection{Terra Soils}

In dieser Gruppe findet man in Malta Terra rossa- und Terra fusca-Böden sowie Zwischenstadien beider Ausprägungen. Sie sind Reliktböden, die nicht unter dem Einfluss des heutigen Klimas entstanden sein können, sondern in feuchteren Klimaperioden gebildet wurden ${ }^{14}$.

Die Böden treten in rötlichen Farben zutage und besitzen im Vergleich zu anderen Bodentypen der Inselgruppe mit ca. 4,5\% einen hohen Anteil an organischen Komponenten.

\subsubsection{Xerorendzina}

Der organische Anteil dieser fahlbraunen Böden schwankt zwischen 2-3\%, kann jedoch bei landwirtschaftlich genutzten und regelmäßig gedüngten Flächen bis zu $6 \%$ betragen ${ }^{15}$. Der Calciumkarbonat-Anteil beträgt meist zwischen 50-88\%. Die Böden dieses Typs sind häufig durchsetzt mit groben unverwitterten Gesteinsresten.

10 Nach BoWen-Jones, DeWdney \& Fisher (1961), S. 25; SCHEMBrI (1994), S. 106 f., FARRUGIA (2001), S. 103 f. \& 107

11 Vgl. z. B. ROTHER (1984), S. 64 f.; ROTHER (1993), S. 51 f.

12 Nomenklatur nach World Reference Base for Soil Resources (WRB) (1998), zitiert aus ZECH \& HINTERMAIER-ERHARD (2002), S. 52

13 Vgl. FARRUGia (2001), S. $106 \mathrm{f}$.

14 Vgl. FARRUGIA., S. 107 sowie CyFFKA (2005)

15 FARRUGIA, S. 107 


\subsubsection{Carbonat-Rohböden}

Die Böden dieser Gruppe sind von der Entwicklungsstufe her am jüngsten und weisen daher einen hohen Anteil an Calciumkarbonat auf (50-60\% bei Bodenbildung auf Blue Clay und $80-90 \%$ bei Bodenbildung auf dem weichem Globigerina limestone ${ }^{16}$ ), während der Humusgehalt sehr gering ist (nur durchschnittlich 1$\left.1,5 \%{ }^{17}\right)$. Das äußere Erscheinungsbild der Böden dieser Gruppe changiert im bräunlichen Farbbereich.

\section{$3.4 \quad$ Klima}

Malta befindet sich im Einflussbereich des Etesienklimas ${ }^{18}$ (vgl. Abb. 1). Charakteristisch ist die Unterteilung des Jahres in nur zwei Jahreszeiten, eine Trockenzeit (April-September) und eine Regenzeit (OktoberMärz). Im Winter kommt es zu typischen Starkregenereignissen, im Sommer zeitweilig zu Staub- und Glutwinden. Die Trockenzeit ist jedoch die windstillere Jahreszeit.

Wind ist einer der Haupteinflussfaktoren des maltesischen Klimas. Nur an 10\% der Tage im Jahr herrscht relative Windstille. Die vorherrschenden Winde wurden schon früh mit einheimischen Bezeichnungen belegt ${ }^{19}$ : An durchschnittlich 29\% der Tage im Jahr bläst der kühle Majjistral aus NW. Aus NE kommt an $15 \%$ der Tage des Jahres der trockene Grigal. Gefürchtet sind die Xlokks, die auf ihrem Weg nach Malta viel Feuchtigkeit vom Meer aufgenommen haben ${ }^{20}$ und im Sommer schwül-heißes Wetter verursachen.

Im Sommer gibt es besondere Windereignisse, so z. B. den Nofsinhar, der direkt aus S weht und als Stauboder sog. "Glutwind“ die Inseln heimsucht. Im Winter kommt der Tramuntana aus $\mathrm{N}$ und bringt für die geographische Lage der Inseln ungewöhnlich kalte Temperaturen mit sich.

Die Höchsttemperaturen werden im Juli und August (normal ist $>30^{\circ} \mathrm{C}$, Extremwerte von bis zu $43^{\circ} \mathrm{C}$ sind keine Seltenheit), die Tiefsttemperaturen im Januar, Februar und März erreicht (vgl. Abb. 1). Normalerweise wird bei den tiefsten Werten eine Lufttemperatur von $10^{\circ} \mathrm{C}-15^{\circ} \mathrm{C}$ nicht unterschritten. In Bodennähe kann es aber an einzelnen Tagen leichten Frost geben, der jedoch nur sehr kurze Zeit anhält ${ }^{21}$.

Auch die Jahresmitteltemperatur von rund $18,7^{\circ} \mathrm{C}$ (vgl. Abb. 1) deutet auf das milde Klima der Inseln hin. Die relative Luftfeuchtigkeit weist mit $65-80 \%$ aufgrund des allgegenwärtigen maritimen Einflusses über das gesamte Jahr hohe Werte auf. Die Sonnenscheindauer beträgt im Jahresmittel 8,355 Stunden ${ }^{22}$.

Die mittlere Jahresniederschlagsmenge beträgt durchschnittlich rund $500 \mathrm{~mm}$ (vgl. Abb. 1). Eine wichtige Rolle spielt, ähnlich wie in anderen Gebieten des Mittelmeerraums, der Morgentau, der in den trockenen Sommermonaten die einzige natürliche Feuchtigkeitsquelle für die Vegetation darstellt.

\footnotetext{
16 FARRUGIA, S. 106
}

17 Ebd.

18 Nach der Klimaklassifikation von KÖPPEN zitiert aus: BLÜTHGEN \& WEISCHET (1980), S. 667-671

19 Vgl. auch FARRUGIA (1993) \& BLOUET (2004)

20 Vgl. BLOUet (2004), S. 11

21 Vgl. FARRUGIA (2001), S. 106

22 Vgl. AZZOPARDI (2002), S. 113-114 


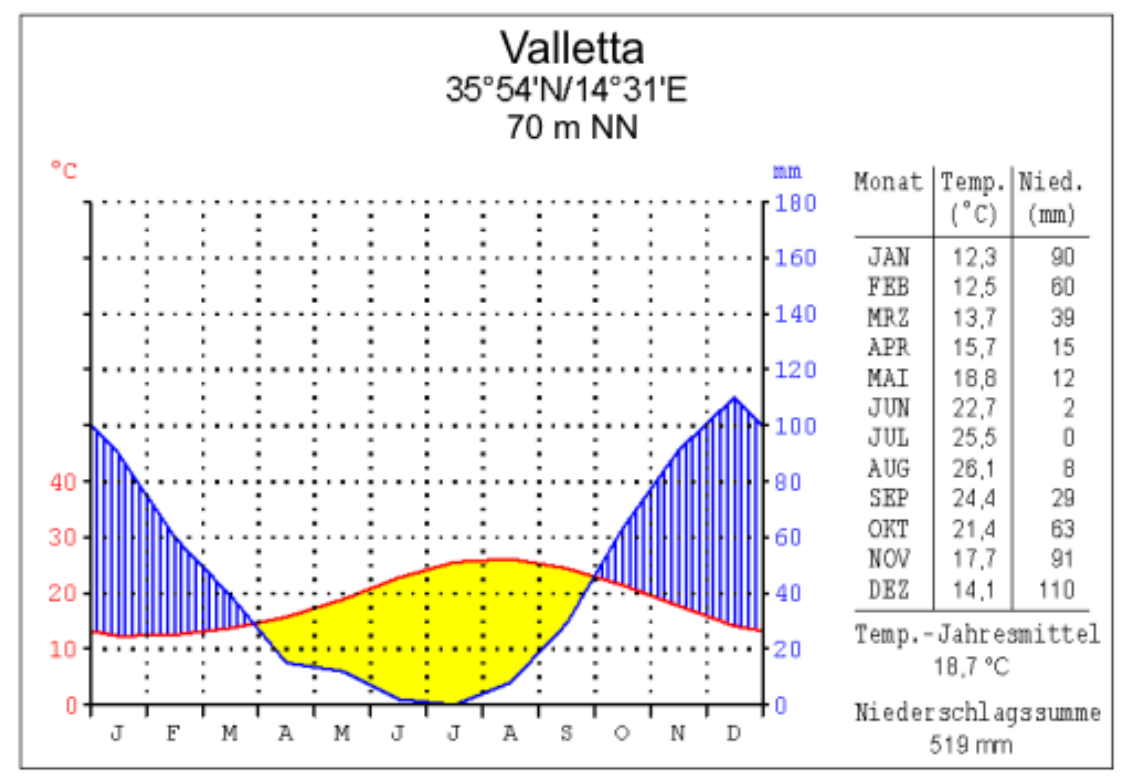

Abb. 1: Klimadiagramm Valletta/Luqa Airport aus dem Jahr 2000. Quelle: www.ilexikon.com/Malta.html. Stand: 08.09.2005, überarbeitet: ASCHEMEIER

\subsection{Wasservorkommen}

Auf den Maltesischen Inseln gibt es keine perennierenden Flüsse oder andere Gewässer ${ }^{23}$. In der Regenzeit dienen die Wiedien (malt. singular: Wied) als Abflusstäler. Die Wiedien sind überall auf der Insel vorkommende Talsysteme, die teils tektonischen (entlang von Verwerfungen), teils erosiven (Karst) Ursprungs sind.

Die geologische Schicht des Blue Clay wirkt wasserstauend, sodass sich über ihr ein Süßwasserreservoir bilden kann. Man spricht in diesem Fall vom oberen Aquifer. Diese wasserführende Schicht wird speziell im südlichen bis südwestlichen Teil Maltas (insbesondere in der dem Ort Dingli und den gleichnamigen Klippen vorgelagerten Region) und auch Gozo in starkem Maße für die landwirtschaftliche Bewässerung genutzt. Aus dem Gestein austretende temporäre Süßwasserquellen gibt es aus diesem Grund immer weniger, zumal sie auch von Natur aus schon Seltenheitswert besitzen ${ }^{24}$.

Der untere Süßwasseraquifer wird durch Regenwasser gebildet, das in Spalten im Karstgestein einsickert und durch seinen Dichteunterschied zum stark salzigen Meerwasser im Untergrund der Inseln eine Süßwasserlinse ausbilden kann. Diese Schicht wird noch intensiver beansprucht als der obere Aquifer und ist neben entsalztem Meerwasser ein Hauptbestandteil der öffentlichen Wasserversorgung. Aber auch in den landwirtschaftlich genutzten Bereichen des Globigerina Bassins im Nordosten wird auf diesen Vorrat zugegriffen, da sie unterhalb des Blue Clay liegen und somit der obere Aquifer nicht verfügbar ist.

\subsection{Vegetation}

Durch lange Trockenphasen, fremd eingeführte Arten und hohe Besiedlungsdichte kann die Flora Maltas als gestört bezeichnet werden ${ }^{25}$. Einer der wichtigsten Störfaktoren für die Vegetation ist der allgegenwärtige Einfluss der Meeresluft. FARRUGIA schreibt dazu: „The ecology of the Maltese Islands is very much affected by the proximity of any area to the sea. In fact, the furthermost point in Malta away from the sea is only $6.5 \mathrm{~km}$. According to weather conditions salt spray may be blown on to any vegetation on the island. Thus all species of plants growing on the island must be more or less salt-tolerant." (FARRUGIA (2001), S. 108).

23 Vgl. AZZOPARDI (2002), S. $184 \mathrm{ff}$.

$24 \mathrm{Vgl}$. Ebd.

25 BENDIX, BENDIX \& REUdENBACH (2002), S. 15-18 sowie CYFFKA (2005), S. $199 \mathrm{ff}$. 
Die Vegetation der Maltesischen Inseln besteht im Wesentlichen aus unterschiedlichen Garigue- und Macchie-Stadien, die laut SCHEMBRI (1994) im mediterranen Vergleich artenreich sind ${ }^{26}$. Sie weist über 2000 Arten auf, von denen viele endemisch $\operatorname{sind}^{27}$. Im Folgenden werden die Hauptvergesellschaftungen der Maltesischen Inseln beschrieben. Als Datengrundlage dienten die Arbeiten von BOWEN JONES, DEWDNEY \& FISHER (1961); NEHRING (1966); SCHEMBRI (1994); FARRUGIA (2001) SOWIE BENDIX, BENDIX \& REUDENBACH (2002).

Die Garigue besteht aus Pflanzengesellschaften niedriger (< 1m) aromatischer Kräuter, Gräser und Sträucher. Sie ist die am häufigsten vorkommende Biozönose der Maltesischen Inseln. Typische Vertreter der maltesischen Garigue sind: Kopfiger Thymian (Coridothymus capitatus), Maltesisches Heidekraut (Erica peduncularis), Harzklee (Psoralea bituminosa), Milchfleckdistel (Galactites tomentosa) und Gedrehtes Federgras (Stipa tortilis spinosa).

Die Macchie - in Malta ist die Bezeichnung Maquis gebräuchlich - besteht aus mehr oder weniger dicht wachsenden, immergrünen Gehölzen. Einzelne Pflanzen erreichen Höhen von 1-3m. Jedoch dominiert auf der Inselgruppe eine Macchie aus niedrigen Gewächsen. Man unterscheidet laut ScHEMBRI (1994) die halbnatürliche und sekundäre Macchie ${ }^{28}$. Sog. halb-natürliche Formen entstehen hauptsächlich an unzugänglichen Stellen wie den Abflusstälern, den bereits erwähnten Wiedien, und den rdum-Bereichen ${ }^{29}$ während künstliche oder sekundäre Macchien oft aus Aufforstungen und künstlichen Baumanpflanzungen (z. B. Eukalyptuspflanzungen) hervorgehen.

Typische Vertreter der maltesischen Macchie sind hohe Sträucher und kleine Bäume. Die wichtigsten Arten sind: Johannisbrotbaum (Ceratonia siliqua), Ölbaum (Olea europea), Immerblühende Akazie (Acacia retinodes), Schreckliche Akazie (Acacia karoo), Mastix-Strauch (Pistacia lentiscus), Baumförmige Wolfsmilch (Euphorbia dendroides), Gliederzypresse (Tetraclinis articulata), Aleppo-Kiefer (Pinus halepensis) sowie diverse Kräuter.

Eine weitere Biozönose ist als Steppic Grassland bekannt. Man versteht darunter in diesem Fall baum- und strauchlose Pflanzengesellschaften, die von Gräsern, Disteln und Geophyten dominiert werden. Sie entstehen aus der Degradierung von Macchie- und Garigue-Gesellschaften, hauptsächlich hervorgerufen durch Beweidung. Auf den Maltesischen Inseln findet Beweidung vor allem durch Ziegen statt, die starke Landschaftsdegradationen hervorrufen können.

Steppic Grasslands sind eine typische Pflanzengesellschaft der Karstzonen. Im Frühjahr kommt es dort zum Aufblühen einer Krautsteppe mit anspruchslosen Knollen- und Zwiebelgewächsen. Einzelne Arten von Garigue und Macchie kommen vor. Charakteristisch für die Steppic Grasslands ist die Weißblühende Meerzwiebel (Urginea maritima).

Neben diesen Hauptvergesellschaftungen existieren einige Biozönosen, die sich auf kleinste Verbreitungsräume und Extremstandorte wie z. B. Höhlen, Kliffs, Mauern, Salzböden, etc. spezialisiert haben.

Auch gestörte Vergesellschaftungen mit einer hohen Anzahl fremder Arten konnten sich aufgrund der vielen Eroberungen durch fremde Kulturvölker, der intensiven Besiedlungsdichte, der landwirtschaftlichen Aktivität und der hohen Bautätigkeit der letzten Jahrzehnte ausbreiten. Vom Menschen eingeführte Arten wirken häufig landschaftsbestimmend. Ihre wichtigsten Vertreter sind: Aleppo-Kiefer (Pinus halepensis) (s. o.), Feigenkaktus (Opuntia Ficus Indica), Agave (Agava americana) und Oleander (Nerium oleander). Hinzu kommen die zahlreichen eingeführten agrarischen Nutzpflanzen, die in einem gesonderten Kapitel behandelt werden (s. Kap. 13).

\footnotetext{
26 Vgl. SCHEMBRI (1994), S. 111

27 Vgl. FARRUGIA (2001), S. 103

28 Nach SCHEMBRI (1994), S. 115

29

Dies sind fruchtbare Zonen unterhalb Kliff-artiger Hänge
} 
Bereits seit der Antike ist Malta entwaldet ${ }^{30}$. Eine quasinatürliche ${ }^{31}$ Biozönose stellt der Baumbestand Buskett Gardens (malt.: il-Buskett) dar. Er ist laut herrschender Literaturlage das einzige noch zusammenhängende Waldgebiet der Hauptinsel Malta, wird mittlerweile aber durch einige in den 1960er Jahren angepflanzte zusammenhängende Bestände auf Malta und Gozo ergänzt. Buskett Gardens stellt eine künstliche Anpflanzung mit den eingeschleppten Aleppo-Kiefern als dominierender Baumart dar. Es gibt dort aber auch alte Steineichen, die auf den Maltesischen Inseln nur noch selten zu finden sind sowie unterschiedliche Anpflanzungen von Obstbäumen. Natürliche Waldgesellschaften existieren auf der Inselgruppe nur noch in kleinsten Überresten, die sich sämtlich auf der Hauptinsel Malta befinden. Aufforstungen der 1950er- und 1960er-Jahre entwickeln sich derzeit zu mediterranen Wäldern und ergänzen somit den Baumbestand der Inselgruppe insgesamt.

Für die Vegetationszusammensetzung der Insel kann i. Allg. ein hoher Verwandtschaftsgrad zu SüdostSizilien, aber auch den benachbarten Pelagischen Inseln Lampione, Pantelleria und Lampedusa festgestellt werden $^{32}$.

\subsection{Die Fauna und ihre Stellung in Alltags- und Landeskultur}

Die Tierwelt der Maltesischen Inseln ist aufgrund der extremen Besiedlung und damit einhergehender Lebensraumverknappung arten- und individuenarm. Aber auch die kritischen Wasser- und Vegetationsverhältnisse begünstigen nicht das Vorkommen einer reichen Fauna. Markante Arten existieren überwiegend unter den Kleintieren und umfassen Eidechsen, Schlangen, Mäuse, Igel, Wiesel und Kaninchen. Kaninchen ist die Grundlage für das in vielen Variationen auftretende maltesische Nationalgericht Fenkata.

Eidechsen kommen in Malta in hoher Individuenzahl vor. Besonders hervorzuheben ist die Maltesische Mauereidechse, die mit drei Arten (Podarcis maltensis, P. kieselbachi und P. laurentiimuelleri) in der Verbreitung auf den Maltesischen Inselraum und die Pelagischen Inseln begrenzt ist. Podarcis filfolensis, eine vierte Art, ist endemisch für die Maltesischen Inseln.

Begriffe wie "Ökologie“ und „Naturschutz" wurden in Malta anfangs mit Skepsis aufgenommen. Als in den 1980ern jedoch die Bausünden der Vergangenheit und die zunehmende Landschaftsdegradierung durch die schwindende Beschäftigung in der Landwirtschaft offenbar wurden, wurden endemische Arten (insbesondere Tierarten) bewusst instrumentalisiert, um ein Interesse für den Naturschutz zu wecken.

Die vielen Zugvogelarten, die die Inseln im Herbst und Frühjahr als Rastplatz auf ihrem Weg nach Afrika bzw. Europa aufsuchen, erlangten durch das in Malta weit verbreitete „Hobby“ der Vogeljagd traurige Berühmtheit. Viele Naturschutzorganisationen ${ }^{33}$ prangern den Abschuss von Singvögeln seit Jahren an, zumal dieser den maltesischen Gesetzen zuwider betrieben wird. Allerdings ist der Rückhalt in der Bevölkerung so groß, dass ein Verbot der Vogeljagd mit Schusswaffen bisher wirkungslos verhallte ${ }^{34}$.

Auch mit dem EU-Beitritt ändert sich das vorerst nicht. Die Europäische Union räumte eine bis 2010 befristete „Übergangszeit“ ein, die zur Einführung eines Vogelzuchtprogramms dient ${ }^{35}$. Malta wird also von der EU zur Zucht der Anzahl an Singvögeln verpflichtet, die im Frühjahr und Herbst durch Freizeitjäger getötet werden. Der Bestand an einheimischen Vögeln ist gering. Dennoch gibt es in Malta einen Nationalvogel, die Blaumerle (Monticula solidarius).

30 Vgl. z. B. Bendix, Bendix \& REUdenBaCH (2002), S. 15-16; AzZOPARDI (2002), S. 53-56

31

Begriff im terminologischen Gebrauch nach LESER et al. (1997)

32 Vgl. auch NeHRING (1966), S. 30-32; SCHEMBRI (1994), S. 112

33 Darunter die „Heinz Sielmann Stiftung“, „Greenpeace“ und der „WWF“

34 Vgl. SCHIFFARTH (2002), S. 6

35 KOMmISSION DER EUROPÄISCHEN GEMEINSCHAFTEN (2003), S. 48 
Ein wichtiger Aspekt der maltesischen Fauna wird durch die Artenvielfalt der Insekten und Mollusken gebildet. Wohl aufgrund ihrer relativen Unauffälligkeit wurde dieser Teil der Inselfauna in früheren geographischen Arbeiten (so z. B. bei NEHRING (1966) und BoWEN JONES et al. (1961)) völlig ausgeklammert. Es kommen aber acht endemische Schneckenarten vor sowie ca. 40 endemische Insektenarten. Hingegen gibt es je nur eine endemische Säugetier- und Reptilienart auf der Inselgruppe. Auch der gesamte Bestand ist sehr hoch: Es gibt 67 Mollusken- und ca. 4500 Insektenarten. Dazu kommen über 200 Spinnenarten. Insekten, Spinnen und Mollusken sind heute Gegenstand engagierter Naturschutzbemühungen. Auch die Tiere des Meeres erreichen eine hohe Artenvielfalt, die jedoch bis auf einige wenige endemische Vertreter nicht von der typischen Meeresfauna des Mittelmeers abweicht.

Wild lebende große Säugetiere gibt es auf Malta seit Jahrhunderten nicht mehr. Ihren Platz nehmen traditionell zahlreiche domestizierte Haus- und Nutztierarten ein. Die wichtigsten unter innen sind Ziege, Esel, Maultier und Rind. Aufgrund der Pferderennbegeisterung der Malteser und dem Gelderwerb durch Kutschfahrten für Touristen ist die Pferdehaltung, oft unter unwürdigen Bedingungen, weit verbreitet. Kaninchen und Hühner werden ebenfalls als Nutztiere gezüchtet und gehalten.

Einen besonders wichtigen Aspekt der Nutztierhaltung stellen Ziegen dar, deren Verbreitung wegen eines allgemeinen Bedeutungsverlusts der Landwirtschaft zwar abgenommen hat, die jedoch noch häufig in kleinen bis mittleren Herden von 20-80 Tieren in den ländlichen Regionen der Inselgruppe anzutreffen sind (vgl. Foto 1). Ziegen sind historisch gesehen wahrscheinlich die Hauptverantwortlichen für große Teile des maltesischen Landschaftsbilds, weil sie mit ihrem anspruchslosen Fressverhalten die Ausbildung von Garigue- und Steppic Grassland-Formationen begünstigen. Ziegenkäse und Ziegenmilch waren in Malta lange Zeit wichtiger als die Milchprodukte von Hausrindern und stellen heute noch eine wichtige Nahrungsquelle dar.

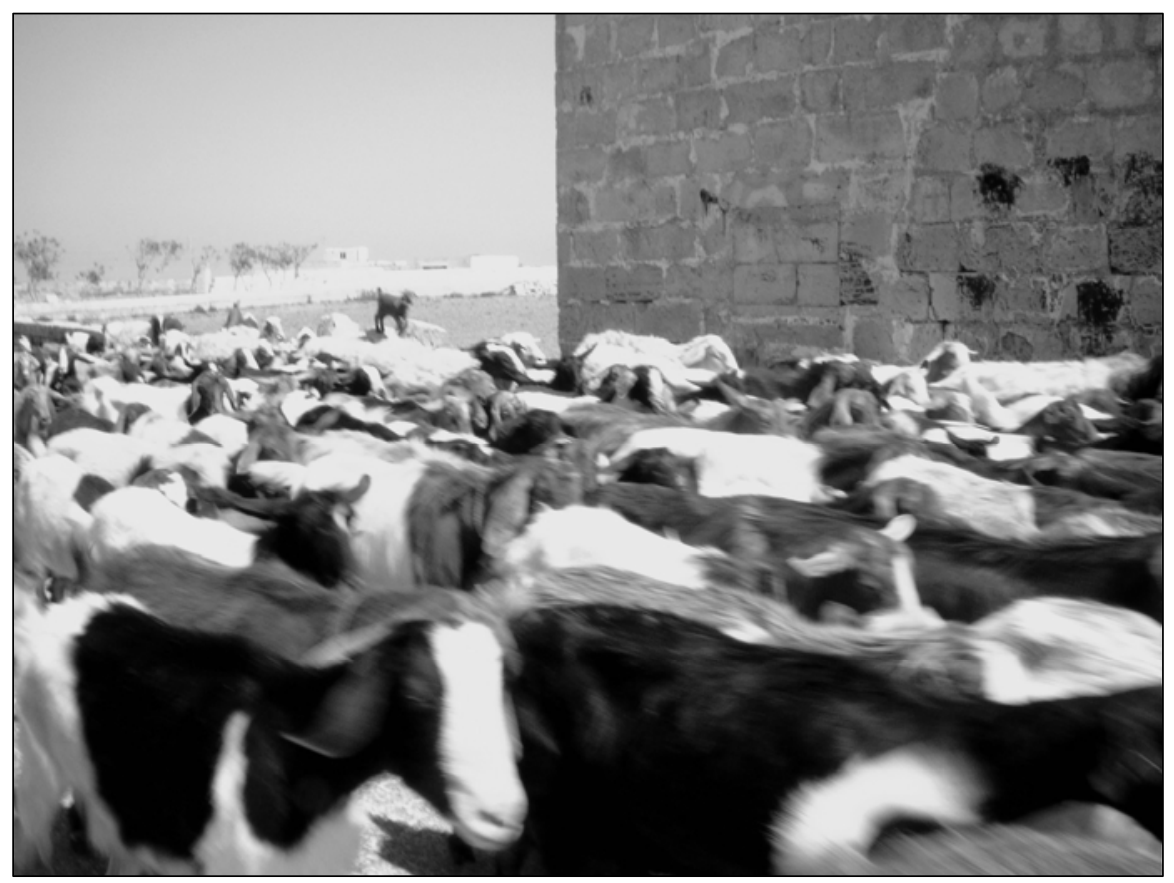

Foto 1: Ziegenherde nahe der Ortschaft Qrendi. Foto: ASCHEMEIER 


\section{Entwicklungsgeschichte der Maltesischen Inseln}

Die historische Entwicklung der Maltesischen Inseln ist geprägt von ständigem Wandel. Eine Unzahl von Eroberern beherrschte die Inseln von der Antike bis in das 20. Jh. hinein. Karthager, Phönizier, Griechen, Römer, Araber, Spanier, Normannen, Türken, die Johanniter, Franzosen und Briten sind nur einige der vielen Fremdherrscher, unter deren Einfluss die Inselgruppe stand. Da die historische Entwicklung in zahlreichen ausführlichen Abhandlungen hinlänglich aufgearbeitet wurde, wurde im Rahmen der vorliegenden Dissertation lediglich ein Abriss der geschichtlichen Vorgänge auf den Maltesischen Inseln aufgenommen. Die Angaben im Folgenden beruhen, soweit nicht anders angegeben, auf den Veröffentlichungen von HYDE (1953), BOWEN JONES, DEWDNEY \& FISHER (1961), EVANS (1963), NeHRING (1966), LUKE (1968), Ministry OF YOUTH AND THE ARTS (1994), AZZOPARDI (1994), CASSAR (2000), ATTARD (2002), GRECH (2003) und BLOUET (1987 \& 2004). Zur weiteren Vertiefung empfiehlt der Autor insbesondere LUKE (1968), CASSAR (2000) und BLOUET (2004).

Die jüngere Geschichte bzw. die Geschichte von Maltas EU-Beitritt wird in Kapitel 5 bearbeitet. Auch die geschichtliche Entwicklung der Landwirtschaft stellt einen eigenen Schwerpunkt dar (s. Kap. 12.1).

Es ist davon auszugehen, dass die Erstbesiedlung der Maltesischen Inseln etwa 5200 bis 4500 v. Chr. von Süditalien/Sizilien aus erfolgte ${ }^{36}$, das einst durch eine Landbrücke mit dem heutigen Malta verbunden war, zur Zeit der Erstbesiedlung jedoch bereits nur über den Seeweg erreicht werden konnte ${ }^{37}$. Verschiedentlich wird aber auch eine Besiedlung der Inselgruppe während der letzten Eiszeit diskutiert ${ }^{38}$, jedoch auf theoretischer Basis. Eindeutige Nachweise für eine derart frühe Besiedlung gibt es nicht.

Indizien für den Ursprung der ersten neolithischen Malta-Bewohner findet man in ihren Kulturrelikten, die beispielsweise Schneidewerkzeuge aus Obsidian umfassen, ein Vulkanit, der auf Malta nicht vorkommt und vermutlich aus der Region der heutigen liparischen Inseln eingeführt werden musste. Insbesondere lassen jedoch Keramikfunde, die typisch sizilianische Muster aufweisen, darauf schließen ${ }^{39}$, dass die Erstbesiedlung von Sizilien aus erfolgte.

Die frühen maltesischen Siedler waren - wenn man das moderne Wort in diesem Zusammenhang gebrauchen will - im weitesten Sinne „Farmer“. Sie betrieben Ackerbau und Viehzucht ${ }^{40}$. Eine über 700 Jahre währende kulturelle Persistenz ermöglichte es ihnen, einen hohen zivilisatorischen Stand zu erreichen. Ihre besondere Errungenschaft sind erstaunlich zahlreiche zyklopische Tempelbauten, die mit der Radiokarbonmethode als die ältesten frei stehenden von Menschenhand errichteten Strukturen aus Stein weltweit ${ }^{41}$ datiert werden konnten. Diese Tempelkultur hinterließ ihre Spuren von ca. 4100 bis $2500 \mathrm{v}^{.} \mathrm{Chr}^{42}$

Die Bronzezeit ist kaum dokumentiert. Sie wird für die Maltesischen Inseln von 2500 bis 800 v. Chr. datiert ${ }^{43}$. Nur wenige archäologische Relikte bieten ein lückenhaftes Gesellschaftsbild. Es wird als herrschende Meinung angenommen, dass die erfolgreiche neolithische Periode nicht fortgeführt werden konnte ${ }^{44}$. Als Grund hierfür werden erste Überfälle von See verantwortlich gemacht. Damit begann eine Bedrohung, die Malta über die kommenden zwei Jahrtausende nicht zur Ruhe kommen lassen sollte.

36 Vgl. CASSAR (2000), S. 47 \& BLOUet (2004), S. $21 \mathrm{ff.}$

37 CASSAR (2000)

38 Vgl. BONANNo (1994), S. 81 \& EVANS (1963), S. 29

39 Vgl. CASSAR (2000), S. 48 \& EVANS (1963), S. 32 f.

40 Vgl. Kapitel 12.1

41 ATTARD (2002), S. 5

42 BLOUET (2004), S. 21

43 Ebd.

44 Ebd. (2004), S. 29 
Der Geograph BLOUET spricht von der Bronzezeit als einer Zeit kriegsähnlicher Zustände, die alle Bereiche des täglichen Lebens in Mitleidenschaft $\mathrm{zog}^{45}$. Andererseits markiert die Bronzezeit den Beginn der maltesischen Tradition der Textilherstellung ${ }^{46}$.

Diese Kunstfertigkeit im Bereich der Textilfertigung und die qualitativ hochwertige Keramik maltesischer Kunsthandwerker wurde von phönizischen Kaufleuten geschätzt ${ }^{47}$, die sich vermutlich ab dem 9. Jh. v. Chr. auf Malta anzusiedeln begannen. Sie wurden im 8. und 7. Jh. v. Chr. schließlich die dominanten Siedler auf den Inseln ${ }^{48} .814$ v. Chr. gründeten Angehörige der Volksgruppe in Nordafrika die Stadt Karthago. Die Phönizier oder Punier sind von diesem Zeitpunkt an als Karthager bekannt. Malta mit seiner Lage im Zentrum des Mittelmeeres - „vor den Toren“ von Karthago - wurde in die punischen Kriege verwickelt und schließlich 257 v. Chr. gewaltsam von römischen Truppen eingenommen ${ }^{49}$.

Unter römischer Herrschaft erlebten die Inseln eine Blütezeit. Die Römer etablierten ein Netz von landwirtschaftlichen Gutshöfen. Die Gutsherren widmeten sich der Produktion von Getreide, Früchten, Wein und Olivenöl. Der Honig der Maltesischen Inseln wurde berühmt - sogar so berühmt, dass sein Diebstahl durch den sizilianischen Praetor CAIUS VERRES den Anlass zu einer außenpolitischen Krise gab ${ }^{50}$.

Die byzantinische und arabische Periode Maltas ab 494 ist schlecht dokumentiert, da kaum Quellen und Funde existieren. Es gibt viele Widersprüche und Gegensätzlichkeiten, welche die Interpretation dieser beiden historischen Phasen schwierig machen ${ }^{51}$. Gesichert ist, dass Malta von der arabischen Volksgruppe der Aghlabiden von Tunesien aus um das Jahr 870 erobert wurde ${ }^{52}$. Zeitgenössische Geschichtsschreiber berichten in der Folge von einer fast kompletten Entsiedlung der Hauptinsel Malta. Während der arabischen Herrschaftsperiode soll die Insel zeitweise nahezu menschenleer gewesen sein ${ }^{53}$. Andere Quellen aus der gleichen Zeit berichten von einer florierenden Handelsgesellschaft auf der Inselgruppe ${ }^{54}$ mit einer Bevölkerung von insgesamt rund 15.000 Einwohnern.

Die Normannen um Graf RogER vertrieben die Araber im Jahr 1090 im Rahmen der sog. Kreuzzüge wieder von den Inseln ${ }^{55}$, errichteten jedoch zunächst keine Garnison. Es folgte eine chaotische Zeit von Überfällen, Eroberungen und kurzfristigen Herrschaftsepisoden, die 1127 von einem weiteren normannischen Eingriff unterbrochen wurden. Da der letzte normannische König keinen Thronfolger hinterließ, fiel die Inselgruppe der Reihe nach an die Hohenstaufen (1194), die Anjou (1268), die Aragonesen (1283) und schließlich an Kastilien (1410). Die Bevölkerung der Inseln wurde christianisiert, Muslime wurden ausgewiesen. Königliche Gutshöfe entstanden, die sich fast vollständig dem Baumwollanbau widmeten.

Im Jahr 1522 wurde der Orden der Johanniter-Ritter von der türkischen Streitmacht des Sultans Suleiman von der Insel Rhodos vertrieben. KARL V., König von Spanien und Vizekönig von Sizilien, gewährte dem Orden zunächst Unterschlupf in Sizilien und überließ ihm als cleveren Schachzug 1524 die Maltesischen Inseln als neuen Stützpunkt ${ }^{56}$. So sicherte er sich Malta als stark verteidigtes christliches Bollwerk gegen die

45 Vgl. BLOUEt (2004), S. 29 f.

46

Vgl. Evans (1963)

47 Vgl. AtTARD (2002), S. 9

48 Vgl. Evans (1963), S. 159

49 Vgl. ATtARD (2002), S. 13 ff.

50 Vgl. Kap. 14

51 Vgl. BLOUet (2004), S. 40 ff.

52 Vgl. AZZOPARDI (1994), S. 6

53 Vgl. CASSAR, S. 4 \& S. 97 f.

54 Vgl. Blouet (2004), S. 42 f. \& AZZOPARDi (1994), S. 6

$55 \mathrm{Vgl}$. ATtARD (2002), S. $34 \mathrm{f}$.

56 Vgl. AZZOPARDI (1994), S. 9 
gefürchteten muslimischen Angreifer. Als symbolische Jahrespacht verlangte KARL V. jedes Jahr zu Allerseelen einen „Malteserfalken“.

Im Jahr 1530 landeten die Johanniter auf Malta. Schon 1547 erfolgte ein weiterer Angriff der Türken auf den Orden, 1551 der nächste ${ }^{57}$. Diese Entwicklung gipfelte in der „Großen Belagerung“ durch die Türken im Jahr 1565, bei der etwa 20.000 türkische Soldaten ${ }^{58}$ gegen ca. 9.000 Mann - größtenteils schlecht bewaffnete Bauern - auf Seiten des Ritterordens kämpften. Die Johanniter waren siegreich, nicht zuletzt durch einen unerwarteten Wetterwechsel ${ }^{59}$ und die späte Unterstützung durch sizilianische Söldner.

Nach der „Großen Belagerung“ entwickelte der Orden eine 268 Jahre währende autokratische Herrschaft, die nach dem türkischen Großangriff weitestgehend ungefährdet blieb. Malta wurde zu einem wichtigen Baumwollexporteur und erlebte eine lange Phase des Wohlstands und Handels, unterbrochen durch wiederholte Seuchen und Pestepidemien, denen sich damals kein Land Europas entziehen konnte. Die hohe Symbolwirkung der türkischen Belagerung von 1565 führte dazu, dass sie bis heute als eines der national wichtigsten historischen Ereignisse hochstilisiert wird. Schon VOLTAIRE schrieb: „Nichts ist besser bekannt als die Belagerung von Malta“ (zitiert in AzzOPARDI, A. E. (1994), S. 9). AzzoPARDı fügte hinzu: „(...) und für die Malteser gilt diese Behauptung heute noch". (ebenda).

1798 landete Napoleon auf seinem Weg nach Ägypten in Malta. Der Johanniterorden war geschwächt und hatte den Franzosen kaum Widerstand entgegenzusetzen, zumal auch die Bevölkerung nicht mehr hinter der Ritterherrschaft stand. Die kurze Herrschaftsperiode der Franzosen (1798 bis 1800) war geprägt durch bürgerkriegsähnliche Zustände ${ }^{60}$. Innerhalb von nur zwei Jahren kam der Baumwollhandel zum Stillstand die Briten kontrollierten und blockierten die Schifffahrtswege rund um das nun französische Malta ${ }^{61}-$ und die maltesische Bevölkerung rief die Briten zu Hilfe ${ }^{62}$. Die Inselbevölkerung wurde durch die Franzosen unterdrückt und die Kirchen systematisch ausgeplündert ${ }^{63}$. Der Adel wurde abgeschafft.

Die folgende britische Kolonialzeit (bis 1964) erlaubte Malta ab 1921 in Teilen die Selbstverwaltung mit einer Regierung und einem Premierminister, überwacht durch britische Gouverneure, deren Herrschaftsanspruch, zunächst in der Tradition der Großmeister des Johanniterordens, von der maltesischen Bevölkerung von Beginn an akzeptiert wurde ${ }^{64}$.

Die britischen Kolonialherren änderten die ökonomische Struktur der Inseln von Grund auf. Malta wurde zum wichtigsten Handelsstützpunkt der Briten in Südeuropa. Die Einführung der englischen Sprache als Amtsund Schulsprache erfolgte zwar zunächst gegen den Willen der Bevölkerung, die italienisch als Amtssprache bevorzugt hätte ${ }^{65}$, jedoch ergab sich aus dieser "Gleichschaltung“ der offiziellen Kommunikation eine Erweiterung des Arbeitsmarkts. Traditionelle Berufe wurden nun durch Arbeitsmöglichkeiten in der öffentlichen Verwaltung, die Beschäftigung von Lehrern in britischen Schulen und den Betrieb der in britischem Auftrag ausgebauten Werften ergänzt.

57 Diese und vorherige Angaben: ATTARD (2002)

58 Diese Zahl ist die jüngste Schätzung (BLOUET (2004), S. 76). Zeitgenössische Chroniken variieren in den Angaben zwischen 15.000 und 40.000 türkischen Besatzern. Die Zahl der Kämpfer auf der Seite des Ordens gilt als zuverlässig.

59 Vgl. BLOUET (2004), S. 74

60 Vgl. CASSAR (2000), S. 141-144

61 Vgl. AZZOPARDI (1994), S. 12

$62 \mathrm{Vgl}$. ATtARD (2002), S. 144-161

$63 \mathrm{Vgl}$. AZZOPARDI (1994), S. $11 \mathrm{f}$.

64 Vgl. BLouet (2004), S. $149 \mathrm{ff}$.

65 Vgl. OECKL (1980) 
Im zweiten Weltkrieg hatte Malta stark unter den Bombardierungen italienischer und deutscher Luftstreitkräfte zu leiden. Die Inseln durchlitten eine schwere Hungersnot in der Zeit der sog. „Abessinischen Krise“66, die häufig als Maltas „Zweite Große Belagerung“ bezeichnet wird ${ }^{67}$. Der maltesischen Bevölkerung wurde 1942, nach dem Sieg der britischen Truppen in Nordafrika, der durch die Verteidigung Maltas erst möglich wurde ${ }^{68}$, der wichtigste Orden des britischen Königreichs verliehen: Das George Cross. Der Orden wurde nur ein einziges Mal in der bisherigen Geschichte der gesamten Bevölkerung eines Staats verliehen, und das war die Bevölkerung Maltas. Mit dem Wiedereintritt der Selbstverwaltung Maltas unter britischer Aufsicht im Jahr 1947 - in den Kriegsjahren wurde Malta als britisches Territorium betrachtet und die Selbstverwaltung wurde ausgesetzt - wurde beschlossen, das George Cross in der maltesischen Nationalflagge und im Nationalwappen zu führen.

Mit der Selbstverwaltung wurde von der Bevölkerung eine Labour-Regierung gewählt. DOMINIC MINTOFF wurde Parteichef, Dr. PAWLU BofFA ${ }^{69}$ war Regierungschef. Beide zählen zu den Personen, denen der letztendliche Erfolg der Unabhängigkeit Maltas von der britischen Kolonialverwaltung zugeschrieben wird. Diesen Bemühungen ging 1955 ein förmlicher Antrag voraus, in dem die maltesische Regierung die offizielle Integration der bisherigen Kolonie als staatsrechtlichen Teil des Territoriums von Großbritannien wünschte ${ }^{70}$. Der Antrag wurde abgelehnt. Der Antrag war, wie sich herausstellte, ein kluger Schachzug MiNTOFFs - zu der Zeit Premierminister im kolonialen Malta. Da die Briten die Integration abgelehnt hatten, war nun der Weg offen für einen förmlichen Antrag auf Unabhängigkeit ${ }^{71}$.

Am 21. September 1964 wurde Malta schließlich unabhängiger Staat im Verbund des Commonwealth of Nations. Seit einer Verfassungsänderung im Jahr 1974 ist der Inselstaat eine Republik. Die detaillierten politischen Vorgänge dieser Zeit sind Kap. 5 zu entnehmen.

Malta war von Beginn an ein Kleinstaat im Sinne der Politischen Geographie ${ }^{72}$ und der Staatengeographie ${ }^{73}$. Auch im außenpolitischen Umgang kann Malta im Sinne der Kleinstaaten-Forschung ${ }^{74}$ als Kleinstaat bzw. Mikrostaat definiert werden ${ }^{75}$. Mit seiner Diskrepanz aus äußerst geringer Staatsfläche und beachtlicher Bevölkerungsdichte stellt Malta einen Sonderfall im Netzwerk der internationalen Staaten dar und dies beschert der Mittelmeer-Republik auch eine strukturelle Ausnahmestellung unter den Mikrostaaten der Welt.

In den 1970er- und 1980er-Jahren entwickelte sich in Malta der Massentourismus und wurde schließlich zum wichtigsten wirtschaftlichen Standbein der Inselrepublik. In den 1990ern wurde der Höhepunkt von fast zwei Millionen Touristen pro Jahr erreicht. Seitdem befindet sich die Entwicklung des Touristenzustroms in Richtung Malta im Abwärtstrend, was zu einer Suche nach neuen politischen und wirtschaftlichen Konzepten führte.

66 Nach LUKE (1968), S. 250

67 Vgl. z. B. LUKE (1968), CASSAR (2000), ATTARD (2002), BLOUET (1987 \& 2004)

68 S. dazu ausführlich z. B. ATTARD (1980). Malta lag auf dem Weg der deutschen Nachschubeinheiten für Nordafrika. Deutsche UBoote belagerten Malta und versuchten die Insel, die für schwere Verluste unter den Nachschubflugzeugen sorgte, auszuhungern. Dies gelang beinahe, doch ein Schiff eines britischen Konvois durchbrach schwerbeschädigt die deutschen Linien und sicherte die Versorgung Maltas, sodass die alliierten Truppen in Nordafrika schließlich den Sieg davon trugen. BLOUET bezeichnet Maltas Rolle im zweiten Weltkrieg als „kritisch“: „Malta's role in the European War was crucial. Had Malta submitted in the spring of 1942, the North African campaign might have been lost. The invasion of Italy, and her exist from the war might have been delayed. (...). Malta, by engaging Axis resources, shortened the European War by a year." (BLOUET (2004), S. 233).

69 PAWLU BOFFA wurde später von der britischen Königin zum „Sir“ geadelt.

70 Vgl. BLOUET (2004), S. 243

$71 \mathrm{Ebd}$.

72 Vgl. z. B. MAULL (1956)

73 Vgl. z. B. ScHWIND (1972)

74 Zum Terminus technicus „Kleinstaaten-Forschung“ vgl. KIRT \& WASCHKUHN (2001b)

75 Vgl. z. B. VITAL (1967) oder GeSER (2001) 
Dieser Umstand im Zusammenspiel mit der langjährigen Regierung von Premierminister Dr. EDWARD FENECH ADAMI - seit Beginn seiner Amtszeit ein starker Befürworter der EU-Annäherungspolitik - führten zu Maltas Integration in die Europäische Union am 01. Mai 2004 im Rahmen der historischen sog. „EUOsterweiterung“, an der mit Zypern und Malta auch zwei südeuropäische Staaten beteiligt waren. Als Schauplatz für die offiziellen Festlichkeiten wurde Fort St. Angelo auf der Landzunge von Vittoriosa im Becken des Grand Harbour ausgewählt. Dies war der Ort der Entscheidung bei der Schlacht gegen die Türken im Jahr 1565. Offizielle Stimmen wiesen darauf hin, dass man mit der Wahl dieses Veranstaltungsorts die europäischen Wurzeln Maltas besonders hervorheben wolle.

Zur zusammenfassenden Veranschaulichung der geschichtlichen Ereignisse vgl. Abb. 2 auf der folgenden Seite. 


\section{Zeittafel zur Geschichte der Maltesischen Inseln}

vermutl. $5200-4500$ v. Chr

Erstbesiedlung der Maltesischen Inseln vermutl. von Sizilien ausgehend

vermutl. $4100-2500$ v. Chr

Tempelkultur

mit verschiedenen Entwicklungsstadien

$$
2500-800 \text { v. Chr }
$$

Bronzezeitliche Kultur

vermutl. $900-257$ v. Chr

Schrittweise Ansiedlung phönizischer Händler

257 v. Chr. -494 n. Chr.

Römische Herrschaft, Griechischer Einfluss

$$
494 \text { - ca. } 600
$$

Byzantinische Periode

$600-$ ca. 870

Keine gesicherten Erkenntnisse

ca. $870-1090$

Eroberung durch die Aghlabiden, in der Folge arabische Herrschaft

$$
1090-1194
$$

Normannische Herrschaft

$$
1194-1268
$$

Maltesische Inseln im Besitz von Hohenstaufen

\section{$1268-1283$}

Maltesische Inseln im Besitz von Anjou

$1283-1410$

Maltesische Inseln im Besitz von Aragon

$1410-1524$

Maltesische Inseln im Besitz von Kastilien

1524

Schenkung der Maltesischen Inseln an die Johanniter durch Karl V.

$$
1530-1798
$$

Herrschaft des Johanniterordens, später: Ritter des Malteserordens

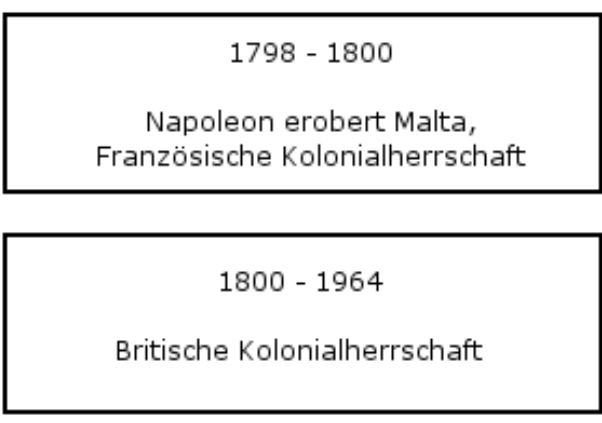

$1964-1974$

Unabhängiger Staat im Commonwealth, Staatsoberhaupt: Queen Elisabeth II.

\section{seit 1974}

Unabhängige Republik

\section{4}

Beitritt der Republik Malta zur Europäischen Union

Abb. 2: Zeittafel zur Geschichte der Maltesischen Inseln. Entwurf \& Grafik: AsCHEMEIER 


\section{Malta und die Europäische Union}

Die jüngere Geschichte Maltas gipfelte im Jahr 2004 im Beitritt des unabhängigen Staats zur Europäischen Union. Die ersten Beitrittsbemühungen erfolgten noch vor der Unabhängigkeit von Großbritannien. Eine Darstellung der Geschichte von Maltas EU-Beitritt kann daher gleichzeitig als eine historische Darstellung von Maltas Außenpolitik angesehen werden. Aus diesem Grund erfolgt an dieser Stelle eine Zusammenfassung der Ereignisse, die eine Ergänzung und Fortschreibung des vorangegangenen entwicklungshistorischen Abschnitts darstellt.

In die folgenden Ausführungen sind einzelne Textabschnitte eines Beitrags von ASCHEMEIER \& CYFFKA (2004a) eingeflossen.

Malta hat in historischer Hinsicht oft eine bedeutende Rolle im europäischen Kontext gespielt. Trotzdem haben sich auf der Inselgruppe bis heute auch zahlreiche Traditionen und Produkte arabisch-orientalischen Ursprungs erhalten, die dem Festlandseuropäer mithin fremdartig erscheinen mögen.

Für viele Einwohner der bis dato 15 Mitgliedsstaaten der EU war es vielleicht auch deshalb eine „Überraschung“, dass neben den acht neuen osteuropäischen EU-Zugängen und dem südöstlichen Beitrittsanwärter Zypern auch Malta dem europäischen Staatenbund angegliedert wurde. Über kaum eines der neuen Beitrittsländer wurde in der deutschen Presse so oft berichtet wie über Malta. Ist dies ein Zeichen dafür, dass der Kleinstaat bisher kaum in der öffentlichen Berichterstattung Deutschlands wahrgenommen wurde?

Dabei haben die Kontakte zwischen der EU und Malta bereits sehr früh begonnen. Es ist wohl den vielen Rückschlägen auf dem langen Weg zum Beitritt zuzuschreiben, dass die Republik Malta lange Zeit nicht richtig im europäischen Bewusstsein verankert war.

Bereits ein Jahr nach dem EU-Beitritt sind mittelbare wie auch unmittelbare Auswirkungen auf die Hersteller und Verkäufer regionaler traditioneller Produkte auf den Maltesischen Inseln zu spüren gewesen. Im Folgenden werden daher Maltas Weg zum EU-Beitritt, sowie die jüngsten Entwicklungen nach dem Beitritt am 01.05.2004 betrachtet.

Die historischen Entwicklungen während des gesamten Zeitraums seit Maltas erster EG-Annäherung sind in umfangreichen Abhandlungen beschrieben worden ${ }^{76}$. Eine Darstellung des geschichtlichen Verlaufs bis zum endgültigen Beitritt kann an dieser Stelle nur in Kürze und überblicksartig erfolgen. 


\subsection{Die Periode 1964 bis zum Ende der 1980er-Jahre}

Im Jahr 1958 schlug Premierminister DOMINIC MINTOFF erstmals öffentlich eine enge Verbindung zur EG vor. Er entwickelte in diesem Zusammenhang die Vision und den Begriff „a little Switzerland in the Mediterranean“. Diesen Bemühungen ging 1955 ein Antrag voraus, anhand dessen die maltesische Regierung die offizielle Integration der bisherigen Kolonie als staatsrechtlichen Teil des Territoriums von Großbritannien wünschte ${ }^{77}$. Wie in Kap. 4 beschrieben, diente dieser Antrag als Vorbereitung auf Maltas förmlichen Unabhängigkeitsantrag, der 1962 erfolgte.

Der Unabhängigkeitsantrag löste innenpolitische Debatten aus, da Malta Mitte der 1960er-Jahre wirtschaftlich in stärkstem Maße von Großbritannien abhängig war. Die EG-Annäherung Maltas in dieser Zeit muss daher in enger Verknüpfung mit der Europa-Politik Großbritanniens im selben Zeitraum betrachtet werden. Im Zusammenhang mit Maltas Annäherungsbemühungen wird oft eine Rede ${ }^{78}$ von WINSTON CHURCHILL erwähnt, in der dieser die Vision der „United States of Europe“ entwickelte mit dem Hinweis „The structure of the United States of Europe, if well and truly built, will be such as to make the material strength of a single state less important. Small states will count as much as large ones and gain their honour by their contribution to the common cause“. (CHURCHILL (1946)). MINTOFF scheint seinerzeit bei der Regierungspolitik nachhaltig von dieser Rede beeinflusst gewesen zu sein ${ }^{79}$.

Am 21.09.1964 erlangte Malta den Status eines souveränen Staats im Verbund des Commonwealth of Nations. Bereits ein Jahr später engagierte sich der Inselstaat im Europarat ${ }^{80}$. Malta folgte damit den folgenden Empfehlungen eines UNO-Reports ${ }^{81}$ :

1. Malta sollte enge Verbindungen zu Großbritannien unterhalten, auch nach der Unabhängigkeit.

2. Ähnlich enge Verbindungen wurden mit Italien empfohlen.

3. Ein Beitritt zur oder zumindest eine enge Verbindung mit der EG wurden nahe gelegt.

4. Die Mitgliedschaft in der EFTA (Europäische Freihandelszone) wurde vorgeschlagen.

Diese Handlungsempfehlungen konnten nicht in vollem Umfang erfüllt werden. Die Mitgliedschaft in der EFTA hätte ein Ausbleiben von wichtigen Zöllen bedeutet, die seinerzeit für Maltas Finanzsystem als unerlässlich angesehen wurden. Auch war Italien zunächst nicht an engen Bindungen zu Malta interessiert, bis Ende der 1960er-Jahre die britische Militärpräsenz auf Malta zu verschwinden begann. Italien befürchtete nun ein sicherheitspolitisches Machtvakuum im zentralen Mittelmeerraum und bemühte sich um die Vertiefung der Zusammenarbeit beider Länder $^{82}$.

\footnotetext{
77 Vgl. BLOUET (2004), S. 243

78 CHURCHILL (1946): „Something to astonish you!“ (Rede vom 19.09.1946, gehalten in Zürich)

79 Vgl. PACE (2001), S. 79 f.

$80 \mathrm{Vgl.} \mathrm{SEILER} \mathrm{(1995),} \mathrm{S.} 142$

81 Stolper; Hellberg \& CALLANDER (1964)

82 Vgl. HAUSMANn (1997)
} 
Am 05.12.1970 wurde ein Assoziationsvertrag zwischen Malta und der EG vereinbart ${ }^{83}$. Dieser Vertrag trat im April 1971 in Kraft und bezog sich im Wesentlichen auf gegenseitige Zollminderungen. 1976 und '77 wurden verschiedene EG-Malta-Protokolle auf den Weg gebracht, deren Inhalte sich später als problematisch erweisen sollten. Diese Protokolle umfassten im Wesentlichen EG-Verpflichtungen:

- Finanzhilfe für Malta: 26 Mio. Ecu (teilweise als Darlehen)

- Zulassung weiterer maltesischer Produkte im EG-Raum

- Erhöhung der EG-Einfuhrquote für maltesische Agrarprodukte

- Adaption der ursprünglichen Vertragsvereinbarung von 1970 auf die Situation nach der ersten EG-Erweiterung

Die Auswirkungen dieses ersten Zusammenarbeitsvertrags und der folgenden Protokolle werden heute mehrheitlich als der wesentliche Motor für Maltas wirtschaftliche Boomphase in den 1970ern betrachtet ${ }^{84}$.

Maltas Wirtschaft wandelte sich und wurde zunehmend exportorientiert ${ }^{85}$. Von 1971-1981 verzehnfachte sich die Exportrate des Landes und vervierfachte sich die Zuwachsrate von Maltas BSP $^{86}$. Die absolute Beschäftigung von Arbeitern im sekundären Sektor stieg in dieser Zeit von 20.662 auf $32.923^{87}$. Anfang der 1980er setzte sich dieser Trend fort und Malta erweiterte seine Palette an Exportgütern beträchtlich.

Im selben Zeitraum kündigten sich Differenzen mit der EG an. Bereits seit 1971 hatte Maltas Regierung das Assoziationsabkommen nicht mehr als Vorbereitung zum Beitritt verstanden, sondern als „ökonomisches Werkzeug" um den wirtschaftlichen Schaden durch den fortschreitenden Abzug der britischen Luftstreitkräfte zu kompensieren. Die maltesische Regierung hatte sich von der Zusammenarbeit mit der EG mehr versprochen, vor allem auf dem Investitionssektor.

1977 wurde daher in großem Umfang versucht, Handelsabkommen mit Staaten der Arabischen Liga abzuschließen. Allerdings konnten nur mit Saudi-Arabien, Kuwait und Libyen erfolgreiche Verträge verzeichnet werden (vgl. Karte 2).

83 Vgl. KOMMISSION DER EUROPÄISCHEN GEMEINSCHAFTEN (2003a), S. 5 f.

84 Vgl. PACE, R. (2001), S. 151-152

85 Vgl. Metwally (1977), S. 57

86 Vgl. PACE (2001), S. 156-157

$87 \mathrm{Ebd}$. 


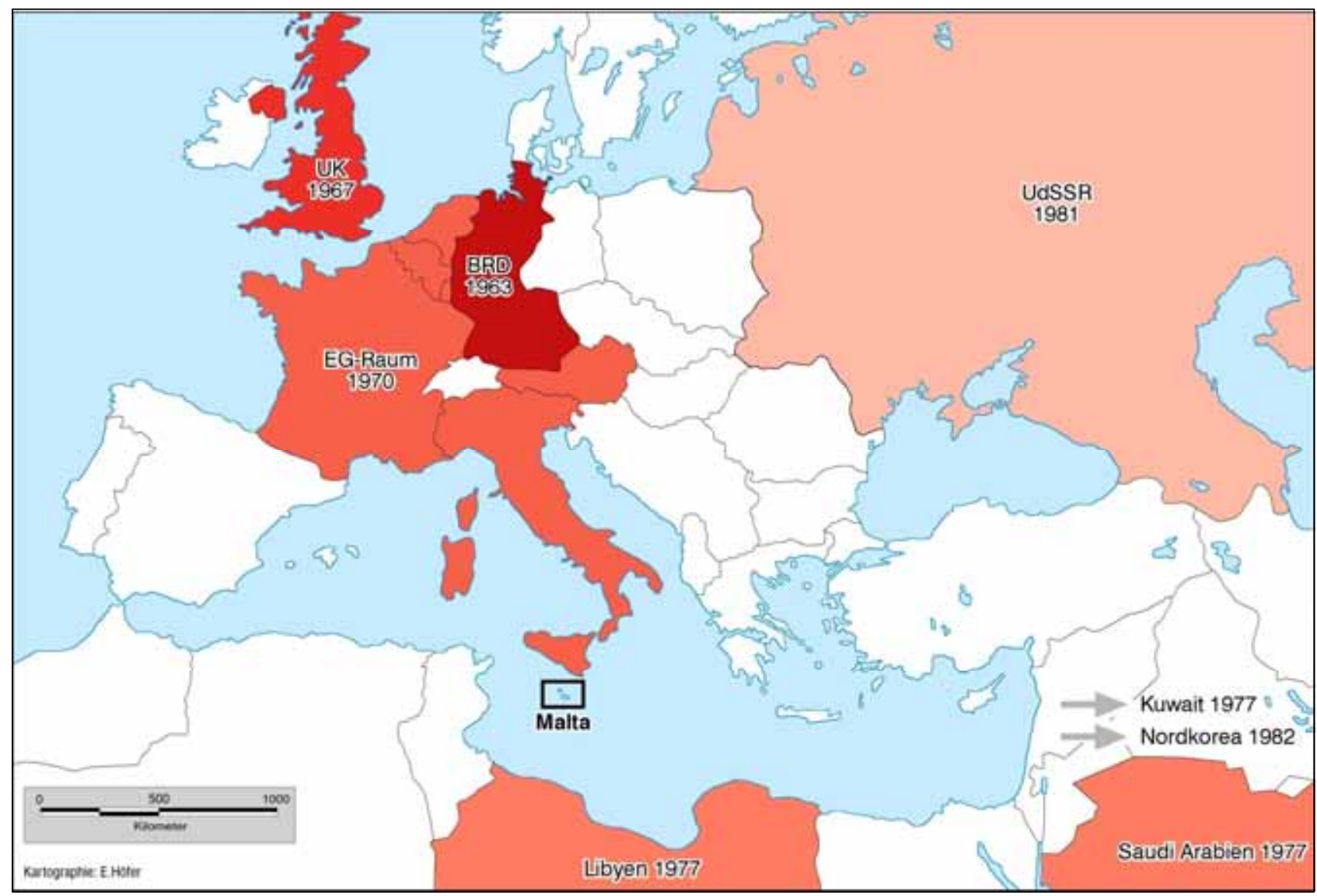

Karte 2: Maltas Verträge mit dem Ausland in der Zeit von 1963-1982. Entwurf: ASCHEMEIER; Grafik: HÖFER

Malta beanspruchte seit der Verfassungsänderung von 1974 einen europapolitisch nicht unproblematischen Neutralitätsstatus. 1981 bot MINTOFF (Partit Laburista = Malta Labour Party (MLP)) der EG eine „Special Relationship“-Politik an ${ }^{88}$. Diese sollte auf zwei Grundlagen beruhen:

1. Maltas Neutralitätsstatus müsse „zum Frieden und zur Einheit aller Mittelmeerstaaten“ in vollem Umfang erhalten bleiben.

2. Finanzielle, ökonomische und politische Verpflichtungen aus den Protokollen von 1976 und '77 müssten von der EG in stärkerem Maße akzeptiert werden, um Maltas neuen Status zu unterstützen.

Obwohl die Regierung MINTOFF das Gegenteil beteuerte, wurde schnell klar, dass Malta sich zunehmend von der Europäischen Gemeinschaft und Großbritannien zu lösen begann ${ }^{89}$. MiNTOFFs selbstbewusster Vorschlag ließ die EG-Malta-Verhandlungen fast zum Stillstand kommen.

Es kam zu außergewöhnlichen Vorgängen in Maltas Innenpolitik, die europäische Analysten als akute Gefährdung von Demokratie und Menschenrechten deuteten ${ }^{90}$. Vor allem ein Gesetz - der Foreign Interference Act von 1982 - sorgte für Empörung. Das Gesetz verbot es Ausländern, sich an politischen Vorgängen in Malta zu beteiligen. Der Zugang der Oppositionspartei Partit Nazzjonalista (Nationalist Party

88 Vgl. POLLACCO (2004)

$89 \mathrm{Vgl}$. SEILER (1995), S. 149

90 Vgl. z. B. Rossı (1986), der Malta zu dieser Zeit als „Libyan Sattelite“ bezeichnete. 
(PN)) zum diplomatischen Korps wurde damit stark eingeschränkt ${ }^{91}$. Außerdem war es maltesischen Staatsbürgern ab sofort verboten, in TV- und Radioübertragungen aus dem Ausland mitzuwirken.

Das Haus von PN-Leader DR. EDWARD FENECH ADAMI wurde von Anhängern der MLP verwüstet, der PNnahe Verlag „Progress Press“ fiel einem Brandanschlag zum Opfer, die Zentrale der PN wurden mit Billigung der Regierung MiNTOFF ${ }^{92}$ im Rahmen einer Polizei-Razzia durchsucht. Die Kirche als wichtige maltesische Institution wurde fast vollständig enteignet; kirchliche Schulen wurden geschlossen. Bis heute sind daher viele ehemals kirchliche Grundstücke in Staatsbesitz, was gegenwärtig aufgrund fehlender Finanzen und Pflegemaßnahmen zunehmend zum Problem wird ${ }^{93}$. Die Krise rund um den Foreign Interference Act wird von SEILER als der Höhepunkt der innenpolitischen Auseinandersetzungen in Maltas bisheriger Geschichte gedeutet $^{94}$. Auch außenpolitisch hat Malta zuvor und danach selten mehr Aufsehen verursacht.

Darüber hinaus verfolgte die Regierung MINTOFF dubiose Beziehungen zu Nordkorea (zwei Geheimverträge 1982) und Libyen (Freundschafts- und Sicherheitsvertrag 1984). Hochgestellte maltesische Politiker plädierten in der Öffentlichkeit für die Einführung eines Ein-Parteien-Systems nach osteuropäischem Vorbild. 1982 wurde auch der Arabischen Liga ein „Special Relationship“-Abkommen angeboten, aber in der Folge abgelehnt.

Die EG sah die Entwicklung mit Besorgnis und machte der maltesischen Regierung unter Zuhilfenahme von Berichten einschlägiger Analysten vor allem drei Hauptvorwürfe ${ }^{95}$ :

1. Graduelle Drift Maltas von einem demokratischen System hin zum sozialistisch geprägten EinParteien-Staat

2. Verschlechterung der allgemeinen Menschenrechtssituation

3. Attacken auf die Pressefreiheit

Das Europäische Parlament verhängte letztlich eine Resolution mit der Drohung eines Stopps der EGFinanzhilfe. Malta drohte daraufhin mit dem Abzug seiner Botschafter aus dem EG-Raum und mit dem Austritt Maltas aus dem Europarat ${ }^{96}$. Die Beziehungen zwischen der Inselrepublik und der Europäischen Gemeinschaft waren auf einem Tiefpunkt angelangt und blieben vorerst angespannt ${ }^{97}$. Nach 1985 normalisierten sich die Beziehungen nur allmählich ${ }^{98}$.

\footnotetext{
91 Noch weit drastischer sieht dies PACE, R. (2001), S. 174, der die Abkoppelung der Opposition von der Außenwelt als einziges und hauptsächliches Ziel des Gesetzes betrachtet.

92 Vgl. SEILER (1995), S. 165

93 The TIMES OF MALTA, 08.08.2000, S. 17

$94 \mathrm{Vgl.} \mathrm{SEILER} \mathrm{(1995),} \mathrm{S.} 165$

95 Nach PACE (2001), S. 169-177

96 Vgl. SEILER (1995), S. 157

97 Ebd., S. 159

98 Ebd., S. 169-174
} 


\subsection{Maltas Europa-Politik von 1987-2003}

Nachdem die Jahre 1980-1987 von wirtschaftlicher Rezession geprägt waren, konnte im Mai 1987 die PN die Wahlen für sich entscheiden. DR. FENECH ADAMI wurde Premierminister. Im Europarat engagierte sich Malta nun für die Belange des gesamten Mittelmeerraums: „Wichtigstes Thema der maltesischen Politik in Strassburg [sic] war stets das Mittelmeer und die Politik des Europarates in dieser Region. Die Lage Maltas „am Rande Europas“, aber im Zentrum des Mittelmeers, führte dazu, dass alle maltesischen Politiker, insbesondere auch dessen [sic] Vertreter in der Parlamentarischen Versammlung des Europarates, dem Mittelmeer und der Frage der Zukunft dieser Region grosse [sic] Bedeutung beimassen [sic]." (SEILER (1995), S. 174).

Der innenpolitische Machtwechsel führte auch zu einer Verbesserung in den Beziehungen Maltas zur EG. Das Projekt der 1960er-Jahre wurde wieder aufgegriffen ${ }^{99}$, und ein Beitritt wurde angestrebt. Dies geschah mit Verzögerungen, da die neue Regierung die Wirtschafts-, Innen- und Außenpolitik zunächst grundlegend reformierte. Weitere Reformen im Jahr 1994 wurden fast sämtlich im Hinblick auf den anvisierten EU-Beitritt durchgeführt. Wirtschaftlich gesehen hatte Großbritannien als Handelspartner mittlerweile stark an Bedeutung eingebüßt.

Der Kollaps des europäischen Kommunismus in den 1990ern kam dem Kleinstaat einerseits zugute, da der Neutralitätsstatus des Landes nun mit neuen Augen gesehen wurde, andererseits ergaben sich dadurch heftige interne Diskussionen über die künftige Linie der Außenpolitik (s. u.; Zitat von CALLEYA). Die politische Kooperationsfähigkeit Maltas wurde von der EU nach wie vor infragegestellt. Als Resultat der Bemühungen Griechenlands um einen EU-Beitritt Zyperns im Jahr 1994 wurde auch der Beitrittsprozess Maltas wieder aufgerollt ${ }^{100}$. Dieser Umstand erscheint insofern paradox, als die Beziehungen zwischen Malta und Griechenland auf politischer Ebene bis dahin eher schleppend verlaufen waren. ${ }^{101}$

1996 löste die MLP mit Premierminister DR. ALFRED SANT kurzfristig die PN als Regierungspartei ab. Die zwei Jahre dieser MLP-Regierung waren geprägt von der Stagnation der Beitrittsbemühungen. In dieser Zeit war von Seiten der EU Skepsis und Unsicherheit gegenüber der Labour-Regierung von ALFRED SANT zu spüren. Es war schließlich die MLP, die in den frühen 1980ern zum (Beinahe-)Bruch mit dem Europarat geführt hatte.

SEILER schreibt im Hinblick auf Maltas Integration in den europäischen Staatenbund aber schon vor dem Regierungswechsel im Jahr 1995: „Bezeichnend für die Mitarbeit Maltas im Europarat ist indes auch, dass insbesondere auf Regierungsebene - die Präsenz des Inselstaates in Strassburg [sic] stets bescheiden blieb. (...). Die Situation hat sich seit dem Regierungswechsel 1987 und der darauf folgenden Normalisierung der Beziehungen zum Europarat zwar auch hier verbessert. Wie die Beispiele anderer, zum Teil kleinerer Staaten aber zeigen, bleibt für Malta in Strassburg [sic] noch viel zu tun." (SEILER (1995), S. 177).

Die Regierung SANT scheiterte noch vor Ende ihrer Legislaturperiode. Seit 1998 dominiert daher die PN wieder die politische Szene Maltas. Die PN deutete diesen Wahlsieg als klares Votum der Wählerschaft für einen EU-Beitritt ${ }^{102}$ (s. Foto 2) und hat es trotz heftiger Dispute in der politischen Öffentlichkeit Maltas zwischen 1998 und 2004 geschafft, den EU-Beitritt einzuleiten. Dabei wurde mit deutlich erhöhten Bemühungen eine Verbesserung der Beziehungen zum Europarat und zur EU angestrebt und letztendlich zu weiten Teilen erreicht.

99 Allerdings in modifizierter Form

100 Vgl. Baier-Allen (2001)

101 Vgl. Ebd.

102 Vgl. CALleya (2002), S. 8 
Der EU-Beitritt muss vor allem im Hinblick auf die geänderte weltweite politische Szene - in der Malta seinen Neutralitätsstatus völlig neu überdenken musste - gesehen werden. Der maltesische Politikwissenschaftler CALLEYA schrieb zwei Jahre vor Maltas EU-Beitritt einige symptomatische Sätze für diese Zeit, die für Außenstehende zur Verdeutlichung der Handlungsweise der maltesischen Regierung nach der Jahrtausendwende beitragen können:

„As Malta deliberates over its foreign policy goal of becoming a European Union member it is worth reminding everyone of a few basics that are perhaps quite often forgotten. The post-Cold War era that we are living in is a very different time from the Cold War days after the Second World War to 1989. In a world dominated by two superpowers, countries such as Malta, were able to play one superpower off against the other. Foreign policy strategy could be formulated accordingly. In a world where only one superpower remains, such strategic thinking is obsolete. While the number of states has continued to increase, so have the number of regional and global institutions. (...). Those that are best able to navigate through such a web of dynamics are the actors that will prosper the most in the twenty-first century. Those that do not, will wither away and could disappear altogether." (CALLEYA (2002), S. 11).

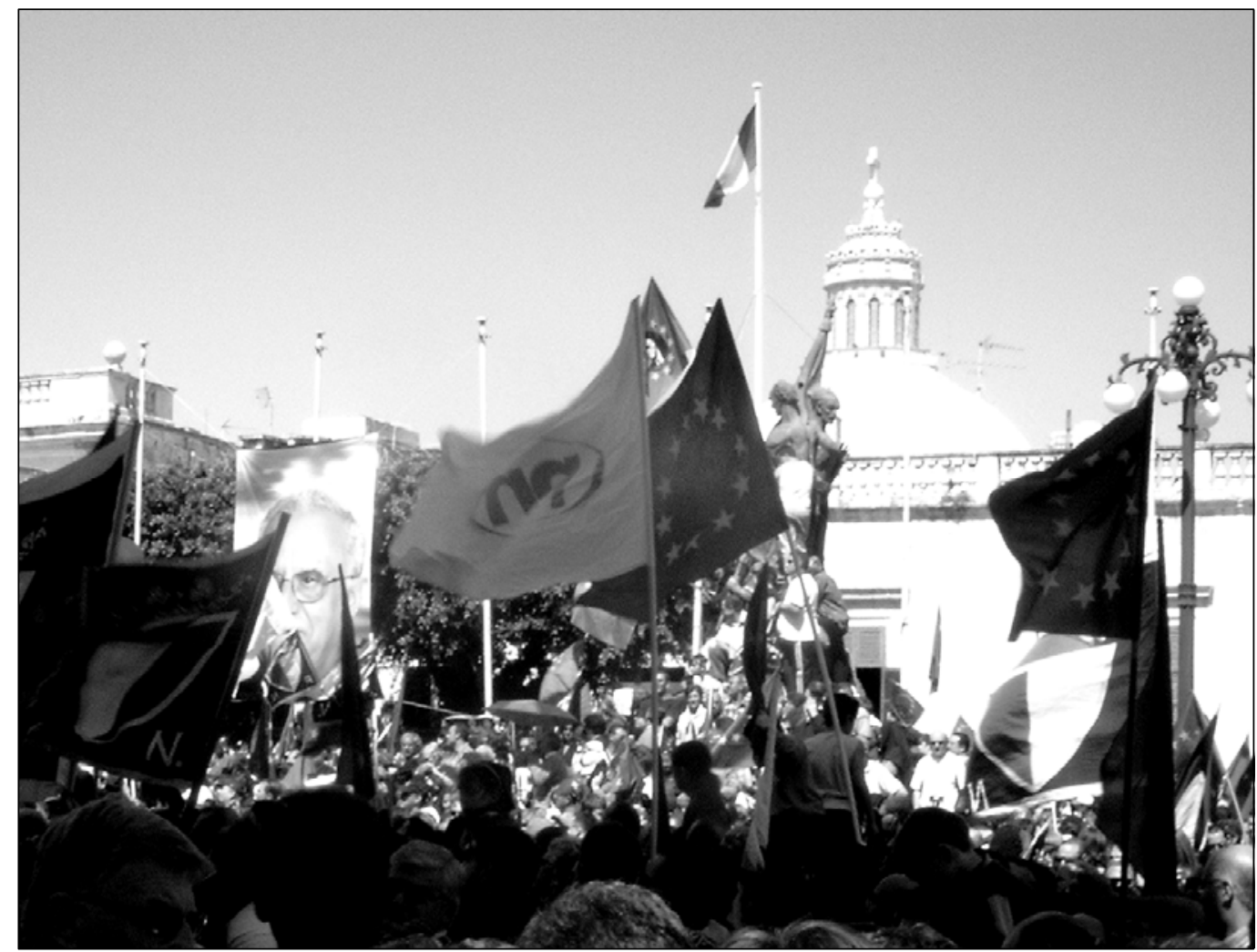

Foto 2: Wahlfeier im Jahr 2003 in Valletta. Die Wahl wurde als „Stunde der Entscheidung“ aufgefasst, ob Malta der EU beitreten würde oder nicht. Fahne und Logo der PN dominieren das Bild. Im Hintergrund ist ein Transparent mit dem Konterfei EdWARD FENECH AdAMIs zu sehen. Foto: AschemeIER

Am 01. Mai 2004 wurde Malta offizielles EU-Mitglied. Dabei konnte die maltesische Regierung üppige Zugeständnisse erwirken: Über 80 Sonderregelungen sollten Malta den Beitritt zur EU erleichtern.

Der ehemalige Premierminister FENECH ADAMI löste nach dem Beitritt, der in Maltas politischer Szene vor allem als sein persönlicher Triumph gedeutet wurde, im April 2004 GUIDO DE MARCO als Staatspräsident Maltas ab. Neuer Premierminister und PN-Leader wurde DR. LAWRENCE GONZI.

Am 05.05.2004 wurden die neuen Mitglieder für die EU-Kommission aus den 10 Beitrittsstaaten vereidigt, darunter auch die maltesische Delegation. 


\subsection{Protest der Malta Labour Party gegen den EU-Beitritt}

Die MLP propagierte noch bis zum 01.05.2004 eine enge Zusammenarbeit mit der EU jedoch ohne Beitritt. Diese Haltung änderte sich auch nach der Eingliederung Maltas in die Europäische Union zunächst nicht. Die Unumkehrbarkeit des politischen Prozesses wurde aber schließlich eingesehen.

Noch am Tag des EU-Beitritts kam es zu einer Protestkundgebung der MLP in Valletta mit einer klaren AntiEU-Aussage, die jedoch als Versammlung zum „Tag der Arbeit“ ausgegeben wurde ${ }^{103}$. „Super 1 television", ein Fernsehsender, der sich faktisch im Besitz der Labour Party befindet, widmete am 01.05.2004 die ersten 15 Min. seiner Hauptnachrichten dem „Tag der Arbeit“, während der Anlass des EU-Beitritts in weniger als 60 Sekunden abgehandelt wurde ${ }^{104}$. Unmittelbar nach dem 01. Mai schrieb LEO BRINCAT, Sprecher für Außenpolitik der MLP-Opposition, einen polemischen Leserbrief in der Tageszeitung The Malta Independent in der der EU-Beitritt pessimistisch ausgelegt ${ }^{105}$ und mit harscher PN-Kritik nicht gespart wurde.

Diese Gesinnung wurde auch von dem der MLP nahe stehenden Teil der Presse mit Kritik aufgenommen ${ }^{106}$. Insbesondere der Umstand, dass die Malta Labour Party auch über eine Woche nach dem EU-Beitritt ihres Landes diesen noch nicht offiziell anerkannt hatte ${ }^{107}$, veranschaulicht die harte Linie der Partei.

Ein Hauptgrund für die EU-kritische Politik der MLP war in den Jahren vor dem Beitritt die Befürchtung, dass mit einem EU-Beitritt Maltas auch die Importkontrollen gegenüber Waren aus anderen EU-Mitgliedsstaaten nicht aufrechterhalten werden könnten ${ }^{108}$ und sich außerdem eine Verteuerung der Lebenshaltungskosten bemerkbar machen würde.

In der Tat wurden erste Änderungen schon wenige Tage nach dem EU-Beitritt für alle Bürger des Landes spürbar. So berichtete die Presse über neue Steuern, die auf 400 Gebrauchsgegenstände erhoben wurden, darunter Medikamente und Bücher, aber auch bestimmte Lebensmittel wie z. B. Fruchtsaft ${ }^{109}$.

\subsection{Ausblick}

Während die MLP auch gegenwärtig noch eine kritische Haltung ggegenüber der EU vertritt - im Mai 2004 aber trotzdem Kandidaten für die Europawahl aufstellte - sind sich die meisten maltesischen Geschäftsleute einig, dass der EU-Beitritt langfristig einen positiven Einfluss für den Kleinstaat bringen wird. In zahlreichen Gesprächen und Experteninterviews mit maltesischen Staatsbürgern ist der Autor der vorliegenden Arbeit auf teilweise überschwängliche Äußerungen gestoßen.

Es gibt in Malta Menschen, die sehr viel vom EU-Beitritt erwarten. Ihre Äußerungen waren äußerst selbstbewusst. Wieder andere waren strikt gegen den Beitritt und versuchten auch die negativen Auswirkungen der EU-Erweiterung nicht in Vergessenheit geraten zu lassen.

103 THE MALTA INDEPENDENT, 04.05.2004, S. 7

104 Ebd.

105 Ebd., S. 6

106 Ebd., S. 7

107 MALTA TODAY, 09.05.2004, S. 4

108 Vgl. CASSAR (2000), S. 249-250

109 The MALTA INDEPENDENT , 05.05.2004, S. 8 sowie THE TIMES OF MALTA, 06.05.2004, S. 44 
Die erste Wahl nach dem EU-Beitritt Maltas war die Wahl zum Europäischen Parlament im Juni 2004. Ähnlich wie im übrigen Europa wurde auch in Malta die Regierungspartei von den Wählern „abgestraft“. Den Wahlsieg errang die MLP. Überraschend war der Wahlerfolg der "grünen“ Partei Alternativa Demokratika (AD), die über 1000\% Stimmenzuwachs (!) im Vergleich zur Parlamentswahl vom Frühjahr 2003 verbuchen konnte und anteilig 10\% der gesamten Stimmen erreichte. Dieser Vorgang ist beispiellos für die bisherige politische Geschichte Maltas seit der Unabhängigkeit im September 1964. Die politische Landschaft des Inselstaats scheint vor einer Wende zu stehen. In den Zeitungen wird erstmals offen über eine Änderung des Wahlrechts diskutiert, das den Blockparteien MLP und PN bisher ihre absoluten Mehrheiten sicherte.

Im Folgenden erfolgt eine Auswahl von Meinungen und Zitaten, aus denen der Leser sich ein eigenes Bild machen kann, in welche Richtung sich Maltas Dasein als Mitgliedsland der EU in Zukunft entwickeln könnte.

Bereits im Vorfeld der EU-Erweiterung wurde in der maltesischen Presse die besondere Rolle des Kleinstaats im Hinblick auf wirtschaftliche Beziehungen zu Nordafrika und dem arabischen Raum angesprochen ${ }^{110}$ : "(Prime Minister DR. EDWARD FENECH ADAMI) added that he also saw advantages for companies that wish to operate "from" Malta rather than "in" Malta. "Wether it is marketing, sales and distribution, project management, costumer training or after sales support, we believe that it can be done most effectively from Malta and at a lower cost than most alternative solutions. "During the reception many potential investors and businessmen talked freely to the journalists present. (...) The managing director of the company Brecks Heating Services, Brett Ainsworth, said that they looked at Malta because of their client base in Libya and Dubai."

Aus touristischer Perspektive ein Zitat aus einem Bericht über den Hotelmanager CHRISTOPHE LAURE ${ }^{111}$ :

„Mr. Laure is „very enthusiatic“ about the future. „First of all, because of EU membership and enlargement", he says. "This will open new doors and build business. We shall continue and concentrate on Europe, because that is our feeder market (...)"." (In: THE TIMES OF MALTA, 06.05.2004. Business section, S. 3).

Des Weiteren folgt ein Zitat eines nicht-maltesischen Politikers. Es stammt vom israelischen Botschafter in Malta, EHUD GOL:

„Let's be realistic. Malta outside the EU had no political power unlike now that you're inside the Union. Being a member of the EU, Malta cannot play a part in a unilateral policy but has to align itself with the EU. And because of Malta's special relations with the Arab world I believe Malta can influence. And I am not exaggerating. Being part of the EU club, you don't take account of the size. Malta is four percent of the EU." (In: THE TIMES OF MALTA, 08.05.2004. S.4).

Auch EU-kritische Meinungen sind in Malta nicht verstummt. Dazu ein Auszug aus dem erwähnten Leserbrief (s. o.) vom Sprecher für außenpolitische Angelegenheiten der MLP, LEO BRINCAT:

„Certain economists I have spoken to in recent weeks seem to have already given up. Not because they harbour any anti-EU sentiments but simply because they happen to be appalled at our country's economic indicators, particularly the gargantuan proportions of our national debt and fiscal deficit, our bureaucracy and red tape as well as the dearth of foreign direct investment. (...). Unless we make a real success of our membership, not only will we be surpassed in terms of job creation and foreign direct investment by the central and eastern Europeans that joined the EU with us on Saturday, but we will find that even Romania and Bulgaria, who are next in line to join, will be a direct threat to us. " (In: THE MALTA INDEPENDENT, 04.05.2004. S. 6).

110 BALZAN in: MALTA TODAY (Onlineausgabe vom 23.02.2003)

111 LAURE ist General Manager des Hotels Intercontinental in Malta 
Ganz im Gegensatz zu BRINCATs Äußerungen stehen Teile der deutschen Presse, die Malta als „reichstes der 10 neuen Beitrittsländer" bezeichnen:

„(...) das Land gilt als das reichste aller EU-Beitrittsstaaten ${ }^{112 ، ~(N E U E ~ W E S T F A ̈ L I S C H E, ~ A u s g a b e ~ v o m ~}$ 29.04.2004, S. 3)

Der Arbeitgeberverband Maltas (Malta Employers' Association - MEA) stellt ein Merkmal von Maltas Ökonomie besonders heraus - die Anpassungsfähigkeit:

„A famous quote from Darwin states that it is not the strongest of species that survive, but the ones most adaptable to change. In a sense this can be applied to Malta's membership in the European Union. As a small state with hardly any natural resources, Malta cannot be termed a strong country in this sense. Its main strength lies in the fact that, because of its size, it should be able to adapt more rapidly to change. (...). The main challenge facing us, as a member of the EU, will be our flexibility and the extent to which we can develop a culture of innovation and proactivity. History has proved that, with focus and determination, we can pass this test." (In: The EMPLOYER (editorial), zitiert in: THE TIMES OF MALTA, 05.07.2004, S. 2).

Der Ökonom und Kleinstaatenforscher MANUEL BORDA zieht ein ähnliches Fazit und führt einen auch in Deutschland in der Zeit nach der Fußballeuropameisterschaft 2004 viel strapazierten Vergleich ${ }^{113}$ zwischen $^{2}$ Fußball und Wirtschaft an:

"The list of small successful states competing with larger and more endowed countries is lengthening. Flexibility has proved to be a key factor to their positive trends. But the greatest contribution to their success is human resources development and their successful fight against complacency. This has also shown in Euro 2004. Malta, too, even though smaller than any of those that participated in the tournament, can be a typical success story in any activity taken on board." (BORDA in: THE TIMES OF MALTA, 11.07.2004, S. 43).

DR. MICHAEL BARTOLO, Maltas früherer UNO-Botschafter und jetziger Chairman der World Intellectual Property Organisation (WIPO) sieht hingegen Malta in der „Bringpflicht“:

„In fact, preparations are well in hand to ensure that Malta would exploit its newly attained status. We have already learned some lessons and it is hoped that within a relatively short time Malta's administrative infrastructure will be geared and up-graded to enable us to participate in the European institutions and to leave our mark there, as we have already done elsewhere. It is therefore not too early to ask ourselves what contribution Malta can make to the EU. It is not only important to see what we can receive, but also to see what we can give." (BARTOLO in: THE SUNDAY TIMES, 18.07.2004, S. 14).

Zuletzt ein Zitat aus der erst nach der EU-Wahl erschienenen Neuauflage des Buchs "The Story of Malta" des Geographen Prof. DR. BRIAN BLOUET. Als abschließende Anmerkung vereint es Pro und Kontra:

"Integration into the EU will take years. In aggregate, the islands should benefit from increased economic interaction with Europe but there will be frustrating episodes as life in Malta is brought into line with European law and the Brussels regulations." (BLOUET (2004), S. 258).

112 Vgl. z. B. NeUE WeStFäLISCHE, Ausgabe vom 29.04.2004, S.3 sowie FAZ, Ausgabe vom 13.01.2003, S. 14

113 Vgl. u. a. DeR SPIEgeL, 28/2004, S. 132-134 


\section{Allgemeine Wirtschaftssituation der Republik Malta zum Zeitpunkt der EU-Erweiterung 2004 - Eine Kurz- darstellung:}

Malta galt in der Berichterstattung der deutschen Medien zum Zeitpunkt seines EU-Beitritts und davor als eine der "Gewinner-Nationen“ der neuen EU der 25 Mitgliedsstaaten ${ }^{114}$. Besonders das hohe relative ProKopf-Einkommen sowie die niedrige Arbeitslosenquote gaben den deutschen Printmedien den Anlass, Malta als wohlhabende Inselrepublik darzustellen: „(...) das Land gilt als das reichste aller EU-Beitrittsstaaten ${ }^{115 \text { “ }}$ (NeUE WESTFÄLISCHE, Ausgabe vom 29.04.2004, S. 3).

Die außerordentlich hohe Auslandsverschuldung des Landes wurde vielfach weniger beachtet. In den maltesischen Medien war sie hingegen der ausschlaggebende Kritikpunkt der Berichterstattung kurz nach der Europawahl 2004. Zudem wurde von der maltesischen Presse die immer noch niedrige Arbeitslosenrate des Kleinstaats nicht gewürdigt, sondern ihr leichter Anstieg im Vergleich zu den Vorjahren kritisiert.

Im Folgenden wird eine Kurzdarstellung von Maltas wirtschaftlicher Ausgangssituation zur Zeit des EUBeitritts stattfinden. Dies ist notwendig um die wirtschaftliche Stellung regionaler traditioneller Produkte im weiteren Verlauf der Arbeit verdeutlichen zu können.

Als wichtigste Literaturquelle fungierte dabei eine Studie zu Maltas Wettbewerbsfähigkeit. Sie wurde 2003 vom Institute for Islands and Small States Studies der University of Malta erarbeitet und 2004 in Form von zwei Bänden ${ }^{116}$ publiziert. Nähere Informationen dazu finden sich im laufenden Text. Die Studie hat schon kurz nach der Publikation den Eingang in die öffentliche Diskussion in Malta gefunden und wird derzeit als aktuellste und umfassendste Studie zu Maltas Wirtschaftssituation gewertet ${ }^{117}$.

\subsection{Wirtschaftliche Grunddaten Maltas im Sommer 2004}

Die Volkswirtschaft der Republik Malta ist weltweit eine der kleinsten. Betrachtet man die Gesamtsituation jedoch unter der Vorgabe „Bruttosozialprodukt pro Kopf“, wird klar, dass Malta den internationalen Vergleich nicht zu scheuen braucht. Seine wirtschaftliche Gesamtsituation zum Zeitpunkt des EU-Beitritts zeichnete sich allerdings durch die Diskrepanz zwischen der relativ positiven Entwicklung der inländischen Wirtschaft und der äußerst hohen Auslandsverschuldung aus.

Zur Mitte des neuen Jahrtausends zeigt Maltas Wirtschaftsentwicklung alle Anzeichen einer Krise ${ }^{118}$ von der zum Zeitpunkt der Schriftsetzung der vorliegenden Arbeit noch nicht abzusehen war, wie sie sich in der Folgezeit entwickeln wird. Offenbar ist die marode Weltwirtschaftslage teilweise für diese Krise verantwortlich ${ }^{119}$.

Die weitgehend positiven Wirtschaftsindikatoren wurden durch diese negative Entwicklung bislang noch nicht in starkem Ausmaß beeinflusst ${ }^{120}$ : Das relative Pro-Kopf-Einkommen Maltas lag 2004 bei $70 \%$ des Durchschnitts der „alten“ EU-Länder, eine der höchsten Raten unter den 10 Beitrittsnationen. Die Arbeitslosenquote, die in den letzten Jahren zwischen 3,7\% und 5,3\% schwankte, gehörte dagegen zum Zeitpunkt des EU-Beitritts zu den niedrigsten der neuen Mitgliedsländer. Die maltesische Wirtschaft stellt

114 Im folgenden Text kurz als „EU-25“ bezeichnet.

115 Vgl. z. B. NeUe WeStFälisChe, Ausgabe vom 29.04.2004, S.3; FAZ, Ausgabe vom 13.01.2003, S. 14

116 Briguglio, BARE, Cordina \& Endeley (2004) \& in Teilen BRIGUglio \& CoRdina (2004a)

117 Vgl. u. a. GRIP Magazine, H. 2/2004, S. 45

118 Vgl. BRIGUGLIO \& CORDINA (2004b), S. 58

119 Vgl. BRIguglio, BARE, Cordina \& ENDELEY (2004), S. 32 f.

120 Die folgenden Angaben beruhen auf BRIGUGLIO, BARE, CORDINA \& ENDELEY (2004), S. $21 \mathrm{ff}$. 


\section{0,04\% der Gesamtökonomie der EU-25.}

$40 \%$ des Bruttosozialprodukts (BSP) Maltas wurden im Dienstleistungssektor erwirtschaftet - ein Ergebnis der ausgeprägten Tourismuswirtschaft des Landes. Der öffentliche Sektor steht mit 23\% des BSP an zweiter Stelle. Seit den frühen Tagen der britischen Kolonialzeit hat das eine gewisse "Tradition" in Maltas Ökonomie ${ }^{121}$. Mit nur einem Prozent weniger $(22 \%)$ schlägt der sekundäre Sektor zu Buche - u. a. ist dies das Ergebnis erfolgreicher Projekte ausländischer Großinvestoren ${ }^{122}$ und der Malta Drydocks. Lediglich $5 \%$ des BSP stammen aus Fischerei, Landwirtschaft und Bauwesen. Die verbliebenen $10 \%$ resultieren beachtlicherweise - aus den Einkünften durch so genanntes „domestic property income“. In Malta bedeutet das, es handelt sich weitgehend um Einkommen aus Immobilienspekulationen.

Dem steht eine der geringsten Wirtschaftswachstumsraten der EU-25 (2003: 1,7\%) und eine sehr hohe Auslandsverschuldung gegenüber: Untersucht man das Verhältnis von Staatsschulden zu BIP, so ergibt sich ein Wert von rund $60 \%{ }^{123}$. Seit 1996 verging kaum ein Jahr ohne ein jährliches Haushaltsdefizit von $10 \%{ }^{124}$.

Ein weiteres Problem der maltesischen Wirtschaft ist das unausgewogene Verhältnis zwischen Exporten und Importen des primären Sektors, das insbesondere auch der EU im Zuge der Beitrittsbemühungen Maltas unangenehm auffiel ${ }^{125}$. Während die Agrarimporte im Jahr 2000 aus der EU 209,5 Mio. $€$ ausmachten, wurden aus der maltesischen Landwirtschaft nur 7,7 Mio. $€$ Warenwert in die bisherigen 15 EU-Staaten exportiert ${ }^{126}$.

Der sekundäre Sektor hingegen ist traditionell exportorientiert. Waren im Wert von 254,4 Mio. $€$ wurden im Jahr 2003 in die Bundesrepublik Deutschland exportiert. Im Gegenzug wurden deutsche Waren im Wert von 288 Mio. $€$ von Malta eingekauft. ${ }^{127}$ "Malta's small land area, coupled with its small domestic market, renders it difficult to reap the benefits of large scale production in domestically oriented industries. This tends to push up unit costs and inhibits the use of modern technology. For this reason, a large proportion of Malta's output has to be exported, giving rise to a very high degree of dependence on conditions in the rest of the world." (BRIGUGLIO (1994), S. 242). Dieser Umstand bedingt eine Behinderung der Diversifizierung der Wirtschaft. Maltas Export im sekundären Sektor konzentriert sich derzeit überwiegend auf Elektronik ${ }^{128}$.

Der tertiäre Sektor ist einerseits durch Dienstleistungen für den Massentourismus - der seit langem Maltas wichtigste Einkommensquelle darstellt ${ }^{129}$ - andererseits durch den öffentlichen Sektor - der bereits seit den frühen Jahren der britischen Kolonialherrschaft einen wirtschaftlich unvorteilhaften Bedeutungsüberschuss genießt - stark ausgeprägt. Dagegen ist der quartäre Sektor nur in minimalem Umfang vorhanden.

Im Bereich des tertiären Sektors kommt dem Tourismusbetrieb erhebliche Bedeutung zu, denn er ist nicht nur für sich allein gesehen schon einer von Maltas wichtigsten Devisenbringern, sondern von inm hängen auch zahlreiche Aufträge und somit Arbeitsplätze in anderen Branchen $a^{130}$. Solche Vernetzungen sind sehr vielfältig. Sie reichen von der Bauindustrie mit ihren Hotel- bzw. Ferienwohnungs-Neubauten sowie Renovierungs- und Reparaturarbeiten, über die Gastronomie - die in Malta erst durch den Tourismus als

121 Vgl. BLOUET (2004), S. 159 ff.

122 Dazu gehören z. B. Playmobil, Lloyd Schuhe, Mc Neal Schulranzen und Sony

123 FAZ, Ausgabe vom 13.01.2003, S. 14

$124 \mathrm{Ebd}$.

$125 \mathrm{Vgl}$. ASCHEMEIER \& CYFFKA (2004b)

126 Vgl. EUROPÄISCHE KOMMISSION (2001), S. 45 f.

127 Vgl. MÜNCHENER MERKUR, Onlineausgabe (www.merkur-online.de/nachrichten/wirtschaft/aktuell/art279,335462.html)

128 VgI. BRIGUGLIO \& CORDINA (2004c)

129 Vgl. u. a. Besenthal (1994), BRiguglio (1996), Pollacco (2003), Aschemeier (2005)

130 Vgl. Pollacco (2003) sowie Aschemeier (2005) 
eigenständiges Element der nationalen Ökonomie entstand ${ }^{131}$ - bis hin zur Produktion von Kunsthandwerksartikeln und Lebensmitteln, die im Verkauf an Touristen Gewinne einbringen. Darüber hinaus gibt es noch viele weitere Beispiele, auf welche Weise der Tourismus als „Perpetuum mobile“ Maltas „Wirtschaftsmaschinerie“ in Gang hält.

\subsection{Ausblick und Prognose für Maltas Wirtschaftsentwicklung nach dem Beitritt zur EU und die Konsequenzen für die Vermarktung regionaler traditioneller Produkte}

2001 und 2002 war Malta die am langsamsten wachsende Ökonomie der 25 Staaten, die gegenwärtig die EU-25 bilden ${ }^{132}$. Das gleichbleibend geringe Wachstum, das sich auch im Sommer 2004 unverändert abzeichnete, führt langsam aber sicher zum Umdenken in Maltas Wirtschaftswelt: „There is a strong realisation among Malta's business community that the domestic market is saturated and presents relatively few opportunities compared to the substantially larger export markets. Exporting requires identifying and exploiting profitable market niches, which in turn, necessitates a cost-conscious culture in an international business environment that is becoming ever more competitive. For Malta this is all the more crucial because the country is not competing merely for export sales, but also for the attraction of foreign investment." (BRIGUGLIO \& CORDINA (2004), S. 59).

Die unvorteilhafte Kombination aus nachhaltig schwachem Wirtschaftswachstum, hoher Auslandsverschuldung und persistenten makroökonomischen Unausgeglichenheiten stellt in Malta zur Zeit das zentrale Problemfeld dar, das nicht mehr nur die größeren Betriebe betrifft. Auch kleine Familienunternehmen spüren derzeit zunehmend die Verschlechterung der ökonomischen Gesamtsituation.

Hinzu kommt eine Stagnation der touristischen Entwicklung, die bereits seit Jahren anhält und sich nicht wesentlich verbessert. Es hat den Anschein, dass sich die Touristenankünfte auf einen mehr oder weniger stabilen Level „eingependelt“ haben. Ein reelles Wachstum in diesem Bereich findet seit Mitte der 1990er in Malta nicht mehr statt ${ }^{133}$. Die Regierungsbehörden streben seit spätestens 2002 eine umfassende Reform des Tourismussektors an, die sich in Qualitätssteigerung und Nachhaltigkeit ausdrücken soll ${ }^{134}$. Eine ökonomische Studie von BLAKE, SINCLAIR \& SUGIYARTO (2003) extrapoliert für die Zeit nach Maltas Beitritt zur EU langfristig eine moderate Abnahme des im Urlaub ausgegebenen Geldes pro Malta-Tourist von ca. $2 \%{ }^{135}$. Dies wird insbesondere für die unmittelbar vom Tourismus abhängigen Wirtschaftszweige spürbare Konsequenzen haben.

Es ist nicht unwahrscheinlich, dass nach der ersten Phase der Exportorientierung der maltesischen Wirtschaft in den 1970 ern $^{136}$ sich in den kommenden Jahren des neuen Jahrtausends eine ähnliche Phase anbahnen könnte. Zu diesem Ergebnis kommen auch BLAKE et al. (2003). Diesmal könnte die Entwicklung jedoch auch von mittelständischen und kleinen Unternehmen getragen werden, die aufgrund der rasanten technologischen Entwicklungen der letzten Jahre im Bereich Online-Business nun auch am europäischen und globalen Handel partizipieren wollen ${ }^{137}$. Malta ist schon heute eines der exportorientiertesten Länder der Europäischen Union ${ }^{138}$.

131 Vgl. BESENTHAL (1994)

132 Vgl. BRIGUGLIO \& CORDINA (2004c)

133 Vgl. POLLACCO (2003)

134 Ebd.

135 Vgl. BLAKE, SINCLAIR \& SUGIYARTO (2003), S. 28

136 Vgl. Metwally (1977)

137 Dieses Vorgehen würde in Übereinstimmung mit den Richtlinien für mehr Wettbewerbsfähigkeit in Maltas Wirtschaft nach BRIGUGLIO, BARE, CORDINA \& ENDELEY (2004) erfolgen.

138 Vgl. BRIGUglio \& CORDINA (2004b), S. 61 und s. o. 
Der Verfasser deutet die Entwicklung als Vorteil für Maltas regionale traditionelle Produktpalette: Viele Waren können gegenwärtig auch von personalschwachen Betrieben ohne größeren Aufwand und nahezu problemlos über die von der Topographie gesetzten, insulären Grenzen hinaus vermarktet und versendet werden. Der Schlüssel dazu liegt im Online-Business ${ }^{139}$.

Gerade im Bereich des technologisch innovativen Marketings ist in Malta noch viel nachzuholen. Dem Inselstaat wird aber auch großes Potenzial in diesem Sektor bescheinigt ${ }^{140}$ : "Malta scores relatively well in the field of information society, reflecting the wide use of computer and IT and the development of the relative infrastructure with a sore close to that of the 15 "old" member states." (BRIGUGLIO \& CORDINA (2004c), S. 45). Die Verbreitung der Internetnutzung ist jedoch noch ausbaufähig: $39 \%$ der maltesischen Bevölkerung haben bisher kaum oder keine Erfahrungen mit Internetnutzung gemacht ${ }^{141}$.

Auch der überaus größte Teil der lokalen Erzeuger hat noch nicht die Chance zum Online-Business ergriffen. Insbesondere im Bereich des Kunsthandwerks ist diese Möglichkeit erst durch einen einzigen Anbieter aufgegriffen worden ${ }^{142}$.

Der stagnierende Tourismusbetrieb, im Zusammenspiel mit der nachlassenden Bereitschaft der Reisenden, während des Urlaubs Geld auszugeben, könnte die Hersteller früher oder später zur Innovationsbereitschaft und möglicherweise auch zum Export ihrer Produkte „Zwingen“143. Ein solcher Schritt, der von vielen kleineren Betrieben häufig mit eher skeptischen Augen betrachtet wird, weil unberechtigterweise große Investitionskosten hinter dem Schlagwort „Online-Business“ vermutet werden, könnte sich letztendlich positiv für die Erzeuger von Maltas regionalen traditionellen Produkten auswirken. Dass ein solches Szenario realistisch ist, zeigt die Nachbarinsel Sizilien, die im Internet mit einem Angebot von rund 20 Online-Shops aufwartet, die regionale Kunsthandwerksprodukte und Lebensmittelspezialitäten vertreiben.

\subsection{Wettbewerbsstärken und -schwächen}

Maltas Wettbewerbsfähigkeit wurde im Jahr 2003 nach Angaben des World Economic Forum unter den Staaten der EU-25 an 19. Stelle ${ }^{144}$ gelistet. Unter den 10 neuen Beitrittsländern wird Maltas Wettbewerbsfähigkeit an vierter Stelle angeführt ${ }^{145}$. Weltweit konnte sich der Inselstaat im Rahmen von aktuellen Studien ${ }^{146} \mathrm{im}$ oberen Viertel der wettbewerbsfähigsten Nationen der Erde positionieren.

Die Ausgangsbedingungen für einen wirtschaftlichen Aufschwung werden dementsprechend als zufrieden stellend beurteilt, bedürfen jedoch eines umfassenden Reformschubs unter der Einbeziehung der LissabonAgenda der Europäischen Union, die anhand von Empfehlungen die Weichen für die weltwirtschaftliche Vormachtstellung des Europäischen Verbunds bis 2010 stellen soll $^{147}$.

$139 \mathrm{Vgl}$. ASCHEMEIER (2005)

140 Vgl. BRIGUglio \& CoRdinA (2004b) sowie BRIGUgLIO, BARE, CoRDINA \& ENDELEY (2004)

$141 \mathrm{Vgl}$. NSO (2002b)

142 Empire Arts \& Crafts Centre, Rabat (Malta). http://www.empiremalta.com

143 Vgl. auch AsCHEMEIER (2005)

144 Vgl. BRIGUgLIO \& CORDINA (2004b), S. 73

145 Vgl. BRIGUGLIO \& CORDINA (2004c)

146 Federation of Industry (2003); Blake, Sinclair \& Sugiyarto (2003); BRIguglio, BARE, Cordina \& Endeley (2004) sowie BRIGUGLIO \& CORDINA (2004b)

147 Vgl. BRIGUgLIO \& CoRdina (2004a) 
Die acht Hauptbestandteile der EU Lissabon-Agenda können wie folgt zusammengefasst werden:

- Schaffung der Informationsgesellschaft für alle EU-Bürger

- Weiterentwicklung von Innovation und Forschung

- Fertigstellung der Entwicklung des EU-Binnenmarkts sowie fortdauernde Neubewertung von staatlicher Wettbewerbs- und Subventionspolitik

- Entwicklung von Netzwerkindustrien, insbesondere in den Bereichen Telekommunikation, Transport und öffentlichen Einrichtungen

- Schaffung von effizienten und integrierten Finanzdienstleistungen

- Verbesserung der Wirtschaftslandschaft durch die Simplifizierung der Gesetzgebung und Ermutigung zu „Business Start-ups“

- Erweiterung der sozialen Einbindung durch die Wiederbeschäftigung von Arbeitslosen, der Weiterbildung im Beruf und dem modernisierten Sozialstaat

- Förderung nachhaltiger Entwicklung

Betrachtet man diese Auflistung, so muss angemerkt werden, dass Malta in den Bereichen Innovation, Forschung und Entwicklung wenig vorteilhafte Werte aufweist ${ }^{148}$. Das scheinbare Paradoxon zwischen Maltas gutem Rang im Bereich Informations- und Telekommunikationsvernetzung und einer gleichzeitig schlechten Einstufung bei Innovation, Forschung und Entwicklung wird von BRIGUGLIO \& CORDINA so erklärt: „This is breaking the link often observed in other countries between the development of the information society and the ability to innovate. (...). It is, however, to be pointed out that it is difficult for an economy of Malta's size to develop the critical mass necessary to undertake research and development activities to any significant extent. In the case of Malta the adaptability and creative skills in the labour force are perhaps more important contributors to competitiveness." (BRIGUGLIO \& CORDINA (2004c), S. 45).

Neue Technologien und Innovationen sind jedoch für einen Staat wie Malta besonders wichtig. Sie ermöglichen es, sowohl kleinen wie großen Betrieben viele Unzulänglichkeiten des wirtschaftlich unvorteilhaften kleinstaatlichen Daseins zu umgehen. So ist durch Online-Business z. B. nicht nur der Bestell- und Versandvorgang optimierbar, sondern es erweitert sich zudem auch der Kreis potenzieller Kunden. Das Internet ist weltweit verfügbar. Somit werden auch im Internet angebotene Produkte weltweit verfügbar. Nach Statistiken der Malta Tourism Authority (MTA) sucht ein Großteil der internationalen MaltaReisenden vor allem im Internet nach weiterführenden Informationen über die Inselgruppe: „Thirty-six per cent of travellers to Malta and Gozo use the internet to find information about the islands (...). " (DEBATTISTA in THE TIMES OF MALTA (Beilage: i-tech), Ausgabe vom 22.07.2004, S. 2). Legt man diese Zahlen zugrunde, so informieren sich jährlich ca. 432.000 Menschen im Internet über die Maltesischen Inseln.

Die Vorteile für regionale traditionelle Produkte liegen auf der Hand: Ein kleiner strukturell eingeschränkter Binnenmarkt kann mit wenig Aufwand zu einem globalen „virtuellen Verkaufsstand“ ausgebaut werden. Maltas bereits bestehende Exportorientierung kann dabei helfen, da oft schon erschlossene - aber noch nicht ausgelastete - Märkte vorliegen.

Der Inselstaat begäbe sich damit allerdings weiter in die Abhängigkeit von weltwirtschaftlichen Entwicklungen, sodass ein neu errungener Erfolg im Exportbereich relativ instabil wäre und leicht ins Wanken geraten könnte. Im Rahmen der EU wird Maltas Rolle als kleinster Mitgliedsstaat helfen, diese Gefahren von Zeit zu Zeit abzufedern. Auszuschließen sind sie jedoch nicht. Malta weist damit das typische Paradoxon der Globalisierungsdebatte auf, in dem die globale Öffnung der Märkte sowohl negativ wie auch positiv beurteilt werden kann ${ }^{\uparrow 9}$.

148 Vgl. BRIGUGLIO, BARE, CORDINA \& ENDELEY (2004) sowie BRIGUGLIO \& CORDINA (2004c)

149 „Liberalisten erheben Marktentscheidungen zum Modell individueller wie politischer Freiheit schlechthin. Sie interpretieren die "Globalisierung als Chance, die Märkte aus staatlicher Regulierung zu lösen - ein Freiheitsgewinn, der langfristig allen zugute kommen soll. Demokratietheoretiker befürchten dagegen, dass staatliche Politik immer weniger in der Lage ist, Marktversagen zu korrigieren." (MüLLER (2002), S. 43). Vgl. ferner: MüLLER, S. 43 ff. 
Aktuelle Studien bewerten Maltas Exportabhängigkeit jedenfalls nicht grundsätzlich negativ ${ }^{150}$. Vielmehr muss darauf geachtet werden, dass der „EU-Vorteil“ in einer günstigen Art und Weise genutzt wird. Dabei ist auch die hohe Abhängigkeit Maltas von Importen ${ }^{151}$ wichtig: Ähnlich wie bei den Exporten ergeben sich dadurch einerseits strukturelle Unsicherheiten, andererseits aber auch Vorteile, da man als "guter Kunde" ein „Druckmittel“ in der Hand hat. 15,2\% aller Importe Maltas sind deutsche Produkte ${ }^{152}$. 26,2 \% aller maltesischen Exporte gehen nach Deutschland ${ }^{153}$. Deutschland ist damit, neben Italien, Maltas wichtigster Außenhandelspartner.

BRIguglio, BARE, CoRdina \& Endeley gaben in ihrer Studie ${ }^{154}$ eine Zusammenfassung von Maltas Wettbewerbsschwächen und -stärken an, die wie folgt aussieht ${ }^{155}$ :

Maltas Wettbewerbsstärken:

- Offenheit gegenüber internationalem Handel

- Hohe Währungsstabilität der Maltesischen Lira

- Telekommunikationsinfrastruktur ist gut ausgebaut

- Bisherige ökonomische Performance

- Erfolge im Bereich der Steuerreformen

- Flexibler und gut ausgebildeter Arbeitnehmerpool

- Gesetzesinfrastruktur weitgehend kompatibel mit der EU-Legislative

- Qualitäts- und Umweltstandards überwiegend kompatibel mit den EU-Standards

- Nähe zum und Kenntnis im Umgang mit dem nordafrikanischen Markt

- Kulturelle Grundwerte in Übereinstimmung mit den europäischen Wirtschaftsnationen

- Relativ stabile politische Entwicklung

- Natürliche ${ }^{156}$ sowie in langjähriger Arbeit geschaffene Vorteile im Bereich des Tourismus

Maltas Wettbewerbsschwächen:

- Schwächen im Bildungssystem

- Starrer Arbeitsmarkt, insbesondere im öffentlichen Sektor

- Keine Bereitschaft in der unternehmerischen Kultur Risiken zu tragen, Innovation zu wagen und neue Exportmärkte zu erschließen

- Exzessive ${ }^{157}$ Interventionen der Regierung in ökonomische Angelegenheiten

- Ineffizienz bei der Nutzung von Geldmitteln im öffentlichen Sektor

- Niedrige Verknüpfungstendenz zwischen den Industrien

150 Vgl. Briguglio, BARE, CoRdinA \& ENDELEY (2004), S. 22 ff.

151 Vgl. BRIGUGLIO (1994), S. 242 f.

152 Ebd., S. 244

153 Ebd.

154 Vgl. Briguglio, Bare, Cordina \& Endeley (2004)

155 Nach Briguglio \& Cordina (2004b) sowie Briguglio, BaRe, Cordina \& Endeley (2004).

156 Unter „natürlichen Vorteilen“ wird von den Autoren u. a. die klimatische Gunstlage der Maltesischen Inseln verstanden.

157 Der Ausdruck "exzessiv" ist originaler Wortlaut der Autoren. 
- Insuffiziente Forschungs- und Entwicklungsaktivität

- Natürliche Rohstoffarmut

- Limitierte Möglichkeiten, das Prinzip der economies of scale zum eigenen Vorteil zu nutzen, bedingt durch die eingeschränkte Größe des Binnenmarkts

- Insularität und damit erhöhte Kosten in vielen Bereichen

Beim Lesen der Wettbewerbsschwächen Maltas fällt auf, dass viele der verzeichneten Schwachstellen, als strukturelle Probleme aufzufassen sind, die - die nötigen Reformen vorausgesetzt - in einem absehbaren Zeitraum aus der Welt geschafft werden könnten. Es sind dies keine massiven irreparablen Schwächen außer der topographisch bedingten Insularität und Rohstoffarmut - die mancher vielleicht bei einem Kleinstaat erwartet hätte.

Die Wettbewerbsvorteile Maltas sind positiv zu bewerten: Währungsstabilität und funktionierende Exportwirtschaft sind nicht zu unterschätzen. Die Kenntnis und der tägliche Umgang mit den Besonderheiten des nordafrikanischen Markts verschaffen Malta eine Ausnahmestellung unter den EU-Staaten. Malta kann u.U. als „Brücke" zu den Märkten der südlichen Mittelmeer-Anrainer fungieren ${ }^{158}$. Informations- und Telekommunikationssektor sind verhältnismäßig gut ausgeprägt ${ }^{159}$.

BRIGUGLIO, BARE, CORDINA \& ENDELEY kamen in ihrer Studie zu einem Katalog „strategischer Direktiven“, die Maltas Wettbewerbsfähigkeit erhöhen und die genannten Wettbewerbsvorteile ausbauen sollen. Die Direktiven wurden im Rahmen einer Konferenz zur Maximierung von Maltas Wettbewerbspotenzial unter der Beteiligung von „(...) Maltese decision-makers in the public and private sectors“" BRIGUGLIO, BARE, CORDINA \& ENDELEY (2004), S. 95) beschlossen. Die zentralen Punkte dieses Direktivenkatalogs sind unterteilt in 10 Kategorien, von denen jede Kategorie einem wirtschaftswirksamen Bereich des politischen und sozialen Backgrounds von Maltas Ökonomie entspricht.

Die zehn Einordnungskategorien ${ }^{160}$ sind:

1. Wirtschaftspolitik

2. Gesetzgebung

3. Infrastruktur und Finanzsektor

4. Bildungssektor

5. Innovation, Forschung und Entwicklung

6. Öffentliche Verwaltung

7. Löhne und Produktivität

8. Probleme im Sozialbereich und beim Umweltschutz

9. Ausbeutung der Möglichkeiten, die der Markt bietet

10. Werbung für Investment

158 S. dazu auch MALTA TODAY, Onlineausgabe vom 23.02.2003: "Malta as Gateway to the South for EU Investors".

159 Vgl. dazu auch BRIGUGLIO \& CORDINA (2004c)

160 Nach BRIGUglio, BARE, CoRdinA \& ENDELEY (2004), S. $137 \mathrm{ff}$. 
Die in diesem Rahmen gegebenen Handlungsdirektiven wurden von den Autoren der Studie nochmals als „Main Proposed Implementation Arrangements“ zusammengefasst ${ }^{161}$. Diese Auflistung lautet wie folgt ${ }^{162}$ :

- Aufstellung eines nationalen technischen Wettbewerbsfähigkeitskomitees, um Wettbewerbsfähigkeit als fortwirkenden Prozess zu institutionalisieren und Kohärenzeffekte in seiner Implementation zu pflegen

- Verantwortlichkeiten identifizieren und Strukturen spezifizieren, um doppelte Anstrengungen zu vermeiden und Synergien zwischen Interessensvertretern zu maximieren

- Wettbewerbsfähigkeits- und Produktivitätsindikatoren entwickeln, wobei das nationale technische Wettbewerbsfähigkeitskomitee eine Schlüsselrolle bei diesen Bemühungen spielt und eng mit dem National Statistics Office (NSO) kooperiert

- Weitere Forschungsaktivitäten im Bereich der speziellen Voraussetzungen und Vorteile kleiner Ökonomien in Hinblick auf Produktivität und Wettbewerb

- Adäquate Finanzierung des nationalen technischen Wettbewerbsfähigkeitskomitees, um es zu befähigen, die Implementation der erarbeiteten Wettbewerbsstrategie zu überwachen

Bei der Betrachtung der aufgeführten Punkte ist zu bedenken, dass die Prozesse für jede einzelne der 10 Unterkategorien spezifiziert wurden.

Das „nationale technische Wettbewerbsfähigkeitskomitee“, von dem in den o.a. Punkten die Rede ist, ist im Umfeld der erwähnten Studie gebildet worden. Bislang ist jedoch noch unklar, ob seine Arbeit weitergeführt werden wird.

BRIGUGLIO \& CORDINA kamen in einem Zeitschriftenartikel, der ihre Studie in aller Kürze zusammenfasst, zu dem Ergebnis: "This simple analysis yields two evident prescriptions to unleash Malta's potential economic growth. One is to remove the constraints being posed by an unduly large public sector with significant pockets of inefficiency. The other is to focus critical attention to better develop Malta's most abundant and potentially productive resources, namely its human capital and its natural environment. These prescriptions are to be viewed within the context of a holistic drive to be inculcated at all levels of policy towards enhancing Malta's economic competitiveness, to the benefit of its society as a whole." (BRIGUGLIO \& CORDINA (2004c), S. 45).

161 Briguglio, BARE, CoRdina \& ENDELEY (2004), S. 140

162 Vom Autor der vorliegenden Dissertation vom Englischen ins Deutsche übersetzt 


\section{Auswertung einer touristischen Befragung zum Thema „regionale maltesische Produkte“}

Wie im weiteren Verlauf der Abhandlung zu zeigen sein wird, gibt es in Malta zahlreiche regionale traditionelle Produkte in vielfältigen Produktkategorien. Unter der einheimischen Bevölkerung werden diese gut aufgenommen und regelmäßig konsumiert. Doch wie steht es mit der touristischen Kundschaft? Ist sich diese wichtige Konsumentengruppe der Alleinstellungsmerkmale oder ganz einfach nur der Existenz der einzelnen regionalen traditionellen Produkte Maltas bewusst?

Da zu dieser Problemstellung bislang kein Datenmaterial zur Verfügung stand, wurde im Rahmen der Erhebungsphase angestrebt, eine eigene Datengrundlage zu schaffen. Als Methode kam eine quantitative Befragung der touristischen Kundschaft in Betracht. Aus organisatorischen wie finanziellen Gründen, konnte jedoch keine zahlenmäßig repräsentative Stichprobe erhoben werden. Bei ca. 1,2 Mio. Reisenden pro Jahr in Malta, hätte eine Stichprobengröße mehrere tausend Probanden umfassen müssen, damit die Repräsentativität in adäquatem Maße gewährleistet gewesen wäre.

Im Rahmen des „großen Geländekurses Malta“, den das Geographische Institut der Universität Göttingen im Frühjahr 2003 durchführte, wurde eine touristische Befragung begonnen und vom Autor bei späteren MaltaAufenthalten fortgeschrieben. Die Probandenzahl betrug letztendlich 131 durch Remote Sampling ausgewählte Personen. Aufgrund dieser Werte, wird die folgende Befragungsauswertung keine repräsentativen Ergebnisse liefern.

Sie kann u.U. aber Trends und Strömungen anzeigen, die von den Malta-Touristen in Bezug auf das Verständnis von regionalen traditionellen Produkten an den Tag gelegt werden.

Wie REEH in seiner Dissertation (2005) feststellte, können auch kleine Stichproben unter bestimmten Bedingungen aussagekräftige Ergebnisse liefern. REEH stellte dies für den folgenden Fall fest: Wenn bestimmte Kennwerte sowohl für die Grundgesamtheit als auch für die Stichprobe bekannt sind, können diese Werte unter bestimmten Bedingungen miteinander in Beziehung gesetzt werden. Das heißt, wenn die abprüfbaren Ergebnisse der Stichprobe annähernd oder genau mit den bekannten Daten der Grundgesamtheit korrelieren, kann die (zu) kleine Stichprobe jedenfalls als „Trendanzeiger“ gewertet werden.

Bei den demographischen Aspekten „Altersverteilung“, "Geschlechterverteilung“ und „Herkunft der Befragten" zeigten sich große Ähnlichkeiten der Stichprobe mit den offiziellen Tourismus-Statistiken des NSO, die in diesem Fall die Grundgesamtheit repräsentieren. Dies bedeutet, dass die Stichprobe als Trendanzeiger eine befriedigende Datengrundlage liefern kann. Im Übrigen ist es wichtig zu erwähnen, dass die Stichprobe im Rahmen der vorliegenden Schrift nicht in die wissenschaftliche Auswertung eingeht, sondern eher als Anstoß zur Diskussion und zur weiteren Forschung zu verstehen ist.

In diesem Zusammenhang ist es interessant nachzufragen, inwiefern sich die Malta-Touristen, die eine der wichtigsten Konsumentengruppen für den maltesischen Einzelhandel stellen, über die Produkte informieren, die sie im Urlaub erwerben. Es drängen sich Fragen auf wie: Fühlt sich der durchschnittliche Malta-Tourist gut informiert über die regionalen traditionellen Produkte an seinem Reiseziel? Würde er womöglich gern besser oder anders informiert werden? Was bestimmt seine Kaufentscheidung? Und vor allem: Was ist in den Augen eines Touristen überhaupt ein regionales maltesisches Produkt?

Diese aufschlussreichen Unbekannten wurden in dem erwähnten Erhebungsbogen abgefragt. Im nachfolgenden Text erfolgt die Auswertung dieser Ergebnisse.

Die folgende Auswertung ausgewählter Fragen aus der Erhebungsaktion erfolgt aus didaktischen Gründen nicht in der Reihenfolge, wie sie ursprünglich auf dem Fragebogen angeordnet waren ${ }^{163}$. 
Frage 1: „Fühlen Sie sich ausreichend über maltesische Produkte informiert?“

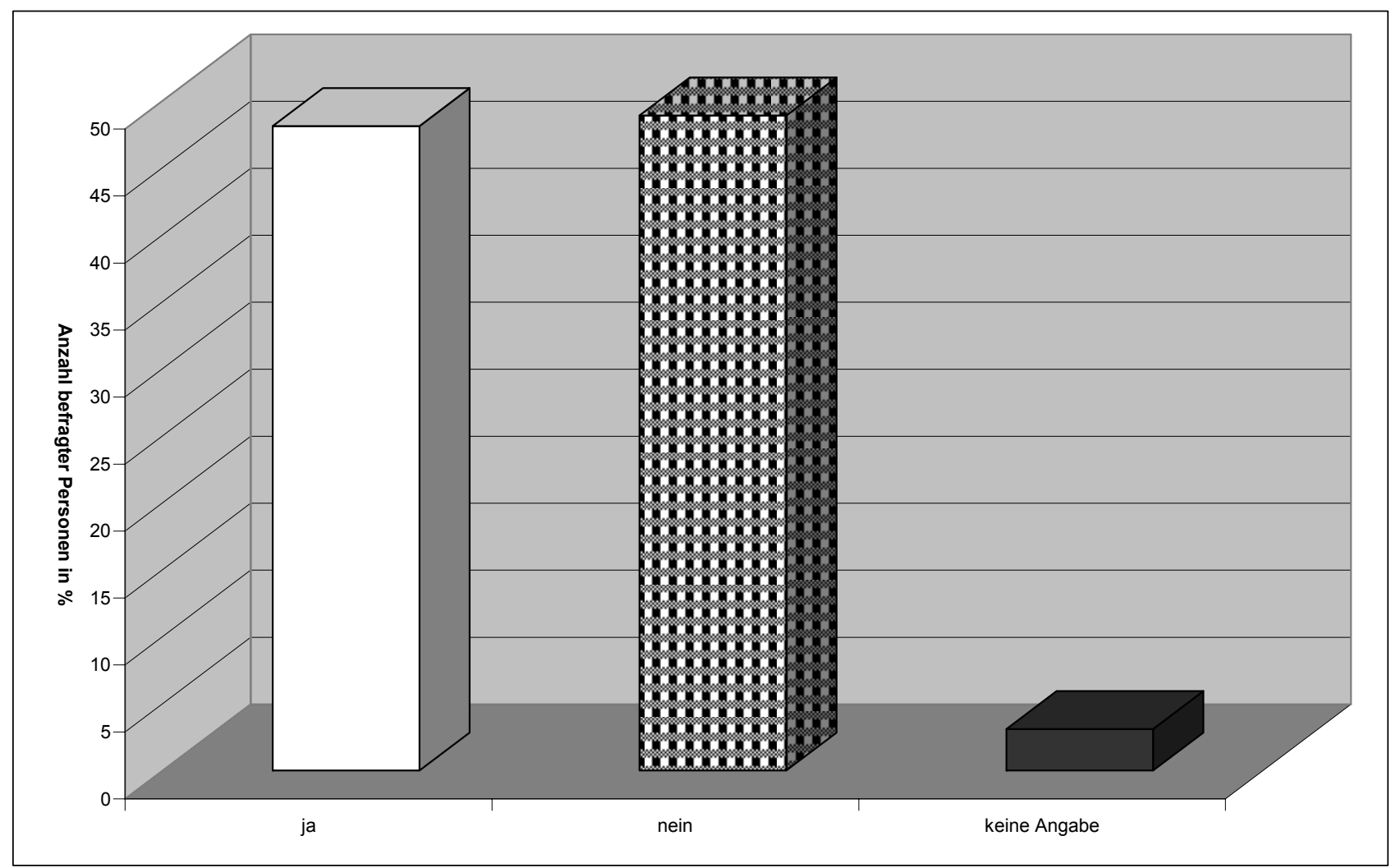

Abb. 3: Statistische Auswertung der Frage: „Fühlen Sie sich ausreichend über maltesische Produkte informiert?" Entwurf \& Grafik: ASCHEMEIER

Die Frage ist essenziell, wenn man ergründen möchte, nach welchen Kriterien die touristischen Konsumenten regionale traditionelle Produkte ableiten, definieren und letztendlich konsumieren.

Kunden, die nicht oder nur wenig über ein bestimmtes Produktsortiment informiert sind, können die Produkte, bei denen ein Informationsdefizit besteht, nicht kaufen. Der Grund ist, dass sie gar nicht wissen, dass es diese Produkte gibt oder sie wissen nicht, was sie mit bestimmten Angeboten anfangen sollen und entscheiden sich dann aus Unsicherheit gegen einen Kauf.

Abb. 3 zeigt, dass sich rund die Hälfte aller Befragten nicht genügend über maltesische Produkte informiert fühlt. Es besteht also offenbar, zumindest unter der befragten Stichprobengruppe, ein erhebliches Informationsdefizit in Bezug auf die Produkte ihres Reiselands.

Argumentationskette zu knüpfen, die anhand des in der Auswertung gewonnenen Datenmaterials ersichtlich wurde. 
Demgegenüber steht:

Frage 2: „Kennen Sie typisch maltesische Produkte?“

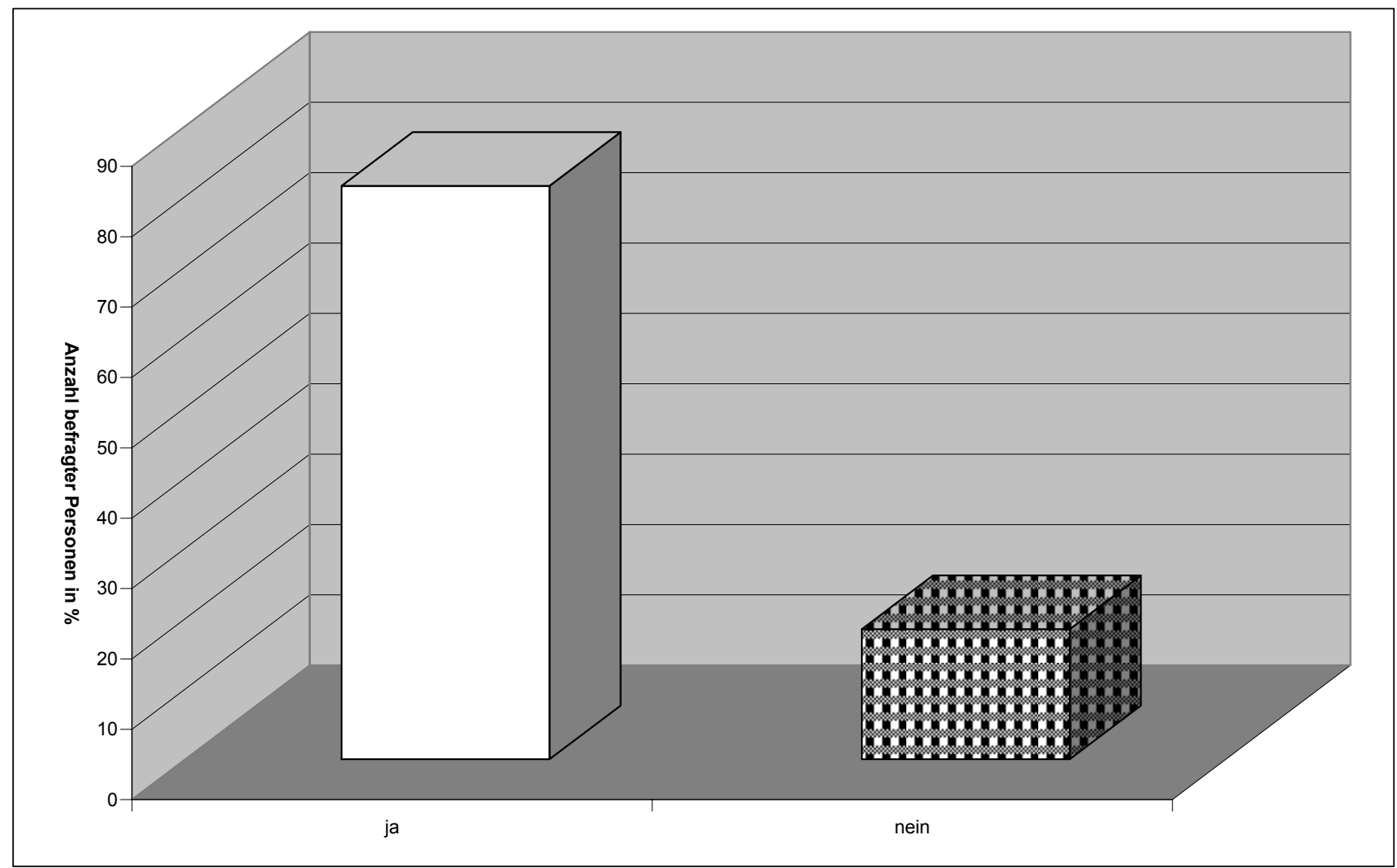

Abb. 4: Statistische Auswertung der Frage: „Kennen Sie typisch maltesische Produkte?“ Entwurf \& Grafik: AsCHEMEIER

Mehr als $80 \%$ der Befragten sahen sich dazu in der Lage, ein oder mehrere traditionelle maltesische Produkte zu identifizieren, wie Abb. 4 veranschaulicht.

Dies zeigt ganz allgemein, dass traditionelle maltesische Produkte den Reisenden auffallen. Sie begegnen Touristen stets und ständig am Urlaubsort. Dies führt dazu, dass fast jeder Malta-Reisende früher oder später einige „typische“ maltesische Produkte erkennt, die aufgrund ihrer ungewöhnlichen Alleinstellungsmerkmale im Gedächtnis haften bleiben.

Es stellt sich aber im Anschluss an diese Erkenntnis die Frage: Was ist in den Augen der Reisenden ein typisch maltesisches Produkt? 
Frage 3: „In welchen dieser Produktkategorien würden Sie am ehesten typisch maltesische Produkte erwarten?"

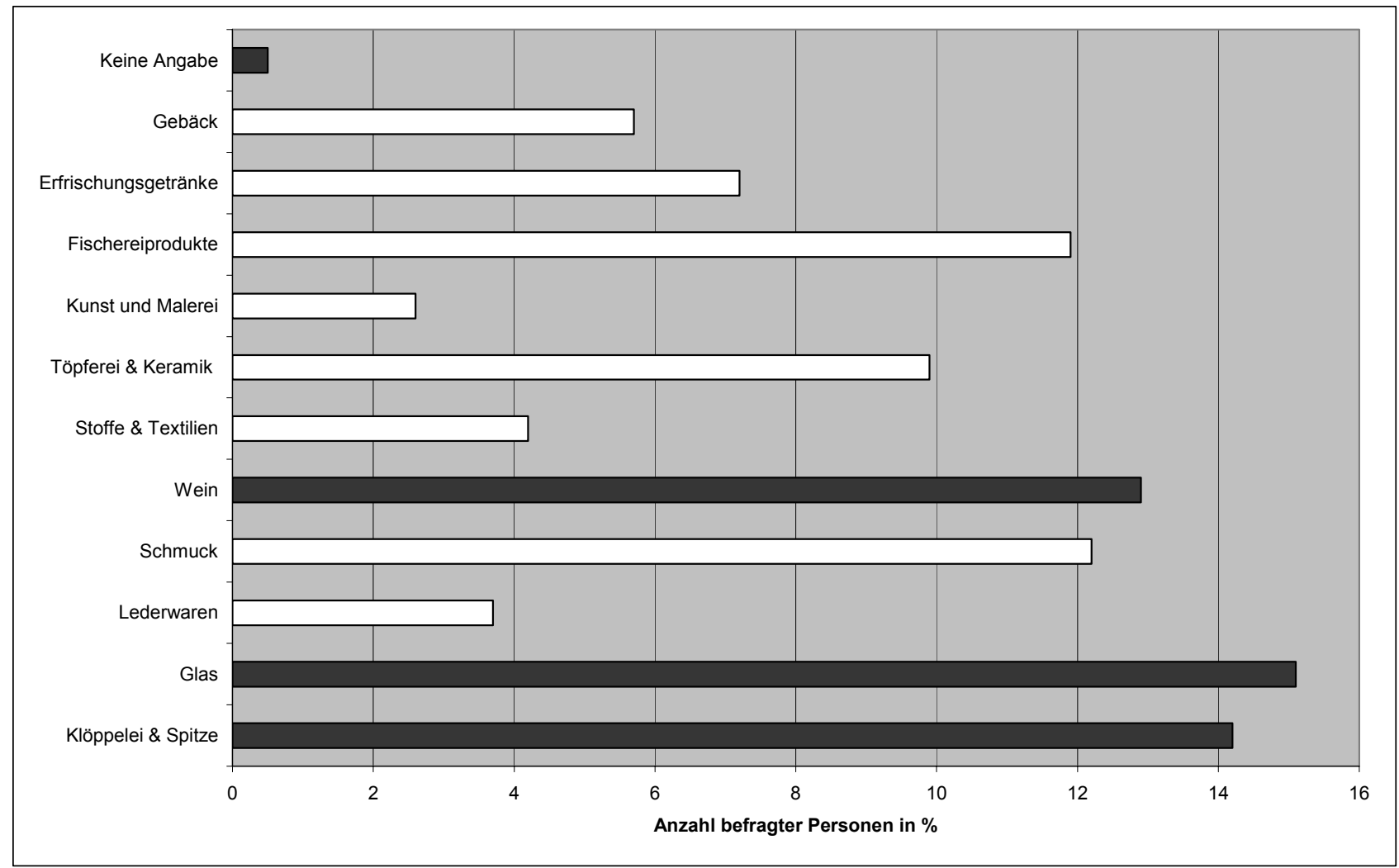

Abb. 5: Statistische Auswertung der Frage: „In welchen dieser Produktkategorien würden Sie am ehesten typisch maltesische Produkte erwarten?" Entwurf \& Grafik: ASCHEMEIER. Die drei am meisten beantworteten Kategorien wurden farblich hervorgehoben.

Die Ergebnisse in Abb. 5 zeigen: Typisch maltesische Produkte werden von den Touristen vor allem in den Bereichen „Glas" (15,1\%), „Klöppelei \& Spitze“ (14,2\%), „Wein“ (12,8\%), „Schmuck“ (12,2\%) und „Fischereiprodukte“ $(11,9 \%)$ erwartet.

Dies zeigt, dass offenbar eine verzerrte Wahrnehmung besteht, was den Begriff „typisch maltesisches Produkt" angeht. Im weiteren Verlauf der Arbeit wird sich zeigen, dass Glas - mit 15,1\% die meist genannte Kategorie zum Thema „typisch maltesisches Produkt“ - zu den artifiziell eingeführten Wirtschaftszweigen Maltas gehört und nicht zu den traditionellen Handwerkszweigen Maltas gehört.

Die äußerst traditionsreichen Produktkategorien „Töpferei \& Keramik“ $(9,9 \%)$, „Stoffe \& Textilien“ $(4,2 \%)$ sowie „Gebäck“ $(5,7 \%)$ sind in der touristischen Wahrnehmung deutlich unterrepräsentiert.

Für den weiteren Verlauf der Arbeit leitet der Verfasser hieraus die Notwendigkeit ab, Begriffe wie „typisch“, "authentisch“, „regional“ und "traditionell“ genauer zu hinterfragen und eine möglichst widerspruchsfreie Nomenklatur zu entwickeln. Dieser Prozess erfolgt im Rahmen der Kap. 9 bis 10. 
Des Weiteren fiel in der Auswertung auf, dass Frauen offenbar zugänglicher für maltesische Produkte sind als Männer.

Frage 4: („Fänden Sie es gut, wenn Sie maltesische Produkte auch in Ihrem Heimatland kaufen könnten?") sollte ermitteln, wie beliebt maltesische Produkte bei den Touristen sind und ob maltesische Waren Chancen im europäischen Export haben. Sind maltesische Produkte beliebt genug, damit sich der touristische Konsument wünscht, dass er sie auch zu Hause erwerben kann?

In Abb. 6 zeigt sich: Der Konsument kann gut auf maltesische Produkte im Alltagsleben verzichten (64,5\% Ablehnung), die Konsumentin dagegen spricht sich zu rund $58 \%$ dafür aus, dass maltesische Produkte auch zu Hause Einzug halten.

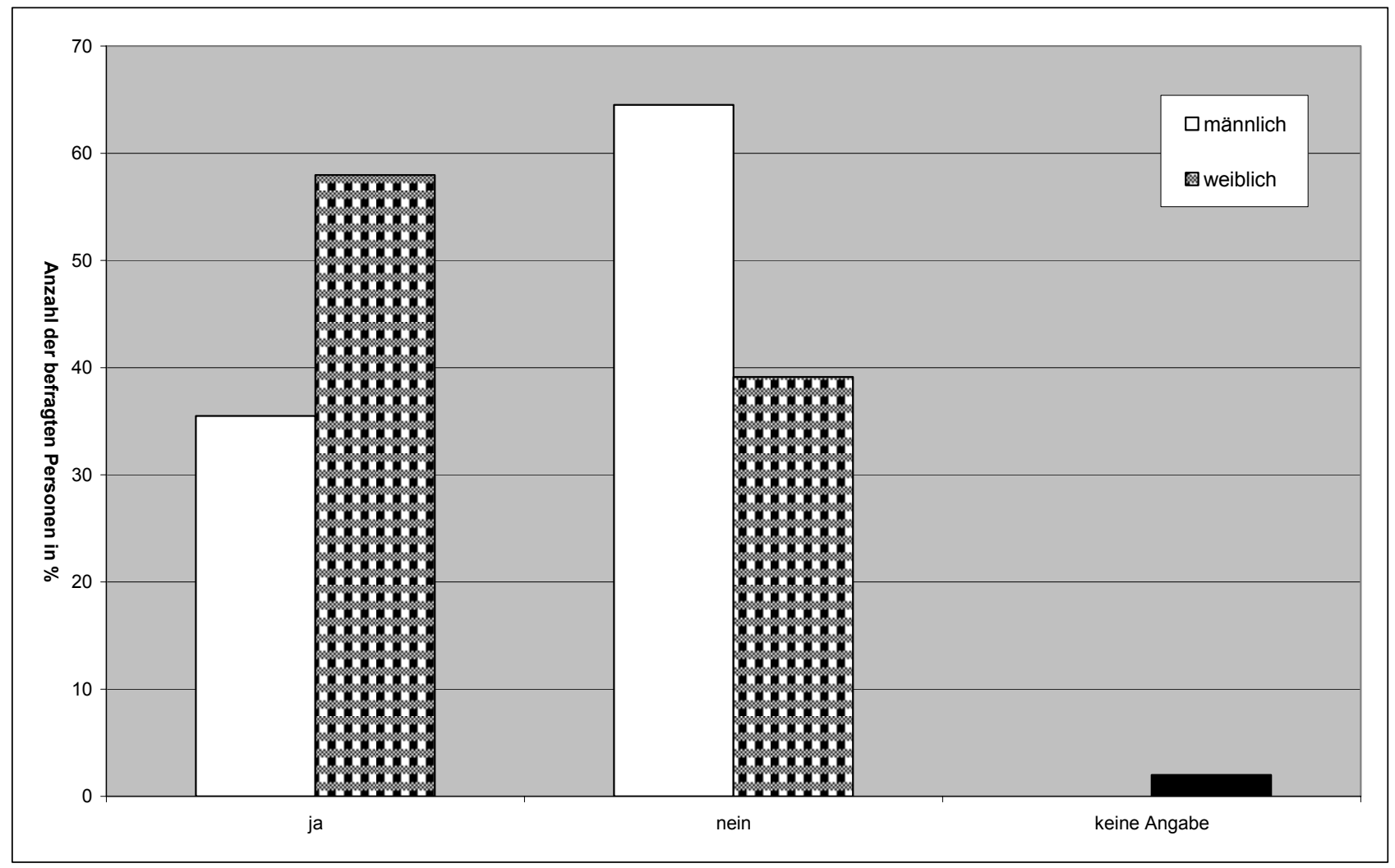

Abb. 6: Statistische Auswertung der Frage: „Fänden Sie es gut, wenn Sie maltesische Produkte auch in Ihrem Heimatland kaufen könnten?" nach geschlechterbezogenem Antwortverhalten. Entwurf \& Grafik: ASCHEMEIER

Dieselbe Frage wurde mit der Altersstruktur der Befragten verknüpft, was, wie in Abb. 7 zu ersehen ist, ebenfalls aufschlussreiche Ergebnisse erbrachte. 


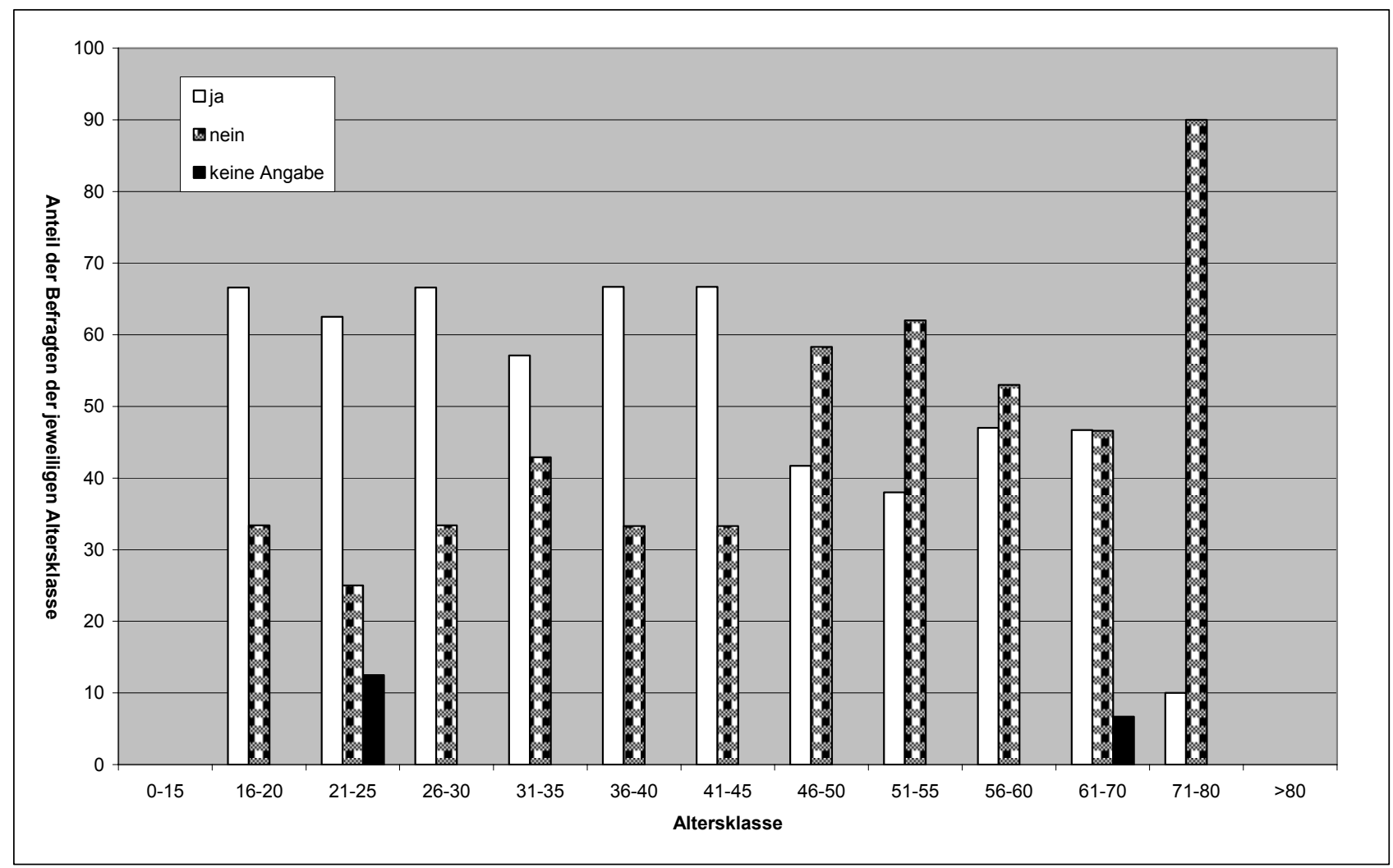

Abb. 7: Statistische Auswertung der Verknüpfung von Alterszusammensetzung der Befragten und der Auswerung der Frage „Fänden Sie es gut, wenn Sie maltesische Produkte auch in Ihrem Heimatland kaufen könnten?". Entwurf \& Grafik: AsCHEMEIER

Im Rahmen dieser Verknüpfung fällt auf, dass mit steigendem Alter offenbar auch die Skepsis gegenüber den maltesischen Produkten größer wird. In der Altersklasse der 21- bis 25-Jährigen würden 62,5\% der Befragten maltesische Produkte im Heimatland begrüßen. 25\% der Befragten dieser Altersgruppe würden maltesische Produkte im Heimatland nicht gutheißen, 12,5\% machten keine Angabe. Auf dieselbe Frage antworteten in der Altersklasse der 51- bis 55-Jährigen nur 38\% zugunsten maltesischer Waren, während sich $62 \%$ dagegen aussprachen, dass maltesische Produkte zukünftig auch im Heimatland der Reisenden erhältlich wären.

Das Phänomen der zunehmenden Skepsis gegenüber maltesischen Produkten mit zunehmendem Alter der Befragten wird noch deutlicher, wenn die Ergebnisse als Kurve visualisiert werden. In Abb. 8 ist deutlich erkennbar, dass ab der Altersklasse der 41- bis 45-Jährigen ein Meinungsumschwung stattfindet. Vorher verläuft die Kurve derer, die maltesische Produkte im Heimatland begrüßen würden mehr oder weniger stabil, während sie an dieser Stelle abfällt. Gleichzeitig ist auffällig, dass die Kurve für die ablehnende Haltung nahezu von Beginn an mit jeder neuen Altersgruppe etwas mehr ansteigt.

Damit ist als Trend erkennbar: Je älter die Befragten sind, desto ablehnender stehen Sie der Vorstellung gegenüber, maltesische Produkte bald auch im Heimatland kaufen zu können.

Setzt man in der Schlussfolgerung voraus, dass diese Ergebnisse auch ein Anzeiger für die allgemeine Beliebtheit der maltesischen Erzeugnisse sind - was wahrscheinlich ist - dann muss festgestellt werden, dass die Produkte der Maltesischen Inseln bei älteren Menschen weniger beliebt sind, als bei jüngeren. Dies wiederum hat Konsequenzen für spätere Überlegungen bzgl. der Vermarktungsstategien (vgl. Kap. 22), denn laut Angaben des National Statistics Office (NSO) sind rund die Hälfte der Malta-Reisenden ist über 40 Jahre alt und älter. 29\% aller Malta-Urlauber gehören Altersklassen über 55 Jahre $^{164}$ an.

\footnotetext{
${ }^{164}$ Nach NSO (2004c)
} 


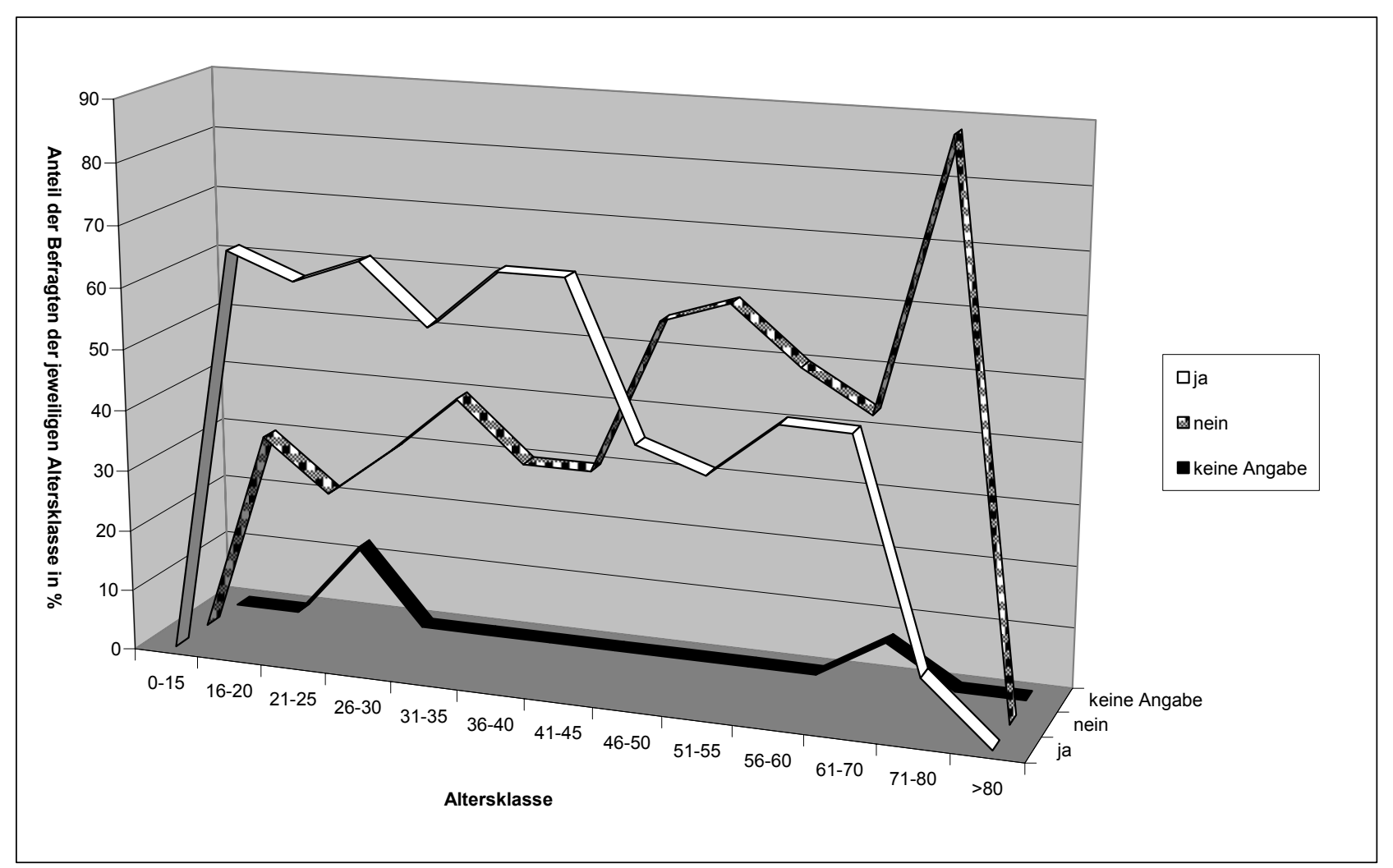

Abb. 8: Statistische Auswertung der Verknüpfung von Alterszusammensetzung der Befragten und der Auswerung der Frage „Fänden Sie es gut, wenn Sie maltesische Produkte auch in Ihrem Heimatland kaufen könnten?", hier als Kurve aufgetragen. Entwurf \& Grafik: ASCHEMEIER

\subsection{Fazit}

Diese kleine Auswahl von Statistiken lässt Trends und Entwicklungen erkennen, die Fragen aufwerfen, aber auch Einiges vorab beantworten.

Die Ergebnisse der Befragung können wie folgt als Trends zusammengefasst werden:

- Die Interviewten haben eine selektive Wahrnehmung von typisch maltesischen Produkten. Während einige Produktkategorien, wie z. B. „Glas“ und „Klöppelei“ sehr stark wahrgenommen werden, sind andere, wie z. B. „Gebäck“ und „Textilien“, in der Wahrnehmung durch die touristische Kundschaft offenbar benachteiligt.

- Touristen akzeptieren auch solche Produktkategorien als „typisch“, die erwiesenermaßen nicht zu den traditionellen maltesischen Produkten gezählt werden können.

- Maltesische Produkte sind bei Frauen beliebter als bei Männern.

- Maltesische Produkte sind bei Touristen bis zu einem Lebensalter von 45 Jahren beliebter als bei älteren.

- Mehr als $80 \%$ der Befragten kennen traditionelle maltesische Produkte, doch nur etwa $48 \%$ der Befragten fühlen sich ausreichend über maltesische Produkte informiert. Das heißt: Die Aufmerksamkeit der Kunden ist geweckt, doch es besteht ein Informationsdefizit. 
Alles in allem zeigen diese Daten, dass es offensichtlich Klärungsbedarf in folgenden Punkten gibt:

1. Wie können "echte“ regionale traditionelle Produkte definiert werden und aus welchen Wirtschaftszweigen stammen sie für das Fallbeispiel der Maltesischen Inseln?

2. Wie kann die Informationsvermittlung in Bezug auf regionale traditionelle Produkte optimiert werden?

3. Welche Bestandteile des Produktmarketings müssen verbessert, angepasst oder völlig neu etabliert werden, damit Ungleichheiten (z. B. bei der geschlechterbezogenen Produktbeliebtheit) ausgeschlossen werden können?

4. Welche regionalen traditionellen Produkte müssen gefördert werden?

5. Welche regionalen traditionellen Produkte sind besonders entwicklungsfähig?

6. Kann ein Produktkategorien-übergreifendes Marketingkonzept für maltesische regionale traditionelle Produkte entwickelt werden?

Im Folgenden wird diesen Fragen nachgegangen werden, wobei zunächst geklärt werden muss, wie der neu eingeführte Begriff des regionalen traditionellen Produkts definiert werden kann.

Eine Annäherung mit dem Ziel der Begriffsdefinition muss zunächst über eine Analyse der Strukturen von Maltas "Alltagskultur" erfolgen, damit erfasst werden kann, was der Sinngehalt der Begriffe „regional“ und „traditionell“ in Bezug auf die Maltesischen Inseln ist. Dieser Ansatz erfolgt im Rahmen von Kap. 8. 


\section{Kultur und Folklore Maltas}

\section{1 Äußere Einflüsse und indigene Volkskultur}

Folklore und Kultur der Maltesischen Inseln sind trotz der stark eingeschränkten Größe der Inseln außergewöhnlich vielgestaltig. Der maltesische Anthropologe, Soziologe und Folklorist ĠUŻÉ CASSAR PULLICINO schreibt: "The field of Maltese Folklore is so extensive as to be almost limitless. It is as vast as life itself. The subject allows enough room for a division of labour, if only enough workers were to come forward, (...), in order to turn up the virgin soil of which only the surface has yet been scratched." (CASSAR PULLICINO (1992), S. 14).

Aus dieser außergewöhnlichen Diversität von landestypischen Eigenarten ragt auch eine große Anzahl von regionalen Produkten heraus. Diese können zwar ihre Beeinflussung durch zahlreiche Eroberer und Besatzer nicht verleugnen, besitzen aber dennoch ein hohes Maß an Eigenständigkeit, weswegen man sie keinesfalls lediglich als "Imitate“ oder „landesübliche Erscheinungsformen“ von fremd eingebrachten Kulturrelikten verstehen darf. Vielmehr führten Eroberungen und Besatzungen von historischem Ausmaß, die die Geschichte Maltas nachhaltig prägten, erst zu einer sehr eigenständigen Volkskultur ${ }^{165}$ mit einem außerordentlich kleinen Verbreitungsgebiet: "The geographical position of the islands affected Malta's cultural tradition, since this tended to develop in relative isolation. (...) Malta was essentially a self-contained island with its own language, customs and archaic economy and for centuries, it managed to preserve its own form of civilization." (CASSAR (2000), S. 1 f.).

BLOUET führt nachvollziehbare Gründe für das Entstehen einer eigenständigen Volkskultur der maltesischen Bevölkerung an und sieht diese in der Herrschaftsperiode der Ritter vom Johanniterorden begründet: „Under the Order, Malta's sense of identity had grown. Daily the distinctively attired knights made in plain that they were the exclusive, autocratic, ruling class. The sharp social and political distinctions between the Maltese people and the Order meant that the islanders built upon their own traditions and culture, although assimilating new ideas (...)." (BLOUET (2004), S. 125 f.).

POLLACCO schreibt, der "Mix“ fremder und indigener Einflüsse habe als Ergebnis eine ungewöhnlich eigenständige maltesische Kultur hervorgebracht: „Since those early days of the first colonists Malta was made a colony several times by several powers: the Phoenicians, the Carthaginians, the Romans, the Arabs, the Knights of St. John, the French, until its last colonial masters, the British, granted it its independence in 1964 and departed conclusively in 1979. During this long period, Malta has accumulated a cultural heritage that is uniquely its own (...)." (POLLACCO (2003), S. 5).

Dieses maltesische Kulturerbe war im Lauf der Geschichte stets großen Wandlungen unterworfen, steht aber heute im Zuge beschleunigter Globalisierung ${ }^{166}$ und kultureller Angleichung an die mitteleuropäische Lebensart vor besonders großen Herausforderungen.

Durch die Entwicklung des Fernreiseverkehrs und der damit zunehmenden kulturellen Kontakte, wurde die Abgeschiedenheit der Inseln als einstmals dominierender Faktor beim Erhalt indigener Bestandteile der Volkskultur und Folklore unbedeutend. Dies bestätigt ZARB, der in seiner Arbeit über die folkloristischen Gebräuche der Maltesischen Inseln den 2. Weltkrieg als einschneidenden Wendepunkt angibt: „The Second World War was decisive in the changes brought about in patterns of behaviour and the conception of life in general." (ZARB (1998), S. 28). In Bezug auf die Etablierung des Massentourismus während der letzten vierzig Jahre, war eine klar erkennbare Änderung der sozialen Strukturen der maltesischen Gesellschaft inklusive ihrer Gebräuche und ihrer Folklore zu beobachten. Es ist anzunehmen, dass dieser Trend in

165 Zum Term „folk-culture“vgl. CASSAR PULLICINO (1994), S. 181: „The result has been an extremely variegated form of folk-culture constituting a distinctive element of Malta's national identity."

166 U. a. hervorgerufen durch Maltas Beitritt zur Europäischen Union im Mai 2004 
Zukunft nicht nur anhält, sondern sich noch beschleunigen wird, denn es ist „(...) zu berücksichtigen, daß nach Dieter Kramer jeder Tourismus kulturellen Wandel induziert, was auch heißt, daß jede Begegnung als Kulturkontakt zu bewerten ist und Einfluß und Veränderung bedeutet und zur Entstehung neuer kultureller Muster führen kann." (ScHRUTKA-RECHTENSTAMM (1994), S. 85).

\subsection{Zur Vergleichbarkeit des Untersuchungsraums mit anderen Regionen in Europa}

In der Literatur finden sich Beispiele, die zunächst vergleichbar mit den Maltesischen Inseln erscheinen. Erwähnt sei an dieser Stelle das Beispiel der Algarve in Portugal. Die Algarve ist ebenfalls eine räumlich eng begrenzte Region, die über längere Zeiträume ihrer geschichtlichen Entwicklung unter arabischem Einfluss stand. Heute weist der Landstrich einen hohen Grad an Eigenständigkeit, auch im Bereich der lokalen Traditionen und Produkte auf ${ }^{167}$.

Beispiele wie dieses legen den schnellen Schluss nahe, dass Malta nicht grundlegend andersartig sei. Die potenzielle Vergleichbarkeit von Regionen untereinander wird bekanntlich in der wissenschaftlichen Forschung nicht ausgeschlossen.

Der Verfasser gibt jedoch zu bedenken, dass Maltas Abgeschiedenheit als Inselgruppe und die damit einhergehenden ständig wechselnden Machthaber viel weit reichendere Folgen auf Tradition, Kultur und Produkte hatten, als dies in Bezug auf eine festländische Region angenommen werden könnte.

Ist Malta dann aber u.U. mit anderen mediterranen Inseln vergleichbar? Die Antwort muss lauten: nur eingeschränkt! Während sich die Vergleichbarkeit mit Sizilien, Kreta, Sardinien und Korsika schon allein wegen der Inselgröße schwierig gestaltet ${ }^{168}$, sind Inseln oder Inselgruppen mit zu Malta vergleichbaren Flächenausmaßen ${ }^{169}$ oft nachhaltig von einem großen festländischen (Kolonial-)Staat geprägt gewesen. Am ehesten könnte noch Zypern zum Vergleich mit Malta herangezogen werden, zumal sich in diesem Fall bestimmte historische Vorgänge vom Ansatz her ähneln. Die politische Teilung Zyperns ist jedoch ein einmaliger Vorgang in der Geschichte des Mittelmerraums, wirkt sich auf alle Lebens- und Wirtschaftsbereiche der Zyprer aus und schränkt deshalb die Vergleichbarkeit Zyperns mit den Maltesischen Inseln wieder in starkem Maße ein.

Die Maltesische Inselgruppe bleibt im Prinzip ein Sonderfall und muss auch dementsprechend behandelt werden. Die Vergleichbarkeit Maltas mit anderen europäischen Regionen und/oder Inseln ist nur in Teilbereichen möglich, wie auch der folgende einführende Abschnitt über die indigene Kultur der Maltesischen Inseln zeigen soll.

\subsection{Lokale Erzeugnisse als Bestandteil von Kultur und Folklore Maltas}

In der vorliegenden Arbeit werden verschiedene Erzeugnisse als Bestandteil der maltesischen Folklore verstanden. Worin die Berechtigung zu dieser Ansicht und die wissenschaftliche Herangehensweise liegt, bedarf einiger Erläuterungen:

Folgt man den Ausführungen des sizilianischen Folklore-Forschers GIUSEPPE PITRĖ, so ist Folklore eine Symbiose aus den verschiedensten Alltagsphänomenen einer Bevölkerung: „Fiabe e favole, racconti e leggende, proverbi e motti, canti e melodie, enigmi e indovinelli, giochi e passatempi, giocattoli e balocchi, spettacoli e feste, usi e costumi, riti e cerimonie, pratiche, credenze, superstizioni e ubbie, tutto un mundo palese e occulto, di realtà e d'immaginazione, si muove, si agita, sorride, geme a chi sa accostarvisi e comprenderlo. "i70 (PITRE, mündlicher Vortrag an der Universität zu Palermo vom 12.01.1911. Zitiert in

\section{Vgl. METZGeR (2004)}

168 „Große“ Inseln können sich autonomer versorgen und waren in der Vergangenheit besser zu verteidigen. Sizilien, Kreta, etc. wurden nie so nachhaltig, kurzfristig und intensiv bis in die Tiefen des Landesinneren von fremden Kulturen beeinflusst wie Malta, wenngleich eine bedeutsame Beeinflussung von außen in den genannten Fällen historisch unstrittig sein muss.

169 Ein „Paradebeispiel“ für einen solchen Fall wäre z. B. die Insel Elba vor der Westküste Italiens.

170 Englische Übersetzung (mit Ergänzungen) s. http://www.aboutmalta.com/gozo/folklore1.html. Stand 11/2003: „(...) legends, fables, popular stories, proverbs and sayings, songs, melodies and folk-ballads, nursery rhymes, folk-games and pastimes, children's words, riddles, folk-toys and playthings, superstitions, the occult, popular spectacles and festivals, rites and ceremonies, marriages, 
Additiv zu dieser umfassenden Behandlung des Terminus technicus, dabei mit Bezug auf die konkrete Situation Maltas, ergänzt CASSAR PULLICINO die kritische Bemerkung: "The majority of historians have concerned themselves more with the chronological, politico-ecclesiastical account of events, the petty quarrels between Grand Masters, politicians, bishops and inquisitors, the erection of monasteries and public buildings, than they have with the inner life of the people, with their domestic worries, their traditional callings, food and dress, their popular pastimes, beliefs, sayings, and usages, or the manner in which they reacted to such national calamities as Moslem raids and drudgery at the Turkish oar." (CASSAR PULLICINO (1992), S. 20).

Damit werden landestypische Lebensmittel, Gerichte, Spezialitäten, etc. dem Feld der Folklore zugeordnet. In einer späteren Veröffentlichung schreibt derselbe Autor: „Even a short stay in Malta will enable visitors especially those from a northern climate - to notice certain features of the local scene. (...) Besides, there are numerous items of local craft produce to choose from, including silver and gold filigree work, (...), and handmade lace (...)." (CASSAR PULLICINO (1994), S. 181). CASSAR PULLICINO zählt auch die landestypischen Handwerksprodukte zur überlieferten Folklore Maltas. Auch ZARB bindet beide Elemente der maltesischen Volkskultur in seine Arbeit ein und sieht sie als wesentlichen Bestandteil in einem Netz von psychologischen und sozialen Verflechtungen ${ }^{171}$.

LANFRANCO, offensichtlich auf PITRE aufbauend, bringt schließlich eine aktuelle Definition ein, die eine kompakte Verschmelzung der erwähnten älteren Definitionen repräsentiert: „The word Folklore has had several meanings over the years, but we now understand it to stand for all that the people of a country say, think and do in everyday life, and this includes their language, proverbs, tales, legends, devotions, superstitions, pastimes, song, music, costume, food, customs in birth, marriage and death, popular medicine, trades and crafts, and many other aspects, including utensils, tools, amulets and other objects." (LANFRANCO (2003), S. 2).

\subsection{Der Begriff „Folklore“ in der wissenschaftlichen Forschung}

GeORGE LAURENCE GOMME schrieb 1890 das erste Standardwerk der Folkloreforschung ${ }^{172}$, 44 Jahre nachdem der Begriff "Folklore ${ }^{173 ،}$ erstmals in einem Artikel des Literaturwissenschaftlers und Antiquariaten WILLIAM JOHN THOMS auftauchte ${ }^{174}$. Wichtiger jedoch war ALEXANDER HAGGERTY KRAPPES Abhandlung „The Science of Folk-lore“, womit Folklore als Term erstmals sowohl Untersuchungsgegenstand als auch Wissenschaftszweig umfasste.

Seinem viel zitierten Buch folgten zahlreiche weitere Veröffentlichungen bis in die jüngste Zeit ${ }^{175}$, womit aufgezeigt werden soll, dass Volkskultur und Folklore noch immer Gegenstand der wissenschaftlichen Forschung sind. Sie fallen heute aber überwiegend in die Fachbereiche von Ethnologie und Anthropologie und werden nur noch selten als eigener Wissenschaftszweig aufgefasst. In Deutschland war Folklore von jeher der Volkskunde beigeordnet. Gegenwärtig fällt die Thematik in Deutschland aber in den Bereich der Ethnologie sowie der Kultur- und Sozialgeographie. Auch international spricht man nicht mehr von der "Science of Folk-lore“ wie sie einst KRAPPE definierte. Dazu LANFRANCO: „Folklore nowadays forms part of the sciences of ethnology, anthropology and similar subjects." (LANFRANCO (2003), S. 2).

birth and death, popular beliefs, dress and costumes, domestic and religious usage, food and drink, local and traditional crafts, occupations both in villages and in cities, and other aspects of daily life." (ANTON F. ATTARD)

171 Vgl. ZARB (1998), u. a. S. 87-121

172 GOMME (1890)

173 Der Begriff „Folklore“, begründet von THOMS (1856), setzt sich aus den englischen Wörtern „folk“ und „/ore“ zusammen, könnte also ins Deutsche übertragen als „Volksüberlieferung“ übersetzt werden.

174 Begriffsschöpfung durch THOMS in einer regelmäßigen Kolumne in „The Athenaeum Journal“, Ausgabe vom 22.08.1846

175 Für Veröffentlichungen jüngeren Datums s. insbesondere DORSON (1972); CASSAR PULLICINO (1992 \& 1994); GEORGES \& OWEN JONES (1995), ZARB (1998) 
Die frühen Autoren (z. B. Gomme (1890); KRAPPE (1930) und O'SUILLEABHAIN (1942 \& 1963)) verstanden handwerkliche Produkte und Lebensmittel zunächst nicht als Untersuchungsgegenstand der Folklore, sondern grenzten diesen Bereich zur Volkskunde ab.

Andere (z. B. Roberts (1972); CASSAR Pullicino (1992 \& 1994); GEORges \& OWEN Jones (1995); ZARB (1998) und LANFRANCO (2003)) sprachen sich später für einen Einbezug von Handwerk und Lebensmittelproduktion in die Folkloreforschung aus. Dies mag daraus resultieren, dass sowohl Folkloreforschung als auch Volkskunde mittlerweile von anderen Wissenschaftszweigen assimiliert wurden und daher in diesem „großen Topf“ nun „verschmolzen“ sind.

Dieser disziplininterne Streit soll für die vorliegende Arbeit keine weitere Rolle spielen, da im Folgenden eine geographische Sichtweise vertreten wird. Der Verfasser hielt die einleitenden Bemerkungen für notwendig um die Bedeutung von Handwerk und Lebensmittelproduktion als Forschungsgegenstand in anderen Wissenschaftszweigen hervorzuheben, was zur selbstverständlichen Legitimierung des Forschungsobjekts der regionalen traditionellen Produkte in der Geographie führen sollte. 


\section{Die Debatte um Authentizität und Identität im Bereich der regionalen Produkte}

Die bisherigen Ausführungen haben gezeigt, dass die Zugehörigkeit zur Folklore/zur Volkskultur einer bestimmten Region ein wesentliches Merkmal vieler regionaler traditioneller Produkte darstellt. Als Bestandteil der regionalen Kultur fungiert ein Produkt auch als Träger von regionalen Identitätswerten, da seine Herstellung und sein Konsum über längere Zeiträume von großen Teilen der in der Region ansässigen Bevölkerung durchgeführt worden sind. Das Ergebnis ist: Das Produkt wird allgemein als "typisch" für die Region betrachtet und die Bevölkerung „identifiziert sich mit dem Produkt“.

Diese Überlegungen legen nahe, ein solches Produkt als „authentisch“ für die Region zu bezeichnen. Dass dieser Ansatz große Schwierigkeiten mit sich bringt, zeigten u. a. ROBERTS (1972); COHEN (1988); LITTRELL, ANDERSON \& BROWN (1993); HeNNIG (1997); MACCANNelL (1999) und MARKWICK (2001a).

LITTRELL et al. haben nachgewiesen, dass Käufer von touristischen Souvenirs quasi automatisch die Produktauthentizität mit regionalen Traditionen verknüpfen.

HENNIG hinterfragte inwiefern etwas als authentisch bezeichnet werden kann, was offensichtlich für den Tourismus inszeniert wurde. Er beschrieb das Beispiel eines italienischen Gasthauses, in dem der Gastwirt „in traditioneller Art und Weise“ Schinken unter die Zimmerdecke hängt ${ }^{176}$. Dieses Procedere wird jedoch eigentlich nur für die Touristen inszeniert um die Gaststätte in den Augen der Reisenden „authentisch italienisch“ erscheinen zu lassen: „Die Nachfrage nach Ursprünglichem ist viel größer ist [sic] als das Angebot; daher werden Bodenständigkeit und Originalität - für die Touristen kaum durchschaubar - bewußt erzeugt. Die urige Trattoria hängt die Schinken nur deshalb malerisch an die Decke, weil es den ausländischen Gästen so gefällt;(...)“(HENNIG (1999), S. 170).

Der Gastronom „benutzt“ demzufolge eine alte Tradition der Region um seinen eigenen Gewinn zu maximieren. Für inn spielt es keine Rolle ob die Tradition des „Schinken-Aufhängens“ an seinem Wohnort u.U. bereits als altmodisch oder überkommen gilt, oder, ob diese „Tradition“ sogar schon längst nicht mehr praktiziert und in diesem Fall vielmehr künstlich wiederbelebt wurde. Dies führt zu der Frage warum der Aufwand der Vorspiegelung und Inszenierung von Authentizität immer wieder auftaucht.

Der Tourismuswissenschaftler DEAN MACCANNELL geht noch einen Schritt weiter und behauptet: „Tourists demand authenticity (...)." (MACCANNELL (1999), S. 104). Doch warum?

Als Gründe dafür nennt MACCANNELL vielfältige Prozesse: Sie beinhalten einerseits das generelle menschliche Bedürfnis der Neugier in von MACCANNELL so genannte „back regions“ vorzustoßen: Regionen oder Orte, die normalerweise der Öffentlichkeit - in diesem Fall den Touristen - vorenthalten sind. Die Erfahrung in einer „urigen Trattoria“ mit Einheimischen (scheinbar) „zusammen zu tafeln“, ist eine innere Bestätigung dafür, dass man das „Authentische“ im Urlaub gefunden hat. Die Reisenden sind damit ihrem normalen Bedürfnis nach dem Vorstoß in eine „back region" nachgegangen, oder wie MACCANNELL es auch ausdrückt: Dem Bedürfnis nach dem „authentischen Erlebnis“: „Touristic Consciousness is motivated by its desire for authentic experiences, and the tourist may believe that he is moving in this direction, but often it is very difficult to know for sure if the experience is in fact authentic. It is always possible that what is taken to be entry into a back region is really entry into a front region that has been totally set up in advance for touristic visitation." (MACCANNELL (1999), S. 101).

Dieser „Trieb“ wird von Geschäftsleuten ausgenutzt: Authentizität wird künstlich inszeniert. MARKWICK betonte "diesen Aspekt, indem sie den Begriff „,backstage realities" statt „back region“ verwendete ${ }^{177}$. Zudem, so MACCANNELL, sei das Wort "Tourist“ in unserer modernen Wahrnehmung zunehmend negativ geprägt. Unter "Tourist" werde der "typische Pauschaltourist" verstanden, der sich in glanzvollen Hotelanlagen von der Außenwelt abschotte und wenn er sich dann doch nach außen begibt, in klimatisierten Bussen „durch

176 Vgl. HENNIG (1997), S. 170

177 MARKWICK (2001a), S. 432 
die Gegend kutschiert" werde. Fazit: "The term "tourist" is increasingly used as a derisive label for someone who seems content with his obviously inauthentic experiences" (MACCANNELL (1999), S. 94). Daraus kann bei Menschen, denen diese Art des Tourismus zuwider ist, eine regelrechte Protesthaltung entstehen ${ }^{178}$. Auch HENNIG ist sich dessen bewusst und schreibt von "Strategien nicht Tourist zu sein ${ }^{\text {“179 }}$.

LITTRELL, ANDERSON \& BROWN (1993) stellten klar, dass die Authentizitätsdebatte sich nicht nur auf die inszenierte Umwelt beschränkt, sondern sich auch auf die touristische Produkt- und Souvenirvermarktung übertragen lässt: „As part of their travels, tourists purchase crafts and other souvenirs that serve as tangible evidence of having found the authentic (...)."“80 (LITTRELL, ANDERSON \& BROWN (1993), S. 198). Sie haben anhand von touristischen Befragungen ermittelt, inwieweit Touristen selbst „Authentizität" in Bezug auf handwerkliche Erzeugnisse definieren. Das Ergebnis war, dass der Begriff „Authentizität" ein „fließender“ Begriff ist, der von Menschen unterschiedlicher Alterszusammensetzung, Herkunft oder Interessensausprägung auch unterschiedlich definiert wird: „(...) authentic crafts can be products that are unique or original, exhibit a handmade appearance and high quality workmanship, meet aesthetic criteria for color and design, are functional and useful, illustrate cultural and historical ties, are made with local materials by local craftpersons, and/or are sold with information about the craftperson or written evidence of genuineness." (LITTRELL, ANDERSON \& BROWN (1993), S. 210).

Setzt man in der Schlussfolge dieser Ausführungen voraus, dass "regionale Produkte“ im Sinne der vorliegenden Arbeit auch „authentische Produkte“ sein müssen, so könnte man nun die Annahme vertreten, dass eine sichere Bestimmung kaum noch möglich ist.

Es stellt sich einerseits die Frage, ob eine Ware, die nur noch für den Konsum durch Touristen inszeniert wird trotzdem noch regional und traditionell sein kann. Andererseits ist fraglich wie das Authentische an einem regionalen traditionellen Produkt klar erkannt werden kann, wenn es doch so viele unterschiedliche Auffassungen der Authentizität an sich gibt.

Es gibt somit bislang keine zufrieden stellende Definition in welchem Fall genau ein Produkt als „authentisch“ bezeichnet werden könnte. Die vorherrschende Meinung jedoch besagt, dass es zumindest notwendig ist, dass sich die einheimische Bevölkerung am Erzeugungsort mit dem Produkt identifiziert und es als Teil des kulturellen Erbes ihrer Region begreift. Um mit den Worten von CASSAR PULLICINO zu sprechen: Das Produkt muss Bestandteil der "folk-culture" der Region sein. Diese Meinung kann als allgemein anerkannt angesehen werden, wie auch verschiedene andere Autoren (vor allem MACCANNELL und LITTRELL et al.) ausführten.

Um die Lage in Malta anzusprechen, so muss gesagt werden, dass viel Trivial- und populärwissenschaftliche Literatur von maltesischen Autoren und Verlagen vorliegt, die sich ausschließlich mit maltesischer Folklore und traditionellen Produkten befasst. Allein durch die bloße Existenz dieser Bücher - von denen viele in maltesischer Sprache publiziert wurden - kann schon angenommen werden, dass ein Interesse von Teilen der maltesischen Gesellschaft am Themenbereich der „regionalen Produkte“ besteht.

Eine Umfrage des Autors im Rahmen einer geographischen Exkursion im Frühjahr 2003 hat zudem gezeigt, dass Malta-Touristen ohne größere Probleme in der Lage sind eine Meinung darüber zu äußern, inwiefern sie bestimmte Produkte als "typisch maltesisch“ betrachten oder nicht ${ }^{181}$. Dies zeigt, dass es offensichtlich etwas geben muss, was einige maltesische Produkte von anderen abhebt was sie im Sinne der Reisenden „authentischer" erscheinen lässt als andere.

178 Vgl. MacCANNeLL (1999), S. 98

179 Vgl. HENNIG (1999), S. 20 ff.

180 LITTRELL et al. führten ihre Untersuchungen unter sog. „,interstate-tourists“ in den USA durch, wiesen aber darauf hin, dass die Ergebnisse ihrer Forschungsarbeit universelle Gültigkeit beanspruchen können.

181 Vgl. Kap. 7 


\section{Regionale traditionelle Produkte - Definition und Erläuterung}

Mit den vorangegangenen Ausführungen wurde erwiesen, dass es offenbar bestimmte Merkmale gibt, die ein Produkt „authentischer“, "traditioneller" oder „regionaler" erscheinen lassen. Aufgabe des folgenden Abschnitts wird es sein, die Problembereiche Authentizität, Regionalität, Traditionalität und Identität in einer Definition so zu arrangieren, dass das Ergebnis eine genaue Einordnung eines Produkts in eine vorher zugewiesene Kategorie zulässt. Diese „Kategorie“ wird mit der Bezeichnung „regionales traditionelles Produkt" festgesetzt, da angenommen wird, dass sich in diesen Sektor besonders viele und wichtige maltesische Produkte einordnen lassen. Zudem versucht der Verfasser, eine Abgrenzungsmöglichkeit zu der unüberschaubaren Vielfalt von in Malta hergestellten Billigsouvenirs ${ }^{182}$ zu erreichen.

\subsection{Definition}

Es werden als "regionale traditionelle Produkte" fortan solche Erzeugnisse verstanden, die für den zu untersuchenden geographischen Raum neben ihrer Funktion als Produkt im Sinne des Ver- oder Gebrauchs auch Funktionen der nationalen bzw. regionalen Identifikation ausüben. Der Autor der vorliegenden Arbeit führte den Begriff im Jahr 2005 im Rahmen eines Fachbeitrags ${ }^{183}$ ein und definierte ihn wie folgt:

A regional traditional product is an object which is produced in a delimited spatial unit (= region). Its characteristics include both the production process and consumption, which contribute to the finding of a regional identity for the native population in such a way that the population of the region accepts the product as a carrier of values of the regional identity. Such a progress is not measurable in numbers, but can be presupposed if the product is a traditional ${ }^{184}$ part of the folklore of a region, enjoying sustainable popularity among the native population. ${ }^{185}$ A regional traditional product may be exported in larger quantities beyond the borders of the region as long as this does not have a negative effect on the character of the product as a carrier of values of identity. (ASCHEMEIER (2005), S. 240).

182 Darunter können z. B. verstanden werden: Spielzeugnachbildungen maltesischer Busse, Dgћajsas (maltesisches „Wassertaxi“), Luzzus (maltesisches Fischerboot), etc.; Nachbildungen maltesischer Bauwerke \& Tempel; Geldbörsen, Gürtel, Handtücher, etc. mit Aufdruck eines landesbezogenen Logos wie z. B. dem Malteserkreuz, usw.

183 Vgl. ASCHEMEIER (2005)

184 For a regional traditional product it is not necessarily important to look back on a very long tradition. A product can for example be introduced only a few decades ago, but it can already be regarded as "traditional." Brands like Coca Cola \& Pepsi, for example, are linked to the development of the youth or pop subculture of the $20^{\text {th }}$ century and have in such way developed a form of "tradition" of their own.

185 This can, for example, be suspected, if the product is featured in publications on „typical traditions" of a region, but it can also be presupposed when the mentioned product is so essential or portentous for the everyday life of the mentioned region, that life in that area could hardly be imagined without the product under research. 
Der Verfasser ergänzt im Rahmen dieser Dissertation eine deutschsprachige Version des Terminus technicus, den er als „regionales traditionelles Produkt“ festsetzt und der im weiteren Verlauf der Arbeit einheitlich Gebrauch finden soll. Als deutschsprachige Definition schlägt der Autor Folgendes vor:

Ein regionales traditionelles Produkt ist ein Erzeugnis, das in einer abgegrenzten geographischen Raumeinheit (Region) hergestellt wird. Sowohl sein Herstellungsprozess als auch sein Konsum wirken als Charakteristika, die dazu beitragen, dass sich die Einwohner dieser Raumeinheit in einer Art und Weise mit dem Produkt identifizieren, dass das Produkt als Träger regionaler Identitätswerte akzeptiert wird. Ein solcher Vorgang ist nicht in Zahlen messbar, kann jedoch vorausgesetzt werden, wenn das Produkt erwiesenermaßen zur Tradition ${ }^{186}$ bzw. zur Folklore einer Region gehört und nachhaltige Popularität aufweist ${ }^{187}$. Ein regionales traditionelles Produkt kann ohne weiteres in größeren Stückzahlen über die Regionsgrenzen hinaus exportiert werden, sofern dadurch seine Eigenschaft als Träger regionaler Identitätswerte nicht gestört wird.

Diese Definition steht somit klar den üblichen Definitionen von „regionalen Produkten“ entgegen, deren wesentliche Kernbereiche oft in anderen Schwerpunkten liegen: „Ein regionales Produkt ist dadurch definiert, dass seine Vorprodukte und deren Roh- und Hilfsstoffe weitestgehend aus der Region stammen, in der sie verarbeitet und vermarktet werden. Die Nutzungsphase kann durch ein ebenfalls regionales Angebot für Wartung und Reparatur des Produktes verlängert werden. Nach dem Gebrauch wird das Produkt recycelt und, soweit möglich, wieder als Vorprodukt in die Produktion zurückgeführt. Die Abfallbeseitigung erfolgt ebenfalls in der Region. Im Idealfall verbleibt ein so definiertes Produkt also "von der Wiege bis zur Bahre" in der Region." (NABU \& DLV (2002), S. 3. Eingebettet ist ein Bestandteil eines Zitates von KINDERMANN (1997)).

Die NABU-Definition bestimmt nicht, ob ein Produkt traditionell ist oder nicht. Sogar wenn die Produktentstehung und -einführung gänzlich in artifizieller Art und Weise erfolgte, kann das Ergebnis laut der NABU-Definition noch immer ein "regionales Produkt" sein, da in diesem Fall der Produktlebenszyklus und die Vermarktung innerhalb der Region als wichtiger angesehen wird als die regionale Identität.

Auch die bekannten EU-Denominationskategorien ${ }^{188}$, die für die Kategorisierung regionaler Produkte zunächst nahe liegend scheinen, sind nicht verwendbar. Sie decken ebenfalls nicht alle Aspekte ab, die in dem neuen Begriff "regionales traditionelles Produkt" enthalten sind.

Das Hauptproblem für den Forschenden, der sich der EU-Denominationen bedienen will, ist, dass die EUKategorien als rein politisches Instrument verstanden werden müssen. Sie sind Bestandteil der agrarpolitischen Vorgänge in den EU-Mitgliedsstaaten, indem die Europäische Union mit der Einführung der genannten Kategorien versuchte, eine Art "copyright" für bestimmte regionale Produkte zu schaffen. Das Ergebnis ist, dass nur einige wenige Produkte wirklich mit diesen Bezeichnungen versehen werden, was zudem als politischer Akt des Protektionismus erfolgt.

Aus diesem Grund können die EU-Zertifizierungen nicht als wissenschaftliche Benennungen akzeptiert werden, was sie demzufolge auch für die Verwendung beim wissenschaftlichen Arbeiten problematisch macht.

186 Ein regionales traditionelles Produkt muss nicht zwangsweise eine lange Tradition aufweisen. Ein Produkt kann z. B. bereits als "traditionelles Produkt" bezeichnet werden, obwohl seine Markteinführung erst einige Jahrzehnte zurückliegt. Marken wie Coca Cola, Pepsi oder (in Deutschland) Mars und Kinderschokolade haben in großem Umfang zur Entwicklung bestimmter Jugend- und Subkulturprozesse des 20. Jh. beigetragen und dadurch eine ganz eigene „Tradition“ entwickeln können, jedoch ist ihre Verbreitung oder Herstellung nicht regional begrenzt.

187 Dies kann z. B. dann vorausgesetzt werden, wenn das Produkt in Publikationen über „typische Traditionen“ einer Region auftaucht, doch es kann auch dann angenommen werden, wenn das bewusste Produkt so essenziell oder bedeutungsvoll für das alltägliche Leben der untersuchten Region ist, dass das Leben in dieser Raumeinheit kaum ohne das bewusste Produkt denkbar wäre.

$1881^{\text {st }}$ category: DOP (Denominazione di origine protetta), $2^{\text {nd }}$ category: IGP (Indicazione geografica protetta), $3^{\text {rd }}$ category: STG (Specialità tradizionale garantia). 


\subsection{Einschränkungen}

Wenn ein ursprünglich traditionsreiches Produkt zum gewöhnlichen, quasi belanglosen Gut wird - also die regionale Identität der Ware verloren geht - kann es auch nicht mehr als regionales traditionelles Produkt angesehen werden. Beispiele hierfür sind schwer zu finden, weil dies in vielen Fällen eine Definition des Regionsbegriffs nötig machen würde. Es wird jedoch zum besseren Verständnis folgendes Beispiel vorgeschlagen:

Die allseits bekannten „Pommes Frites“ sind eine belgische Erfindung. Von Belgien aus begannen sie ihren Siegeszug um die Welt, wurden in Amerika (fälschlicherweise) als „French Fries“ bekannt, und sind heutzutage immer und überall erhältlich. Ihr Charakter als regionales traditionelles Produkt Belgiens ist völlig verblasst, und kaum jemand würde heute noch behaupten wollen, dass "Pommes Frites" in Belgien wesentlich „authentischer" oder "traditioneller" erzeugt würden als anderswo. Auch ist es nicht anzunehmen, dass sich die belgische Bevölkerung zu großen Teilen mit dem Produkt identifiziert oder stolz auf die belgische Herkunft des Produktes ist.

\subsection{Zusammenfassung}

Der Vergleich zeigt, dass für maltesische Produkte der Begriff des „regionalen Produkts“ nicht ausreicht. Der Autor schlägt daher den Begriff "regionales traditionelles Produkt" vor. Dieser Begriff impliziert regionale Herkunft, Traditionalität/Authentizität und regionale Identität, schließt dabei aber eine nach außen gerichtete Vermarktung nicht mehr aus.

Identitätsstiftende Produkte in diesem Sinne sind damit ferner klar von subjektiven Wahrnehmungen (z. B. von der touristischen Wahrnehmung) abgrenzbar, die unter dem Begriff "regional“ mitunter auch Produkte akzeptiert, die eine falsche Authentizität vorspiegeln (z. B. Billigsouvenirs mit dem Aufdruck von regionsbezogenen Symbolen, Bauwerken, etc.). Solche von Außenstehenden oft fälschlicherweise als „landestypisch“ interpretierten Souvenirs können durchaus räumlich begrenzt auftreten. Ihnen fehlt jedoch in den allermeisten Fällen die breite Akzeptanz in der ansässigen Bevölkerung, weswegen sie nicht in derem alltäglichen Gebrauch auftreten und somit keine identitätsstiftende Wirkung entfalten können.

Regionale traditionelle Produkte müssen nicht unbedingt alten Traditionen entspringen. Wichtig für ihr Verständnis ist vor allem die räumlich begrenzte Erzeugung und die erwähnte identitätsstiftende Leistung ${ }^{189}$. Allerdings erstaunt es nicht, wenn die meisten regionalen traditionellen Erzeugnisse einer Region aus der Folklore entspringen, denn altüberlieferte Waren haben meist einen höheren Identifikationswert. Ihre Herstellung wird seit Generationen betrieben, und damit gehört auch der Konsum, z. B. im Familienkreis, zur (oft als Selbstverständlichkeit wahrgenommenen) Tradition.

Der indigene Ursprung eines Produkts bedeutet außerdem nicht, dass es infolge von Marketingbemühungen keine überregionale oder sogar globale Verbreitung erfahren könnte. Bestes Beispiel dafür sind einige regionale traditionelle Produkte aus Italien, z. B. „Parma-Schinken“, Parmesan-Käse oder Marsala-Wein, die trotz ihrer mittlerweile weltweiten Verfügbarkeit ihren regionalen Charakter durch eine stark verankerte Position in der Volkskultur der Erzeugergebiete bewahren konnten. Ob hierbei übrigens die Zertifizierung durch eine EU-Denomination bei einigen Objekten eine Rolle gespielt haben könnte, ist fraglich, da die "Labelisierungspolitik" der EU von vorn herein bereits enge Maßstäbe setzt, in denen regional nicht etablierte Produkte kaum eine Chance haben.

189 Ein gutes Beispiel für ein modernes regionales traditionelles Produkt ist z. B. die maltesische Kräuterlimonade „Kinnie“, die alle Bedingungen eines nationalen Identifikationsprozesses und dazu einer räumlich begrenzten Herstellung erfüllt. Vgl. Kap. 14.1.3. 


\title{
11 Maltesische Produkte in den Augen der einheimischen Bevölkerung
}

\begin{abstract}
Versteht man Maltas regionale traditionelle Produkte im Sinne der soeben vorgestellten neuen Definition, so muss unbedingt hinterfragt werden, inwiefern diese Erzeugnisse innerhalb der eigenen Bevölkerung akzeptiert sind oder nicht. So wären beispielsweise auch Waren vorstellbar, die zwar auf eine lange Tradition in der Herstellung auf den Maltesischen Inseln zurückblicken können, die aber im eigenen Land von einem Großteil der Bevölkerung nicht akzeptiert werden. Der Hauptabnehmer-Markt wäre dann z. B. der Tourismus, und es würde sich wieder die Frage nach der Authentizität des Produkts stellen (s. o.).
\end{abstract}

Es ist jedoch vom Ansatz her nicht einfach, die Beziehung der maltesischen Bevölkerung zu ihren Lebensmitteln und Kunsthandwerksartikeln zu veranschaulichen. Dennoch ist der Autor der festen Überzeugung, dass regionale traditionelle Produkte maltesischen Ursprungs eine Schlüsselrolle im Selbstverständnis der Inselbevölkerung spielen. Sie wirken als Identifikationsobjekte nach innen und nach außen. Dabei können im Bereich der Identifikationsleistung, für die die Produkte als Träger fungieren, zwei grundsätzliche Sichtweisen unterschieden werden: eine ablehnende und eine zustimmende Haltung.

\subsection{Die zustimmende Haltung}

Große Teile der maltesischen Bevölkerung schätzen und konsumieren regionale traditionelle Produkte ihres Heimatlands regelmäßig und aus eigenem Antrieb heraus. Das ist eine Identifikationsleistung "nach innen“. Auf der anderen Seite werden maltesische Erzeugnisse als wichtiger Bestandteil der Landeskultur verstanden. Sie werden in vielen Fällen direkt mit der nationalen Identität in Bezug gesetzt und dienen so als Zusammengehörigkeitsmerkmal für die maltesische Bevölkerung sowie als nach außen gerichtetes Einzigartigkeitsmerkmal.

Ein anschauliches Beispiel hierfür lieferte ein Tageszeitungsartikel in der HAMBURGER MORGENPOST vom 01.05.2004 ${ }^{190}$. In diesem Beitrag wurde über einen in Deutschland lebenden Geschäftsmann aus Malta berichtet. Der gebürtige Malteser STEPHAN AQUILINA war in dem Artikel mit einem Foto abgebildet, auf dem er regionale traditionelle maltesische Produkte - das Getränk Kinnie und das runde Gebäck Qagћaq ta'lghase $I^{191}$ - in die Kamera hält. Auf psychologischer Ebene identifiziert sich Herr Aquilina mit den Produkten aus der Heimat. Oder anders ausgedrückt: Die Erinnerung an regionale traditionelle Produkte, die in Deutschland nicht erhältlich sind, dient Herrn Aquilina in diesem Fall zur Identifikation mit der eigenen Herkunft als gebürtiger Malteser ${ }^{192}$.

In dieser Assoziation sind maltesische Produkte in vielerlei Hinsicht in der Bevölkerung nicht nur akzeptiert, sondern werden zusätzlich als Bestandteil ihres geschichtlichen und kulturellen „Erbes" verstanden. So bildet sich in der Wahrnehmung von außen ein „Abziehbild“ der inneren Realität, indem sich die komplexe Sozialstruktur und -geschichte eines Landes auf ein oder wenige typische Produkte reduzieren lässt und somit oft zum weithin akzeptierten "Klischee“ wird. Derartige „Beispiel-Klischees" für angeblich "typische“ Produkte gibt es viele: Niederlande und Schweiz: Käse, Großbritannien: Fish \& Chips, Irland: Whiskey \& Guinness, USA: Hamburger \& Hot Dogs, Italien: Pizza \& Nudeln, Frankreich: Baguette \& Rotwein, Spanien: Paella \& Wein, Russland: Borschtsch \& Wodka, Deutschland: Weißwurst \& Bier, usw. Und so ließe sich eben auch die Identität Maltas auf eine „Formel“" reduzieren, die z. B. heißen könnte: Kinnie \& Qagћaq ta'lgћasel aber z. B. auch Fenkata \& Ǵbejniet ${ }^{193}$. Es wundert daher kaum, dass der erwähnte MORGENPOSTArtikel auch in Malta Aufsehen erregte und mit einer Randnotiz in der Presse bedacht wurde ${ }^{194}$.

190 HAMBURGER MORGENPOST vom 01.05.2004. Beitrag: „Wir haben zu wenig Platz auf der Insel“

191 Zu beiden Produkten s. Einträge im Verzeichnis Maltesischer Produkte, Kap. 14

192 Dabei bildet Herr AQUILINA keine Ausnahme. Es ist bekannt, dass in Australien, dem Zentrum der größten ausgewanderten maltesischen Population, die Bräuche und Produkte aus der Heimat ebenfalls aufrecht erhalten werden. In Australien leben insgesamt über 150.000 gebürtige Malteserinnen und Malteser mit ihren Familien.

193 Vgl. Kap. 14.1 \& 14.1 .2

194 THE MALTA INDEPENDENT vom 05.01.2004, Beitrag: „Maltese featured in Hamburg newspaper“ 
Als sich Malta als touristisches Reiseziel in der Schweiz vorstellte, organisierte die Malta Tourism Authority ein Event mit Produktbezug: Durch Zürich fuhr eine „Tram“, geschmückt in den maltesischen Landesfarben. $\mathrm{Zu}$ bestimmten Uhrzeiten war Personal an Bord, das den Fahrgästen regionale traditionelle maltesische Lebensmittel als "Snack" zur Verkostung anbot ${ }^{195}$. Auch hierin zeigt sich zum wiederholten Mal der Bezug von nationaler Identität zum regionalen traditionellen Produkt. Der Autor behauptet, dass dieser Bezug, vielleicht aufgrund der geringen Staatsgröße Maltas, vielleicht aufgrund der großen Vielzahl regionaler traditioneller Produkte ${ }^{196}$, auf den Maltesischen Inseln besonders ausgeprägt ist.

\subsection{Die ablehnende Haltung}

Zusätzlich muss erwähnt werden, dass ein ebenfalls nicht geringer Teil der maltesischen Bevölkerung ein völlig konträres Einkaufsverhalten an den Tag legt. Sie kaufen lieber alles, was importiert ist, weil sie der Qualität der einheimischen Waren nicht vertrauen, oder weil sie diese als „altmodisch“ betrachten. Ähnlich wie bestimmte exklusive Lebensmittel und Produkte in Deutschland einst als "Yuppie-Zubehör" verschrieen waren, gibt es auch in Malta unter bestimmten Bevölkerungsgruppen einen Trend zum schwerpunktmäßigen Kauf ausländischer Waren, um auch im alltäglichen Leben auf gesellschaftliche "Statussymbole“ nicht verzichten zu müssen.

Eine vorsichtige Einschätzung der Ursachen dieses Minderheitenphänomens gab Frau DR. GIGQUEL, Geschäftsführerin des Maltesischen Wein- und Spirituosenherstellers MASTER WINE GROUP aus Naxxar, im Experteninterview:

„You have to go back to the History of Malta where for many years the Government had stopped the imports. And they had the idea that all basic products of the daily life could be produced and offered on the Maltese market. Back then they were offering on the market extremely low quality products. And from these times on some Maltese have attached the idea that what is historic is still there. As a local manufacturer of wine we are still fighting this mentality that grew back in the Seventies. Even now some Maltese have the idea "what is foreign is better". We are in a process of educating the people that what we are producing locally is now as good as the foreign product. But of course there is a certain layer of the society who understood a long time ago that Malta is being very competitive on certain sectors. Also in food it is very competitive." (DR. GIGQUEL im Experteninterview vom 08.06.2004).

\subsection{Effekte des EU-Beitritts auf die Haltung der Bevölkerung zu maltesischen Produkten}

Im Rahmen von Maltas EU-Beitritt am 01. Mai 2004 war im Nachhinein eine große Unsicherheit bezüglich des Fortbestehens einiger regionaler traditioneller Produkte spürbar. In Malta, das zur Zeit der EUErweiterung Ansätze einer ökonomischen Krise und steigende Arbeitslosenzahlen aufwies ${ }^{197}$, äußerte sich dies in der Angst vor der möglichen Dominanz ausländischer Produkte, insbesondere ausländischer Agrarprodukte. Ein Auszug aus einem Leserbrief an die Tageszeitung THE TIMES OF MALTA kann exemplarisch für die Stimmung im Land nach dem 01. Mai 2004 herangezogen werden: „In no way am I saying that joining Europe was the wrong thing to do...on the contrary joining Europe will help us achieve progress and protect worker's rights. Let us be true Maltese and continue to look after the Maltese industry. For every foreign product we buy, let us buy another or two local products. This will, no doubt, help our economy and keep our colleagues, relatives and friends in employment." (Leserbrief in THE TIMES OF MALTA vom 07.06.2004, S. 9).

Der Leserbrief führt vor Augen, dass nicht nur Nationalstolz und regional erzeugte Produkte in Malta miteinander in Beziehung gesetzt werden, sondern dass der Erwerb von maltesischen Produkten auch als ökonomischer Faktor verstanden wird. Beim Erwerb maltesischer Produkte versteht sich der Verfasser des Leserbriefs nicht nur als „Käufer“, sondern darüber hinaus als aktiver ökonomischer Faktor mit

195 Vgl.: ThE TIMES Of MALtA, Ausgabe vom 25.05.2004, S. 6

196 Vgl. Kap. 14

197 Vgl. u. a. BRIGuglio, BARE, CoRdinA \& ENDELEY (2004) 
nachvollziehbarem Einfluss auf die Sicherstellung von Arbeitsmarkt und Wohlstand seines Heimatlandes. Als Anmerkung sei noch erwähnt, dass der in Auszügen vorgestellte Leserbrief mit der Schlagzeile „Proud to be Maltese“ überschrieben war.

Alles in allem zeigen die Beispiele, dass die Identifikation mancher Malteser mit den regionalen traditionellen Produkten der Inselgruppe sehr groß ist. Zwar ist auch in Deutschland, Großbritannien, Frankreich oder in fast jedem europäischen Land der Faktor der Herstellung im eigenen Land oft ein wirkungsvolles Marketinginstrument. Die Identifikation der maltesischen Bevölkerung mit ihren heimischen Produkten erscheint jedoch in ihren Dimensionen außergewöhnlich.

\subsection{Fazit und Ausblick}

Regionale traditionelle Produkte gehören untrennbar sowohl zu Maltas Folklore als auch zur Ökonomie. Sie fungieren in vielgestaltiger Weise als Träger regionaler Identitätswerte. In den einheimischen Produkten spiegelt sich viel Nationalstolz der Malteser wider. Es sind Produkte, die in ihrer Art und Ausprägung oftmals einzigartig im gesamten Mittelmeerraum sind und von Reisenden als „typisch maltesisch“ betrachtet werden. Sie sind „authentisch“ und fest verankert im alltäglichen Leben der Bevölkerung. Gerade das macht sie auch als Vermarktungsobjekt für ausländische Touristen wertvoll, denn diese suchen - man erinnere sich an das Zitat von MACCANNELL (s. o.) - Authentizität ${ }^{198}$.

Auch für den Export könnten sich Vorteile durch die erwähnte Situation ergeben, da Produkte mit „urigem“ Lokalkolorit in der Vergangenheit oft zu „Exportschlagern“ wurden. In dieser Hinsicht sei nochmals der Hinweis auf den viel gepriesenen „Parma-Schinken“ erlaubt.

Traditionelle Produkte, die auf eine lange Geschichte von Entstehung und Erzeugung zurückblicken, wirken in vielen Fällen schon „von sich aus“ als Qualitätsgarant. Nach dem Motto: „Was seit sehr langer Zeit immer wieder produziert und konsumiert wird, muss auch qualitativ seinen Wert haben“, können solche Erzeugnisse, trotz geringerem Werbeaufwand als bei vergleichbaren modernen Produkten, gute Verkäufe erzielen $^{199}$.

Aber die Verankerung im maltesischen Alltagsleben hat als Kehrseite der Medaille bisher eine weitere Verbreitung über die Landesgrenzen hinaus oder einen ökonomischen Bedeutungsgewinn für maltesische Erzeugnisse verhindert. Die Produkte werden eben als „alltäglich“ wahrgenommen. Bis auf einschlägige Buchautoren $^{200}$ hat man in Malta noch nicht erkannt, dass das Allägliche im eigenen Land oft das Besondere in einem anderen Land, bzw. das Besondere für Besucher aus einem anderen Land sein kann. Dies ist um so erstaunlicher, weil festgestellt wurde, dass die Identifikation der maltesischen Bevölkerung mit heimischen Produkten sehr hoch ist. Das müsste in der Folge eigentlich eine Außenwirkung nach sich ziehen, einen „natürlichen Präsentationswillen“ für die Erzeugnisse, die man gerne konsumiert und auf die man stolz ist, dass sie im eigenen Land erzeugt werden.

Die Vermarktungsmöglichkeiten sind jedenfalls nahe liegend:

Die im Durchschnitt hohe Qualität der maltesischen Produkte trifft auf einen gesunden touristischen Markt und neuerdings auch auf einen sich ausweitenden EU-Markt. Die Produktionskapazitäten sind darüber hinaus in fast allen Bereichen noch längst nicht ausgeschöpft. Es ist unverständlich, warum nicht schon vor Jahren verstärkte Bemühungen um eine Steigerung des Exports, vor allem von Seiten der kleineren Hersteller, in Angriff genommen wurden oder warum bisher nur wenige und dazu eher halbherzige Vorsichtsmaßnahmen ${ }^{201}$ im Bereich des Produktschutzes unternommen worden sind.

198 Vgl. MACCANNELL (1999), S. 91-107.

199 Dies bestätigen auch Befragungsergebnisse unter Käufern touristischer Souvenirs durch LITTRELL et al. (1993).

200 Vgl. z. B. DARMANIN (1991) \& CREMONA (2003) in englischer Sprache, BORG (2000 \& 2001) \& LANFRANCO (2002) in maltesischer Sprache

201 Dazu könnten z. B. die official Standards der MSA (2003a \& 2003b) gezählt werden, sowie das „Genuinely Maltese“-Label des Malta Crafts Council, welches im weiteren Verlauf der Arbeit näher vorgestellt werden wird. Aber auch die geschützten Herkunftsbezeichnungen der EU dienen, wie bereits erläutert wurde, dem Produktschutz. 
Die im angesprochenen Leserbrief geäußerte Besorgnis betreffs ausländischer Importe ist eine Sorge. Jedoch könnte dies die kleinere Besorgnis sein, falls es sich zeigen sollte, dass Firmen aus dem Ausland (z. B. aus Süditalien) beginnen würden, maltesische Produkte zu kopieren. U.U. dürften in so einem Fall sogar regionale Herkunftsbezeichungen verwendet werden, wie z. B. "Gozitan Cheeselets", falls die zuständigen Akteure nicht frühzeitig um die Zertifizierung per EU-Denomination ${ }^{202}$ ersucht haben.

Seit Jahren bereits führen Schweizer Käsehersteller aus dem Emmental gerichtlich Klagen gegen italienische Käsereien, die ihren Käse ebenfalls „Emmentaler“ nennen, obwohl er nicht in der betreffenden Region erzeugt wird. Sollte nun ein ausländischer Hersteller den sehr einfach herzustellenden ${ }^{203}$ gozitanischen Käse Ǵbejniet tal-bżar in Malta vermarkten, bleibt nur zu hoffen, dass die regionale Bezeichnung „Gozitan Cheeselets" mittlerweile bei der EU als geschützte Herkunftsangabe beantragt wurde. Sonst droht den maltesischen Herstellern nicht nur Konkurrenz durch ausländische Produkte, sondern auch eine bizarre Situation der Konkurrenz einheimischer Produkte, die aber im Ausland hergestellt werden.

Nachdem die theoretischen Grundlagen der vorliegenden Untersuchung dargelegt worden sind, sollen im Folgenden die konkreten regionalen traditionellen Produkte vorgestellt und analysiert werden. Der Bereich der regionalen traditionellen Produkte ist in Malta in zwei Großbereiche aufteilbar: primäre Produkte des primären Sektors (d. h. die Feldfrüchte der maltesischen Landwirtschaft) und sekundäre Produkte (d. h. solche Produkte, die aus mehreren Zutaten zubereitet werden).

Zunächst werden im Folgenden die Primärprodukte der Agrarwirtschaft behandelt. Bevor dies geschieht, wird eine Einführung in die Geschichte der maltesischen Landwirtschaft erfolgen. 


\section{Maltas Landwirtschaft}

Malta weist, trotz seines über große Teile agrarisch geprägten Landschaftsbilds, eine in vielen Punkten eher spärlich ausgeprägte Landwirtschaft mit vielen Strukturproblemen auf. Dieses Paradoxon wird von dem Leiter des Plant Biotechnology Centre Malta, VICTOR FARRUGIA, wie folgt kommentiert: „Though the economy of the Maltese Islands can be by no means described as agricultural, agriculture has played a significant role in economic development." (FARRUGIA (2001), S. 109).

Ursprünglich dem Subsistenzprinzip dienend ${ }^{204}$ und durch Eroberungen immer wieder in ihrer Entwicklung zurückgeworfen, hatte die insuläre Landwirtschaft Maltas und Gozos ihre höchste Bedeutung in der ersten Hälfte des 20. Jh. bereits überschritten. Schon 1955 erwähnte BOWEN JONES einen drastischen Rückgang der Beschäftigung in der Agrarwirtschaft ${ }^{205}$ der Inselgruppe, dessen fortlaufende Beschreibung in späteren Aufsätzen verschiedener Autoren ein Gemeinplatz der Malta-Forschung wurde ${ }^{206}$.

\subsection{Abriss der historischen Landwirtschaftsentwicklung und Diskussion der historischen Ursachen der heutigen sekundären Position des Agrarsektors in Maltas Volkswirtschaft}

\subsubsection{Maltas Landwirtschaftsentwicklung von der Steinzeit bis zur Ritterherrschaft}

Der folgende Textabschnitt bezieht sich auf die historische Entwicklung der Landwirtschaft auf den Maltesischen Inseln. Zur historisch-politischen Entwicklung s. Kap. 4 sowie Kap. 5.

Malta war nachweisbar schon im Neolithikum besiedelt. Zahlreiche Funde und Tempelanlagen, darunter die teilweise zum Weltkulturerbe der UNESCO gehörenden Tempel Faġar Qim/Mnajdra, Ggantija, Fal-Tarxien und das unterirdische Hypogäum von Hal-Saflieni belegen dies. Bereits die frühen Siedler betrieben eine rege Landwirtschaft ${ }^{207}$. Der maltesische UN-Beauftragte SALVINO BUSUTTIL gibt in seiner Arbeit eine Einleitung, die Maltas landwirtschaftliche Bedeutung in Steinzeit und Antike zusammenfasst:

"The origins of agriculture in Malta are as old as man's presence on the archipelago. Judging by the quality, size and numbers of the megalithic temples on the islands, those origins are indeed remote. Tools and agricultural equipment discovered in Malta express neolithic man's vocation to husbandry and his dedication to agricultural life. That the Maltese were advanced in their knowledge of agricultural science and of related pursuits has been well documented. Roman historians refer to the islanders' prowess in producing cotton and honey. (...). Seafaring nations, and seafarers generally were, since antiquity, lured to Malta's shores not only by the sheltering natural coves and harbours but also by the abundance of agricultural produce to replenish their stores." (BUSUTTIL, S. (1993), S. 10).

Ausgrabungen im Bereich der Tempel brachten zahlreiche Keramikplastiken unterschiedlicher Größe hervor. Darstellungen einer beleibten Fruchtbarkeitsgöttin werden, auch in wissenschaftlichen Kreisen, als "fat ladies" bezeichnet $^{208}$. Auch Reliefdarstellungen von domestizierten Hausschweinen, Rindern und Ziegen wurden in den Tempeln gefunden ${ }^{209}$.

204 Vgl. NeHRing (1966), S. 87 f.

205 Vgl. Daten in BOWEN JONES (1955), später eingegangen in das umfassende Grundlagenwerk von BOWEN JONES; DEWDNEY \& FISHER (1961)

206 Vgl. z. B. NeHRING (1966), Biagini (1974), CHARLton \& BEELEy (1987), CHARLton \& BeEley (1993), MizZI (1993), BeSENTHAL (1994), SHORT (1997), FARRUgia (2001), PACE (2001), AZZOPARDI (2002), BEndIX, BENDIX \& REUDENBACH (2002), AschemeIER \& CYFFKA (2004) Sowie CYFFKA (2005)

207 Vgl. u. a. CASSAR (2000), S. 47-51

208 Vgl. z. B. GRECH (2003) \& BLOUET (2004), S. 22

209 Vgl. Evans (1963), S. 125 f. 
Dies sind weitere Indizien dafür, dass Malta schon sehr früh in der Menschheitsgeschichte Schauplatz landwirtschaftlicher Aktivitäten war, denn die Verehrung einer Fruchtbarkeitsgöttin deutet auf das Bitten um klimatisch günstige Faktoren für eine ertragreiche Ernte hin, während die Tierdarstellungen auf die frühe Verbreitung der Nutztierhaltung hinweisen.

\section{Der Archäologe TRUMP schreibt über die frühen landwirtschaftlichen Aktivitäten:}

„The earliest inhabitants were farmers, and to have reached Malta they must have been sailors too. They brought with them crops like barley, two primitive forms of wheat, emmer and club wheat, and lentils. Remains have been found at Skorba. Their boats were large and seaworthy enough for the transportation of domestic animals, like cattle, sheep, goats and pigs, doubtless securely trussed to prevent accidents." (TRUMP (1972) zitiert in POLLACCO (2003), S. 5)

Wie diese früheste Form der Landwirtschaft auf den Inseln ausgesehen haben könnte, beschreibt BLOUET anschaulich und stellt gleichzeitig eine Theorie zur Entwaldung der Maltesischen Inseln noch vor dem Beginn der klassischen Antike auf:

„The simple subsistence farmers, (...), probably utilised a system of shifting agriculture in which land was roughly cleared of vegetation, cultivated for a few years and then allowed to recover fertility by a natural process of bush and forest regeneration but we can assume that over time, on an island, shifting cultivation results in the clearance of woodland.

The early subsistence farmers probably lived in small communities in simple stone or wattle huts; there is no evidence of large villages. (...). Excavation at the skorba site, undertaken by DAVID TRUMP, revealed that the religion of the farmers was linked to fertility cults." (BLOUET (2004), S. 21).

Doch schon während der Entwicklung im Neolithikum mussten die maltesischen Erstbesiedler offenbar von außerhalb mit Waren beliefert werden, welche die natürliche Umwelt Maltas nicht bot. BLOUET (2004) vermutet eine Tauschkultur mit Sizilien und den liparischen Inseln, bei der Produkte der damals schon vergleichsweise weit entwickelten Textilherstellung und Wollbearbeitung Maltas gegen Obsidian, Feuerstein und Ocker eingetauscht wurden ${ }^{210}$. Diese Darstellung ist jedoch noch weitgehend unbewiesen. Es wurden zwar Funde der genannten Minerale in Malta gemacht, es gibt jedoch bislang noch keine Hinweise auf Textil- und Wollexporte in „retour“. EVANS (1963) vermutet hingegen umfangreiche Pilgerströme, die über Jahrhunderte von Süditalien und Sizilien aus in Richtung von Maltas mystischer Tempelkultur über das Mittelmeer segelten. Dies würde auch wertvolle Gegenstände wie Schneidewerkzeuge aus Obsidian erklären, die von EVANS als Opfergaben der Pilger gedeutet werden.

Die Bronzezeit ist gekennzeichnet durch ein Nachlassen der landwirtschaftlichen Aktivität ${ }^{211}$. Insgesamt existieren aber nur wenige archäologische Funde aus der Zeit, sodass sich kein verlässliches Bild der bronzezeitlichen Landwirtschaft in Malta zeichnen lässt. Es gilt jedoch als gesichert, dass in dieser Epoche die Tierhaltung weit wichtiger war als der Anbau von Feldfrüchten. Es wird angenommen, dass Wolle, Leder und fertige Textilien die Hauptprodukte der bronzezeitlichen Agrarwirtschaft waren.

Mit der fortschreitenden Entwicklung der Seefahrt mehrten sich nachweislich die Handelskontakte. Hierbei spielten vor allem phönizische Kaufleute aus dem Gebiet des heutigen Libanon eine Rolle ${ }^{212}$, die sich teilweise auf den Maltesischen Inseln ansiedelten ${ }^{213}$.

Es wird vermutet, dass die Phönizier, später die Karthager, Malta und Gozo hauptsächlich als strategische Handels- und Flottenstützpunkte nutzten. Das Handwerk dieser Periode war geprägt von Töpferarbeiten und Textilien. Es ist unwahrscheinlich, dass die Landwirtschaft wiederbelebt wurde. Nichtsdestotrotz trieben die Phönizier die bereits bestehende Imkerei auf Malta voran und verhalfen den Inseln somit zu einem Produkt, das in der gesamten antiken Welt bekannt wurde.

210 Vgl. BLOUET (2004), S. 24 f.

211 Ebd., S. 29 f.

212 Vgl. FRENDO, H. (1994), S. 2

213 Vgl. Bonanno, A. (1994), S. 93 
Die römische Herrschaftsperiode auf den Maltesischen Inseln ist gut dokumentiert. Viele Funde belegen, dass die Landwirtschaft auf Malta unter römischer Herrschaft in bisher nicht gekanntem Ausmaß aufblühte. Dabei wird angenommen, dass die Römer eine Art Gutsherrenverwaltung einrichteten. BLOUET schreibt: „The sites of over thirty Roman villas are known in Malta. The villas were part of agricultural estates which probably produced grains, grapes, olives, fruit and vegetables. (...). It may be that much of the arable land in the islands was cultivated in the estates of villas. No evidence has emerged so far of agricultural hamlets and villages occupied by small-scale peasant farmers." (BLOUET (2004), S. 37). Die Latifundienwirtschaft konnte für die römische Periode nicht nachgewiesen werden, es gibt jedoch Quellen aus der Zeit der sizilianisch-spanischen Herrschaft (s. u.), welche die Existenz einer Latifundialökonomie erwähnen ${ }^{214}$. Ob diese auf römische Ursprünge zurückgeht, oder erst später aus Sizilien „eingeführt“ wurde, ist bislang ungeklärt.

Die byzantinische Zeit Maltas ist so gut wie unerforscht ${ }^{215}$. Auch die über 200 Jahre andauernde arabische Herrschaft hinterließ kaum oder wenn, dann nur widersprüchliche Spuren. So gibt es verschiedene antike Berichte, die Malta zur gleichen Zeit einerseits als völlig entsiedelt darstellen - eine Inselgruppe die man nur aufsuchte um Honig zu „ernten“ oder verwilderte Esel und Schafe einzufangen ${ }^{216}$ - andererseits dagegen als florierende viel bevölkerte Kulturregion bezeichnen. Die Wahrheit wird wohl dazwischen liegen. Es gibt Hinweise auf eine dauerhafte arabische Besiedlung ${ }^{217}$. Einige Flurnamen stammen vermutlich aus der arabischen Zeit ${ }^{218}$, und ihre Bezeichnungen deuten auf landwirtschaftliche Aktivität hin.

Es wird vermutet, dass die Araber die Agrarwirtschaft hinsichtlich des Obstanbaus umgestalteten. Das Auftreten von Zitrusfrüchten in Malta wird erstmals während ihrer Herrschaftsepoche beschrieben. Außerdem wurden vermutlich Zuckerrohr und Baumwolle in größerem Maßstab angebaut. Die einst verbreitete Olivenöl-Erzeugung aber verfiel. Oliven waren zwar weiterhin vorhanden, jedoch eher als verwilderter Restbestand denn als kontrollierter Anbau. Der größte Verdienst der Araber in Malta war die Etablierung völlig neuer revolutionärer Bewässerungstechniken, die den Anbau von Obst und Zitrusfrüchten erst ermöglichten.

Die Araber wurden durch die Normannen in der Herrschaft abgelöst. Die Bevölkerung blieb aber weitgehend arabisch, was dazu führte, dass sich die landwirtschaftlichen Methoden nur wenig änderten. Zwar sind auch für diese Herrschaftsperiode die Quellen rar, jedoch gibt es eine gute Beschreibung der maltesischen Landwirtschaft in normannischer Zeit durch IDRISI, die in verschiedenen Auflagen des Buchs "The Story of Malta" des Geographen BLOUET wiedergegeben wird. Das wichtigste, im weitesten Sinne landwirtschafliche Gut war wiederum Honig: „Another account written by IDRISI, the famous Moslem geographer who served ROGER II, includes the statement that Malta „abounds in pasture, flocks, fruit and, above all, honey“. (BLOUET (1987), S. 36).

Die Erwähnung von Herden in der zuvor genannten Quelle deutet auf Weideflächen hin, hinter der der Ackerbau zurückstand. Weitere Einzelheiten aus dem IDRISI-Bericht gibt BLOUET in der neuesten Auflage seines Buchs: „From the IDRISI account we can infer three categories of land use on the islands. Firstly, in the spring-fed valleys, irrigation allowed the cultivation of good quality fruit and vegetables. Secondly, in the central part of the island of Malta, much land which is cultivated today, and has been for many centuries, was used for pasture and the production of livestock. Thirdly, to the north, in the region of ridges and plateau topped by hard, karstic, upper coralline limestone, the land was covered in herbs, wild flowers, and xerophytic (drought resistant) shrubby vegetation. (...). In the era of IDRISI, such land was used for rough grazing and the collection of honey produced by bees that thrived on herbs and wild flowers. Away from irrigated valleys land use was not intensive. There is no mention in Idrisi's account of sugar cane (...) or cash crops, like cumin, cotton and grains that were exported by the end of the thirteenth century. "(BLOUET (2004), S. 45).

214 Vgl. CASSAR (2000), S. 5

215 Vgl. u. a. ATTARD (2002)

216 Vgl. CASSAR (1994), S. 4

217 Vgl. BLOUET (2004), S. 40-45

218 Aber auch in diesem Punkt gibt es Unstimmigkeiten: Es ist möglich, dass einige Flurnamen mit landwirtschaftlichem Bezug erst später entstanden. Die ersten schriftlichen Quellen in denen sich Flurnamen belegen lassen, stammen aus dem 14. Jh. - also lange nach dem Untergang der arabischen Herrschaft auf Malta. 
Der Vertreibung der Normannen folgte in Malta eine chaotische Zeit von Überfällen, Eroberungen und kurzfristigen Herrschaftsepisoden, die in der sizilianisch-spanischen Besatzung der Inseln gipfelte.

Bemerkenswert waren in dieser Zeit die Christianisierung oder Ausweisung von Arabern und die Ansiedlung von Aufständischen aus verschiedenen Bereichen Italiens, Spaniens und Siziliens. Dies hatte nicht nur folgenreiche Auswirkungen auf die maltesische Sprache (die nun viele italienische Einflüsse aufnahm), sondern auch auf die Landwirtschaft der Inselgruppe. Ihre praktische Ausführung blieb aufgrund der rigiden Wasserknappheit der Inseln harte Arbeit, zumal einige der arabischen Bewässerungsmethoden wieder in Vergessenheit gerieten. Die maltesische Schriftstellerin CREMONA schreibt: „In the days before drip irrigation, pumps and hoses, watering the crops meant hard, hot hours of drawing water from wells and filling buckets from natural springs. Whole fields were painstakingly watered, bucketful by bucketful, in the early evening when the sun's rays were dying and the water could be allowed to seep into the earth throughout the night." (CREMONA (2003), S. 7).

Sklavenarbeit war weit verbreitet, und der königliche Gutsbesitz machte reichen Gebrauch davon, wie eine Quelle um 1241 erwähnt ${ }^{219}$. Für den Export wurden Getreide und Kümmel ${ }^{220}$ produziert und immer mehr Baumwolle, die mit der Zeit das wichtigste Element der maltesischen Landwirtschaft wurde ${ }^{221}$. Alle anderen Faktoren des Anbaus befanden sich auf unterem Subsistenzniveau.

Insbesondere der Baumwollanbau veränderte nicht nur das Bild der Landwirtschaft Maltas, sondern auch die Sozialstruktur: Der Anbau von Baumwolle ist sehr arbeitsaufwändig und ermöglicht darüber hinaus noch die Etablierung von Handelsberufen. Es gab nun Bürger, die nicht mehr direkt in den Anbau integriert waren, sondern als eine Art frühmittelalterliche „Sales Manager" fungierten. Sie teilten Baumwolle in Güteklassen ein, machten sie transportfähig und beförderten sie zum Hafen. Wie tief greifend diese Veränderung in der Gesellschaft war, ist nachvollziehbar: „Malta went from predominantly subsistence agricultural, with some grain beeing produced for export on the royal estates, to an economy in which small amounts of cash flowed to the countryside as a result of cotton production. Farmers continued to produce subsistence crops for their families but now a cash crop was widespread in the countryside. Another cash crop was cumin." (BLOUET (2004), S. 55).

\subsubsection{Unstimmigkeiten in der Darstellung der Landwirtschaft zur Zeit der Johanniterherrschaft}

Der heute spürbare Bedeutungsverlust der Agrarwirtschaft wird von einigen Autoren sehr früh in der geschichtlichen Entwicklung Maltas angesetzt, nämlich zur Zeit der Johanniterherrschaft. Der Autor der vorliegenden Dissertation versucht dagegen eine andere Sichtweise auf die Agrargeschichte Maltas zu eröffnen.

BESENTHAL ${ }^{222}$ und NEHRING ${ }^{223}$ gaben an, die Landwirtschaft sei aufgrund einer von den Johannitern erstmals etablierten Dienstleistungsgesellschaft - BESENTHAL nennt sie nach HUGHES „,fortress economy“ - teilweise bereits im 16. Jh. völlig bedeutungslos gewesen. Die Genannten sehen hier bereits, zumindest mittelbar, den Ursprung für den desolaten Zustand der Agrarwirtschaft im modernen Malta. NEHRING z. B. schreibt in seiner Länderkunde: „Besonders folgenschwer für die spätere wirtschaftliche Entwicklung der Inseln ist die Tatsache, daß sich zur Zeit des Ordens eine vorwiegend agrarisch ausgerichtete Bevölkerung - in einer von der Natur nur dürftig ausgestatteten Landschaft - mehr und mehr auf Dienstleistungen für eine fremde Macht (...) einstellte (...). Diese Wirtschaftsstruktur, so gewinnbringend sie zeitweilig sein kann, ist äußerst krisenanfällig, weil sie einseitig ist und ihr eigentlich die natürlich gewachsenen Grundlagen auf den Inseln selbst fehlen."(NEHRING (1966), S. 41).

219 Vgl. CASSAR (2000), S. 5

220 Kümmel ist bis heute ein wichtiger Bestandteil der Gewürzmischung Curry und war u. a. deswegen im Mittelmeerraum zur Zeit des florierenden Gewürzhandels sehr begehrt.

221 Vgl. BLOUET (2004), S. 55

222 Vgl. BeSENTHAL (1994), S. 37, S. 58 \& S. 146

223 Vgl. NeHRING (1966), S. $87 \mathrm{ff}$. 
Der Verfasser der vorliegenden Abhandlung vertritt die Meinung, dass die Agrarwirtschaft durch die Herrschaftsübernahme der Johanniter in Teilbereichen nicht abgebaut, sondern nachweislich gestärkt wurde, während sie vor dem Eintreffen der neuen Machthaber Überfälle und Raubzüge gegen die Inselgruppe sowie wegen der Bevölkerungsdezimierung aufgrund einer fortdauernden Malariaepidemie ${ }^{224}$ tatsächlich völlig darniederlag. BLOUET zum Zustand der Maltesischen Inseln beim Eintreffen der Johanniter: „From the Order's viewpoint Malta had severe disadvantages: a large part of the island's foodstuffs had to be imported from Sicily; (...) Malta was so lacking in natural resources and industries that everything from timber to nails had to be imported." (BLOUET (1987), S. 46).

Nach der „Großen Belagerung“ von 1565 wandelte sich das Bild der Inseln zwar langsam, aber schließlich von Grund auf: „During the Order's rule the Maltese population increased fivefold, new trades and industries were developed, and the islands became the home of one of the most prosperous communities in Europe." (BLOUET (1987), S. 68).

Insbesondere der Weinbau, der zur Zeit des Herrschaftsantritts von den Johannitern gründlich modernisiert und wieder aufgebaut wurde, profitierte von den neuen Machthabern. Dazu BoRG: „Although it is assumed that vines were planted on Malta from the time it was properly settled, the foundations of today's wine industry were laid by the Knights of St. John when they were ceded Malta by the Holy Roman Emperor, Charles $V$ of Spain in 1530. The Knights brought with them vine-cuttings from Europe to revitalize whatever vineyards remained after two centuries of Arab domination.

In the middle ages owing to the recurrent invasions by the Arabs of Barbary, the spread of malaria, the epidemics of fevers and plague, and the emigration to Sicily and Italy, the population became greatly reduced in numbers, and the cultivation of lands in outlying districts was neglected or abandoned. But during the rule of the Order of St. John with the return of comparative security after the defeat of the Turkish invasion in 1565, viticulture once more thrived, and the Island began to produce its own wine, and even exported some to other countries." (BORG (1993), S. 105 f.)

Auch die von BLOUET angesprochene fünffache Bevölkerungszunahme (vgl. o.) konnte realistischerweise nur einhergehend mit einem Aufschwung der Landwirtschaft stattfinden. Ein drastischer agrarwirtschaftlicher Niedergang, wie von NEHRING und BESENTHAL behauptet, kann nur in der unmittelbaren Zeit nach der Großen Belagerung vom Sommer 1565 nachgewiesen werden. Danach entwickelte sich die Landwirtschaft zugunsten des großflächigen Baumwollanbaus, der den Inseln eine ökonomische Hochphase bescherte wie nie zuvor in ihrer Siedlungsgeschichte ${ }^{225}$. BLOUET erwähnt Kümmel und Baumwolle als Hauptwirtschaftsfaktoren der maltesischen Agrarwirtschaft zur Zeit der Ritterherrschaft. Darüber hinaus gibt er den erfolgreichen Anbau von Zitrusfrüchten ${ }^{226}$ und die Existenz von Ziegen, Schafen und Schweinen $\mathrm{an}^{227}$.

Es ist nicht abzustreiten, dass die Malteserritter erste wichtige Dienstleistungen einführten und damit die Ökonomie und die Lebensumstände der Bevölkerung beeinflussten. Es muss jedoch damit einhergehend nur von einer graduellen Verlagerung der Bedeutungsanteile bestimmter landwirtschaftlicher Anbauformen ausgegangen werden, nicht von einem pauschalen agrarwirtschaftlichen Bedeutungsverlust, wie er bei BESENTHAL und NeHRING beschrieben wird.

Einen Nachweis dafür liefert BESENTHAL selbst in ihrer Dissertation, in der sie sich in den 1990er-Jahren noch immer beträchtlichen Anteilen landwirtschaftlicher Nutzfläche gegenüber sieht, von ihrer These der landwirtschaftlichen Bedeutungslosigkeit schon zu Zeiten des Johanniterordens jedoch nicht abweicht. Ergebnisse dieser Betrachtungsweise sind Äußerungen in ihrer Arbeit, die bei kritischer Betrachtung widersprüchlich erscheinen müssen: „Die Landwirtschaft, die über viele Jahrhunderte bis zur Etablierung der Festungsökonomie durch die Johanniter die wichtigste Existenzgrundlage der maltesischen Bevölkerung war, ist, wenn auch mit rückläufigem Anteil, auch heute noch der bedeutendste Flächennutzungsfaktor auf

224 Vgl. BORG (1993), S. $105 \mathrm{f}$.

225 Vgl. BLOUET (2004), S. 115-117

226 „(...) the fruit quality was high.“ (BLOUET (2004), S. 116)

227 Vgl. BLOUET (2004), S. $116 \mathrm{f}$. 
den maltesischen Inseln, insbesondere auf Gozo. (...). Insgesamt umfasst die landwirtschaftliche Nutzfläche heute ca. 12.000 Hektar, das sind etwa 38\% der Gesamtfläche des maltesischen Archipels (...). " (BESENTHAL (1994), S. 146).

Der Grund für die Diskrepanz in BESENTHALs Aussage ist einfach: Die Landwirtschaft wurde durch den Baumwollanbau, der durch die Johanniter weiter angeregt und gefördert wurde, nicht etwa abgebaut, sondern im Gegenteil so erfolgreich ausgebaut, dass im Verlauf der Johanniterherrschaft selbst dort neues Land kultiviert wurde, wo, realistisch gesehen, kaum noch Land urbar gemacht werden konnte. Dazu BLOUET: „In the seventeenth and eighteenth centuries, considerable areas of rocky ground were leased to peasants and brought laboriously into cultivation. The hard surface of the rock was chipped away and a bed of crushed limestone laid to form the foundation of the projected field. Soil was carefully collected from nooks and crannies in the surrounding rocks and spread over the crushed limestone foundation. Then the new field was enclosed with dry stone walls. Often the land being colonised was on a hillside and a stairway of small fields would be created upon the slope." (BLOUET (2004), S. 117).

Die hier von BLOUET beschriebenen mühevollen Versuche der Urbarmachung durch maltesische Farmer können nur damit erklärt werden, dass bessere Standorte für die Landwirtschaft nicht mehr verfügbar waren. Dies wiederum kann nur bedeuten, dass die besseren Standorte sich bereits in Bewirtschaftung befanden. BesentHals und NeHRINGs Meinung, ein landwirtschaftlicher "Bedeutungsverlust“ zur Zeit der Johanniterherrschaft sei der Grund für die heutige sekundäre Stellung der maltesischen Agrarwirtschaft, kann nicht aufrecht erhalten werden und gilt für den Autor der vorliegenden Arbeit als widerlegt.

\subsubsection{Die Landwirtschaft während französischer und britischer Kolonialzeit bis zum Zweiten Weltkrieg}

Am 10. Juni 1798 eroberte NAPOLEON BONAPARTE auf seinem Weg nach Ägypten die Hauptinsel Malta. Der Malteserorden war schon zwei Tage später besiegt und wurde unsanft von der Insel verwiesen. Es folgte die nur zwei Jahre währende Periode französischer Herrschaft über Malta. Diese Zeit war gekennzeichnet durch den tiefen Bruch zwischen den Kolonialherren und der Inselbevölkerung. Die Einwohner Maltas wurden politisch unterdrückt ${ }^{28}$. Es erfolgten keine Investitionen mehr in die Inseln, Maltas Stellung als Handelsstützpunkt verblasste innerhalb kürzester Zeit, ebenso wie der Baumwollanbau, der in nur wenigen Jahren stark an Bedeutung verlor. Die Landwirtschaft diente während der Seuchen und Hungersnöte der Jahre 1799 und 1800 - in denen sich die Sterberate der Bevölkerung vervierfachte ${ }^{229}$ - wieder gänzlich der Eigenversorgung der Bevölkerung. Ein Aufstand gegen die Franzosen wurde blutig niedergeschlagen und führte in der Folge zum „Hilferuf“ der Malteser an die Briten ${ }^{230}$.

Im Jahr 1800 etablierten sich die Briten in Malta. Sie waren von der Bevölkerung selbst gerufen worden und wurden freundlich empfangen. Die Malteser kehrten allmählich wieder zu ihrem gewohnten Lebensgang zurück: „By the end of 1800, with the French gone and commerce rebounding, people returned to the harbour towns, the men in the fighting units went back to the fields, the death rate dropped, marriages surged, and a post-war baby boom was on the way."(BLOUET (2004), S. 134).

Kommerziell war Malta für die Briten in den Folgejahren ein „Lagerhaus im Zentrum des Mittelmeers“231. Malta profitierte davon vor allem während der Blockade der europäischen Häfen durch NAPOLEON ab 1806. Der europäische Handel mit Großbritannien wurde über die Kolonie Malta abgewickelt.

\footnotetext{
228 Vgl. ATTARD (2002), S. 147

229 Vgl. BLOUET (2004), S. 130

230 Vgl. ATTARD (2002), S. 144-161

231 Formulierung nach BLOUET (2004)
} 
Doch alles änderte sich wieder im Jahr 1813: Malta wurde von der Pest heimgesucht. Die Häfen wurden geschlossen, der Handel kam zum Stillstand.

Die Inbetriebnahme des Suezkanals machte Malta wieder zum wichtigen Umschlagplatz, jedoch wurden damit die Baumwollexporte behindert. Die Landwirtschaft Maltas wurde wieder eine rein inländische Angelegenheit: „Cotton exports quickly declined after the French invasion and never recovered. However much of the cotton acreage was planted in wheat helping local food supplies, although villagers lost income from spinning and weaving." (BLOUET (2004), S. 150). "The local industry had lost markets for spun cotton in the Napoleonic Wars and post-war, and American and Egyptian growers dominated the markets. The local cottage spinning and weaving industry could not compete with machine-made products as textile factories became an important element in European industrialisation. Governor MAITLAND tried to establish factories in Malta without success." (BLOUET (2004), S. 157).

Gouverneur SIR ALEXANDER BALL versuchte die Agrarwirtschaft unabhängig vom Verfall des Baumwollanbaus wieder voranzubringen und führte die Kartoffelpflanze ein. Sie wurde in kurzer Zeit populär und ist bis heute Maltas wichtigstes Exportprodukt aus dem primären Sektor. BALL versuchte auch die Orangen- und Zitronenkultivierung wieder zu beleben. Eine Zitrusanpflanzung im ehemaligen Befestigungsgraben von Mdina ist noch heute in Nutzung. 1812 erfolgte erstmals eine sektorale Untersuchung der Maltesischen Inseln durch eine von der britischen Regierung beauftragte Kommission. 1836 lieferte eine zweite Erhebung der Kommission ihren Bericht ab: Die Reformbemühungen in der Landwirtschaft hatten kaum Wirkung gezeigt. Die Farmer waren mehr oder weniger wieder auf dem Level der Subsistenzwirtschaft angelangt. Lediglich Weizen und Gerste wurden kommerziell verwertet.

Erst nach 1850 konnten wieder Erfolge vermeldet werden: Die Förderung der Schweine- und Rinderzucht zeigte gute Erfolge. Es wurden landwirtschaftliche Genossenschaften ins Leben gerufen und Zuchtpreise verliehen. Die Schaffung dieses Fundaments ermöglichte die wichtige Stellung der Viehwirtschaft in der heutigen Agrarstruktur Maltas ${ }^{232}$. Die folgenden Jahre sind geprägt von einem Auf und Ab der Landwirtschaft auf den Inseln. In den 1860er-Jahren des amerikanischen Bürgerkriegs war plötzlich wieder maltesische Baumwolle gefragt. Die Nachfrage ging jedoch so schnell wieder zurück, wie sie gekommen war.

Maltas Agrarwirtschaft verlor jetzt grundlegend an Bedeutung. In der Zeit der britischen Kolonialherrschaft wurde der Dienstleistungs- und Industriesektor ausgebaut. Der Handel florierte, und es erschien den Gouverneuren daher nicht existenziell notwendig, die Landwirtschaft ernsthaft zu fördern. Den "Gnadenstoß“ versetzte der Zweite Weltkrieg Maltas Agrarwirtschaft. In der Zeit andauernder schwerer Bombardierungen durch deutsche und italienische Luftstreitkräfte waren die maltesischen Männer in der Verteidigung ihrer Inseln unverzichtbar. Feldbau konnte unter diesen Umständen nicht aufrechterhalten werden. In der Hungersnot durch die deutsche U-Boot-Belagerung der frühen 1940er-Jahre wurden nur Ziegen als Milchlieferanten geschont. Andere Nutztiere dienten der Versorgung der Bevölkerung mit proteinreichem Fleisch.

\subsubsection{Maltas Agrarwirtschaft in den Nachkriegsjahren}

Seit den ersten modernen wissenschaftlichen Arbeiten über Malta in den frühen 1950er-Jahren war ein dramatischer Bedeutungsverlust der agrarischen Strukturen auf den Maltesischen Inseln feststellbar. BOWEN JONES, DEWDNEY \& FISHER schrieben in ihrer wegweisenden Arbeit: „The relatively small and, until very recently, declining size of the full-time farming population, and its rapid ageing, suggest a decline in the importance and status of agriculture." (BOWEN JONES, DEWDNEY \& FISHER (1961), S. 189).

Dieser Umstand kann nach Einschätzung des Autors der vorliegenden Arbeit vor allem den Nachwirkungen des Zweiten Weltkriegs zugeschrieben werden: Während der massiven Bombardierungen durch deutsche und italienische Luftstreitkräfte in den späten 1930er- und den beginnenden 1940er-Jahren ${ }^{233}$ kam die Landwirtschaft Maltas nachweislich nahezu ganz zum Erliegen und den Inseln drohte eine Hungersnot ${ }^{234}$, die bekanntermaßen erst durch das Eintreffen von Teilen eines britischen Schiffskonvois in den Jahren 1942

232 Vgl. BLOUET (2004), S. 169

233 Vgl. BLOUET (1987), S. 203-208

234 Vgl. ebd., S. 208 
und 1943 eingedämmt werden konnte ${ }^{235}$. Nach dem Krieg diente Malta noch relativ lange Zeit als wichtiger Stützpunkt der britischen Streitkräfte, was wie schon zuvor - nun aber in ungleich stärkerem Ausmaß Arbeitskräfte aus der Landwirtschaft abzog, die nun im Bereich der Dienstleistungen und des öffentlichen Sektors beschäftigt wurden. Die Werftarbeit blühte unter britischer Einflussnahme wieder auf. Die Agrarwirtschaft der Maltesischen Inseln hat sich in der Folge davon nie wieder ganz erholt. Erschwerend kam das Problem kleiner Nutzflächengrößen hinzu, ein Ergebnis der Realerbteilung, die in Malta bis in die Gegenwart praktiziert wird.

C. Pollacco gibt außerdem zu bedenken, dass die Pressefreiheit und der allmähliche Einzug der multimedialen Informationsfülle nach dem Krieg nun auch der Bevölkerung Maltas den Lebensstil der „großen weiten Welt“ vor Augen führten. Junge Leute wollten nicht mehr Farmer werden, sondern strebten andere Berufe an oder wanderten aus, um im Ausland zu arbeiten ${ }^{236}$.

Zwar schrieb noch 1968 Lieutenant-Governor of Malta (1930-38) SIR HARRY LUKE „The principal industry of the Maltese islands is agriculture (...) “(LUKE (1968), S. 237), doch war wohl auch ihm bewusst, dass diese Aussage mehr auf seinem persönlichen Eindruck als auf statistisch belegbaren Grundlagen beruhte. C. POLLACCO weist nach, dass die Landwirtschaft in Malta bis 1951 zwar noch ein wichtiger Arbeitgeber war, jedoch in der Rangfolge der Gesamtbeschäftigung mit 12,9\% Anteil bereits nur noch an dritter Stelle (hinter öffentlichem Sektor und Werften) stand ${ }^{237}$.

Ein Konsens aller Autoren ist, dass ab Mitte der 1960er-Jahre der angesprochene landwirtschaftliche Bedeutungsverlust des 20. Jh. - auch aufgrund des aufkommenden Massentourismus und der damit einhergehenden Attraktivitätssteigerung des tertiären Sektors - rapide Ausmaße annahm: „Fifteen years later, faster development in other economic sectors such as manufacturing and tourism, had pushed agriculture down to sixth place in order of importance. In fact, by 1964 only (...) 7.8 per cent of all gainfully occupied were employed in this primary sector." (POLLACCO, C. (2003), S. 29 f.).

Der Abfall der Beschäftigungszahlen in der Landwirtschaft bedeutete jedoch nicht das Ende ihrer wirtschaftlichen Bedeutung. BESENTHAL gibt an, dass 1957 noch beachtliche $56 \%$ der gesamten Staatsfläche agrarisches Nutzland waren ${ }^{238}$. Ihr wesentlich geringerer Wert aus den 1990er-Jahren (s. o.) wird erklärt durch die Studie von CHARLTON \& BEELEY, die 1993 nachwiesen, dass im Wesentlichen aufgegebene landwirtschaftliche Nutzflächen in großem Stil und erst während der touristischen Boomphase der 1980er in Bauland umgewandelt wurden ${ }^{239}$. Der agrarwirtschaftliche Beitrag zum Bruttosozialprodukt Maltas stieg zeitweise aber trotzdem $a^{240}$. Dies ist das Ergebnis einer neuen Entwicklung: Zwar gab es nach dem Zweiten Weltkrieg viel weniger Farmer als vorher und auch die Flächengrößen schrumpften beträchtlich, jedoch war durch den technischen Fortschritt auch im Bereich der Pflanzenschutzmittel und der Saatgutqualität eine Produktionssteigerung trotz Beschäftigungs- und Flächenverlust möglich. Der Erlass von Fünf-Jahresplänen nach sozialistischem Vorbild in den 1960er-Jahren ${ }^{241}$ führte dagegen eher zu Problemen. Der Beruf des Landwirts, der in den Zeiten der Planwirtschaft nicht auf Wohlstand hoffen durfte, wurde zunehmend unattraktiv. Mit immer älter werdenden Farmern und dem Fehlen von Nachwuchs im primären Sektor ist Maltas Landwirtschaft heute wieder in einem schlechtem Zustand. FARRUGIA wies 2001 den nahezu völligen Bedeutungsverlust der Landwirtschaft im modernen Malta nach ${ }^{242}$. Ihr Beitrag zum Bruttoinlandsprodukt betrug Ende 2003 nur noch $3 \%{ }^{243}$.

235 Vgl. BLOUET (1987), S. 209

236 Vgl. Pollacco, C. (2003), S. $30 \mathrm{f}$.

237 Vgl. Ebd. (2003), S. 29

238 Vgl. BESENTHAL (1994), S. 146

239 Vgl. ChARLton \& BEELEY (1993)

240 Vgl. Pollacco, C. (2003), S. 30

241 Vgl. PollacCo, C. (2003)

242 FARRUGIA (2001), S. $108 \mathrm{f}$.

243 EUROPÄISCHE KOMMISSION (2003b), S. 10 
Die Verknüpfungslinien der landwirtschaftlichen Tradition Maltas mit den unterschiedlichsten Bereichen des Alltagslebens sind jedoch selbst in dieser neuerlichen Phase der ökonomischen Bedeutungslosigkeit immer noch äußerst vielfältig und weiterhin ein wichtiger Bestandteil der Alltagskultur der Bevölkerung, auch der nicht im Agrarsektor Beschäftigten ${ }^{244}$.

\subsubsection{Entwicklungen der letzten Jahrzehnte und Auswirkungen von Tourismus und Erbteilung}

Noch immer wird in der Fachliteratur manchmal im Tourismus mit dem damit einhergehenden Beschäftigungsboom im tertiären Sektor die hauptverantwortliche Kraft für den Untergang der maltesischen Landwirtschaft gesehen ${ }^{245}$. Diese Autoren ignorieren dabei, dass sich schon früh die Erbteilung der landwirtschaftlichen Flächen, die noch heute vielerorts im alten Muster der Realteilung betrieben wird, wohl als mindestens ebenso wichtig für den Prozess des Verfalls herausstellte ${ }^{246}$. AzZOPARDI wies auf ein weiteres Problem hin: „Sometimes, the fields a farmer inherits (as property or as tenancy) are not only small but also distant from one another. This, naturally, increases his difficulty to work on them." (AzZOPARDI (2002), S. 149).

FARRUGIA nahm 2001 in der fortschreitenden Verkleinerung landwirtschaftlicher Flächen durch den Vererbungsprozess den Hauptgrund für den rapiden Niedergang der Zahlen der sog. full-time farmer (also der Vollerwerbslandwirte) an. Er gibt an, dass die durchschnittliche landwirtschaftliche Flächengröße pro Farmer in der Republik Malta nur noch 0,78 ha beträgt ${ }^{247} .70 \%$ der Farmer bearbeiten trotz einer in Malta agrarisch nutzbaren Gesamtgrundfläche von insgesamt rund 7.471 ha $^{248}$ gegenwärtig kleinfleckige und verstreute Flächenansammlungen von einer durchschnittlichen Größe von unter 2 ha $^{249}$. Rentables Wirtschaften, oder die Arbeitserleichterung durch den Einsatz von Maschinen zur Landbearbeitung, ist auf so kleinen Flächen nahezu nicht mehr möglich. Hieraus erklärt sich vermutlich auch der immer noch hohe Anteil der Mischwirtschaft in der maltesischen Agrarstruktur. FARRUGIAs Daten werden gestützt von einer Statistik des NSO, die in Abb. 9 dargestellt ist (s. folgende Seite).

244 CREMONA (2003)

245 Vgl. z. B. Pollacco, J. (2003), PACE (2001)

246 Vgl. z. B. NeHRING (1966), S. 105 f.; Busuttil (1993), S. 18 ff.; FARRUgia (2001), S. 109 f.

247 FARRUGIA (2001), S. 109

$248 \mathrm{Vgl}$. NSO (2003a), S. 37

249 FARRUGIA (2001), S. 111 


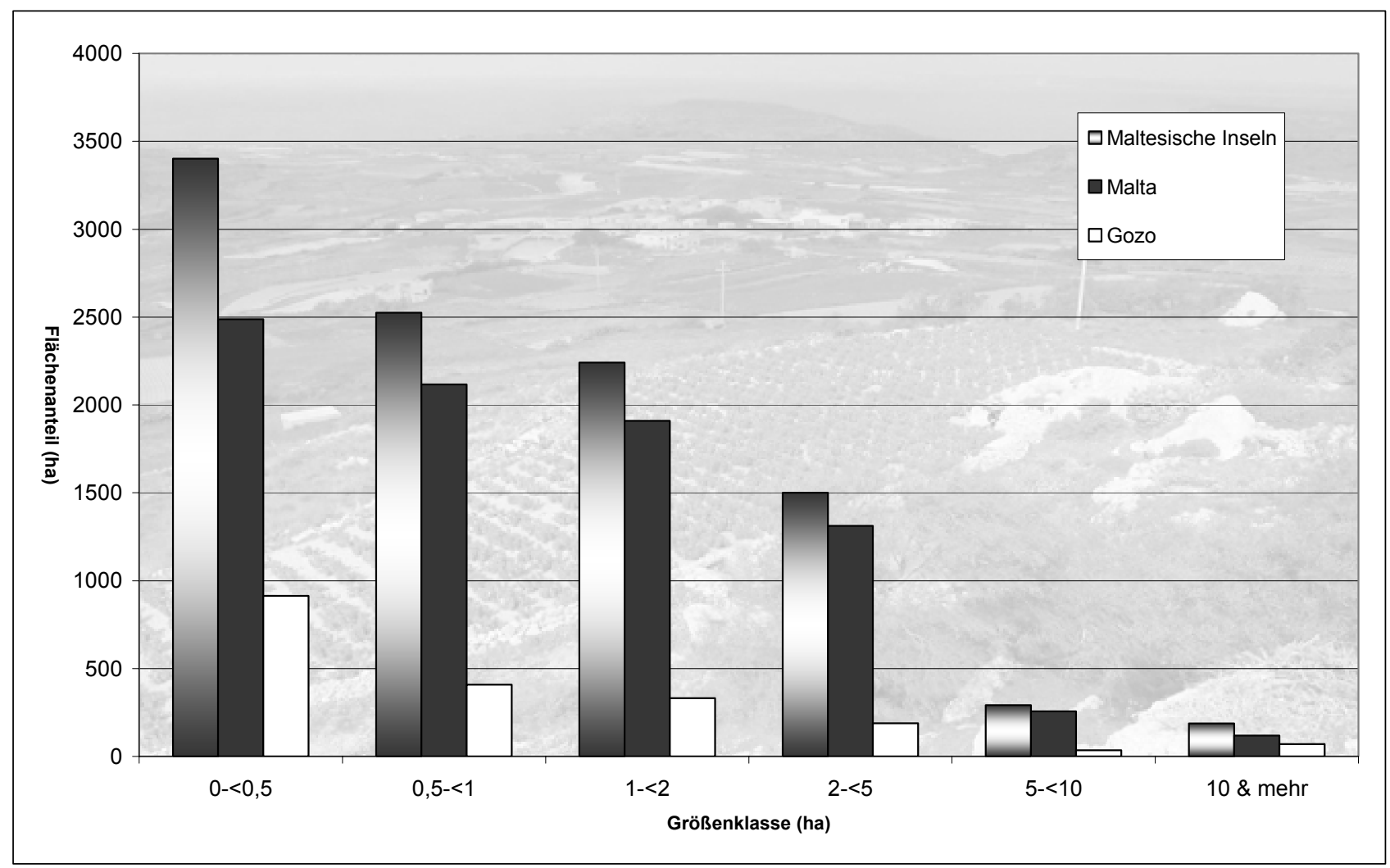

Abb. 9: Größenverteilung der landwirtschaftlichen Nutzflächen auf den Maltesischen Inseln. Entwurf \& Grafik: AsCHEMEIER. Daten: NSO (2003a).

Die Grafik zeigt Daten des National Statistics OfFice (NSO), nach denen sich der überwiegende Teil der nutzbaren Agrarfläche der Maltesischen Inseln aus Arealen von 0-2 ha zusammensetzt. Besonders im landwirtschaftlich aktivsten Teil der Inselgruppe, auf Gozo, ist diese Entwicklung spürbar (vgl. Abb. 9).

Dieser Umstand, im Zusammenspiel mit der Wasserknappheit im Sommer, die zum rentablen landwirtschaftlichen Anbau einen hohen Aufwand in Form von Bewässerungsmaßnahmen notwendig macht, dem durch Düngung, Pestizide, Fungizide, etc. möglich gewordenen Vorsprung der wenigen großen Agrarbetriebe Maltas sowie der weiterhin nicht unwesentlichen Verlockung durch besser bezahlte und weniger arbeitsaufwändige Jobs im Dienstleistungssektor, hat eine Lawine des agrarischen Bedeutungsverfalls ausgelöst. Diese wurde von politischer Seite erst spät erkannt ${ }^{250}$. Zusätzlich sollte noch erwähnt werden, dass der Beruf des Landwirts in Malta mittlerweile ein schlechtes soziales Image hat, was den erwähnten Prozess noch beschleunigt. Das negative Image ist jedoch in Teilen den Landwirten selbst zuzuschreiben, die durch illegale Aneignung von öffentlichem Land in den Medien von sich reden machen sowie zu rigorosen Methoden greifen, um Spaziergänger von ihren Feldern fern zu halten ${ }^{251}$.

250 Die EUROPÄISCHE KOMMISSION spricht in ihrem umfassenden Monitoring-Bericht 2003 noch von „ernsten Sorgen“, was die staatliche Verwaltung der landwirtschaftlichen Flächen angeht. Vgl. EUROPÄISCHE KOMMISSION (2003), S. 32.

251 Vgl. z. B. ZAMmitT TABONA in: THE TIMES OF MALTA, vom 13.07.2004, S. 8: „(...) taking a country walk in Malta can be a hazardous experience. One always feels as if one is trespassing, even if one isn't! One never knows when one is going to be shouted or shot at, or whether one is suddenly going to be confronted by the Hound(s) of the Baskervilles. (...) (I) hope that come next October some authority, having had ample time to do it, will scour the countryside for illegal barriers and actually define where one can walk freely without the fear of being either a sitting target or poor Actaeon himself." Vgl. außerdem CINI in: THE TIMES OF MALTA, vom 22.07.2004, S. 6. 


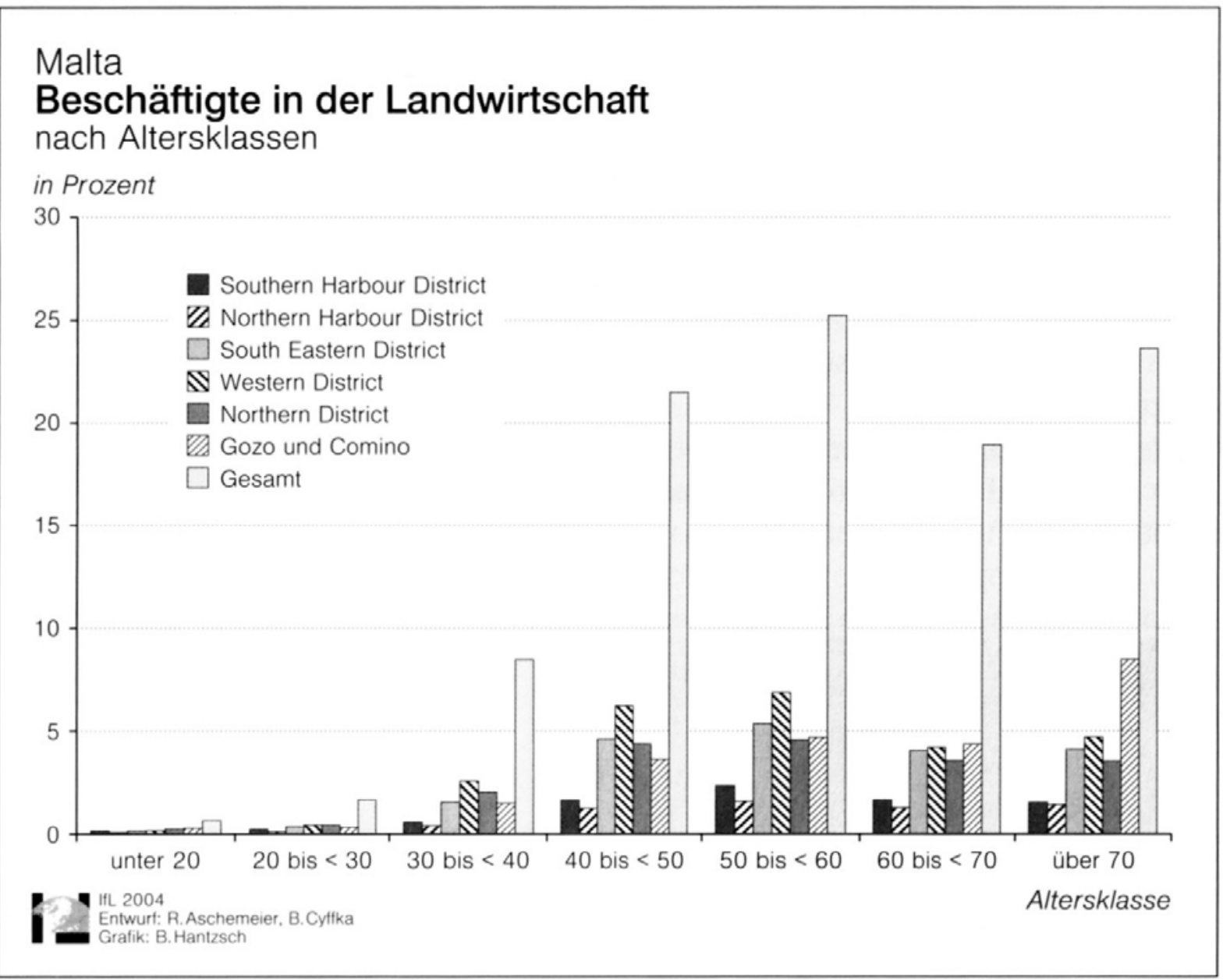

Abb.10: Beschäftigte in der Landwirtschaft nach Altersklassen. Quelle: AsCHEMEIER \& CYFFKA (2004b), S. 109

Malta hat z. Zt. einen Anteil von unter $2 \%{ }^{252}$ Erwerbstätigen in der Landwirtschaft. Dies ist die niedrigste Rate aller neuen EU-Beitrittsländer ${ }^{253}$. Die Auswertung der statistischen Daten des NSO durch ASCHEMEIER \& CYFFKA (vgl. Abb. 10) zeigt drastische Werte für Maltas Agrarstruktur: 67\% der Beschäftigten in der Landwirtschaft sind über 50 und $23,6 \%$ der Landwirte sind gar über 70 Jahre alt!

Dabei muss bedacht werden, dass die gezeigten Werte bereits auch die Nebenerwerbslandwirte umfassen. Das Verhältnis von Vollerwerbs- zu Nebenerwerbslandwirten in Malta liegt bei $11 \%$ zu $89 \%{ }^{254}$.

Trotzdem hat sich bis heute, vor allem im Bereich Dingli und anderer ländlicher Siedlungen, eine hohe Anzahl an ländlichen Bräuchen und Traditionen erhalten ${ }^{255}$. Des Weiteren sind es die alteingesessenen Landwirte, die auch wichtige Landschaftspflegemaßnahmen durchführen, wie z. B. die Erhaltung der Rubble Walls (Geröllmauern), die die agrarisch genutzten Hangterrassen vor Erosion und den Feldanbau in TalLagen vor Wind schützen.

252 Quelle: EUROPÄISCHE KOMMISSION (2002), S. 1

253 LIPPERT (2003), S. 48

254 Vgl. NSO (2003a), S. 129

255 Vgl. Cremona (2003) 


\subsubsection{Maltas Landwirtschaft nach dem EU-Beitritt}

Die drängenden Probleme der maltesischen Agrarwirtschaft haben nach der EU-Erweiterung den Weg für Fördermaßnahmen frei gemacht.

184 Mio. $€$ sollen, von der EU und der maltesischen Regierung zu unterschiedlichen Teilen finanziert, bis zum Jahr 2014 in die maltesische Landwirtschaft fließen ${ }^{256}$. Die ersten drei Jahre des Programms sind dabei mit 67 Mio. $€$ angesetzt, wovon 20,1 Mio. € von der EU übernommen werden.

Darunter fällt auch eine direkte Einkommensunterstützung (SMPPMA-Programm), die es den Landwirten ermöglichen soll, ihre Waren - trotz der im Vergleich zu anderen EU-Ländern teureren Produktionskosten zu konkurrenzfähigen Preisen anzubieten. Auf diese Weise soll eine übermäßige Konkurrenz von Agrarprodukten aus anderen EU-Staaten auf dem maltesischen Markt vermieden werden. Dieses System löst die umstrittene Regelung ab, die bisher Zölle auf Agrarprodukte aus dem EU-Raum vorsah ${ }^{257}$.

Ferner ist Malta von der EU als landwirtschaftliche Ungunstregion anerkannt worden, was mit weiteren Förderungen verbunden ist. Zudem erließ die Regierung einen Rural Development Plan, der u. a. festlegt, wann welche Phase der Förderung in Kraft treten soll.

Es ist interessant zu beobachten, wie viel Geld für welchen Fördersektor ausgegeben wird. Dabei ist nur die Vergabe der EU-Gelder in Höhe von 20,1 Mio. $€$ bekannt, die sich wie folgt aufteilen ${ }^{258}$ :

- 11 Mio. $€$ für die direkte Einkommensunterstützung der Landwirte (SMPPMA)

- 6,6 Mio. € als Unterstützungsmaßnahme für Malta und Gozo als landwirtschaftliche Ungunsträume

- 1,83 Mio. $€$ zur direkten Unterstützung des Tomatenanbaus

- $\quad 0,67$ Mio. $€$ zur Unterstützung der Milchwirtschaft.

Aber die Regierung hat sich mit dem Rural Development Plan noch andere Ziele gesteckt: Durch eine neue Stellung des Landwirts als Landschaftspfleger ${ }^{259}$, der für seine landespflegerischen Maßnahmen von der Regierung Geld bekommt, soll das soziale Image der Landwirte gehoben werden.

Außerdem soll das Erbrecht für landwirtschaftliche Nutzflächen vereinfacht bzw. geändert werden, was sich zur Zeit der Schriftsetzung dieser Arbeit in der Umsetzung befindet. Bereits im Jahr 2000 wurde die Möglichkeit geschaffen, Staatsland als landwirtschaftliche Fläche zu pachten und registrieren zu lassen ${ }^{260}$, ein notwendiger Schritt, da immer noch ca. zwei Drittel der landwirtschaftlichen Nutzfläche dem Staat gehören ${ }^{261}$, der mit ihrer Verwaltung und Pflege zunehmend überfordert zu sein scheint.

Neben den Fördermaßnahmen hat sich die maltesische Regierung durch den EU-Beitritt aber auch zu einschneidenden Anpassungen an EU-Normen verpflichtet. Da dieser Punkt zwar zur Veranschaulichung der derzeitigen Situation der maltesischen Agrarwirtschaft dient, das eigentliche Thema der Dissertation aber nur am Rande berührt, sollen an dieser Stelle nur einige der wichtigsten Probleme angesprochen werden.

256 Vgl. BusutTIL \& DEMICOLI (2003), S. 1

257 Vgl. ASCHEMEIER \& CYFFKA (2004), S.113 f

258 Angaben nach BUSUTTIL \& DEMICOLI (2003), S. 3

259 „In addition to this, during the first three years of membership, additional EU funding will support the implementation of Malta's Rural "Development Programme (e.g. technical assistance and agri-environment measures such as rubble wall maintenance" (BUSUTTIL \& DeMICOLI (2003), S. 3)

260 THE TIMES OF MALTA, 08.08.2000, S. 17

261 Vgl. FARRUGIA (2001), S. 111 
Ein Hauptproblem der Landwirtschaft auf den Maltesischen Inseln ist die Wasserversorgung in der Trockenzeit von Mai bis September ${ }^{262}$. Gegenwärtig dienen als eine der Hauptbezugsquellen für Bewässerungswasser die Klärwerke in Marsaskala und Sant' Antnin. Zusammen stellen sie rund $7.000 \mathrm{~m}^{3}$ an geklärten Abwässern zur Bewässerung der landwirtschaftlichen Flächen zur Verfügung ${ }^{263}$. Die EUNormen werden diese Art der Bewässerung schon in Kürze unmöglich machen, da durch die bisherige Vorgehensweise zu viel Nitrat in den Boden und damit ins Grundwasser eingebracht wird ${ }^{264}$. Malta wurde wegen des anteilig (zu) hohen Wasserverbrauchs seiner Landwirtschaft in dieser Hinsicht aber eine Übergangsfrist eingeräumt.

Maltas Verhältnis von Agrar-Im- und -Exporten ist extrem unausgewogen. Es bewegt sich im Rahmen von 205,9 Mio. $€$ zu 7,7 Mio $€^{265}$. Dieser Zustand wird von der Europäischen Kommission kritisiert ${ }^{266}$, weswegen die maltesische Regierung diverse Maßnahmen in die Wege geleitet hat, um die Beschäftigung und die Produktionsrate im Agrarsektor zu erhöhen ${ }^{267}$.

Der Anteil landwirtschaftlich genutzten Lands nimmt seit Jahrzehnten rapide ab. Während 1956 noch 20.433 ha Land unter agrarischer Nutzung standen, waren es 2001 nur noch $9.563,53 \mathrm{ha}^{268}$. Das stellt eine Abnahme von 53,2 \% innerhalb von 45 Jahren dar. Dagegen nahm der Anteil bebauter Fläche in einem vergleichbaren Zeitraum schnell $\mathrm{zu}^{269}$. Betrachtet man dieses Phänomen unter Berücksichtigung des zuvor genannten Problems, dann wird klar, dass auch hier Reglementierungen der Regierung eingreifen müssen. Im Rural Development Plan sind erste Ansätze dazu festgehalten worden.

262 Vgl. Kap. 3. Vgl. zudem Bendix, Bendix \& ReUdENBACH (2002)

263 Vgl. AZZOPARDI (2002), S. 154

264 Vgl. CORDINA (2002), S. 4

265 Vgl. EUROPÄISCHE KOMMISSION (2001), S. 45 f.

266 Ebd.

267 Vgl. AZZOPARDI (2002), S. 155

268 Ebd.

269 Vgl. CHARLTON \& BEELEY (1993) 


\section{Agrarische Nutzpflanzen der Maltesischen Inseln}

Seit Anbeginn der maltesischen Agrarwirtschaft waren für deren Erscheinungsbild die Kulturpflanzen besonders wichtig.

Im folgenden Abschnitt soll ein Überblick über einige der typisch maltesischen Anbaupflanzen und ihre ökonomischen Hintergründe erfolgen. Dabei ist zu bedenken, dass nicht alle der heute angebauten Nutzpflanzen auch in historischer Hinsicht gleich „wichtig“ waren oder gar als traditionell maltesisch bezeichnet werden können.

Der Autor folgt der Methodik von BOWEN JONES, DEWDNEY \& FISHER, die in ihrer Veröffentlichung aus dem Jahr 1961 erstmals eine umfassende Aufstellung der maltesischen Nutzpflanzen unter Angabe ihres damals aktuellen Status im Rahmen einer Landnutzungsanalyse aufführten ${ }^{270}$.

Seitdem hat sich in Maltas Agrarwirtschaft viel geändert: In Malta werden gegenwärtig z. B. dreimal mehr Flächen im Bewässerungsfeldbau bewirtschaftet als noch vor 20 Jahren $^{271}$. Daraus ergab sich im Laufe der Zeit eine Orientierung hin zu bestimmten Kulturpflanzen, die in historischer Vergangenheit nicht oder nur selten auf der Inselgruppe vorkamen. In gleicher Weise erfolgte eine Verdrängung von alten Nutzpflanzen bzw. Nutzpflanzensorten zugunsten von neu eingebrachten Arten, welche dem, der sie anpflanzte, höhere Erträge und/oder Gewinne garantierten.

\subsection{Begründung für die folgende Nutzpflanzenauflistung im Rahmen der Abhandlung}

Trotz dieser gravierenden Veränderungen wurde die Liste der Nutzpflanzen, wie sie in BOWEN JONES et al. aufgeführt war, seit 1961 bisher nie fortgeschrieben oder aktualisiert. Der Autor versucht mit der folgenden Aufstellung diesem Umstand Rechnung zu tragen und zum ersten Mal seit gut 40 Jahren wieder eine systematische Aufstellung der Nutzpflanzen der maltesischen Landwirtschaft unter geographischem Blickwinkel vorzulegen. Allerdings konnte im Rahmen der Dissertation keine neuerliche umfassende Landnutzungsanalyse durchgeführt werden, weswegen sich die Angaben für die einzelnen Pflanzen primär der derzeitigen kultur- und agrargeographischen Situation widmen. Es wird darauf hingewiesen, dass nach wie vor erheblicher Forschungsbedarf besteht, um die graduelle Veränderung der Landnutzung seit den 1960er-Jahren adäquat abbilden zu können.

Da der nachfolgende Abschnitt als ergänzende Information für die Untersuchung der regionalen traditionellen Produkte dient, wurden vor allem die traditionellen Feldfrüchte berücksichtigt. In vielen Fällen können auch die Früchte vieler Nutzpflanzen allein für sich genommen schon als landwirtschaftliches Produkt bezeichnet werden. Zur modernen Vielfalt an angebauten Gewächsen und ihrem Anteil an der gesamten landwirtschaftlichen Nutzfläche verweist der Autor an dieser Stelle ergänzend auf die Veröffentlichungen des NSO (2001, 2002, 2003a, 2003c \& 2004b), sowie auf die Arbeiten von MELI (1993), FARRUGIA (2001) und AZZOPARDI (2002).

Es liegt derzeit keine umfassende agrargeographische oder agrarwissenschaftliche Arbeit über Malta vor. Informationen müssen aus Artikeln, Sammelbänden und Einzelkapiteln geographischer Werke individuell recherchiert werden. Der Verfasser möchte mit der folgenden Nutzpflanzenaufstellung, die viele eigene Erhebungen und Beobachtungen enthält, einen Beitrag zur Übersichtlichkeit und zur Vereinfachung für nachfolgende Forschende leisten, die an Maltas Agrargeographie interessiert sind. 


\subsection{Auflistung der Anbaupflanzen}

Im Folgenden sind ausgewählte maltesische Nutzpflanzen (vgl. Tab. 1) aufgelistet und nach Gruppen unter Angabe ihrer deutschen, botanischen und maltesischen Bezeichnung (soweit bekannt) geordnet. Nutzpflanzen sind die Grundlage zahlreicher agrarischer Sekundärerzeugnisse, und ihre Früchte können oftmals als allein stehende landwirtschaftliche Produkte aufgefasst werden. Für jede Pflanze erfolgt daher eine Erläuterung, deren Umfang sich nach der ökonomischen und soziokulturellen Bedeutung der jeweiligen Anbaupflanze richtet. In der Aufzählung sind sowohl Gemüse und Obst in Bodenkultur, als auch Nuss- und Obstbäume aufgeführt.

Es kann kein Anspruch auf Vollständigkeit der Aufzählung erhoben werden, da die Liste lediglich einen grundlegenden Überblick über die Kulturpflanzen verschaffen soll, die die Basis für zahlreiche regionale traditionelle Produkte sind.

Zu Angaben zum Prozess der Verarbeitung der hier aufgeführten Kulturpflanzen zu Sekundärerzeugnissen sowie deren Beschreibung s. Kap. 14.1.

\begin{tabular}{|l|l|l|l|l|l|l|}
\hline Obst & Gemüse & Leguminosen & Hackfrüchte & Getreide & Sonstige & $\begin{array}{l}\text { Nüsse \& } \\
\text { Unkontrollierter } \\
\text { Anbau }\end{array}$ \\
\hline \hline Aprikose & Artischocke & Bohnen & Kartoffel & Gerste & Klee & Mandeln \\
Bambinella & Aubergine & Linsen & Knoblauch & Weizen & Ölbaum & Feigenkaktus \\
Birne & Kürbis & & Zwiebel & & Pfeffer & Johannisbrotbaum \\
Erdbeere & Tomate & & & & & \\
Feige & & & & & & \\
Granatapfel & & & & & & \\
Melone & & & & & & \\
Nektarine & & & & & & \\
Pfirsich & & & & & & \\
Wein & & & & & & \\
\hline
\end{tabular}

Tab. 1: Auflistung der Nutzpflanzen, die in Kap. 13 behandelt werden

\subsection{Obst}

\section{- Aprikose (berquq)}

(Prunus armeniaca L.)

Aprikosen sind in Malta nicht weit verbreitet, obwohl sie unter der Bevölkerung beliebt sind und auch einige Gerichte der traditionellen maltesischen Küche Aprikosen als Zutat beinhalten. Sie kommen vor allem im Privatanbau zur Eigenversorgung vor. Die Früchte werden Ende Mai geerntet. Weil sie schnell verderben, findet man Aprikosen nur selten im Obsthandel wieder. Sie sind zudem wind- und salzempfindlich. Dies sind vermutlich die Hauptgründe, warum ein Anbau in größerem Maßstab bisher ausblieb. Traditionell werden Aprikosen in Malta eingeweckt oder zu Marmelade verarbeitet. Es gibt einige Anbieter regionaler traditioneller Produkte, die in kleinem Umfang Aprikosen als Basis für ihre Produkte verwenden. Die kommerziellen Marmeladenhersteller (z. B. „Granny's Pride“ aus Magћtab) verarbeiten importierte Früchte.

- Bambinella (bambinella) und Birne (lanǵa)

(Birne: Pyrus communis L.)

Die Bambinella ist eine harte, aber saftige Frucht. Aussehen und Geschmack ähneln dem einer Birne, jedoch ist die Bambinella viel kleiner. Die Frucht wird nicht weiter verarbeitet, sondern wird als Obst gegessen oder zur Dekoration verwendet. Sie wächst an Bäumen und wird auf Malta und Gozo häufig 
angebaut. Jedoch sinken ihre Verkaufsmengen derzeit rapide. Während 1996 auf dem Binnenmarkt noch $81.915 \mathrm{~kg}$ Bambinelli verkauft wurden, waren es im Jahr 2001 nur noch $24.094 \mathrm{~kg}^{272}$.

Ihre wirtschaftliche Bedeutung ist durchaus noch mit der der Birne vergleichbar, die in den letzten Jahren einen Aufschwung in den Verkaufszahlen erfahren hat. Die Birnenverkäufe stiegen von $12.617 \mathrm{~kg} \mathrm{im}$ Jahr 1996 auf $29.861 \mathrm{~kg}$ im Jahr $2001^{273}$. Bambinelli werden zu einem höheren Preis verkauft, sodass ihre Gewinnspanne trotz geringerer Verkaufsmengen immer noch über der des Birnenverkaufs liegt. Trotzdem ist es auffällig, dass noch vor 8 Jahren Bambinelli in Malta in deutlich größeren Mengen als Birnen verkauft wurden, und dass sich dieses Verhältnis in jüngster Zeit umzukehren scheint. Die Früchte sind, für sich genommen, bereits als agrarische Produkte anzusehen und unterliegen in Malta keiner weiteren Verarbeitung zu Sekundärprodukten.

\section{- $\quad$ Erdbeere (frawli)}

(Fragaria spec.)

War die Erdbeere in der maltesischen Landwirtschaft früher eine verbreitete Pflanze, so ist ihr Flächenanteil heute gering. Nur 19,426 ha agrarischer Nutzfläche werden zur Erdbeerzucht verwendet ${ }^{274}$. CREMONA gibt als Grund dafür steigende Importe aus Frankreich, England und Italien $a^{275}$.

Es gibt eine endemische Erdbeersorte in Malta, die jedoch - wenn überhaupt - nur noch in privaten Gärten gezogen wird. Ihre Früchte sind klein und flach. Sie sollen sehr süß und schmackhaft sein. Ihr ungewöhnliches Aussehen, ihre schnelle Verderblichkeit und ihre äußerst geringe Ergiebigkeit schließen jedoch eine kommerzielle Nutzung nach heutigen Maßstäben aus.

Noch bis kurz nach dem Zweiten Weltkrieg wurden die maltesischen Erdbeeren auf dem Wochenmarkt in speziellen Körben verkauft (malt.: Qaleb tal-frawli). Nach mündlicher Auskunft der Schriftstellerin MATTY CREMONA und des Landwirts RENO AgIUS sind diese Körbe für Dekorationszwecke heute begehrter als die Erdbeeren selbst ${ }^{276}$. Erdbeeren spielen unter den regionalen traditionellen Produkten nur eine untergeordnete Rolle. Gelegentlich findet man hausgemachte Erdbeermarmelade auf dem Wochen- oder Fischmarkt, doch die kommerziellen Hersteller importieren ihre Früchte aus anderen Ländern.

\section{- Feige (tin, farkizzan oder bajtar)}

(Ficus carica L.)

Der Feigenbaum ist eine der ältesten bekannten Nutzpflanzen der Maltesischen Inseln. Anhand eines archäologischen Funds konnte festgestellt werden, dass Feigenbäume bereits vor 4.500 Jahren auf der Inselgruppe wuchsen 277 . Feigen sind zweihäusig. Nur die „weiblichen“ Sträucher bringen essbare Früchte hervor. Die „männlichen“ Früchte hingegen reifen nie aus und sind nicht essbar ${ }^{27}$.

Gegenwärtig nimmt das ausufernd strauchartig wachsende Gehölz im maltesischen Anbau eine Zwischenstellung ein: Einerseits kann es nicht als Pflanze bezeichnet werden, die keinem kontrollierten Anbau unterläge, andererseits wächst es auf aufgegebenen landwirtschaftlichen Gehöften und Flächen häufig doch wild und tritt auch in Städten an verwahrlosten Gebäuden zutage ${ }^{279}$. Anfang der 1960er-Jahre ordneten BOWEN JONES, DEWDNEY \& FISHER die Feigenbäume in Malta eindeutig den kultivierten

272 Vgl. NSO (2004b), S. 31

273 Ebd.

274 Vgl. NSO (2003a), S. 104 f.

275 Vgl. CREMONA (2003), S. 118

276 Aussagen in Experteninterviews mit dem Autor vom 12.10.2003 (R. AGIUS) und 25.05.2004 (M. CREMONA)

277 Vgl. CREMONA (2003), S. 14

278 Vgl. ebd., S. 134

279 So findet man z. B. in den Mauern von Fort St. Angelo, welches sich an der Spitze der Landzunge von Vittoriosa (malt.: Birgu) mitten in der städtischen Agglomerationszone rund um die Hauptstadt Valletta befindet, etwa 5-10 Feigenbäume, die aus den Befestigungsmauern und den Schlaglöchern in den asphaltierten Flächen wuchern. Auch im Bereich der Zitadelle von Victoria auf Gozo kommt es zum Wildwuchs von Feigenbäumen. Dies sind nur zwei exemplarische Beispiele von vielen auf der Inselgruppe. 
Nutzpflanzen $z^{280}$. CREMONA (2003) schreibt, dass die Feigenfrucht auch gegenwärtig verbreitet genutzt wird.

In Malta sind drei Sorten bekannt, die mit maltesischen Bezeichnungen belegt sind: Die Früchte der Sorte farkizzan sind von außen schwarz-violett. Das Fruchtfleisch ist rötlich. Die Früchte sind klein und sie tendieren zu trockenem Fruchtfleisch. Bäume dieser Sorte wurden meistens aus landschaftsgestalterischen Gesichtspunkten gepflanzt. Die Früchte der Sorte bajtar werden früh geerntet, da sie sonst schon in einem frühen Reifestadium von selbst vom Baum fallen und dann schnell verderben. Die meist genutzte und beliebteste Sorte ist daher tin (auch bekannt als tina oder tena). Die Früchte sind mittelgroß, grün-gelblich bis bernsteinfarben und sehr süß. Nahezu alle kommerziellen Produkte zu denen Feigen verarbeitet werden, basieren auf der Sorte tin.

Frische Feigen und in Blockform konservierte, getrocknete Feigen (tin tać-ćappa bzw. tin tal-balla) sind unter der Bevölkerung sehr beliebt und haben auch wirtschaftliche Bedeutung für den maltesischen Binnenmarkt. 2001 wurden in Malta und Gozo $99.523 \mathrm{~kg}$ frische Feigen verkauft. Getrocknete Feigen brachten es auf einen Gesamtverkauf von $3.312 \mathrm{~kg}$ im selben Jahr. Der Verkauf von Feigen und deren Folgeprodukten schwankt jedoch von Jahr zu Jahr sehr stark ${ }^{281}$. Fast jeder Malteser mit Zugang zu einem Garten oder einer Landparzelle besitzt auch einen Feigenbaum. Da die direkten Folgeprodukte nicht schwierig herzustellen sind, unterliegt der freie Verkauf jährlichen Schwankungen. Getrocknete Feigen werden in traditionellen Backwaren verwendet.

\section{- Granatapfel (rummien)}

(Punica granatum L.)

Granatäpfel kommen in Malta vereinzelt im kommerziellen und privaten Anbau vor. Sie werden ab September geerntet. Wirtschaftlich spielen sie nahezu keine Rolle. Es ist jedoch interessant, dass es einst eine endemische Sorte gab, den „Maltesischen Granatapfel“. Diese Varietät ist in Malta jedoch nicht mehr zu finden. Es wird vermutet, dass einige Setzlinge des Maltesischen Granatapfels, der als ausgestorben gilt, in der Vergangenheit nach Sizilien verbracht wurden und daher vielleicht dort noch zu finden sind ${ }^{282}$.

\section{- $\quad$ Melone (bettieћ)}

(Cucumis melu \& Citrullus vulgaris)

In Malta werden vor allem Wassermelonen (dullieg $\hbar$ ), aber auch andere Melonensorten angebaut. Sie gehören zwar zu den traditionellen Feldfrüchten, konnten jedoch wegen ihres hohen Wasserbedarfs früher nur unter großem Aufwand angebaut werden. Erst mit der Einführung der Windpumpe wurde inr Anbau verbreiteter. Die Ernte erfolgt im August. Melonen werden im Allgemeinen nicht weiter verarbeitet, sondern dienen als Dessert oder Sorbet.

Ihr heutiger Anbau hat beachtliche Flächenanteile erreicht. 33,231 ha Wintermelonen, 180,384 ha Sommermelonen und 113,526 ha Wassermelonen ${ }^{283}$ sind für maltesische Verhältnisse ein hoher Wert, der erst in den 1980er-Jahren etabliert werden konnte ${ }^{284}$. Die Sommerfrüchte werden nur für den einheimischen Markt gezogen, exportiert werden sie bislang nicht.

280 Vgl. BOWEN JONES, DEWDNEY \& FISHER (1961), S. 218

281 Vgl. NSO (2004b), S. 31

282 Vgl. Galea de GIOVANNI (o. J.), S. 8

283 Vgl. NSO (2003a), S. 102-104

284 Ebd., S. 4 


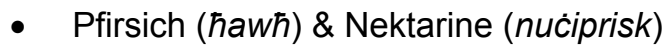

(Prunus persica (L.) Batsch. \& Prunus spp.)

Im August werden in Malta Pfirsiche geerntet. Der Anbau dieser Steinfrucht hat, zusammen mit dem Anbau von Aprikosenbäumen, in den letzten zehn Jahren zugenommen und hält im Plantagenbetrieb mit 152,602 ha mittlerweile flächenmäßig höhere Anteile an der gesamten landwirtschaftlichen Nutzfläche als die Kultivierung von Zitrusfrüchten ${ }^{285}$. Auch der Anbau von Nektarinen kommt vor.

Pfirsiche und Nektarinen gehören zu den begehrtesten Früchten in Malta und versprechen vergleichsweise hohe Gewinnspannen. Es gibt jedoch keine regionalen traditionellen Produkte, die auf der Basis von Pfirsichen erzeugt werden.

\section{- Wein (gћeneb)}

(Vitis vinifera)

In Bezug auf den Weinbau der Maltesischen Inseln muss zwischen dem Anbau von Tafeltrauben und dem Anbau von Trauben zur Weinherstellung unterschieden werden. Von den vielen kleinen und größeren Weinbauflächen, die wie aus Karte 3 ersichtlich ist, mosaikartig über die Inseln verstreut sind, dienen die meisten heute zur Erzeugung von Trauben, die zu Wein weiterverarbeitet werden.

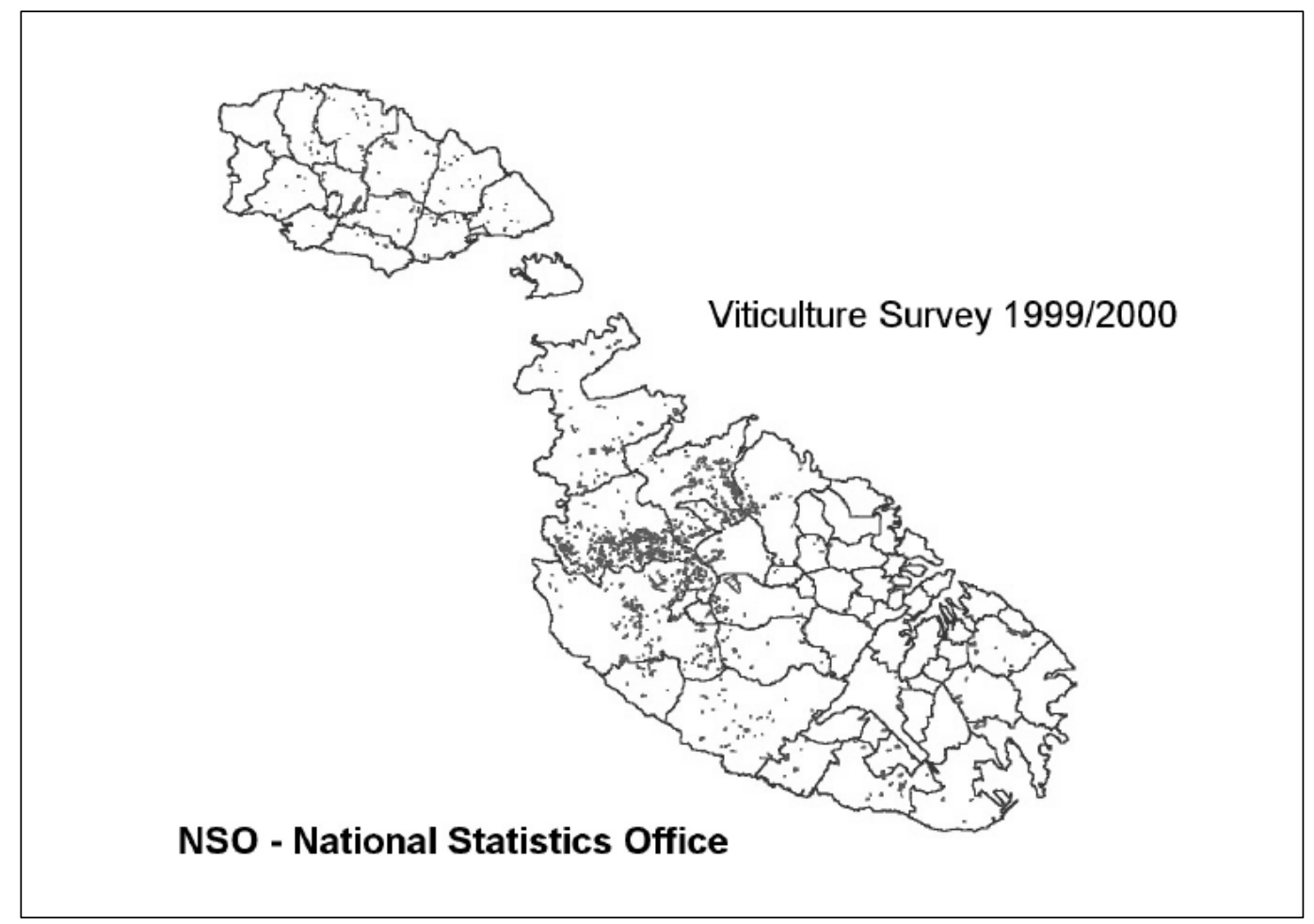

Karte 3: NSO Viticulture Survey 1999/2000: die Verbreitung des Weinbaus auf den Maltesischen Inseln. Jeder dunkle Fleck ist ein vineyard. Die eingezeichneten Grenzen sind die Verwaltungsbezirke des Staates Malta. Quelle: NSO (2002a), S. 31

Die gesamte Anbaufläche für Weintrauben beträgt 481,669 ha $^{286}$, von der sich der größere Teil $(443,965$ ha) auf Malta befindet. Das sind rund $5 \%$ der gesamten agrarischen Nutzfläche des Kleinstaats.

142,891 ha davon werden für den Anbau von Tafeltrauben verwendet. Der Rest der Fläche umfasst Trauben für die Weinproduktion. Auch bei diesen Trauben kann wieder unterschieden werden zwischen dem Anbau von Qualitätswein ${ }^{287}$, der 129,231 ha Anbaufläche umfasst, und dem Anbau aller anderen Sorten ${ }^{288}$, der

285 Vgl. NSO (2003a), S. 108-111

286 Dieser und die folgenden Werte aus NSO (2004b), S. 12, Tab. 2.4

287 Darunter sind im Rahmen der NSO-Statistik alle internationalen und DNA-geprüften Sorten wie z. B. Chardonnay und Sauvignon Blanc oder Merlot und Cabernet Sauvignon zu verstehen. Vgl. NSO (2004b), Einleitung zu den Kapiteln, ohne Seitenzahl. 
209,546 ha ausmacht. Der hohe Wert der anderen Sorten kommt durch die große Anzahl von Feldern mit einheimischen Weinsorten zustande, auf denen die unüberschaubare Anzahl von nicht weniger als 120 unterschiedlichen Rebsorten gedeiht ${ }^{289}$. Von 38 dieser Varietäten wird angenommen, dass sie endemisch für Malta und Gozo sind, während nur 20 Sorten insgesamt in größerem Stil angebaut werden ${ }^{290}$. Diese Werte beinhalten sowohl Tafeltrauben als auch Trauben zur Weinherstellung.

Der Weinbau in Malta wurde vermutlich von den Phöniziern initiiert ${ }^{291}$. Der Anbau, der sich im Lauf der Zeit verändert hat, weist stellenweise noch heute typische Anbaumethoden der frühen phönizischen Siedler auf, weitere Kultureinflüsse deuten auch auf griechische Ursprünge hin. Der Anbau von Wein war in Malta nie sehr verbreitet. Erst mit dem Auftreten kommerzieller Hersteller breitete sich die Anbaufläche aus. Davor konzentrierten sich die Anpflanzungen kleinräumig: ,In ancient days, Qormi was the land of vineyards and extensive vineyards must have existed also at Zabbar and in the hilly western districts, as shown by the characteristically long troughs excavated in the rock for the reception of the vine, and these are met with everywhere it was planted." (DEGUARA (1979), S. 1).

Der Schwerpunkt des heutigen Weinbaus liegt im Norden und Westen der Insel Malta. In diesen Landesteilen finden heute gut $83 \%$ des gesamten Weinbaus des Kleinstaats statt ${ }^{292}$. Durch den großen Erfolg des maltesischen Weins in den letzten 20 Jahren wird auch der Weinbau auf den Maltesischen Inseln immer wichtiger. AZZOPARDI bringt es auf den Punkt wenn er schreibt: „Of all the fruit that is produced locally, the most abundant and important is the fruit of the vine, the grape." (AZZOPARDI (2002), S. 166).

Es gibt auf der Inselgruppe vier Weinhersteller, die den Binnenmarkt dominieren: Marsovin Ltd., Master Wine Group (umfasst Camillieri Winery), Meridian Wine Estate und Emmanuel Delicata Winemaker. Zur Zeit der Schriftsetzung von CAMILLIERIS Arbeit (1994) gab es nur zwei große Hersteller, die den Wettbewerb miteinander ausgefochten haben: Marsovin und Delicata ${ }^{293}$. Heute verzeichnet auch Meridiana trotz exklusiver Preise steigende Verkäufe, und Master Wine Group ist in den 1990ern als dritter Großanbieter dazugekommen: Ein Indikator für die steigende Beliebtheit maltesischer Weine.

Darüber hinaus gibt es eine große Zahl ${ }^{294}$ von privaten Herstellern, die ihre Weine auf Tages- und Wochenmärkten, in Obst- und Gemüsegeschäften, am Straßenrand, in der Nähe touristisch hoch frequentierter Sehenswürdigkeiten oder direkt ab Erzeuger feilbieten. Aufgrund der hohen Qualität der kommerziell erzeugten Weine nimmt die Zahl der privaten Kleinhersteller seit Jahren ab, zumal der Wein der Kleinerzeuger auch unter der maltesischen Bevölkerung keinen guten Ruf hat. „Minor manufacturers producing what is known as local "artificial" wine seems [sic] condemned to extinction as the Maltese customer becomes more appreciative of quality. In a survey conducted in 1986, it was found that DIY (do-ityourself) wine making is also losing popularity." (CAMILLIERI (1994), S. 4 f.).

Ein anderer wichtiger Faktor war die Etablierung großer zusammenhängender vineyards in der jüngsten Vergangenheit. Dies wurde hauptsächlich von den Firmen Marsovin, Delicata und Meridiana vorangetrieben. In CAMILLIERIS Arbeit von 1994 werden diese Flächen noch nicht erwähnt. Damals war der Weinbau im „Sammelanbau“ auf kleinen Flächen, die im Besitz von mehr als 160 verschiedenen Farmern waren, üblich. Diese Methode führte in der Vergangenheit zu schwankender Qualität des Endprodukts. Daher wurde seit den späten 1980ern der Import von Trauben aus Italien zur Kelterung in Malta die Regel. Dies musste jedoch zunächst von der Regierung genehmigt werden, was aufgrund von Missernten der maltesischen

288 Darunter werden in der Statistik des NSO die indigenen maltesischen Sorten verstanden, die bisher nicht DNA-geprüft wurden. Vgl. NSO (2004b), Einleitung zu den Kapiteln, ohne Seitenzahl.

289 Vgl. CAMILLIERI (1994), S. 3

290 Ebd.

291 Deguara (1979), S. 1

292 Vgl. NSO (2004b), S. 12

293 Vgl. CAMILLIERI (1994), S. 10 ff. Es sollte an dieser Stelle erwähnt werden, dass Meridiana zwar 1994 schon existierte, jedoch die großen Quantitäten schon damals von Marsovin und Delicata stammten. Bis heute kann in Bezug auf Verkaufsquantitäten Meridiana nicht als ernsthafte Konkurrenz der drei großen Anbieter (Master Wine kam in den 1990ern dazu) gesehen werden, wenngleich die Weine dieses Herstellers von sehr ansprechender Qualität sind und sich immer größerer Beliebtheit erfreuen.

294 AZZOPARDI (2002) spricht in seinem Buch von insgesamt 20 Weinherstellern. Diese Zahl ist nicht belegt und kann, auch aufgrund der lückenhaften Datengrundlage die das NSO dazu bietet, angezweifelt werden. 
Bestände zu Beginn der 1990er durchgesetzt werden konnte: „In recent years, there have been a number of poor harvests which, in turn, induced the government to allow companies like Marsovin Ltd. and Emmanuel Delicata Winemakers to import grapes. This has allowed companies to produce high quality wine, which is competing favourably with imported wines as well as meeting consumer demands. Companies are allowed to import grapes on the condition that they purchase all the local production of grapes, unless the health of the grapes is not of acceptable standards. In turn, this has instigated local farmers to produce grapes of consistent quality." (CAMILLIERI (1994), S. 4).

Was sich in CAMILLIERIS Arbeit so einfach anhört und in sich schlüssig wirkt, funktioniert in der Realität leider nicht. Als die großen Hersteller erkannten, dass die Weine aus ausländischen Trauben teilweise besser waren als die bisher produzierten, setzte man alles daran, diese Qualität aufrechtzuerhalten. Als Ergebnis wurden die angesprochenen großen vineyards ausgebaut, die die "lokale Produktion" heute selbst übernehmen. Auch der Aufkauf von Weintrauben aus dem Ausland zeigt trotz Schwankungen eine insgesamt weiterhin steigende Tendenz (vgl. Abb. 11). Vorreiter dieser Politik ist Marsovin, während Delicata und Master Wine erst seit Beginn des 21. Jh. dem Vorbild Marsovins folgen. Meridiana hingegen konzentrierte sich von Beginn an auf die Herstellung von Qualitätswein geringer Quantitäten aus eigenem Anbau.

Es gibt durch die angesprochenen Entwicklungen mittlerweile Weine von maltesischen Herstellern, in denen fast keine oder sogar gar keine Anteile von in Malta gezogenen Trauben enthalten sind ${ }^{295}$. Zur weiteren Diskussion dieser Problematik wie zur Geschichte des Weinbaus und zur näheren Produktbeschreibung vgl. Kap. 14.1.3.

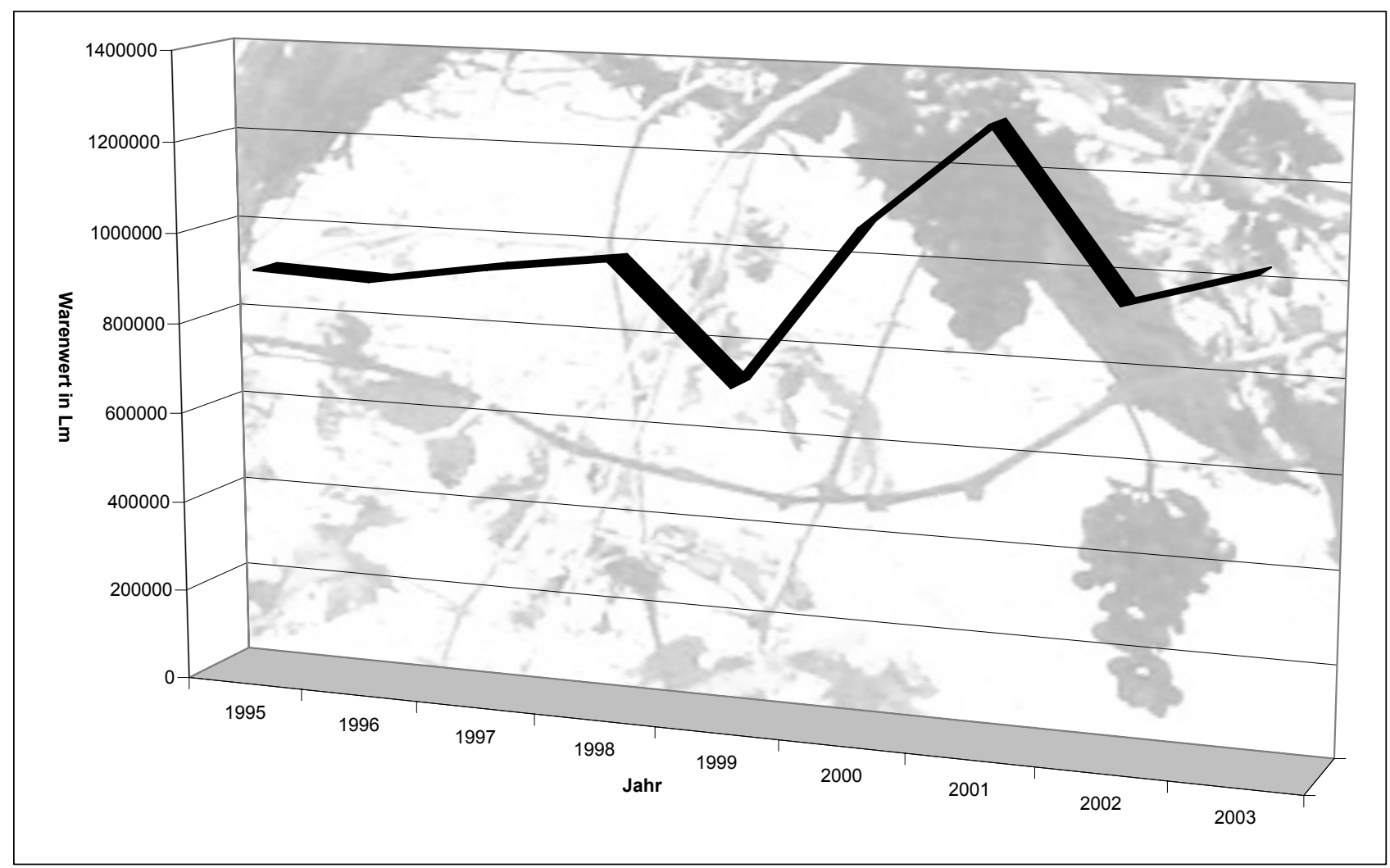

Abb. 11: Maltas Ausgaben für den Import von Weintrauben (Tafeltrauben und Trauben für die Weinherstellung) in Lm. Entwurf: ASCHEMEIER. Quelle: NSO-Daten, die individuell für den Autor zusammengestellt wurden.

295 z. B. Trebbiano von Marsovin. Die Trauben für diesen Wein werden fast zur Gänze in Sizilien geerntet. In Fällen wie diesem muss das Ursprungsland der Trauben auf dem Flaschenetikett angegeben werden. 


\subsection{Gemüse}

- Artischocke (qaqoćć)

(Stachys affinis Bunge)

Die Artischocke ist eine der alten Nutzpflanzen Maltas. Schon zu Zeiten der Ritter vom Johanniterorden wird berichtet, dass die Inselbevölkerung Maltas und Gozos sich von „wilden Artischocken“ ernährt habe. Die moderne Botanik kennt aber keine Wildform der Artischocke ${ }^{296}$. Vermutlich stammt die Kulturform, die aus Amerika eingeführt wurde ${ }^{297}$, von der Distelart $C$. cardunculus $L$. ab. In der Vergangenheit Maltas war nur eine der Artischocke ähnliche Distelart in Gebrauch ${ }^{298}$, die nur wenige Tage im Jahr genießbar war und aufwändig zubereitet und konserviert werden musste. Wahrscheinlich handelt es sich dabei um die Milchfleckdistel (Galactites tomentosa L.), die den alten Beschreibungen am ehesten entspricht.

Die domestizierte Artischocke wird im August gepflanzt (es werden Ableger benutzt, keine Sämlinge). Das Gemüse kann im Dezember zum ersten Mal geerntet werden. Die Pflanze blüht jedoch noch bis ins Frühjahr hinein.

Artischocken sind auf den Maltesischen Inseln nur vereinzelt auf größeren Flächen zu sehen, wirtschaftlich sind sie, auch bedingt durch soziokulturelle Umstände (vgl. Kap. 20.2), bisher unwichtig.

\section{- Aubergine (brungiiel)}

(Solanum melongena var. Esculentum N.)

Der Anbau von Auberginen ist in Malta mit 19,240 ha Gesamtflächenanteil ${ }^{299}$ eher unbedeutend. Möglicherweise liegt dies an der Nähe zu Sizilien, das ein Hauptproduzent für Auberginen im Mittelmeerraum ist. Der Anbau in Malta erfolgt in den meisten Fällen nicht im Gewächshaus ${ }^{300}$. Die in Sizilien in großer Zahl vorhandenen regionalen traditionellen Sekundärprodukte (z. B. eingelegte Auberginenscheiben, Auberginenmus, etc.) existieren in dieser Form in Malta nicht.

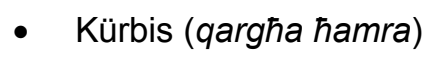

(Cucurbita moschata (Duch))

Die Kürbisse Maltas werden im August und September geerntet. Nachdem die grünen Anteile der Kürbispflanze vollständig vertrocknet sind, wird die Kürbisfrucht abgetrennt. Es folgt ein Nachreifeprozess, bei dem die Kürbisse in langen Reihen auf Hausdächern und/oder Mauern der Sonne ausgesetzt werden ein typisches Bild der maltesischen Kulturlandschaft zum Ende jedes Sommers. Der Kürbis dient als Grundlage der typisch maltesischen Gemüsesuppe Minestra und wird auch in anderer Form (z. B. eingekocht) verwendet.

Gegenwärtig werden Kürbisse vor allem im Norden der Insel Malta und in Gozo angebaut ${ }^{301}$. Mit insgesamt 50,12 ha Gesamtflächenanteil ${ }^{302}$ gehört der Kürbis aber nicht zu den wichtigsten Kulturpflanzen.

\footnotetext{
296 Vgl. SCHÖNFELDER \& SCHÖNFELDER (1999), S. 244

297 Vgl. AZZOPARDI (2002), S. 164

298 Vgl. CREMONA (2003), S. 117

$299 \mathrm{Vgl}$. NSO (2003a), S. 94 f.

300 Vgl. FARRUGIA (o. J.)

301 Vgl. NSO (2003a), S. 100

302 Ebd.
} 
(Lycopersicon esculentum M.)

Die Tomate ist eine der wichtigsten Kulturpflanzen der Maltesischen Inseln. Sie dient zudem als Lebensgrundlage einer ganzen Gruppe von Landwirten, die sich schwerpunktmäßig dem Tomatenanbau gewidmet haben. Schon 1966 schreibt NEHRING: „Unter den wichtigsten agrarischen Produkten für den einheimischen Markt und in der Ernährung der Bevölkerung, sowie für eine industrielle Verarbeitung, sind Tomaten zu nennen (...)." (NEHRING (1966), S. 112). Daran hat sich bis heute nichts geändert ${ }^{303}$.

Flächenmäßig nahm der Anbau im Verlauf der letzten zwei Dekaden allerdings rapide von 562 ha in den Jahren 1982/83 auf 351,7 ha in den Jahren 2000/01 $\mathrm{ab}^{304}$. Der Ertrag an Tomaten nahm im selben Zeitraum trotz des Flächenverlusts aber zu, so wie es auch bei einigen anderen Nutzpflanzen in Malta der Fall war. GODFREY CAMILLIERI vom staatlichen Agricultural Research and Development Centre in Lija erklärt das Phänomen folgendermaßen: „There's a simple word: efficiency! Efficiency is obviously going up. For example in agriculture drip irrigation has increased efficiency enormous in this part of the world. Also people are more and more orientated to bring in high quality seeds which can produce higher outputs. They make better use of the land, manure it properly, cultivate it properly, etc.. There is quite an amount of very intensive cultivation locally. Also the increase of glasshouses increased the amount per hectar." (GoDFREY CAMILLIERI im Experteninterview vom 02.06.2004). Der zuletzt genannte Faktor, in Verbindung mit der Verfügbarkeit von einheitlich qualitativem Saatgut, der Tröpfchenbewässerung und erhöhter Düngung, dürfte es ermöglicht haben, dass sich die Fläche des Tomatenanbaus stark verringern konnte, während der Ertrag immer mehr zunahm. Zur Zeit von NEHRINGs Arbeit wurden nur 1,23 ha des Tomatenanbaus unter Glas bewerkstelligt $^{305}$. Heute findet nahezu der gesamte Tomatenanbau in Gewächshäusern oder unter Folien statt.

Tomaten werden, vor allem industriell, aber auch alltäglich in der Bevölkerung, zu zahlreichen regionalen traditionellen Produkten weiterverarbeitet. Die kommerziell bedeutsamste Rolle spielt dabei die Tomatenpaste Kunserva, die schon vor der Zeit der Johanniterritter ein typischer Bestandteil der maltesischen Ernährung war. Kunserva wurde im Rahmen der EU-Verhandlungen von der Europäischen Union als geschützte Herkunftsbezeichnung anerkannt ${ }^{306}$. Bereits in den 1960er-Jahren wurden sieben Fabriken für die Erzeugung von konservierten Tomatenprodukten erwähnt ${ }^{307}$. Heute sind nach Kenntnisstand des Autors davon noch drei Produktionsstätten übrig geblieben, diese sprengen jedoch die einstigen Maßstäbe.

Der größte Hersteller für Sekundärprodukte der genannten Art ist die gozitanische Firma Magro Bros. Mit Sitz in Xewkija, für die exklusiv 400 maltesische Farmer arbeiten. Dabei wird das Saatgut gestellt und die Pflanzzeit einheitlich vorgegeben. Insgesamt verarbeitete die Magro Company für ihre Hausmarken Mayor (Saucen, Tomatenmark, passierte Tomaten, etc.), Three Hills (Kunserva und Ketchup) im Jahr 2003 Erträge im Wert von Lm 700.000 (ca. 1,64 Mio. €). Tomaten im Wert von über Lm 1 Mio. (ca. 2,34 Mio. $€$ ) wurden aus dem von der Firma gestellten Saatgut erzeugt. 10.000t Tomaten insgesamt wurden 2003 auf den Maltesischen Inseln verkauft, von denen $65 \%$ von Magro abgenommen wurden ${ }^{308}$.

Maltesische Tomaten werden bis heute ausschließlich von Hand gepflückt. Maschinelle Pflücktechnik hat bisher noch nicht Einzug gehalten, obwohl es mittlerweile Tomatenanbau unter großen Folienflächen und vor allem in Gewächshäusern gibt. Der Gewächshausanbau dient vor allem dem Windschutz, da starker Wind - mit dem in Malta auch im Sommer jederzeit gerechnet werden muss - die Pflanzen schädigen kann. Außerdem wird durch die Gewächshäuser ein früherer Erntebeginn und ein verzögertes Ende der Erntesaison möglich.

303 Vgl. BusutTIL \& DEMICOLI (2003), S. 6

304 Vgl. NSO (2003a), S. 4

305 NEHRING (1966), S. 112

306 Vgl. BusutTIL \& DEMICOLI (2003), S. 6

307 NEHRING (1966), S. 112

308 Angaben nach MASSA in: THE TIMES OF MALTA vom 13.07.2004, S. 5 
Der Tomatenanbau wird seit dem EU-Beitritt Maltas subventioniert. 2004 griff erstmals das Special Market Policy Program der Regierung ${ }^{309}$ (vgl. Kap. 12.1.6), das sich direkt auf die großen Verarbeiter auswirkte: Magro musste 2004 seine Abnahme von Tomaten im Vergleich zu 2003 um weitere 25\% erhöhen ${ }^{310}$, wobei zu beachten ist, dass sich dieser Wert auf die Gesamtabnahme des Herstellers und nicht auf die Gesamtsumme der national erzeugten Tomaten bezieht. Dank der Unterstützung durch das EU-Programm wird damit gerechnet, dass der Tomatenanbau seinen Output in den nächsten Jahren mehr als verdoppeln kann. Erste Schätzungen gehen von 27.000t Gesamtertrag im Jahr 2010 aus $^{311}$.

Der Export soll vorangetrieben werden, was zumindest aufgrund der hohen Qualität des Produkts nicht unrealistisch erscheint. Firmeneigner JOHN MAGRO äußerte in einem Zeitungsinterview: „The Maltese tomato has a taste unequalled by others." (THE TIMES OF MALTA, 13.07.2004, S. 5).

In der traditionellen Küche dienen Tomaten meistens als Saucengrundlage, als Beilage, als Salat oder sie werden (sonnen-)getrocknet. Sie sind unter der Bevölkerung extrem beliebt. Dies ist nicht nur der außergewöhnlich guten Qualität der maltesischen Tomaten zuzuschreiben, sondern vor allem auch der Tatsache, dass Tomaten eine der wenigen Gemüsesorten sind, die im Juli und August geerntet werden; in einer Zeit in der normalerweise aufgrund der Dürre des südmediterranen Sommers die meisten anderen Feldfrüchte nicht mehr zur Verfügung stehen. Während diese in der Zeit zwischen Juli und August immer teurer und qualitativ schlechter werden, werden Tomaten qualitativ immer besser und gleichzeitig billiger. Dies macht sie zum „Verkaufsschlager" unter der einheimischen Bevölkerung und soll auch im Export Wunder wirken.

CREMONA beschreibt die Situation - sowohl heute als auch in historischer Zeit - zur Erntesaison: „While the vine tomatoes go into the ground, the season for non-vine tomatoes is at its height. The crop is abundant, the tomato is at its tastiest, and prices fall as they ever get. Crates of the fruit are sold cheaply from trucks or taken off to the canning factories. Yet before canning, other means were devised to preserve the tomato for use in the winter months, and many of those methods, including sun-drying, are still used today. The results are now seen to be a particular delicacy, rather than as a straightforward preserved tomato and the next best thing to a fresh one." (CREMONA (2003), S. 11).

Tomaten sind auch Bestandteil der maltesischen Folklore mit ihren traditionellen Gebräuchen und Verhaltensweisen. Ein Aberglaube z. B. verbot es maltesischen Frauen, in der Schwangerschaft in Salzlake konservierte Tomaten zu verzehren ${ }^{312}$. Der maltesische Name tuffie $\hbar$ tadam bedeutet soviel wie „Apfel Adams" und bezieht sich somit mystizistisch auf das biblische Buch Genesis. Dies zeigt, wie sehr die rote Frucht auch im Volks-Aberglauben der Malteser Spuren hinterlassen hat. Tomaten werden unter der Bevölkerung als "typisch maltesisch" wahrgenommen, und darüber hinaus als qualitativ den gleichartigen Erzeugnissen aus anderen Regionen der Erde überlegen betrachtet. Sie sind als kulturelles Erbe Maltas mindestens so etabliert wie Ǵbejniet und Lampuki (s. Kap. 14.1).

Der Autor betont diesen Umstand deswegen besonders, weil die bisherigen Arbeiten über Maltas Agrarwirtschaft über die wirtschaftliche Bedeutung des Tomatenanbaus hinaus die Identifikation der Bevölkerung mit einer ihrer wichtigsten Feldfrüchte nie erwähnt hatten ${ }^{313}$. Das erscheint im Prinzip logisch, denn der Tomatenanbau ist in jedem südeuropäischen Land etabliert und in dieser Hinsicht keine Besonderheit. Tomaten wurden somit bislang als reine Wirtschaftsware angesehen.

Der Verfasser möchte ausdrücklich betonen, dass Tomaten und ihre Folgeprodukte auch zu den identitätsprägenden Erzeugnissen der maltesischen Landwirtschaft gehören. Ihre Erntezeit wird von der

309 Malta musste mit dem EU-Beitritt auf die bisher üblichen Zölle auf eingeführte Tomaten für die Weiterverarbeitung verzichten. Aus diesem Grund wird jetzt ein Teilsektor des Tomatenanbaus, nämlich nur der Anbau für die Weiterverarbeitung in der Konserven-, Saft- und Ketchup-Industrie, subventioniert. Bis 2014 fließen 31,16 Mio. $€$ finanziert von der maltesischen Regierung und der EU in diesen Teilsektor. Darüber hinaus mussten sich die Weiterverarbeiter zu höheren Abnahmequoten verpflichten (vgl. Text). Zur Vertiefung vgl. BUSUTTIL \& DEMICOLI (2003).

310 MASSA in: THE TIMES OF MALTA vom 13.07.2004, S. 5

311 BuSUTTIL \& DEMICOLI (2003), S. 6

312 Vgl. ZARB (1998), S. 41

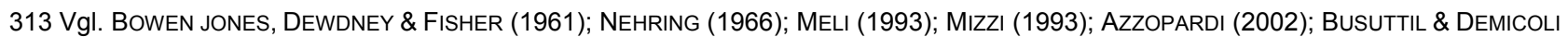
(2003) 
einheimischen Bevölkerung, ähnlich wie die Ernte von Kartoffeln, seit Jahrzehnten als eine Art „nationales Ereignis" wahrgenommen ${ }^{314}$.

\subsection{Leguminosen}

\section{- Bohnen (ful)}

(Vicia spec.)

In Malta werden verschiedene Bohnensorten angebaut. Sie dienen heute meist als Viehfutter ${ }^{315}$, waren in früheren Zeiten, da sie das Protein lugumin enthalten, aber auch wichtige Proteinlieferanten der traditionell proteinarmen maltesischen Küche.

Die vorherrschende Bohnensorte ist die Saubohne (engl:: broad bean; botan.: vicia faba minor). Bohnen werden meist auf kleinen Flächen angebaut. Sie kommen auch vereinzelt im Verbund mit Gerste vor. Von den beachtlichen 358,337 ha Anteil des Bohnenanbaus an der gesamten landwirtschaftlichen Nutzfläche des Landes, sind 78,53\% kleiner als 0,336 ha. Der Rest der gesamten Bohnenanbaufläche gedeiht derzeit sogar auf Flächengrößen von durchschnittlich weniger als 0,112 ha ${ }^{316}$. Dies lässt ahnen, dass der Bohnenanbau trotz gesamtanteilig hohem Flächenverbrauch keine nennenswerte kommerzielle Rolle spielt, sondern hauptsächlich zur Futternutzung und zur Eigenversorgung von nebenberuflichen Landwirten dient.

Dennoch gibt es neben zahllosen lokalen Gerichten einige regionale traditionelle Produkte, die auf Bohnenbasis hergestellt werden. Das bekannteste dieser Produkte ist die Bohnenpaste Bigilla, die als Dip oder Brotaufstrich verwendet wird ${ }^{317}$.

\subsection{Hackfrüchte}

\section{- Kartoffel (patata)}

(Caprium annum)

Vom wirtschaftlichen Standpunkt aus betrachtet, ist die Kartoffelpflanze die wichtigste Kulturpflanze der Maltesischen Inseln. Sie ist mit derzeit $1.153,5$ ha Flächenanteil ${ }^{318}$ nach Weizen die Feldfrucht mit der größten Verbreitung ${ }^{319}$. Der Kartoffelanbau in Malta war von Beginn an eine Erfolgs-Story. Fast alle traditionellen maltesischen Gerichte (mit Ausnahme der Nudel- und Reisgerichte) werden mit Kartoffeln serviert. Dennoch wurde die Kartoffelpflanze auf den Maltesischen Inseln erst im Januar 1805 eingeführt ${ }^{320}$. Es gibt zwei Sorten im Anbau: Die „gelbe Kartoffel“ (malt.: Patata Safra) und die „weiße Kartoffel“ (malt.: Patata Bajda). Beide Sorten werden überwiegend im Dezember und Januar in den Boden ausgebracht. Im März erfolgt die Blüte und im April/Mai kann geerntet werden.

Die erste Ernte erfolgt hauptsächlich für den inländischen Verkauf aber schon während der Hauptpflanzzeit im Dezember zum Weihnachtsgeschäft ${ }^{321}$. Diese Kartoffeln sind bereits im August gesät worden ${ }^{322}$. Die

314 Vgl. TASTE MAGAZINE 1/2004, S. 44 f.

315 Vgl. AZZOPARDI (2002), S. 160

$316 \mathrm{Vgl}$. NSO (2003a), S. 96 f.

317 Vgl. Kap. 14.1

318 Vgl. NSO (2003a), S. 4

319 Vgl. ebd., S. xxii

320 Vgl. TASTE MAGAZINE 3/2004, S. 17. Bereits im 17. Jh. hatten die Johanniter versucht, die Kartoffel in Malta einzuführen, scheiterten jedoch an den Farmern, die sich weigerten die Knolle anzubauen. Vgl. CREMONA, S. 56

321 Vgl. CREMONA (2003), S. 7

322 Als noch keine flächendeckende Bewässerung möglich war, richtete man sich bzgl. des Zeitpunkts der Kartoffelaussaat nach einem kirchlichen Feiertag: „Our Lady of Victories“ am 08. September war der Tag der ersten Aussaat. Klimatisch gesehen war dies der Zeitpunkt an dem die ersten Regenschauer nach dem Sommer zu erwarten waren. Vgl. CREMONA (2003), S. 7 
zweite Ernte im April dient dann vornehmlich dem Export. Nach wie vor sind Kartoffeln Maltas wichtigster agrarischer Exportartikel ${ }^{323}$. Sie werden vor allem in die Niederlande und nach Großbritannien verschifft ${ }^{324}$. Die Exportrate ist gemessen am Vergleich mit dem Export anderer landwirtschaftlicher maltesischer Erzeugnisse gewaltig. Im Jahr 2004 wurde ein neuer Rekord aufgestellt: Für rund Lm 1,4 Mio. (= 3,29 Mio. €) wurden in jenem Jahr Kartoffeln nach Holland exportiert - Lm 611.384 (= 1,44 Mio. €) mehr als im Vorjahr ${ }^{325}$.

Der derzeitige Plan des Ministeriums für Landwirtschaft und Fischerei sieht vor, den Export auch auf andere EU-Länder auszudehnen. Insbesondere die Schweiz, Dänemark und Deutschland sind dabei im „Visier“ der maltesischen Kartoffelbauern. Im Jahr 2006 wird erstmals der Kartoffelexport nach Deutschland stattfinden $^{326}$.

Regionale traditionelle Produkte auf Kartoffelbasis existieren erstaunlicherweise fast gar nicht. Die Kartoffel wird in Malta allgemein als ein Produkt wahrgenommen auf dessen hohe Qualität man stolz ist ${ }^{327}$. Kartoffeln werden meist als Beilage mit Fenchelsamen gekocht oder gebacken und dann mit viel jungem Olivenöl gegessen.

- Knoblauch (tewm)

(Allium sativum L.)

In Malta ist Knoblauch in zwei Erscheinungsformen bekannt: als getrockneter (malt.: tewn niexef) und grüner Knoblauch (malt.: tewm aћdar). Der Anbau spielt wirtschaftlich gesehen keine Rolle. Knoblauch wird in großen Mengen aus anderen Ländern des Mediterrans importiert. Selbst fahrende Gemüsehändler haben nur selten maltesischen Knoblauch im Sortiment.

Die aromatische Pflanze ist einer der wichtigsten Bestandteile der sog. „Maltesischen Soße“, die als Grundlage für viele Gerichte der maltesischen Küche unverzichtbar ist.

- Zwiebeln (basal)

(Allium capa L.)

Die Flächenanteile des Zwiebelanbaus waren in der Vergangenheit größeren Schwankungen unterworfen. Der Anbau von Zwiebeln in Malta ist seit über 30 Jahren ein kontinuierliches „Auf und Ab“. Zusammen mit Bohnen und Tomaten gehören Zwiebeln zu den wichtigsten Feldfrüchten der Maltesischen Inseln ${ }^{328}$. Sie sind jedoch durch die Dominanz des Kartoffelanbaus in ihrer Entwicklung eingeschränkt. Im Bereich der regionalen traditionellen Produkte spielen sie zur Weiterverarbeitung kaum eine Rolle. Für sich allein genommen sind Zwiebeln jedoch weit verbreitet und gehören zum umsatzstärksten Gemüse des Maltesischen Binnenmarkts. Wie aus Abb. 12 ersichtlich ist, unterliegt auch der Verkauf von Zwiebeln aktuell starken Schwankungen.

323 Vgl. AZZOPARDI (2002), S. 162

324 Ebd., S. 162

325 Vgl. TIMES OF MALTA, Ausgabe vom 28.05.2004, S. 16

326 Vgl. Rundbrief 2/2005 der Deutsch-Maltesischen Gesellschaft

$327 \mathrm{Vgl}$. TASTE MAGAZINE 3/2004, S. $16 \mathrm{f}$.

328 Vgl. NSO (2003a), S. xxii 


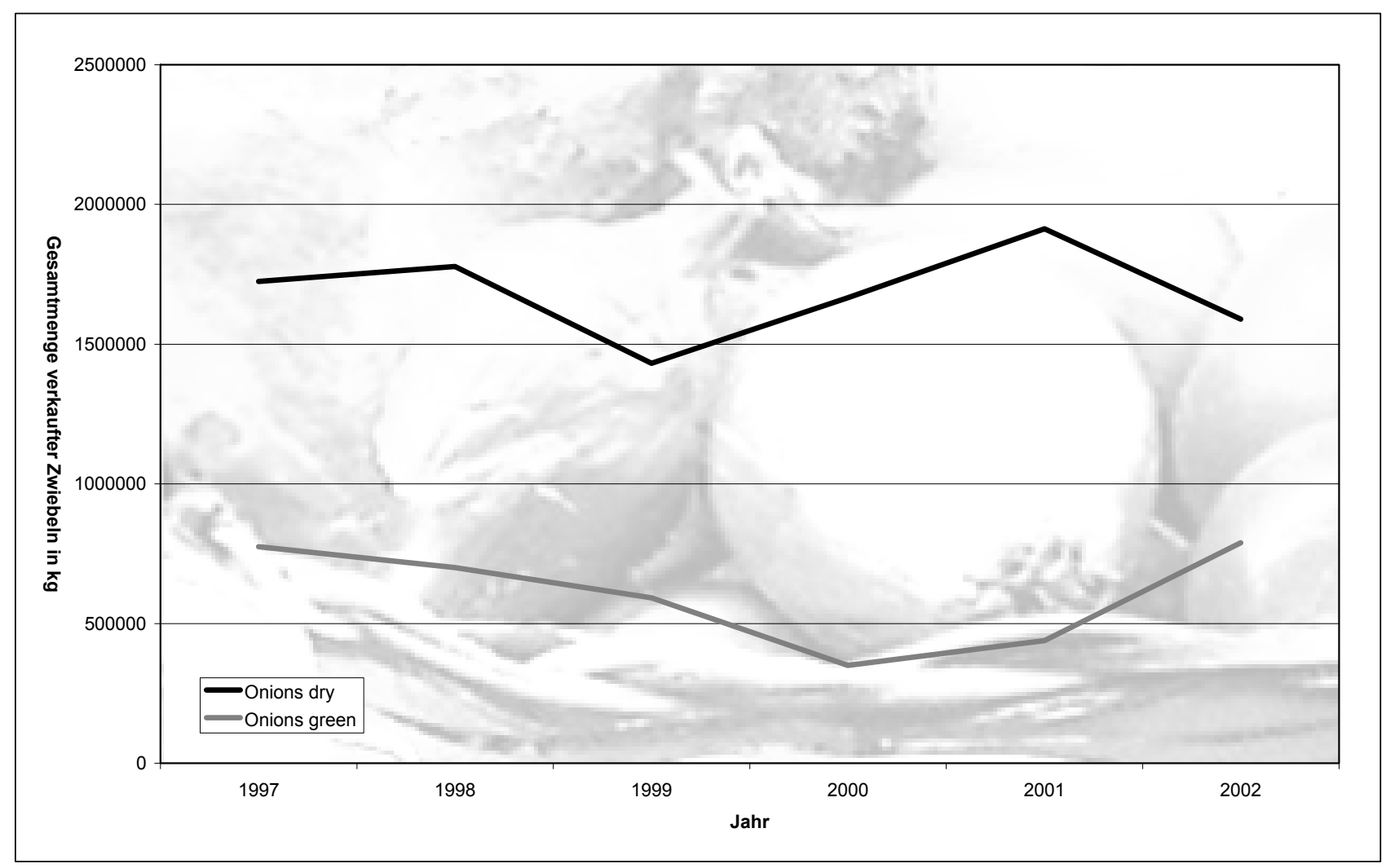

Abb. 12: Die Schwankungen beim Verkauf von Zwiebeln in Malta 1999-2002. Entwurf: AsCHEMEIER. Daten: NSO (2004d)

\subsection{Getreide}

- Gerste (xejr bzw. xghir)

(Hordeum vulgare L.)

Der Anbau von Gerste hat innerhalb der letzten zwanzig Jahre stark abgenommen. Während zwischen 1970 und 1983 ihre Flächenanteile im landwirtschaftlichen Anbau sich jeweils im Rahmen von 900-1.100 ha bewegten, beträgt der Gesamtflächenanteil des Gerstenanbaus heute nur noch 426 ha $^{329}$.

Maltesische Gerste wird häufig in einer Mischung mit Weizen ausgesät ${ }^{330}$. Solche Saatmischungen heißen auf Maltesisch maћlut, meslin oder mixta. Sofern die Bodenfeuchtigkeit in einem bestimmten Jahr nicht für den Weizen ausreicht, kann immer noch die Gerste geerntet werden. Daher muss von einer großen Unsicherheit der statistischen Daten für beide Getreidearten ausgegangen werden.

Gerste ist in der NSO-Statistik, ebenso wie Weizen, als Futterpflanze angegeben. Getreide wird in Malta seit Jahrzehnten fast nur zur Futterverwertung angebaut. Für die Nahrungsmittelindustrie wird es importiert ${ }^{331}$. 167,685 ha des derzeitigen Gerstenanbaus befinden sich auf Feldern, die 1 ha oder größer sind ${ }^{332}$, was im Prinzip die maschinelle Landbearbeitung ermöglicht. Der Schwerpunkt liegt mit 332,323 ha Anteil an der Gesamtanbaufläche in Gozo ${ }^{333}$. Der Weizenanbau konzentriert sich aber eindeutig auf die Insel Malta ${ }^{334}$.

329 Vgl. NSO (2003a), S. 4

330 Vgl. AZZOPARDI (2002), S. 160; BOWEN JONES, DEWDNEY \& FISHER (1961), S. 195

$331 \mathrm{Vgl}$. ebd.

332 Vgl. NSO (2003a), S. 83

333 Vgl. ebd., S. 84

334 Vgl. ebd., S. 83 
- Weizen (qamћ)

(Triticum spec.)

Im Gegensatz zu Gerste hat der Anbau von Weizen während der letzten 20 Jahre deutlich zugenommen. In der Dekade von $1972 / 73$ bis $1982 / 83$ war ein Anstieg von 917,1 ha auf 1.949 ha mit Weizen bestellter Fläche zu verzeichnen. Von 1982/83 bis 2000/01 wuchs sie auf $3.572,2$ ha $\mathrm{an}^{335}$. Damit ist Weizen die bedeutendste Anbaufrucht im Futterpflanzensektor, der insgesamt eine Fläche von 4.464 ha umfasst ${ }^{336}$. Getreide wird in Malta vor allem zur Futterverwertung angebaut. Getreide für die Nahrungsmittelindustrie wird fast ausschließlich importiert ${ }^{337}$.

Maltesischer Weizen wird häufig in einer Mischung mit Gerste ausgesät (s. o.). Daher sind die statistischen Angaben über den Weizenanbau oft ungenau (s. o.). Aber auch wenn die Weizensaat in reiner Form vorliegt, ist die Bestimmung schwierig, weil häufig maltesische Bastard-Sorten im Anbau vorkommen, die (gewollt oder ungewollt) mit anderen Getreidearten gekreuzt wurden ${ }^{338}$.

Weizen ist nach der offiziellen Statistik das Getreide, welches die anteilsmäßig höchsten Flächengrößen einzelner Anbauareale aufweist. 2.044,337 ha, also mehr als die Hälfte der mit Weizen bestellten Felder, weisen eine durchschnittliche Flächengröße von 1 ha oder mehr auf ${ }^{339}$, was für maltesische Verhältnisse bedeutet, dass der Anbau in "großem Stil“ stattfinden kann und Landbearbeitungsmaschinen zum Einsatz kommen. Der Weizenanbau konzentriert sich auf die Hauptinsel Malta. Vor allem im Norden und Westen Maltas liegt mit 1.862,54 ha Anbaufläche das Zentrum der Weizenerzeugung ${ }^{340}$. Insgesamt befinden sich 3.064,971 ha des gesamten Anbaus in Malta ${ }^{341}$. Gozo dominiert hingegen beim Anbau von Gerste (s. o.).

\subsection{Nüsse}

\section{- Mandeln (lewż)}

(Prunus amygdalus Batsch.)

Als typischer Vertreter des südlichen Mittelmeerraums ist der Mandelbaum auch in Malta zu finden. Jedoch ist seine Verbreitung so spärlich, dass selbst viele Insulaner kaum wissen wie der Baum aussieht: „The Almond is a pure native of the Mediterranean, but how many of us know what the nuts look like as they grow on the tree? Like the city children who think that potatoes are made in factories, we're too accustomed to seeing them only in little plastic packets or ready-roasted in branded tins." (TASTE MAGAZINE (1/2004), S. 41).

Nichtsdestotrotz finden sich in der Nähe von Dingli im ländlichen Südwesten Maltas auch größere heckenartige Anpflanzungen, die als Windschutz angelegt wurden. Der Mandelbaum wird in Malta kaum genutzt. Mandeln werden aus Marokko, Sizilien und Spanien eingeführt. Die Nuss kann im Herbst geerntet werden.

335 Vgl. NSO (2003a)., S. 4

336 Vgl. ebd., S. xxii

337 Vgl. AZZOPARDI (2002), S. 160; BOWEN JONES, DEWDNEY \& FISHER (1961), S. 195

338 Vgl. BOWEN JONES, DEWDNEY \& FISHER (1961), S. 195

$339 \mathrm{Vgl}$. NSO (2003a), S. 83

$340 \mathrm{Vgl}$. ebd.

$341 \mathrm{Vgl.} \mathrm{ebd.}$ 


\subsection{Sonstige Anbaupflanzen}

- Klee (silla)

(Linnaeus Hedysarium conorarium)

Klee als Futterpflanze hat stark an Bedeutung eingebüßt. Waren 1972/73 noch 2.391,5 ha agrarischer Fläche mit Klee bepflanzt, so waren es 2000/01 nur noch 363,2 ha $^{342}$. Dies liegt im Trend der anderen Futterpflanzen, die ebenfalls nahezu sämtlich (mit Ausnahme von Weizen) an Flächenanteilen verloren haben. Unklar ist, ob die NSO-Statistik nur den kontrollierten Anbau umfasst, oder auch die immer noch zahlreich vorhandenen aufgegebenen Hangterrassen, auf denen der Klee nun wild wächst.

Klee für Futterzwecke wurde in der Vergangenheit Ende Mai gemäht. Die Mahd wurde auf den AnbauArealen belassen und durch Luft und Sonne getrocknet. Klee, der wieder zur Saat verwendet werden sollte, wurde länger im Wachstum belassen und dann bei einbrechender Dunkelheit gemäht, sodass der Tau der Nacht die Pflanzen feucht und weich werden ließ. Am darauf folgenden Tag wurde der so behandelte Klee gedroschen und die Samen gesammelt, um sie im August auszusäen ${ }^{343}$.

\section{- Ölbaum/Olive (żebbuǵ/żebbuǵa)}

(Olea europea L.)

Der Ölbaum wurde in Malta, ähnlich wie im gesamten Mediterran, schon seit der Antike kultiviert. Olivenöl scheint aber insbesondere während der römischen Periode Maltas (ab 218 v. Chr. bis ca. 535 n. Chr.) eine Schlüsselrolle in der Wirtschaft der Inselgruppe gespielt zu haben ${ }^{344}$. Eine detaillierte Schilderung des historischen Hintergrunds der Olivenölproduktion auf den Maltesischen Inseln ist deshalb als gesondert abgefasstes Kapitel 20.3 zu finden.

Trotz der langen Tradition kam die Kultivierung von Olivenbäumen in späteren Jahrhunderten nahezu vollständig zum Erliegen. Eine genaue Datierung dieses Verfallsprozesses ist unklar. Es gibt in der einschlägigen Literatur auch verschiedene Erklärungen für den Grund der Einstellung der Ölbaumkultivierung. Es kann daher an dieser Stelle keine genaue Angabe gemacht werden. CREMONA schreibt zu diesem Problem lediglich: „We do know that the production of olive oil ceased at some point in the "dark age" after the end of Roman rule, and that the olive trees that once covered the islands were cut down, (...)." (CREMONA (2003), S. 32). NEHRING berichtete von 80.000 Ölbäumen, die noch im 19. Jh. auf den Maltesischen Inseln gefällt worden seien ${ }^{345}$. Die von ihm zu dieser Behauptung angegebene Literaturquelle erwies sich bei neuerlicher Durchsicht durch den Verfasser der vorliegenden Arbeit jedoch als unsicher: Es findet sich dort kein Hinweis auf ein solches Ereignis. NEHRINGs Angabe ist damit nicht belegbar. BowEN JONES et al. schrieben, der Olivenanbau sei früher offenbar verbreitet gewesen, zur Zeit der Schriftsetzung ihrer Arbeit sei er jedoch nahezu nicht mehr existent ${ }^{346}$.

Zusammengefasst lässt sich sagen, dass der Verfallsprozess des Olivenanbaus in Malta von verschiedenen Autoren unterschiedlich interpretiert wird. Es gibt gegenwärtig keine herrschende Meinung.

Im Jahr 2000 wurde die Olivenölproduktion wieder in kommerziell verwertbare Bahnen gelenkt ${ }^{347}$. Seitdem ist auf den Inseln ein wahrer „Boom“ spürbar. Leider gibt es kaum nachprüfbare statistische Datengrundlagen, anhand derer dieser Prozess nachvollziehbar wäre. Es scheint tatsächlich so zu sein,

342 Vgl. NSO (2003a), S. 4

343 Vgl. CREMONA (2003), S. 112

344 Vgl. Kap. 20.3.2

345 Vgl. NeHRING (1966), S. 32

346 Vgl. BOWEN JONES, DEWDNEY \& FISHER (1961), S. 213

347 Vgl. Kap. 20.3 
dass die Ölbaumanpflanzung so lange nicht betrieben worden ist ${ }^{348}$, dass sie in den statistischen Erhebungen des National Statistics Office gar nicht als ausgewiesener Bereich auftaucht ${ }^{349}$.

Die jüngste Veröffentlichung des NSO zeigt in Bezug auf den direkten Verkauf von Oliven auf organisierten Märkten seit 2000 rückläufige Werte ${ }^{350}$. Ob dies eine direkte Konsequenz der Möglichkeit ist, die Oliven seit dem betreffenden Jahr auch zu Olivenöl weiterverarbeiten zu lassen ${ }^{351}$, ist nicht bekannt.

Jedoch kann der Autor anhand persönlicher Expertengespräche ${ }^{352}$ nachweisen, dass die Ölbaumkultivierung auf den Maltesischen Inseln derzeit schnell ansteigende Flächenanteile und damit einhergehende Bedeutungsgewinne aufweist. Neue Anpflanzungen sind vor allem in Malta zu beobachten und konzentrieren sich auf die Regionen Dingli, Sigggiewi/Zurrieq, Wardija und Marfa Ridge. Sie sind professionell angelegt, mit „drip irrigation“-Systemen versehen und besetzen immer größere Flächenanteile.

AZZOPARDI schreibt diesbezüglich: „It is only in recent years that the oil-producing industry has again been taken up seriously. In the year 2000, at Wardija alone, 17 tons of olives, extracting 2,600 kg of pure olive oil, of which $600 \mathrm{~kg}$ were put on the market, were produced. This same industry now hopes to be in a position to press 100 tons of olives in a few year's time. Other olive oil-producing industries have now began [sic] also in other parts of Malta." (AZZOPARDI (2002), S. 166).

Diese Entwicklung ist vor allem die Konsequenz der exquisiten Qualität des gewonnenen Olivenöls der letzten fünf Jahre. In jüngster Vergangenheit wurden von Seiten der Investoren, in Zusammenarbeit mit maltesischen und italienischen Forschungsinstituten, Bestrebungen unternommen, endemische Varietäten des Ölbaums in Malta wieder zu entdecken und für die Olivenölproduktion zu nutzen.

Zur Geschichte der Olivenölproduktion in Malta, sowie zur weiteren thematischen Vertiefung s. Kap. 14.1 sowie 20.3.

\section{- Pfeffer (bżar aћdar)}

(Piper spec.)

Pfeffer ist eine durchaus wichtige Pflanze im Anbau. 32,593 ha Nutzfläche werden zum Pfefferanbau genutzt $^{353}$. Er bildet eine wichtige Grundlage für zahlreiche Produkte und Gerichte der maltesischen Küche. Pfeffer ist neben Kümmel und Fenchelsaat das typische Gewürz der Maltesischen Inseln und taucht auf der Zutatenliste zahlreicher regionaler traditioneller Produkte auf.

Die o. a. Auflistung umfasst ausgewählte Anbaupflanzen. In Malta kommen ferner alle gängigen Gemüsesorten sowie eine große Vielfalt an bewässerten Obstkulturen, teilweise unter Folie oder im Gewächshaus, im Anbau vor.

Darüber hinaus werden diverse Steinobstsorten, z. B. Kirschen, Pflaumen und Mirabellen, angebaut. Vereinzelt wachsen auch Edelkastanien.

348 CREMONA ((2003), S. 28-35) spricht von einem Verfall der Ölbaumkultivierung der bereits vor einigen hundert Jahren begonnen habe und gibt an, dass schon durch die Einführung des Baumwollanbaus durch die Araber erste Verfallserscheinungen im Bereich der Ölbaumkultivierung zu spüren gewesen sein müssten. Diese Meinung, obschon sie nicht unwahrscheinlich scheint, entbehrt jedoch der wissenschaftlichen Grundlage.

349 Vgl. z. B. NSO (2003a, 2003c)

350 Vgl. NSO (2004b), S. 31

351 Vgl. Kap. 14.1

352 Experteninterviews mit SAM CREMONA (Olivenölerzeuger aus Wardija) vom 13.10.2003 und 25.05.2004; Experteninterview mit Prof. Dr. CHARLES AGIUS (privater Investor einer im Jahr 2002 neu angepflanzten Ölbaumplantage in der Gemarkung Dingli) vom 12.10.2003, Experteninterview mit VICTOR FARRUGIA (Direktor des Plant Biotechnology Centre in Lija (Malta) und Autor verschiedener wissenschaftlicher Fachbeiträge (vgl. Literaturverzeichnis)) vom 24.05.2004. Vgl. ferner Interview mit SAM CREMONA in MALTA TODAY, Onlineausgabe vom 28.10.2001.

353 Vgl. NSO (2003a), S. 94 f. 
Der Autor empfiehlt zur weiteren Vertiefung die Arbeiten von BOWEN JONES, DEWDNEY \& FISHER (1961); MELI (1993); MizZI (1993); GATT, MERCIECA \& FARRUgIA (1998); FARRUGIA (2001); AZZOPARDI (2002); CREMONA (2003); ASCHEMEIER \& CYFFKA (2004b) sowie CYFFKA (2005).

\subsection{Nutzpflanzen, die keinem Anbau unterliegen}

Neben den im vorangegangenen Abschnitt beschriebenen Kulturpflanzen gab und gibt es daneben in Malta auch Pflanzen, die aufgrund ihrer allgemeinen Verbreitung im ländlichen Raum nicht einem kontrollierten Anbau unterliegen, jedoch als Grundlage für regionale traditionelle Produkte dienen oder dienten.

Diese Pflanzen sollen im nachfolgenden Abschnitt beschrieben werden, wobei in der Auflistung dieselbe Methodik wie bei den Kulturpflanzen angewendet wurde.

- Feigenkaktus (engl.: Prickly Pear, malt.: bajtra tax-xewk)

(Opuntia ficus indica)

Kaktusfeigen entstehen aus der Blüte des überall auf den Maltesischen Inseln verbreiteten Feigenkaktus, einer Opuntienart. Eine weitere international gebräuchliche Bezeichnung für diese Pflanze ist Nopal-Cactus.

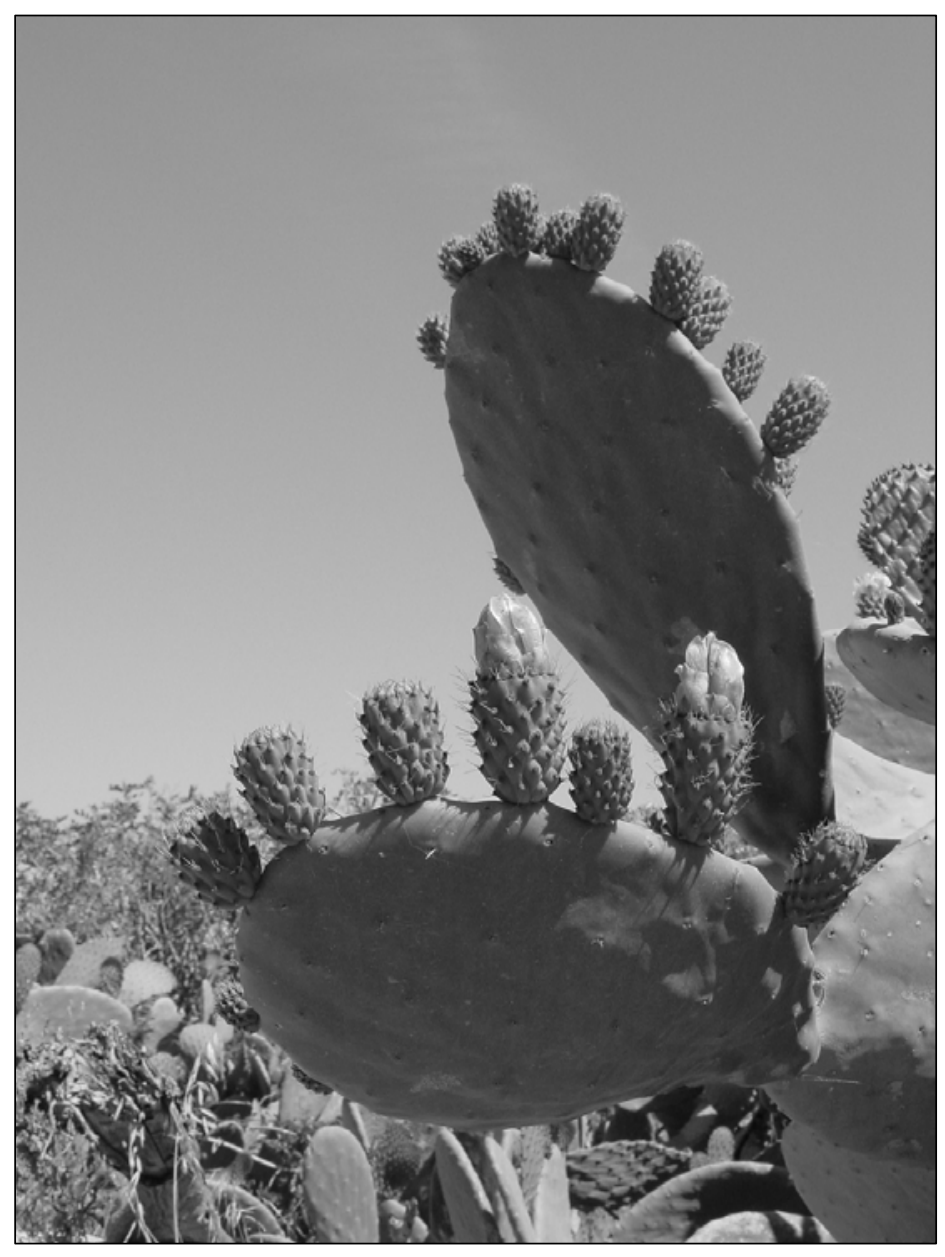

Foto 3: Detailaufnahme eines Feigenkaktus der Sorte aћmar ingliż bei Bidnija. Der Blüten tragende Teil wird zur Kaktusfeige. Foto: AsCHEMEIER 
Opuntia ficus indica wurde im 17. Jh. aus Mittel- und Südamerika eingeschleppt ${ }^{354}$ und wuchert seitdem beinahe unkrautartig im gesamten ländlichen Raum Maltas und Gozos. Ursprünglich wurde die Kaktuspflanze als Sicht- und Windschutz für die Abraumhalden (malt.: il-mandra) der traditionellen maltesischen Farmhäuser genutzt ${ }^{355}$. Doch sie verbreitete sich von dort aus unkontrolliert weiter.

Große Opuntienhecken, die in manchen Landesteilen auch zur Begrenzung der Felder angelegt wurden ${ }^{356}$, verwildern gegenwärtig, verbrauchen unnötig viel Land und entziehen dessen benachbarten Flächen in größerem Umfang Wasser ${ }^{357}$. Es gibt in Malta drei Varietäten ${ }^{358}$ der Kaktusfeige: isfar ta'Malta (Maltesische Gelbe), abjad ta'Franza (Französische Weiße) und aћmar ingliż (Englische Rote).

Die erste Ernte erfolgt im Sommer. Eine zweite Ernte im Herbst kann durch das Entfernen der ersten Blüten erreicht werden, welche die Pflanze dazu veranlassen, eine zweite, spätere Blüte auszubilden. Zur Zeit der Reife fallen dann so viele Kaktusfeigen an, dass sie nicht alle geerntet werden können.

Die Frucht des Feigenkaktus dient als Grundlage für den traditionellen Kaktuslikör Bajtra sowie als Obst und Grundlage von Desserts, Sorbets, Marmeladen und Süßspeisen. Die restlichen Bestandteile der Kaktuspflanze wurden früher als Viehfutter verwendet ${ }^{359}$. Diese Form ihrer Nutzung wird jedoch nicht mehr praktiziert ${ }^{360}$, sodass die unkontrollierte Verbreitung der Opuntien mittlerweile zum Problem wird. Oft sieht man ausgedehnte Kaktushecken, die gerodet, in der Sonne getrocknet und dann ungenutzt verbrannt werden.

Leider gibt es bislang keine statistischen Daten über die flächenhafte Verbreitung des Feigenkaktus auf der Inselgruppe. Deren Erhebung würde sich auch sehr aufwändig gestalten, da die Pflanze „an jeder Ecke“ wächst. Als möglichen Forschungsansatz für die Zukunft schlägt der Verfasser vor, die heckenartigen Anpflanzungen von Opuntia ficus indica im Rahmen einer Kartierung zu erfassen, weil sie in nicht unerheblicher Weise den natürlichen Bodenwasserhaushalt stören (s.o.).

Eine ernst zu nehmende kommerzielle Nutzung der Kaktusfeige erfolgt z. Zt. als Grundlage für den traditionellen maltesischen Likör Bajtra von der Master Wine Group ${ }^{361}$ und zur Herstellung eines medizinischen Anti-Stress-Präparats ${ }^{362}$. Für den Likör werden nur Kaktusfeigen der Sorte aћmar ingliż (Englische Rote) verwendet, die ausschließlich in Malta geerntet werden. Dies hat einen besonderen Grund: Während Master Wine Group das Fruchtfleisch für den Likör verwendet, wird die Schale vom Institute for Cellular Pharmacology zu dem erwähnten Anti-Stress-Präparat weiterverarbeitet. Dieser Herstellungsprozess besitzt Vorbildcharakter in puncto Nachhaltigkeit und Umweltschutz und wurde deshalb in dieser Dissertation in einem eigenen Abschnitt hervorgehoben (vgl. Kap. 19.1).

354 Vgl. CREMONA, S. 13

355 Vgl. BOWEN JONES, DEWDNEY \& FISHER (1961)

356 Vgl. NEHRING (1966), S. 31

357 Vgl. BOWEN JONES, DEWDNEY \& FISHER (1961), S. 217

358 Vgl. CREMONA (2003), S. 13

359 Vgl. BOWEN JONES, DEWDNEY \& FISHER (1961), S. 217

360 Vgl. CREMONA (2003), S. 13

361 Es gibt auch einen Bajtra-Likör des Herstellers M. Demajo Group, jedoch werden die Früchte hierfür nicht in Malta geerntet, sondern importiert.

362 Vgl. zu beiden Produkten Kap. 14.1.3 \& 15.1 
- Johannisbrotbaum (II-Harruba)

(Ceratonia siliqua, L.)

Der Johannisbrotbaum blickt in Malta als Nutzpflanze auf eine lange Tradition zurück, wurde jedoch nie planmäßig angebaut. Er ist u. a. fester Bestandteil populärer Volkssagen und Legenden aus Gozo ${ }^{363}$ und Malta $^{364}$. Einer der ältesten Bäume der Maltesischen Inseln ${ }^{365}$ ist ein Johannisbrotbaum, der an der gut erhaltenen römischen Straße oberhalb von Xemxija, heute bekannt als "The Xemxija Heritage Walk“, wächst.

SULTANA schlug 1988 vor, den Johannisbrotbaum als „Nationalbaum“ Maltas auszuweisen ${ }^{366}$, es gäbe ja schließlich auch den „Nationalvogel“ und die „Nationalblume“ der Maltesischen Inseln. Sein Vorschlag verhallte bisher zwar ungehört, jedoch stellt seine Forderung die Bedeutung des Johannisbrotbaums als Bestandteil der nationalen Identitätsfindung im Selbstverständnis der maltesischen Bevölkerung heraus.

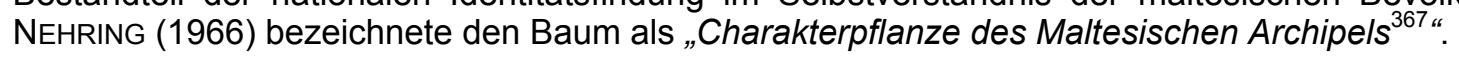

Der Johannisbrotbaum gehört zur Famile der Johannisbrotgewächse (Caesalpiniceae) und ist der einzige Vertreter der Gattung Ceratonia. Alte Bäume können mehrere Meter große Flächen bedecken, da die ausladenden Äste, auf die Erde aufgestützt, ein „Dach“ formen können ${ }^{368}$. Seine Früchte als lange, zunächst hell- bis mittelgrüne Schoten sehr auffällig. Die einzelnen Samen innerhalb der Schote werden als Karoben bezeichnet. Im August reift die Schote und wird schokoladenbraun. Wenn die Reife erreicht ist, trocknet sie aus und fällt zu Boden. Sie ist in diesem Zustand trocken und kann ohne Mühe gemahlen werden. Die Karoben besitzen eine natürliche Süße, die als Grundlage verschiedener Produkte dient, so z. B. für das traditionelle Volksheilmittel Gulepp tal Harub (vgl. Kap. 14.1) und den Likör Leila (vgl. Kap. 15.1).

$\mathrm{Zu}$ den Eigenschaften der Johannisbrotbaumschote äußerte sich im Zeitungsinterview der Wein- und Likörhersteller MARK MICELI-FARRUGIA: "The carob is a naturally sweet, almost honey-like fruit which is high in essential minerals and salts. It is inherently free from any addictive or allergenic stimulants such as caffeine (found in coffee and tea), theobromine (found in tea and cocoa), or phenylethylamine (found in cocoa). In a nutshell: the carob soothes both our stomach and taste buds with no undesirable side-effects." (NAUDI in: THE TIMES ON SUNDAY, 18.07.2004, S. 65).

Im 19. Jh. wurden die Karoben gebacken und ohne weitere Behandlung zerkaut. Sie waren „Süßigkeiten für arme Leute“, wie der Autor GEORGE FRENCH ANGUS im Rahmen seiner Malta-Reise anschaulich beschrieb: „The „Carrubae“ or beans of the locust tree, are when baked, a very common article of food amongst the poor; and in passing through the villages, travellers are often annoyed by children holding out their hands, and begging for „habba, harroob“, or a grain to buy locusts with“. (ANGUS (1841) zitiert in CREMONA (2003), S. 25). In der durch die deutsche U-Boot-Belagerung der Inseln im Zweiten Weltkrieg hervorgerufenen Hungersnot wurde der Bevölkerung von staatlicher Seite wegen ihres hohen Protein- und Fettgehalts der Verzehr von Johannisbrotbaumschoten empfohlen ${ }^{369}$. NEHRING erwähnt dagegen einen hohen Reichtum an Kohlenhydraten ${ }^{370}$.

Johannisbrotbäume kommen auf der Inselgruppe nicht in zusammenhängenden Anpflanzungen vor. Sie werden von der Bevölkerung heute nicht als „Nutzpflanzen“ im engeren Sinne betrachtet und auch nicht mehr systematisch abgeerntet ${ }^{371}$ wie es in der Vergangenheit üblich war. Früher wurden die Schoten als

363 Vgl. z. B. BEZZINA (1992)

364 Vgl. STUMmE (1904a\&b) und ILG (1906a\&b)

365 Laut TEUMA (2003)

366 Vgl. SULTANA (1988), S. 47

367 Vgl. NEHRING (1966), S. 31

368 Dazu DARMANIN: „Der Johannisbrotbaum wächst nie sehr hoch, anstatt dessen (sic!) entfaltet er dichtwachsende Äste die zusammen einen Schirm erbilden (sic!) die den Stamm vor dem Wind schützen." (DARMANIN (1991), S. 115).

369 Vgl. AZZOPARDI (2002), S. 166

370 Vgl. NeHRING (1966), S. 31

371 Vgl. CREMONA (2003), S. 25 
Viehfutter, vor allem für Schweine, verwendet ${ }^{372}$, während das Holz des Baums nahezu das einzig verfügbare Brennholz war. Johannisbrotbäume wurden außerdem als Wind- oder Sichtschutz angepflanzt. Eine weitere Nutzung erwähnt AzZOPARDI: „It is often planted (...) to disintegrate the rock and boulders, for its roots are very strong, penetrate into the fissures of the rock and break it up. In this way, new soil is added." (AZZOPARDI (2002), S. 166). Auch NEHRING schrieb über diese Eigenschaft der Johannisbrotbaum-Wurzeln in seiner Arbeit ${ }^{373}$

In einigen öffentlichen Gärten von Valletta und Floriana (insbesondere in Hastings Gardens oberhalb von St. John's Cavalier in Valletta und in den Argotti Botanical Gardens in Floriana) sind prächtige Exemplare des Johannisbrotbaums zu finden. Dies zeigt, dass der Baum in Malta stets nicht nur als nützlich, sondern auch als dekorativ empfunden wurde.

\section{- Kapern}

(capparis spinosa)

Kapern sind die Knospen des Kapernstrauchs, der an Mauern, Bastionen und den unzugänglichen Klippen der Maltesischen Inseln wächst. Die Blüten des Strauchs sind weiß. Sie weisen längliche violette Staubfäden auf. Die Knospen sind von Ende Mai bis Ende Juli zu finden, da sich die Blüten der Pflanze nicht alle zur selben Zeit, sondern erst nach und nach öffnen.

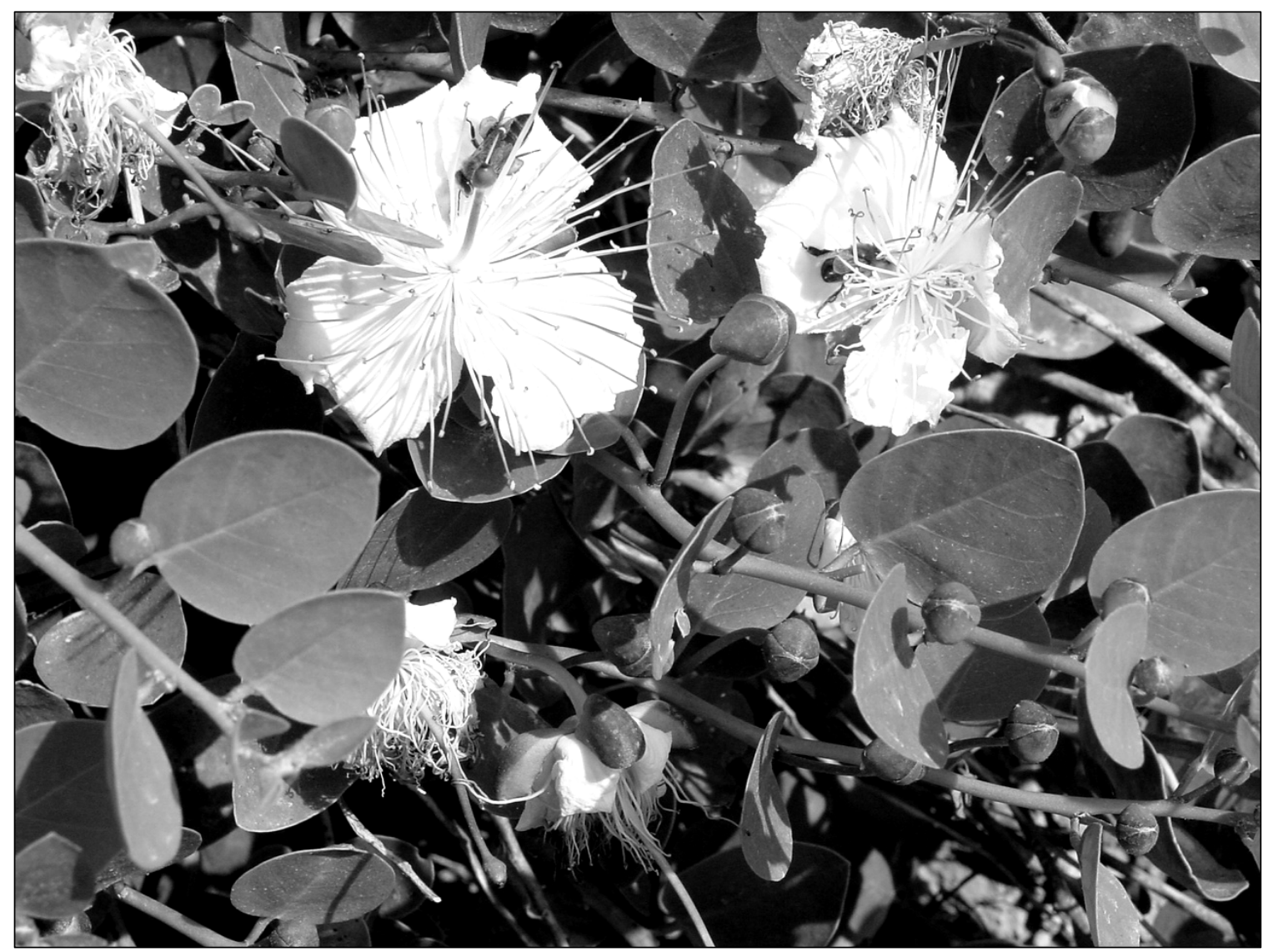

Foto 4: Detailaufnahme einer Kapernpflanze bei Xemxija. Einige Blüten blühen bereits und dienen als Bienenweide (s. Mitte oben), während die noch geschlossenen Knospen gepflückt werden können, um sie als Kapern einzulegen. Foto: ASCHEMEIER 
Die Kapern werden in dieser Zeit gesammelt, zwei Wochen lang in starkem Meersalz gesalzen und anschließend in einer Essiglösung eingelegt. Sie sind ein würziger Bestandteil vieler maltesischer Gerichte und gelten in Malta als besondere Delikatesse ${ }^{374}$.

Kapern werden auf Wochenmärkten in Gläsern zu ca. Lm 1,50 verkauft und sind ein beliebtes Touristensouvenir sowie auch ein tägliches Lebensmittel der maltesischen Bevölkerung.

374 Vgl. DARMANIN (1991), S. 8 


\section{Regionale traditionelle Produkte Maltas und Gozos}

In Malta gibt es eine Vielzahl von regionalen traditionellen Produkten. Sie entstammen fünf unterschiedlichen Erzeugerzweigen (vgl. Abb. 13 auf der folgenden Seite), von denen vier besonders hervorzuheben sind, während der fünfte eher zu Missverständnissen führt. In Malta tragen das regionale Kunsthandwerk, die von der Landwirtschaft abhängige und weitgehend unabhängige Lebensmittelindustrie sowie die bildende Kunst zur Produktion von regionalen traditionellen Produkten bei.

Als fünfter Faktor sind „neue Strömungen“ zu nennen. Dabei handelt es sich um Erzeuger, die keiner Herstellertradition folgen, sondern ihre eigene innovative Nische entwickelt haben und erst vor relativ kurzer Zeit mit der Produktion ihrer Produkte begonnen haben. Jedoch können auch in einer solch relativ kurzen Zeitspanne der Erzeugung sehr charakteristische Produkte entstanden sein, bei denen im Endeffekt strittig ist, ob es sich nach der in dieser Arbeit vorgestellten Definition (vgl. Kap. 10.1) um „echte“ regionale traditionelle Produkte handelt oder nicht. Dies muss für jeden Einzelfall individuell entschieden werden.

Der folgende Abschnitt soll Klarheit schaffen und listet alle wichtigen regionalen traditionellen Produkte Maltas, nach Erzeugerzweigen und alphabetisch sortiert, auf. Zu jedem Produkt erfolgt eine Erörterung. Am Schluss werden typische Streitfälle, wie z. B. das maltesische Glasbläserhandwerk, behandelt. Es wird erläutert, aus welchem Grund und inwiefern diese Produkte der Definition eines regionalen traditionellen Produkts wie sie im Rahmen dieser Abhandlung aufgestellt wurde (vgl. Kap. 10.1), entsprechen oder nicht. 


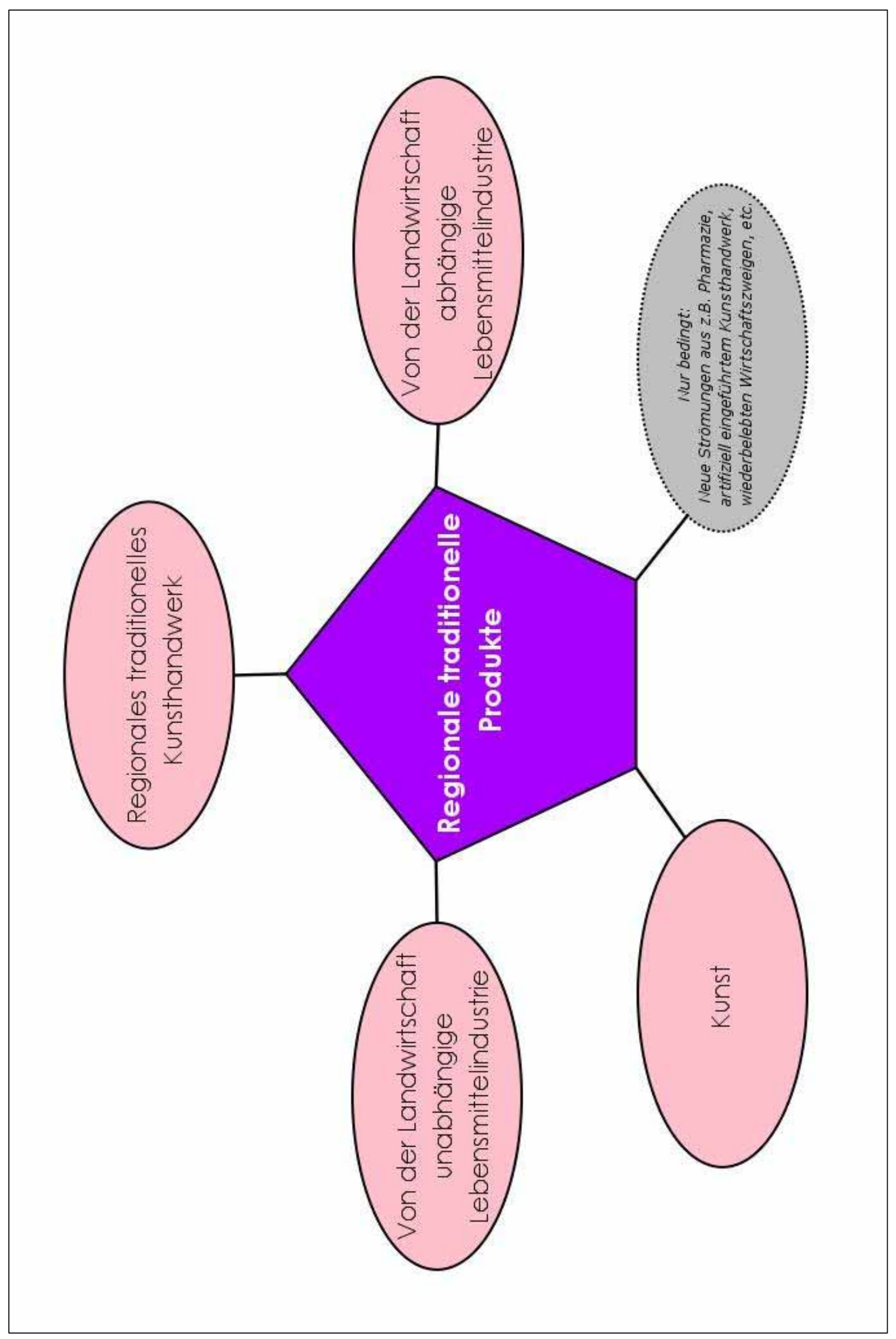




\subsection{Lebensmittel}

Die Erzeugung von Lebensmitteln ist die ultimative Grundlage jeder Gesellschaft, die lebensfähig bleiben will. Trotz der Abhängigkeit von anderen Mittelmeer-Anrainern, die bis in das Neolithikum zurückreicht ${ }^{375}$, hat es in Malta immer eine Subsistenzwirtschaft zur Erzeugung von Lebensmitteln gegeben. Doch schon zu Zeiten der Johanniterritter wurden bestimmte Lebensmittel aus Malta auch in kleinen Mengen exportiert, so z. B. Wein und Zitrusfrüchte.

Die heutige Vielfalt von Produkten aus dem Lebensmittelsektor ist das Ergebnis jahrhundertelanger Entwicklung und Assimilation von Traditionen sowohl Südeuropas als auch Nordafrikas durch die maltesische Folklore. Mit dem Einzug der Briten um 1800 entfaltete sich ein weiterer wichtiger Einfluss, der noch heute spürbar ist und zur Etablierung von Produkten wie z. B. Bier führte, das vor der britischen Kolonialisierung nahezu unbekannt war, heute aber Maltas meistkonsumiertes alkoholisches Getränk ist.

Traditionelle Lebensmittel der Maltesischen Inseln sind oft an ihren einfachen Zutaten und am geringen Proteingehalt zu erkennen. Dieses Phänomen hat seine Ursache in der extremen Trockenheit des maltesischen Sommers, gegen die in der Vergangenheit nicht viele Schutzmaßnahmen möglich waren. Damit einher ging eine karge und unsichere Ernte auf einem nicht minder kargen und unzuverlässigen Boden. Die Zutaten, die sicher und ständig verfügbar waren, kamen von anspruchslosen Gewächsen, die an Trockenheit adaptiert waren und deren Früchte auch in extrem trockenen Sommern nicht ausblieben.

Zudem findet man unter den maltesischen Lebensmitteln oft Zutaten wie Kapern, Pfeffer, Fenchelsaat oder Kaktusfeigen: Allesamt sind dies essbare Pflanzen, die auf Allmende-ähnlich genutzten Flächen wild wuchsen, nicht kultiviert werden mussten und die es in jeder Hinsicht "gratis“ gab. Die Nutzung wirklich jeder Proteinquelle verdeutlicht auch die traditionelle Vorliebe der Malteser für Schnecken.

Im folgenden Produktverzeichnis der maltesischen Lebensmittel sind ausführlich und ausschließlich diejenigen Produkte aufgeführt und mit ihrem traditionellen Herstellungsprozess beschrieben, die dem Produktverständnis der vorliegenden Arbeit (vgl. Kap. 10) entsprechen. Es sind somit fertig produzierte und unmittelbar nach dem Erwerb konsumierbare Lebensmittelprodukte, die vor dem Genuss keiner weiteren Zubereitung mehr bedürfen ${ }^{376}$. Gerichte - die erst noch aus Einzelzutaten zubereitet werden müssten wurden nicht unmittelbar in die Auflistung aufgenommen, werden jedoch in Kapitel 14.1.2 der Vollständigkeit halber und zur besseren Unterscheidbarkeit von den Produkten mit Kurzbeschreibungen erwähnt.

\section{- Bigilla}

Bigilla ist eine traditionelle maltesische Speise, deren Hauptbestandteil broad beans (malt.: ful) - eine Art dicker Bohnen, in Deutschland am ehesten als „Saubohnen“ bekannt - ist. Der Bohnenbrei war einst ein wichtiger Bestandteil der Ernährung der Insulaner, weil er einen sehr hohen Proteingehalt aufwies. Proteine waren in der Vergangenheit ein Mangelfaktor der Ernährung auf der Inselgruppe. Denn aufgrund der naturräumlichen Gegebenheiten und der Armut der Bevölkerung war Fleisch ein sehr seltener Nahrungsbestandteil, während Fisch nur den Bewohnern der Küstenregionen zugänglich war. Bohnen waren ursprünglich auch Bestandteil des Viehfutters. Nur übrig gebliebene Bohnen wurden zu bigilla verarbeitet ${ }^{377}$. Bigilla wird noch heute vielfach in Heimarbeit hergestellt.

Die Bohnen werden dabei über Nacht kalt gewässert. Am nächsten Tag werden sie in frischem Wasser gekocht und anschließend zusammen mit Pflanzenöl, Knoblauch, Pfeffer und Petersilie zu einem groben Brei verrührt bzw. durchgedreht. Bigilla wird als Gemüse-Dip oder Brotaufstrich genutzt ${ }^{378}$, manchmal auch pur verzehrt.

Eigentlich ein Gericht, das der Herstellung bedarf (und somit nicht in vollem Umfang dem Produktverständnis dieser Dissertation entspräche), wird bigilla heute aber von mehreren Firmen (z. B. Discus Products und Camel Brand) als abgepacktes Produkt verkauft. Auf Wochenmärkten sieht man bigilla selten im Verkauf, da die Lagerung schwierig ist. Auch die Optik der Speise wirkt aufgrund ihrer braun-

\footnotetext{
375 Vgl. EVAns (1963)
}

376 Zum Produktverständnis bzw. zur Definition des Terminus technicus „regionales traditionelles Produkt“ s. Kap. 10

377 Vgl. CREMONA (2003), S. 28

378 Vgl. DARMANIN (1991), S. 27 f. 
olivgrauen Färbung zunächst wenig ansprechend, was den Verkauf an Touristen erschwert, während Einheimische bigilla entweder selbst herstellen oder billig im Supermarkt kaufen.

\section{- Ǵbejniet}

Ǵbejniet, Singular: Ǵbejna, ist die bekannteste und beliebteste Käsesorte in Malta und Gozo. Viele Familien stellen ihn selbst her. Die Herstellung ist in Gozo verbreiteter als in Malta, weswegen auf den Verpackungen kommerzieller Hersteller häufig von "Gozo Cheeselets“ die Rede ist ${ }^{379}$. Der geschichtliche Ursprung des Produkts liegt weitgehend im Dunkeln. Der Name Ǵbejniet deutet laut Meinung von Sprachwissenschaftlern darauf hin, dass das Produkt bereits vor der arabischen Herrschaftsperiode bekannt war ${ }^{380}$. Eine Legende besagt, Ǵbejna sei während der großen Belagerung durch die Türken im Jahr 1565 entstanden, als die einzigen verfügbaren Lebensmittel Milch, Essig und Pfeffer gewesen seien (s. u.). Dies ist jedoch unwahrscheinlich, weil für die Herstellung von Ġbejniet Milch von hohem Fettgehalt und guter Qualität benötigt wird ${ }^{381}$.

Während früher der komplette Vorgang der Käseherstellung in der Hand der Familien lag, wird heute meist der Grundstoff, ein Frischkäse, baqta genannt, eingekauft und pur verzehrt oder weiterverarbeitet.

Ǵbejna besteht in seiner streng traditionellen Form aus Schafsmilch. Diese wird pasteurisiert und zu einem Frischkäse verarbeitet, der in kleine Körbchen ${ }^{382}$ gepresst wird, woraus sich die charakteristische Form der kleinen Käserundlinge ergibt. Der frische, ungetrocknete Käse wird nur mit etwas Salz bestreut und sehr frisch verzehrt, da er nur wenige Tage haltbar ist.

Der getrocknete Ǵbejna ist haltbarer. Im frischen Zustand wird er in einem luftdurchlässigen Behältnis (malt:: qafas) an einem kühlen und schattigen Ort aufgehängt. Der Käse wird dabei durch Lufttrocknung hart und kompakt. Zur weiteren Aufbewahrung muss das Milchprodukt nicht einmal mehr gekühlt werden. Der getrocknete Ǵbejna wurde früher traditionell in einem Baumwollsack an einem schattigen Ort aufbewahrt. Modern verpackte, eingeschweißte Käserundlinge sind gekühlt bis zu 180 Tage haltbar ${ }^{383}$.

Ǵbejna gibt es in zwei Variationen: entweder pur oder eingelegt. Soll der Käse pur gegessen werden, so ist er nach einer der oben beschriebenen Prozeduren fertig zum Verzehr. Pikanter ist die eingelegte Variante. Ursprünglich war dies eine Methode, um zu trockene Ǵbejniet wieder „aufzufrischen“. Sie werden für mindestens zwei Stunden, besser aber mehrere Tage, in ein Glas mit Salzlauge und Essig eingelegt. Anschließend werden die noch vom Essig feuchten Ǵbejniet in ein Glas mit grob zerstoßenem Pfeffer gegeben und das Glas wird so lange geschüttelt, bis der Käse überall gut mit Pfeffer bedeckt ist. Diese Variante nennt man Ǵbejniet tal-bżar, (Ǵbejniet mit Pfeffer).

379 S. Verpackungen von Camel Brand, Gozo und Discus Products, Mosta

380 Vgl. GaLEA de GiovanNi (o. J.), S. 9

381 So Käsehersteller CHRIS ZAHRA im Experteninterview vom 29.05.2004

382 Die traditionellen Behältnisse bestanden aus einem Rohrgeflecht. Heute werden wieder verwendbare Plastikkörbchen genutzt.

383 Telefonische Information des Herstellers Discus Procucts, Mosta. 


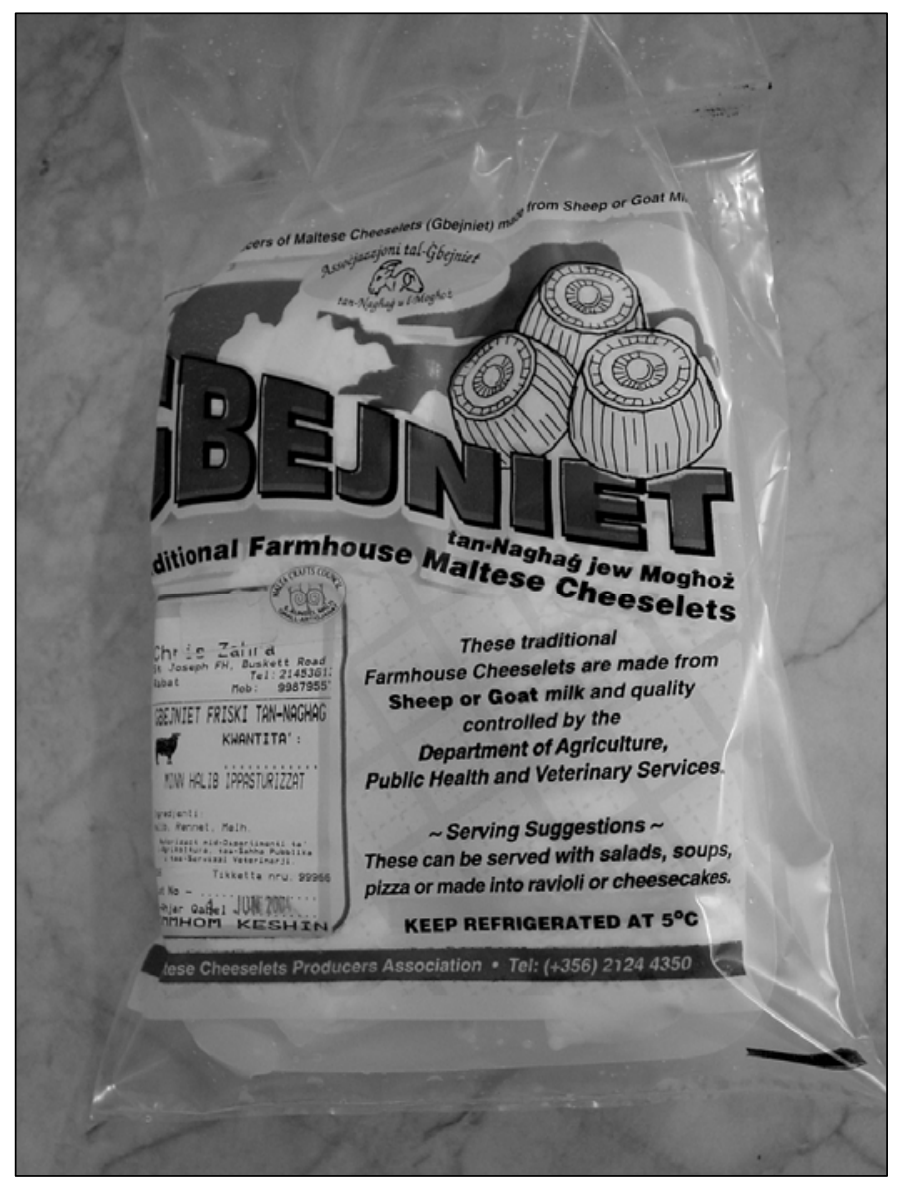

Foto 5: Verpackte Ġbejniet (frisch). Foto: AsCHEMEIER

Zum Einlegen werden je nach Familienrezept aber auch andere Zutaten eingesetzt (z. B. ganze Chilischoten). Auch die Sorte des verwendeten Essigs differiert von Fall zu Fall. Es ist nicht übertrieben zu behaupten, dass fast jeder Ǵbejna, den man in Malta bekommen kann, anders schmeckt.

Obwohl Ǵbejna noch häufig in Heimarbeit angefertigt wird, lohnt sich offenbar auch die kommerzielle Herstellung. Drei Firmen bieten die Käsesorte als industriell erzeugtes und in Plastikfolie eingeschweißtes Produkt an. Dabei ging man mittlerweile auch dazu über, Ǵbejniet aus Kuhmilch herzustellen (Hersteller: Benna). Diese Variante lässt den ursprünglichen geschmacklichen Charakter des Produkts vermissen.

Viele Kleinhersteller haben sich in der Maltese Cheeselets Producers Association (malt.: Assoćjazzjoni talǴbejniet) zusammengeschlossen, um einheitliche Qualitätsstandards zu erreichen. Durch das Logo des Verbands kann zudem eine kostengünstige, weil einheitliche, Verpackung (vgl. Foto 5) und Vermarktung stattfinden. Außerdem sind die Mitglieder des Verbands berechtigt, das Logo des Malta Crafts Council für regional erzeugte Produkte zu führen (vgl. Kap. 18.1.2).

Doch auch andere, in Heimarbeit hergestellte Ǵbejniet sind in Malta und Gozo bei jedem Obst- und Gemüsehändler und auch in vielen Souvenir- und Andenkenläden im Überfluss erhältlich. 
Ǵbejna gehört als Milchprodukt zum Alltag der maltesischen Bevölkerung und wird auf den Verpackungen der Hersteller beworben (vgl. Foto 6).

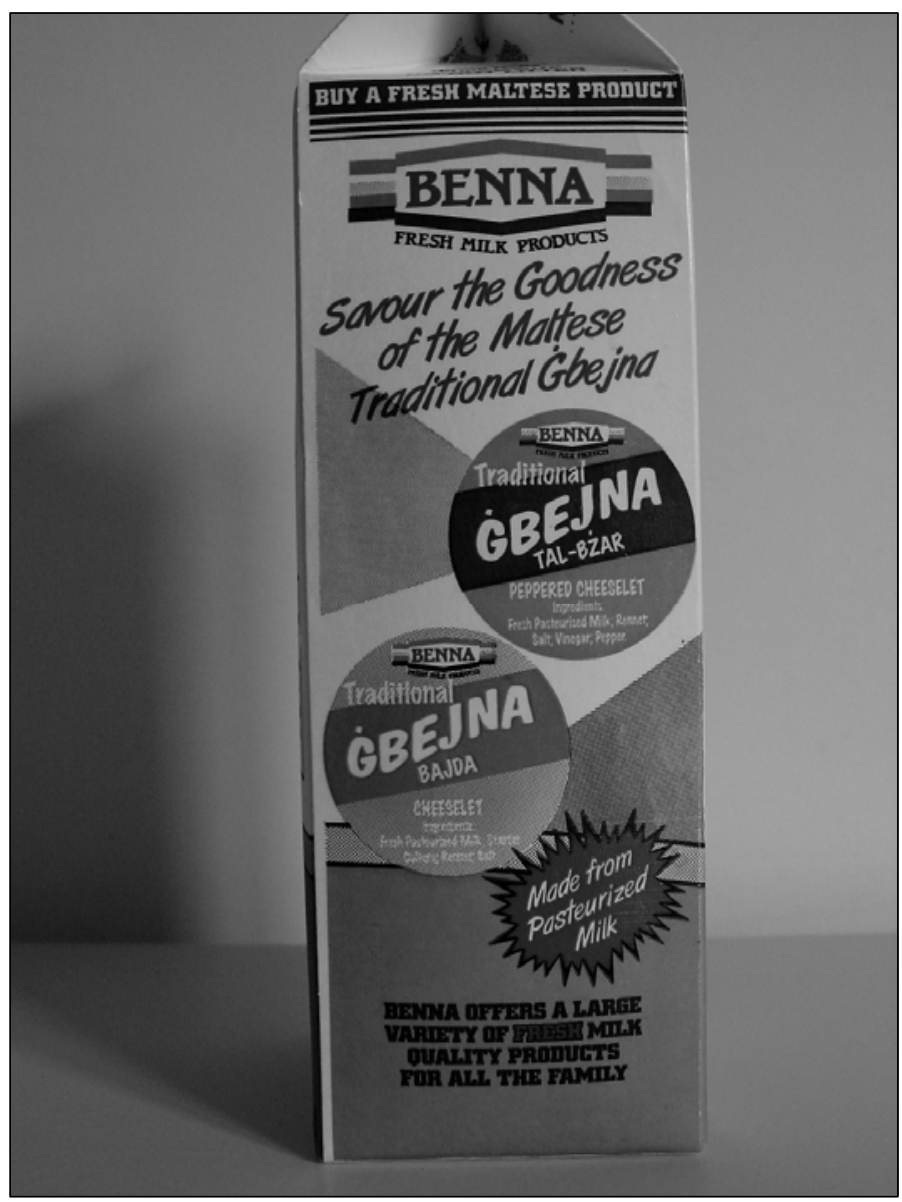

Foto. 6: Werbung für Ǵbejna auf der Milchpackung des Herstellers Benna. Foto: AsCHEMEIER

Ǵbejniet werden pur verzehrt, in den Salat geschnitten, oder in der Pfanne mit viel Olivenöl in Scheiben geschnitten frittiert. In Südfrankreich und Korsika ist unter dem Namen crottin de chevre ein vom Aussehen her ähnlicher Käse bekannt, der sich jedoch in Geschmack und Herstellung vom Maltesischen Ǵbejna grundlegend unterscheidet.

\section{- Gulepp tal Harub}

Der Johannisbrotbaum (malt.: Il-Harruba) blickt als Nutzpflanze in Malta auf eine lange Tradition zurück. Er ist $u$. a. auch Bestandteil vieler Volkssagen und Legenden aus Gozo ${ }^{384}$. Zum Anbau und zur Botanik vgl. Kap. 13.10.

Der Sirup aus den Karoben des Johannisbrotbaums ist eines der ältesten maltesischen Lebensmittel. Er wird als Süßstoff oder als Brotaufstrich (ähnlich dem in Deutschland bekannten Zuckerrübensirup) genutzt. Vor allem wird er aber als Volksmedizin zur Linderung von Husten und Halsweh pur eingenommen ${ }^{385}$. Auch zum Backen von Süßwaren wird er verwendet. Gulepp tal Harub wird auf den Maltesischen Inseln bislang nicht von kommerziellen Herstellern vertrieben. Es gibt jedoch Delikatessen- und Spezialitätengeschäfte, die den Sirup entweder selbst herstellen oder inn von lokalen Landwirten beziehen.

384 Vgl. z. B. BEZZINA (1992), S. 41

385 Vgl. DARMANIN (1991), S. 115; CREMONA (2003), S. 16 
Der Herstellungsprozess ist vergleichsweise einfach, wenngleich arbeitsreich. Die reifen Schoten des Johannisbrotbaums werden zunächst gewaschen und in der Sonne getrocknet. Danach werden sie nochmals im Backofen getrocknet, bis sie keine Feuchtigkeit mehr enthalten. Die Schoten werden dann in kleine Stücke zerbrochen, die über Nacht in kaltem Wasser quellen müssen. Am nächsten Morgen wird dieser Aufguss kurz aufgekocht und dann auf kleiner Flamme weiter erhitzt. Es werden Gewürznelken, Zimt und Zitronenschale zugegeben. Die Flüssigkeit wird nun gefiltert, wobei die festen Bestandteile, insbesondere die Schotenteile, nochmals ausgepresst werden. Anschließend setzt man Zucker zu und kocht die Flüssigkeit wieder, bis der erwünschte honigartige Sirup erreicht ist. Dieser wird in kleine Flaschen oder Gläser abgefüllt.

Gulepp tal Harub war in den 1990er-Jahren etwas in Vergessenheit geraten ${ }^{386}$. Durch die zunehmende Erkenntnis, dass sich regionale traditionelle Produkte für den Tourismus vermarkten lassen, kam der Sirup wieder in die Auslagen der Geschäfte und ist zur Zeit auf jedem Wochenmarkt, in vielen Geschäften und sogar in den größeren Supermärkten erhältlich. Die vorteilhafte Doppelfunktion als Lebensmittel und Volksheilmittel verspricht nachhaltige Beliebtheit auch beim touristischen Publikum.

\section{- Honig (ghasel)}

Wenn man das älteste traditionelle Produkt Maltas sucht, kommt man am Honig kaum vorbei. Es ist unbekannt, wer die Imkerei in Malta etabliert hat ${ }^{387}$. Es ist jedoch sicher, dass schon zu Zeiten der ersten Kontaktaufnahme Maltas mit phönizischen Händlern (vermutl. um 900-800 v. Chr.) Honig als Handelsware eine nicht unbedeutende Rolle spielte.

Der Honig der Maltesischen Inseln war in der Antike im ganzen Mittelmeerraum berühmt. Auch der Name Malta leitet sich, wie mittlerweile als sicher angenommen wird ${ }^{388}$, vom griechischen Melita (sinngemäße Übersetzung: „Ein Land, reich an Honig“) ab. Der maltesische Honig wurde in der Zeit der Sklaverei und der Piratenüberfälle, die der Herrschaft der Johanniter vorausging ${ }^{389}$, als Beutegut geraubt, weil er als sehr wertvoll galt ${ }^{390}$. Römische Geschichtsschreiber erwähnen den Diebstahl von 400 Krügen maltesischen Honigs durch CAIUS VERRES ${ }^{391}$. Zusammen mit anderen Verfehlungen war dies der Anlass für CICEROS Kampagne gegen den korrupten sizilianischen Prätor ${ }^{392}$. Auch BusUTTIL erwähnt in seinem Artikel über die historische Entwicklung der maltesischen Landwirtschaft: „Roman historians refer to the islanders' prowess in producing cotton and honey." (BusuTTIL (1993), S. 10). Dies wird untermauert durch Quellen aus dem 11. Jh., die Maltas Imkerei während der normannischen Herrschaft hervorheben ${ }^{393}$.

Auf den Inseln gibt es noch einige antike Bienenstöcke (malt:: Mǵieba $)$, die sich teils durch Nutzung natürlicher Höhlen, teils durch Ergänzung dieser Höhlen durch Mauerwerk und deffun (malt.), einem primitiven Zement aus zerstoßener Keramik auszeichnen. Ihre Geschichte reicht teilweise bis in die römische Zeit zurück (Ancient Beehives bei Xemxija), aber auch bis ins 17. und 18. Jh., also in die Zeit der Herrschaft der Johanniter auf Malta. Einige dieser Bienenstöcke werden noch heute genutzt ${ }^{394}$, wenngleich sich die Arbeitsweisen der Honiggewinnung verändert haben. Viele Orts- und Flurnamen deuten auf die lange Tradition der Honigproduktion hin, so z. B. Wied Mġiebaћ (malt. sinngemäß: „Tal der Bienenstöcke“) und Wied il-Ghasel (malt. sinngemäß: „Tal des Honigs“) ${ }^{395}$.

386 Vgl. DARMANIN (1991), S. 115

387 So die Imkerin und ehemalige Präsidentin der Malta Beekeeper's Association MARICA GATT im Experteninterview vom 08.06.2004

388 Vgl. TEUMA (2003), S. $27 \mathrm{ff}$.

389 Vgl. dazu z. B. BEZZINA (1992), S. 29 \& BLOUET (1987), S. 35-45

390 So die Imkerin und ehemalige Präsidentin der Malta Beekeeper's Association MARICA GATT im Experteninterview vom 08.06.2004

391 Vgl. AtTARd (2002), S. 17 \& Galea de GiovanNi (o. J.), S. 9 (Letzterer gibt statt „400 Krügen“ Honig „13 Amphoren“ an.)

392 Vgl. ATtARD (2002), S. 17

393 Vgl. CASSAR (2000), S. 4 f.

394 So die Imkerin und ehemalige Präsidentin der Malta Beekeeper's Association MARICA GATT im Experteninterview vom 08.06.2004

395 Vgl. Teuma (2003), S. 34 f. \& Galea de Giovanni (o. J.), S. 9 
Die moderne Honigproduktion unterliegt der Obhut der Malta Beekeeper's Association und dem Department of Agriculture der maltesischen Regierung. Es werden regelmäßig Kontrollen unter den Imkern durchgeführt, denn Gesetze regeln die Arbeitsweise der Imker. So ist z. B. per Gesetz die alte römisch/phönizische Methode der Bienenhaltung in Tongefäßen ${ }^{396}$ (malt.: Qolla) verboten ${ }^{397}$, weil sie die Ausbreitung von Krankheiten unter den Bienenvölkern begünstigt.

Ganz ließen sich Krankheiten unter den maltesischen Bienen aber auch durch Gesetze nicht vermeiden, was der Imkerei im Jahr 1992 einen drastischen Rückgang von über 80\% der Bienenvölker innerhalb von nur 6 Monaten beschert hat. Grund war eine Milbe (Varroa jacobsonii), die, ähnlich wie in vielen anderen Ländern, großen Schaden unter den Bienenvölkern Maltas angerichtet hat. Von über 1.000 maltesischen Imkern sind seit 1992 nur noch einige Hundert übrig geblieben. Durch den Rückgang der bestäubenden Bienen litt auch die Erzeugung von Zitrusfrüchten erheblich.

In Malta gibt es eine endemische Subspezies der Honigbiene (Apis mellifera ruttneri). Sie wird derzeit erforscht. Die Art ist von dunkler Farbe, fast schwarz, von kleiner Körpergröße und sehr aktiv ${ }^{398}$. Sie wird jedoch kaum von Imkern genutzt, weil diese Subspezies aggressiv ist. Ihre starke Abwehrkraft gegen Krankheiten und Seuchen machte sie jedoch zum idealen Kreuzungsobjekt. Apis mellifera ruttneri wurde mit neuseeländischen Arten gekreuzt. Die Kreuzung ist nun in Malta weit verbreitet und zeichnet sich vor allem dadurch aus, dass sie so aktiv und widerstandsfähig ist, wie die maltesische Honigbiene, jedoch weniger angriffslustig.

Honig ist in Malta nach wie vor ein hochqualitatives regionales traditionelles Produkt. Der Preis wird von der Regierung festgelegt ${ }^{399}$ und beträgt zur Zeit teure $\mathrm{Lm} 3$ für $500 \mathrm{~g}$ Honig. Umgerechnet sind das ca. $7 €$. Die Gläser sind fast immer von einheitlichem Aussehen, nur die Etikettierung variiert von Hersteller zu Hersteller. ${ }^{400}$ Die maltesischen Imker sind mit der derzeitigen Situation selbst unzufrieden, zumal unter der maltesischen Bevölkerung importierter Honig aufgrund des niedrigeren Preises immer beliebter wird. Der hohe Preis des einheimischen Produkts ist bedingt durch Protektionismus während der Krise von 1992 und wurde seitdem nur minimal geändert. Die einheimischen Hersteller könnten mittlerweile wieder billiger produzieren, dürfen aber nicht. Die Zeiten, als maltesischer Honig noch in 0,75 I-Whiskeyflaschen verkauft werden konnte ${ }^{401}$, sind vorbei. Die Imker versuchen daher andere Wege der Vermarktung. So wird maltesischer Honig z. B. als Arzneimittel in Apotheken verkauft. Die ehemalige Präsidentin der Malta Beekeeper's Association, MARICA GATT, hierzu im Experteninterview:

"Honey in Malta is quite used. But the most common sort of use of honey is - since we have imported honey which is cheap - most housewives and confectioneries, they use the imported honey in cookery and the Maltese honey is mostly used to keep as a medicinal for cough, for sore throats, for colds. Nearly every family has a jar of honey in the cupboard which they will use when some of the children or the parents are sick. (...). In fact we sell our honey all year round, but in autumn and winter we will have tremendous calls for urgent needs of honey, because the people are sick." (Experteninterview mit MARICA GATT vom 08.06.2004).

396 Es handelte sich dabei um amphorenartige Tongefäße, die im Bienenstock auf der Seite liegend gelagert wurden. Der Öffnung des Bienenstocks zugewandt befand sich die schmale Öffnung, durch welche die Bienen ein- und ausflogen. Im hinteren Teil war der Boden des Gefäßes gekappt und mit einem Holzbrett oder einer flachen Steinplatte verschlossen. Diese Platte ermöglichte den problemlosen Zugriff auf die Honigwaben im Inneren, und sogar eine Vergrößerung des Tongefäßes durch das Anstecken eines weiteren hohlen Zylinders an den ersten war dadurch möglich. Der Nachteil dieser Methode ist, dass der Imker außer der hinteren Öffnung keine Möglichkeiten hat, die Vorgänge im Inneren des Baus zu beobachten. Der Ausbruch von Krankheiten und Seuchen, bzw. die Invasion von Milben wird also erst erkannt, wenn es bereits zu spät ist.

397 Vgl. CREMONA (2003), S. 140

398 Vgl. auch GaLEA DE GIOVANNI (o. J.), S. 9

399 So die Imkerin und ehemalige Präsidentin der Malta Beekeeper's Association MARICA GATT im Experteninterview vom 08.06.2004

400 Alle Informationen des folgenden Textabschnitts sind dem Experteninterview mit MARICA GATT, Imkerin und ehemalige Präsidentin der Malta Beekeeper's Association, vom 08.06.2004 entnommen

401 Vgl. DARMANIN (1991), Bildteil zwischen S. 86 \& 87, Foto 6 
Ein anderer Vermarktungsweg ist der Verkauf von Honig als touristisches Souvenir. Dies ist dann allerdings ein Souvenir im oberen Preissegment maltesischer Lebensmittel. Man halte sich vor Augen, dass für Lm 3 auch schon hochwertige lokale Weine und Spirituosen erhältlich sind. Für Lm 3 erhält man z. B. auch fast $1 \mathrm{~kg}$ (!) maltesischen Nougat, eigentlich ein ebenfalls sehr teures Produkt mit einem durchschnittlichen Preis von Lm 1 pro $300 \mathrm{~g}$ Nougat. Der hohe Honig-Preis ist also ein Problem, das sich allmählich auf die Vermarktungsmöglichkeiten des Produkts auszuwirken beginnt.

\section{- Kunserva}

Der traditionelle Brotaufstrich der Maltesischen Inseln wird seit Jahrhunderten aus Tomaten, Salz und Zucker hergestellt. Je nach Rezept wird noch Zitronensaft oder Olivenöl zugesetzt.

Kunserva ist noch immer eines der beliebtesten und vielseitigsten Lebensmittel unter der maltesischen Bevölkerung. Für die touristische Vermarktung spielt das Produkt bisher nur eine untergeordnete Rolle. Dies ist jedoch nicht der Fall was den Exportmarkt betrifft. Kunserva gehört zu den Exportschlagern der maltesischen Nahrungsmittelindustrie und wird, in Gläsern oder Dosen verpackt, vor allem nach Nordafrika exportiert.

Die Herstellung in der Vergangenheit unterschied sich von der heutigen Zubereitungsmethode: Die Tomaten - nur die großwüchsige Sorte tadam ćatt (malt.) wurde verwendet - wurden in Stücke geschnitten und dann in einen groben Sack gegeben. Während eine Hand den Sack oben zuhielt, knetete man mit der anderen Hand den Inhalt zu Brei. Dieser Brei wurde danach in einen feinporigen Baumwollsack gefüllt, der an einem windigen Ort (z. B. in der Krone eines Baums, der an einer windigen Stelle wuchs) aufgehängt wurde. Die Flüssigkeit lief heraus und gleichzeitig trat ein Trocknungsprozess ein. Das so gewonnene Tomatenpüree wurde mit Salz versetzt und in Gläser gefüllt, die im oberen Teil mit einer breiten Schicht Olivenöl aufgefüllt wurden. Auf diese Art und Weise war eine Konservierung bis über den Winter hinaus gewährleistet.

Heute ist Kunserva der beliebteste Brotaufstrich Maltas und wird von mehreren Produzenten industriell hergestellt. Der größte Produzent ist das gozitanische Unternehmen Magro Bros., das in Malta der unangefochtene Marktführer bei Tomatenprodukten ist. Der Herstellungsprozess beinhaltet aus technischen Gründen statt der manuellen Knetung mittlerweile einen Erhitzungsprozess, wodurch der ursprüngliche Charakter des Produkts jedoch nicht beeinträchtigt wird.

Kunserva zählt zu den traditionellen maltesischen Lebensmittel ${ }^{402}$. Es war das erste maltesische Produkt, für welches bei der EU eine geschützte Herkunftsbezeichnung beantragt und auch vergeben wurde. Noch im Beitrittsjahr 2004 wurde Kunserva von der EU der Status D.O.P. (Denominazione Origine Protetta) zuerkannt. Damit kann ein Tomatenprodukt, das die Bezeichnung „Kunserva“ trägt, nur noch auf den Maltesischen Inseln legal produziert werden ${ }^{403}$. Es ist anzunehmen, dass Kunserva zukünftig auch auf dem EU-Binnenmarkt eingeführt werden soll.

In jedem Fall gehört Kunserva zu den ursprünglichsten maltesischen Produkten und ist unter der Bevölkerung nicht aus dem täglichen Gebrauch wegzudenken. Die Einstufung als regionales traditionelles Produkt ist zweifelsfrei gerechtfertigt.

\section{- Maltesische Würstchen}

Ein typisches Produkt der Maltesischen Inseln sind kleine Würstchen, in der Konsistenz vergleichbar mit der in Deutschland bekannten „Groben Bratwurst“. Das Besondere an den Würstchen sind die Gewürze und die Herstellung. Maltesische Würstchen werden außer mit Salz, Zucker und Pfeffer auch mit einem hohen Anteil Koriander und Petersilie gewürzt. Insbesondere der Koriander trägt zum süßlich-würzigen Geschmack des Produkts bei.

Der Herstellungsprozess ist ungewöhnlich: $60 \%$ fettarmes Schweinefleisch wird mit $40 \%$ Speck gehackt und mit den Gewürzen vermischt. Die Würstchen müssen in diesem Zustand einige Tage bei Zimmertemperatur „reifen“, um den Geschmack der Gewürze anzunehmen ${ }^{404}$. Dadurch wird ein „Gärungsprozess“ eingeleitet,

402 Vgl. CREMONA (2003), S. 9

403 Zu den Formalia der D.O.P.-Klassifizierung vgl. GRIENBERGER (2000) 
der ebenfalls wesentlich zum typischen Geschmackscharakter beiträgt. Erst nach dieser „Reifezeit“ kommen die Würste in die Auslagen der Metzgerläden und werden verkauft.

${ }^{405}$ Die Mehrheit der maltesischen Bevölkerung isst die Würstchen roh. Aus gesundheitlichen Gründen gehen jedoch immer mehr Malteser, besonders jene der jüngeren Generation, dazu über, die Würstchen in der Pfanne zu braten oder bei Barbecues zu grillen. Das maltesische Gesundheitsministerium warnt regelmäßig in Tageszeitungen vor Sommerbeginn vor dem Verzehr roher Würstchen. Es ist auch üblich, den Inhalt der Wurst zu entfernen und inn in der Art von gewürztem Hackfleisch als Grundlage für Saucen oder als Belag für Pizza, Ftira und Brote zu verwenden.

Schweinefleisch spielt in Malta heute eine große Rolle, obwohl die hier erwähnten Würstchen das einzige wichtige regionale traditionelle Produkt in diesem Sektor sind. Schon in steinzeitlichen Reliefs des neolithischen Tempels von Hal-Tarxien sind Schweine als Nutztiere dargestellt ${ }^{406}$.

Schweine stellen gegenwärtig den größten Anteil der in Ställen gehaltenen Nutztiere auf den Maltesischen Inseln ${ }^{407}$. Zur Zeit wird mehr Schweinefleisch erzeugt, als in Malta verbraucht wird. Teile des Fleisches gehen daher in den Export ${ }^{408}$. Die Schweinefleisch verarbeitende Industrie in Malta ist jedoch eher klein. Es gibt zwar größere Schlachthäuser und Fleischfabriken, jedoch ist es in Malta noch üblich, bei den örtlichen Metzgern - von denen es sehr viele gibt - zu kaufen. 1978 erlitt die maltesische Schweinezucht einen herben Rückschlag: Das Afrikanische Schweinefieber hatte sich auf den Inseln ausgebreitet und sämtliche Bestände mussten geschlachtet und entsorgt werden. Da Comino die einzige Insel ohne verseuchten Schweinebestand war, wurde auf dieser kleinsten Insel der Inselgruppe die neue Schweinezucht aufgebaut ${ }^{409}$. Heute ist die Nachzucht per Gesetz nur mit Tieren erlaubt, deren Herkunft aus der Schweinefarm von Comino verbürgt ist. Das Fehlen von regionalen traditionellen Produkten auf Schweinefleischbasis lässt sich mit der Ärmlichkeit der Lebensverhältnisse auf der Inselgruppe erklären, an der sich erst nach dem Zweiten Weltkrieg grundlegend etwas änderte.

\section{- $\quad$ Nougat (Qubbajt) und Helwa tat-Tork}

Nougat (malt:: Qubbajt) und die Süßspeise Helwa tat-Tork wurden vermutlich zur Zeit der arabischen Herrschaftsperiode auf der Maltesischen Inselgruppe etabliert. Leider existieren aus dieser Zeit kaum Aufzeichnungen. CREMONA bezeichnet die arabische Epoche als „dark age ${ }^{\text {“410 }}$, weil nur wenige Quellen überliefert sind.

Auch im Experteninterview mit MIRIAM VeLLA, Inhaberin der Nougatfirma Frans Vella \& Sons in Qormi (Malta), konnte kein Licht in die Angelegenheit gebracht werden. Hier bezieht man sich auf die Familientradition, die bis ins Jahr 1902 zurückreicht. Und überhaupt habe es „schon immer“ Nougat in Malta gegeben $^{411}$. Literatur über Maltesischen Nougat gibt es faktisch nicht, und so muss sich der Autor im Folgenden vollständig auf die in Gesprächen mit Mrs. VELLA erhobenen Informationen, und einer kurzen Eintragung in einem Kochbuch (DARMANIN (1991), S. 116) stützen.

Zur Zeit gibt es in Malta fünf Nougat-Produzenten. Zum größten Teil gehören sie zur Verwandtschaft der Familie VELLA. Frans Vella \& Sons Company in Qormi ist der älteste und größte Betrieb und offeriert die meisten Geschmacksvarietäten. Die Geschichte der Firma begann im Jahr 1902, als der Stammvater JOHN "ĠANNI" VELLA von Gozo nach Malta übersiedelte und die Produktion von Nougat begann, welche er, laut Aussage von Frau VELLA, durch einen arabischen Händler erlernt hatte. Fast alle nachfolgenden Nougatproduzenten Maltas gingen aus dem Stammbaum von JOHN VELLA hervor. Um die besondere Stellung der Frans Vella Company als „Ursprung“ der Nougatproduktion in Malta hervorzuheben, führt die

405 Alle Angaben der folgenden beiden Abschnitte nach einem Mitarbeiter von Starlite Butcher, Bugibba im Experteninterview vom 14.06.2004

406 Vgl. Galea de Giovanni (o. J.), S. 5

407 Vgl. AZZOPARDI (2002), S. 173 f.

408 Vgl. ebd.

409 Vgl. ebd.

410 CREMONA (2003), S. 21

411 So Geschäftsführerin MIRIAM VELLA im Experteninterview vom 16.06.2004 
Familie im Firmennamen den Hinweis „Ta'Ǵanni L-Ghawdxi“ (malt. sinngemäß: „Von Ǵanni, dem Gozitaner“) (vgl. Foto 7).

Man unterscheidet in Malta soft Nougat und hard Nougat. Zwar sind beide Erzeugnisse mit dem Namen "Nougat" verbunden, jedoch handelt es sich bei dem harten und bei dem weichen Produkt in puncto Erscheinungsbild Geschmack um unterschiedliche Erzeugnisse.

Der weiche Nougat besteht aus einer Art zähem Schaum aus Zucker, Glucosesirup, Honig und Eiweiß. Dieser Grundmasse, genannt plain Nougat, werden nach dem Erhitzen auf eine bestimmte Temperatur je nach Sorte unterschiedliche Zusatzstoffe beigefügt. Dabei handelt es sich sowohl um Nüsse und Mandeln, als auch um Aroma- und Farbstoffe. Einigen Sorten werden getrocknete Früchte, Schokolade, Minzaroma, Orangeat/Zitronat oder aromatische Kräuter zugesetzt. Insgesamt produziert die Vella Company acht verschiedene Sorten soft Nougat. Der Produktionsvorgang ist Familiengeheimnis und kann deswegen an dieser Stelle nicht beschrieben werden. Der Nougat wird in Riegeln zu $100 \mathrm{~g}, 250 \mathrm{~g}$ und $500 \mathrm{~g}$ verkauft. Die besondere Eigenschaft der Produktbeschaffenheit macht nach DARMANIN das Besondere am Maltesischen Nougat aus: „Das Geheimnis liegt nicht nur am Geschmack sondern auch an der Beschaffenheit. Qubbajt sollte nie so hart sein, daß man nicht hineinbeißen kann, immerhin muß er solide und nicht zu weich sein." (DARMANIN (1991), S. 116).

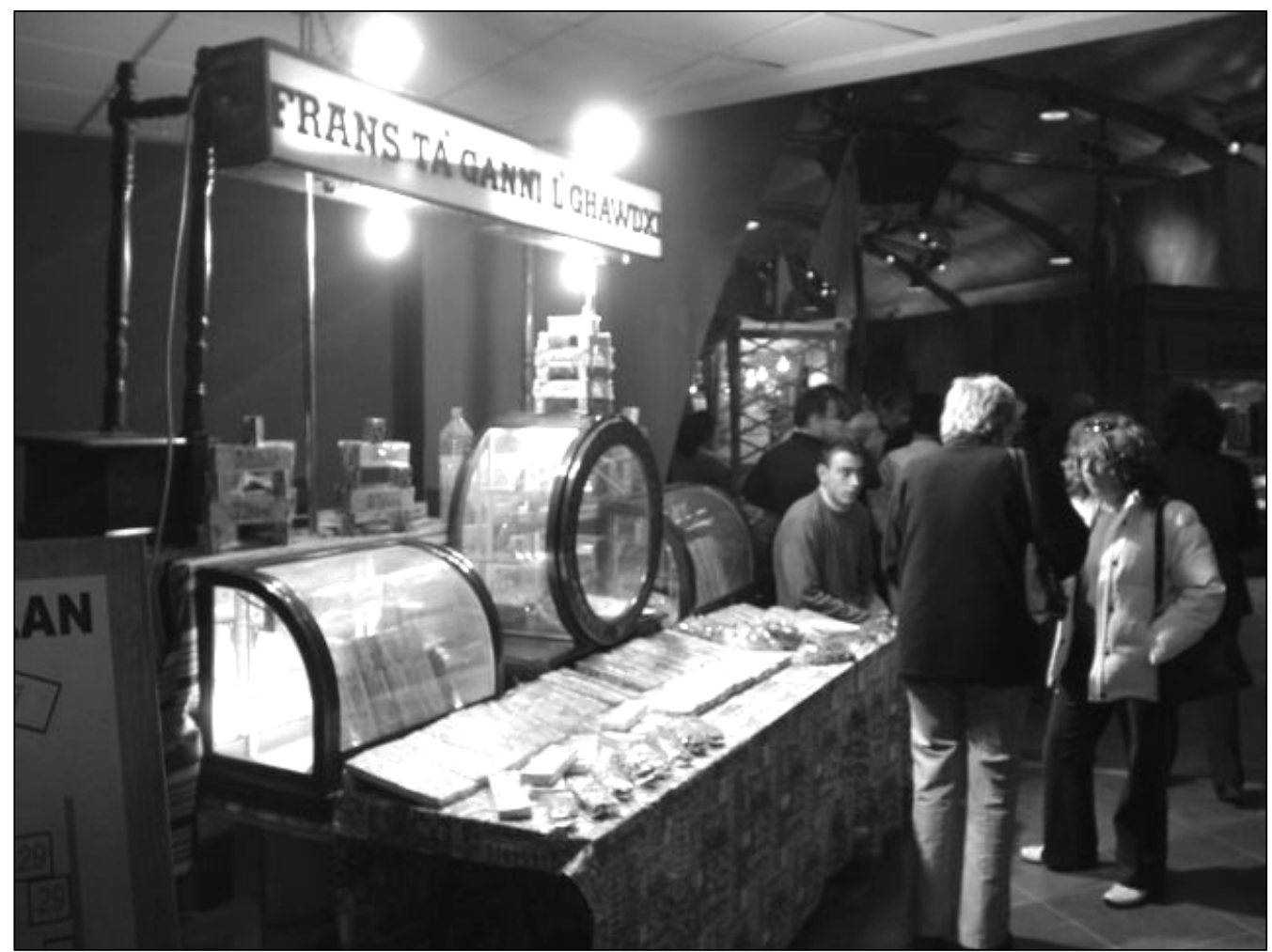

Foto 7: Typischer maltesischer Nougatverkaufsstand der Firma Frans Vella beim Mediterranean Food Festival in Paceville, Malta. Jeder Nougathersteller benutzt ähnliche Verkaufsstände, die vor allem auf den Dorf-Festas zum Einsatz kommen. Foto: ASCHEMEIER

Dem hard Nougat wird kein Eiweiß zugesetzt. Er besteht nur aus Zucker, Glucosesirup, Vanillearoma und einem hohen Nussanteil. Im Prinzip handelt es sich somit nach der Produktion (die auch in diesem Fall geheim gehalten wurde) um einen extrem harten Riegel aus Karamell mit eingeschlossenen Nüssen. Von der Konsistenz ist der harte Nougat den in Deutschland bekannten „gebrannten Mandeln“ vergleichbar und schimmert „glasig“. Die Riegel sind so hart, dass sie zerstoßen werden müssen, bevor sie verzehrt werden können. Hard Nougat gibt es in drei Variationen: mit Erdnüssen oder Mandeln und Erdnüssen sowie mit Mandeln, Haselnüssen und Sesamkörnern.

Hard und soft Nougat werden pur als Süßigkeit verzehrt. Zu besonderen Anlässen, z. B. bestimmten Feiertagen, Muttertag oder zu Festas (den maltesischen Dorffesten), werden Herzen aus Nougat hergestellt, die mit einer üppigen Dekoration aus buntem Zuckerguss verziert werden. 
Dem Nougat äußerlich nicht unähnlich ist die Süßspeise Helwa tat-Tork (malt. sinngemäß: „Süßspeise der Türken/Türkischer Honig“). Helwa tat-Tork sieht aus wie heller soft Nougat, weist jedoch einen völlig anderen Produktcharakter auf. Es wird z. B. auch nicht von der Vella Company produziert, die sich als reine Nougathersteller verstehen.

Helwa tat-Tork besteht aus einer Creme aus gemahlenen Sesamkörnern, Eiweiß und Zucker. Die Masse ist kein zäher „Schaum“ wie beim hellen Nougat, sondern eher eine kristalline, brüchige und weiche Masse, die im Übrigen nicht in Riegeln, sondern in kleinen Töpfchen verkauft wird. Im Gegensatz zum Nougat, der nicht gekühlt werden darf, muss Helwa tat-Tork gekühlt werden. Einer der größeren Produzenten für diese Süßspeise ist die Firma E.M.S. aus Żebbug (Malta) als Zulieferer der Camel Brand Co. (s. auch Einträge Ǵbejniet und Bigilla). Helwa tat-Tork wird entweder als Süßigkeit pur zur "Tea Time" verzehrt, anderen Süßspeisen wie z. B. Speiseeis zugesetzt oder in Scheiben geschnitten als Brotbelag verwendet. Auch dies unterscheidet das Produkt vom Maltesischen Nougat, der immer pur verzehrt wird.

Helwa ist in verschiedenen Variationen im gesamten Mittelmeerraum etabliert. Als „Aladdin's Dessert“ ist es ein Inbegriff für orientalische Süßspeisen. Im Gegensatz zum Maltesischen Nougat ist das maltesische Helwa tat-Tork anderen mediterranen Helwa-Varietäten sehr ähnlich, was seinen Charakter als regionales traditionelles Produkt aber nicht schmälert.

\section{- $\quad$ Olivenöl (żejt taż-żebbuǵ)}

Ölbäume wurden in Malta seit Menschengedenken angepflanzt (zur Historie des Ölbaums auf den Maltesischen Inseln s. Kap. 13.9 und 20.3).

Im 20. Jh. war die Ölbaumkultivierung verfallen ${ }^{412}$. Im 1961 erschienenen Grundlagenwerk „Malta Background for Development" ist folgender Eintrag zu finden:

„Malta must be the only mediterranean land where the tradition of olive cultivation has almost disappeared. Vegetable oil consumption is high and has to be supplied by imports costing on average nearly $£ 300,000$ per annum. (...). Various reasons for this strange situation have been mooted, ranging from the completely invalid belief that the olive cannot stand sea-air to the inadequate charge that tenurial security is insufficiently great. The matter is still a mystery." (BOWEN JONES, DEWDNEY \& FISHER (1961), S. 213).

Im Jahr 2000 wurde die kommerzielle Olivenölproduktion wieder aufgenommen. In Malta ist diese Initiative einem einzelnen Mann zu verdanken: SAM CREMONA.

CREMONA schaffte es bereits im ersten Jahr seiner Unternehmensgründung 17t Oliven zu pressen, wobei insgesamt $2.600 \mathrm{~kg}$ hochwertiges Olivenöl gewonnen werden konnten ${ }^{413}$. Laut seiner Auskunft im Rahmen eines vom Autor geführten Experteninterviews vom Oktober 2003, ist es sein Ziel, bis zum Jahr 2010 eine Presskapazität von 100t Oliven zu erreichen.

Es lohnt sich darüber hinaus einen Blick auf das wirtschaftliche Konzept der maltesischen Olivenölproduktion zu werfen: CREMONAs zukunftsweisende Idee um die Ölbaumkultivierung in Malta anzukurbeln, war es, den Service des Ölpressens den Landwirten Maltas und Gozos „kostenlos“ zur Verfügung zu stellen. Als Lohn für die Dienstleistung behält CREMONA 20\% des gepressten Olivenöls ein, das er seit 2001 unter dem Label Wardija Premium Brand vermarktet.

Das maltesische Olivenöl ist hochwertig und weist kaum nachweisbare Säurewerte auf. CREMONA dazu in einem Zeitungsinterview:

„Our soil is alkaline, which renders an olive oil with a low acidity level. Olives coming from older trees are practically devoid of acidity. The Sicilians are proud to have $1 \%$ acidity which can then be called „extra virgin", "first press" or "fine oil". Ours had 0,03\% acidity when we took it over to Sicily to be tested!" (MALTA TODAY, Onlineausgabe vom 28.10.2001.)

412 Vgl. BOWEn JONES, DEWDNEY \& FISHER (1961), S. 213; AZZOPARDI (2002), S. 166

413 Experteninterview mit SAM CREMONA in Wardija vom 13.10.2003 \& MALTA TODAY, Onlineausgabe vom 28.10.2001; AZZOPARDI (2002), S. 166 
Das maltesische Olivenöl aus Wardija wurde im Rahmen eines Treffens der EU-Agrarminister in Taormina als das beste der dort präsentierten Olivenöle des gesamten Mittelmeerraums ausgezeichnet ${ }^{414}$. Es wird seit 2005 in kleinen Mengen nach Großbritannien exportiert. Das Öl ist in Flaschen zu 0,25 I erhältlich und rangiert im exklusiven Preisbereich ${ }^{415}$. In relativ kurzer Zeit ist die Olivenölproduktion in Malta vom Status eines verfallenden Wirtschaftszweigs zu neuen Höhen aufgestiegen und produziert ein wegweisendes regionales traditionelles Produkt ${ }^{416}$ mit einem hohen Grad an Exklusivität.

Das Wardija-Olivenöl wird deshalb im Rahmen dieser Dissertation nochmals in Kapitel 20.3 unter dem Blickwinkel der Entwicklungsfähigkeit maltesischer Produkte betrachtet.

\subsubsection{Backwaren}

In Malta existiert ein großes Sortiment an Backwaren und Konditorei-Artikeln. Jedoch enthalten viele dieser Produkte Schlagsahne und sind somit leicht verderblich. Sie werden im Rahmen dieser Arbeit nicht aufgeführt, weil ihre Vermarktung unter dem Aspekt des Exports sowie für den touristischen Bedarf die Schwierigkeiten der Konservierung mit sich bringt. Es ist anzunehmen, dass der durchschnittliche MaltaReisende in der Regel selten in eine der schmucklosen, oft von normalen Wohnhäusern kaum zu unterscheidenden Konditoreien geht. Dieser Umstand und die große Vielfalt von allgemein sehr ähnlichem Sahnegebäck sind die Gründe, warum diese Backwaren im vorliegenden Produktverzeichnis nicht auftauchen.

Weitere Backwaren, die in touristisch viel frequentierten Geschäften angeboten werden, stammen meistens aus den Bereichen Brot oder Kekse. Kekse existieren in Malta in einer fast unüberschaubaren Sortenvielfalt. Sie werden von großen und kleinen Konditoreien hergestellt, industriell oder „hausgemacht“.

Unter den vielen Kekssorten sind nur einige wenige als regional traditionell zu erwähnen. In das Produktkataster dieser Arbeit fallen Pasti tal-lewz (Mandelplätzchen), weil fast alle anderen Produkte dieses Bereichs, z. B. die sehr beliebten Pasti tal-jam (Marmeladenplätzchen), aus Rezepten anderer Kulturkreise abgeleitet wurden.

Aus dem Konditoreibereich sind Qaghaq ta'l-ghasel („Honigringe“) eindeutig hervorzuheben, die eines der traditionellsten Produkte der Maltesischen Inseln sein dürften (s. u.). Die Palette weiterer Konditoreiprodukte umfasst u. a. Feigenrollen und Dattelplätzchen, die zwar in jeder Konditorei verkauft werden, deren Ursprungsland jedoch nicht Malta ist. Es handelt sich hierbei um eingeführte Rezepte aus der LevanteRegion bzw. aus Nordafrika.

Sehr charakteristisch ist das maltesische Weizenbrot (Malt.: Hobż), wegen der harten Brotkruste auch „crusted bread“ genannt. Es ist ein regionales traditionelles Produkt und wird nach Kenntnisstand des Autors in der in Malta bekannten Form nirgendwo sonst im Mittelmeerraum gebacken. In Rezepten traditioneller maltesischer Gerichte (z. B. Hobż biż-żejt) wird die Notwendigkeit von original maltesischem Brot als Grundlage hervorgehoben ${ }^{417}$.

414 Angabe von SAM CREMONA im Experteninterview vom 25.05.2004.

415 0,25 I Wardija Premium Brand werden in Malta zum Preis von etwa LM 4 verkauft. Das sind umgerechnet etwa $10 €$.

416 Die Ausweisung als regionales traditionelles Produkt ist möglich aufgrund der Tatsache, dass privates Pressen von Olivenöl auch im 20. Jh. nie wirklich verschwunden war. Vor allem in Gozo gibt es noch heute viele ländliche Familien, die mit kleinen Drehpressen ihr eigenes Öl herstellen.

417 Vgl. z. B. DARMANIN (1991), S. 101 


\section{- Qaghaq ta' l-ghasel}

Hierbei handelt es sich um ein kreisrundes Gebäck mit hohem Honig- bzw. Sirupanteil. Die ungefähre Übersetzung des maltesischen Namens ist „Ring aus Honig“, wobei sich der Name auf den sog. „schwarzen Honig" (malt.: ghasel iswed) bezieht. Diese Substanz ist kein Honig im eigentlichen Sinn, sondern eine Art von Melasse, womit sich die dunkle Farbe der Füllung erklärt ${ }^{418}$.

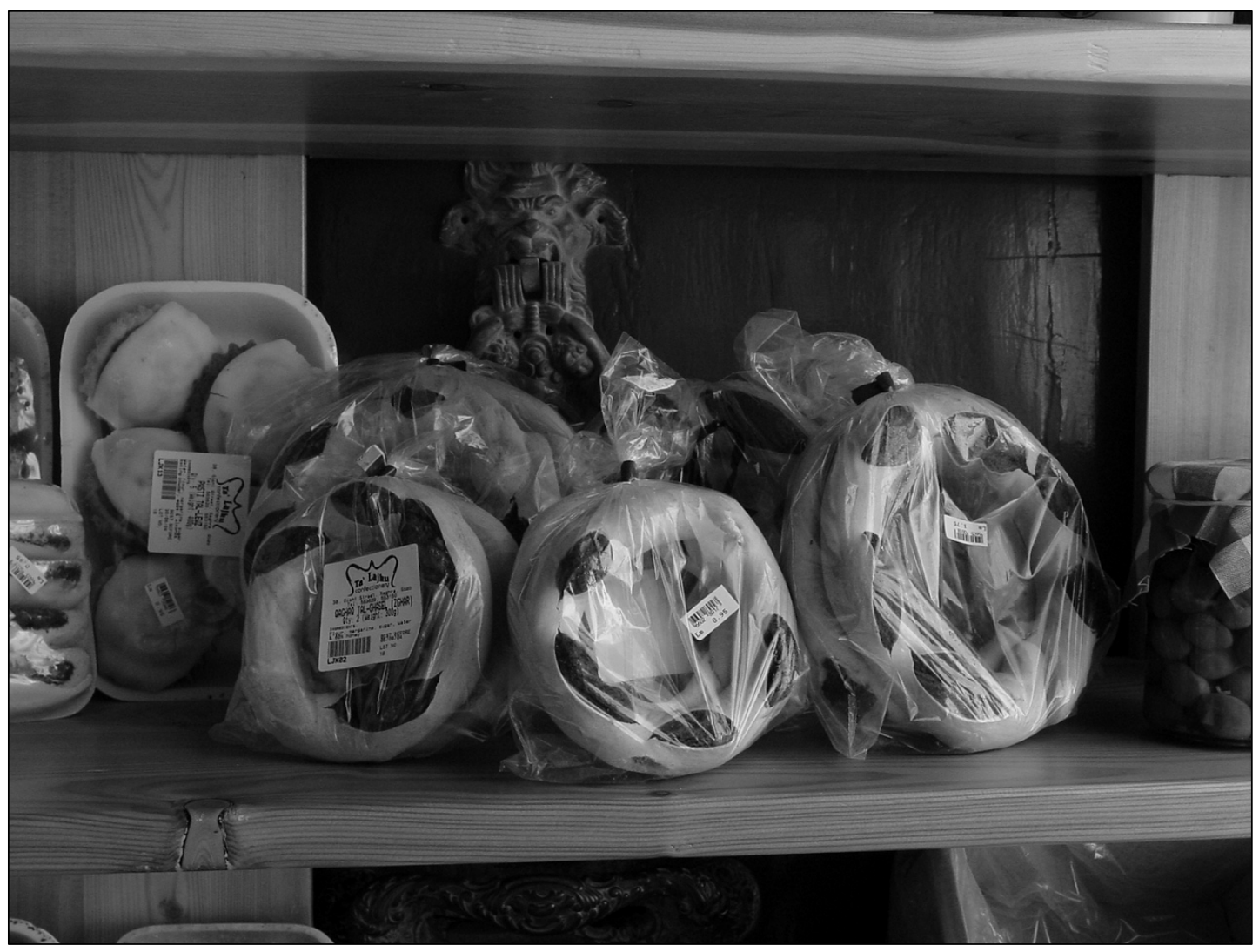

Foto 8: Qaghaq ta'l-ghasel in der Auslage eines Spezialitätengeschäfts in Mdina, Malta. Im Hintergrund sind weitere Backwaren zu sehen, so z. B. (v.I.n.r.) Dattelrollen und Mandelplätzchen. FOTO: AsCHEMEIER

Die ringförmige Backware (s. Foto 8) besteht hauptsächlich aus der Füllung aus Melasse, Wasser, Zucker und Grieß sowie aus einem dünnen Teigüberzug aus Mehl, Eigelb, Wasser und Butter. Der Teigmantel ist an einigen Stellen eingeschnitten, sodass im Verlauf des Backprozesses die Füllung durch die schlitzartigen Öffnungen als dunkelbraune Masse nach außen tritt. Der Geschmack ist bittersüß, ähnlich dem des in Deutschland bekannten Lebkuchens bzw. Honigkuchens, jedoch ohne den zusätzlichen hohen Anteil an Gewürzen und anderen Zusatzstoffen.

Die Süßspeise gehört zu den traditionellsten Erzeugnissen der Maltesischen Inseln und wurde am 01.05.2004 um Mitternacht bei den Festlichkeiten zum EU-Beitritt als Laserdarstellung an die Festungsmauern von Fort St. Angelo in Vittoriosa projiziert. In der Literatur werden Qaghaq tal-gћasel eindeutig zu den ursprünglichsten Lebensmitteln Maltas gezählt ${ }^{419}$. Die Laserdarstellung bei der EUBeitrittsfeier zeugt von der Eigenschaft dieser Backware als Identitätsträger. Das Produkt ist damit ein regionales traditionelles Produkt im Sinne der vorliegenden Dissertation. 


\subsubsection{Gerichte}

Gerichte aus der Maltesischen Küche werden im Rahmen dieser Arbeit nicht als Produkte verstanden. Aufgrund ihres Charakters als meist warme Mahlzeit, die erst mit einigem Aufwand angefertigt und aus Einzelzutaten zubereitet werden muss, ist der Produktcharakter, der nach Auffassung des Verfassers einen hohen Grad an unmittelbarer Konsumierbarkeit voraussetzt, nicht gegeben (vgl. Kap. 10). Dennoch wird im Folgenden eine Auswahl von Gerichten in Kurzform beschrieben, damit ausgeschlossen werden kann, dass die folgenden Speisen missverständlicherweise als Produkte betrachtet werden.

Braǵioli, auch unter dem englischen Pseudonym Beef Olives bekannt, ist eine Roulade aus Rindfleisch, gefüllt mit Oliven. Das Gericht ist vermutlich aus Süditalien nach Malta gekommen und ist mittlerweile auch als Fertiggericht erhältlich.

Fenkata ist das maltesische Nationalgericht. Der Name Fenkata bedingt kein spezielles Gericht bzw. Rezept, sondern bedeutet lediglich, dass es sich um ein Essen mit Kaninchenfleisch (malt.: Fenek = Kaninchen) handelt. Es sind viele verschiedene Variationen bekannt.

Lampuki, die Goldmakrele, ist Grundlage verschiedener maltesischer Fischgerichte. Besonders bekannt ist die Fischsuppe Aljotta, die große Mengen Knoblauch und Salz enthält.

Ross II-Forn bedeutet übersetzt „Reis aus dem Ofen“. Es handelt sich dabei um einen in der Zubereitung zeitaufwändigen Reisauflauf, der mit zahlreichen Zutaten aus dem landwirtschaftlichen Sektor zubereitet wird.

Schnecken (malt.: bebbux) sind die Basis für einige der traditionellsten Speisen in Malta. Die Schnecken werden entweder vom Wegesrand, von Rubble Walls oder von Opuntienhecken aufgesammelt. Man verarbeitet sie zu kleinen Beilagen (in Salzwasser gekocht), als Zutat in Eintöpfen oder frittiert sie als Snack. Die typische Zeit zum Schnecken sammeln ist nach den ersten Regenfällen im Spätsommer, da sie zu diesem Zeitpunkt angeblich am besten schmecken ${ }^{420}$. Schnecken stellten in der Vergangenheit eine proteinreiche Ergänzung der traditionell proteinarmen maltesischen Ernährung dar.

Eine der traditionellsten Suppen Maltas ist die Gemüsesuppe Minestra. Sie enthält Kohlrabi, Möhren, Zwiebeln, Sellerie, Blumenkohl, Kartoffeln, Weißkohl, Kürbis, Kunserva (s. o.) und żibeǵ $(\mathrm{ca} .1 \mathrm{~cm}$ lange Nudeln). Im Vergleich zur italienischen Minestrone ist die maltesische Minestra dickflüssig und cremig.

Die Ftira ist eine typisch gozitanische Spezialität. Sie ist der italienischen Pizza vergleichbar. Statt mit Tomatensoße wird sie jedoch mit frischem Ǵbejniet (s. o.) bestrichen. Der Belag besteht im Wesentlichen aus mit Fenchelsaat gewürzten dünnen Kartoffelscheiben. 


\subsubsection{Getränke}

Die Vielfalt traditioneller Getränke der Maltesischen Inseln ist begrenzt. Wo Süßwasser als Grundlage fehlt, kann sich keine umfassende Folklore um Getränke entwickeln.

Neben Wasser und Milch besitzt daher Wein die längste Tradition in Malta. Der Weinbau wurde von den Phöniziern und Griechen eingeführt ${ }^{421}$. Tee und Kaffee kamen aufgrund der regen Handelstätigkeit der Briten auf die Inselgruppe. Der maltesische Kaktuslikör bajtra wurde von französischen Johannitern etabliert, die Herstellung von Bier erfolgte erstmals unter britischer Verwaltung. Unter maltesischen Farmern war die Herstellung eines Anisschnapses beliebt. Moderne Getränke, wie Kinnie und andere Limonaden, kamen erst Mitte des 20. Jh. auf die Inseln. Die 1970er- und 1980er-Jahre brachten die Squashes - Getränkesirups zum Verdünnen mit Wasser - aus Großbritannien und Südeuropa auf die Inselgruppe.

Das folgende Produktverzeichnis der regionalen traditionellen maltesischen Getränke orientiert sich methodisch am Verzeichnis der Lebensmittel (s. o.) und erfolgt wie dieses alphabetisch.

\section{- Kinnie}

Kinnie ist eine herb-süße Limonade mit Auszügen aus Bitterorangen und Kräutern. Der Getränkekonzern Simonds Farsons Ćisk, ein Tochterunternehmen der Farsons Group of Companies, führte die Limonade in direkter Konkurrenz zu amerikanischen Produkten wie z. B. Coca Cola oder Pepsi im Jahr 1952 auf dem maltesischen Markt ein. 1984 kam Diet Kinnie, eine zuckerfreie Variante, dazu. Der große Erfolg unter Einheimischen und Touristen führte dazu, dass Kinnie mittlerweile in sieben Länder ${ }^{422}$ exportiert wird.

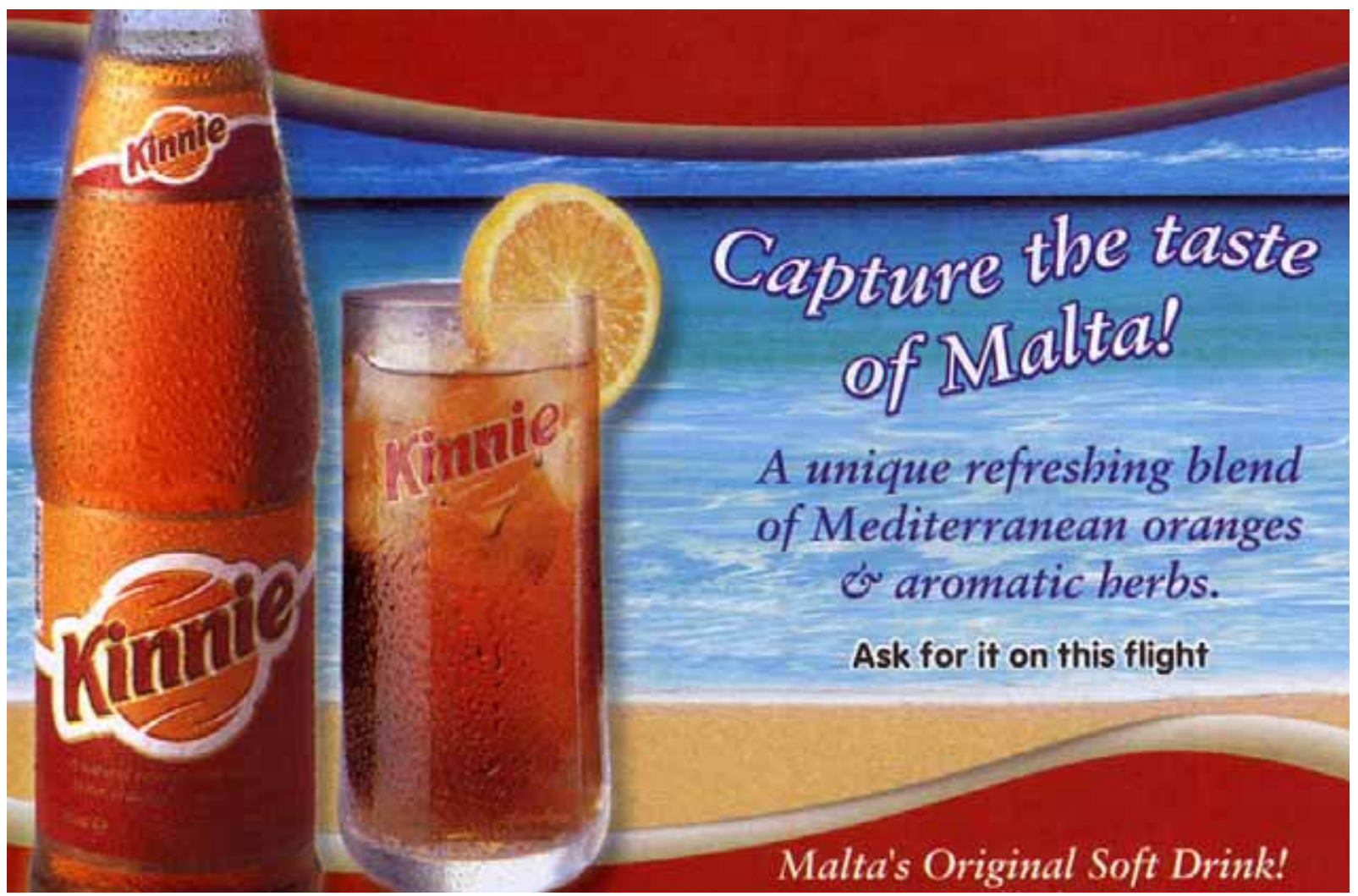

Abb. 14: Kinnie-Werbung im Magazin von „Air Malta“. Kinnie wird als „Malta’s Original Soft Drink“ beworben.

\section{Vgl. DEguARA (1979)}

422 Laut Angaben des Herstellers wird Kinnie nach Australien, Belgien, Luxemburg, Schweden, Großbritannien, Deutschland und in die Niederlande exportiert. 
Kinnie ist nach Angaben des Herstellers seit seiner Einführung Maltas erfolgreichster Softdrink mit einem höheren Marktanteil als die Produkte der Coca Cola-Company deren Lizenzinhaber ${ }^{423}$ in Malta das KinnieImitat Krest herstellt und vertreibt.

Zum 50-jährigen Jubiläum der Markteinführung von Kinnie veranlasste Farsons ein dreitägiges Open AirSpektakel auf dem Gelände des ehemaligen Royal Opera House in Valletta vom 09.-11.05.2002. Teile dieser Jubiläumsfeierlichkeiten wurden im maltesischen Rundfunk und Fernsehen übertragen. Dies zeigt, dass das Jubiläum den Charakter einer landesweit beachteten Veranstaltung hatte, von der die Medien in großem Umfang berichteten.

Das gesamte Kinnie-Marketing basiert seit Jahren darauf, die Limonade als typisch maltesisches Produkt zu verkaufen (vgl. Abb. 14). Diese Art der Werbung funktioniert sowohl im Inland als auch im Ausland. Kinnie ist ein moderner Identitätsträger maltesischer Werte und war für die Entwicklung der Jugendkultur in Malta ähnlich wichtig, wie z. B. Coca Cola oder Pepsi in Mitteleuropa. Kinnie kann zweifellos als vollwertiges regionales traditionelles Produkt eingestuft werden.

Anders als andere Getränke des Herstellers Farsons, hat Kinnie seine eigene, von der Firmen-Homepage unabhängige, Internetpräsenz ${ }^{424}$.

\section{- Wein}

Der heutige große Erfolg, der zum florierenden Export des maltesischen Weins führte, ist nicht zu vergleichen mit der Situation in der Vergangenheit. Berichte aus dem 16. Jh. attestierten maltesischem Wein höchstens mittlere Qualität, und Autoren zur Zeit der Johanniterritter weisen darauf hin, dass die maltesischen Trauben als Tafeltrauben besser zu genießen seien als in gekeltertem Zustand ${ }^{425}$. Antike Berichte loben hingegen den maltesischen Wein, so z. B. HOMERs „Odyssee“, in der sich der Held Odysseus in der Gegenwart der Nymphe Calypso über den wohlschmeckenden Wein der Insel Ogygia (wahrscheinlich gleichzusetzen mit dem heutigen $\mathrm{Gozo}^{426}$ ) äußert ${ }^{427}$.

Die Geschichte des Weinbaus in Malta reicht zurück bis in die phönizische Periode Maltas, in der die Weinpflanze vermutlich von griechischen Händlern eingeführt wurde ${ }^{428}$. Die Tradition des Weinbaus hat seit der Antike zu vielen Volkssagen und Legenden beigetragen, in denen Wein als besonders wertvolle Pflanze hervorgehoben wurde ${ }^{429}$. Die Römer übernahmen die Kultivierung des Weins in Malta von den Phöniziern und Griechen. In der Villa Rustica von San Pawl Milqi, eines der wichtigsten Zentren der Herstellung von Olivenöl des antiken Malta ${ }^{430}$, wurden nachweislich auch Weinpressen betrieben ${ }^{431}$.

Während der Zeit der arabischen Herrschaft, der Piratenüberfälle und der mittelalterlichen Seuchen wurde der Weinbau in Malta - wie viele landwirtschaftliche Nutzungsformen - nur in einer stark reduzierten Form betrieben. Trotzdem kam es niemals zum völligen Stillstand des Weinbaus. Eine historische Quelle aus dem Jahr 1434 berichtet vom Weinbau als anteilige Lebensgrundlage von knapp 1.000 Farmern. Schon damals war der Verbrauch so hoch, dass sizilianischer Wein importiert werden musste ${ }^{432}$.

423 Lizenzinhaber der Coca Cola-Produkte für Malta: General Soft Drinks Co. Ltd., Qormi.

424 www.kinnie.com

425 Vgl. CREMONA (2003), S. $20 \mathrm{f}$.

426 BRADFORD (1971); CASSAR (2000); ATTARD (2002), TEUMA (2003)

427 Vgl. auch Deguara (1979), S. 1

428 CREMONA (2003), S. 21 \& Deguara (1979), S. 1

429 Vgl. z. B. BEZZINA (1992), S. 13 f.

430 Vgl. Kap. 20.3.2

$431 \mathrm{Vgl}$. Deguara (1979), S. 1

432 Ebd., S. 2 
Erst die Johanniter modernisierten den Weinbau und führten Rebsorten aus Süditalien und Frankreich ein. Trotzdem war der maltesische Wein nur von mäßiger Qualität. Der Grund lag im Klima der Inseln, welches für hochqualitativen Wein bereits zu trocken ist. Um 1920 erlitt der Weinbau in Malta durch die Einschleppung einer Seuche (Phylloxera), die die Weinreben befiel, herbe Rückschläge. Dies war vor allem der Grund, warum der Weinbau zunächst keinen größeren Erfolg hatte, als er zu Beginn des 20. Jh. in Malta eine weitere Wiederbelebungsphase erlebte - zu einer Zeit, in der auch süditalienische Weine schon längst Spitzenqualität aufwiesen. Der meiste Wein für den kommerziellen Handel wurde aus Sizilien importiert und nur einer der damaligen Betriebe ${ }^{433}$ konnte sich bis in die heutige Zeit halten.

Heute ist die Lage anders: Maltesischer Wein ist bekannt für seine gute Qualität, die aber unter hohem Aufwand „erkämpft“ werden muss. Aufwändige Bewässerungsmaßnahmen gehen Hand in Hand mit der Auswahl der richtigen Lagen und Böden - und der Hoffnung auf einen nicht allzu trockenen Sommer. Die Firmen Marsovin und Meridiana Wine Estate beschäftigen ganzjährig Oenologen, während Emmanuel Delicata und Master Wine saisonal Experten aus Italien und Frankreich einfliegen lassen ${ }^{434}$. Wein wird seit Generationen aber vor allem von den Familien der Farmer in Heimarbeit hergestellt. Diese Tradition war über Jahrhunderte trotz Dürren und Seuchengefahr kontinuierlich aktiv, da auch der Bedarf von Seiten der Bevölkerung stets vorhanden war. Wer Wein anbaute, verschaffte sich damit nicht nur ein SubsistenzLebensmittel, sondern auch eine Erwerbsgrundlage. Wein ist ein regionales traditionelles Produkt der Maltesischen Inseln. 


\subsection{Produkte des Kunsthandwerks}

\subsubsection{Kunsthandwerk allgemein}

Das Kunsthandwerk in Europa entwickelte sich aus der Produktion von oftmals alltäglichen Gebrauchs- oder Dekorationsgegenständen, die durch die herausragende Fähigkeit und den Ideenreichtum ihres Schöpfers über das normale und "nötige“ Maß hinaus entwickelt wurden. In der Renaissance erkannte man erstmals einen Unterschied zwischen dem Kunsthandwerk und den „schönen Künsten“, wobei letztere in der Folge lange Zeit als dem Kunsthandwerk überlegen angesehen wurden.

Erst durch die Phase der Industrialisierung im 19. Jh. und der damit einhergehenden „Maschinisierung“ der Produktionsprozesse von ehemals reinen Handwerksprodukten wurde die Uniformität der Qualität und der begrenzte Formen- Farben- und Ideenreichtum maschinell erzeugter Produkte infrage gestellt. Je mehr sich die Welt industrialisierte und je mehr auch neue Produkte wie Kunststoffe und Kunstfasern auftauchten, desto wertvoller erschien das Kunsthandwerk, das natürliche Werkstoffe und herausragende Fähigkeiten des Erzeugers voraussetzt.

Es muss zusätzlich ein psychologischer Faktor genannt werden, der Produkte des Kunsthandwerks für Laien dieses Sektors besonders hochwertig erscheinen lässt: „Crafts are a true expression of a people's cultural identity. Artisans understand how the limited resources of their natural environment can be used to maintain the authenticity of their craft. Sometimes, the crafts are purely artistic expressions of the innate skills and creativity of the artisans or are created to emphasise the natural beauty of the materials used." (CARABOTT (1998), S. 1).

Dieser Prozess ermöglicht es dem Kunsthandwerker, einem von ihm erzeugten Gegenstand „Persönlichkeit“ zu verleihen. Der an sich leblose Gegenstand wird zum Ausdruck der Schaffenskraft eines Einzelnen, aber auch zum Ausdruck der Kultur eines Landes oder einer Region. Dies wirkt auf den Käufer assoziativ, indem er sich beim Erwerb und bei der Betrachtung/Benutzung des kunsthandwerklichen Artikels wieder an bestimmte Ereignisse in Zusammenhang mit ihm erinnert fühlt $\mathrm{t}^{435}$. Dass das Kunsthandwerk der früheste traditionelle Zulieferer der Souvenirverkäufer für den touristischen Markt in aller Welt war, ist, aus diesem Blickwinkel betrachtet, kein Wunder. „Tourists are often particularly interested in culturally diverse people and objects and will purchase local crafts." (CARABOTT (1998), Abstract).

\subsubsection{Kunsthandwerk Maltas und Gozos}

Das maltesische Handwerk ist ähnlich wie die Fülle an regionaltypischen Lebensmitteln sehr vielfältig. Die Literaturlage in Bezug auf maltesisches Handwerk ist sehr dünn, sodass sich der folgende Abschnitt mit Ausnahme weniger Arbeiten über Glasbläserei und Klöppelei ${ }^{236}$ auf eigene Erhebungen und Experteninterviews mit maltesischen Kunsthandwerkern stützt.

Im folgenden Kapitel wurde ausschließlich das Kunsthandwerk der Inselgruppe berücksichtigt. Selbstverständlich gibt es auch in Malta Handwerkssektoren wie z. B. das Maurerhandwerk, das Malerhandwerk, usw. Diese Handwerkszweige spielen jedoch für die Vermarktung auf touristischer Ebene oder auf dem EU-Binnenmarkt verständlicherweise keine Rolle, weswegen sie an dieser Stelle nicht erwähnt werden - obwohl auch sie durchaus regionale Besonderheiten aufweisen, denen es wert wäre nachzugehen. Sie erzeugen aber keine Produkte im engeren Sinne, was ein weiterer Grund ist, sich in diesem Abschnitt ausschließlich dem Kunsthandwerk zu widmen.

Tab. 2 lässt bereits erkennen, dass im Bereich des Kunsthandwerks deutlich weniger Erzeugnisse in die Kategorie der regionalen traditionellen Produkte einzuordnen sind als bei den Lebensmitteln. Eine Begründung für den Einzelfall findet sich im jeweiligen Abschnitt (s. u.).

\footnotetext{
435 Vgl. diesbezüglich auch die Ergebnisse der Untersuchung von LITTRELL ET. AL. (1993) bei der festgestellt werden konnte, dass touristische Kunden einen Zusammenhang zwischen der Einmaligkeit bzw. der Produktionsauflage eines Erzeugnisses (z. B. Unikat) und dem Grad seiner „Authentizität“ knüpfen.
} 


\begin{tabular}{|l|l|}
\hline $\begin{array}{l}\text { Handwerkszweige aus denen } \\
\text { regionale traditionelle Produkte } \\
\text { hervorgehen }\end{array}$ & $\begin{array}{l}\text { Streitfälle und Handwerkszweige aus } \\
\text { denen nicht-regionale-traditionelle } \\
\text { Produkte hervorgehen }\end{array}$ \\
\hline \hline Töpferei & Glasbläserei \\
Filigranarbeiten & Steinmetzarbeiten \\
Wollverarbeitung und Webarbeiten & Metallverarbeitung \\
Klöppelei & Bodenkachel-Herstellung \\
Ganutell & \\
\hline
\end{tabular}

Tab. 2: Auflistung der Handwerksprodukte, die in Kap. 14.2.2 behandelt werden

- Metallverarbeitung und Bodenkacheln

Beide genannten Handwerksformen werden in dieser Arbeit nicht (mehr) zu den regionalen traditionellen Kunsthandwerksprodukten Maltas gezählt. Hierzu einige Erläuterungen:

Metallverarbeitung als Kunsthandwerk ist für die Maltesischen Inseln eine relativ junge Erscheinung, die erst mit dem 20. Jh. einsetzte. Die Anfertigung von Metallartikeln als Kunsthandwerk und somit "Luxusgut“ war im ländlichen Malta der Vergangenheit undenkbar. Brennholz und Kohle zur Metallschmelze waren auf den Maltesischen Inseln nie in ausreichender Menge vorhanden und mussten schon sehr früh importiert werden ${ }^{437}$. Eisenerz gehörte darüber hinaus nicht zu den äußerst spärlichen Bodenschätzen Maltas.

Der Dorfschmied (malt.: ћaddied) betrieb also ein Handwerk, das auf streng limitiert verfügbaren Rohstoffen beruhte. Unter diesen Umständen war lediglich die Anfertigung der unbedingt notwendigen Gebrauchsgegenstände, z. B. Hufeisen, Fensterrahmen, Maschinen- und Kutschenteile, Werkzeuge, Schlösser und Schlüssel, etc. möglich ${ }^{438}$. Metallartikel, die verziert waren, einen eingeschränkten oder überhaupt keinen Nutzen aufwiesen, waren in Malta bis ins 19. Jh. hinein nahezu unbekannt ${ }^{439}$.

Metallobjekte als kunsthandwerkliche Produkte wurden bis in die jüngste Zeit vor allem importiert und können demnach nicht als regional traditionell angesehen werden. Nichtsdestotrotz gibt es heute auf den Inseln eine achtbare Anzahl von metallverarbeitenden Betrieben, die sich in ihrem Sortiment auf die Fertigung von Kunsthandwerk und verzierten Gebäudeteilen (z. B. für Pergolen, Balkone, etc.) spezialisiert haben. Auch Inneneinrichtungsgegenstände der modernen Gesellschaft (z. B. CD-Ständer, Stehlampen, etc.) werden von diesen Firmen angefertigt. Schwerpunkte dieser Handwerkszweige finden sich in Ta'Qali und Ta'Dbiegi, aber auch in Valletta.

Zur Metallbearbeitung gehören auch die „traditionellen“ Messing-Türklopfer, die im Laufe der Zeit zu einem der begehrtesten touristischen Souvenirs geworden sind. Es gibt $u$. a. einen Roman von CAROLINE HARVEY („The Brass Dolphin“), der die Messingtürklopfer Mdinas zum Anlass nimmt, einen Kriminalfall im Malta der frühen britischen Kolonialzeit zu konstruieren.

Zweifellos sind die reich verzierten Messingtürklopfer, die überall in Malta in mehr oder auch weniger guter Qualität erhältlich sind, mittlerweile zu einem typischen Landessymbol geworden. Ihre Einführung auf der Inselgruppe erfolgte jedoch artifiziell durch die britischen Kolonialherren. Nur diese waren lange Zeit auch in der Lage, sich solche Luxusgüter leisten zu können. Sie brachten die Türklopfer aus Großbritannien mit. Noch heute gibt es nur wenige Türklopfer, die mit typisch maltesischer Symbolik (z. B. dem erwähnten „Brass Dolphin ${ }^{40 \text { “) }}$ aufwarten. In vielen Fällen wirkt diese Symbolik aufgesetzt, so z. B. wenn billig hergestellte Türklopfer in Form eines Malteserkreuzes angeboten werden.

437 Vgl. BLOUET (2004), S. 105

438 Vgl. LANFRANCO (2003), S. 9

439 Mit Ausnahme von Macht- und Statussymbolen der Besatzer, so z. B. verzierte Kanonen und Rüstungen der Johanniter, sowie die Errichtung der ersten Bronzestatuen durch die Briten. Die Aussage im Text bezieht sich auf den Großteil der Malteser, die überwiegend in ländlichen Dorfgemeinschaften wohnten.

440 Der Delphin ist das Wappentier der Maltesischen Inseln 
Im Zuge der Definition, die in Kap. 10.1 der Arbeit aufgestellt wurde, können auch Nachahmungen der antiken Türklopfer nicht als regionale traditionelle Produkte bezeichnet werden.

In den Crafts Villages und in Valletta finden sich auch Betriebe zur Produktion von „Floortiles“ (Bodenfliesen bzw. -kacheln). Dieses Handwerk gehört tatsächlich zu den traditionellen maltesischen Handwerkszweigen. Bis auf wenige Firmen (wie z. B. Bristow Pottery, Ta'Qali) wurde die Produktion jedoch weitestgehend maschinisiert und ist heute somit mit nahezu jedem Bodenfliesenproduzenten in anderen Teilen Europas vergleichbar. Handgefertigte Fliesen mit traditionellen Mustern sind als dekorative Einzelstücke zu verstehen und werden von Touristen und Einheimischen auch als solche gekauft. Ihr hoher Preis schließt eine flächenhafte Verlegung von vornherein aus.

In diesem Fall sieht der Autor das Beispiel eines zwar traditionellen Handwerks, das aber nicht mehr in der aktuellen Volkskultur verankert ist. Es gibt keinen spezialisierten Hersteller mehr und vor allem: Es gibt keine Kundschaft, die handgefertigte dekorierte Bodenfliesen in größeren Mengen abnehmen würde. Somit entspricht das beschriebene Produkt nicht den definierten Vorgaben für ein regionales traditionelles Produkt.

\section{- Klöppelei/Spitzenherstellung}

Textilherstellung hat in Malta eine lange Tradition. Schon in römischen Quellen wird Malta als Standort qualitativ hochwertiger Textilerzeugung hervorgehoben ${ }^{441}$. Die Johanniter versuchten sich 1833 sogar in der Herstellung von Seide und pflanzten in größerem Maßstab Maulbeerbüsche in den Regionen Valletta, Floriana und Mdina, die in der Folge mit Seidenraupen besetzt wurden. „The main aim was to create a local silk industry whose products could compete in the commercial markets of London, Lyons, Germany and Russia."(CARABOtT (1998), S. 6).

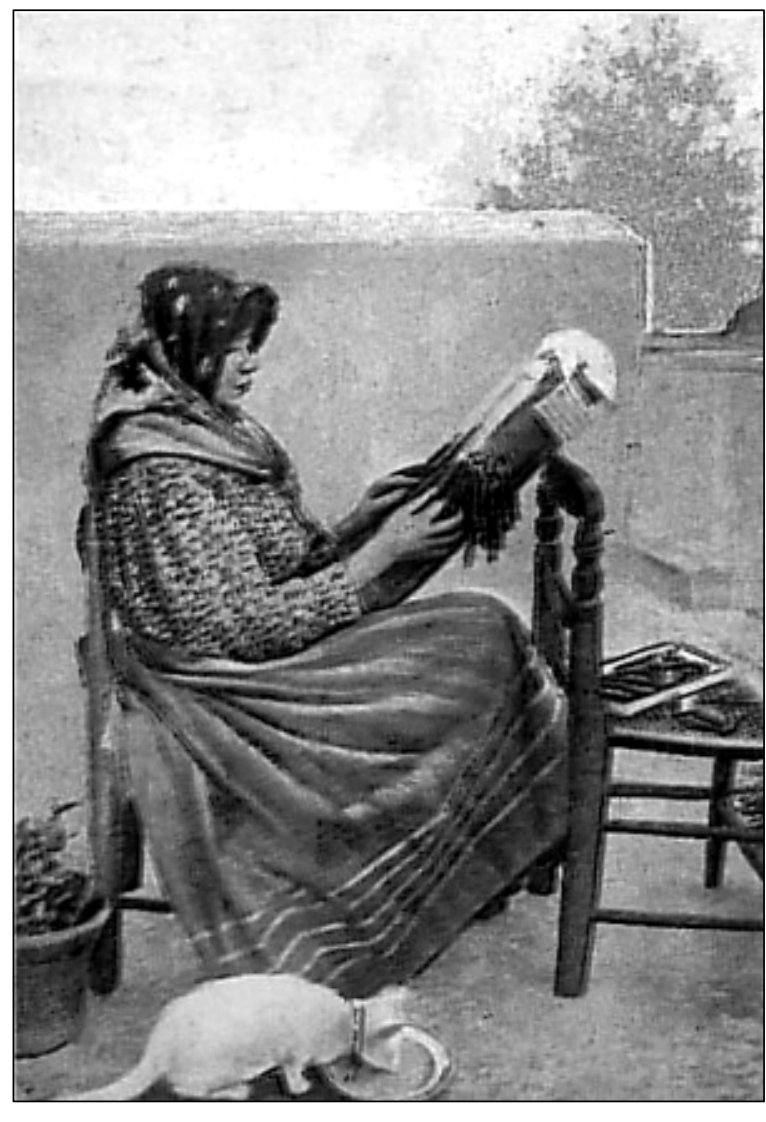

Abb. 15: „Lace-Making“ in Malta im 19. Jh. Quelle: ZARB (1998), Bildteil 
Der Anbau von Baumwolle war in Malta zu dieser Zeit der Hauptexportfaktor der damaligen Agrarwirtschaft der Inseln: „In country districts the growing and processing of cotton was an important source of income. The industry depended upon exports (...). In the eighteenth century, cotton became so important, in terms of employment and foreign exchange that the industry was regulated. In 1728 Maltese spun cotton was allowed continued entry into Spain provided it was "properly packed, sealed and certified to be of Maltese origin" "“ (BLOUET (2004), S. 109, mit einem eingebetteten Zitat von VASSALLO (1997)).

Es kann also festgehalten werden, dass zwei Werkstoffe in Malta existierten: Seide und Baumwolle. Einer davon, Baumwolle, sogar im Überfluss. Durch diese gute Ausstattung entwickelte sich die Klöppelei zur Erzeugung der sog. „Spitze“ (engl.: lace; malt.: bizilla).

„Lace is a decorative openwork fabric, but not all decorative openwork fabrics are lace. A true lace is one in which the pattern and any ground which links the pattern parts, are built upgradually by the interlacing of free threads. Therefore this excludes textiles such as embroidered net in which decoration is added to a premade fabric." (TOAMER (1989) zitiert in CARABOTT (1998), S. 8).

Die Klöppelei als Kunsthandwerk kennt verschiedene Techniken. In Malta wird die sog. „Pillow Lace“Technik angewandt (vgl. Abb. 15). Sie hat ihren Ursprung vermutlich in derselben Zeit, in der die Johanniter die Herrschaft über die maltesische Inselgruppe antraten. Ähnliche Spitze, wie sie in Malta noch heute erzeugt wird, wurde erstmals in Genua im Jahr 1530, also zur Zeit der Renaissance, nachgewiesen.

1640 ist das Jahr der ältesten nachgewiesenen Spitzen-Produktion in Malta ${ }^{442}$. Im Laufe des in diesen Zeitraum eingebetteten Jahrhunderts muss die Technik von Italien bis nach Malta vorgedrungen $\operatorname{sein}^{443}$. Die Klöppelei in Malta war nicht nur ein willkommener Zeitvertreib für die weibliche Bevölkerung ${ }^{444}$, sondern umfasste auch bedeutende Hersteller, die zu Hoflieferanten der Großmeister der Johanniter und später des britischen Königshauses wurden. Spitze war sowohl für Frauen wie auch für Männer ein wesentlicher Bestandteil der höfischen Kleidung des 17. und 18. Jahrhunderts ${ }^{445}$. Doch auch die Tracht der

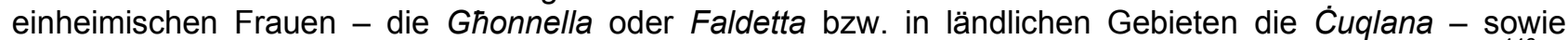
bestimmte Kleidungsstücke und Gegenstände des kirchlichen Bereichs waren oftmals mit Spitze verziert ${ }^{46}$.

CARABOTT verdeutlicht in ihrer Arbeit die vielfältige Verbreitung von Spitze in den Textilartikeln der damaligen Zeit: „A passion to be well dressed was one of the privileges of the nobility and this keenness to show off, was seen in the way the Knights displayed lace in their attire, in precious fibres, in embroideries, in collars, in neck ruffs and cloth trimmings. Also prominent was the extensive use of ecclesiastical vestments and altar cloths." (CARABOTT (1998), S. 13).

Die Maltesischen Inseln waren zeitweise als „The Islands of Lace“ in der Welt bekannt ${ }^{447}$.

$\mathrm{Zu}$ Beginn bis Mitte des 19. Jh. wurde eine Absatzkrise dieses traditionellen Handwerks spürbar. Als „Protektionsmaßnahme“ bestellte Queen VICTORIA persönlich im Jahr 1838 „eight dozen pairs long and eight dozen pairs short mits, besides a scarf" (LUKE (1968), S. 138). Seit dieser Zeit war die traditionelle Herstellung von Spitze in Malta immer wieder Krisen und Phasen des Niedergangs unterworfen. Aber der Bezug zum englischen Königshaus blieb bestehen: Im April 1951 bestellte QUEEN ELISABETH II. eine größere Menge maltesischer Spitze, 1968 wurden PRINCE CHARLES im Rahmen eines Malta-Besuchs als Geschenk verschiedene maltesische Klöppeleiarbeiten übergeben ${ }^{448}$.

In jüngster Zeit erlebt das Handwerk wieder eine Blütezeit, hervorgerufen durch staatliche Fördermaßnahmen und die Einrichtung eines Lehrstuhls für Klöppelei an der University of Malta und der Gozo School of Art. Dieser Studiengang umfasst die historische Entwicklung des Handwerks ebenso wie die praktische Ausführung, womit auch dem Nachwuchsproblem effektiv begegnet werden konnte.

\footnotetext{
442 Angaben nach CARABOTT (1998), S. 13 auch nicht bewiesen.

444 Vgl. AZZOPARDI (1991), S. 19

445 Vgl. CARABOtT (1998), S. 13

446 Vgl. CASSAR PuLLICINO (1992) und ZARB (1998)

447 Vgl. LUKE (1968) und AZZOPARDI (1991)

448 Vgl. AzZOPARDI (1991), S. 18
}

443 AZZOPARDI (1991) schreibt die Einführung der Spitze den Johanniterrittern zu. Diese Annahme ist nicht unwahrscheinlich, aber 
Dieser erfolgreichen Reaktivierungspolitik ging eine Phase des Verfalls voraus, in der es versäumt wurde, den Nachwuchs auf das Klöppeleihandwerk aufmerksam zu machen. In den 1970er- und 1980er-Jahren strebten viele junge Erwerbstätige, darunter erstmals in größerem Maße auch Frauen, in die Dienstleistungsund Tourismuswirtschaft, wodurch das Klöppeleihandwerk in Mitleidenschaft gezogen wurde. Viele alte Formen und Techniken starben mit den älter werdenden Handwerkerinnen. Erst Ende der 1980er wurde dieser Prozess erkannt, und es wurden die o. a. Gegenmaßnahmen eingeleitet, die dazu führten, dass die Spitzenherstellung heute als Maltas am besten erforschtes und beschriebenes Handwerk gilt. Es begeistert zunehmend auch wieder junge Leute und es können gute Verkäufe verzeichnet werden. Bezeichnend hierfür ist ein Zitat von C. AZZOPARDI: „(...) there is still enough enthusiasm in the local people who want to learn lace-making and charish the old patterns as a challenge and pride in their work. In this way lace-making is being revived in our islands, and with the increasing demand from the tourists who visit the Islands of Lace, it will flourish again and will have an even better reputation." (AZZOPARDI, C. (1991), S. 19).

Maltesische Spitze war vom Beginn ihrer Herstellungsgeschichte an auch im ländlichen Raum etabliert und wird noch immer mit großem Enthusiasmus von der maltesischen Bevölkerung hergestellt. Es gibt eigene Familienmuster, auf die man stolz ist und die der geklöppelten Spitze ein hohes Maß an Individualität verleihen. Demnach ist maltesische Spitze zweifelsfrei ein Träger regionaler Werte. Alles in allem steht die sichere Stellung als regionales traditionelles Produkt fest.

\section{- Töpferei}

Die Töpferei ist gemeinsam mit der Textilherstellung vermutlich die älteste Form des Kunsthandwerks auf der Inselgruppe 49 . Die Wurzeln für diese Form des Handwerks liegen bereits im Neolithikum. Spätestens zur Zeit der phönizischen Siedler, die Tongefäße für ihre Waren und die Imkerei benötigten, wurden auch Gebrauchsgegenstände aus Keramik angefertigt.

Die an vielen Stellen der Inseln offen zutage tretende geologische Schicht des formbaren Blue Clay hat die Entwicklung der Töpferei auf den Maltesischen Inseln schon in frühester Zeit begünstigt. Maltas bekanntestes archäologisches Ausstellungsstück ${ }^{450}$, die "Sleeping Lady" - auch bekannt als "Venus von Malta" - ist eine getöpferte Figur. Sie wurde von den Erbauern der Tempel von Tarxien im Neolithikum geschaffen.

Im Laufe der Jahrtausende wurde die Töpferei in Malta nie aufgegeben, und es entwickelten sich landestypische Formen für unterschiedlichste Arten von Gebrauchsgegenständen. Traditionelle Gegenstände aus Ton waren z. B. Öllampen (malt.: imnara bzw. fuћћar), Koch- und Backtöpfe, dekorative Pflanzgefäße (z. B. für Erdbeeren), Flaschen zur Aufbewahrung von Speiseöl, Tongefäße zur Bienenzucht ${ }^{451}$ (malt.: qolla), Salzaufbewahrungsgefäße (s. Foto 9) sowie Krüge aller Art ${ }^{452}$. Ihre Erzeugung und Nutzung reichte noch bis ins frühe 20 . Jh. hinein.

\footnotetext{
449 Vgl. BLOUET (2004). Töpferei war überall im Mittelmeerraum sehr früh verbreitet. Der Archäologe EvANS (1963) berichtete jedoch von einem besonders hohen Verbreitungs- und Qualitätsniveau in Malta im Vergleich mit anderen Mittelmeerinseln, was u. a. zu der Annahme führte, Malta sei eine Pilgerstätte des Neolithikums gewesen. Viele Keramikfunde seien von maltesischen Handwerkern erzeugte und dann von den Pilgern des Kontinents erworbene Symbol- bzw. Opfergaben gewesen.
}

450 Ausgestellt im National Archeological Museum in Valletta

451 S. dazu auch den Eintrag „Honig“ im vorliegenden Produktverzeichnis.

452 Viele dieser ursprünglichen Gegenstände sind heute im staatlichen Folklore-Museum in den Gemäuern der Zitadelle von Victoria auf Gozo ausgestellt. 


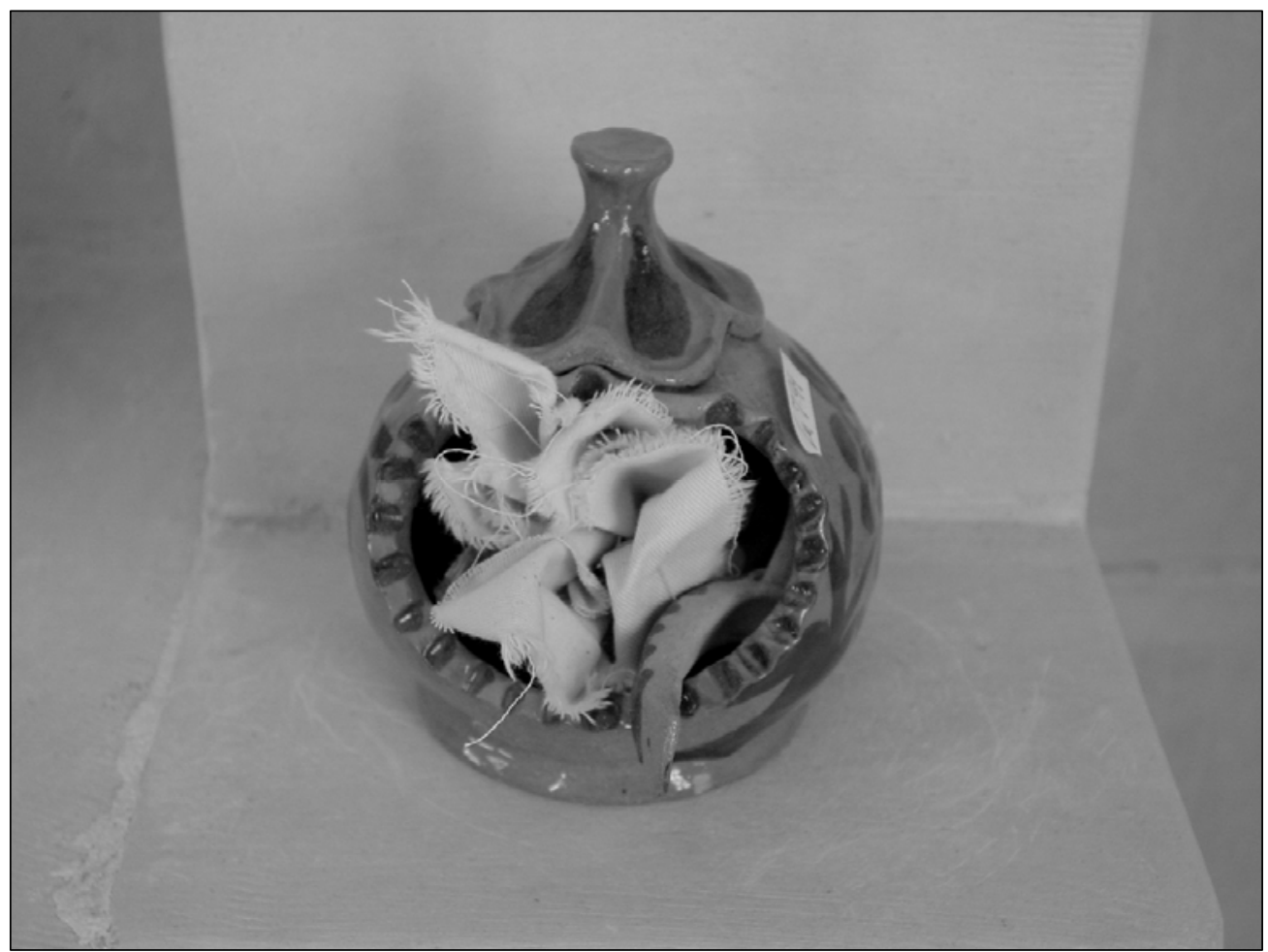

Foto 9: Traditioneller gozitanischer Salztopf in der Auslage der „Gozo Pottery Barn“ in Ta'Dbieġi. Foto: ASCHEMEIER

Weitere Besonderheiten waren ein kannenähnlicher Krug für Ziegenmilch mit eingearbeitetem Milchmaß $\beta^{453}$ (malt.: Satal il-Halib) sowie eine getöpferte Kochstelle ${ }^{454}$ (malt.: kenur).

Die Zentren der Töpferei in heutiger Zeit sind - ähnlich wie bei vielen anderen Kunsthandwerkszweigen der Inselgruppe - die Crafts Villages in Ta'Qali (Malta) und Ta'Dbieġi (bei San Lawrenz auf Gozo). Daneben gibt es in Sliema, Mdina, Lija und Rabat Töpfereien. Das Geschäft mit den Touristen wird jedoch in den Crafts Villages gemacht, und die Töpferwerkstätten in anderen Landesteilen dienen oft nur als Produktionsstätten für den Nachschub, der in Ta'Qali verkauft wird ${ }^{455}$.

Maltesischer Ton wird bereits seit Jahrzehnten nicht mehr zur Töpferei benutzt. Sein Salzgehalt ist zu hoch und die Qualität der getöpferten Waren wird dadurch beeinträchtigt. Die meisten Betriebe beziehen ihren Rohstoff in Form von Tonplatten aus Großbritannien und Italien. Es wird dabei in Tonsorten für Steingut, Terrakotta oder Tongeschirr unterschieden. Durch den importierten Rohstoff ist die maltesische Keramik qualitativ hochwertig und kann jedem internationalen Vergleich standhalten. Außerdem werden gegenwärtig alle möglichen Arten und Formen von Keramik und Töpfereiwaren in Malta erzeugt: „In the Maltese Islands, the pottery industry is small, but it produces all sorts of earthenware and chinaware, vases, pots, some tableware, ornamental, china, statues of plaster of Paris, glazed tiles and so on." (AzZOPARDI (2002), S. 264).

453 Dieser Gegenstand wurde von wandernden Milchhändlern benutzt. Sie kamen mit einer Ziege ins Dorf und verkauften die Milch frisch gemolken aus dem beschriebenen Krug an ihre Kundschaft (zur Vertiefung s. u. a. CASSAR PULLICINO (1992), BORG (2001), LANFRANCO (2003)).

454 Diese Kochgestelle sind den heute noch von Nomaden in Nordafrika verwendeten Kochstellen ähnlich (vgl. CREMONA (2003), S. 50). Die ursprüngliche Form war getöpfert, es gab aber auch kenure aus Globigerinenkalkstein. Gekocht wurde ausschließlich in tönernen Gefäßen. Das typische Gericht, welches mit einem kenur zubereitet wurde, war die maltesische Gemüsesuppe Minestra (nicht zu verwechseln mit der italienischen Minestrone, die ein völlig anderes Gericht ist). Maltesische Farmhäuser besaßen aus Mangel an Holz keine feste Feuerstelle. Je nach Jahreszeit und Wetter wurde der kenur drinnen oder draußen zum Kochen verwendet.

455 Das anschaulichste Beispiel dieser Art ist die Firma Peter The Potter, die in Rabat ihre Werkstatt unterhält, während sich die Verkaufsräumlichkeiten in Ta'Qali befinden. 
Der größte und wirtschaftlich bedeutendste Töpfereibetrieb ist Bristow Pottery mit 25 fest angestellten Mitarbeitern. Weitere bedeutende Töpfereien sind Peter The Potter, Alka und in Gozo die Gozo Pottery Barn. Alle Betriebe sind in Crafts Villages angesiedelt und verkaufen dort ihre Waren. Während Bristow in Ta'Qali auch die Werkstatt unterhält, wird Peter The Potter von Rabat auf Malta aus beliefert. Bei Alka konnte nicht in Erfahrung gebracht werden, ob die Produkte an Ort und Stelle erzeugt werden.

In Gozo dürfte die Gozo Pottery Barn die wirtschaftlich wichtigste Stellung einnehmen. In diesem Fall sind Werkstatt und Verkaufsräume in Ta'Dbieg̉i. In Gozo gibt es auch in den Dörfern noch kleine Töpfereibetriebe.

Die traditionelle Keramik und Töpferei der Maltesischen Inseln war stets ursprünglich und einfach. Produkte aus Ton waren die traditionellen Gebrauchsgegenstände der armen Landbevölkerung. Ergebnis dieses Umstands war die nahezu völlige Beschränkung auf den Nutzwert der getöpferten Gegenstände. Verzierungen, Bemalungen, etc. kamen bei den traditionellen Töpfereiwaren Maltas kaum vor. Außerdem ist die Zerbrechlichkeit tönerner Gegenstände hoch. Deswegen wurden sie im täglichen Gebrauch häufig erneuert. Die Tonwaren Maltas waren somit nicht nur schlichte Produkte, sondern sie wurden zudem auch noch in einer Art früher "Massenfertigung" erzeugt.

Ausnahmen umfassten nur Gegenstände, die traditionell zur Aussteuer gehörten, oder zusätzliche dekorative Zwecke erfüllten. In Gozo war z. B. ein Gefäß zur Aufbewahrung von Salz Bestandteil der Aussteuer, das dekorativ bemalt wurde (s. Foto 9). Tönerne Löffel und Teller wurden häufig mit Mustern verziert. Dies war gewissermaßen eine natürliche Folge der notwendigen Glasur des Essgeschirrs.

Einfache Gegenstände eignen sich heute nur eingeschränkt zur Vermarktung für den touristischen Markt. Außer einigen klassischen tönernen Amphoren und Krügen werden kaum noch traditionelle Tongegenstände hergestellt. Ausnahmen wie der dekorative Salztopf aus dem Sortiment der Gozo Pottery Barn sind sehr selten. BRIAN GRIMA, Geschäftsführer des maltesischen Marktführers Bristow Pottery auf die Frage, welche traditionellen maltesischen Gegenstände heute noch getöpfert werden und im Angebot sind:

„We've got the Maltese Buses, the Maltese balconies, Maltese doors. Typically Maltese are the cooking pans. But people don't look for traditional Maltese items much. What people look for is houseware, household items and souvenirs. Typical traditional Maltese is the Maltese Falcon which I have over there. The Americans would go everywhere to find one ${ }^{456}$ ! It's a very popular item. As you can see there, all these are made out of the traditional Maltese scenes ${ }^{457}$, you know, like the knights, the Triton Fountain and so on. Then I've got the candle holders which are traditional, too. And the olive oil bottles and obviously the traditional cross which we have over there. There are no more things which are typical Maltese but they are not beeing asked for. So we stopped producing them. It's unbelievable: People sometimes see something attractive and that's what they go for so we have eliminated a lot of traditional Maltese items, like the oil lamps, like the cooking pots... quite a few things." (BRIAN GRIMA im Experteninterview vom 07.05.2004).

Auffällig an der zitierten Aussage ist, dass Herr GRIMA bei der Frage nach traditionellen Gegenständen zunächst die typischen Touristensouvenirs erwähnt. Diese sind zwar maltesischen Wahrzeichen nachempfunden (Malteserkreuz, Malteserfalke) oder stellen traditionelle Landschaften und Behausungen dar, ihre Existenz ist jedoch dem touristischen Geschäft zu verdanken, und ihre Markteinführung dürfte nicht länger etabliert sein als etwa 15-20 Jahre.

Gegen Ende seines Statements nennt GRIMA Gebrauchsgegenstände (Flaschen für Olivenöl, Kerzenhalter), diese aber nur am Rande. Der letzte Abschnitt ist bemerkenswert und informationsträchtig: Traditionelle maltesische Töpferei verkauft sich nicht und wird daher auch nicht mehr hergestellt. Das Sortiment ist in der Folge zwar ohne Frage qualitativ hochwertig (vgl. Foto 11), aber die Formen und Designs sind „austauschbar" und könnten aus jedem mediterranen Ursprungsland stammen. Das „typisch Maltesische“ beschränkt sich auf die Motivwahl, nicht auf den historischen Ursprung des Objekts.

\footnotetext{
456 Herr Grima spricht hier die Nachbildung der aus dem Humphrey Bogart-Film „Die Spur des Falken“ bekannten Statue des Malteserfalken an.
}

457 Herr GRIMA spricht hier Miniaturabgüsse von Maltesischen Farmhäusern, Ritterrüstungen, Windmühlen, etc. an. 
Andere Hersteller, insbesondere Peter the Potter (vgl. Foto 10), haben mehr traditionelle Gegenstände im Sortiment, doch insgesamt ist die Ausmerzung traditioneller Waren im Töpfereisektor in Malta besonders gründlich vollzogen worden. Keramik nach traditionellen maltesischen Originalen stirbt in beeindruckender Geschwindigkeit aus. Was bleibt, ist ein Angebot der Hersteller: Man könne jeden Gegenstand, auch Traditionelles, bei innen bestellen - als teure Einzelanfertigung.

Es wird darauf hingewiesen, dass im Bereich der maltesischen Töpferei- und Keramikwaren derzeit ein rasanter Kulturverlust stattfindet. Traditionsgegenstände wurden für das touristische Geschäft kritiklos gegen bunte Souvenirs und multikulturelle Designs ausgetauscht, was jedoch nicht heißen soll, dass die Qualität gelitten hätte. Es sollte darauf geachtet werden, dass alte Formen und Herstellungsmethoden nicht verloren gehen und vielleicht in wenigen Generationen schon nicht mehr zum lebendigen maltesischen „Kulturerbe“ gehören.

Dies hat dazu geführt, dass im Rahmen dieser Arbeit viele Keramikprodukte nicht oder nicht mehr als regional traditionell bezeichnet werden können, was im Hinblick auf die jahrtausendealte Tradition maltesischer Töpferei durchaus alarmierend ist. 


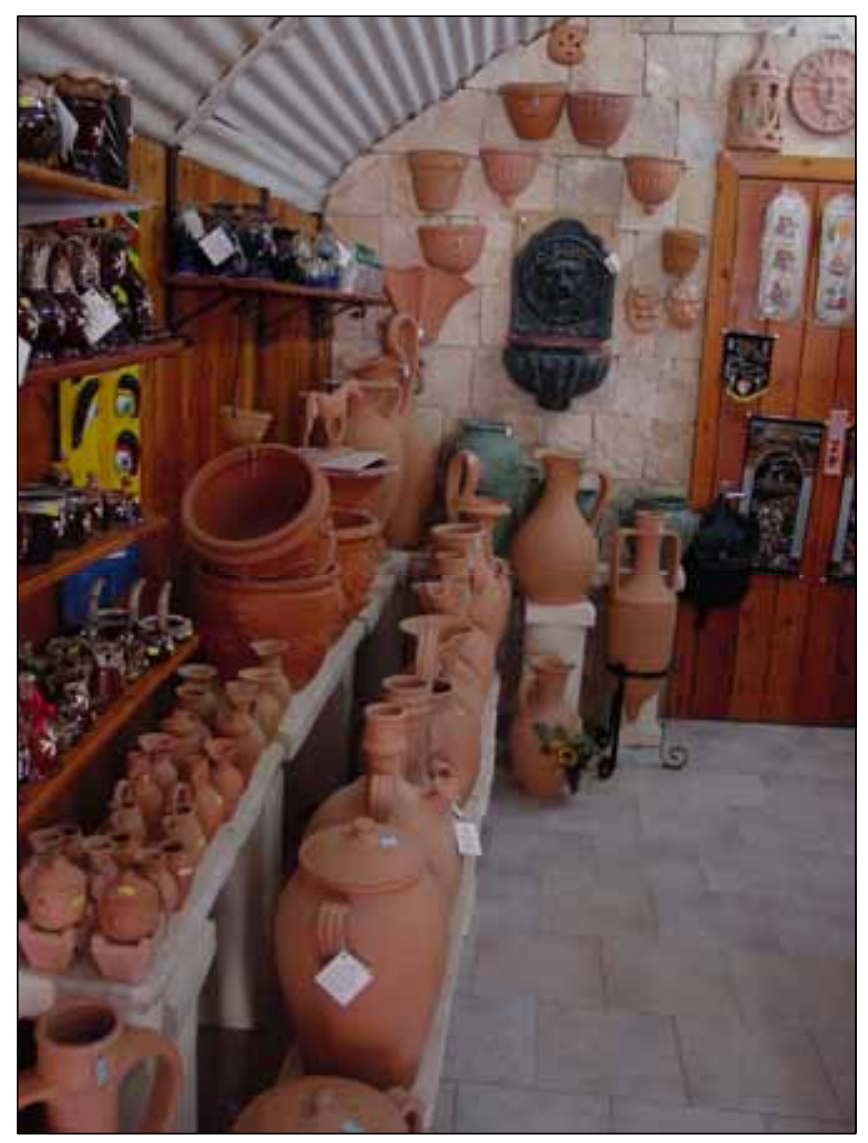

Foto 10: In den Geschäftsräumen von „Peter the Potter“, Ta'Qali: Souvenirs, Kitsch und Traditionsreiches eng beieinander. Im Vordergrund links: Miniaturnachbildungen des kenur mit Tongefäß. Im Hintergrund: Amphoren. Foto: AsCHEMEIER

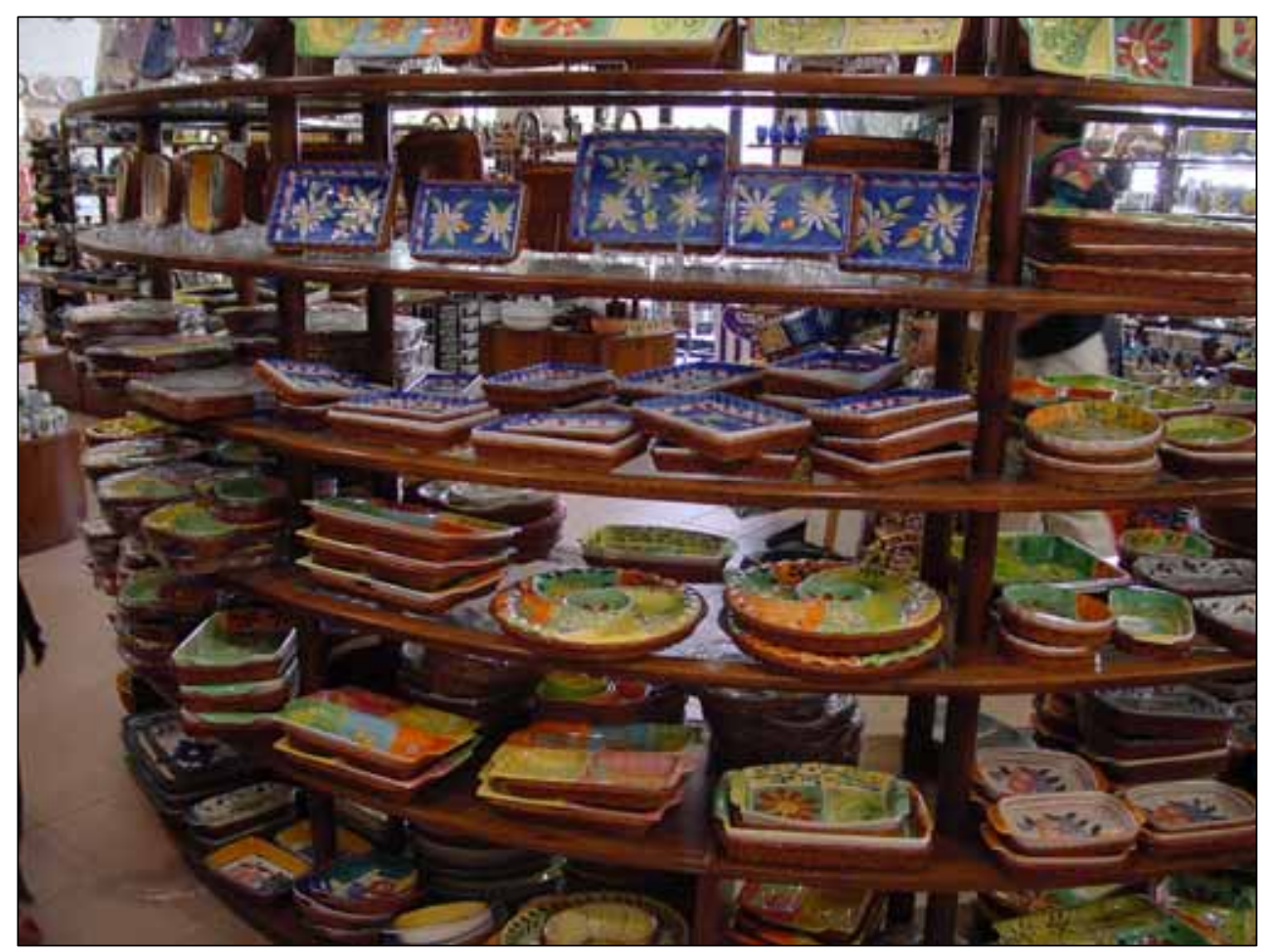

Foto 11: Die Geschäftsräume von „Bristow Pottery“, Ta'Qali. Qualitativ hochwertige Keramik, jedoch weitgehend ohne traditionellen Bezug. Foto: Aschemeier 


\section{- $\quad$ Maltese Filigree}

Eine der wichtigsten Kategorien unter den typisch maltesischen Touristensouvenirs sind Filigranarbeiten aus Silber, sog. „Silver Filigree“" (s. Foto 12).

Leider ist aber zur Historie dieses Handwerks keine gedruckte Literatur verfügbar, nicht einmal in maltesischer Sprache. Es existieren Internetquellen von Hobby-Folkloristen und gewerblichen Anbietern, die jedoch nur eingeschränkt Informationen bieten können. Trotz mehrerer persönlicher Gespräche mit Kunsthandwerkern, die Filigranarbeiten selbst herstellen, konnte vom Verfasser nicht in Erfahrung gebracht werden, wann und von wem das Handwerk in Malta eingeführt wurde. Die folgenden Angaben beruhen auf mündlichen Informationen verschiedener Personen, darunter Buchautoren ${ }^{458}$ und Kunsthandwerker aus Ta'Qali.

Die ältesten bekannten Filigranarbeiten der Weltgeschichte stammen aus Ägypten und wurden ca. 5.000 Jahre vor unserer Zeitrechnung angefertigt. In den folgenden Jahrtausenden wurden Filigranarbeiten zu einem typischen Kunsthandwerk des Mittelmeerraums. Da Malta im Zentrum des Mittelmeers liegt und im Laufe seiner Geschichte vielen Eroberern unterworfen war, entwickelte sich bereits sehr früh eine Tradition in diesem Kunsthandwerkssektor, die innerhalb der Familien vor allem von den männlichen Mitgliedern ausgeübt und vom Vater auf den Sohn „weiter vererbt“ wurde. Heute ist diese Praxis nicht mehr gebräuchlich. Interessierte lernen das Kunsthandwerk in Abendschulkursen.

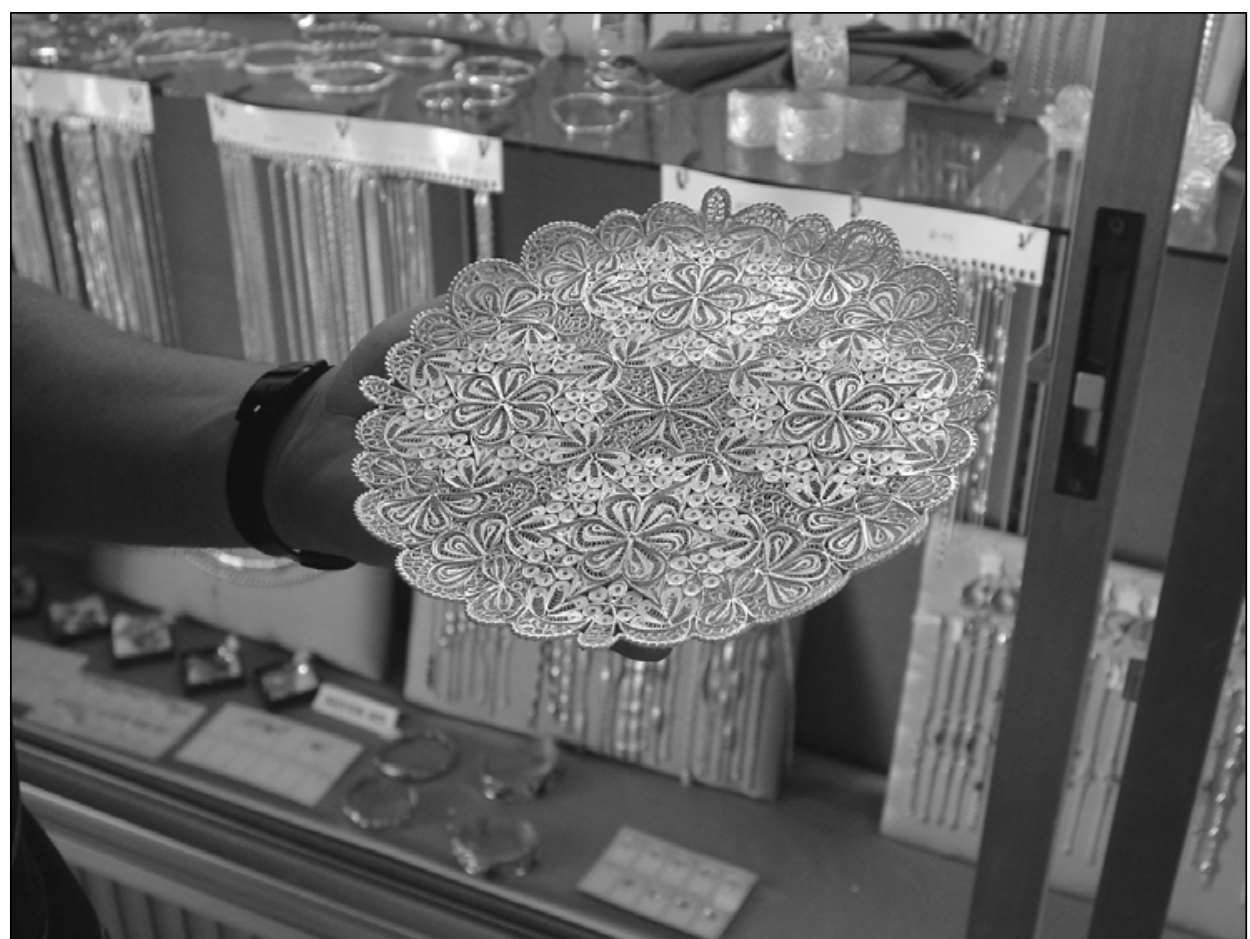

Foto 12: Silver Filigree im Juweliergeschäft „Denis Jewellery“, Ta'Qali. Foto: ASCHEMEIER

${ }^{459}$ Der Herstellungsprozess beginnt mit der Schmelze von Silber- oder Goldkörnchen (traditionell wird in Malta eher Silber verwendet). Wenn das Metall flüssig ist, wird es zu kleinen Barren oder Plättchen gegossen. Diese Barren werden unter Druck mit einer Walze gepresst und gerollt, und danach mit einem Zieheisen bearbeitet, sodass als Endergebnis Silber- oder Golddraht von unterschiedlicher Stärke entsteht.

\section{MATTY CREMONA Und FRANS SCERRI}

459 Die Angaben zum Herstellungsprozess entstammen zum größten Teil einem Experteninterview welches vom Autor mit dem Geschäftsführer des Betriebs „Denis Jewellery“ (Ta'Qali, Malta) geführt wurde (10.05.2004). Weitere Details wurden dem Text über Silver Filigree auf der Internetseite des Betriebs „Empire Arts \& Crafts Centre“ (Rabat, Malta) entnommen (Link: http://www.empiremalta.com). Zudem sind in den Text Angaben aus dem nationalen Standard für traditionelle handgearbeitete maltesische Filigranarbeiten der MALTA STANDARDS AUTHORITY (MSA (2003b), S. 9) eingeflossen. 
Dieser Prozess der Silber- und Golddrahtherstellung wird nur noch von exklusiven Juwelieren in Eigenarbeit durchgeführt. Viele Kunsthandwerker, die „Massenware“ für den touristischen Betrieb anbieten, sind dazu übergegangen, industriell hergestellten Draht zu benutzen. Er ist häufig gleichmäßiger geformt, robuster und biegsamer. Verfechter der traditionellen Herstellungsmethode sehen durch den Einsatz von industriell hergestelltem Draht ein typisches Charakteristikum von Maltese filigree in Gefahr, da die leichten Unregelmäßigkeiten des selbst hergestellten Ausgangsmaterials eigentlich zum typischen Erscheinungsbild der Filigranarbeiten gehören.

Dickere Drähte (malt.: vultar) werden zur Herstellung des Außenskeletts des Filigranobjekts verwendet. Die Füllung des Rahmens mit Ornamenten verlangt dem Kunsthandwerker hohe technische Fertigkeiten ab. Die feinsten Drähte werden vorher einer aufwändigen Prozedur unterzogen: Zwei extrem feine Silber- oder Golddrähte werden nach Art eines Seils zusammengeflochten, erhitzt und geplättet. Das erhöht ihre Festigkeit bei gleichzeitiger Beibehaltung ihrer feinen Struktur. Man nennt die so bearbeiteten Drähte nun rizzol (malt.).

Nach dem Abkühlen werden sie von Hand zu runden Mustern geformt und in den Rahmen eingelötet. Auch diese geflochtenen Feindrähte liegen in unterschiedlichen Stärken vor. Für die spiralartigen Muster im Inneren der Ornamentik wird der feinste Draht benötigt. Diese Füllung kann nur mit speziellen Werkzeugen angefertigt werden.

Das fertige Objekt wird in ein Bad aus einer erhitzten Lösung aus Wasser und Schwefelsäure getaucht, um die durch die Bearbeitung entstandenen oxidierten Stellen zu beseitigen. Nach einem Trocknungsprozess muss die Filigranarbeit poliert werden. Zum Schluss erfolgt ein Feingehaltsstempel. Auf Wunsch kann ein Echtheitszertifikat durch die Association of Gold and Silver Manufacturers ausgestellt werden.

Zusätzlich zum reinen Drahtwerk können auch feine Kügelchen, Ringe, Häkchen oder Plättchen eingearbeitet werden, welche die maltesischen Bezeichnungen Koććli, Granelli, Malji tas-Sinna und Kazzott tragen.

Laut nationalem Standard der Malta Standards Authority (MSA) für traditionelle handgemachte maltesische Filigranarbeiten ${ }^{460}$ werden folgende Formen als „landestypisch“ bezeichnet:

- das Malteserkreuz,

- die Dghajsa (ein maltesisches gondelartiges Boot),

- das Papiermesser/der Brieföffner,

- der Anhänger.

Sie bestehen aus insgesamt 37 typischen Ornamentformen ${ }^{461}$, die zusammen das gewünschte Objekt ergeben. Jedes der 37 Ornamente hat eine traditionelle maltesische Bezeichnung.

Maltese Filigree ist ein typisches Touristensouvenir, aber auch von einheimischen Maltesern begehrt ${ }^{462}$. Filigranarbeiten sind tief in der Tradition der Maltesischen Inseln verankert und darüber hinaus kulturelle Identitätsträger. Deswegen wird im weiteren Verlauf dieser Arbeit dafür plädiert, dass Maltese Filigree zu den regionalen traditionellen Produkten zu zählen ist.

460 MSA (2003b), S. 8

461 Vgl. MSA (2003b), S. 12 f.

462 Information eines Angestellten von „Denis Jewellery“ (Ta'Qali, Malta) im Experteninterview vom 10.05.2004. 


\section{- Ganutell}

„The craft of Ganutell, which is enjoying a revival in Malta but is little known in other countries, consists of making decorative flowers using silk thread, beads and metal wires." (MENHENNET \& COHEN auf www.ganutell.com, Stand: 11.07.2005).

Der maltesische Begriff „Ganutell“ stammt vom italienischen „Canutiglia“ ab. Das Wort beschreibt nicht nur das Kunsthandwerk, sondern auch den wichtigsten Werkstoff: Einen spiraligen Seidenfaden, der um einen dünnen Gold- oder Silberdraht gewickelt wird. Weitere maltesische Bezeichnungen für das Handwerk sind z. B. „Ganitill“ oder „Ganutil“.

Obwohl das Kunsthandwerk ursprünglich vermutlich aus dem italienischen Raum stammte, waren die Maltesischen Inseln bereits im 18. Jh. berühmt für ihre Ganutell-Arbeiten. Dabei handelte es sich um aus Seidenfäden, Glaskugeln, Perlen, Silber- und Goldfäden gefertigte „künstliche“ Blumenarrangements. Sie wurden zunächst fast ausschließlich im kirchlichen Bereich zur Feiertagsdekoration von Altären, Gemälden, Statuen, etc. eingesetzt (vgl. Foto 13). Die „Kunsthandwerkerinnen“ waren vor allem Konventsschwestern, die Ganutell als klösterliche Arbeit ausführten. Sie erreichten im Laufe der Zeit eine außerordentliche Meisterschaft, was den Ganutell-Arbeiten Maltas im 18. und 19. Jh. in ganz Europa Aufmerksamkeit verschaffte.

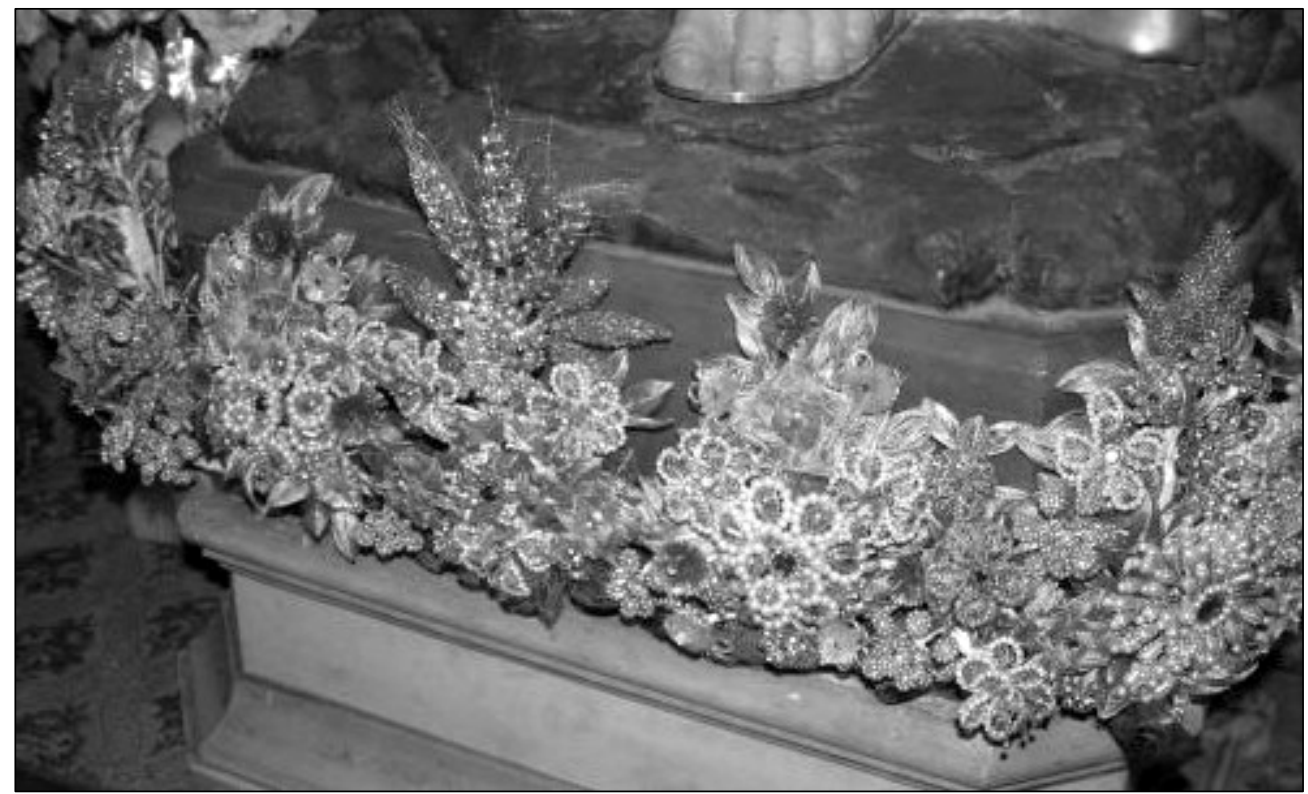

Foto 13: Ganutell-Dekoration im Dom von Mosta. Foto: MENHENNET auf http://www.ganutell.com

In heutiger Zeit findet Ganutell vor allem im sakralen Umfeld der „Festas“ der Maltesischen Inseln Verwendung; es wird jedoch auch zunehmend im Privatbereich Ganutell gefertigt, vor allem zur Dekoration der Weihnachtskrippe, und mitunter findet man Objekte auch im Verkauf an Touristen. Vor allem im Bereich des touristischen Souvenirverkaufs sind mittlerweile Objekte für säkulare bzw. profane Dekorationszwecke festzustellen.

Das Ganutell-Handwerk wird z. Zt. vom Ministry for Tourism and Culture der maltesischen Regierung gefördert, u. a. durch Abendkurse, die von der Bevölkerung gut aufgenommen werden. Diese Entwicklung wird vom Verfasser begrüßt, da es sich bei Ganutell zweifellos um ein regionales traditionelles Handwerk im Sinne der vorliegenden Dissertation handelt.

Als Produkt für den Verkauf steht Ganutell stilistisch in einer Reihe mit Silver Filigree und Klöppeleiarbeiten, sodass eine weitere Verbreitung der Erzeugnisse im Verkauf für den touristischen Markt als ausbaufähig einzuschätzen ist. 


\section{Streitfälle und nicht-regional-traditionelle Produkte}

Unter den Produkten, die in Werbung und Literatur als „typisch maltesisch“ bezeichnet werden, sind auch solche, die laut der in der vorliegenden Arbeit erarbeiteten Definition nicht als regionale traditionelle Produkte eingestuft werden können. Bei einigen Objekten ist der Grund offensichtlich, bei anderen ist die Einstufung schwieriger. Die wichtigsten dieser Produkte sollen im Folgenden vorgestellt werden:

\section{- Bier}

Bier ist Maltas beliebtestes alkoholhaltiges Getränk ${ }^{463}$. Größter Fabrikant ist der Getränkekonzern Simonds Farsons Cisk. Sein Biersortiment umfasst die Labels Cisk Lager, Cisk Export, Hopleaf Ale, Blue Label Ale und Farsons Shandy. Letzteres ist ein Mischgetränk aus Bier und Apfellimonade. Damit ist die Farsons Group Of Companies der führende Anbieter im Biersektor ${ }^{464}$. Die Cisk-Brauerei hält außerdem die Lizenz für die Marke Carlsberg ${ }^{465}$.

Ein anderer einheimischer Brauereibetrieb ist $L B M$ Breweries $^{466}$, die ein eigenes Bier mit dem Namen 1565 Victory anbieten und die Lizenzbrauerei der Marke Löwenbräu sind.

Das älteste Bierlabel Maltas ist Farsons Pale Ale (heute unter dem Namen Hopleaf bekannt), das erstmals 1928 auf einem Dorffest (malt.: festa) in Qormi ausgeschenkt wurde ${ }^{467}$.

Das in Malta erzeugte Bier ist britischen Bieren nachempfunden. Die Markteinführung erfolgte artifiziell als „Imitat" britischer Biere. Es kann nicht als eigenständiges regionales traditionelles Produkt angesehen werden. Die Beliebtheit unter der Bevölkerung ist jedoch hoch.

Der Exporterfolg kann hingegen nicht mit dem der Kräuterlimonade Kinnie (s. u.) verglichen werden. Cisk Export und Cisk Lager werden, wenngleich mit steigenden Exportraten, nur nach Australien und Italien exportiert ${ }^{468}$. Dies ist vermutlich eine Folge der Konzentration von ausgewanderten maltesischen Staatsbürgern in Australien, deren Population ca. 150.000 Individuen umfasst ${ }^{469}$.

463 Vgl. MızZı (1993)

464 Vgl. MALTA TODAY, 09.05.2004, S. 20

465 Vgl. TRUe, 09.05.2004, S. 22 f.

466 Ein Tochterunternehmen des Weinherstellers Marsovin

467 Schriftliche Information des Herstellers

468 Vgl. MızZı (1993)

469 Vgl. AsCHEMEIER (2005), S. 244 


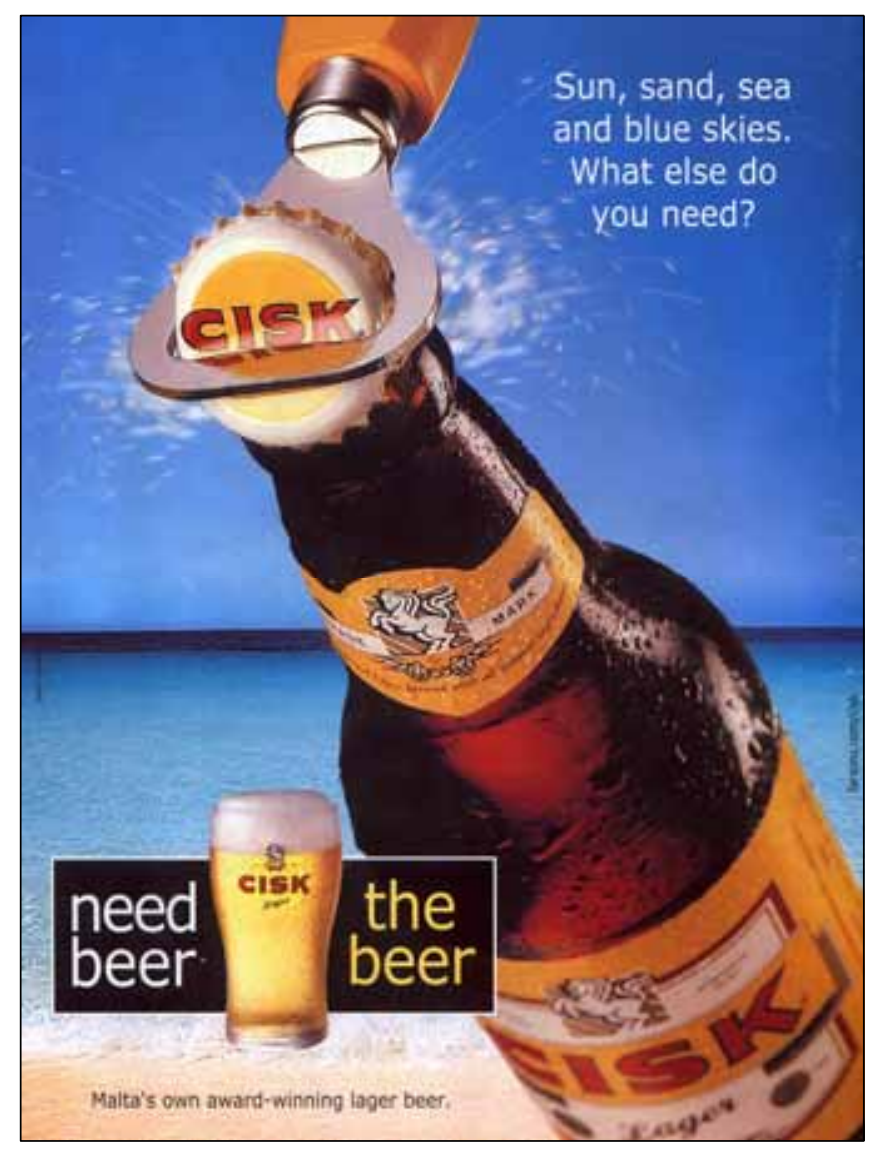

Abb. 16: Ganzseitige Zeitschriftenwerbung für Ċisk Lager aus dem Jahr 2004

Die Vermarktung der Cisk-Labels erfolgt in Malta vor allem in Hinblick auf den Freizeitcharakter des Produkts (vgl. Abb. 16). Im Gegensatz zur Kinnie-Werbung (s. Kap. 14.1.3) wird nicht versucht, dem Produkt einen nationalen Charakter zu geben. Wohl aber werden bestimmte feierliche Anlässe wahrgenommen, zu denen jeweils ein spezielles Bier in limitierter Auflage hergestellt wird. So gab es z. B. anlässlich des EUBeitritts Maltas am 01. Mai 2004 sowie zum eine Woche später anberaumten Malta Fireworks Festival eine limitierte Auflage Cisk Celebration Brew ${ }^{470}$.

Anders ist die Werbung bei der Marke 1565 Victory Bier des Herstellers LBM Breweries, eine Tochtergesellschaft des Weinherstellers Marsovin. Der Produktname nimmt Bezug auf das bis heute meistzelebrierte Ereignis der maltesischen Geschichte, die „Große Belagerung“ von 1565.

Die Printwerbung greift diesen Gedanken auf und zeigt kampfbereite Johanniterritter in Rüstung oberhalb des Bierlabels. In diesem Fall wird einem höchstens regional gefärbten Produkt, nämlich maltesischem Bier, durch seinen Namen und seine Marketingstrategie ein nationaltypischer/nationaler Charakter „aufgestempelt". Es handelt sich um eine Projektion von landestypischen Charakteristika auf ein Produkt, das im Sinne der vorliegenden Arbeit kein regionales traditionelles Produkt sein kann. 


\section{- Glasbläserei}

(The) „glass industries in our country are small and of relatively small importance economically. However, they produce a wide variety of objects which serve as souvenirs for the many tourists that visit our country." (AZZOPARDI (2002), S. 266).

Das Zitat von AzZOPARDI veranschaulicht die Situation auf dem Glassektor in Malta kurz, aber treffend. Glasbläserei ist kein ursprünglich maltesischer Handwerkszweig. Alle Rohstoffe für die Glasherstellung müssen importiert werden. Unter diesen Umständen war die Glaserzeugung, die zudem sehr energieintensiv ist, auf der Inselgruppe lange Zeit undurchführbar. Erst in den 1960ern wurde die Glasbläserei in Malta von britischen Geschäftsleuten artifiziell eingeführt und etabliert. Als „Blueprint“ für den Aufbau der Industrie dienten Erfahrungen von der Isle of Wight ${ }^{471}$. Mdina Glass war der erste Betrieb, der sich im Bereich des heutigen Crafts Villages Ta'Qali (Malta) ansiedelte. Dies geschah im Jahr 1968. Erst ca. 8 Jahre später folgte der Ausbau der restlichen Handwerkskonzentration auf dem Gebiet des ehemaligen Militärflugplatzes.

Der ökonomische Erfolg von Mdina Glass war von Beginn an gut. Die hohe Verarbeitungs- und künstlerische Qualität der Erzeugnisse wurde durch ein eigenes „maltesisches“ Produktdesign ${ }^{42}$ ergänzt. Das kam bei Touristen und Einheimischen gut an und führte in der Folge zur Schaffung von drei weiteren konkurrierenden Glasbläserei-Betrieben auf Gozo und Malta ${ }^{473}$.

Gegenwärtig werden mehr als 60\% der Produktion direkt ins Ausland verschickt. Die restlichen $40 \%$ verbleiben für den Verkauf am Standort Ta'Qali in Malta. Insgesamt wurden auf diese Weise Ende der 1990 er rund $98 \%$ der Gesamtproduktion an ausländische Kunden verkauft ${ }^{474}$.

\subsection{Moderne Produkte mit traditionellen Ausgangsstoffen}

Ein weiterer Faktor in Maltas Produktpalette sind solche Produkte, die zwar aus regionalen traditionellen Rohstoffen bestehen, ihrem Charakter nach jedoch neue, teils artifiziell eingeführte, Produkte sind. Sie können auf keine historische Herstellungstradition zurückblicken. Sie beruhen jedoch z. B. auf traditionellen Rezepten oder Wirkstoffen, die dem neuen Produkt eine „Aura“ des Traditionellen verleihen und damit für Touristen oft ansprechend wirken.

Diese Produktgruppe stellt auf den ersten Blick ein Problem dar, wenn sie mit der in der vorliegenden Arbeit gegebenen Definition von regionalen traditionellen Produkten konfrontiert wird: Einerseits haben die Waren dieser Kategorie keine Tradition, weil es sich um Neuentwicklungen handelt. Sie wären in dieser Hinsicht laut der gegebenen Definition nicht als regionale traditionelle Produkte zu beurteilen. Andererseits bauen sie aber auf traditionellen Zutaten und Rezepten auf, die bereits in Maltas Vergangenheit bekannt waren, aber für den Produktionsprozess der neuen Produkte modernisiert oder abgewandelt wurden. Sie stellen somit Streitfälle dar, bei denen durchaus diskutiert werden kann, ob eine Einordnung als regionales traditionelles Produkt gerechtfertigt wäre oder nicht. Der Autor plädiert in den beiden vorgestellten Fällen gegen die Klassifizierung als regionales traditionelles Produkt, was im Folgenden für den jeweiligen Einzelfall erläutert wird.

471 Vgl. MARKWICK (2001), S. 45 \& ASCHEMEIER (2005), S. 241

472 Einer der Unternehmensgründer, JOSEPH SAID, schuf das sog. „blue glass“ als charakteristisches Produktdesign. Dabei handelte es sich um zweifarbiges Glas, in dem sich Blautöne und Erdtöne ineinander verwoben. Die Erdtöne sollten die karge Landschaft Maltas symbolisieren, während die Blautöne metaphorisch für das Meer standen (mündliche Auskunft am 14.10.2003 von OLIVIA SAID, Mdina Glass). Diese Produktedition ist noch heute erhältlich und gilt seither als das „typisch maltesische“ Glasdesign, das auch von anderen Herstellern übernommen wurde.

473 Namentlich sind dies Mtarfa Glass Blowers, Phoenician Glassblowers und Gozo Glass.

474 Vgl. MARKWICK (2001), S. 46 
MARK MICELI-FARRUGIA, Geschäftsführer des maltesischen Herstellers von Qualitätsweinen Meridiana Wine Estate, entwickelte zwischen 1988 und 2004 das Konzept eines Digestifs auf der Basis von Auszügen der Schote des Johannisbrotbaums. Im Juli 2004 wurde der Likör, genannt „Leila“, auf dem maltesischen Markt eingeführt.

Der Digestif beruht auf den natürlichen Wirkstoffen und der natürlichen Süße der Schote des Johannisbrotbaums (s. Kap. 13.10). Diese Grundlage wurde mit verschiedenen mediterranen Kräutern und Aromen angereichert. Sinn war es, einen Magenlikör zu produzieren, der nicht bitter schmeckt ${ }^{475}$. MICELIFARRUGIA charakterisiert seine Schöpfung im Interview mit der maltesischen Sonntagszeitung THE SUNDAY TIMES: „We are convinced that we have created a one-of-a-kind digestive liqueur which both tastes good like a traditional liqueur - and does you good - like a Mediterranean digestivo! (...). During small group taste trials we observed that the product appealed especially to regular drinkers of the leading digestivi on the market." (MICELI-FARRUGIA in: THE SUNDAY TIMES, 18.07.2004, S. 65).

Die Produktentwicklung erscheint gut durchdacht: Die Form der Flasche wurde dem touristischen Konsumenten angepasst, indem eine möglichst flache, rechteckige Form gewählt wurde, die problemlos auch in voll gepackte Koffer passt ${ }^{476}$. Es wurde von Anfang an darauf geachtet, auch die Möglichkeiten zur Verwendung von Leila als Longdrink-Grundlage anzupreisen ${ }^{477}$. MICELI-FARRUGIA verwendet im Zusammenhang mit dem Mix aus Leila und Kaffee die ansprechende und originelle Bezeichung „café maltais“478. Leila wird laut EU-Reglementierung als maltesisches Produkt vermarktet werden, obwohl die Ernte der Johannisbrotbaumschoten aus Kostengründen nicht in Malta stattfindet ${ }^{499}$. Auch die ökonomische Seite der Produkteinführung scheint wohl überlegt zu sein. Mit dem Meridiana-Ableger Terrae Reditus wurde eine eigene Firma für die Produkteinführung von Leila geschaffen.

Die traditionelle Grundlage, auf die der Leila-Likör offensichtlich zurückgreift, ist das traditionelle maltesische Volksheilmittel Gulepp tal Harub (s. o.). Der offensichtliche Bezug zu diesem traditionsreichen Sirup - der u. a. bei Halsschmerzen und Magenschmerzen von der maltesischen Bevölkerung konsumiert wird und auch als natürlicher Süßstoff Verwendung findet - rechtfertigt die Erwähnung von Leila in Zusammenhang mit der vorliegenden Arbeit.

Das Produkt beruht auf der Tradition eines ähnlichen, aber alkohol- und kräuterzusatzfreien, Volksheilmittels. Leila ist jedoch bislang kein regionales traditionelles Produkt: Die Markteinführung und die Entwicklung des Produkts waren weitgehend künstlich initiierte Prozesse. Die Rohstoffe stammen nicht aus Malta. Es ist noch nicht abzusehen, ob das Produkt als Träger regionaler Werte fungieren wird. Das Getränk hat noch keine Tradition.

Bei einer Erhebung durch den Autor im März 2005 zeigte sich aber, dass der Likör eine rasante Ausbreitung in Geschäften und Gastronomiebetrieben verzeichnen konnte. Im Touristenort Bugiibba, dessen Strandpromenade mit Spirituosen- und Weinhandlungen übersät ist, konnte festgestellt werden, das nur ein dreiviertel Jahr nach der Markteinführung nahezu 85\% aller Geschäfte mit einer Angebotsspezialisierung auf Alkoholika auch Leila führten. In den Supermärkten und Lebensmittelhandlungen ist das Produkt gegenwärtig ebenfalls immer häufiger zu finden ${ }^{480}$. Sollte dieser Erfolg anhalten und die Ernte der Johannisbrotbaumschoten nun noch im eigenen Land erfolgen, steht einer zukünftigen Einstufung Leilas als regionales traditionelles Produkt wenig im Weg.

475 Vgl. Zeitungsinterview mit MARK MICELI-FARRUGIA in: THE SUNDAY TIMES, 18.07.2004, S. 65

476 Vgl. Zeitungsinterview mit MARK MICELI-FARRUGIA in: THE SUNDAY TIMES, 18.07.2004, S. 65

477 Vgl. TASTE MAGAZINE 1/2004, S. 2

478 Vgl. Zeitungsinterview mit MARK MICELI-FARRUGIA in: THE SUNDAY TIMES, 18.07.2004, S. 65

479 Mündliche Information des Herstellers

480 Leila wurde z. B. in das Angebot der größten maltesischen Supermarktkette „Arkadia Foodstores“ aufgenommen, deren Angebot in jeder Filiale bis auf wenige Ausnahmen einheitlich ist. 


\section{- Preservation}

Das Produkt "Preservation“ ist laut maltesischem Recht ein Arzneimittel, das nur in Apotheken verkauft werden darf, dies jedoch rezeptfrei. In Deutschland ist das Produkt noch nicht zugelassen. Es wäre aber denkbar, dass es als Nahrungsergänzungsmittel eingestuft werden könnte.

Das pharmazeutische Erzeugnis wird aus dem Extrakt der Schale der Kaktusfeige gewonnen. Das maltesisch-französische Pharmaunternehmen ICP Ltd. ist der Hersteller. Im Rahmen einer medizinischen Studie des Tulane University Health Science Centre in New Orleans stellte sich im Jahr 2003 heraus, dass in der Schale der Kaktusfeige Stoffe enthalten sind, die den menschlichen Körper auf Enzymbasis beim Abbau von Stresshormonen und radikalen Kohlenwasserstoffen unterstützen.

Die Wirkungsweise der Kaktusfeigenschale war bereits zur Zeit der Johanniter bekannt, die ebenfalls ein Extrakt anfertigten ${ }^{481}$. Das Produkt „Preservation“ besitzt jedoch eine ungleich höhere Konzentration, die durch Gefriertrocknung und Granulation erreicht wird.

Die Anwendungsgebiete der Arznei „Preservation“ sieht der Hersteller ICP Ltd. vor allem bei Sportlern und Menschen, die häufig körperlich anstrengenden Arbeiten ausgesetzt sind. Schlagzeilen in der maltesischen Presse ${ }^{482}$ machte das Produkt jedoch vor allem wegen seiner Eigenschaft, dem sog. "Kater" nach übermäßigem Alkoholgenuss vorzubeugen.

„Preservation" wird gegenwärtig in Malta produziert und in mehrere europäische Länder sowie in die USA exportiert. In den USA ist das Mittel als „Prepare“ bekannt. Zur Zeit laufen weiterführende Tests, die untersuchen, ob das Mittel dank seiner unterstützenden Eigenschaften beim Abbau von Stresshormonen auch bei der Krebsvorsorge helfen kann.

Des Weiteren ist der Herstellungsprozess des Produkts hervorzuheben, da er auf ökologisch schonende und nachhaltige Weise stattfindet (vgl. Kap. 19.1).

Das Produkt "Preservation“ ist in Belangen des Produktcharakters, der Wirkungsweise wie auch des Herstellungsprozesses mittlerweile so weit von der ursprünglichen Kaktusfeigenschalen-Essenz der Johanniter entfernt, dass eine Klassifizierung des Arzneimittels als regionales traditionelles Produkt derzeit nicht sinnvoll erscheint.

\footnotetext{
481 Vgl. CREMONA (2003)

482 Vgl. The Times of MaltA, Ausgabe vom 05.07.2004, S. 1 \& S. 7
} 


\section{Agrargeographische Landnutzungsanalysen}

Auf Basis der erhobenen Daten aus dem vorangegangenen agrargeographischen Teil der vorliegenden Abhandlung, wurde im März 2005 eine Landnutzungsanalyse agrarwirtschaftlich geprägter Flächen der Hauptinsel Malta erstellt. Dabei handelt es sich um vier separate Teilerhebungen, die die Hauptanbauzonen der Insel Malta repräsentieren (s. u.).

Die Erhebungen beinhalten:

1) Eine vollständige Geländeaufnahme eines maltesischen Valleys, in dem vornehmlich landwirtschaftlicher Anbau zur kommerziellen Auswertung der Feldfrüchte erfolgt

= Analyse 1: II-Miżieb

2) Eine Erhebung im kleinbäuerlichen Anbau in Tal- und flacher Hanglage, auf denen vornehmlich kleinbäuerlicher Anbau zur Eigenversorgung erfolgt

= Analyse 2: II-Hofra/Mellieћa Isthmus

3) Eine Erhebung in flacher, gut zugänglicher, aber stark witterungsexponierter Hanglage

= Analyse 3: Santi-Binġemma Uplands

4) Eine Erhebung in steiler, schlecht zugänglicher, jedoch geschützter Hanglage

= Analyse 4: Ǵnien Busewdien

\subsection{Die Bedeutung der Erhebungen im Rahmen der Hauptthematik}

Warum sind die an diesem Punkt der Arbeit vorgestellten Landnutzungsanalysen im thematischen Hauptzusammenhang der vorliegenden Dissertation wichtig?

Für die regionalen traditionellen Produkte aus dem Bereich Agroindustry bzw. deren Roh- und Ausgangsstoffe bedeuten die folgenden Nutzungskartierungen eine Analyse ihres Nachhaltigkeitspotenzials. Dies kann wie folgt erklärt werden: Wie bereits an anderer Stelle gezeigt wurde ${ }^{483}$, sinken die Beschäftigungszahlen im primären Sektor in Malta seit Jahren rapide. Zugleich stieg das Durchschnittsalter der Landwirte im selben Zeitraum erheblich. Diese Entwicklung hatte unmittelbare Folgen auf die Agrarflächenzusammensetzung, die gegenwärtig, wie insbesondere CHARLTON \& BEELEY (1993) sowie CYFFKA (2005) dargestellt haben, einen immer gravierenderen Anstieg brachliegender Nutzflächenanteile aufweist.

Im Hinblick auf die regionalen traditionellen Produkte heißt das: Die Nachhaltigkeit ihrer Erzeugung ist gefährdet. Denn wenn aufgrund des Fehlens agrarischer Rohstoffe bestimmte Produkte nicht (mehr) hergestellt werden können, wird ihnen die Basis entzogen. Ihre Grundstoffe müssen in einem solchen Fall u.U. aus anderen Ländern importiert werden. Damit wäre die Stellung des Enderzeugnisses als regionales traditionelles Produkt akut infragegestellt.

Z. Zt. sind von diesem Problem z. B. verschiedene maltesische Tomatenprodukte betroffen ${ }^{484}$, weil der Tomatenanbau der Maltesischen Inseln gegenwärtig zu wenig Output erzeugt, um die Nachfrage abzudecken. Aus diesem Grund ist ein EU-Förderprogramm in Kraft getreten, das speziell die Stellung des Tomatenanbaus auf der Inselgruppe stärken soll ${ }^{485}$.

483 Vgl. Kap. 12.1.4 bis 12.1 .6

484 Darunter auch die von der EU mit der regionalen Herkunftsbezeichnung D.O.P. geschützte Tomatenpaste Kunserva.

485 Vgl. BUSUTTIL \& DEMICOLI (2003) 


\subsection{Zur Methodik}

Die folgenden Kartierungen basieren auf zwei Grundlagen. Die erste Grundlage ist eine IKONOSSatellitenbildszene vom 28.03.2004 (Bodenauflösung 4m), welche den Nordwesten Maltas abdeckt (vgl. Abb. 17). Die östliche Grenze der Satellitenaufnahme erstreckt sich linienhaft zwischen Ta'Qali und Maghtab. Die südliche Grenze verläuft auf einer Geraden annähernd zwischen Ta'Qali und Baћrija. Die nördliche und westliche Grenze ist durch den Küstenverlauf bedingt.

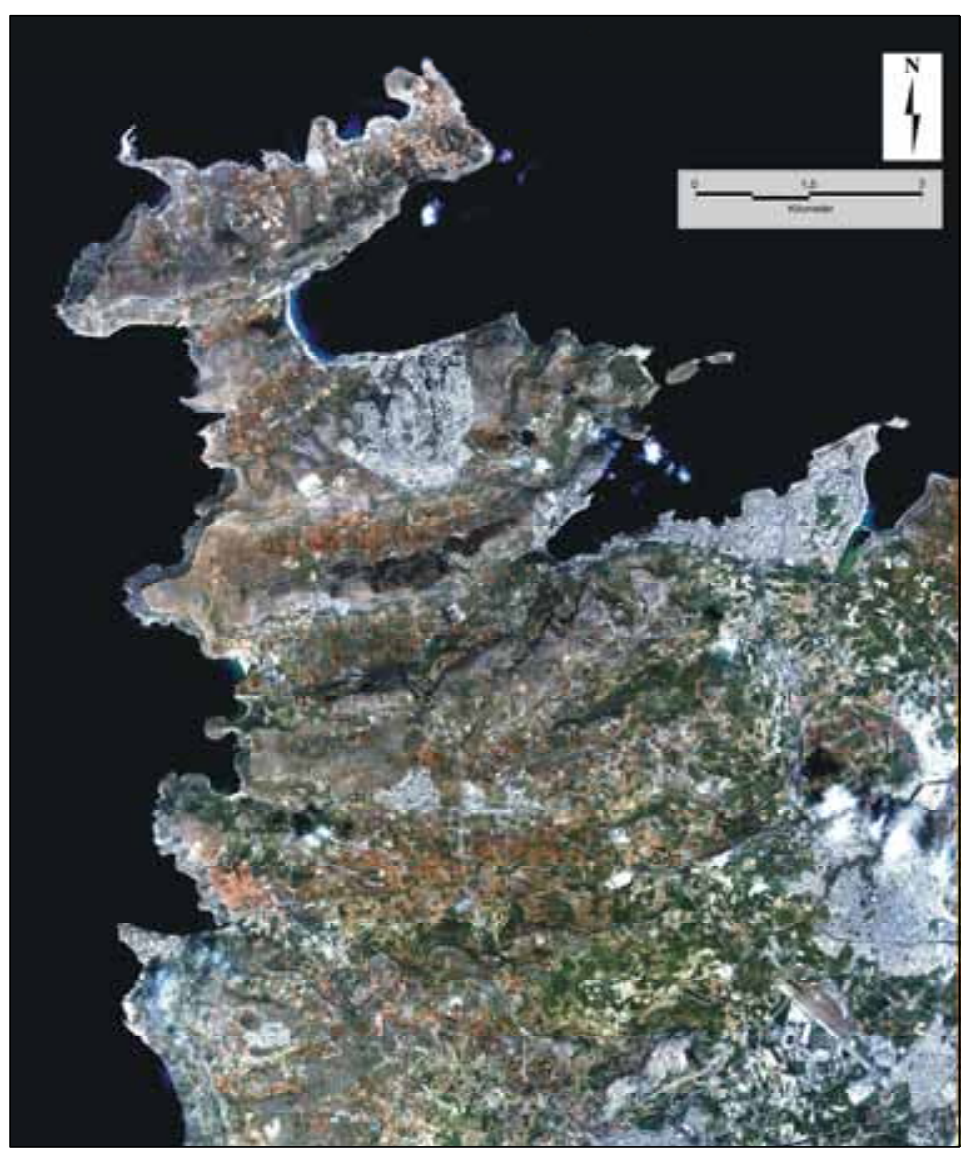

Abb. 17: IKONOS-Satellitenbildszene (28.03.2004) vom nordwestlichen Teil Maltas, Wiedergabe stark verkleinert

Die zweite Grundlage bilden einige über den Mapserver ${ }^{486}$ der Malta Environment and Planning Authority (MEPA) frei zugängliche und im UTM-Gitter kartierte Ausschnitte topographischer Karten.

Auf der Basis dieser Daten wurde für jedes einzelne agrarisch genutzte Feld im jeweiligen Untersuchungsgebiet eine Landnutzungsanalyse nach einem vorher bestimmten Schlüssel erstellt. Er enthält Daten über die Anbauweise (z. B. Trockenfeldbau, Bewässerungsfeldbau, „Fallow Land“ (s. u.), etc.) sowie die jeweilige Anbaukategorie (z. B. Getreide, Gemüse, Weinbau, etc.). Der Schlüssel setzt sich somit aus zwei Variablen zusammen, deren Auflistung in der Legende der jeweiligen GIS-Auswertung einsehbar ist.

Das Endergebnis ist eine Analyse der kartierten Flächen mithilfe der Software Maplnfo GIS. Anhand der GIS-Auswertungen wurden Rückschlüsse auf Anbauverteilung und -auslastung möglich.

Alles in allem ergibt sich somit eine stichprobenhafte Erhebung der landwirtschaftlichen Nutzflächen im Nordwesten Maltas (vgl. Karte 4), deren Ergebnisse vom Prinzip auf den Rest der Insel übertragen werden

486 Öffentlich zugänglich über http://www.mepa.org.mt/Planning/index.htm?MapServer.htm\&1 
können. Das Resultat kann nicht auf die Nachbarinsel Gozo bezogen werden, da diese eine weitgehend autonome Agrar- und Reliefstruktur aufweist, die sich von dem landwirtschaftlichen Anbau, so wie er sich in Malta darstellt, in wesentlichen Punkten unterscheidet ${ }^{487}$.

Leider konnte aber im Rahmen der vorliegenden Dissertation keine zusätzliche Landnutzungsanalyse der Insel Gozo erstellt werden, weil sowohl eine topographische Kartenstaffel als auch eine etwaige alternative Kartierungsgrundlage (z. B. Luft- oder Satellitenbild) nicht verfügbar waren.

Die vier Teilkartierungen stellen, wie bereits eingangs erwähnt, repräsentative Anbaugebiete dar. Diese Äußerung gilt wohlgemerkt nicht quantitativ im Sinne der Repräsentativität einer typischen Anbauflächenzusammensetzung im Vergleich mit der Grundgesamtheit. Vielmehr äußert sich der Autor diesbezüglich qualitativ im Sinne der Vergleichbarkeit von typischen Landschaftsbildern, in denen landwirtschaftlicher Anbau in Malta vorkommt. Andere agrarisch genutzte Landschaftsbilder wie die der vier Teilkartierungen gibt es in Malta bis auf einige Ausnahmen ${ }^{488}$ effektiv nicht $^{489}$.

\subsection{Zur Auswahl der Teilerhebungen}

\section{Analyse 1: Il-Miżieb}

Die Auswahl der Erhebungen umfasst Beispiele der typischen Anbauzonen der maltesischen Landwirtschaft (vgl. Karte 4). Die wichtigste Rolle spielen dabei die Valleys, weil sie den Hauptanteil der kommerziell verwertbaren landwirtschaftlichen Nutzflächen beinhalten ${ }^{490}$. Die Flächengrößen in den Valleys sind in den meisten Fällen um ein Mehrfaches umfangreicher als an den Hängen, wo das Relief wechselhaft ist und die genutzten Hangterrassen durch Rutschungen gefährdet sind.

Dies bedeutet, dass die Tallagen weniger aufwändig und dadurch Kosten sparender zu bewirtschaften sind. Einige Flächen in den Tälern werden auch maschinell bearbeitet. Nur durch die Lage im Valley ist eine dafür ausreichende Bodenmächtigkeit gewährleistet ${ }^{491}$. Man findet deswegen an diesen Standorten eine große Anzahl von Flächen mit relativ anspruchsvollem Bestand (z. B. Gemüse und Obst) sowie ausgeprägten Bewässerungsfeldbau. Des Weiteren treten in den Tälern die Getreidefelder in wesentlich größerem Maßstab auf und sind, im Gegensatz zu Getreide in Hanglage, viel häufiger bewässert. Valleys sind die Standorte, an denen die Agrarprodukte erzeugt werden, die später in den Gemüseläden der ganzen Insel zu kaufen sind oder von den zahlreichen fahrenden Händlern angeboten werden.

Für die erste Beispielerhebung wurde ein Valley nördlich von Bajda Ridge gewählt. Der Flurname der Region ist II-Miżieb (vgl. Karte 4). Topographisch gesehen ist das Valley das obere Bassin des Wied talMistra $^{492}$. Es verläuft parallel zum Bajda Ridge und ist zusammen mit dem Wied tal-Pwales (Pwales Valley) eines der wichtigsten Täler für die agrarische Nutzung im Verwaltungsbereich San Pawl il-Bahar im maltesischen Nordwesten ${ }^{493}$.

487 Z. B. ist in Gozo der Kartoffelanbau im Vergleich zur Hauptinsel Malta stark unterrepräsentiert: „Distance from Malta's harbours put Gozo virtually out of the potato export picture, and in the sister Island early potato cultivation is consequently of lesser importance (...)." (BUSUTTIL (1993), S. 16). Im Bereich der Geomorphologie bedingen Tafelberge, die auf Malta in der Form nicht anzutreffen sind, auf Gozo eigenständige Anbaubereiche. Dies sind nur zwei von vielen Faktoren, die Gozos Landwirtschaft von der Maltas abgrenzen.

488 Hierzu zählen z. B. der kleinbäuerliche Anbau im direkten Umfeld städtischer Bereiche, der Gartenbau in den Innenhöfen einzeln stehender Häuser und die nur noch vereinzelt vorkommende Bewirtschaftung extremer Lagen, wie $\mathbf{z}$. B. Kliffs oder extremer Karstflächen.

489 Vgl. MELI (1993)

$490 \mathrm{Vgl}$. AZZOPARDI (2002)

491 Vgl. ebd., S. 150

492 Vgl. BOWEN JONES et al. (1961), S. 236 \& S. 241

$493 \mathrm{Vgl}$. TEUmA (2003) 
Das Tal II-Miżieb wird topographisch im Süden von Bajda Ridge, im Norden von Mellieћa Ridge, im Osten von der Mistra Bay und im Westen wiederum von Bajda und Mellieћa Ridge eingegrenzt.

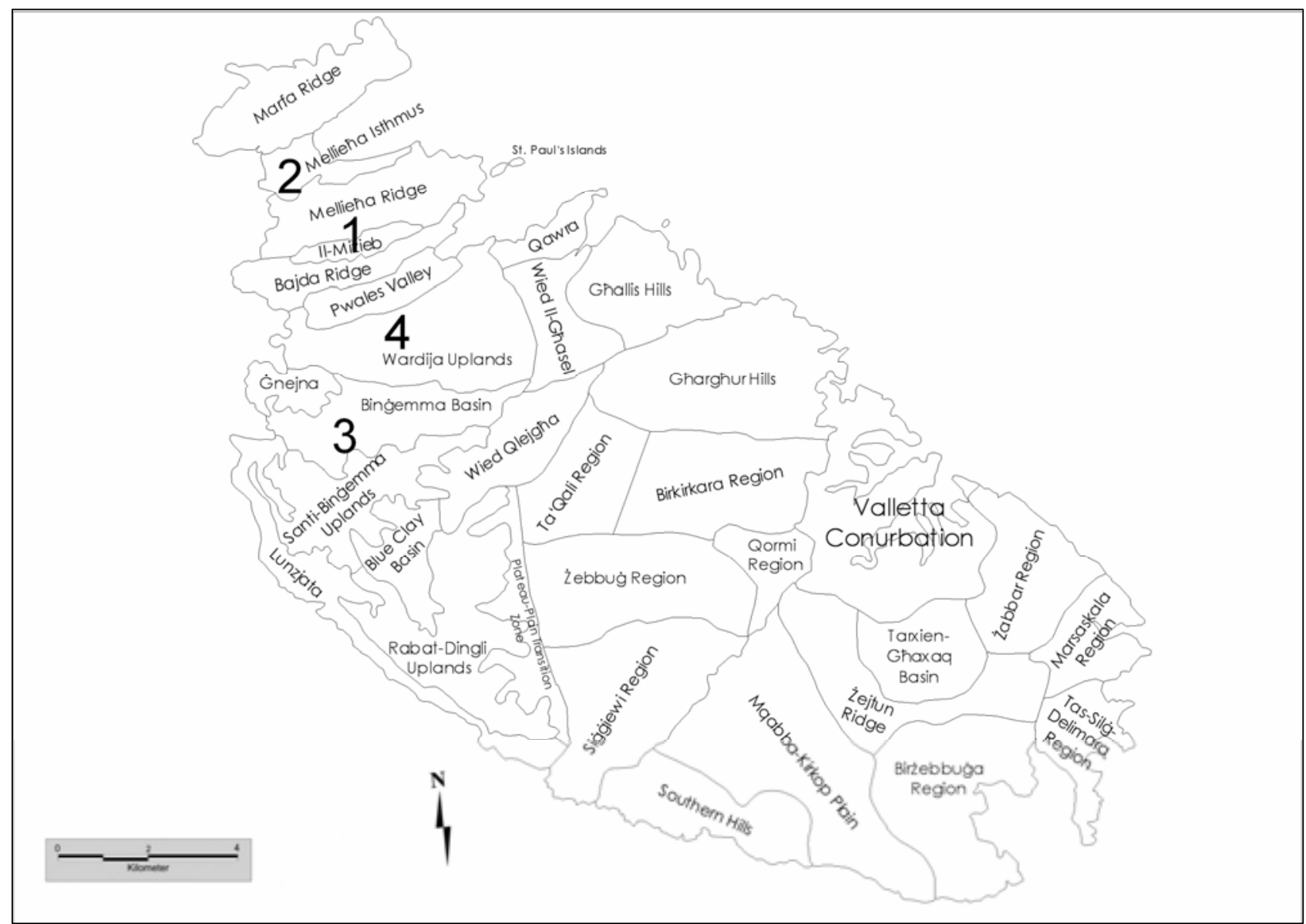

Karte 4: Die landwirtschaftlichen Anbaugebiete der Insel Malta. Eingetragen sind die Orte der Geländeaufnahmen: 1=II-Miżieb; 2=IIHofra/Mellieћa Isthmus; 3=Santi Binġemma Uplands; 4=Ġnien Busewdien. Entwurf \& Grafik: AschEMEIER in Anlehnung an BowEN JONES, DEWDNEY \& FISHER (1961), S. 236.

\section{Analyse 2: II-Hofra/Mellieћa Isthmus}

Der Mellieћa Isthmus ist die Landenge zwischen Marfa Ridge und Mellieћa Ridge (vgl. Karte 4). Aufgrund der natürlichen Begrenzung möglicher Nutzflächenausdehnungen ist die Landwirtschaft in diesem Bereich benachteiligt und traditionell nur in kleinbäuerlichem Rahmen anzutreffen. In der Mitte der Region befindet sich ein Karsthügel, der oberhalb der Anchor Bay das Panorama für die touristisch genutzte Filmkulisse „Popeye Village" bietet. Die Hauptanbauflächen liegen nordwestlich und südöstlich des Hügels. Ihre Flurnamen sind II-Ghadira und II-Hofra.

Für die zweite Geländeaufnahme wurde ein Ausschnitt aus II-Hofra im Südosten kartiert. Die Flächengrößen in diesem Bereich rangieren für maltesische Verhältnisse in den Klassen mittelgroß $\left(\sim 270 \mathrm{~m}^{2}\right)$ bis sehr klein $\left(\sim 6 \mathrm{~m}^{2}\right)$. Es dominiert der typische kleinbäuerliche Anbau zum Zweck der Eigenversorgung. Auf mittelgroßen Flächen kommt nur vereinzelt kommerzieller Anbau vor. Noch seltener sind Folien- und Gewächshauskulturen.

Der kleinbäuerliche Anbau spielt im wirtschaftlichen Bereich nur eine untergeordnete Rolle. Er stellt jedoch die ursprünglichste Form der Landwirtschaft auf Malta dar, deren Tradition bis weit vor die Zeit der Johanniter zurückreicht (vgl. Kap. 12.1). Für das Landschaftsbild ist diese Form der Bewirtschaftung ein prägender Faktor. 


\section{Analyse 3: Fort Binġemma}

Erhebung 3 umfasst einen Ausschnitt aus dem Bingemma-Hochland in der Geologie des Upper Coralline Limestone, südlich des fruchtbaren, gewächshausdurchsetzten Binġemma Bassins (vgl. Karte 4). „It is the wildest and most isolated district of Malta" schrieben BOWEN JONES, DEWDNEY \& FISHER im Jahr $1961^{494}$. An der Feststellung von BOWEN JONES et al. hat sich bis heute nichts Wesentliches geändert. Der Grund dafür ist die extreme Windexposition der in Meeresnähe auf bis zu 234m NN gelegenen Hangterrassen. Der Anbau auf den flachen Terrassen ist durchsetzt mit nischenhaftem Vorkommen von Obstbäumen in windstillen Bereichen. Im Wesentlichen dominiert aber der Getreideanbau.

Im Bereich der Hänge ist der große Anstieg von aufgegebenen und brach liegenden Feldern im Verlauf der letzten Jahrzehnte nicht zu übersehen. Die Hangterrassen bilden zwar relativ große Flächen, jedoch sind sie mit ihren asymmetrischen Feldgrenzen, die gezwungenermaßen isohypsenparallel verlaufen, und ihrer extremen Witterungsexposition praktisch nicht mehr wirtschaftlich nutzbar. Das hatte den Verfall vieler Hangterrassen und die Abspülung der Bodenauflage zur Folge womit diese Bereiche Maltas z. Zt. Anlass zur Sorge geben ${ }^{495}$.

\section{Analyse 4: Ǵnien Busewdien}

BOWEN JONES et al. (1961) definierten den Begriff Ǵnien noch als Zone überaus intensiven Anbaus und stellten die Ǵnien lands ranggleich zu den rdum-Bereichen der flachen Hänge und Valleys ${ }^{496}$. Diese Sicht der Dinge kann heute nicht mehr in allen Belangen aufrechterhalten werden.

Bestes Beispiel hierfür sind die steilen Hänge des Ġnien Busewdien im Bereich der Wardija Uplands (vgl. Karte 4). Die Ǵnien lands waren in der Vergangenheit vor allem deswegen Zonen der intensiven Bearbeitung, weil sie fruchtbare Nischen in karstigen Bereichen darstellten. In Zeiten der noch wenig durchprofessionalisierten Landwirtschaft der 1960er-Jahre, in der für hohe Erträge auch hohe Flächenanteile notwendig waren, waren die Ǵnien lands begehrte Anbaustandorte.

Im Verlauf der fortschreitenden Flurzersplitterung, der Maschinisierung der Landwirtschaft sowie der Einführung hoch produktiver Nutzpflanzensorten und den entsprechenden Düngemitteln, wurden die Ǵnien lands zunehmend unwirtschaftlich. Zu der Unwirtschaftlichkeit kam die Problematik der steilen Hanglage und die damit einhergehende schlechte Zugänglichkeit der Anbauflächen. Die Ǵnien lands wurden damit auch zunehmend unpraktisch, sodass sich der Anbau von intensiv mit Gemüse und Hackfrüchten bebauten Bereichen (wie 1961 bei BOWEN JONES et al. beschrieben) zu extensiv bewirtschafteten Flächen wandelte, auf denen gegenwärtig vor allem der Weizenanbau, der kleinbäuerliche Weinbau und der Viehfutteranbau vorkommt. Zu diesen in der Bewirtschaftung wenig aufwändigen Nutzungsformen kommt ein hoher Anteil aufgegebener Flächen, der aufzeigt, dass mit der zunehmenden Reliefenergie der Hänge auch der Anteil der offen gelassenen Hangterrassen zunimmt.

494 Vgl. BOWEN JONES, DEWDNEY \& FISHER (1961), S. 251

$495 \mathrm{Vgl}$. ASCHEMEIER \& CYFFKA (2004b)

496 Vgl. BOWEN JONES, DEWDNEY \& FISHER (1961), S. 235 


\subsection{Zur Legende}

Die Legende der folgenden Kartierungen setzt sich aus Angaben über die Art des Anbaus und die Landnutzungskategorie zusammen. Im Folgenden werden die einzelnen Abstufungen erläutert.

\section{a) Art des Anbaus}

- Trockenfeldbau: Feldbau, der ohne dauerhafte Bewässerung auskommt und den Wasserbedarf hauptsächlich aus dem natürlichen Niederschlag bezieht. Periodische Bewässerung kommt vor. Auch dry farming ${ }^{497}$ fällt im Rahmen der Kartierungen unter diese Kategorie.

- Bewässerungsfeldbau: „Form der landwirtschaftlichen Bodennutzung, bei der den Nutzpflanzen in niederschlagsfreien oder -armen Zeiten der Vegetationsperiode durch angeeignete technische Maßnahmen ausreichende Wassermengen zugeführt werden." (LESER (1997), S. 84).

- „Fallow Land“: Ehemalig bewirtschaftete Nutzflächen, die von ihren Besitzern seit ca. einem Jahr nicht mehr bearbeitet oder gepflegt wurden und in einem Zustand beginnender Verwilderung vorliegen. A. AZZOPARDI (2002, S. 167) definiert „Fallow Land“ wie folgt: „Fallow Land is land which neither carries a crop nor has a prepared soil in readiness for a crop, but which appears to have been unworked for not more than about a year. Such land is considered to be in its first stage of abandonment (...). "Eine dominierende Feldfrucht ist noch erkennbar, oft sind dies Reste einer nicht eingebrachten Ernte.

- Nicht bestimmbar: Diese Einordnung wurde für die Flächen der Landnutzungskategorie „Brache" verwandt, da lediglich ein gepflügter Acker erkennbar war. Mögliche Bewässerungsmechanismen wurden noch nicht installiert oder bereits wieder abgebaut. Es wäre daher spekulativ, einzuschätzen, ob die bewussten Flächen Trockenfeldbau- oder Bewässerungsfeldbauflächen werden bzw. waren.

\section{b) Landnutzungskategorie}

- Gemüse: Auf Feldern dieser Kategorie wird der Anbau von Gemüse betrieben. Dazu zählen in Malta z. B. Artischocken, diverse Kohlarten, Paprika, Spinat, Mohrrüben, etc. Um die totale Anzahl der Abstufungen gering zu halten, wurden auch Leguminosen - in Malta sind dies vor allem Bohnen, Erbsen und Linsen - in diese Kategorie mit einbezogen, weil ihr Anbau in Methodik, Aufwand und Ausprägung grundsätzlich ähnlicher Natur ist.

- Getreide: In diesen Fällen wird der Anbau von Getreide betrieben. In Malta wird vor allem Hartweizen (Winterweizen) und Gerste angebaut, Hafer kommt nur selten vor. Verschiedentlich säen maltesische Farmer auf Flächen mit kritischer Wasserversorgung eine Mischung von Weizen und Gerste (Malt: $m a \hbar / u t)$ aus ${ }^{498}$. Aus diesem Grund ist eine Einteilung der Anbauflächen in die einzelnen Getreidearten ungenau. Es wurde für die Legende daher die allgemeine Bezeichnung "Getreide" gewählt.

- Wein: Auf Flächen der Kategorie Wein wird die Kultivierung von Weinreben betrieben. Im Rahmen der Geländeaufnahme konnte aufgrund des jahreszeitlichen Wuchsstatus nicht unterschieden werden, ob es sich um Tafelweintrauben handelte oder um Trauben für die Herstellung von Wein.

- Hackfrüchte: Hierbei wird der Anbau von Hackfrüchten betrieben. Im Untersuchungsraum handelte es sich dabei vor allem um Kartoffeln - das Hauptexportprodukt der maltesischen Landwirtschaft. Aber auch verschiedene Arten von Zwiebeln und Knoblauch fallen unter diese Einordnung.

\footnotetext{
497 Vgl. LESER (1997), S. 150: „Dry Farming: Anbaumethode im Bereich der Trockengrenze. Wegen des zu geringen jährlichen Niederschlags wird das Niederschlagswasser durch Bearbeitung des Bodens meist mehrerer [sic] Jahre im Boden angereichert. (...)."
} 
- Obstbäume: Auf Flächen, die unter diese Einteilung fallen, dominieren Obstbaumkulturen im Oberbau. Die Festsetzung der Obstbaumkulturen als Flächennutzungskategorie wird erschwert, weil im Unterbau häufig weitere Nutzpflanzen kultiviert werden (z. B. Hackfrüchte) und somit Sonderformen des stufigen Anbaus zutage treten.

- Aufgegebene Flächen: Im Rahmen der vorliegenden Erhebung fällt unter diese Abstufung Land, bei dem aufgrund längerer Nicht-Bewirtschaftung kein geordneter Anbau mit einer dominierenden Nutzpflanze mehr erkennbar war. Die Flächen waren zumeist mit Wildkräutern und Gräsern überwuchert. Die äquivalente englischsprachige Bezeichnung in der Malta-Forschung für Flächen dieser Art ist „Abandoned Land“ (vgl. z. B. BOWEN JONES et al. (1961) sowie CHARLTON \& BEELEY (1993)).

- Folienkultur: In diesem Fall wird der Anbau als Folienkultur betrieben. Vor allem Gemüse und Obst werden in Folienkulturen herangezogen. Gründe für die Einrichtung von Folienkulturen in Malta sind vor allem der Schutz vor den Witterungseinflüssen des mediterranen Winters, der Schutz vor Salzaerosolen in der Luft, die Erzielung eines Treibhauseffekts zur Anzucht von wärmebedürftigen Nutzpflanzen und der Transpirationsschutz.

- Sonstiges: Unter „Sonstiges" werden im Rahmen der vorliegenden Kartierung alle Nutzpflanzenkategorien verstanden, die nicht zu den anderen in der Legende angegebenen Einordnungen gezählt werden können. Ein Beispiel hierfür ist z. B. der Anbau von Ölsaat wie z. B. Raps. Aber auch die Kultivierung von Ölbäumen würde zu dieser Kategorie zählen. Im Einzelfall sind deshalb zu dem Bereich "Sonstiges" detaillierte Angaben im Erläuterungstext der jeweiligen Kartierung zu finden. Als „Sonstiges" wurden auch diejenigen Flächen klassifiziert, bei denen nicht eindeutig bestimmt werden konnte, welche Nutzpflanzenkategorie angebaut wurde.

- Brache: Anbauflächen dieser Einstufung waren entweder abgeerntet oder frisch beackert, sodass nicht festgestellt werden konnte, welche Nutzpflanzenkategorie auf dem Areal angebaut wurde oder in Zukunft angebaut wird. Die Abstufung unterscheidet sich von den „Aufgegebenen Flächen" dadurch, dass die ihr zugeordneten Felder in gutem Zustand sind, regelmäßig beackert werden und somit „in Kultur" stehen. Bewässerungsmechanismen waren entweder abgebaut oder (noch) nicht installiert, sodass alle Flächen dieser Kategorie im Bereich „Art des Anbaus" (s. o.) als „nicht bestimmbar" eingestuft werden mussten.

- Gewächshaus: Auf Flächen dieser Kategorie erfolgt der Anbau von Gemüse und/oder Obst in Gewächshauskultur. Vor allem salzempfindliche und wärmeliebende Nutzpflanzen werden in Malta auf diese Art und Weise bewirtschaftet. Gewächshauskultur ist die materiell und wirtschaftlich aufwändigste Anbaumethode der Maltesischen Inseln. Deswegen werden vor allem ökonomisch interessante Nutzpflanzen wie z. B. Tomaten, Auberginen, Erdbeeren, etc. in diesem Verfahren bewirtschaftet. Auch die Anzucht kältempfindlicher Setzlinge erfolgt im Gewächshaus.

Nicht in der Legende enthalten ist der Anbau von Blumen. Diese Wirtschaftsweise erfreut sich in Malta seit Jahren wachsender Beliebtheit. Ökonomisch gesehen ist der Anbau von Schnittblumen vor allem deswegen interessant, weil die Vegetationsperiode aufgrund des milden Klimas der Maltesischen Inseln früher einsetzt als auf dem europäischen Festland und in Großbritannien. Blumen sind daher ein nicht unbedeutender Exportfaktor der maltesischen Landwirtschaft geworden ${ }^{499}$. Ihr Anbau erfolgt jedoch in vielen Fällen in der Umgebung der Siedlungen und weniger auf den ausgedehnten Landwirtschaftsflächen. Im Rahmen der erhobenen Landnutzungsanalyse tauchte die zugehörige Landnutzungskategorie nicht auf und wurde aus diesem Grund auch nicht in der Legende berücksichtigt. 


\section{Die Ergebnisse der Landnutzungsanalysen im Einzelnen}

Nachdem im vorangegangenen Text die Methodik der Landnutzungskartierungen, die Erläuterung ihrer Legende und die Auswahlkriterien für die einzelnen Teilbereiche angesprochen wurden, soll im folgenden Abschnitt auf die Ergebnisse der GIS-Analyse der erhobenen Daten näher eingegangen werden.

Für jede der Teilerhebungen wurden thematische Karten erstellt, anhand derer unterschiedliche geographische Phänomene dargestellt werden. Für jede der Einzelaufnahmen wurde zunächst eine Karte erstellt, anhand derer die Landnutzungskategorie ersichtlich ist (Karten im Anhang). Die weiteren thematischen Kartierungen beziehen sich auf die Art des Anbaus der jeweiligen Landnutzungsanalyse.

\subsection{Ergebnisse - Analyse 1: Il-Miżieb}

Analyse 1 (s. Karte I, Anhang) ist die umfangreichste der vier Teilerhebungen. Die Landnutzungskartierung von II-Miżieb beinhaltet 508 landwirtschaftlich genutzte Flächen. Die kleinsten Flächengrößen betragen ca. $20 \mathrm{~m}^{2}$. Die größten Flächen umgrenzen ca. $1.000 \mathrm{~m}^{2}$. Die Umrisse der einzelnen Felder sind in der Talmitte vorwiegend symmetrisch, während sie an den Talrändern hangaufwärts dem Relief angepasst werden mussten und somit häufig terrassiertere und unsymmetrische Strukturen aufweisen. II-Miżieb gilt in der Literatur als eines der fünf fruchtbarsten Anbaugebiete der Hauptinsel Malta ${ }^{500}$.

Im südwestlichen Bereich und in der südlichen Talmitte ist das Valley geprägt von Längsfluren, die am nördlichen Talrand sowie an den östlichen und äußersten westlichen Ausläufern des Anbaugebiets in Blockfluren übergehen. Dies ist eine Folge des Reliefs, das nur in den relativ flachen Lagen die Anlage von Längsfluren zulässt. Beide Flurformen werden in gleichem Maße genutzt. Es ist jedenfalls nicht zweifelsfrei belegbar, dass die ökonomisch günstigeren Längsfluren in stärkerem Ausmaß genutzt werden. Der Getreideanbau ist die einzige Landnutzungskategorie bei der erkennbar ist, dass der Anbau auf Längsfluren dem Anbau auf Blockfluren vorgezogen wird. (vgl. Karte I, Anhang).

\section{Brachen und aufgegebene Flächen}

Die meisten kartierten Flächen (Anzahl: 166) des Valleys können als aufgegebene Flächen bezeichnet werden. Zu diesen offensichtlich verlassenen Ländereien kommen 99 beackerte Brachen, bei denen entweder die Ernte bereits erfolgt war oder die Vegetationsperiode der dort angebauten Nutzpflanzen noch nicht begonnen hatte. Die Anzahl der in Nutzung befindlichen Areale beträgt insgesamt 342. Das heißt, dass beinahe die Hälfte aller zur Verfügung stehenden Felder nicht mehr genutzt werden.

Dieser Eindruck wird bestätigt durch eine Satellitenbildszene im infraroten Bereich vom März 2004 (vgl. Abb. 18). In dieser Aufnahme stellen alle roten Flächen aktive Vegetation dar, während die grünen Flächen anzeigen, dass überwiegend der „nackte" Ackerboden vorliegt. 


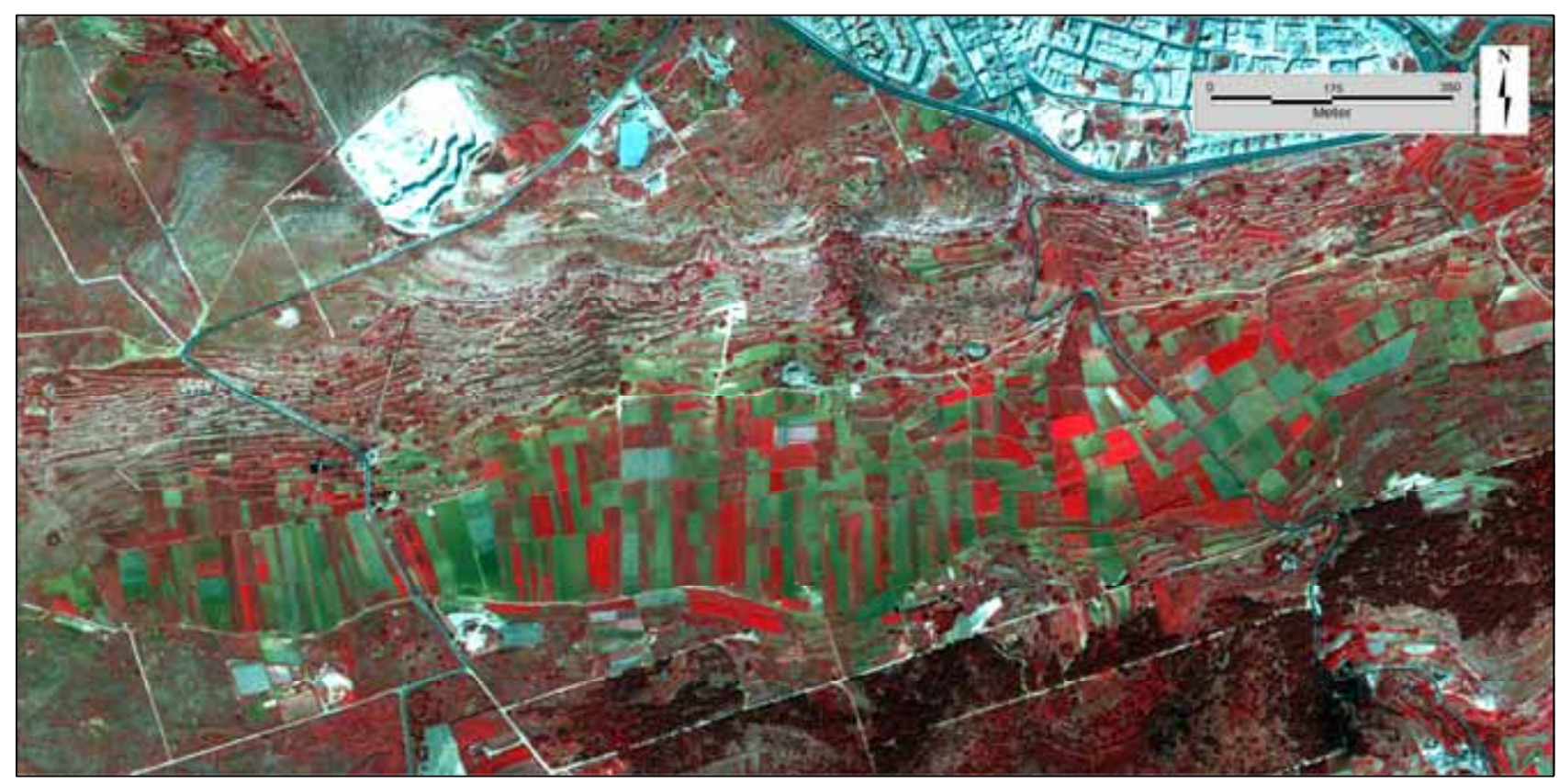

Abb. 18: Anbauzone II-Miżieb im IKONOS-Satellitenbild, Channel 3, Infrarot. Die grünen Flächen zeigen die spärlich oder gar nicht bepflanzten Areale, die in dem Tal überwiegen.

Die Ergebnisse spiegeln die aktuellen Zustände der gesamten maltesischen Landwirtschaft wider, in denen die Vollerwerbslandwirte nur noch eine untergeordnete Rolle spielen ${ }^{501}$ und der Anteil des aufgegebenen Lands an der Grundgesamtheit seit Jahrzehnten zunimmt, während die Gesamtnutzfläche als solche im selben Zeitraum vornehmlich durch Bebauung dezimiert wurde: „Many dry-farmed fields have been abandoned and it probably has to be accepted that, for both economic and sociological reasons, this trend is unlikely to be reversed." (CHARLTON \& BEELEY (1993), S. 325). "The build-up area increased from six per cent to 27 per cent; (...) as a result of building and abandonment, the cultivated area shrank by 37 per cent. (...) These figures reflect important social and economic processes which have been at work during the 30-year period."(CHARLTON \& BEELEY (1993), S. 327).

Die große Anzahl an aufgegebenen Flächen ist in II-Miżieb aber auch durch die topographischen Gegebenheiten bedingt. Die östlichen und westlichen Ausläufer des Gebiets gehen in verkarstete Zonen über, in denen ein Anbau aus heutiger Sicht unmöglich erscheint. Deshalb finden sich vor allem im östlichen Ausläufer des Tals viele aufgegebene Areale (vgl. Karte 5). Nichtsdestotrotz sind auch die Karstzonen an den Rändern des Valleys mit Kulturrelikten von Rubble Walls, ehemaligen Bewässerungsanlagen, antiken Bienenstöcken und Girnas ${ }^{502}$ durchsetzt. Dieser Umstand kann als Hinweis gedeutet werden, dass auch jene Flächen einst mit fruchtbarer Bodenauflage überzogen waren ${ }^{503}$. Noch erkennbare, klar abgegrenzte Grundstückslinien deuten ebenfalls auf eine Bearbeitung dieser Felder in früherer Zeit hin.

Allerdings muss man sich auch vor Augen halten, dass zur Zeit von Maltas Baumwollwirtschaft auch solche Areale erschlossen wurden, die unter normalen Umständen als nicht kultivierbar gegolten hätten. Sie wurden unter großen Mühen künstlich hergestellt.

\section{Vgl. AsCHEMEIER \& CYFFKA (2004b), S. 109}

502 Die Girna (malt.) ist ein runder Steinbau, welcher in Art der Rubble Walls völlig ohne Mörtel aus aufgeschichteten Steinen errichtet wird. Das Dach ist ebenfalls aus lockerem Geröll in Kuppelbauweise aufgeschichtet. Dieser handwerklich kunstvoll gefertigte Unterstand diente maltesischen Landwirten zum Lagern von Gerätschaften zur Feldbearbeitung sowie als Unterschlupf bei schlechtem Wetter. Die Girnas sind nahezu ausschließlich in Maltas Nordwesten zu finden und bilden ein wertvolles, regional eng begrenztes Kulturrelikt. Gegenwärtig werden immer mehr Girnas wieder instand gesetzt um als illegale Wochenendhäuser oder Jagdansitze zu dienen.

503 Vgl. hierzu auch TEUMA (2003) 


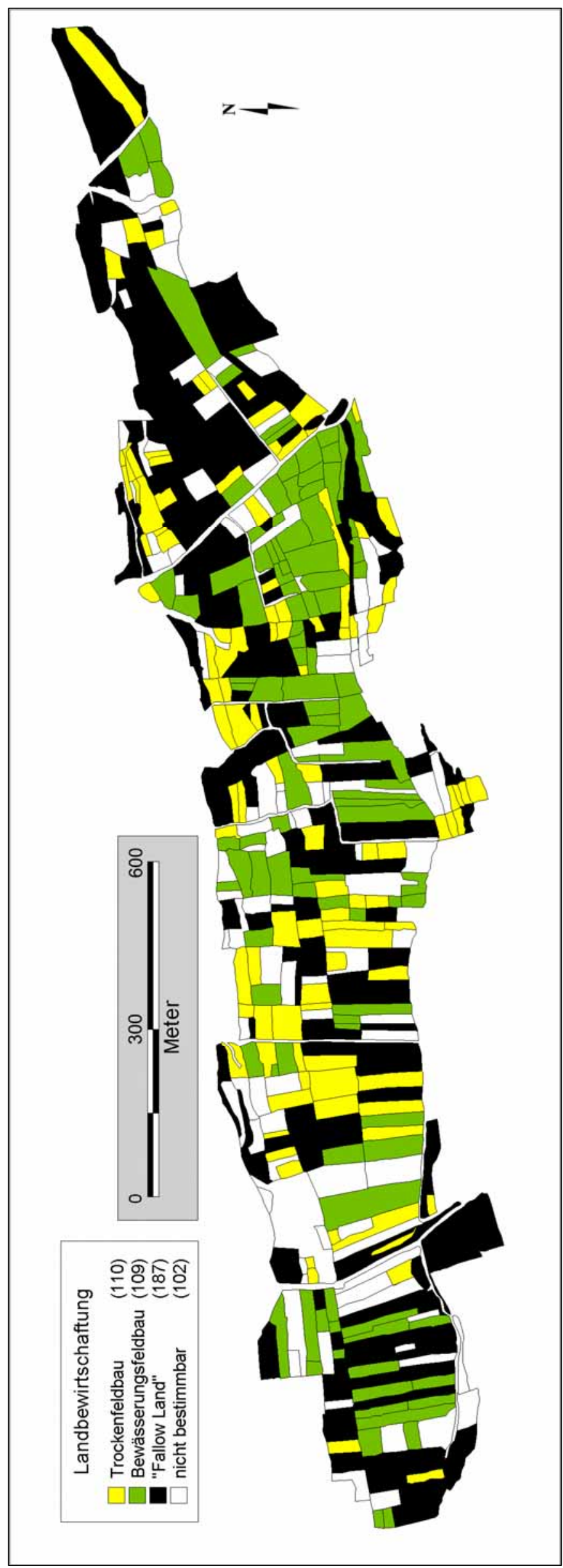

Karte 5: Landbewirtschaftung in IIMiżieb. Alle schwarzen Flächen sind aufgegebene Nutzflächen, die brach gefallen oder aufgegeben worden sind.

Entwurf \& Grafik: AsCHEMEIER (unter Verwendung von mapinfo GIS) 
Die große Anzahl von Brachen in der Region II-Miżieb ist zu hoher Wahrscheinlichkeit dem Kartoffelanbau zuzuschreiben. In Malta gibt es drei Erntezeiten für Kartoffeln im Jahr: Spring crop, Summer crop und Winter crop $^{504}$. Die Frühjahrsernte ist dabei die qualitativ hochwertigste: "The Spring crop [is] sown in December or early January and lifted in March-April. The produce is large and the quality is rich." (AzZOPARDI (2002), S. 162). Aus diesem Grund ist die Frühkartoffel Maltas bedeutendster agrarischer Exportartikel. Doch schon im Dezember wird ein großer Anteil der Jahresernte eingefahren, weil der maltesische Binnenmarkt zum Weihnachtsfest in starkem Maße Kartoffeln nachfrag ${ }^{505}$. Hier sieht der Autor den Hauptgrund für viele Brachen.

Die Gesamterntemenge an Kartoffeln im Jahr 2001 betrug knapp $4 \mathrm{t}^{506}$. Es ist anzunehmen, dass die meisten der in der Kartierung verzeichneten Brachen (vor allem die größeren Flächen) abgeerntete Kartoffeläcker sind. Aber auch die einjährige Brache des Dry Farming erzeugt vorübergehend stillgelegtes Ackerland ${ }^{507}$. Vor allem jedoch die Tatsache, dass noch nicht abgeerntete Felder der Kategorie „Hackfrüchte“ vor allem Zwiebel- und Knoblauchanpflanzungen aufwiesen, stärkt die These, dass es sich bei den Brachen vormals um Kartoffeläcker handelte.

\section{Nutzland}

Im Valley II-Miżieb waren zur Zeit der Geländeaufnahme 243 Flächen mit Feldfrüchten bestellt. 110 davon wurden im traditionellen Trockenfeldbauverfahren bewirtschaftet. Etwa die gleiche Anzahl (109) wurde bewässert.

Die Differenz von 24 Feldern ist eine Anzahl nicht mehr in Nutzung befindlicher Areale, die aber noch nicht verwahrlost waren und somit die vormals angebaute Feldfrucht zweifelsfrei erkennen ließen. Diese Gebiete wurden an dieser Stelle als „Fallow Land“ ausgewiesen, da sie nach Einschätzung des Autors mit verhältnismäßig wenig Aufwand wieder reaktiviert werden könnten. Die meisten dieser Flächen sind in IIMiżieb ehemalige Obstbaumanpflanzungen und Weinbauareale.

Mit insgesamt 69 Nutzflächen stellt der Gemüse- und Leguminosenanbau den größten Anteil an der Landwirtschaft in diesem Valley. Hackfrüchte werden auf 35 Grundstücken angebaut. Geht man davon aus, dass der überwiegende Anteil der 99 Brachen eigentlich zum Anbau von Kartoffeln dient (s. o.), ändert sich der Hauptanteil zugunsten der Hackfrüchte. Die dritt wichtigste Anbaukategorie stellen 51 Getreideflächen dar, von denen rund $50 \%$ bewässert sind. Mit 30 Arealen, davon viele nicht mehr in Nutzung, ist der Anteil des Weinbaus in II-Miżieb durchschnittlich ausgeprägt.

Obstbaumanpflanzungen beschränken sich auf wenige Grundstücke im östlichen Bereich des Tals. Dort ist der Windschutz im Vergleich zum Westen durch den Höhenzug Bajda Ridge im Süden und die Siedlungsausläufer von Mellieћa im Norden gewährleistet.

Folienkulturen kommen auf 13 unterschiedlich großen Arealen vor. Sie dienen in der Region II-Miżieb hauptsächlich der Anzucht von Gemüse.

Nur zwei Grundstücke mit Gewächshäusern sind untypisch wenig für ein verhältnismäßig großes landwirtschaftliches Gebiet wie II-Miżieb. In anderen Tallagen, so z. B. im Binġemma Bassin, befinden sich oft 50 und mehr Gewächshäuser. Dort dominieren sie aufgrund ihrer großen Flächenanteile sogar das Landschaftsbild. Es kann davon ausgegangen werden, dass die absoluten Flächengrößen in II-Miżieb, die durch Realerbteilungsprozesse bereits teils stark zusammengeschrumpft sind, eine Erhöhung des Gewächshausanteils verhindern. Gewächshäuser sind teuer in Anschaffung, Aufbau und Unterhaltung. Sie rechnen sich nur bei adäquaten Flächengrößen. Diese sind im Untersuchungsraum jedoch nur in geringer Anzahl verfügbar.

504 Vgl. AZZOPARDI (2002), S. 162

$505 \mathrm{Vgl}$. TASte Magazine, H. 3/04, S. $16 \mathrm{f}$.

506 Vgl. NSO (2004b), S. 27

507 Vgl. AZZOPARDI (2002), LeSER (1997) 
31 Flächen sind mit der Ausweisung „Sonstiges" gekennzeichnet. Oft handelt es sich dabei um Rapsfelder. Es gab aber auch einige Areale, deren Bewirtschaftung nicht bestimmt werden konnte. Der Grund war in solchen Fällen zumeist ihre eingeschränkte Zugänglichkeit. Einige Grundstücke waren in Gartenbauart aufgeteilt, sodass in unmittelbarer Nähe zueinander kleine Beete aus z. B. Hackfrüchten, Gemüse, Folienkulturen und Obstbäumen vorkamen. Solche Felder wurden ebenfalls mit der Ausweisung "Sonstiges" belegt.

\section{Zusammenfassung}

II-Miżieb ist eine für die maltesische Agrarlandschaft typische Valley-Zone. Neben vielen Brachflächen dominiert der Anbau von Hackfrüchten, Gemüse und Getreide. Alle anderen Nutzpflanzen und Nutzungsweisen spielen nur eine untergeordnete Rolle.

Die Flächengrößen sind im Vergleich zu anderen zusammenhängenden landwirtschaftlich genutzten Regionen im Durchschnitt nur mittelgroß (in den meisten Fällen ca. $50-150 \mathrm{~m}^{2}$ ). Dies könnte ein Grund sein, warum im Vergleich mit z. B. Pwales Valley oder Binġemma Bassin nur wenige Gewächshäuser vorzufinden sind, die üblicherweise zum Landschaftsbild der maltesischen Valleys gehören.

Auffällig ist die Konzentration von Brachland im östlichen Talbereich. Hier beginnen karstige Zonen, die sich bis zur Hauptstraße Xemxija-Mellieћa erstrecken. Viele Obst- und Weinbauflächen sind aufgegeben worden und drohen zur Ausdehnung der Sukzessionsflächen in der Talregion beizutragen. Wie die meisten landwirtschaftlichen Bereiche Maltas braucht II-Miżieb eine Flurbereinigung.

Die Landnutzungsanalyse von II-Miżieb zeigt deutlich, dass die Valleys die Standorte sind, in denen ein Großteil der regionalen traditionellen Produkte der Maltesischen Inseln ihren Ursprung haben. Die Tallagen sind aus heutiger Sicht die produktivsten und professionellsten Anbauregionen der Inselgruppe. Doch selbst sie drohen teilweise zu verfallen. Fast die Hälfte aller Nutzflächen in II-Miżieb werden nicht mehr bewirtschaftet.

Durch besseres Saatgut und wirksamere Düngemittel ist der Verzicht auf Nutzland, das zu klein, zu trocken, zu wenig tiefgründig, etc. ist, kompensierbar geworden. Auf einem Minimum an Fläche kann heute ein Maximum an Ertrag erreicht werden. Die Nachhaltigkeit der regionalen traditionellen Produkte ist aus Sicht der Landnutzungsanalyse des Valleys II-Miżieb noch nicht gefährdet. Die Degradation der Kulturlandschaft aus kulturgeographischer Perspektive schreitet jedoch rasch voran und führt zu einem Verlust alter Anbautechniken und Feldfrüchte.

\subsection{Ergebnisse - Analyse 2: II-Hofra/Mellieha Isthmus}

Erhebung 2 (s. Karte II, Anhang) repräsentiert eine der typischen Anbauzonen der Insel Malta: Es ist die Zone der kleinbäuerlich bewirtschafteten Areale, die vom äußerlichen Anschein her fast in den Bereich „Gartenbau" eingeordnet werden könnten ${ }^{508}$. Interessant an der vorliegenden Landnutzungskartierung ist die Tatsache, dass sowohl kleine und kleinste Flächen für die Eigenversorgung, als auch mittelgroße Flächen für den kommerziellen Anbau im Untersuchungsbereich vorkommen.

Insgesamt sind 154 Grundstücke im Rahmen der Teilanalyse II-Hofra kartiert worden. Die kleinsten Flächengrößen betrugen dabei ca. $6 \mathrm{~m}^{2}$. Die größten Flächen umgrenzten ca. $270 \mathrm{~m}^{2}$. Nahezu alle kartierten Felder sind mehr oder weniger symmetrisch gegliedert, was die landwirtschaftliche Bearbeitung erleichtert.

508 Vgl. auch AsCHEMEIER \& CYFFKA (2004b): Der deutsche Botschafter in Malta GEORg MERTEN bezeichnete im persönlichen Gespräch die Landwirtschaft Maltas ironisch als „kollektive Gartenwirtschaft". Als Karikatur der maltesischen Gegebenheiten taucht der Vergleich mit dem Gartenbau immer wieder auf, z. B. auch bei LUKE (1968). 


\section{Brachen und aufgegebene Flächen}

Unter den vier Teilerhebungen ist II-Hofra die einzige, bei denen die Brachflächen nicht das Landschaftsbild dominieren. Es wurden jedoch unter den 154 kartierten Flächen trotzdem 29 aufgegebene verzeichnet. Das heißt, ca. ein Fünftel der Gesamtfläche ist derzeit unbestellt und könnte nur mit großem Aufwand wieder kulturfähig gemacht werden.

44 Brachen repräsentieren, wie schon bei der vorangegangenen Analyse von II-Miżieb, offenes Ackerland, bei dem die Ernte bereits erfolgt war oder die angebauten Feldfrüchte noch nicht erkennbar waren. Die Flächengrößen dieser Brachen schwanken zwischen $12 \mathrm{~m}^{2}$ und $168 \mathrm{~m}^{2}$. Es ist anzunehmen, dass auf Flächen über $50 \mathrm{~m}^{2}$ durchaus kommerzieller Anbau erfolgt. Vermutlich ist daher ein Großteil der 44 verzeichneten Areale dem Kartoffelanbau zuzuschreiben, bei dem die Ernte bereits im Winter 2003 erfolgt ist. Diese Annahme wird von BOWEN JONES, DEWDNEY \& FISHER's Landnutzungskartierung von 1961 gestützt, in dem die Region II-Hofra als ein Bereich gekennzeichnet ist, in dem der Kartoffelanbau die dominierende Wirtschaftsweise darstellt ${ }^{509}$.

Insgesamt gesehen weist der kartierte Ausschnitt aus der Region II-Hofra eine vergleichsweise gute Bilanz beim Verhältnis von Brache zu Nutzland auf.

Die Theorie, die sich aus diesem Umstand ableiten ließe, ist interessant: Wirkt der kleinbäuerliche Anbau in Malta in größerem Maße landschaftserhaltend als andere Wirtschaftsformen?

Eine genaue Analyse dieses Ansatzes würde den Rahmen der vorliegenden Abhandlung deutlich sprengen. Dennoch kann nach Meinung des Autors davon ausgegangen werden, dass die kleinbäuerliche, gartenbauähnliche Bewirtschaftungsweise Vorteile für die Persistenz eines bestimmten Kulturlandschaftstyps mit sich bringt. Hier sieht der Verfasser einen lohnenden Ansatz für künftige Forschungsvorhaben. Zumindest ist in der Region II-Hofra das Fortschreiten der Sukzessionsfläche deutlich geringer als in allen anderen kartierten Anbauzonen.

Was bedeutet diese Feststellung für die Analyse der Verbreitung und Vermarktung regionaler traditioneller Produkte? Auf kleinen Flächen, die vornehmlich zum Zweck der Selbstversorgung bestellt werden, wird nicht zwangsläufig auf eine wirtschaftlich ausgeglichene Ökonomie zwischen Aufwand und Ertrag geachtet werden müssen. Es ist daher möglich, dass auf kleinen Feldern endemische Anbautechniken und auch Nutzpflanzen (z. B. im Bereich des Weinbaus ${ }^{510}$ ) erhalten bleiben. Dies stellt eine Bereicherung unter den regionalen traditionellen Agrarprodukte dar. Auf diese Weise werden auch gegenwärtig noch Feldfrüchte angebaut, die im Rahmen einer vollständig ökonomisch orientierten Landwirtschaft möglicherweise bereits „ausgestorben“ wären. Im folgenden Abschnitt sollen daher die vorkommenden Nutzpflanzenkategorien genauer betrachtet werden:

\section{Nutzland}

Zieht man die oben beschriebenen Brachflächen von der Gesamtzahl der Anbauareale ab, bleiben 81 Felder, die mit Nutzpflanzen bestellt waren. Der überwiegende Teil davon (70) unterliegt dem aufwändigen Bewässerungsfeldbau (vgl. Karte 6). Im traditionellen Trockenfeldbauverfahren werden nur 11 Flächen bewirtschaftet. Das ist der niedrigste Wert aller vier Teilkartierungen.

Die dominierende Nutzungskategorie ist "Gemüse und Leguminosen". Auf 40 Feldern II-Fofras werden Pflanzen dieser Einstufung angebaut, 38 davon sind bewässert. Vor allem auf den kleinen und kleinsten Flächen dominiert der Gemüse- bzw. Leguminosenanbau. Dies legt nahe, dass der Anbau vornehmlich der Eigenversorgung dient.

Auf 21 Arealen sind Hackfrüchte im Anbau vorzufinden, vor allem Zwiebeln und Knoblauch. Auch diese Flächen sind in fast allen Fällen bewässert und weisen mittlere bis größere Flächenmaße auf. Wie bereits im vorausgegangenen Abschnitt angesprochen, ist auch hier davon auszugehen, dass ein großer Teil der Brachen dem Anbau von Hackfrüchten dient. 
Alle anderen Landnutzungskategorien spielen kaum eine Rolle. Es gibt 6 Folienkulturen und jeweils zwei Getreidefelder, kleine vineyards, Obstbaumanpflanzungen und Gewächshäuser, die jedoch nur auf kleinen Flächen von $14 \mathrm{~m}^{2}$ bzw. $29 \mathrm{~m}^{2}$ untergebracht sind und somit ehrgeizigen kommerziellen Interessen nicht genügen dürften.

Auf den 6 Arealen, die mit der Bezeichnung „Sonstiges" ausgewiesen sind, wird Raps angebaut.

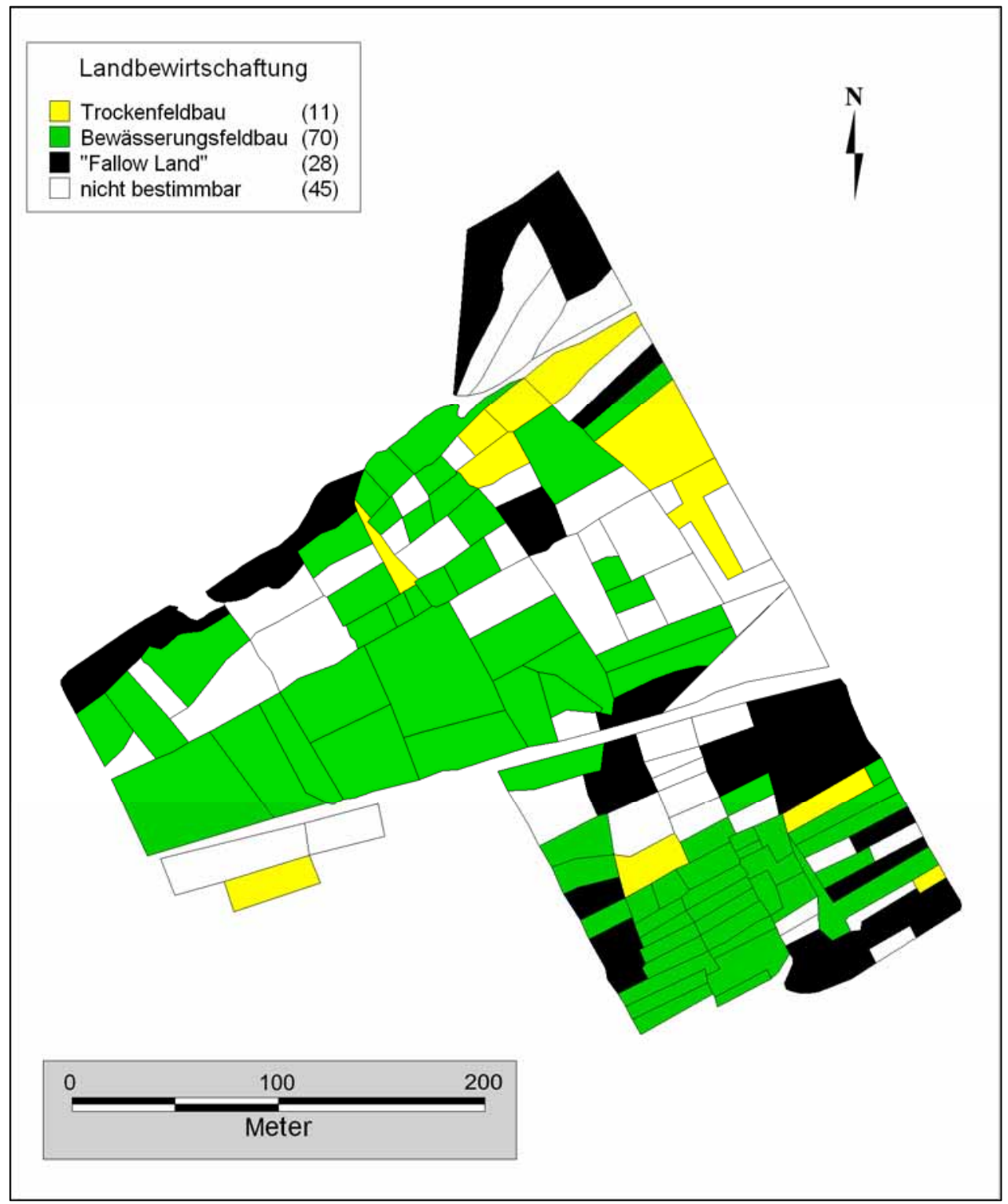

Karte 6: Landbewirtschaftung in II-Hofra: Viel Bewässerungsfeldbau. Der Kartierungsbereich umfasst die niedrigsten Trockenfeldbau- und Bracheanteile aller vier Teilanalysen. Entwurf \& Grafik: ASCHEMEIER (unter Verwendung von MapInfo GIS)

\section{Zusammenfassung}

II-Hofra ist ein typisches Beispiel für eine kleinbäuerliche Anbauzone, so wie sie in Malta vor allem im Nordwesten (Marfa Ridge, Mellieћa Isthmus) und rund um Dingli, die Dingli Cliffs und Buskett Gardens vorgefunden werden kann.

Der Anteil aufgegebener Flächen dieser Teilaufnahme ist der niedrigste von allen in dieser Arbeit vorgestellten Landnutzungsanalysen. Gleichzeitig ist der Anteil von Flächen des Bewässerungsfeldbaus in IIHofra am höchsten. Gemüse, Leguminosen und Hackfrüchte sind die dominierenden Feldfrüchte im Anbau. Alle anderen Anbaupflanzen sind nicht von Bedeutung. 
II-Hofra kann trotz seiner kleinen Flächengrößen und seiner insgesamt begrenzten Ausmaße als Zone regelmäßiger und intensiver Landbewirtschaftung gedeutet werden. Der Brachlandanteil konnte zugunsten vieler kleiner bewirtschafteter Flächen bisher gering gehalten werden. Als Kulturlandschaftstyp wirkt die Region durchaus auch optisch ansprechend, zumal die kleinen Grundstücke in diesem Bereich Maltas noch mit den traditionellen Rubble Walls ${ }^{511}$ (auch in Tallage) umgrenzt werden. Die Rubble Walls sind in II-Hofra häufig in gutem Zustand und werden regelmäßig gepflegt. II-Hofra, in direkter Nähe zum touristischen Vergnügungspark „Popeye Village" gelegen, ist daher als Kulturlandschaft auch für den Fremdenverkehr interessant.

Die Bewässerung vieler kleiner Areale, die zudem häufig noch mit alten Methoden bewerkstelligt wird (z. B. großflächige, offene Wassertanks, Windpumpen und Zisternen) ist allerdings ein transpirationsintensiver Anbau. Dies stellt bei Maltas Süßwasserknappheit ein ernsthaftes Problem dar und führt dazu, dass die Landwirtschaft Maltas zusammen mit der Industrie der größte Wasserverbraucher ist ${ }^{512}$. In dieser Beziehung repräsentieren kleine bewässerte Felder im Vergleich zu großen einen Umweltnachteil.

Die These am Ende dieser Teilanalyse lautet: Kleinbäuerlicher Anbau wirkt kulturlandschaftserhaltend. Im Bereich der regionalen traditionellen Produkte bedeutet das: Nicht nur ein agrarisches Produkt und/oder seine Ausgangsstoffe sind gesichert, sondern es werden oft auch alte Nutzpflanzensorten in den Anbau integriert, die in der konventionellen Landwirtschaft kaum noch eine Chance haben.

Hier sieht der Verfasser einen lohnenden Forschungsansatz für künftige Projekte.

Kleinbäuerliche Bereiche wie II-Hofra sind deswegen wichtig, weil durch sie die Existenz zahlreicher agrarisch geprägter traditioneller regionaler Produkte gefördert und ermöglicht wird. Der kleinbäuerliche Weinbau z. B. ist ein wichtiger Gen-Pool für alte endemische Sorten. Dennoch sind alle regionalen traditionellen Produkte des maltesischen Nahrungsmittelsektors auch ohne den kleinbäuerlichen Anbau denkbar. Soltten die "gartenbauähnlichen" Strukturen dieser Bewirtschaftungsmethode jedoch eines Tages ganz wegfallen, ist auch die Vielfalt der regionalen traditionellen Produkte gefährdet.

\subsection{Ergebnisse - Analyse 3: Fort Binġemma}

Die nördliche Randzone der Santi Binġemma Uplands ist eine typische Zone des Anbaus auf flachen bis mäßig steilen Hangterrassen, wie er in Malta und Gozo an vielen Standorten vorkommt. Als Ausläufer des agrarisch intensiv genutzten Binġemma Bassins im Westen der Insel bei Mǵarr sind auch die Santi Binġemma Uplands noch zu weiten Teilen vom landwirtschaftlichen Anbau geprägt. Das Untersuchungsgebiet für Erhebung 3 (vgl. Karte III, Anhang) befindet sich am der JohanniterBefestigungsanlage „Fort Binġemma" nordwestlich gegenüberliegenden Hang.

Die Region gilt als einer der witterungs- und windexponiertesten Standorte der Maltesischen Inseln. Dies wirkt sich auf die agrarischen Nutzungsweisen aus: „In general, the quality of farming in this region is affected by remoteness and exposure. (...) It is the wildest and most isolated district of Malta. "(BOWEN JONES et al. (1961), S. 251). Einerseits ist die Wasserversorgung durch natürliche Niederschläge gesichert, andererseits ist die Gefahr der Transpiration und auch der direkten Pflanzenschädigung durch den extremen Windeinfluss sehr hoch. Möglicherweise liegen hier die Gründe, warum von 127 insgesamt in der Teilerhebung kartierten Flächen keine einzige bewässert war.

Die Flächengrößen der hier vorgestellten Anbauzone liegen im Bereich von $\sim 10 \mathrm{~m}^{2}$ bis $\sim 280 \mathrm{~m}^{2}$. Somit sind sie auf den ersten Blick vermeintlich mit denen in II-Hofra vergleichbar. Die strukturelle Zusammensetzung ist jedoch grundlegend anders: Im Bereich Fort Binġemma dominieren die größeren Nutzflächen, während in II-Hofra eindeutig kleine und kleinste Areale die Mehrheit stellen.

$511 \mathrm{Vgl}$. ASCHEMEIER \& CYFFKA (2004a \& 2004b)

512 Vgl. KRetschmeR (1980); Bendix, Bendix \& Reudenbach (2002); Aschemeier \& CyFFKA (2004a \& 2004b) 


\section{Brachen und aufgegebene Flächen}

Die Santi Binġemma Uplands sind eine Zone des nachhaltig aktiven Anbaus. Nur knapp ein Viertel aller kartierten Flächen (34) gehören zur Kategorie „Aufgegebene Flächen“. Jedoch wurden im Rahmen der Kartierung der Anbauweise noch weitere 12 unbewirtschaftete Felder verzeichnet. Zusammen befinden sich damit derzeit 46 Flächen nicht in Nutzung. Folglich gehört die Region zu den Anbauzonen mit durchschnittlichen bis leicht erhöhten Anteilen an aufgegebem Land.

30 Areale fallen in die Kategorie der Brache, der Kategorie des offenen Ackerlands. Die Brachen in dieser Region sind vermutlich wieder dem in Malta weit verbreiteten Kartoffelanbau zuzuschreiben (s. o.).

\section{Nutzland}

Das Nutzland um Fort Binġemma ist geprägt von der nahen Nachbarschaft des fruchtbaren Binġemma Bassins, in dem Gemüseanbau und Hackfrüchte dominieren. Demzufolge findet man in den Hanglagen des Erhebungsgebiets kaum Flächen mit Gemüse und Hackfrüchten (3 und 7), da es nicht notwendig ist, die empfindlichen Feldfrüchte den extremen Witterungseinflüssen am Hang auszusetzen.

Auch Wein spielt mit 6 Grundstücken, davon 4 z. Zt. nicht bewirtschaftet, kaum eine Rolle. Doch der Getreideanbau ist mit 26 Feldern stark vertreten. Fast ebenso viele Areale, nämlich 21, beansprucht der Obstanbau. Bei beiden Kategorien waren jedoch jeweils 4 Felder in verwahrlostem Zustand, wonach man von 22 effektiv bewirtschafteten Arealen beim Weizenanbau bzw. von 17 Grundstücken in der Obstanpflanzung sprechen kann.

Getreideanbau auf großflächigen Hangterrassen ist ein vertrautes Bild auf den Maltesischen Inseln: Die ertragreichen Böden der Tallagen sind zumeist mit Gemüse, Leguminosen und Hackfrüchten bestellt. Die weniger tiefgründigen, steinigen Böden der Hangterrassen werden dagegen vom Getreideanbau geprägt.

Der verstärkte Obstanbau ist jedoch eine spezifische Besonderheit der an dieser Stelle vorgestellten Teilkartierung. Literatur und Statistiken weisen für die Insel Malta allgemein nur mäßig intensiven Obstanbau aus $^{513}$. Da die Region, in der kartiert wurde, zu den windigsten der gesamten Insel gehört (vgl. o.) und der Hang, an dem kartiert wurde, in einer windstillen Nische lag, ist anzunehmen, dass sich im Rahmen dieser Teilkartierung der Obstanbau überproportional stark ausgeprägt darstellt und vermutlich eher die Ausnahme als die Regel repräsentiert.

Keine Nutzfläche war bewässert, auch nicht der Obstanbau. 52 Areale wurden im Trockenfeldbauverfahren bewirtschaftet, 30 Flächen waren Brachen und konnten nicht erhoben werden und 45 Flächen wurden in der Kartierung als „Fallow Land" ausgewiesen (vgl. Karte 7). 


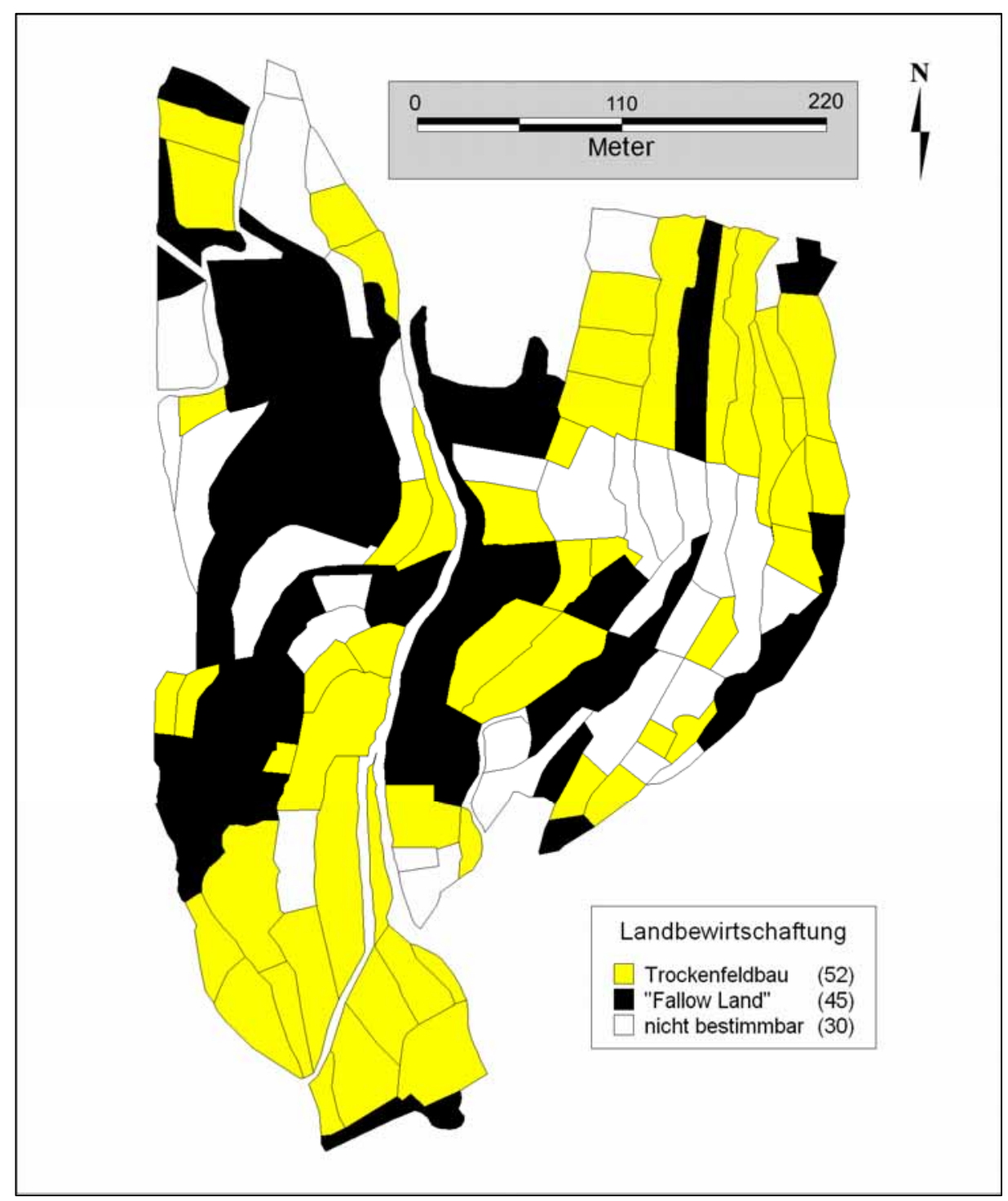

Karte 7: Landbewirtschaftung am Rande der Santi-Binġemma Uplands, nordwestlich von Fort Binġemma. Es konnte kein Bewässerungsfeldbau verzeichnet werden. Entwurf \& Grafik: ASCHEMEIER (unter Verwendung von MapInfo GIS)

\subsection{Ergebnisse - Analyse 4: Ǵnien Busewdien}

Die Teilerhebung von Ǵnien Busewdien (vgl. Karte IV, Anhang) wurde nordwestlich der Ortschaft Bidnija am Südhang des Wardija Plateaus, ca. $700 \mathrm{~m}$ südwestlich von Wardija Hilltop Village erhoben. Während das Plateau von Wardija Ridge ein ausgesprochen karstiger Bereich ist, der kaum in größerem Maße landwirtschaftliche Aktivitäten zulässt, sind die Hänge terrassiert und fruchtbar, weisen aber grobe, steinige Böden auf. Die 85 kartierten Areale des Ǵnien Busewdien zeigen Flächengrößen von ca. $30 \mathrm{~m}^{2}$ bis $550 \mathrm{~m}^{2}$ und liegen für maltesische Verhältnisse damit im mittelgroßen Bereich.

Der Begriff „Ǵnien" bezeichnet im Maltesischen ursprünglich eine Zone intensiver Nutzung - ähnlich intensiv wie man es sonst nur in den Valley- und rdum-Bereichen vorfindet. Ein Ǵnien muss jedoch als "Landnutzungs-Oase" in einer für den landwirtschaftlichen Anbau weitgehend ungeeigneten Umgebung verstanden werden ${ }^{514}$. Dass diese Einordnung vielfach nicht mehr in der Form zutrifft, wie BOWEN JONES, DEWDNEY \& FISHER dies 1961 für die Ǵnien-Bereiche definiert hatten, zeigt die an dieser Stelle vorgestellte 
Teilkartierung des Ǵnien Busewdien. Die vorliegende Darstellung stützt infolgedessen auch die Untersuchung von CYFFKA (2005), der "Land Use-Change“ als Ursache für jüngste Degradierungserscheinungen ehemaliger agrarischer Flächen verantwortlich macht.

\section{Brachen und aufgegebene Flächen}

Der landwirtschaftliche Anbau ist im Bereich Ġnien Busewdien starken Einschränkungen unterworfen. Durch die Steilheit des Hangs, der kein flach auslaufendes Relief aufweist wie z. B. in Analyse 3 (Fort Bingemma), sind die Hangterrassen in besonderem Maße durch Rutschungen gefährdet. Im Spätsommer 2003 zerstörte ein Unwetter mit Starkregen landwirtschaftliche Nutzflächen in der Region Wardija/Burmarrad (vgl. Fotos 14 $\& 15)$.

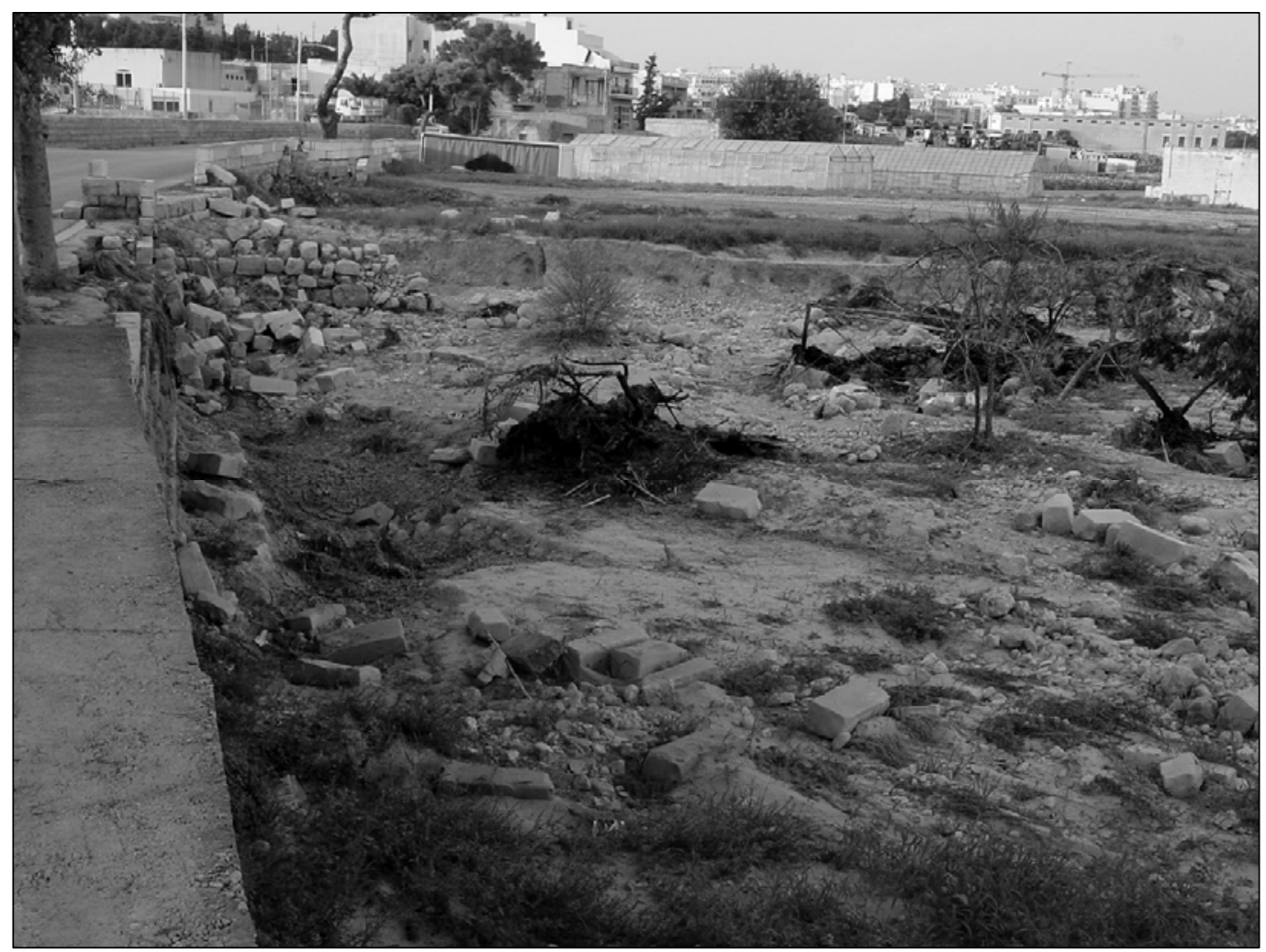

Foto 14: Schäden des Unwetters vom Oktober 2003 am Rand der Ortschaft Burmarrad. Selbst mit Mörtel gefugte Straßenbegrenzungsmauern wurden weggespült. Dahinter eine vernichtete Obstbaumanpflanzung. Der Landwirt hat die Überreste seiner vom Wasser entwurzelten Bäume abgebrannt. Foto: ASCHEMEIER 


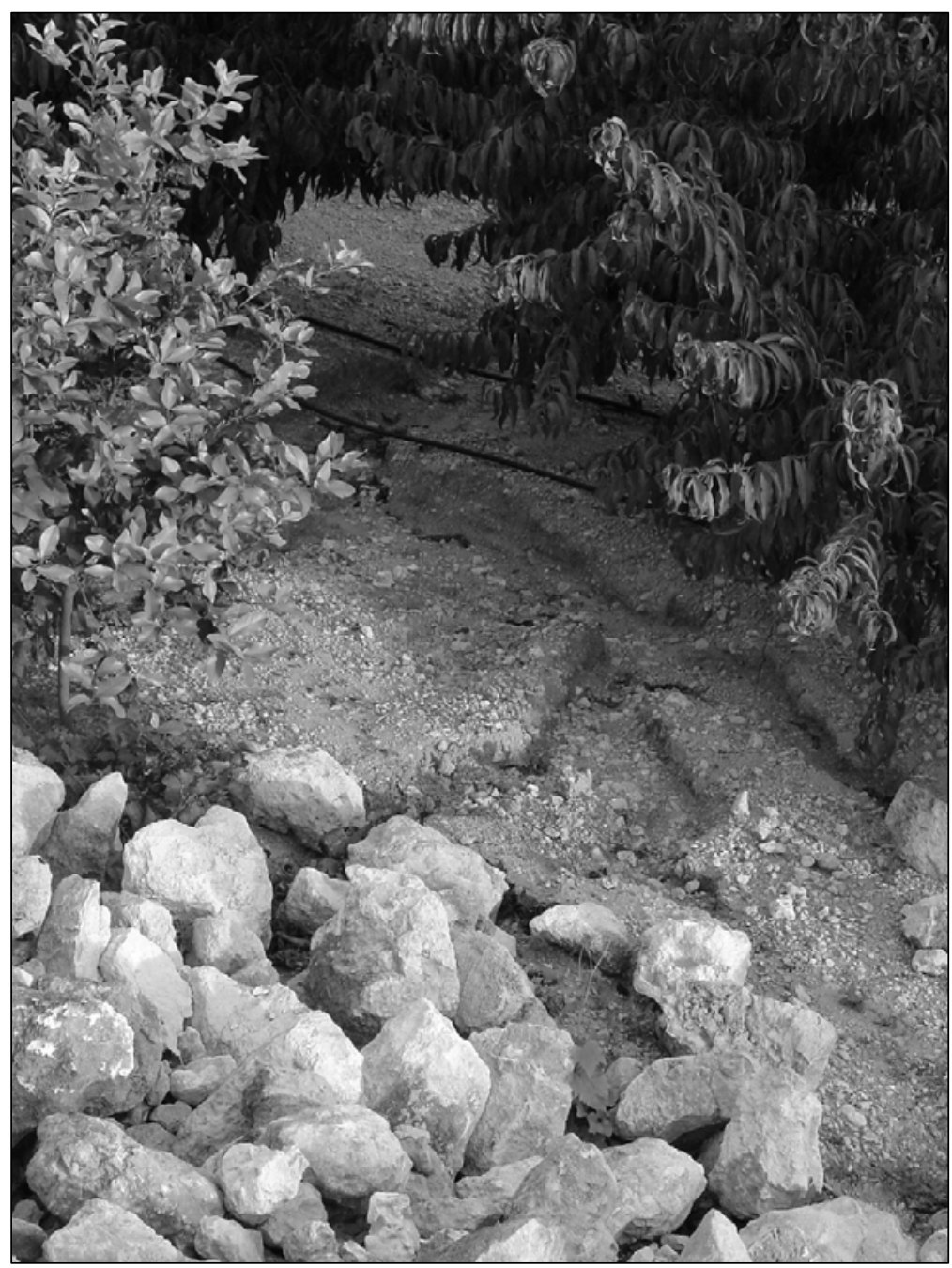

Foto 15: Erosionsrinnen auf einer Hangterrasse in der Nähe von Ġnien Busewdien nach dem Unwetter im Oktober 2003. Im Vordergrund eingestürzte Rubble Wall. Foto: ASCHEMEIER

Des Weiteren müssen die agrarisch genutzten Hangterrassen in ihrer Form isohypsenparallel dem Hangverlauf angepasst werden, was zu schlecht bearbeitbaren, asymmetrischen Feldern führt. Der Anteil der aufgegebenen Flächen ist daher groß: Von den 85 landwirtschaftlichen Flächen insgesamt im Ǵnien Busewdien wurden 25 Areale kartiert, die als ,verlassen“ klassifiziert werden mussten. Das sind mehr als ein Drittel aller Felder, wobei 23 davon in einem so schlechten Zustand waren, dass keine dominierende Feldfrucht mehr erkannt werden konnte.

Im Fall des Ǵnien Busewdien sind auch die als „Sonstiges“ bezeichneten Flächen vermutlich als verlassen anzusehen. Bei der Geländeaufnahme konnte im Fall der vier Areale dieses Typs nicht bestimmt werden, ob es sich um geregelten Anbau handelte oder nicht. Die Flächen waren in schlechtem Zustand und am Übergang zur Verwilderung. Nichtsdestotrotz lag die letzte Landbearbeitung vermutlich noch kein ganzes Jahr zurück.

Die aufgegebenen Areale konzentrieren sich vor allem auf die Mitte des Hangbereichs, in dem eine steile Abflusskerbe den Anbau unmöglich macht. Doch auch in der östlichen Hangregion, sogar nahe der Landwirtschaftsstraße, finden sich in zunehmendem Maße aufgegebene Nutzflächen.

Die Anzahl von 20 Landwirtschaftsbrachen gliedert sich in den vorherrschenden Getreideanbau ein. Es ist also möglich, dass diese Areale brachliegende Getreidefelder sind. Diese Annahme wird gestützt durch BOWEN JONES et al., die Ǵnien Busewdien 1961 als Zone intensiven Getreideanbaus auswiesen ${ }^{515}$. Es wäre 
jedoch auch möglich, dass es sich bei den Brachflächen um Felder handelt, die gegenwärtig für den Weinbau vorbereitet werden: Im Zuge der Geländeaufnahme ergab sich der Eindruck, dass viele der im Ġnien Busewdien kartierten vineyards frisch angelegt bzw. wiederhergestellt worden waren.

\section{Nutzflächen}

Getreideanbau (17 Flächen) und Weinbau (12 Flächen, davon allerdings 3 nicht in Nutzung) dominieren das Bild der landwirtschaftlichen Zusammensetzung im Ǵnien Busewdien. Alle weiteren Kategorien spielen kaum eine Rolle und sind vermutlich der Eigenversorgung zuzurechnen. Der Gemüseanbau beansprucht vier Areale, Hackfrüchte werden auf drei Flächen angebaut und auf zwei Grundstücken finden sich kleinere Gewächshäuser.

Führt man sich diese Landnutzungszusammensetzung vor Augen, ist es verständlich, dass der Trockenfeldbau mit 24 Flächen zu 12 bewässerten Arealen (vgl. Karte 8) die vorherrschende Anbauweise darstellt. Bewässert werden hauptsächlich die vineyards und die Gewächshäuser. Getreide und Hackfrüchte werden vollständig im Trockenfeldbauverfahren bewirtschaftet.

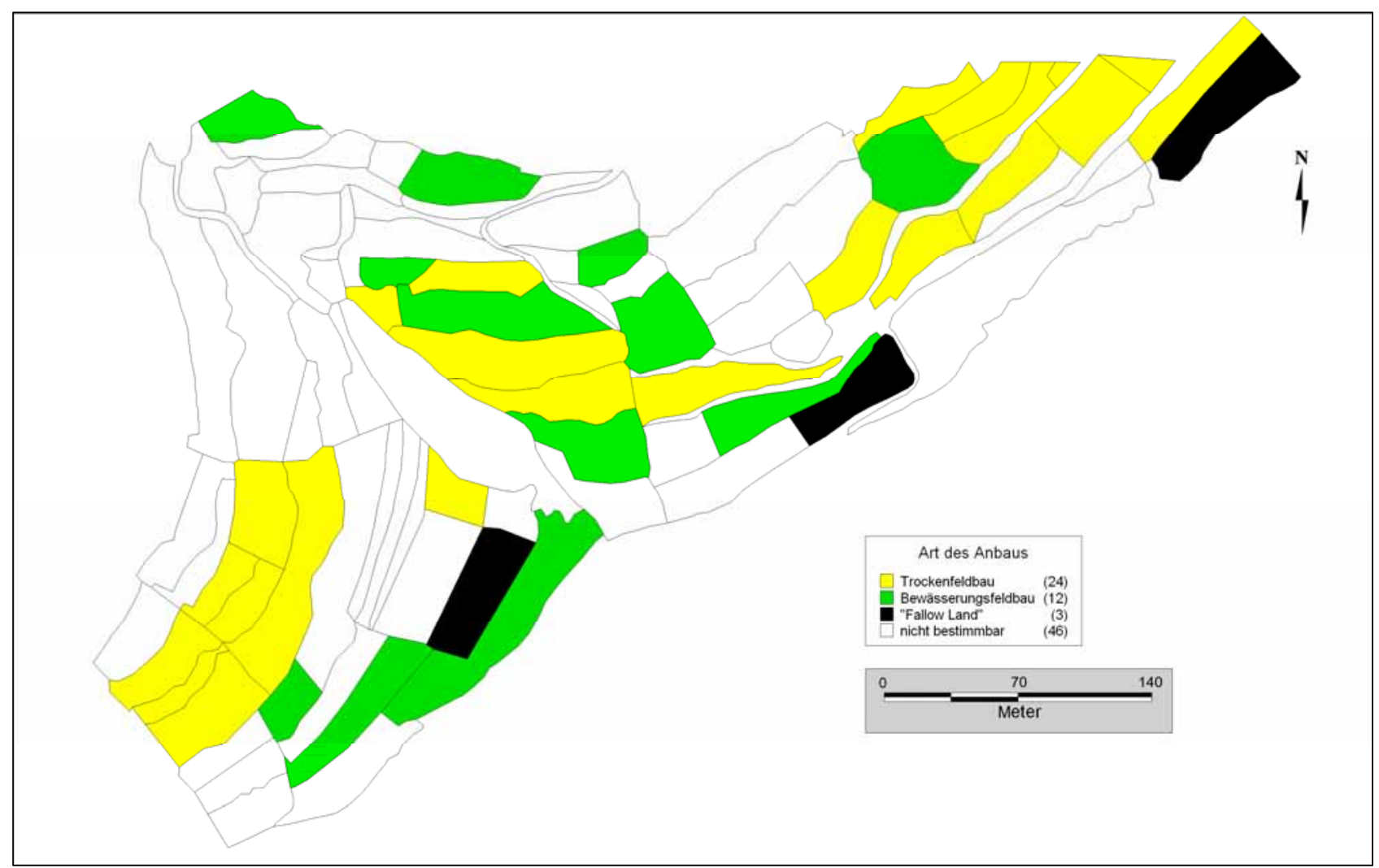

Karte 8: Landbewirtschaftung im Bereich Ġnien Busewdien. Wenig „Fallow Land“, dafür ein großer Anteil Brachland sind erkennbar. Entwurf \& Grafik: AsCHEMEIER (unter Verwendung von mapinfo GIS)

Für die regionalen traditionellen Produkte der Maltesischen Inseln ist Ǵnien Busewdien ein vergleichsweise unproduktiver Bereich. Getreide wird in Malta seit Jahrzehnten fast ausschließlich verfüttert. In die Backwarenproduktion geht nur ein verschwindend geringer Anteil ${ }^{516}$. Die vorherrschende Feldfrucht dieser Anbauzone ist also von der Produktion regionaler traditioneller Produkte völlig ausgeschlossen.

Lediglich die vineyards, die besonders in den oberen Hangbereichen überwiegend großflächig und in gutem Zustand vorliegen, lassen erahnen, dass hier Weintrauben für den Verkauf erzeugt werden. U.U. dient die Produktion auch der Erzeugung von Tafel- bzw. Landweinen, die von der maltesischen Farmer's Coop. vertrieben werden. 


\section{Maltas Produzenten regionaler traditioneller Produkte}

Maltesische Produkte, speziell solche mit traditionellem und regionalem Hintergrund wie sie in dieser Arbeit behandelt werden, entspringen einer unüberschaubaren Anzahl von Privaterzeugern, Kleinbetrieben, aber auch veritablen Konzernen. Es erscheint daher zunächst schwierig, einen allgemeinen Überblick zu geben.

Einer der wichtigsten Teilbereiche in der Vielfalt der regionalen traditionellen Produkte Maltas ist der Lebensmittelsektor. Dies ist im Erhebungsteil der vorliegenden Abhandlung klar geworden ${ }^{517}$.

Die Erzeugung von Lebensmitteln aus landwirtschaftlichen Ausgangsprodukten des inländischen Anbaus wird in Malta als ein Teil der sog. Agro-Industry betrachtet ${ }^{518}$. Trotz der verwirrenden Situation im Herstellerbereich, in dem Hunderte von maltesischen Familien und Heimerzeugern in teilweise minimalen Ausmaßen am Markt partizipieren, gehört der Lebensmittelsektor zu den profitabelsten Teilbereichen in Maltas Ökonomie. „Utilising the yardstick of the ratio of gross trading profits to value added, the Central Bank of Malta Annual Report for 1991 outlines that the second most profitable sector (after machinery and equipment) was the food, beverages and tobacco sector with an estimated gross profits ratio of $0.65^{\prime}$." (MIzzI (1993), S. 93).

Nicht minder verwirrend ist die Lage im Bereich des maltesischen Kunsthandwerks. Auch in diesem Sektor dominieren Klein- und Kleinstbetriebe, die sich überall in Gozo und Malta etabliert haben. Allerdings ist im Kunsthandwerkssektor eine räumliche Konzentration auf die Crafts Villages Ta'Qali (Malta) und Ta'Dbieġi (Gozo) zu beobachten. Das älteste Crafts Village Ta'Qali wurde von der Maltesischen Regierung Mitte der 1970er-Jahre ins Leben gerufen ${ }^{519}$. Ta'Qali und Ta'Dbieǵi entstanden auf ehemaligen Militärflughäfen der britischen Kolonialregierung und sind heute die unbestrittenen Zentren für maltesisches Kunsthandwerk, wenngleich ihr Angebot in der Hauptsache touristisch orientiert ist und der Verkauf von Souvenirs vorherrscht. Ta'Qali ist dabei zweifelsfrei die kommerziell wichtigere Einrichtung und wird regelmäßig auf den Sightseeing Trips der großen Reiseveranstalter aufgesucht. Ta'Dbieġi führt dagegen vergleichsweise ein Schattendasein, liegt etwas abseits der touristischen Routen, und kann, vom kommerziellen Standpunkt aus gesehen, nicht mit Ta'Qali verglichen werden. Es ist daher nachvollziehbar, dass sich in Gozo auch in der Hauptstadt Victoria noch viele Handwerksläden befinden, während in Malta Ta'Qali klar das Handwerkszentrum der Insel darstellt, neben dem Geschäfte in anderen Teilen der Insel deutlich im Nachteil sind.

Diese Entwicklung hatte mittelbare und unmittelbare Auswirkungen auf den Kunsthandwerkssektor der Maltesischen Inseln: Während vor der Etablierung der Handwerksdörfer und der damit gebündelt auftretenden touristischen Nachfrage kaum über eine Massenproduktion von Kunsthandwerksartikeln nachgedacht wurde, stellt sich seit einigen Jahren vielfach die Situation ein, dass es vermehrt zu maschinell erzeugter oder aus dem Ausland importierter Billigware komm ${ }^{520}$. Dies umfasst nahezu alle Handwerksbereiche.

Die Konzentration der maltesischen Handwerksbetriebe in den Crafts Villages macht es außerdem möglich, dass billig erzeugte Massenware in unmittelbarer räumlicher Nähe zu hochqualitativen Artikeln angeboten wird.

Profitieren können davon in der Regel die Anbieter von Billigwaren, weil sie durch diese ungewöhnliche Angebotssituation in der Lage sind, an der „exklusiven Aura“ der Qualitätsanbieter in direkter Nachbarschaft zu partizipieren. Dabei sind Qualitätsunterschiede zweifellos erkennbar, jedoch wird hierbei die oft (zu) unkritische Kaufbereitschaft der Touristen ausgenutzt, die im flüchtigen Augenblick - zudem oft in Eile bei

517 Vgl. Kap. 14

518 Vgl. Mizzı (1993)

519 Vgl. MALTA TODAY, Onlineausgabe vom 16.02.2001

520 Derartige Angaben liegen dem Autor in Form von ihm im persönlichen Interview aufgezeichneten Aussagen bestimmter Kunsthandwerker vor. Um diese Handwerker zu schützen und teilweise auf persönlichen Wunsch der betreffenden Personen, können an dieser Stelle weder Zitate noch detaillierte Quellenangaben zur Thematik erfolgen. Der Autor bittet um Verständnis. Es sei in diesem Zusammenhang auch auf die Arbeiten von MARKWICK (1999) und BESENTHAL (1994) verwiesen, die Ähnliches andeuten. Auch diese Autorinnen verzichteten in vergleichbaren Fällen auf Quellenangaben und/oder Zitate. 
ihrem Einkauf, weil sie sich im Crafts Village nur auf einem Zwischenstopp einer Sightseeing-Busrundfahrt befinden - nicht zwischen Handarbeit und maschinell erzeugter Ware unterscheiden wollen oder können.

Dies ist auch der Grund, warum einige Hersteller sich bewusst nicht in den Crafts Villages angesiedelt haben. In den meisten Fällen handelt es sich dabei um Künstler oder Kunsthandwerker mit qualitativ sehr hochwertigen und eigenständigen Produkten. Mit der Ausweisung von Ta'Qali und Ta'Dbieġi als Crafts Villages konnte der kränkelnde Kunsthandwerkssektor in Malta wiederbelebt werden ${ }^{521}$. Allerdings geschah dies auf Kosten von Originalität und oft auch Qualität der angebotenen Erzeugnisse. Künstler wollen sich zunehmend nicht in der Umgebung von Ta'Qali und Ta'Dbieǵi präsentieren. Sie bevorzugen exklusivere Standorte wie Mdina, die Nähe von St. Anton Gardens mit seinen ihn umgebenden exklusiven Wohnvierteln oder schlicht ihr Heimatdorf, um sich von dem "Qualitäts-Misch-Masch" der Crafts Villages bewusst abzuheben ${ }^{522}$.

\subsection{Einteilung nach Herstellergruppen}

Es gilt also festzuhalten: Der Pool der Produzenten, aus denen sich der maltesische Markt zusammensetzt, ist vielgestaltig. Er lässt sich jedoch anhand bestimmter Merkmale kategorisieren.

Im Folgenden werden deswegen Herstellergruppen unterschieden. Nach Einschätzung des Autors muss in Malta für den Bereich der regionalen traditionellen Produkte von vier Hauptgruppen ausgegangen werden:

1. Einzelpersonen oder kleinste (teils informell organisierte) Familienbetriebe

2. Einzelpersonen, Familien- und Kleinbetriebe, die in Verbänden organisiert sind

3. Betriebe mit mindestens 10 Angestellten und mehr

4. „Alternative Hersteller“ mit eigenem Geschäftskonzept sowie Künstler

Die einzelnen Schnittmengen werden in den folgenden Abschnitten erläutert.

521 Vgl. MARKWICK (1999)

522 Vgl. z. B. Bildhauer JOE XUEREB, der sein Atelier völlig abgelegen von touristischen Routen in seinem gozitanischen Wohnort Ghajnsielem hat. Vgl. auch Experteninterview mit JOE XUEREB, vom 13.05.2004. 


\subsubsection{Gruppe 1: Einzelpersonen oder kleinste Familienbetriebe}

Zwar hat es den Anschein, dass dieser Gruppe die meisten maltesischen Produzenten angehören, vielmehr ist es aber so, dass die meisten Kunsthandwerker und Lebensmittelhersteller als Mitglieder in der Datenbank des Malta Crafts Council eher zu Gruppe 2 zu zählen sind.

Gruppe 1 umfasst also ausschließlich diejenigen Hersteller, die ihre Produkte hauptsächlich als „Hobby“ oder Nebenerwerb erzeugen. Diese Waren kommen entweder gar nicht oder nur in sehr kleinen Mengen auf den Markt, von einem Export ganz zu schweigen.

Hersteller dieser Gruppe sind in der Regel ältere Menschen, die ihr Handwerk zum Zeitvertreib ausüben und sich damit „die Rente aufbessern“. Diese Herstellergruppe ist heute fast nur noch in Gozo zu finden. Zu ihr zählen viele Herstellerinnen von Klöppelei- und Webarbeiten, die sog. Lace Ladies (vgl. Foto 16). Man kann sie in Victoria auf Gozo von Zeit zu Zeit an der Straße sitzen sehen, wo sie ihre Handarbeiten ausführen und Touristen gerne ihre Fragen beantworten. Im Gegensatz zur Literatur der letzten 20 Jahre, die ein graduell ansteigendes Verschwinden der Spitzenherstellung annimmt, muss im Moment eher von einer „Wiederentdeckung“ dieses traditionell maltesischen Handwerkszweigs ausgegangen werden ${ }^{523}$.

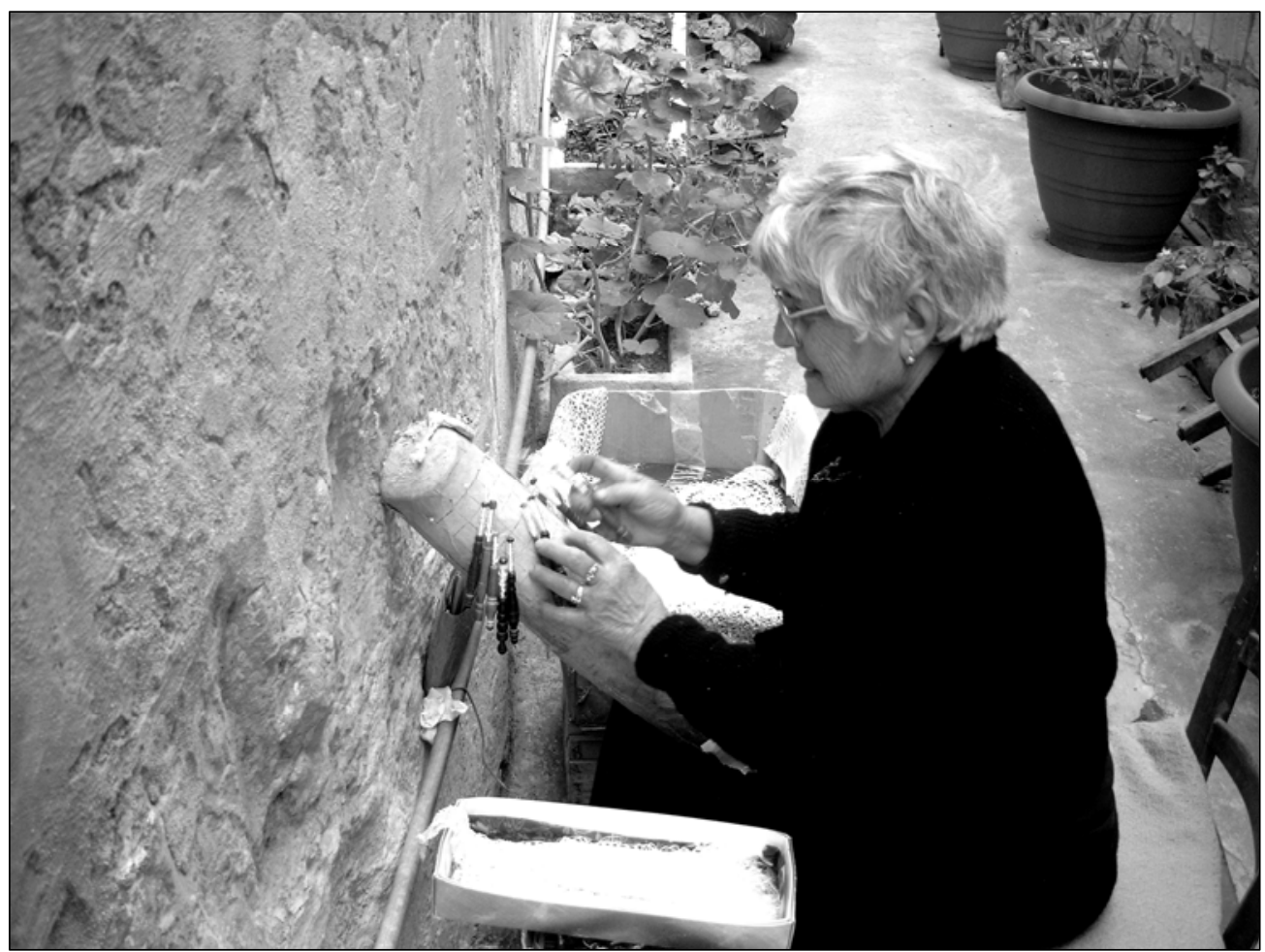

Foto 16: „Lace Lady“ bei der Arbeit. Eine typische Szene im Straßenbild von Victoria auf Gozo. Foto: ASCHEMEIER

Auch die Jugend engagiert sich wieder in der Herstellung von Spitze, was sicherlich dadurch bedingt sein dürfte, dass die Universität Malta (Abteilung Gozo) seit einigen Jahren Kurse zur Erlernung und Verfeinerung dieses Handwerks anbietet, die auf Wunsch auch benotet und zertifiziert werden.

Trotzdem bleibt Gruppe 1 die Gruppe der Eigenversorger und Hobbyhandwerker. Da jüngere Geschäftsleute sehr wohl das Logo des Malta Crafts Council auf ihren Artikeln zu schätzen wissen und sich durch ihre Handarbeit auch einen nicht unerheblichen Nebenverdienst erhoffen, sind sie in Gruppe 1 meist nicht zu finden. Die Gruppe ist somit eine völlig unterrepräsentierte Kraft in der Erzeugung regionaler traditioneller Produkte und spielt für die vorliegende Arbeit ökonomisch eine untergeordnete Rolle. 
Gruppe 1 ist jedoch aus persönlicher Einschätzung des Autors in anderer Hinsicht wichtig, weil sich in dieser Gruppe alte Herstellungsmethoden und Rezepte bis heute erhalten haben, die aufgrund kommerziell geringer Nachfrage nach speziellen Artikeln durch den touristischen Markt u.U. dem Vergessen preisgegeben sind. Beispiele hierfür finden sich bei BORG (2000 \& 2001), der als einer von wenigen maltesischen Autoren erkannt hat, dass es sich lohnt, die Lebenserinnerungen von Handwerkern aufzuschreiben und für nachkommende Generationen zu erhalten. Sehr aufwändige und teure Muster im Klöppeleibereich, die in der Vergangenheit oft als traditionelles Hochzeitsgeschenk von der Familie hergestellt oder in Auftrag gegeben wurden, werden heute nicht mehr produziert ${ }^{524}$. Oft fehlt jede schriftliche Aufzeichnung über die Methode ihrer Herstellung und den Hintergrund ihrer Geschichte. Dieses „Erbe“ ist in Herstellergruppe 1 noch lebendig und kann nach Einschätzung des Autors Anstöße für weitere historischwissenschaftliche Arbeiten geben. Dazu die Herstellerin und Ladenbesitzerin MAUDIE VELLA: „Here in my shop it is all the hand made lace, but there are some places who do machine made ones as well. It's not so good, you see that. It's because the traditional Maltese one goes down." (Experteninterview mit MAUDIE VELLA Vom 08.10.2003).

\subsubsection{Gruppe 2: Einzelpersonen oder Familienbetriebe, die im Verband organisiert sind}

Gruppe 2 ist zweifelsfrei die größte Herstellergruppe der Maltesischen Inseln. Sie stellt vor allem in der Anzahl der Betriebe die Majorität (s. u.), ist aber vermutlich auch in der absoluten Anzahl der Arbeitskräfte den Gruppe 1, 3 und 4 überlegen.

An dieser Stelle soll auch eine der wichtigsten Einrichtungen für Erzeuger maltesischer Produkte vorgestellt werden: Das Malta Crafts Council. Es ist der Verband, in dem quasi alle der Gruppe 2 zugehörigen Produzenten organisiert sind. In diesem Fall ist es also notwendig, mehr über das Malta Crafts Council zu erfahren, um Einblick in Herstellergruppe 2 zu erlangen.

Das vom Ministry for Finance \& Economical Affairs der maltesischen Regierung am 21.11.2000 per Gesetz ins Leben gerufene Malta Crafts Council (malt.: II-Kunsill Malti Ghall-Artig̉janat) ist seit seiner Entstehung eine bedeutende und unter den Herstellern wohlwollend aufgenommene Einrichtung geworden. Das Malta Crafts Council stellt auf seiner Internetseite Namen und Adressen maltesischer Handwerker zur Verfügung. Ein Auszug aus dem Programm der Behörde lautet: "The aims of the Council are focused on the encouragement, promotion and regulation of crafts and craftsmen and entrepreneurs dealing in Maltese craft products. Crafts forming part of Malta's heritage are given particular importance. ${ }^{525 \text { “ }}$

Bedeutender und auch wirksamer Bestandteil der Erfüllung dieser Gesetzesvorgabe ist $u$. a. ein offizielles Label, das von Handwerkern und Lebensmittelherstellern, die ihre Produkte in Malta herstellen und darüber hinaus beim Malta Crafts Council registriert sind, erworben und zur Vermarktung inrer Produkte eingesetzt werden kann (vgl. Abb. 19).

Es sagt allerdings weder etwas über die Qualität eines Produkts aus ${ }^{526}$, noch darüber, ob das Produkt an sich traditionell maltesisch oder wenigstens handgefertigt ist. Es informiert lediglich, dass das Produkt, zu dessen Vermarktung es verwendet wurde, in Malta hergestellt wurde, der Verkäufer der Ware offiziell beim Malta Crafts Council registriert ist und das Produkt zu den 46 als "craft product" definierten Waren des Gesetzestextes des Act XXI aus dem Jahr 2000 gehört.

BRIAN GRIMA, Geschäftsführer von „Bristow Pottery“ in Ta'Qali: „The Government has set up a council for the craft products and everything that is produced in Malta can be given one of these signs as a certificate. Saying that it is Maltese." (Experteninterview vom 07.05.2004).

524 Angabe von MAUDIE VELLA, Ladeninhaberin von „Weaving \& Lace“ in Rabat im Experteninterview vom 08.10.2003.

525 Vgl. MALTA CRAFTS COUNCIL (2001), S. 88

526 Vgl. zu dieser Problematik auch Interviewauszüge des Künstlers JOE XUEREB in den Ausführungen zu Gruppe 4. 


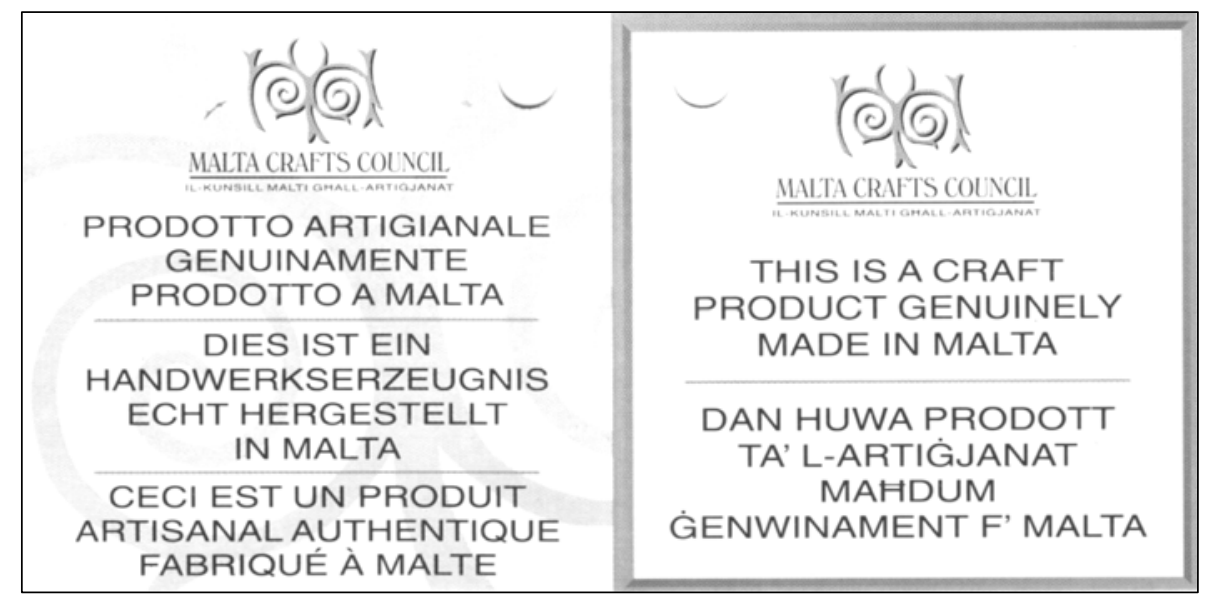

Abb.19: Das Label des Malta Crafts Council das zur Vermarktung von in Malta hergestellten Waren und Produkten Verwendung findet. Quelle: Malta Crafts Council

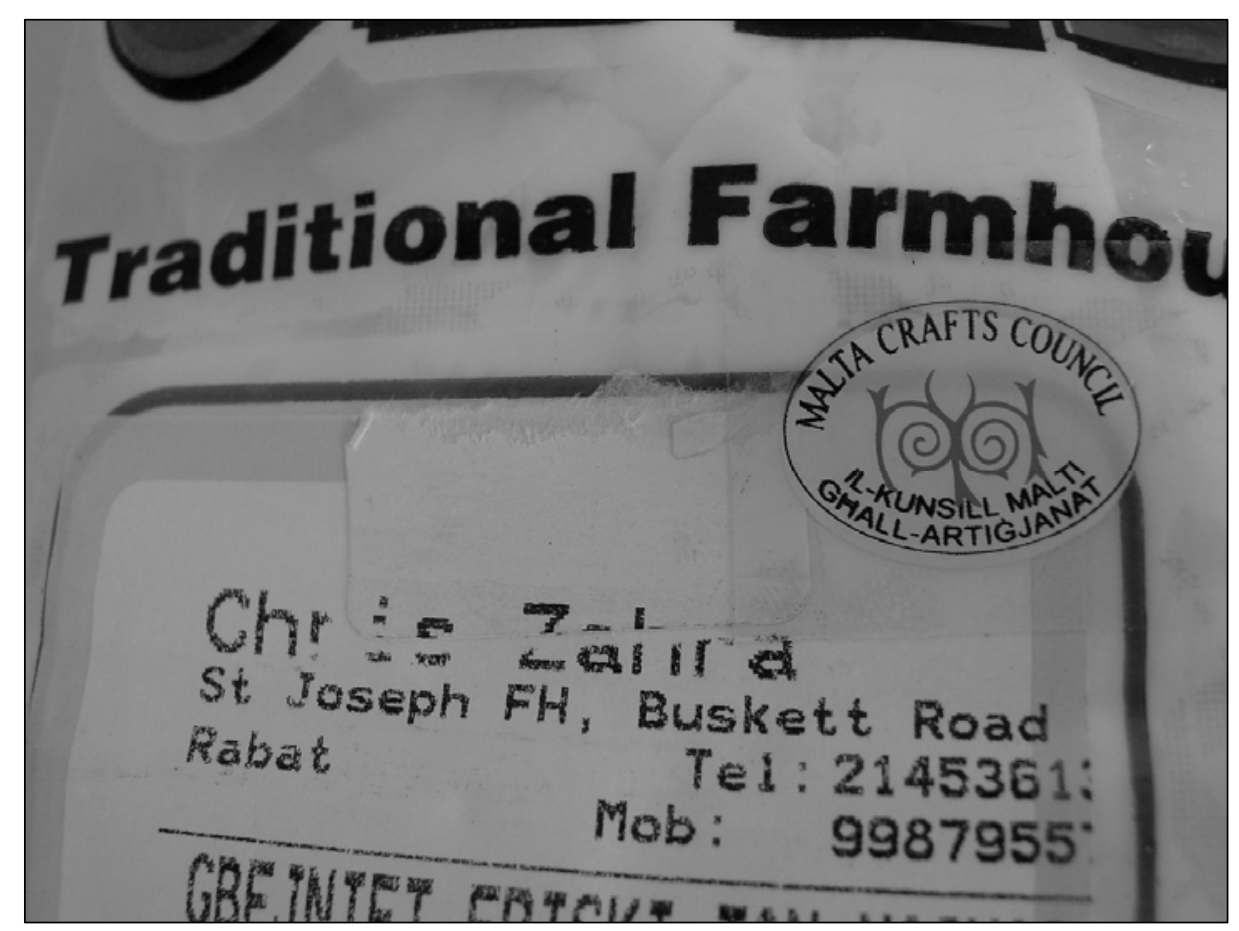

Foto 17: Das Label des Malta Crafts Council als Aufkleber auf der Verpackung von Schafskäse. Foto: ASCHEMEIER

Das „Zertifikat“, wie es von den Herstellern gern bezeichnet wird, kommt bei der Kundschaft gut $a^{527}$. Insbesondere die Crafts Villages Ta'Qali und Ta'Dbieg̉i machen Gebrauch davon. Einige Läden sind mit den kleinen Pappschildchen geradezu übervoll „versorgt“.

Doch auch im Rest Maltas ist das Crafts Council-Etikett immer öfter zu sehen, ob beim Bäcker, im Supermarkt, beim Kiosk oder in der Weinhandlung: Das Label ist insbesondere überall dort zu sehen, wo verstärkt mit touristischer Kundschaft zu rechnen ist. 
Dabei ist interessant, dass das Label nicht nur auf Handwerksgegenständen, sondern auch auf Lebensmitteln auftaucht. Selbst direkt vom Landwirt erworbener Käse kann sich mit dem Logo in Form eines kleinen Aufklebers auf der Verpackung schmücken. (vgl. Foto 17).

Diese Maßnahme der Regierung wird vom Autor der vorliegenden Arbeit positiv eingestuft. Zwar stellt das Label kein Qualitätszertifikat dar, wie gern von den Herstellern behauptet wird. Es ist jedoch ein gut wieder erkennbares Logo mit Eigenschaften des corporate designs. Reisende und solche Personen, die mit der Vielfalt lokal produzierter maltesischer Waren nicht vertraut sind, werden durch die hohe Wiedererkennbarkeit des Logos - auch beim flüchtigen Blick - auf maltesische Produkte aufmerksam.

Allerdings sollte sich wieder vor Augen geführt werden, dass nicht nur regionale traditionelle Produkte das Logo tragen, sondern auch moderne maltesische Handwerkszweige zum Gebrauch des Labels berechtigt sind: Auf der Homepage des Malta Crafts Council (s. o.) sind 46 Produktionszweige eingetragen, deren Hersteller das Logo für ihre Produkte beantragen können. Davon sind nach Einschätzung des Autors höchstens 20 tatsächlich dem regionalen traditionellen Bereich zuzuordnen. Die restlichen Handwerkszweige umfassen auch Einträge wie Tätowierungen, Plastik- und Fiberglasprodukte, Hairstyling oder Blumenarrangements. Diese und andere Gewerbe können also ebenfalls das Logo des Malta Crafts Council beantragen, obwohl sie in keiner Weise als regionaltypisch oder traditionell für die Maltesischen Inseln bezeichnet werden können.

Der Autor stellt dieses Label daher grundsätzlich in Frage und schlägt die Einführung einer eindeutigen Produktkennzeichnung vor (vgl. Kap. 22).

Der überwiegende Prozentsatz maltesischer Handwerker und Hersteller von regionalen traditionellen Produkten ist beim Malta Crafts Council verzeichnet. In dem einzigen bisher publizierten Verzeichnis aus dem Jahr 2001 (vgl. Abb. 20) sind das rund 320 Betriebe und Einzelpersonen, ${ }^{528}$ die nahezu alle zur hier vorgestellten Kategorie 2 gehören.

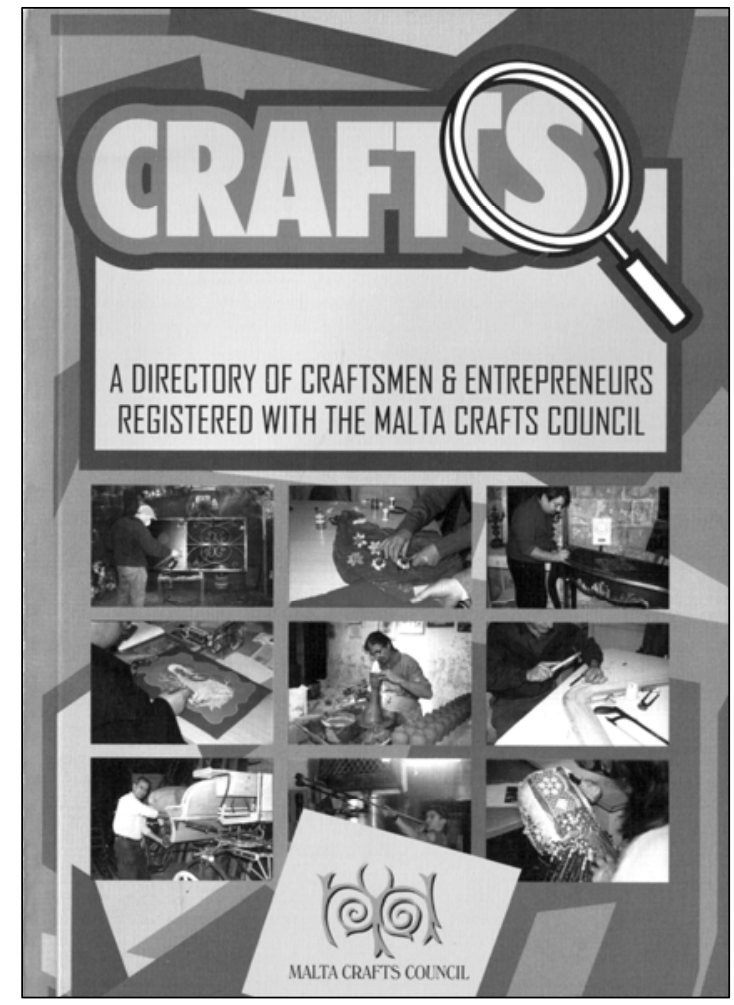

Abb. 20: Buchcover des bisher einzigen in Druckform publizierten Handwerkerverzeichnisses des Malta Crafts Councils. Quelle: Malta Crafts Council 
Neben dem Malta Crafts Council gibt es einzelne Handwerksbereiche, die eigene Verbände aufgebaut haben. Der Verband der Gold- und Silberschmiede hat seinen Sitz in Valletta. Auch die Imker sind in einem Verband organisiert, ebenso wie die Landwirte und die „Hobby-Winzer“.

Von den eben Genannten, kann es nur der Verband der Landwirte in Sachen politischer Meinungsbildung mit dem Malta Crafts Council aufnehmen. In der Tat dürfte der Landwirtschaftsverband zusammen mit dem Verband der Jäger (Association for Hunting \& Conservation Malta) die wichtigste meinungsbildende NGO des Landes darstellen und überflügelt hinsichtlich der politischen Beeinflussungskraft das Crafts Council bei weitem. Für die Hersteller regionaler traditioneller Produkte spielt das jedoch in der Praxis zunächst keine größere Rolle. Sie sind oft bei mehreren Verbänden eingeschrieben.

\subsubsection{Gruppe 3: Betriebe mit 10 Angestellten und mehr}

Diese Gruppe ist in Malta mit zu den einflussreichsten Herstellern zu zählen. Zwar gibt es nur wenige Betriebe ihrer Art, jedoch umfassen diese eine große Anzahl Angestellte und können einen beachtlichen Bekanntheitsgrad aufweisen. Außerdem findet in den Betrieben eine Kapitalkonzentration statt. D. h. die Hersteller dieser Gruppe sind zahlenmäßig gering, verfügen aber über einen großen Anteil des Investitionskapitals im sekundären und oft auch im tertiären Sektor.

Die wichtigsten Hersteller der Gruppe 3 im Bereich der traditionellen maltesischen Produkte sind der Getränkehersteller Simonds Farsons Cisk, der Hersteller von Tomatenprodukten Magro Bros., die Wein produzierenden Firmen Marsovin, Emanuel Delicata Winemarkers, Meridiana Wine Estate und Master Wine Group, u. a.

Auffällig in dieser Gruppe ist die Neigung zur Strukturierung der Betriebe nach dem „Crossover"-Prinzip.

Beispiele: Farsons Group of Companies umfasst neben der Brauerei Cisk auch die maltesischen Lizenzrechte an den Weltmarken Pepsi und $7 U p$, den Bierlabels Carlsberg und Budweiser sowie der Fruchtsaftmarke Sunkist. Außerdem ist Cisk der Hersteller der maltesischen Kräuterlimonade Kinnie. Daneben umfasst die Farsons Group of Companies auch Supermärkte, Fast Food-Restaurants und Hotels.

General Softdrinks hält die Lizenzrechte für Produkte der Coca Cola Company. Wegen des großen Absatzes von Farsons' Limonade Kinnie wird unter dem Schirm der Coca Cola Company das Konkurrenzprodukt Krest hergestellt, eine genaue Nachahmung der Kinnie-Limonade.

Bei der Master Wine Group werden neben Wein auch Spirituosen und alkoholfreie Sirups zur Selbstherstellung von Erfrischungsgetränken (sog. Squashes) hergestellt. Außerdem ist Master Wine Group ein Importeur von ausländischen Spirituosenmarken und will in näherer Zukunft ins Geschäft mit maltesischem Olivenöl einsteigen.

Marsovin ist der Weinhersteller mit den größten Absatzraten auf Malta. Zusätzlich hält Marsovin jedoch diverse Lizenzen für andere Getränke, u. a. die Lizenz für die deutsche Biermarke Löwenbräu.

\section{Lediglich Magro Bros., Delicata und Meridiana bleiben nur einer Produktsparte treu.}

Magro ist der größte Hersteller von Tomatenkonserven in Malta und in diesem Punkt beinahe konkurrenzlos. Die qualitativ hochwertigen Konserven umfassen auch traditionelle maltesische Spezialitäten wie die typische "Maltesische Soße" als Fertigprodukt sowie Kunserva (malt.), eine traditionelle Tomatenpaste (vgl. Kap. 14.1).

Delicata und Meridiana haben sich ganz der Herstellung von Wein verschrieben. Während Delicata, ähnlich wie Marsovin und Master Wine, auch Weine im unteren Preis- und Qualitätssegment herstellt, erzeugt Meridiana Wine Estate (vgl. Foto 18) seit jeher nur exklusive Produkte mit weitaus geringerem Absatz. Nach Angaben der Geschäftsführerin von Master Wine Group wird das Weingeschäft zu 94\% von den Herstellern Marsovin und Delicata ${ }^{529}$ bestritten. Im Spirituosenmarkt hat Master Wine jedoch den höheren Stellenwert. 


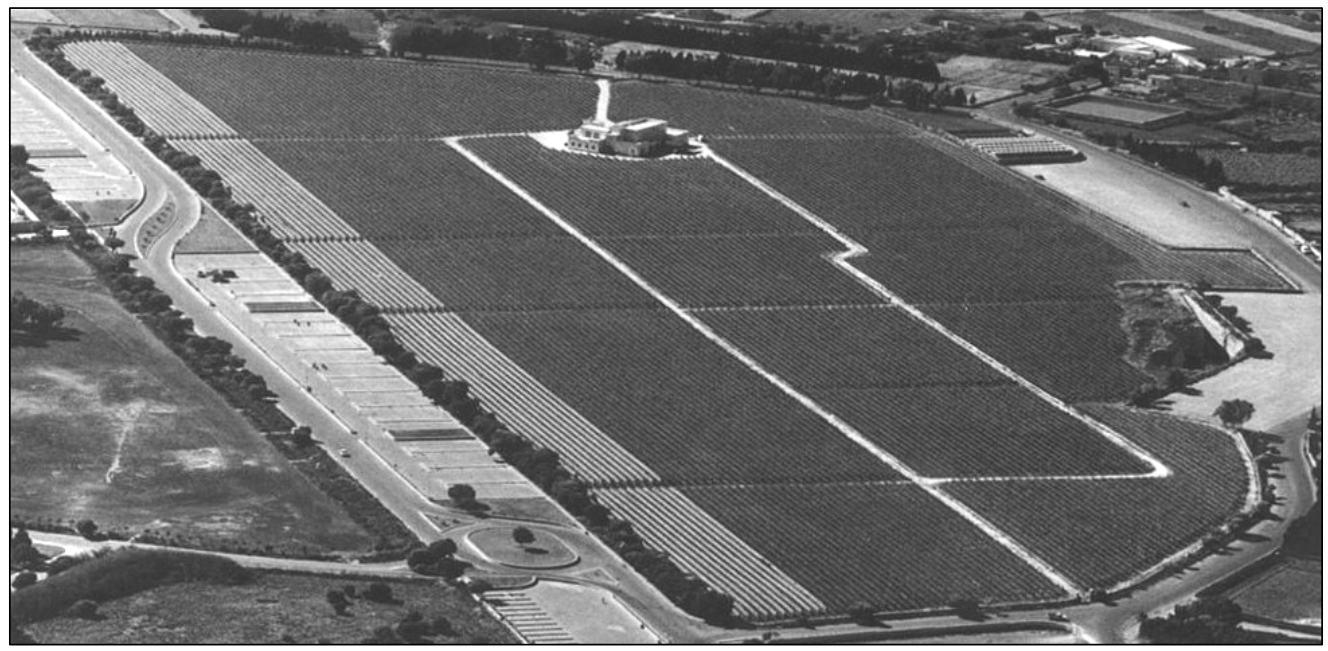

Foto 18: Luftbildaufnahme des Meridiana Wine Estates in Ta'Qali. Das Areal ist in den Bereich des alten Flughafengeländes eingebettet. Im oberen Bereich des vineyards ist das ehemalige Flughafenverwaltungsgebäude zu sehen, heute der Stammsitz des Unternehmens. Foto: MERIDIANA

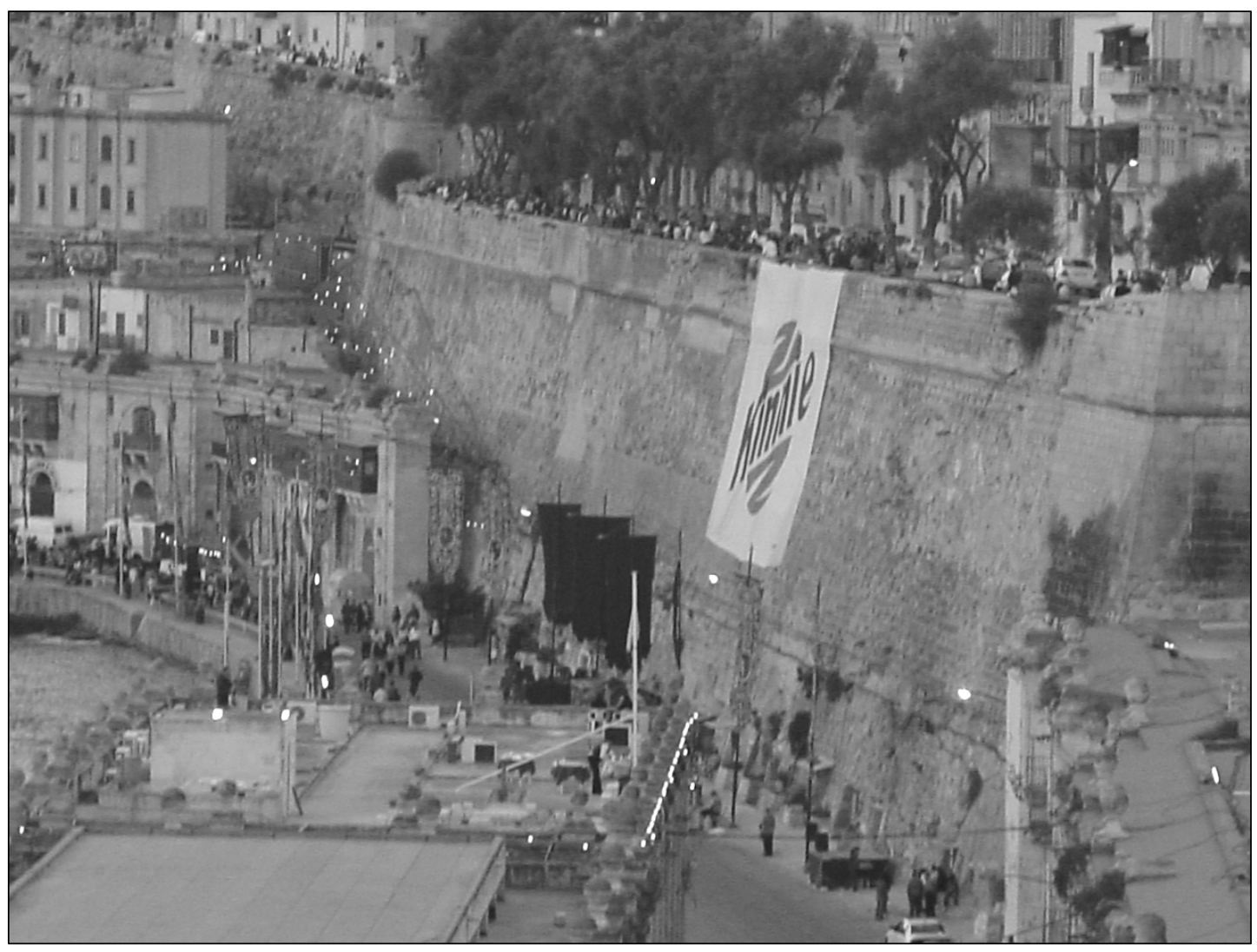

Foto 19: Übergroßes Werbebanner für den Softdrink Kinnie aus dem Hause Cisk während des Fireworks Festivals 2004 am Grand Harbour in Valletta - Beispiel für öffentlichkeitswirksames Sponsoring der Produzenten aus Gruppe 3. Foto: ASCHEMEIER

Es ist nachvollziehbar, dass die o. a. Betriebe den Hauptanteil der Printwerbung für maltesische Produkte bestreiten. Insbesondere Cisk, Delicata und Marsovin sowie die Likörserie Zeppi's von Master Wine sind in fast jeder Illustrierten oder Tageszeitung mit einer oder mehreren Anzeigen vertreten. Auch Magro schaltet Printwerbung, konzentriert sich aber aufgrund der Ausrichtung seines Produktsortiments auf die einheimische Bevölkerung mehr auf Fernsehwerbung in den maltesischen Kanälen. 
Master Wine stellt darüber hinaus in eigenem Aufwand hergestellte touristische Informationsbroschüren her, die über den deutschen Reiseveranstalter FTO, ehemals Frosch Touristik, direkt an die deutschen Reisenden weitergegeben werden ${ }^{530}$. Inhalt dieser Broschüren sind Kurzinformationen zu den wichtigsten Sehenswürdigkeiten Maltas und reichlich Werbung für die Produkte aus dem Hause Master Wine.

Cisk betätigt sich in Malta als Sponsor unterschiedlichster Events. Vom Rockkonzert über das Feuerwerksfestival bis hin zum National Folk Singing Festival ist Cisk überall als Sponsor, oft in Zusammenarbeit mit der Malta Tourism Authority, vertreten. Dabei wird das jeweils der Zielgruppe entsprechende Produkt besonders hervorgehoben. So ist beim National Folk Singing Festival z. B. die Biermarke Cisk gefragt, weil Bier das beliebteste Getränk der maltesischen Bevölkerung ist und ein Volksmusikfestival im Wesentlichen die einheimische Bevölkerung anspricht. Bei touristisch frequentierten Events wird zusätzlich zu Bier auch die Limonade Kinnie promotet, die dadurch den Touristen, die das Produkt zunächst nicht kennen können, nahe gebracht werden soll.

Neben diesen einflussreichen großen Herstellern können in Gruppe 3 auch die Firmen Camel Brand, Benna und Diskus Products erwähnt werden. Sie haben sich auf die Herstellung von maltesischen und mediterranen Spezialitäten ausgerichtet.

Benna ist der in Teilen staatlich finanzierte Hersteller von Milchprodukten. Ein Teil des Sortiments umfasst Ǵbejniet (vgl. Kap. 14.1), die bei Benna jedoch aus Kuhmilch hergestellt werden, was nicht der üblichen Tradition entspricht ${ }^{531}$.

Diskus und Camel vermarkten eine breite Palette mediterraner Spezialitäten, wie z. B. eingelegte Oliven oder Peperoni mit Frischkäse als abgepackte, fertig verzehrbare Produkte. Zusätzlich bieten sie traditionelle maltesische Spezialitäten wie Ǵbejniet und Bigilla an. Nach Kenntnisstand des Autors werden diese Betriebe von externen maltesischen Erzeugern aus dem primären und sekundären Sektor beliefert und fungieren in der Folge als gemeinsamer Vertrieb. Dies erklärt die teils schwankende Qualität der Erzeugnisse.

Es fällt in diesem Zusammenhang außerdem sehr schwer, die Erzeuger in eine Herstellerkategorie einzuordnen. Da der mengenmäßige Output dieses Vertriebskonzepts jedoch beachtlich ist, ist davon auszugehen, dass auch die Herstellung in vergleichsweise großem Stil abläuft und von einer dauerhaften Beschäftigung von mehr als 10 Personen pro Marke in der Herstellung ausgegangen werden kann.

Die großen Hersteller erfahren in der maltesischen Bevölkerung viel Ansehen. Ihre dauerhafte Medienpräsenz macht ihre Produkte auch für die jungendliche Käuferschicht interessant. Darüber hinaus sind die Erzeugnisse der Herstellergruppe 3 bei Touristen sehr beliebt, weil diese durch Werbung auf die Produkte aufmerksam werden. Der erste Kontakt von Reisenden mit einheimischen maltesischen Produkten ist fast immer ein Produkt aus Gruppe 3.

Interessanterweise konnte im Rahmen der persönlichen Gespräche festgestellt werden, dass die Produkte von Camel und Diskus unter einheimischen Lebensmittelherstellern ${ }^{532}$ oft sehr abschätzig beurteilt wurden. In diesem Sektor gibt es einen Teil der maltesischen Bevölkerung, die lieber auf "handgemachte“ Agrarprodukte zurückgreifen. Dies ist vielleicht eine Frage der schwankenden Qualität, denn die stets qualitativ hochwertige Produktpalette von Magro, die ebenfalls viele Erzeugnisse umfasst, die leicht selbst zu Hause hergestellt werden können, wurde in den Interviews fast nie kritisch kommentiert.

530 Angabe von DR. GIGQUEL, Geschäftsführerin Master Wine Group, im Experteninterview vom 08.06.2004

531 Der regionale traditionelle Ġbejna wird aus Schafs- oder Ziegenmilch hergestellt. Vgl. Kap. 14.1

532 Die Interviews inkl. der Namen der interviewten Personen liegen dem Autor vor. Die interviewten Geschäftsleute baten darum in den meisten Fällen nicht zu zitiert werden, wenn es um die Einschätzung anderer einheimischer Anbieter, auch solcher aus anderen Produktkategorien als ihrer eigenen, ging. Der Autor bittet hierfür um Verständnis. 


\subsubsection{Gruppe 4: „Alternative Hersteller" mit eigenem Geschäftskonzept und Künstler}

Wie bereits beschrieben, gibt es in Malta und Gozo auch Hersteller von exklusiven Erzeugnissen, die sich nicht mit den Kunsthandwerkern der Crafts Villages oder den üblichen Lebensmittelherstellern identifizieren können und wollen. Sie fühlen sich in ihrer Tätigkeit und durch die Qualität ihrer Arbeit herausragend und außergewöhnlich und möchten dies - auch wenn dieser Vorgang u.U. unbewusst ablaufen mag - auch durch die äußeren Verhältnisse ihrer Betriebssituation vermitteln.

Zu dieser Kategorie gehören z. B. einige Künstler auf den Maltesischen Inseln ${ }^{533}$, deren Arbeitstechniken sich zwar nicht unmittelbar von denen der Kunsthandwerker der Crafts Villages - sofern diese noch in Handarbeit fertigen - unterscheiden. Das Produkt selbst basiert jedoch auf einem grundlegend anderen Selbstverständnis und wird vom Erzeuger nicht als Kunsthandwerk sondern als originäre Kunst betrachtet.

Diese Künstler sehen den Hauptunterschied in der Kreativität und in der exklusiven Qualität in denen sich ihre Erzeugnisse von denen der Kunsthandwerker unterscheiden. Der international erfolgreiche gozitanische Bildhauer JOE XUEREB im Experteninterview über den Unterschied zwischen seiner Arbeit und dem Angebot der Crafts Villages:

„It's true. One can make a destinction that there ${ }^{534}$ is really good work. But in our days there are so many new ideas caught up which are totally mass produced. The idea is not of exploring new ventures from local traditional craft but mainly more ideas how to make more money and then just giving them some pinch of Maltese history." (Experteninterview mit JOE XUEREB vom 13.05.2004).

XUEREB gab im Interview an, selbst als Kunsthandwerker im Souvenirbereich angefangen zu haben. Er sei aber dann zunehmend von den kreativen Beschränkungen, die dieser Beruf mit sich brachte, und von der allgemeinen Geschäftspraxis des maltesischen Kunsthandwerks enttäuscht gewesen. Über die Crafts Villages Ta'Qali und Ta'Dbiegi hat er seine eigene Meinung und auch zum Label des Malta Crafts Council (s. o.) bezieht er eine kritische Position:

„The idea is good, but sometimes the substance of the crafts involved is not of very good quality. Sometimes visitors come here and they recommend in that direction that they have visited one of these centres and unfortunately they see very very little of really good value. (...). Most of the things there, they have got the postmark of Malta ${ }^{535}$, but there is nothing in substance of the object." (Experteninterview mit JOE XUEREB vom 13.05.2004).

Dabei verleugnet XUEREB seine eigenen gozitanischen Wurzeln im künstlerischen Ausdruck aber nicht, sondern betont sie vielmehr noch. Er fühle sich als einer derjenigen, die Maltas Traditionen im Bereich der Steinbearbeitung in einer Linie mit den überlieferten Traditionen weiterführen und aus dieser historischen Sicht in moderne Skulpturen weiterentwickeln. Die Massenherstellung von z. B. Nachbildungen archäologischer Ausgrabungsgegenstände, die in der Nähe der neolithischen Tempel und in den Crafts Villages verkauft werden oder Nachbildungen von Szenen des maltesischen Alltagslebens, betrachtet er als künstlerische Stagnation.

XUEREB zu seinem besonderen Verständnis der Kunst der Steinbearbeitung im Vergleich mit den für den touristischen Markt produzierten Billig-Souvenirs:

„I think the problem is that the people are not delving enough into the old history of the Maltese Islands. I "mean, if they are doing a bus ${ }^{536}$, the bus is still running on the road! If you try to make small castings of the

533 Die Zahl der bildenden Künstler Maltas ist nicht zuletzt durch die Einrichtung der universitären Kunstakademie in den letzten Jahren gestiegen. Neben Künstlern wie dem Bildhauer JOE XUEREB, die bereits seit Jahrzehnten im In- und Ausland gefragt sind, gehören dazu u. a. viele erfolgreiche „Newcomer“ wie z. B. Kunstschnitzer RAY AZZOPARDI und Maler und Bronzekünstler JOE GRIMA, der im Jahr 2004 u. a. ein riesiges international beachtetes Bronzeportal für die St. Georges Basilica in Victoria herstellte (vgl. THE GOZO TIMES, 12.07.2004, S. 8).

534 In den Crafts Villages, Anm. d. Verf.

535 Der Künstler spielt mit dieser Aussage auf das „Genuinely made in Malta“-Label des Malta Crafts Council an.

536 XUEREB spricht in dieser Äußerung die in maltesischen Souvenirgeschäften weit verbreiteten Nachbildungen der bunt bemalten maltesischen Busse an. 
old buildings ${ }^{537}$... for me: Something not out of this world. And then they are trying to handpaint that object and they make the advertisement it would be something really handmade. To me that does not make sense because it is something molded, castet. And for me: Something out of value! The problem is that they are not making their studies to go back into the Maltese History and try to build on that and try to launch new craftswork on that theme other than making... bubbles." (Experteninterview mit JOE XUEREB vom 13.05.2004).

XUEREB war einer der ersten, der sich von den vorgeformten Schemata der Souvenirindustrie löste und eine Karriere als eigenständiger Künstler begann. Bereits in den 1970er Jahren begann er sein eigenes Selbstverständnis von Bildhauerei in der Tradition der Maltesischen Inseln umzusetzen. Ein Auszug aus seiner Informationsbroschüre:

„Xuereb's art is, (...), closely linked with the prehistoric past of Gozo, a small island with its own distinctive character, going back to Neolithic times. His medium, the soft globigerina limestone of Gozo is highly adapted to his working technique, using traditional tools inherited from his father, a traditional stone mason in his younger days." (JOE ScICLUNA, März 2003 in „Joe Xuereb - Stone Sculptures“ Informationsbroschüre).

\section{An anderer Stelle:}

„Critics have identified ideas in Xuereb's work reminiscent of Henry Moore's sculpture, in which similarities do perhaps exist though more unconscious than through direct imitation. Recently also, an article, in a French magazine hailed Xuereb as "the Botero of Gozo“, due to the similarity of the Peruvian artist's voluptous and squat figures to the Gozitan artist's creations. (...). Xuereb's „oeuvre“ is Gozitan and universal.“ (JOE ScICLUNA, März 2003 in „Joe Xuereb - Stone Sculptures“ Informationsbroschüre).

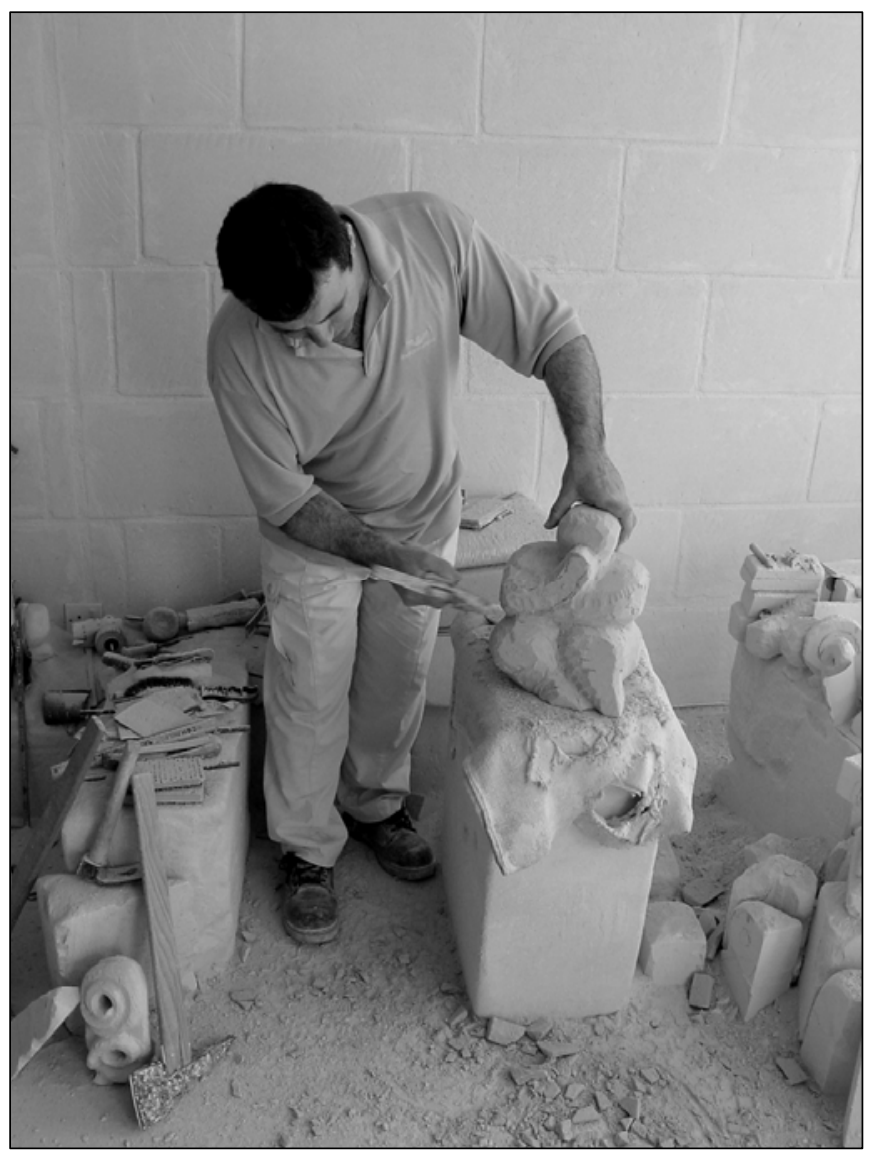

Foto 20: Bildhauer JOE XUEREB bei der Arbeit in seinem Atelier in Ghajnsielem, Gozo. Foto: ASCHEMEIER

537 Mit dieser Äußerung wird auf in maltesischen Souvenirgeschäften erhältliche Miniaturgüsse von geschichtlich bedeutsamen maltesischen Gebäuden oder historisch gefärbten Szenerien aus dem ländlichen Malta angespielt. 
Diese Auszüge belegen, dass es gegenwärtig maltesische Künstler gibt, die zwar traditionelle handwerkliche Techniken nutzen, sich auch durchaus in der Fortsetzung einer Tradition sehen, gerade deswegen aber nicht als „Trittbrettfahrer“ der Souvenir-Industrie eingestuft werden wollen.

Sie haben eine „alternative“ Auffassung von ihrer Tätigkeit und können nicht (mehr) in einem Atemzug mit den Herstellern des traditionellen Kunsthandwerks genannt werden. Der Grund ist, dass sie sich darüber hinaus weiterentwickelt haben und auf ihre eigene Weise neue und außergewöhnliche, wenn man so will, „maltesische Produkte“ herstellen, die einerseits fest in der Tradition des Landes verwurzelt, andererseits aber auch innovativ und modern sind. Neu ist außerdem, dass sie für ihre Erzeugnisse eine universell gültige, internationale Qualität beanspruchen, die sie offensichtlich auch in vielen Fällen erreichen können, wie zahlreiche Ausstellungen maltesischer Künstler im Ausland in jüngster Vergangenheit beweisen.

Was im Kunstbereich am Beispiel des Bildhauers JOE XUEREB veranschaulicht wurde, kann auch vereinzelt im Bereich der Lebensmittelerzeugung beobachtet werden. Zwar kann man hier nicht von „Kunst“ sprechen, dennoch gibt es auch im Lebensmittelsektor Erzeuger, die ein besonderes Selbstverständnis von ihrem Produkt offenbaren, was dieses in den Rang des Besonderen erhebt.

Auch solche Hersteller können nicht ohne weiteres in eine der o. a. Kategorien eingeordnet werden, denn ihre Erzeugerphilosophie ist nicht auf den bloßen Herstellungsprozess und die „übliche" Qualität der betreffenden Ware beschränkt. Vielmehr wollen sie mit ihrem Produkt neue Maßstäbe setzen und - ähnlich wie XUEREB - aus vorhandenen Traditionen neue, qualitativ bisher nicht in dem Maße gekannte Erzeugnisse schaffen. Hierbei wirkt der Prozess der Herstellung des Produkts zu einem Großteil auch als Prozess der Selbstverwirklichung des Herstellers. Der Fabrikant betrachtet sich auf seinem Level auch als „Künstler“, der besonders hochwertige und in gewissem Maße kreative Produkte erzeugt.

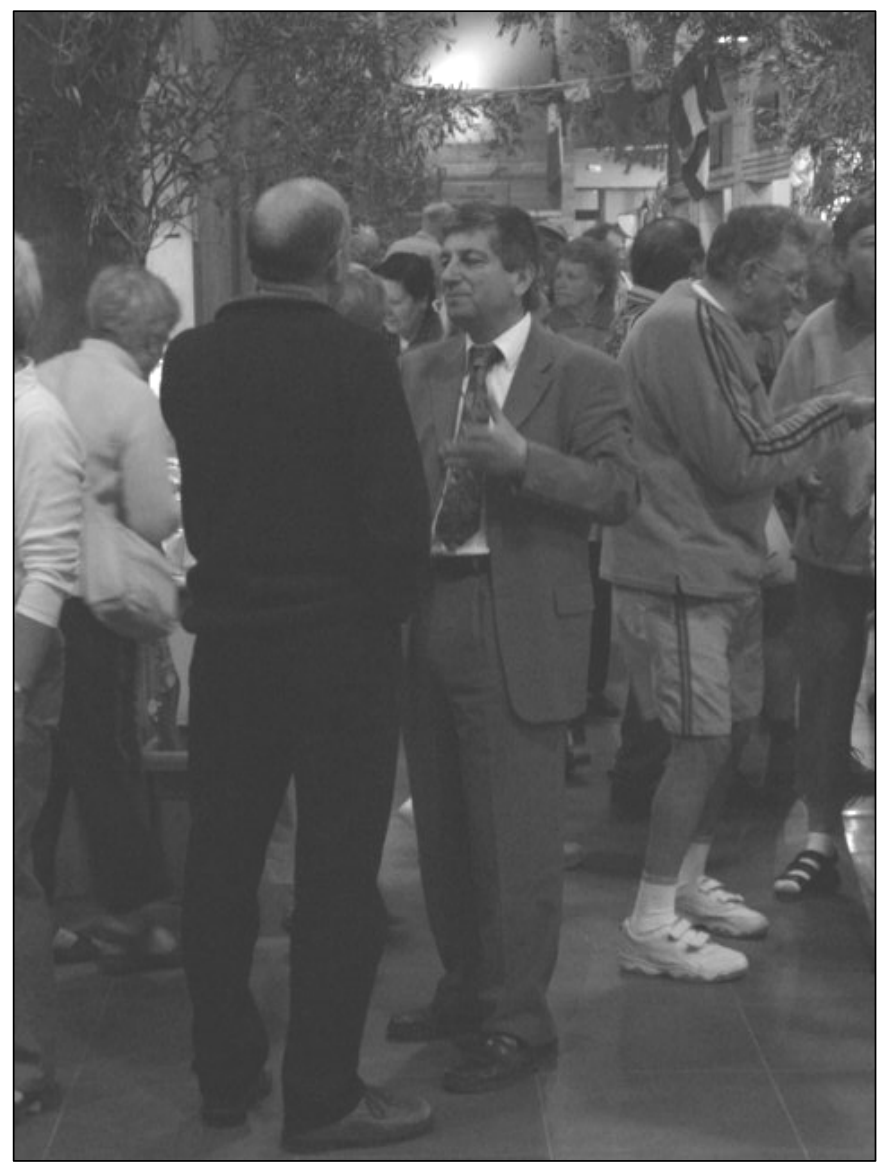

Foto 21: Olivenölhersteller SAM CREMONA (Mitte) im Gespräch mit einem Kunden beim Mediterranean Food Festival, Paceville 2005 Foto: ASCHEMEIER 
Als typisches Beispiel für diese Gruppe sei der Olivenölhersteller ${ }^{538}$ und Juwelier SAM CREMONA (vgl. Foto 21) genannt.

CREMONA behandelt sein Olivenöl in der Herstellung ähnlich aufwändig wie die internationalen Produzenten von Wein, Champagner oder Whiskey es mit ihren Erzeugnissen tun ${ }^{539}$. Das Öl wird z. B. mehrfach dekantiert, nach Jahrgängen und Anpflanzungsgebieten sortiert und ähnlich der Tradition des schottischen Whiskey-Blendings untereinander verschnitten, um ein optimales Resultat zu erzielen. CREMONA lässt verschiedene Olivensorten auf ihre DNA hin untersuchen um festzustellen, ob es möglich ist, ein Öl aus einer endemischen maltesischen Art herzustellen. Sein Ziel dabei ist die Erlangung einer D.O.P.Klassifikation durch die EU. Das von seiner Firma Ghajn Razul Co. vertriebene Olivenöl erhielt bereits hoch dotierte internationale Preise, u. a. die Auszeichnung „bestes Olivenöl des Mittelmeerraums“ auf einer Landwirtschaftsausstellung der EU-Agrarminister in Taormina, Sizilien, im Jahr 2003. Im selben Jahr wurde durch CREMONA der Antrag auf Bio-Zertifizierung seines Öls nach den Richtlinien der EU gestellt.

Beide Beispiele zeigen, dass es in Malta zunehmend Hersteller exklusiver Produkte gibt, die nicht nur um einen international herausragenden Anspruch bemüht sind, sondern sich auch darüber im Klaren sind, dass die Erlangung bzw. die Ausweisung von Alleinstellungsmerkmalen für das Marketing maltesischer Produkte essenziell ist und durch den EU-Beitritt des Kleinstaats in der Zukunft noch wichtiger werden wird.

Insbesondere dieses Bewusstsein ist bei den Herstellern der anderen Produzentenkategorien oft nicht vorhanden, sodass die meisten alternativen Vermarktungskonzepte in der an dieser Stelle vorgestellten Gruppe 4 gefunden werden können. Dies macht Gruppe 4 besonders interessant, weil hier ein „Know-HowPool“" im Entstehen begriffen ist, der sich selber trägt und Lösungen für spezifisch maltesische Probleme sucht, die zunehmend auch gefunden werden.

Ein derartiger Innovationspool kann u.U. als Vorbild für Betriebe der zuvor genannten Herstellergruppen dienen. Daher sollte Herstellergruppe 4 in besonderem Maße die Aufmerksamkeit der öffentlichen Stellen zukommen, was jedoch bislang in keinster Weise umgesetzt worden ist! 


\section{Innovative Konzepte rund um regionale traditionelle maltesische Produkte}

\subsection{Verwertung der Kaktusfeige}

Ein nach Meinung des Autors vorbildliches Beispiel der Ausnutzung wertvoller Rohstoffressourcen ist in Malta die Verwertung der Frucht des Feigenkaktus. Die Verwertung maltesischer Kaktusfeigen erfolgt in gemeinschaftlicher Absprache von Firmen aus drei unterschiedlichen Branchen.

Der aktuelle Verwertungsweg der Früchte ist in den 1990er-Jahren entwickelt worden und in Abb. 21 dargestellt.

- Die Grafik beginnt mit der Ernte der Kaktusfeigen, die per Handarbeit ausschließlich auf Malta und Gozo stattfindet. Die Landwirte, die die Kaktusfeigen anbauen und ernten, greifen dabei auf alte Bestände zurück, die ohne die Verwertung, so wie vielerorts auf den Inseln, verwildert wären. Es wird ausschließlich die Sorte a

- Im nächsten Schritt erfolgt die Trennung von Schale und Fruchtfleisch. Dieser Prozess wird in einer Fabrik der Master Wine Group in Naxxar (Malta) ausgeführt. Das Fruchtfleisch verbleibt an Ort und Stelle, während die rote Schale nach Mosta transportiert wird, um in der Pharmaindustrie zum Einsatz zu kommen.

- In Mosta wird die Schale zu einem Granulat verarbeitet, das der Hauptbestandteil des medizinischen Präparats „Preservation“ des pharmazeutischen Unternehmens ICP Ltd. ist ${ }^{541}$.

- Master Wine in Naxxar verarbeitet das Fruchtfleisch im Rahmen der Herstellung des regionalen traditionellen Likörs Zeppi's Bajtra ${ }^{542}$.

- Die Reststoffe beider Produktherstellungsprozesse, im wesentlichen handelt es sich dabei um Pflanzenbestandteile, aus denen die rote Farbe der Kaktusfeigenschale extrahiert werden kann, wird in der Folge in der kosmetischen Industrie verwendet.

Es bleiben nahezu keine ungenutzten Bestandteile übrig. Drei Unternehmensbranchen haben von dem Rohstoff profitiert. Dabei wurden die Gesetzmäßigkeiten der Nachhaltigkeit und des Umweltschutzes berücksichtigt.

Die Verwertung der Kaktusfeige ist somit ein nach Meinung des Autors positiv hervorzuhebendes Modellprojekt, das auch im Rahmen der Artischockenverarbeitung in ähnlicher Weise wiederholt werden könnte ${ }^{543}$. 


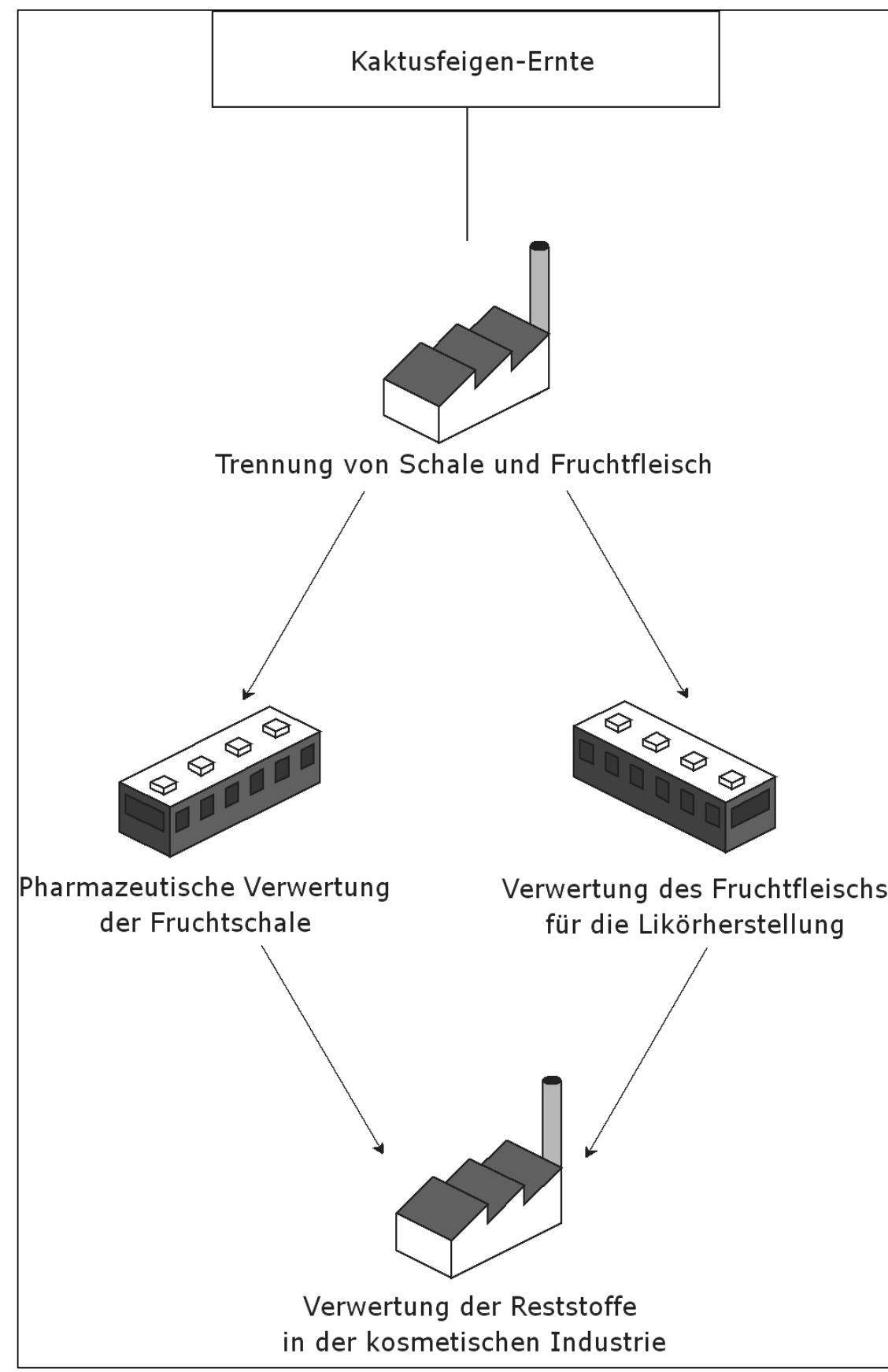

Abb. 21: Verwertungsweg der Kaktusfeige in Malta. Entwurf \& Grafik: AsCHEMEIER

\subsection{Farmhouse cooking}

Das Projekt „farmhouse cooking“ wurde vom deutschen Importeur für Marsovin Weine, Herrn HANS WERNER MORAWETZ und maltesischen Geschäftsleuten ins Leben gerufen. Es handelt sich dabei also um ein deutsch-maltesisches ,joint venture“.

Ein abgelegenes Bauernhaus in den Stadtgrenzen von Qormi (Malta) aus dem Jahr 1743 wurde dabei zu einem exklusiven Restaurant umgewandelt. Dies wäre nichts Besonderes, gibt es solche Bemühungen doch schon länger, auch auf Gozo ${ }^{54}$. Die Besonderheit verbirgt sich im Angebot des Hauses, welches

544 Ein Beispiel wäre z. B. das Restaurant „Ta'Frenć“ auf halber Strecke zwischen Victoria und Marsalforn. Dieses Gasthaus ist ebenfalls in den Gemäuern eines jahrhundertealten traditionellen maltesischen Farmhauses angesiedelt. 
ausschließlich Maltesische Küche serviert. Damit dürfte das Restaurant, welches „Ir-Razzett L-Antik“ benannt wurde, nach Kenntnisstand des Autors das einzige Restaurant Maltas sein, das ohne Ausnahme maltesische Gerichte offeriert.

Zum Angebot des Hauses gehört auch ein Shop für maltesische Spezialitäten. Darüber hinaus werden Happenings wie z. B. Kochkurse mit dem deutschen TV-Koch (TV-Sendung „Kochduell“ des Senders „Vox“) und Buchautor (u. a. „Die Maltesische Mittelmeerküche“) RAINER MITZE angeboten. Speziell für Tagungsund Incentivereisen wurde das Projekt „II-Kćina I-Antika“ aus der Taufe gehoben, bei denen den Gästen ein traditionelles maltesisches Mahl im Stil des 18. Jh. serviert wird. Das Personal des Restaurants ist für diese Anlässe in der zeitgenössischen Kleidung gewandet (s. Foto 22).

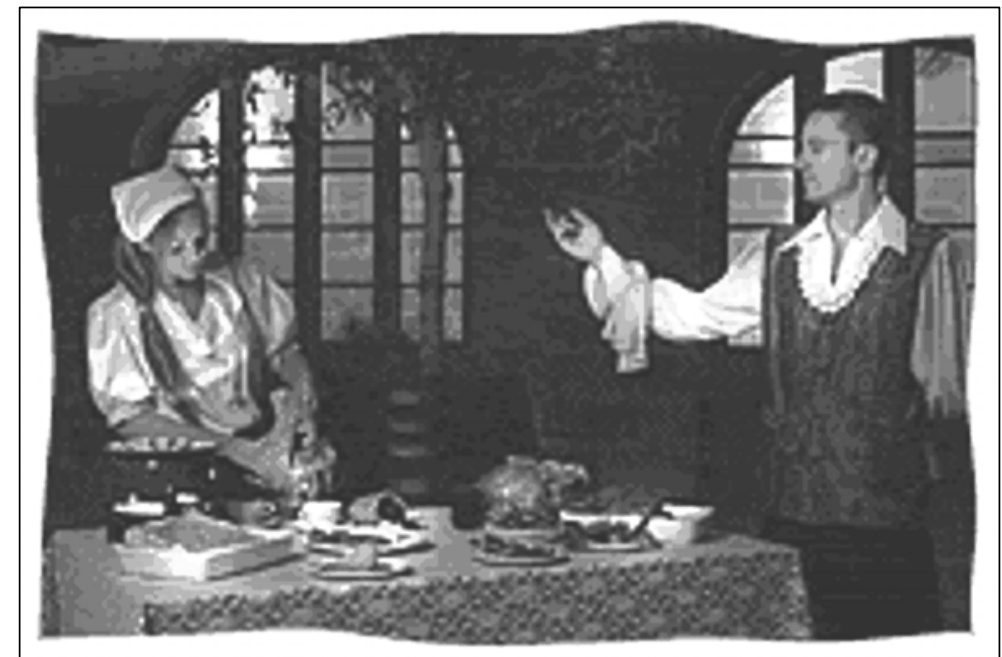

Foto 22: Die Präsentation im Rahmen der Veranstaltung „II-Kċina I-Antika“ im Restaurant „Ir-Razzett L'Antik“ bei Qormi. Das Foto wurde vom Restaurant zur Verfügung gestellt

Das Projekt zeigt, dass die maltesische Küche, die bei vielen Gastronomen im Ruf steht, für Touristen nicht attraktiv zu sein, auch ansprechend und qualitativ hochwertig präsentiert werden kann. Es wäre wünschenswert, dass weitere Restaurants dem beschriebenen Beispiel folgen, ohne das Geschäftskonzept aus Qormi „nur“ zu imitieren.

Besonders die Verbindung von Restaurant und Spezialitätenshop wird vom Autor der vorliegenden Arbeit als vielversprechendes und zukunftsträchtiges Marketinginstrument gedeutet. Auf diese Weise profitieren regionale traditionelle Produkte vom angenehmen Ambiente des Restaurants und werden so in ihrem Imagewert gehoben. 


\section{Ausgewählte Produkte im Hinblick auf ihre Entwicklungsfähigkeit in der Zukunft}

\subsection{Zur Produktpsychologie bestimmter maltesischer Erzeugnisse}

Wie die erfolgte Produktvorstellung gezeigt hat, existiert im Untersuchungsraum - die verhältnismäßig kleine Staatsgröße der Republik Malta in Betracht ziehend - eine beachtliche Vielzahl von regionalen traditionellen Produkten. Nicht alle sind in gleichem Maße für die Vermarktung für Tourismus und EU-Binnenmarkt attraktiv und geeignet.

Neben rein praktischen Problemen, wie Konservierbarkeit, Transport oder Herstellungskapazität der Erzeugnisse, muss vor allem die Konkurrenzfähigkeit der maltesischen Produkte im internationalen Vergleich beachtet werden. Das ist nicht nur der Fall bei einer Betrachtung der Chancen der Waren im Fall eines Etablierungsversuchs auf EU-Binnenmarktebene, sondern vor allem auch bei der inländischen Vermarktung für den Massentourismus, dessen wesentliche Quellgebiete in Überflussländern wie Großbritannien, Deutschland und den USA liegen. Touristen aus diesen Staaten sind reiseerfahren und damit häufig anspruchsvoll, was den Erwerb und den Konsum regionaltypischer Produkte angeht ${ }^{545}$.

Außerdem ist zu beachten, dass vor dem Konsum durch die Käufer eine Hemmschwelle überwunden werden muss: Der Kauf von unbekannter Ware mit für den Konsumenten nicht bekannten Eigenschaften und Formen oder Gerüchen und Geschmäckern soll erreicht werden. Dies bereitet bei einem nicht kleinen Anteil der touristischen Kundschaft durchaus Schwierigkeiten, vor allem dann, wenn dieser Anteil sich u.U. aus wertkonservativen und nicht experimentierfreudigen Personen zusammensetzt.

Malta hat einen hohen Prozentsatz von Touristen in den mittleren und hohen Altersklassen. 55,67\% aller Malta-Reisenden sind über 40 Jahre alt. 28,99\% dieser Reisenden gehören der Altersgruppe 55 Jahre und älter $a^{546}$. Damit bewegt sich Malta als Reiseland im Bereich der prognostizierten touristischen Trends, die eine Erhöhung des Altersdurchschnitts der Reisenden aus den Wirtschaftsländern vorhersagen. Daraus lässt sich ableiten, dass auch die touristische Kundschaft für regionale traditionelle Produkte vor Ort im Durchschnitt immer älter wird.

Trotz zunehmender Individualisierung der touristischen Zielgruppen kann angenommen werden, dass Reisende gehobenen Alters in Bezug auf das Ausprobieren und den Kauf von ihnen unbekannten Lebensmitteln $^{547}$ in der Regel weniger experimentierfreudig sind. Diese These wird gestützt durch die Auswertung der touristischen Befragung im Rahmen der vorliegenden Abhandlung (vgl. Kap. 7).

Im Bereich des Kunsthandwerks muss wieder eine andere Ausgangssituation vorausgesetzt werden. So nehmen z. B. BIEGER \& LAESSER (2003) bei ihrer umfassenden Prognose touristischer Trends an, dass weite Teile der älteren reisenden Bevölkerung aus den Wirtschaftsländern in Zukunft über ausreichend Zeit und Kaufkraft verfügen ${ }^{548}$. Dies würde allgemein eine höhere Konsumfreudigkeit verursachen.

Es kann jedoch vorausgesetzt werden, dass sich die Konsumfreudigkeit, die sich daraus ableitet, in bestimmten Sektoren nicht oder nur eingeschränkt greift. So ist es z. B. durchaus wahrscheinlich, dass Menschen ab einem bestimmten Lebensalter weniger Wert auf die Anschaffung neuer Gegenstände aus dem Bereich Kunsthandwerk legen, weil sich im Laufe ihres bisherigen Lebens aus diesem Sektor schon eine ausreichende Menge in ihrem persönlichen Besitz angesammelt hat.

545 LITTRELL et al. (1993) \& MACCANNELL (1999)

546 Vgl. NSO (2004c), S. 125

547

Vgl. LITTRELL et al. (1993), S. 207 f., die in ihrer Studie zum Konsumverhalten touristischer Souvenirkäufer herausgefunden haben, dass sich mit steigendem Alter der Touristen offenbar deren Wahrnehmung von Produktauthentizität ändert. Die kulturelle und geschichtliche Integrität sowie die „Echtheit“ von Produkten zählt bei älteren Reisenden zu den besonders wichtigen Kriterien. Jüngere Reisende beachten hingegen vor allem die „Einzigartigkeit“, bzw. den Unikatcharakter eines Produkts - unabhängig von dessen Verwurzelung in Tradition, Geschichte oder Landesfolklore.

548 Vgl. BIEGER \& LAESSER (2003) 
Es könnte allerdings auch argumentiert werden, dass Menschen in diesem Lebensabschnitt nun gerade dazu neigen, sich Gegenstände aus dem Bereich Kunst und Kunsthandwerk zu kaufen, weil sie es sich nun leisten können, ohne in hohem Maß über die Konsequenzen der Ausgabe einer größeren Geldsumme nachdenken zu müssen.

Es zeigt sich also, dass die Abschätzung einer nachhaltigen Entwicklung des Marketings für maltesische Produkte grundsätzlich schwierig ist und für den jeweiligen Einzelfall individuell geschehen sollte. Es kann keine Pauschalisierung nach dem Motto "Lebensmittel sind schwieriger zu vermarkten als Kunsthandwerk" erfolgen. Der Einzelfall muss intensiv unter Einbeziehung sowohl historischer als auch aktueller Entwicklungen abgewogen werden.

Der Autor möchte in den folgenden Abschnitten einige ausgewählte Produkte vorstellen und analysieren, die er im Rahmen einer Modernisierung des Marketings für maltesische Produkte für besonders entwicklungsfähig und förderungswürdig hält. Es sind dies die Produkte, die im Rahmen des EU-Beitritts Maltas und der momentanen Situation im touristischen Sektor nach Einschätzung des Verfassers die besten Chancen auf Erfolg haben.

\subsection{Artischocken}

Zum Anbau der Artischockenpflanze in Malta vgl. Kap. 20.2

Die Artischocke wird vom Verfasser zu den regionalen traditionellen Produkten Maltas mit einem hohen Entwicklungspotenzial gezählt. Der Status der essbaren Pflanzenbestandteile als regionales traditionelles Produkt steht außer Frage: Bereits die Johanniter berichteten von Disteln („wilden“ Artischocken) als einem der typischen Nahrungsbestandteile der maltesischen Bevölkerung ${ }^{549}$. Die Pflanze ist heimisch auf den Maltesischen Inseln. Es gibt jedoch keine bekannten Endemismen. Zwar wurden die heute domestizierten Sortenvarietäten aus Amerika eingeführt, jedoch hat dieser Vorgang auch in den anderen Mittelmeerländern in ähnlicher Weise stattgefunden. Die domestizierten Varianten der Artischocke werden gegenwärtig als „authentisch mediterran“ verstanden. Die essbare Artischocke ist im Laufe von vielen Jahrzehnten der Kultivierung vollständig vom kulturellen Bannkreis des Mittelmeerraums assimiliert worden.

Die klimatischen Ausgangsbedingungen für den Anbau dieser Gemüsepflanze sind sehr günstig auf den Maltesischen Inseln. Es kann innerhalb Maltas und Gozos jedoch kein Schwerpunktraum für den Artischockenanbau ausgewiesen werden ${ }^{550}$. Der Anbau richtet sich auf den Inseln insbesondere nach der Verfügbarkeit von Wasser zum Bewässerungsfeldbau und nach der Güte und Tiefgründigkeit der Böden. Unterhalb der Ridges auf Malta bzw. auf Gozo an den Hängen der Tafelberge und in den Valleys sind die Wachstumsbedingungen gut. Daher erfolgt der Artischockenanbau in den Bereichen, in denen traditionell der Gemüseanbau vorkommt: Wied tal-Pwales (Pwales Valley), Il-Miżieb, Wied il-Gћasel, Binġemma Basin, zwischen den Ortschaften Victoria und Marsalforn auf Gozo, auf dem Dingli-Hochland Maltas sowie auf den Terassen unterhalb der Dingli-Cliffs. Auch in der Nähe kleinerer Ortschaften (z. B. bei Bidnija auf Malta) erfolgt kleinflächiger Artischockenanbau in Gartenbaukultur, zumeist für den Verkauf durch fahrende Händler oder für den Eigenbedarf (vgl. Foto 23).

549 Vgl. CREMONA (2003)

550

Mit der Einschränkung, dass der Artischockenanbau, wie jedweder agrarwirtschaftlicher Anbau mit Ausnahme von Oliven, auf Gozo ausgeprägter stattfindet als auf Malta. 


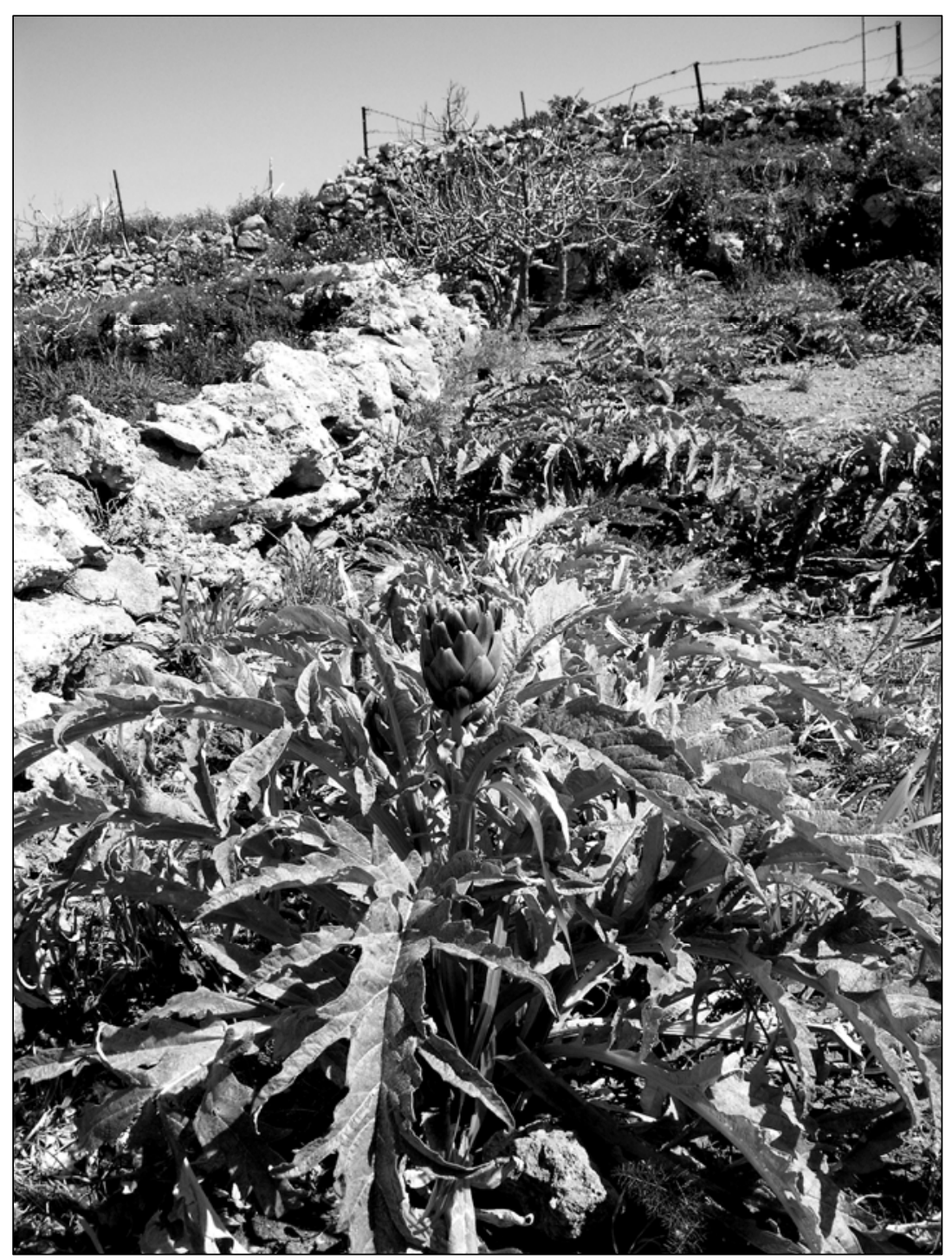

Foto 23: Artischocken im kleinbäuerlichen Anbau in der Region II-Hofra bei Mellieћa (Malta). Foto: KORNITZKY

Die Kultivierung von Artischocken wurde auf den Maltesischen Inseln - trotz guter Voraussetzungen - bisher nicht in vollem Maße ausgeschöpft. Dies ist gerade aufgrund der guten Ausgangsbedingungen unverständlich. Bei gegebenem Klimaoptimum und der Verfügbarkeit von Wasser kann die Artischockenpflanze relativ genügsam und bodenschonend angebaut werden. Zudem sind während der Pflanzenentwicklung nur wenige Kulturmaßnahmen erforderlich.

Das Auftreten möglicher Krankheitserreger hat in vielen Fällen seine Ursache in zu hoher Bodenfeuchtigkeit, zu hohen/zu niedrigen Temperaturen, zu geringer Luftfeuchtigkeit und zu geringem Carbonatgehalt des Bodens ${ }^{551}$. Diese Gefahren können auf den Maltesischen Inseln auf ein Minimum reduziert werden, sodass die meisten Pilzerkrankungen von vornherein unwahrscheinlich sind. Die Gefahr durch Laus- und Schmetterlingsraupenbefall besteht aber auch auf der Maltesischen Inselgruppe.

Der Artischockenabsatz befindet sich auf Malta und Gozo derzeit im Aufwärtstrend, wie Abb. 22 verdeutlicht. Dieser Trend ist einerseits ein Beweis für das Erfolgspotenzial der Distelpflanze, andererseits zeigen die statistischen Daten des NSO aber noch eine hohe Ausbaufähigkeit. 


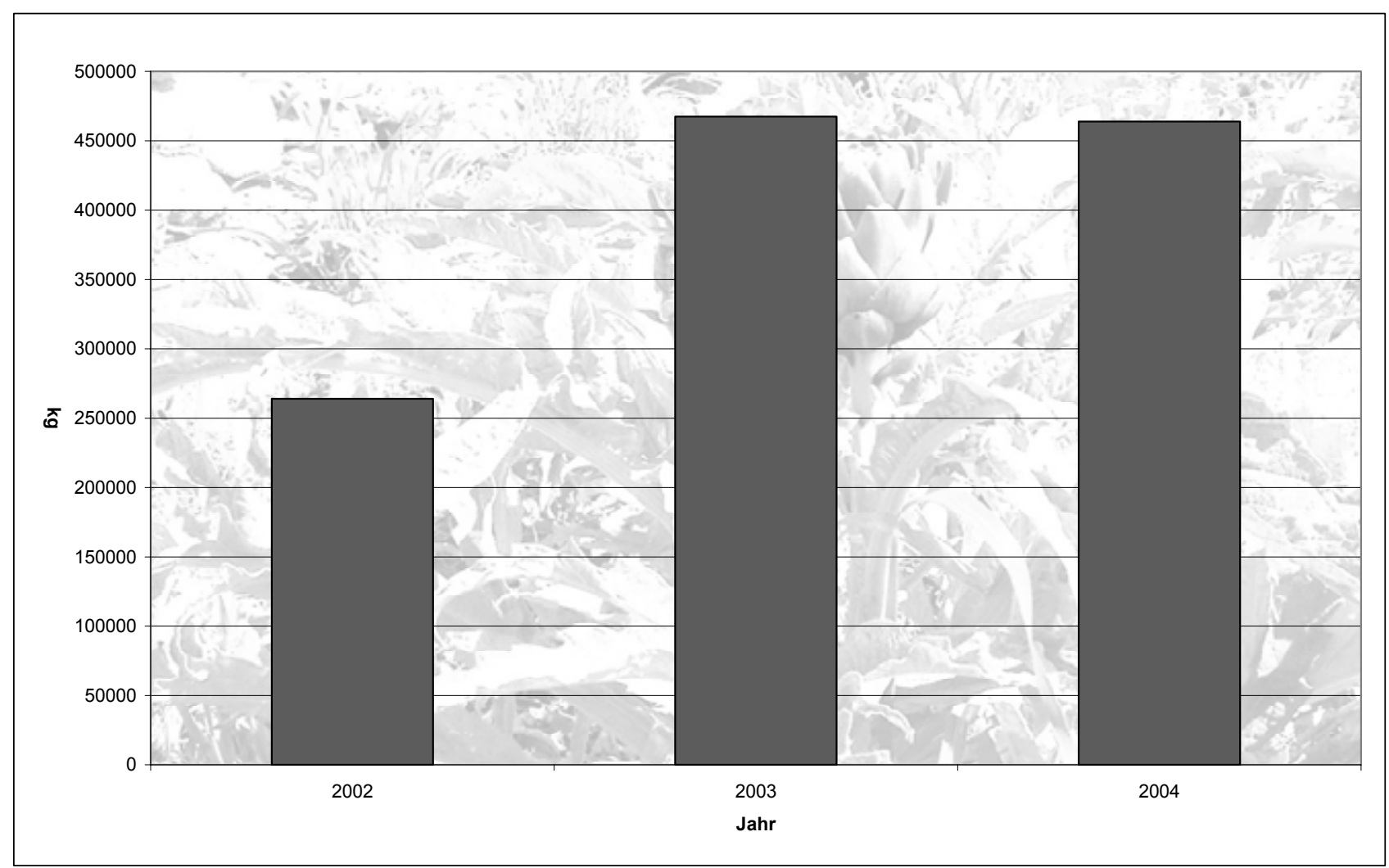

Abb. 22: Gesamtmenge verkaufter Artischocken auf den Maltesischen Inseln pro Jahr von 2002-2004. Es ist davon auszugehen, dass der überwiegende Teil der verkauften Artischocken aus inländischem Anbau stammt. Entwurf: AsCHEMEIER, Quelle: NSO-Daten (individuell für den Autor zusammengestellt)

\subsubsection{Wirtschaftliche Erfolgsaussichten für den Artischockenanbau in Malta}

„Die weltweite Produktion von Artischocken liegt bei 1.198.679 t. Davon werden 60\% allein in Italien und Spanien produziert. Neben dem Anbau im Mittelmeerraum findet man den Anbau von Artischocken in den USA."(HALTER (2001), S. 3).

Die Maltesischen Inseln liegen somit in direkter Nähe der Weltmarktführer für den Anbau und den Export von Artischocken. Dies bedingt einerseits eine Konkurrenzsituation mit übermächtigen Anbietern „direkt vor der Haustür", andererseits können durch die Lage Maltas u.U. bereits bestehende Transportnetzwerke ausgenutzt werden.

Die Maltesischen Inseln sind im Vergleich zu Italien, Spanien und Frankreich keine ernst zu nehmende Konkurrenz in Hinblick auf die Produktion hoher Quantitäten. In Bezug auf die Qualität ist das Gemüse der Maltesischen Inseln jedoch viel gerühmt ${ }^{552}$. Malta könnte, wie im Falle des Olivenöls, zu einem Anbieter von hoch qualitativer Ware werden und somit Vorteile im Bereich der Gourmet- bzw. Slow Food-Kundschaft erreichen ${ }^{53}$.

Als vergleichbares Beispiel sei angeführt, dass sich Artischocken aus der Bretagne europaweit eines besonders guten Rufs erfreuen. Sollte die Qualität der maltesischen Artischocke ähnlich gut oder besser sein, könnte eine Vermarktung des Gemüses als Delikatesse angestrebt werden, zumal Lagerung und Transport von Artischocken problemlos sind ${ }^{554}$ und keine Einschränkung der Qualität bedeuten müssen. In Mitteleuropa müsste sich die maltesische Ware aber gegen die Importe aus den drei Haupterzeugerländern durchsetzen, die ab September auf den Verbrauchermarkt drängen.

\section{Vgl. CREMONA (2003)}

553 Vgl. hierzu die Grundsätze der Slow Food-Bewegung in MARAUHN (2004)

554 Vgl. HALTER (2001) 
Darüber hinaus sind Artischocken seit den 1990er-Jahren ein wichtiger Rohstoff für die Pharmaindustrie. Es konnten positive Wirkungen auf den Blutfettspiegel und verdauungsfördernde Eigenschaften nachgewiesen werden. Im Zuge dieses „Artischocken-Booms“ der letzten Jahre wurden auch in Ländern, die zu den klimatisch ungünstigen Pflanzstandorten zählen, Artischocken in den Anbau eingeführt. In Gewächshäusern geschieht dies z. B. auch in Bayern.

Noch bevor die essbare Knospe entwickelt ist, werden die Blätter zur pharmazeutischen Verwendung geerntet. Auch in diesem Sektor erscheint der Anbau in Malta - evtl. auf ungünstigeren Bodenstandorten vielversprechend. Die Verwertung vor Ort wäre auch gewährleistet, da in Malta einige auf Pflanzenwirkstoffverwertung spezialisierte Pharma-Unternehmen ansässig sind.

Die weltweite Nachfrage nach Artischocken wächst derweil weiter ${ }^{555}$. Der Marktpreis lag in Deutschland im Jahr 2001 bei etwa DM 5,00 pro Kilogramm. Hochwertige Knospen (Gewicht ca. 300 g) wurden mit DM 2,00 pro Stück gehandelt ${ }^{556}$. Seitdem ist im Zuge der Umstellung von DM auf $€$ der Preis gestiegen. Im Internet werden Gourmetprodukte aus Artischocken gegenwärtig zu hohen Preisen von bis zu $10,00 €$ pro $250 \mathrm{~g}$ vertrieben.

Ganz im Gegensatz dazu hat die Artischocke unter der maltesischen Bevölkerung das Image eines „ArmeLeute-Essens" bzw. eines Gemüses, das vornehmlich während der Fastenzeit verzehrt wird ${ }^{557}$. Die Nachfrage auf dem maltesischen Binnenmarkt hält sich daher in Grenzen. Der Autor vermutet, dass die Stellung der Artischocke als „Edel-Gemüse“ in Mitteleuropa bisher in Malta noch nicht in ausreichendem Maße erkannt wurde. Nur so ist auch erklärbar, dass bisher kein staatliches Förderprogramm der maltesischen Regierung zum Anschub eines Ausbaus der Artischockenanpflanzungen ins Leben gerufen wurde. Auch bei der EU wurde nicht um eine Förderung für dieses agrarwirtschaftliche Segment ersucht. Artischocken werden in Malta nicht als kommerziell ertragreiche Anbaupflanze verstanden, sondern dienen nahezu ausschließlich der Versorgung des Binnenmarkts und auch das in eher kleinem Rahmen.

\subsubsection{Fazit}

Die wirtschaftlichen Absatzchancen werden aufgrund der guten Qualität der maltesischen Ware und aufgrund einer möglichen Aufwandsminimierung im dortigen Anbau vom Autor weiterhin positiv eingeschätzt. Die zunehmende Verwertung der Inhaltsstoffe der Artischocke im Pharmabereich stellt eine günstige Ausgangsbasis dar, die auch in näherer Zukunft gute Absatzchancen verspricht. Jedoch vermuten Experten eine Sättigung des Angebots innerhalb der nächsten Dekade ${ }^{558}$.

Artischocken können im Mittelmeerraum günstig und bodenschonend angebaut werden. Ihr Anbau in Malta sollte unter diesem Gesichtspunkt nach einer Prüfung der Marktchancen - auch in Hinblick auf Maltas Status als EU-Mitglied - gefördert werden.

555 Vgl. HALTER (2001)

556 Ebd.

557 Vgl. CREMONA (2003)

558 Vgl. Halter (2001) 
20.2.3 Stärken-Schwächen-Analyse für maltesische Artischocken im Hinblick auf Ihre Vermarktungsfähigkeit als regionales traditionelles Produkt

\begin{tabular}{|c|c|}
\hline Stärken & Schwächen \\
\hline $\begin{array}{l}\text { Ungewöhnliche geographische Herkunft macht } \\
\text { neugierig auf das Produkt => Chancen für originelles } \\
\text { Produktmarketing }\end{array}$ & $\begin{array}{l}\text { Unbekannte geographische Herkunft führt zu } \\
\text { erschwerter Qualitätseinschätzung durch externe } \\
\text { Käufer => erschwertes Produktmarketing }\end{array}$ \\
\hline \multicolumn{2}{|l|}{$\begin{array}{l}\text { Klimatische Faktoren in Malta sind optimal für den } \\
\text { Artischockenanbau => Die Entstehung von } \\
\text { Pilzkrankheiten ist unwahrscheinlich, der Aufwand } \\
\text { bei der Anpflanzung (z. B. greenhouse cultivation) } \\
\text { wird minimiert }\end{array}$} \\
\hline $\begin{array}{l}\text { Anbauprodukt mit langer Tradition, die bis in die } \\
\text { Epoche der Johanniter zurückreicht }\end{array}$ & $\begin{array}{l}\text { Domestizierte Form erst seit ca. } 100 \text { Jahren im } \\
\text { Anbau }\end{array}$ \\
\hline \multirow[t]{2}{*}{$\begin{array}{l}\text { Chance zum Ausbau der Produktion, um höhere } \\
\text { Quantitäten bei gleich bleibend hoher Qualität zu } \\
\text { erzeugen }\end{array}$} & Chance wird derzeit nicht genutzt \\
\hline & $\begin{array}{l}\text { Übermächtige Konkurrenz aus Italien, Spanien und } \\
\text { Frankreich als weltweite Marktführer in unmittelbarer } \\
\text { geographischer Nähe }\end{array}$ \\
\hline \multicolumn{2}{|l|}{ Außergewöhnlich hohe Erzeugerqualität } \\
\hline Bodenschonender Anbau möglich & $\begin{array}{l}\text { Auf ungünstigen Standorten Nachdüngung } \\
\text { notwendig mit u.U. daraus resultierender } \\
\text { Nitratbelastung des Grundwasseraquifers }\end{array}$ \\
\hline $\begin{array}{l}\text { Vermarktung von Artischocken und Folgeprodukten } \\
\text { in Mitteleuropa teilweise als Gourmetprodukt }\end{array}$ & $\begin{array}{l}\text { Schlechtes Image von Artischocken unter der } \\
\text { maltesischen Bevölkerung => „Fastenessen“, „Essen } \\
\text { für arme Leute“ => behindert einen Ausbau des } \\
\text { Anbaus }\end{array}$ \\
\hline $\begin{array}{l}\text { Artischockeninhaltsstoffe finden } \\
\text { Verwendung in der Pharmaindustrie, dies erhöht } \\
\text { zusätzlich den globalen Absatz und den } \\
\text { Weltmarktpreis }\end{array}$ & $\begin{array}{l}\text { Für die pharmazeutische Verwendung können auch } \\
\text { minderwertige Pflanzen verwendet werden, die auch } \\
\text { in klimatischen Ungunstregionen Mitteleuropas } \\
\text { angebaut werden können }\end{array}$ \\
\hline \multicolumn{2}{|l|}{$\begin{array}{l}\text { Artischocken können vor Ort in Malta } \\
\text { pharmazeutisch verwertet werden }\end{array}$} \\
\hline $\begin{array}{l}\text { Der Weltmarktpreis von Artischocken steigt seit } \\
\text { Jahren an }\end{array}$ & $\begin{array}{l}\text { Experten vermuten eine Sättigung des Marktes } \\
\text { innerhalb der nächsten Dekade, was wahrscheinlich } \\
\text { negative Auswirkungen auf den Weltmarktpreis } \\
\text { haben wird }\end{array}$ \\
\hline $\begin{array}{l}\text { Durch Maltas neuen Status als EU-Mitglied } \\
\text { entstehen verbesserte Exportchancen }\end{array}$ & $\begin{array}{l}\text { Artischockenanbau wird im Gegensatz zu anderen } \\
\text { landwirtschaftlichen Produkten nicht von der EU } \\
\text { gefördert }\end{array}$ \\
\hline
\end{tabular}




\subsection{Olivenöl}

\subsubsection{Einleitender Hinweis}

Eingangs sei auf die Kap. 13.9 und 14.1 verwiesen, in denen ebenfalls auf den geschichtlichen Hintergrund, vor allem aber die aktuelle Ausgangssituation der maltesischen Olivenölproduktion im Rahmen der agrarwirtschaftlichen Gesamtlage Maltas eingegangen wurde. Die Angaben a.a.O. ergänzen die Ausführungen im Folgenden.

\subsubsection{Diskussion der historischen Stellung der Olivenölproduktion in Malta und ihrer weit reichenden Ignorierung in wissenschaftlichen Arbeiten des 20. Jahrhunderts}

Ölbaumkultivierung und Verarbeitung von Oliven sind zu den ältesten Beispielen für die Domestizierung von Nutzpflanzen in Malta und im gesamten Mittelmerraum zu rechnen. In der Antike war Malta vermutlich von zusammenhängenden Waldgebieten aus Ölbäumen überdeckt ${ }^{55}$. Heute sind nur noch Relikte dieser Bestände erhalten. Sie gehören zu den ältesten Olivenbäumen im Mittelmerraum.

Die ältesten Ölbäume der Maltesischen Inselgruppe findet man in der Nähe der Ortschaft Bidnija auf Malta. Die dortigen Bäume wurden auf ein Alter von über 1.500 Jahren datiert ${ }^{560}$. Einige Autoren sehen sie sogar im Zusammenhang mit der römischen Periode Maltas ${ }^{561}$. Auch alte Orts- und Flurnamen aus der Zeit vor der arabischen Herrschaftsperiode wie Żebbuǵ ${ }^{562}$ (malt.: żebbuǵa = Olive) oder Żejtun (malt.: żejt = Öl) ${ }^{563}$ weisen auf die lange Tradition der Ölbaumkultivierung hi $^{564}$. Die Wappen der gozitanischen Ortschaften

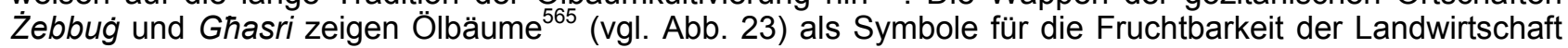
beider Orte (vgl. Wappeninschriften).

In der römischen Periode Maltas und Gozos (ab 218 v. Chr. bis ca. 535 n. Chr.) war Olivenöl nachweislich einer der wichtigsten Wirtschaftsfaktoren der Inseln. Das vermutlich bedeutendste Zentrum der Olivenölproduktion des antiken Malta lag in der Gemarkung der Siedlung Wardija oberhalb des Dorfes Burmarrad im Nordwesten der Insel Malta ${ }^{56}$. Dort, im Bereich des Orts mit dem Flurnamen San Pawl Milqi, wurden von 1962-1967 Ruinen einer römischen „Villa Rustica“ mit angeschlossener Olivenölproduktion ausgegraben $^{567}$ (vgl. Foto 24).

559 Vgl. LocATElLI (2001). BeZZINA gibt an, dass in Gozo einst die Wildform des Ölbaums (olea silvestris) wuchs (BEZZINA (1988), S. 43). Es ist also durchaus nicht unwahrscheinlich, dass LOCATELLIs Vermutung hinsichtlich der Ölbaumwälder stimmt.

560 Vgl. MALTA TODAY, Onlineausgabe vom 28.10.2001

561 Vgl. TEUMA (2003), S. 84

562 Żebbuǵ ist ein Ortsname, der sowohl auf Malta als auch auf Gozo auftaucht und allein dadurch schon annehmen lässt, dass Olivenbäume auf dem gesamten Archipel verbreitet waren.

563 Weitere Orts- und Flurnamen mit Bezug zum Ölbaum sind Birżebbug̉a (sinngemäße Übersetzung vermutl.: Brunnen in der Nähe des Ölbaums), Haż-Żebbuǵ (sinngemäße Übersetzung vermutl.: Mauer am Ort des Ölbaums) und lż-Żebbuǵ.

564 Vgl. BOWEN JONES, DEWDNEY \& FISHER (1961), S. 213

565 Beide Wappen wurden erst 1876 eingeführt (vgl. BEZZINA (1988), S. 7), was zeigt, dass auch zu dieser Zeit der Ölbaum noch als Identitätsträger fungierte. Die Darstellung der Bäume in den Wappen deutet darauf hin, dass die Symbolkraft des Ölbaums ungebrochen war und als geschichtliches Erbe von den Menschen akzeptiert wurde. Es ist daher anzunehmen, dass die Gewinnung von Olivenöl in kleinem Maßstab in diesen Ortschaften auch zum Ende des 19. Jhd. noch praktiziert wurde. Dies kann auch deswegen angenommen werden, weil der Ortsname Ghasri keine Verbindung zu der Thematik der Ölbaumkultivierung erkennen lässt (Ghasri könnte mit „Dämmerung“ übersetzt werden). Der Wappenspruch „Ex Labore Fructus“ deutet auf regen landwirtschaftlichen Anbau hin, der in Verbindung mit dem Wappenmotiv nur den Schluss zulassen kann, dass Olivenbäume in Ghasri zur Zeit der Schöpfung des Wappens noch eine wichtige Rolle in der Agrargeographie gespielt haben müssen.

566 Vgl. AZZOPARDI (2002), S. 166

567 Vgl. u. a. TEUMA (2003), S. 81-94; LoCATELLI (2001) 


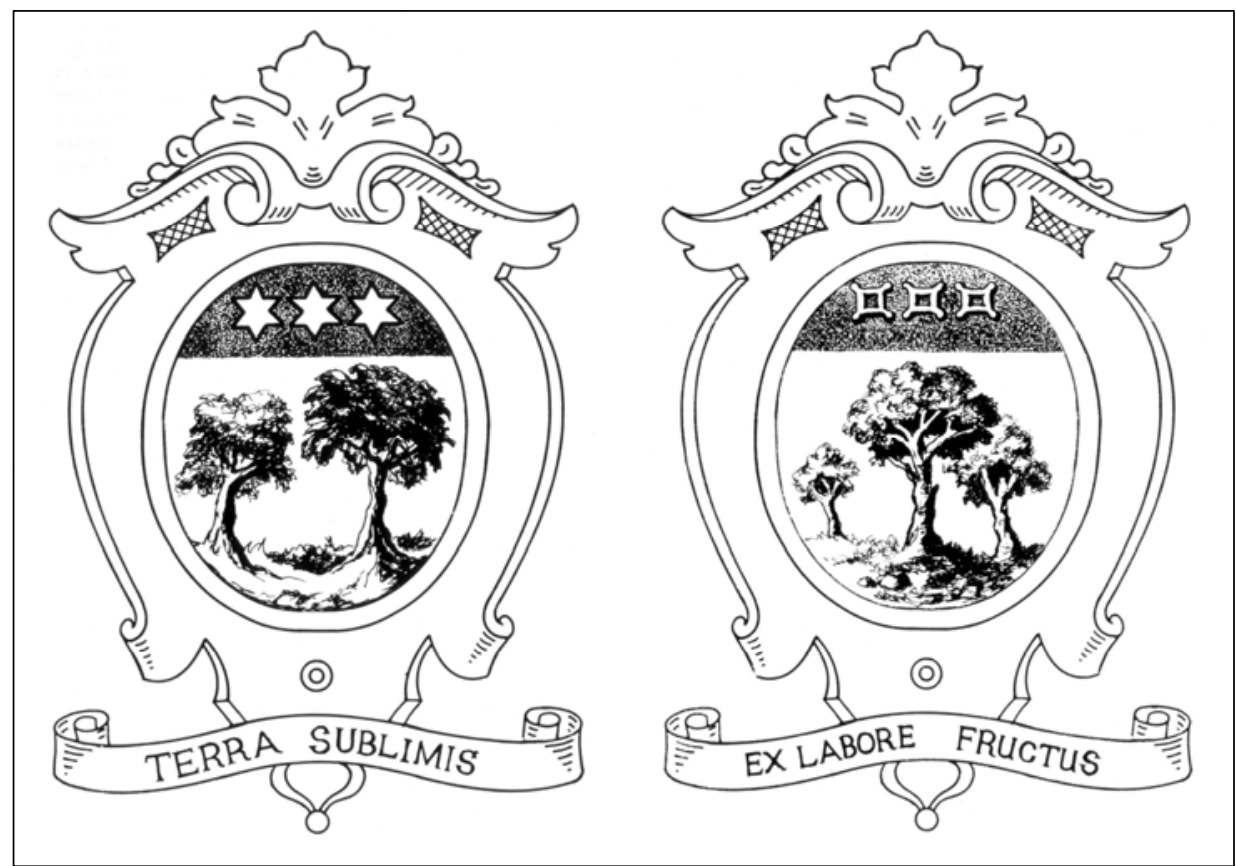

Abb. 23: Die Wappen der gozitanischen Ortschaften Żebbug (links) und Ghasri. Quelle: BEZZINA (1988), S. 20 \& S. 42

Zwar war es nicht der erste Fund der bewies, dass es in vergangener Zeit Olivenölproduktion in Malta gegeben haben musste. Jedoch war diese Entdeckung von herausragender Bedeutung ${ }^{568}$, da die gefundene Produktionsstätte erstaunlich große Ausmaße aufwies. Somit war nun erstmals anzunehmen, dass die Produktion von Olivenöl nicht nur überhaupt stattfand, sondern einer der bedeutendsten Wirtschaftsfaktoren der Insel gewesen sein musste.

Die relativ späte Entdeckung dieser wichtigen archäologischen Stätte erklärt auch, warum in dem Standardwerk von BOWEN JONES, DEWDNEY \& FISHER (1961) das Thema Ölbaumkultivierung und Olivenölproduktion in lediglich 13 Zeilen abgehandelt wurde ${ }^{569}$, denn zum Zeitpunkt der Schriftsetzung ihres Buchs waren nur rudimentäre Hinweise auf die Anlage in San Pawl Milqi vorhanden ${ }^{570}$, und die eigentliche Ausgrabung hatte noch gar nicht begonnen. Es gab auch keine äußeren Hinweise auf die Ausmaße der späteren Fundstätte, da diese unter umgeschichtetem Bodenmaterial begraben lag. Selbst in Quellen aus dem 17. Jh. wurde sie nicht erwähnt und war wohl schon damals in Vergessenheit geraten ${ }^{571}$. Sie wurde erst in den 1960er Jahren, aufgrund eines Zufallsfunds in Zusammenhang mit der ländlichen Kapelle aus dem 17. Jh. ausgegraben, die heute am Ort der römischen Villa steht.

Die angesprochene, extrem kurze Erwähnung der Ölbäume in dem bis heute wichtigen Standardwerk der Malta-Forschung von BOWEN JONES, DEWDNEY \& FISHER - die zwar von ausgedehnterer Produktion von Olivenöl in der Vergangeheit schreiben, aber in keinem außergewöhnlichen Maßstab - führte in der Folge dazu, dass diese Meinung vor allem von nicht maltesischen Autoren als Gemeinplatz in die meisten geographischen Arbeiten über die Maltesischen Inseln weitgehend kritiklos übernommen wurde.

568 Vgl. BONANNO (1994), S. 95

569 Vgl. BOWEN JONES, DEWDNEY \& FISHER (1961), S. 213

570 Vgl. TeUma (2003), S. 84

571 Ebd., S. 81 


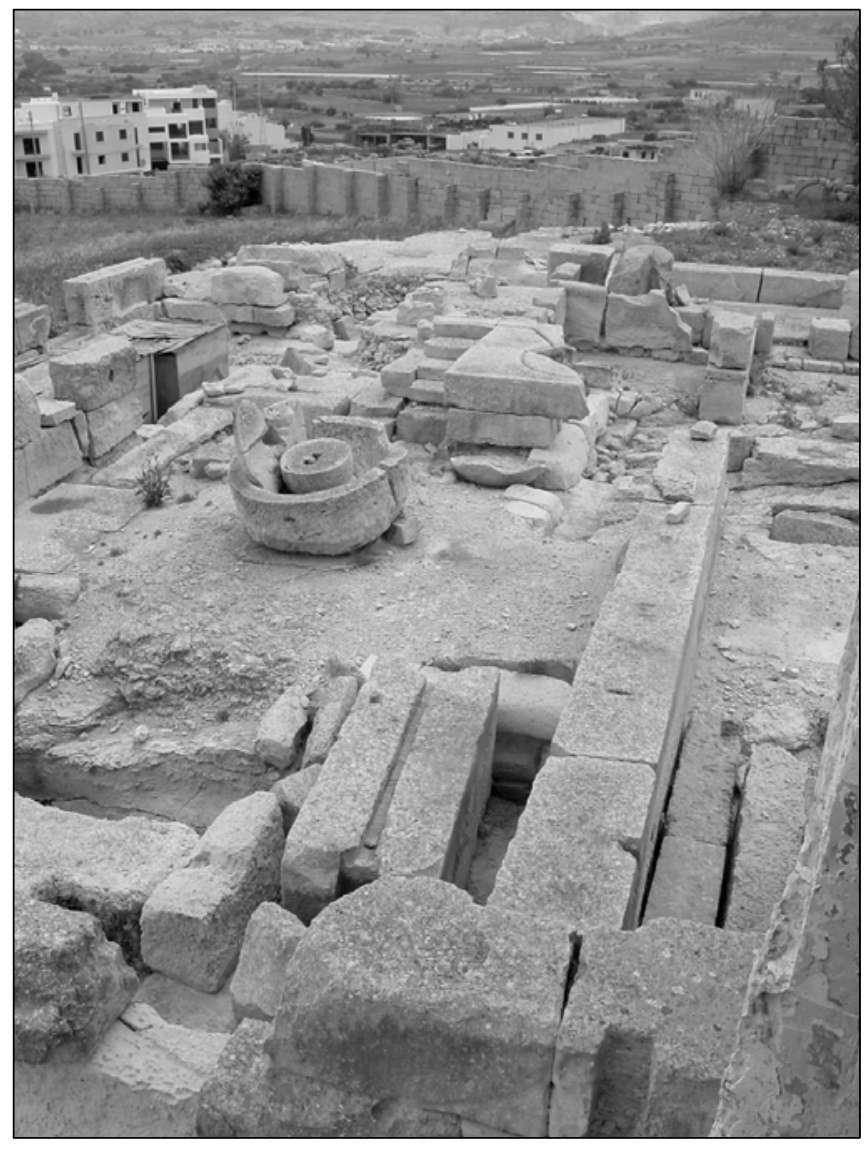

Foto 24: Teilaufnahme der Ruinen der Villa Rustica von San Pawl Milqi. Blickfang Mitte links: ein „Trapezium“ zum Zerstoßen der Oliven. Auch alle anderen auf dem Foto sichtbaren Überreste gehörten zur Olivenölproduktionsstätte der Villa. Es handelt sich dabei u. a. um Dekantiertröge (hinten rechts) und Ölrinnen (vorne links/Mitte). Die Ausgrabungsstätte ist z. Zt. in schlechtem Zustand und für die Offentlichkeit nicht zugänglich. Foto: ASCHEMEIER

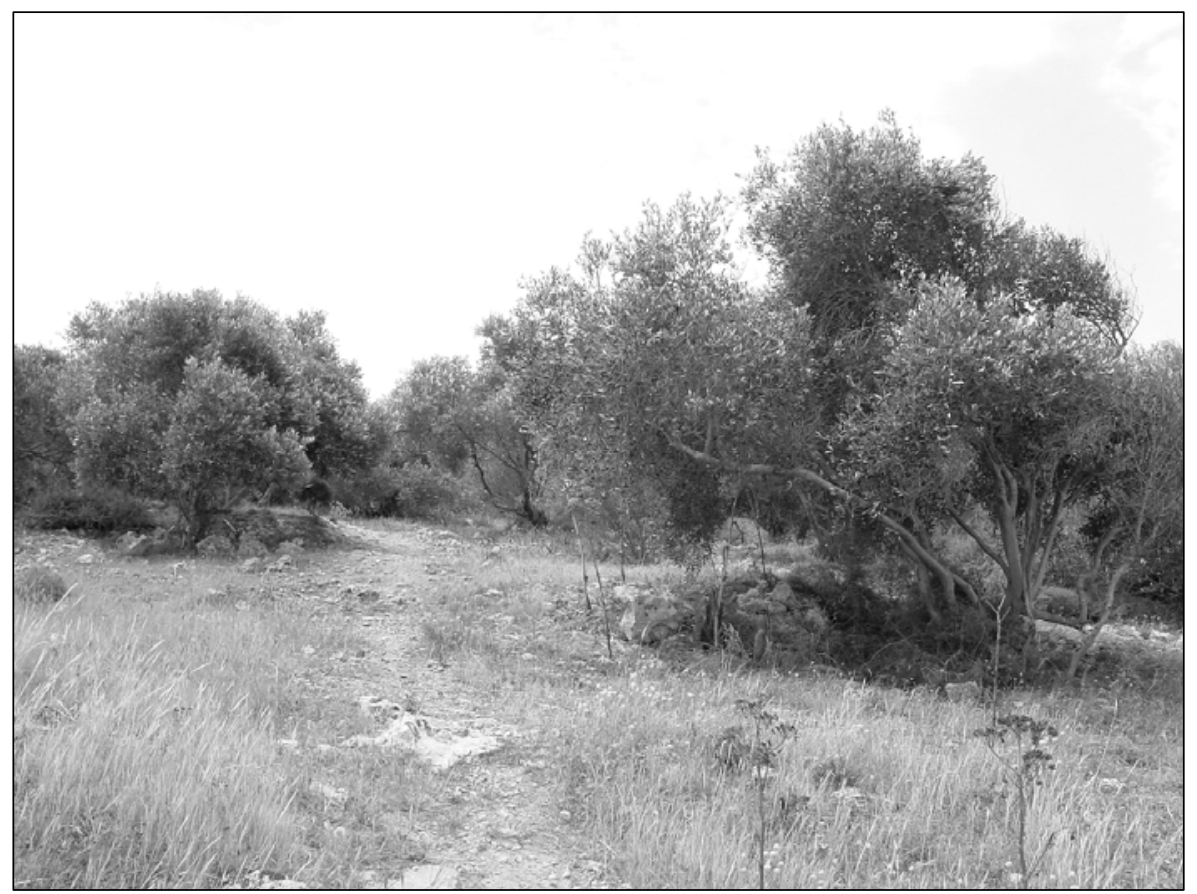

Foto 25: Ölbaumanpflanzung Bajda Ridge. Foto: AsCHEMEIER 
NEHRING (1966) bildete in dieser Hinsicht eine Ausnahme. In seiner Arbeit ist nachzulesen, dass im Rahmen des Aufforstungsprogramms der 1950er- und 1960er-Jahre auch Olivenbäume angepflanzt wurden. Eine der flächenmäßig größten und ältesten dieser Anpflanzungen findet sich oberhalb von Xemxija (Malta) auf dem Bajda Ridge ${ }^{572}$. Mittlerweile sind einige dieser Anpflanzungen zu mediterranen Wäldern herangewachsen (vgl. Foto 25), und es erscheint, nebenbei gesagt, dem Autor der vorliegenden Arbeit unbegreiflich, warum auch in neueren Arbeiten immer wieder von Buskett Gardens als „einzigem zusammenhängenden Waldgebiet Maltas" zu lesen ist.

Heute ist unbestritten, dass Olivenöl eines der wichtigsten Wirtschaftsgüter des antiken Malta war:

"The sites of many villas have been excavated and the remains found indicate that the agricultural system of the day was concerned principally with the production of wheat and olives." (BLOUET (1987), S. 33).

„Olive oil production was a complex and time-consuming work, but was essential to all categories of Roman Social and economic basis. (...). Olive oil weighted its weight in gold, and must have been a backbone of local economy." (TEUMA (2003), S. 91 f.)."

„The evidence for olive cultivation is excellent for many of the pairs of stone discs used to separate the flesh of the olive from the pip have survived. After the pips were extracted, the flesh was pressed to produce oil." (BLOUET (2004), S. 37).

„In the distant past, even during the Roman period, the Maltese Islands were among those Mediterranean countries which had a thriving olive-oil production industry. Olive-oil crushing implements belonging to the Roman period have been excavated in Bidnija, Xemxija, San Pawl Milqi and Tas-Silg in Marsaxlokk. During the following centuries this industry must have grown to a considerable extent (...)." (AZZOPARDI (2002), S. 166).

„The most extensive remains of the agricultural type of villa is that of San Pawl Milqi in the northwest part of Malta, but those of Ta'Kaććatura and Żejtun, close to the southeastern harbour of Marsaxlokk, are also considerable. The distinctive features in these buildings are the surviving stone elements of the apparatus used for the pressing of olive oil." (BONNANNO (1994), S. 95).

Besonderes Aufsehen erregte die Entdeckung von Überresten eines Seehafens aus römischer Zeit im Dorf Burmarrad während der 1980er-Jahre - Nur wenige hundert Meter von der Villa von San Pawl Milqi entfernt.

Das Dorf Burmarrad liegt heute etwa 1,5 km vom Meer entfernt im Landesinneren der Insel. Niemand hätte hier einen Seehafen vermutet. Die Funde ließen aber keinen Zweifel zu. Man fand nicht nur Teile einer antiken Kaimauer aus römischer Zeit, sondern auch Überreste eines antiken Ankers von 12m Länge. Dies ist der bis heute weltweit größte je gefundene Überrest eines Schiffsankers aus römischer Zeit. Als Ursprung wird ein ägyptisches Handelsschiff vermutet (vgl. TEUMA (2003), S. 108).

Große Mengen an Keramik sowie weitere Bleianker (vgl. Foto 26) wurden seitdem ausgegraben. Erst 2002 wurden weitere Teile des ehemaligen Hafenbeckens entdeckt. Sie werden derzeit archäologisch untersucht.

572 Weitere befinden sich bei Xewkija auf Gozo und im Bereich Marfa Ridge (nördlich der Straße nach Cirkewwa) auf Malta. 


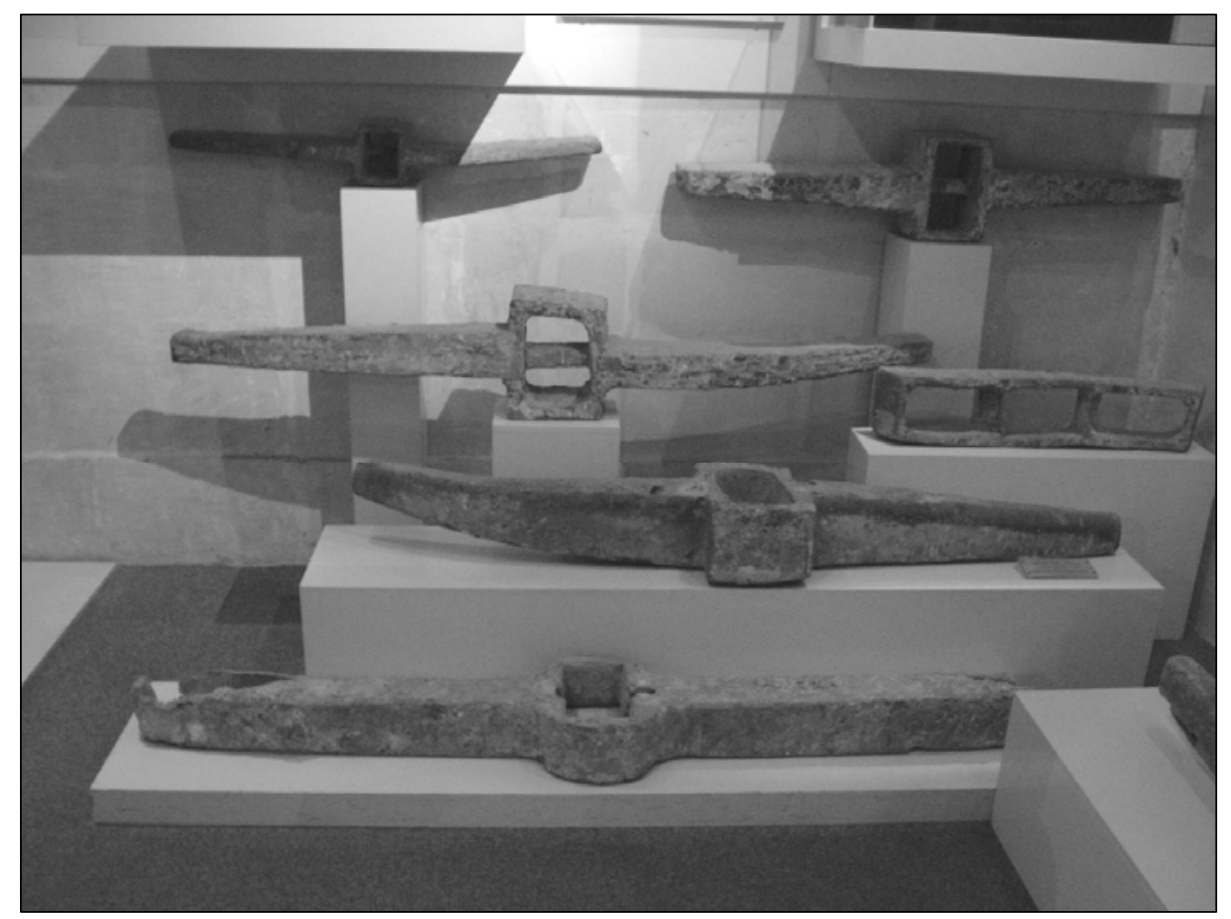

Foto 26: Überreste von römischen und ägyptischen Bleiankern aus der Region Burmarrad in der Ausstellung des Maritime Museum in Vittoriosa (Malta). Foto: AsCHEMEIER

Alles deutet darauf hin, dass die Gegend um Burmarrad einstmals einer der Haupthäfen oder zumindest der wichtigste Nebenhafen der Insel Malta gewesen sein muss, der mit der Zeit vollständig verlandete ${ }^{573}$. U.U. war er in römischer Zeit wichtiger als der große Naturhafen der Insel "Grand Harbour" im heutigen Agglomerationsraum der Stadtregion Valletta ${ }^{5 / 4}$. Dieser war bis zum Bau eines Wellenbrechers durch die britische Kolonialregierung in den Jahren 1903-1906 als ein unsicherer und sturmgefährdeter Hafen bekann ${ }^{575}$ : "The northeast wind, the grigal, blows into the mouth of Grand Harbour and, before the building of the breakwater, at the beginning of the twentieth century, the wind made it dangerous for vessels entering or leaving port." (BLOUET (2004), S. 10 f.).

Fügt man das „historische Puzzle“ zusammen, ergibt sich eine ideale Fabrikationsstätte für den Export von Olivenöl im Bereich Burmarrad/Wardija. TEUMA zur Lage der Ölproduktionsstätte bei San Pawl Milqi in römischer Zeit:

„To seaward the villa would have dominated a large stretch of inland sea. The advantages would have been incalculable, with produce and materials being delivered or exported by sea from the villa's very doorstep." (TEUMA (2003), S. 84).

573 Einen wichtigen Hinweis dazu birgt u. a. der Ortsname Burmarrad, dessen sinngemäße Übersetzung bedeutet: „Das Land, das Krankheit bringt". Der Ortsname, der erst in nach-byzantinischer Zeit auftrat, wird von den Archäologen heute so gedeutet, dass das frühere Hafenbecken mit der Zeit verlandete und zu Marschland wurde (vgl. TEUMA (2003), S. 106). Flächen wie diese waren auch in Malta lange Zeit Brutstätten für die Malaria. Der Hafenname in römischer Zeit war Benwarrad (vgl. CREMONA (2003), S. 31) (Vermutl. Übersetzung: „Erbe des Sohnes“ oder „Sohn des Erbes“ (vgl. TEUMA (2003) S. 81).

574 Diese Einschätzung ist allerdings umstritten. Der Geograph BRIAN BLOUET äußerte sich dahingehend, dass es nur deshalb so viele Funde in der Gegend gegeben habe, weil diese bis heute nur wenig überbaut sei und Grabungen daher unkompliziert (BLOUET (2004), S. 37 ff.). In der Gegend um das heutige Marsa wurden ebenfalls römische Hafenanlagen entdeckt. Auch hierbei handelte es sich um einen verlandeten Hafen, der erst in den 1960er-Jahren ausgegraben wurde. Alles in allem konnte die These von Burmarrad als einem der Hauptwirtschaftshäfen in römischer Zeit (die u. a. von LOCATELLI, BONANNO und TEUMA als wahrscheinlich betrachtet wird) bisher weder eindeutig widerlegt noch bestätigt werden.

575 Vgl. GRECH (2003). Zudem dürfte der Hafen, der eine schmale, weit ins Hinterland reichende Bucht umfasste, einfacher gegen Angriffe von See zu verteidigen gewesen sein, als der breite Naturhafen von Valletta. Diesbezüglich muss nach Einschätzung des Autors daran gedacht werden, dass die umfangreichen Befestigungsanlagen des Grand Harbour in römischer Zeit noch nicht existierten. Die Landzunge mit dem heutigen Valletta ragte als felsige Halbinsel zwischen dem heutigen Marsamxett Harbour und dem Grand Harbour hervor. Dies würde Angriffe von See oder Hinterhalte feindlicher Schiffe eher begünstigen als verhindern. 
Die maltesische Schriftstellerin MATTY CREMONA wird noch deutlicher: „Olive oil is a bulky and heavy commodity, and the oil from San Pawl Milqi could be taken with relative ease down to the harbour, where trade ships would carry it out."(CREMONA (2003), S. 33).

\subsubsection{Olivenöl in der Vermarktung als regionales traditionelles Produkt}

Das Olivenöl der heutigen Ölpresse in Wardija ${ }^{576}$ vereint demnach mindestens drei wesentliche Merkmale, die im Hinblick auf die Vermarktungsfähigkeit als regionales traditionelles Produkt eine positive Prognose erlauben:

1. Die Ware ist von sehr hoher Qualität und kann als exklusiv bezeichnet werden ${ }^{577}$.

2. Olivenöl ist eines der Produkte Maltas, dessen Herstellung eine Tradition seit der Antike umfasst.

3. Der Autor wies nach, dass diese Tradition auch in Zeiten des Verfalls der Ölbaumkultivierung aufrechterhalten wurde und dass der Ölbaum als Wappenpflanze bestimmter maltesischer und gozitanischer Ortschaften in größerem Umfang zur regionalen Identitätsfindung beigetragen hat.

Olivenöl kann demnach als regionales traditionelles Produkt der Maltesischen Inseln bezeichnet werden, wenn auch der Neuaufnahme der Produktion im Jahr 2000 eine lange Periode des Verfalls vorausging ${ }^{578}$.

Zur Qualität des in Malta erzeugten Öls wurde bereits im Rahmen dieser Arbeit Stellung genommen. Das Öl der Marke Wardija Premium Brand ist ein exklusives Produkt.

Im Interview mit SAM CREMONA, dem Betreiber der Ölpresse in Wardija, wurde ersichtlich, dass die Ausschöpfung des Vermarktungspotenzials aber erst am Anfang steht. CREMONA hat weitere Maßnahmen in die Wege geleitet, um die Qualität des Olivenöls auch durch international anerkannte Zertifikate zu untermauern. So plant er, sein Olivenöl als ökologisch erzeugtes Produkt zu vermarkten:

„One thing I have applied for is organic certification. And in the beginning the EU and the Italians were very sceptical. They asked: „How can you achieve this on a small island like Malta?". But as the testing was done they realized that there were no traces of chemical presence at all. And when they saw the quality of the olive oil they were very excited and not only this. They said that the assistance to get a certification - which is very expensive - could be channeled directly through the local councils or the government with the intention of doing the certification."

(Auszug aus einem Experteninterview mit SAM CREMONA vom 25.05.2004).

Weitere Ansätze zur Qualitätssteigerung des Produkts laufen über DNA-Tests alter maltesischer Ölbaumsorten, weil man erkannt hat, dass es in Malta (ähnlich wie im Weinbau) endemische Sorten zu geben scheint, die nirgendwo sonst im Mittelmeerraum auftauchen.

Hintergrund dieser Anstrengungen ist es, die Exklusivität des maltesischen Öls weiter zu erhöhen, indem auf dem internationalen Markt und/oder für den internationalen Tourismus Olivenöl angeboten werden kann, das aus endemischen Ölbaumsorten gepresst wurde. CREMONA dazu:

„We are doing what we are calling a gene bank for these varieties. We try to identify them all. We have already identified three varieties so far. One is called the "Maltese olive“. It has always been called the „Maltese olive" and it has a very particular characteristic. One is called the "Bidnija olive", we got this from the trees there which are 1.000-1.500 years old ${ }^{579}$.

And another variety is the „White olive“ which is very particular. It is a large table olive and it is actually white, completely white. And of course I was very surprised when I saw it for the first time, because it was braught

576 S. zum Thema der Neuaufnahme der maltesischen Tradition der Olivenölproduktion in Wardija Kap. 14.1

577 Vgl. hierzu auch Taste Magazine, H. 2/2004, S. 24-29

578 S. hierzu Kap. $13.9 \& 14.1$

579 S. 0. 
here by people who wanted to press their olives. But in fact this is one of the varieties which a writer called $\mathrm{J}$. BORG had mentioned as typical Maltese during the time of the Knights (...).

But to be able to say that we have our own olive (...) we must propagate a large number. We have to organize not one but ten gene centres. Then we will have to take samples from the same varieties, compare their DNA and see if we do have a variety or not. Once we do have a variety or a sub-variety then we can register it as Maltese and then we can produce Olive oil which is D.O.P., which means „Denominazione Origine Protetta", which means it is from protected origin olives ${ }^{580}$."

(Auszug aus einem Experteninterview mit SAM CREMONA vom 25.05.2004).

Diese Pläne werden laut CREMONA seit November 2004 in die Tat umgesetzt. Im Zuge dieser engagierten Bemühungen soll das Olivenöl, das später aus den neu etablierten Sorten gepresst wird, unter einem anderen Label als bisher verkauft werden.

Damit würden zwei Labels für maltesisches Olivenöl existieren. Das eine, das bereits ein hochwertiges Produkt ist, kann durch die Exklusivität des anderen Öls, das aus Pflanzen mit der im Interviewauszug beschriebenen eng begrenzten Biogeographie gepresst wird, ergänzt werden.

Maltesisches Olivenöl würde infolgedessen mit drei herausragenden Qualitätsausweisungen vermarktet werden können:

1. Es wäre „extra vergine“, also kalt gepresst und nicht nachbehandelt.

2. Es wäre aus organischem Anbau, demnach ein „Bio-Produkt“.

3. Es wäre Öl mit einer geschützten Herkunftsbezeichnung ${ }^{581}$ durch ein DOP-Siegel der EU.

Durch diese Dreifachauszeichnung würde das Image des maltesischen Olivenöls weiter verbessert. Unter kommerziellen Gesichtspunkten wäre ein höherer Preis auf dem internationalen Markt zu erzielen.

\subsubsection{Konsequenz}

Die Konsequenz ist, dass das Produkt, im Gegensatz zu anderen maltesischen Produkten, die ein niedrigeres Prestigelevel erreichen, unterscheidbar beworben und vermarktet werden muss.

Der Autor sieht vor allem in der internationalen Vermarktung erhebliche Ausbaumöglichkeiten. Derzeit wird es kaum ein Delikatessengeschäft außerhalb Maltas geben, das maltesisches Olivenöl führt. Die Gründe werden in der bisherigen kleinräumigen Vermarktung des Produkts gesehen. Die Qualität des Öls ist jedenfalls anerkannt gut. Auch über die mangelnden Quantitäten kann mittlerweile kaum noch argumentiert werden. Laut Aussage von SAM CREMONA wurden 2003 in Wardija mehr Oliven zu Öl verarbeitet als je zuvor:

„Two years ago I bought a much larger machine which crushes 400 kilos an hour, almost half a ton. (...). But this is still not enough. (...). Just handling and working is often from 6 o'clock in the morning to midnight during the pressing season for a whole two months."

"I cannot possibly press everybody's oil. All these olives coming from the new large plantations are a lot. Last year I had more than 225 tons of olives!"

(Auszug aus einem Experteninterview mit SAM CREMONA vom 25.05.2004).

580 Hierbei spielt Herr CREMONA auf eine der drei EU-Denominationen DOP (Geschützte Herkunftsangabe), PDO und AOP an. Zur Vertiefung vgl. GRIENBERGER (2000); VOTH (2003) sowie ASCHEMEIER (2005)

581 Zur Verdeutlichung der Bedeutung dieser Klassifizierung möchte der Autor auf den Stellenwert von Olivenöl geschützter Regionen in Gourmetkreisen hinweisen, die ähnlich einzuschätzen ist, wie der Stellenwert von Wein oder Whisky aus zertifizierten Regionen. Vgl. hierzu auch VOTH (2003) und GRIENBERGER (2000). 


\subsubsection{Einschätzung der nationalen und internationalen Konkurrenz}

Offenbar ist die Herstellung auch größerer Mengen Olivenöl möglich (s. o.). Deshalb wird die Vermarktung auf internationaler, insbesondere - aufgrund der Vorteile Maltas als EU-Mitgliedsstaat - auf europäischer Ebene, empfohlen.

In jüngster Zeit ergab sich jedoch eine bizarre inländische Konkurrenz unter den Ölpressern, welche die Vermarktung des maltesischen Olivenöls als einheitliches Qualitätsprodukt in Frage stellt. CREMONA zur der Frage nach dem Auftauchen weiterer Ölproduzenten in Malta:

"Unfortunatley it happened - but not the way I was expecting it. I was expecting that from a small machine we would go together and get larger and larger. But people simply saw the first one and they bought similar ones which are small ones. Of course small ones are inefficient.

So there are two more now. I know the people who have them and I try to say: "Look, let's get together, let's go for a larger machine!" (...).

Of course I would like to do the marketing together. The pressing I think should be done at various samples. And although Malta is small, I think that I should be pressing the olives that grow in the north of Malta, in the south there should be another one and in the centre there should be another one. And then I think we should get together to market the oil together. The certification should go together, the whole attention. Of course many people don't realize that quality is more important than quantity."

(Auszug aus einem Experteninterview mit SAM CREMONA vom 25.05.2004).

Diese Entwicklung wird im Rahmen der vorliegenden Untersuchung als bedenklich eingestuft, weil sie die Etablierung von maltesischem Olivenöl als einheitliches Qualitätsprodukt bereits im Ansatz unterwandert. Mehrere Hersteller bedeuten fast zwangsläufig unterschiedliche Qualitätsniveaus. Sollten sich in Zukunft neben der Marke „Wardija Premium Brand“ einer oder zwei weitere Hersteller auf den Markt begeben, bleibt nur zu hoffen, dass diese auf ähnlich hohem Qualitätslevel produzieren.

Maltesische Produzenten müssen sich darüber im klaren sein, dass Olivenöl aus Malta nicht „automatisch“ ein exklusives Produkt ist. Öl von guter Qualität wird auch in Spanien und Italien, vor allem aber in Griechenland produziert.

Erst zertifizierte, außerordentliche Qualität in Verbindung mit der ungewöhnlichen geographischen Herkunft macht maltesisches Olivenöl zu etwas Besonderem. Sollte der Markt in Zukunft mit minderwertigem Öl aus Malta beliefert werden, steht der Ruf der gesamten Region auf dem Spiel, was die Vermarktung selbst des Öls der qualitativ hochwertigen Hersteller auf jedem Markt, egal ob touristisch oder international, extrem erschwert, u.U. sogar völlig unrentabel werden lässt.

Die einheitliche Vermarktung eines qualitativ einheitlich hochwertigen Produkts wäre dagegen zu begrüßen, insbesondere wenn es sich bei der Marke um einen Neueinsteiger/Kleinhersteller handelt. 
20.3.6 Stärken-Schwächen-Analyse für maltesisches Olivenöl im Hinblick auf seine Vermarktungsfähigkeit als regionales traditionelles Produkt

\begin{tabular}{|c|c|}
\hline Stärken & Schwächen \\
\hline $\begin{array}{l}\text { Ungewöhnliche geographische Herkunft macht } \\
\text { neugierig auf das Produkt }=>\text { Chancen für originelles } \\
\text { Produktmarketing }\end{array}$ & $\begin{array}{l}\text { Unbekannte geographische Herkunft führt zu } \\
\text { schwieriger Qualitätseinschätzung durch externe } \\
\text { Käufer => erschwertes Produktmarketing }\end{array}$ \\
\hline $\begin{array}{l}\text { Möglichkeit zur Produktion von DOP-klassifiziertem } \\
\text { Öl, d. h. EU-Einstufung als Produkt mit geschützter } \\
\text { Herkunftsbezeichung }\end{array}$ & $\begin{array}{l}\text { DNA-Test als Voraussetzung für DOP- } \\
\text { Klassifizierung noch nicht abgeschlossen }\end{array}$ \\
\hline $\begin{array}{l}\text { Chance der Vermarktung als biologisch-organisch } \\
\text { erzeugtes Naturprodukt }\end{array}$ & $\begin{array}{l}\text { Prozess zur Vergabe des Bio-Status noch nicht } \\
\text { abgeschlossen }\end{array}$ \\
\hline $\begin{array}{l}\text { Produkt mit langer Tradition, die bis in die römische } \\
\text { Periode Maltas zurückreicht }\end{array}$ & $\begin{array}{l}\text { Erst seit } 2000 \text { wieder kommerzielle Herstellung des } \\
\text { Produkts, zuvor lange Verfallsphase des } \\
\text { Wirtschaftszweigs }\end{array}$ \\
\hline $\begin{array}{l}\text { Chance zum Ausbau der Produktion, um höhere } \\
\text { Quantitäten bei gleich bleibendem Qualitätsniveau } \\
\text { zu erzeugen }\end{array}$ & Chance wird derzeit nicht genutzt \\
\hline $\begin{array}{l}\text { Bisheriger Erzeuger als einziger Anbieter auf dem } \\
\text { Markt }\end{array}$ & \\
\hline $\begin{array}{l}\text { Originelles und funktionelles Geschäftskonzept }=> \\
\text { ständiger Nachschub von Oliven als } \\
\text { Ausgangsprodukt ist gesichertt }\end{array}$ & $\begin{array}{l}\text { Hohe Verluste bei der kommerziellen Verwertbarkeit, } \\
\text { weil durch die ungewöhnliche Geschäftsstruktur nur } \\
20 \% \text { des insgesamt gepressten Öls auch auf den } \\
\text { Markt kommen }\end{array}$ \\
\hline & Bisher keine Internetpräsenz des Herstellers \\
\hline $\begin{array}{l}\text { Erzeuger zeigt hohes Engagement in der } \\
\text { Durchsetzung von Zielen sowie ständig neue Ideen } \\
\text { zur Vermarktung seines Produkts }\end{array}$ & $\begin{array}{l}\text { Viele Ideen können aufgrund fehlender betrieblicher } \\
\text { und personeller Infrastrukturen noch nicht umgesetzt } \\
\text { werden }\end{array}$ \\
\hline $\begin{array}{l}\text { Olivenöl hat allgemein nach wie vor ein positives } \\
\text { Image in der Öffentlichkeit (vgl. positive Wirkung im } \\
\text { Rahmen gesunder Ernährung, als Gourmetprodukt, } \\
\text { Assoziierung mit dem Mittelmeerraum, mit } \\
\text { Urlaubserfahrungen, etc.) }\end{array}$ & $\begin{array}{l}\text { Maltesisches Olivenöl im Speziellen hatte bisher gar } \\
\text { kein Image und noch immer wissen die meisten EU- } \\
\text { Bürger nicht, dass es das Produkt überhaupt gibt }\end{array}$ \\
\hline $\begin{array}{l}\text { Aufsehen erregende archäologische Entdeckungen } \\
\text { in Zusammenhang mit der Historie der maltesischen } \\
\text { Ölbaumkultivierung wirken als „,kostenlose Werbung“ } \\
\text { für das Produkt durch die Erwähnung der Stätten der } \\
\text { antiken Olivenölproduktion in Kultur- und } \\
\text { Reiseführern }\end{array}$ & $\begin{array}{l}\text { Archäologisch bedeutsame Stätten der antiken } \\
\text { Olivenölerzeugung sind kaum oder nur unzureichend } \\
\text { für den Tourismus erschlossen (s. z. B. San Pawl } \\
\text { Milqi, welches von der Regierung momentan für } \\
\text { Touristen unzugänglich gehalten wird) }\end{array}$ \\
\hline
\end{tabular}




\section{Die Handwerksdörfer Ta'Qali und Ta'Dbieg̉i}

Nachdem in den zurückliegenden Teilen der Abhandlung die Agrargeographie Maltas und die regionalen traditionellen Produkte der Inselgruppe ausführlich behandelt wurden, soll im Folgenden das Augenmerk verstärkt auf ausgewählte Stätten des Verkaufs und des Marketings gelegt werden. Die Handwerksdörfer Ta'Qali und Ta'Dbieg̉i spielen dabei eine Schlüsselrolle.

Obwohl die Crafts Villages Ta'Qali auf Malta und Ta'Dbieġi auf Gozo zu den in höchstem Maße frequentierten Anlaufstellen für den Tourismus gehören, gibt es über sie bislang verschwindend wenig wissenschaftliche Literatur. Nach Kenntnisstand des Autors gab es bisher zudem keine in der Literatur genannte Datierung, in welchem Zeitraum die Handwerksdörfer entstanden. Auch in dem einzigen ausführlichen Bericht über die Produktions- und Vermarktungsvorgänge in Ta'Qali und Ta'Dbieg̉i von MARKWICK (2001) ist keine korrekte Gründungdsdatierung enthalten.

Bevor im folgenden Abschnitt zwei Geschäftskartierungen im Handwerksdorf Ta'Qali ausgewertet werden, muss also zunächst eine Einführung in die Thematik „Handicraft Village“ erfolgen:

Sowohl Ta'Qali als auch Ta'Dbieġi waren in der Vergangenheit Militärflugplätze der auf Malta stationierten britischen Truppen. Im Zweiten Weltkrieg, während des schweren Bombardements der Inseln und dem damit verbundenen Luftkrieg, der über den Inseln tobte ${ }^{584}$, waren Gozo und Malta mit mehr oder weniger kleinen Flughäfen übersät. Die bekanntesten und größten dieser Startbahnen für Kampfflieger waren Luqa und Ta'Qali. Luqa war der größere der beiden Stützpunkte und wurde deshalb nach dem Abzug der britischen Truppen zum zivilen Hauptflughafen ausgebaut, auf dem gegenwärtig alle Flugzeuge landen, die Malta anfliegen. 


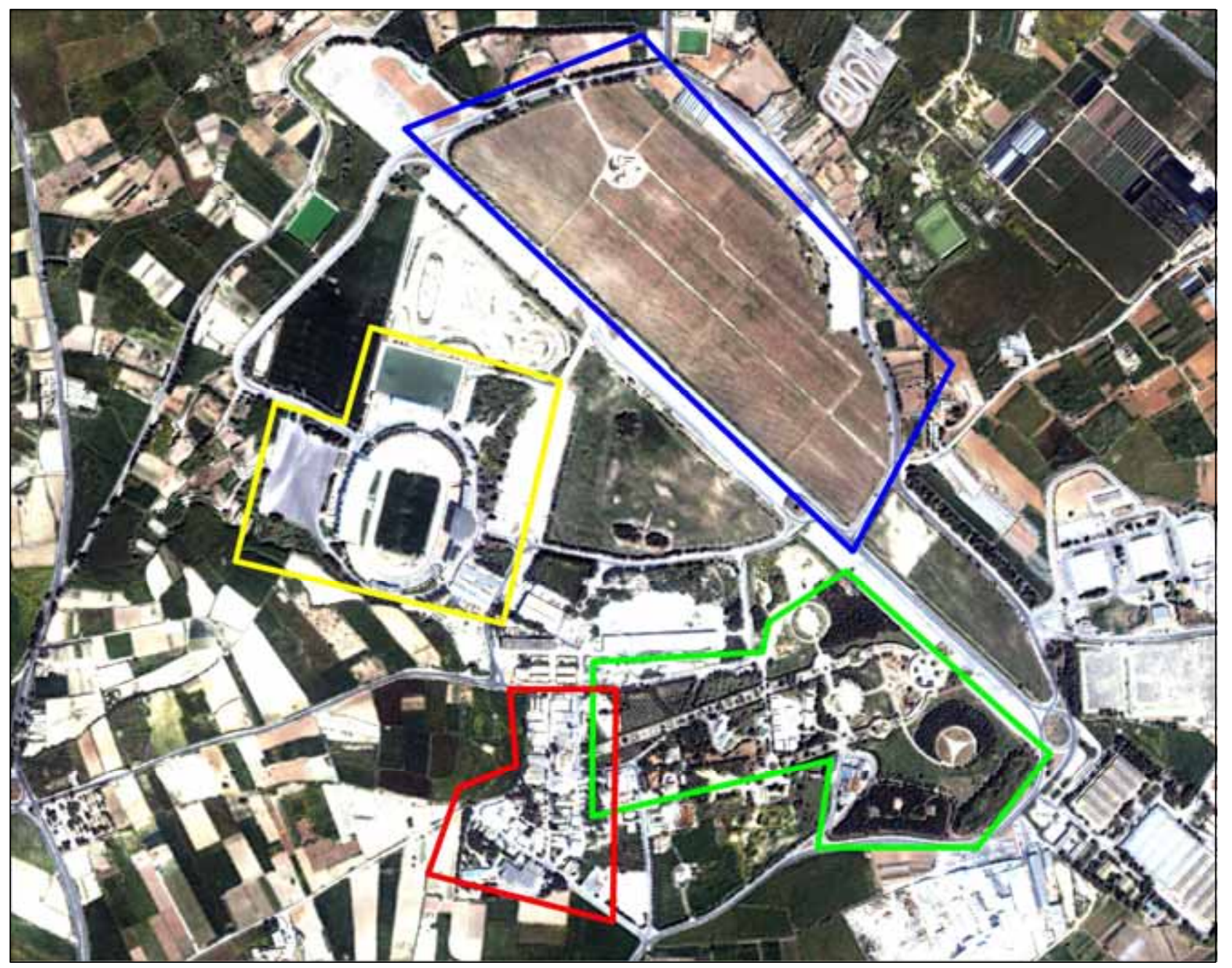

Abb. 24: Der Bereich Ta'Qali auf Malta. Kennzeichnung: rot = Ta'Qali Crafts Village, gelb = National Stadium, grün = National Park, blau = Meridiana Wine Estate. Darstellung ASCHEMEIER auf Basis einer IKONOS-Satellitenbildszene vom 28.03.2004

Ta'Qali wies gleichfalls eine fast zwei Kilometer lange Startbahn mit angrenzenden Gebäuden auf. Ta'Dbieġi dagegen war einer der kleineren Stützpunkte, wie sie fast überall auf den Inseln verteilt vorlagen (so z. B. im Raum Dingli, bei Hag̉ar Qim, usw.). Spätestens nach dem vollständigen Abzug der Briten im März 1979 stellte sich für die maltesischen Raumplaner die Frage der Nachnutzung der Militärbaracken und dem angrenzenden Umland. Während Luqa Airport von Grund auf saniert wurde, wurden in den Airbases von Ta'Qali und Ta'Dbieġi die Armeebaracken zunächst zur Einrichtung von Werkstätten und Läden für das maltesische Handwerk genutzt. Diese Entwicklung fiel zeitlich mit der Ausprägung des Massentourismus auf den Maltesischen Inseln ${ }^{585}$ zusammen.

Aus diesem Grund war die Konzentration von Kunsthandwerksbetrieben in Ta'Qali von Beginn an sehr erfolgreich $^{586}$, und es kam in späterer Zeit nicht zu einer geregelten Planung bzw. zu einer weiterführenden Umgestaltung des Geländes mit den Armeebaracken, welche nun fast vollständig von Handwerkern in Beschlag genommen wurden. Es wurden von öffentlicher Seite drei Zonen ausgewiesen ${ }^{587}$ : Im Westen der ehemaligen Startbahn entstand das „National Stadium “ für Fußball- und Großveranstaltungen (Zone 2), im Nordwesten schließt sich der "National Park" an - eine begrünte Parkanlage mit Freilufttheater (Zone 3). Im Nordosten entstand (erst in den 1990er-Jahren) der ausgedehnte Komplex des Meridiana Wine Estate (vgl. Abb. 24). Im nahe gelegenen Dorf Ta'Qali, das einst die Keimzelle und der Namensgeber für den

585 Vgl. BESENTHAL (1994)

586 Vgl. MARKWICK (2001)

587 Vgl. MALTA TODAY, Onlineausgabe vom 16.02.2001 
Militärflugplatz war, entstand ein gewerblicher Großmarkt für landwirtschaftliche Produkte. Die ehemalige Startbahn selbst wurde je teilweise zur Parkplatzfläche für das Fußballstadion, zum Naherholungsgebiet und als Straßenverkehrsverbindung ausgebaut.

Während also in direkter Umgebung von Ta'Qali das ehemalige Flughafengelände Schritt für Schritt umgestaltet wurde, blieb der Crafts Village Bereich (Zone 1, südlich der Startbahn) über rund vierzig Jahre bis auf wenige Neu- und Erweiterungsbauten nahezu unverändert - bestehen (vgl. Foto 28). Trotz der schlechten Bausubstanz, die im Lauf der Jahrzehnte zusehends verfiel (vor allem im Bereich der abgelegenen leer stehenden Hütten), kamen die Touristen scharenweise, um sich mit maltesischen Produkten sowie mit Kitsch und Souvenirs einzudecken.

In Gozo, wo der Bereich Ta'Dbieg̉i (benannt nach dem gleichnamigen Berg in unmittelbarer Nachbarschaft des ehemaligen Luftstützpunkts) erst Ende der 1980er zum Handicraft Village umfunktioniert wurde, geschah der Funktionswandel deutlich geplanter. Bereits die Standortwahl war im Voraus gut durchdacht, da sich im Ort Gharb, der heute baulich mit Ta'Dbiegi verbunden ist, die Fabrik von Gozo Glass befand. Dieser Betrieb gehörte seit langem zu den etablierten Touristenzielen. Durch die Umgestaltung von Ta'Dbieǵi zum Crafts Village, konnte eine ähnliche räumliche Konzentration von Anbietern erzielt werden wie in Ta'Qali. Zwar hatten auch bereits vor dem Umbau des Geländes einige Handwerksbetriebe die alten Militärbaracken in Beschlag genommen, jedoch wurde erst durch die Ausweisung Ta'Dbiegis als Crafts Village eine Konzentration erkennbar. Das Gelände wurde umzäunt, die Barracken wurden abgerissen und durch Neubauten und Grünanlagen ersetzt, sodass sich gegenwärtig ein baulich „freundlicherer" Gesamteindruck ergibt als in Ta'Qali (vgl. Foto 27).

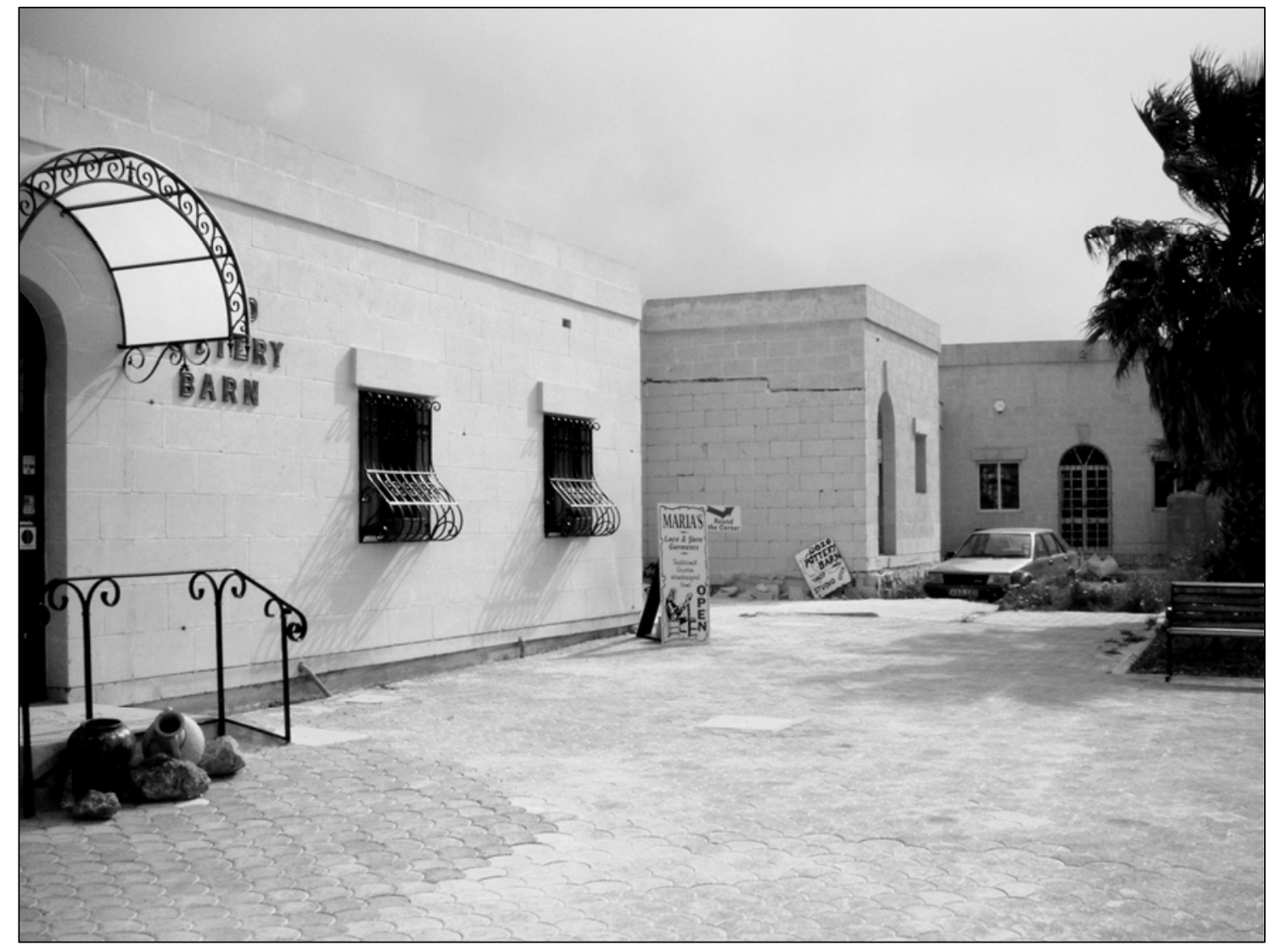

Foto 27: Crafts Village Ta'Dbieǵi auf Gozo: Die ehemaligen Militärbaracken wurden abgerissen und das Gelände wurde von Grund auf neu gestaltet. Foto: ASCHEMEIER 
Im Jahr 2005 beginnt aber auch für Ta'Qali die erste Phase einer groß angelegten Umbaumaßnahme der Regierung, die im Jahr 2000 beschlossen wurde ${ }^{588}$. Im Zuge dieser Umbaumaßnahme soll das Handwerksdorf Schritt für Schritt baulich und infrastrukturell saniert werden, wobei insbesondere auch Sicherheitsmaßnahmen (Brandschutz, Baufälligkeit, etc.) eine Rolle spielen. Es ist daher fraglich, inwiefern sich die im folgenden Abschnitt der Arbeit beobachteten kommerziellen Strukturen dieses Crafts Villages im Zuge des Umbaus wandeln werden. Es ist nicht bekannt, ob von Regierungsseite eine Qualitätssteigerung des Angebots angestrebt wird, u.U. auch unter Verwendung restriktiver Maßnahmen, um ein „authentisches“ regionales traditionelles Produktangebot sicherzustellen. Dies wäre aus Sicht des Autors zu begrüßen.

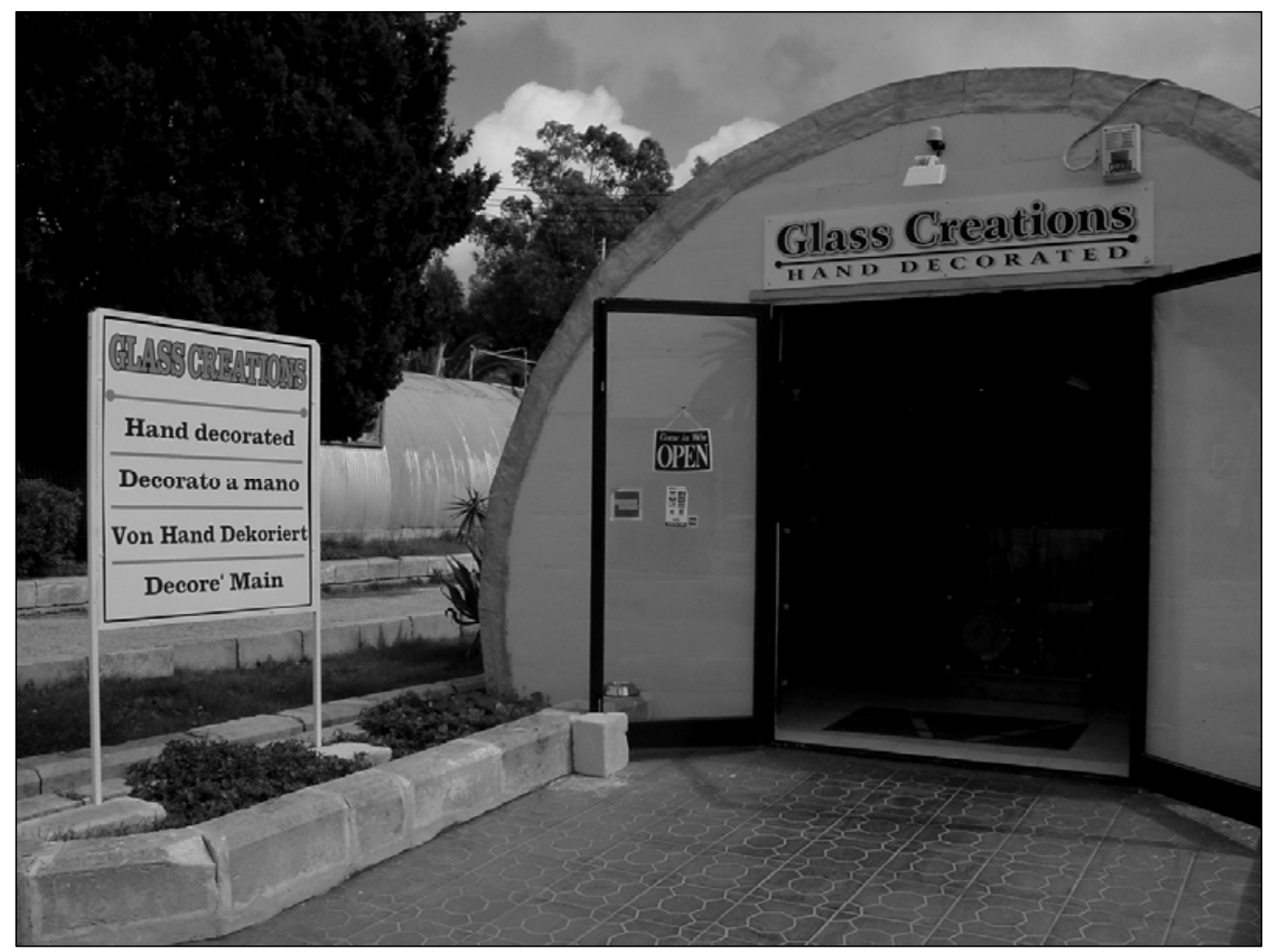

Foto 28: Die typische Bauweise der Shops und Werkstätten in Ta'Qali. Genutzt werden gegenwärtig vor allem alte Wellblech- und Kalksteinbaracken die von den britischen Luftstreitkräften zurückgelassen wurden. Foto: ASCHEMEIER

\subsection{Geschäftskartierungen in Ta'Qali}

Im März 2005 wurden im Rahmen der vorliegenden Arbeit zwei Geschäftskartierungen im Crafts Village Ta'Qali auf Malta durchgeführt. Ziel dieser Kartierungen sollte sein, herauszufinden, wie sich Angebotsstruktur und Preisniveau in dieser Handwerkeransiedlung gestalten. Zentrale Fragen der Dissertation („Was ist regional traditionell maltesisch?" bzw. „Wo und in welcher Form werden regionale traditionelle Produkte Maltas vermarktet?") rückten damit unmittelbar ins Zentrum der Untersuchungen.

Im Rahmen der Erhebungen wurden verschiedene Parameter berücksichtigt. Die Untersuchung gestaltete sich als Geschäftsbegehung, die insgesamt 56 Gebäude umfasste. Darunter waren alle aktuell als Laden oder Werkstatt betriebenen Firmen im zentralen Bereich von Ta'Qali. Einer der größten und wichtigsten Läden (Mdina Glass) lag abseits des Crafts Villages im unmittelbaren Bereich des National Park und wurde daher gesondert berücksichtigt. 


\subsection{Kartierung Nr. 1}

Die erste Kartierung (s. Karte 9) zeigt eine Geschäftskartierung der Einzelunternehmen in Ta'Qali. Die Fragestellungen hierbei waren:

a) Wie gestaltet sich die Angebotsstruktur der Unternehmen in Ta'Qali (z. B. überwiegend regionale Produkte oder überwiegend gemischtes Sortiment)?

b) Wie hoch ist das Preisniveau der Läden in Ta'Qali im Durchschnitt und im Einzelfall?

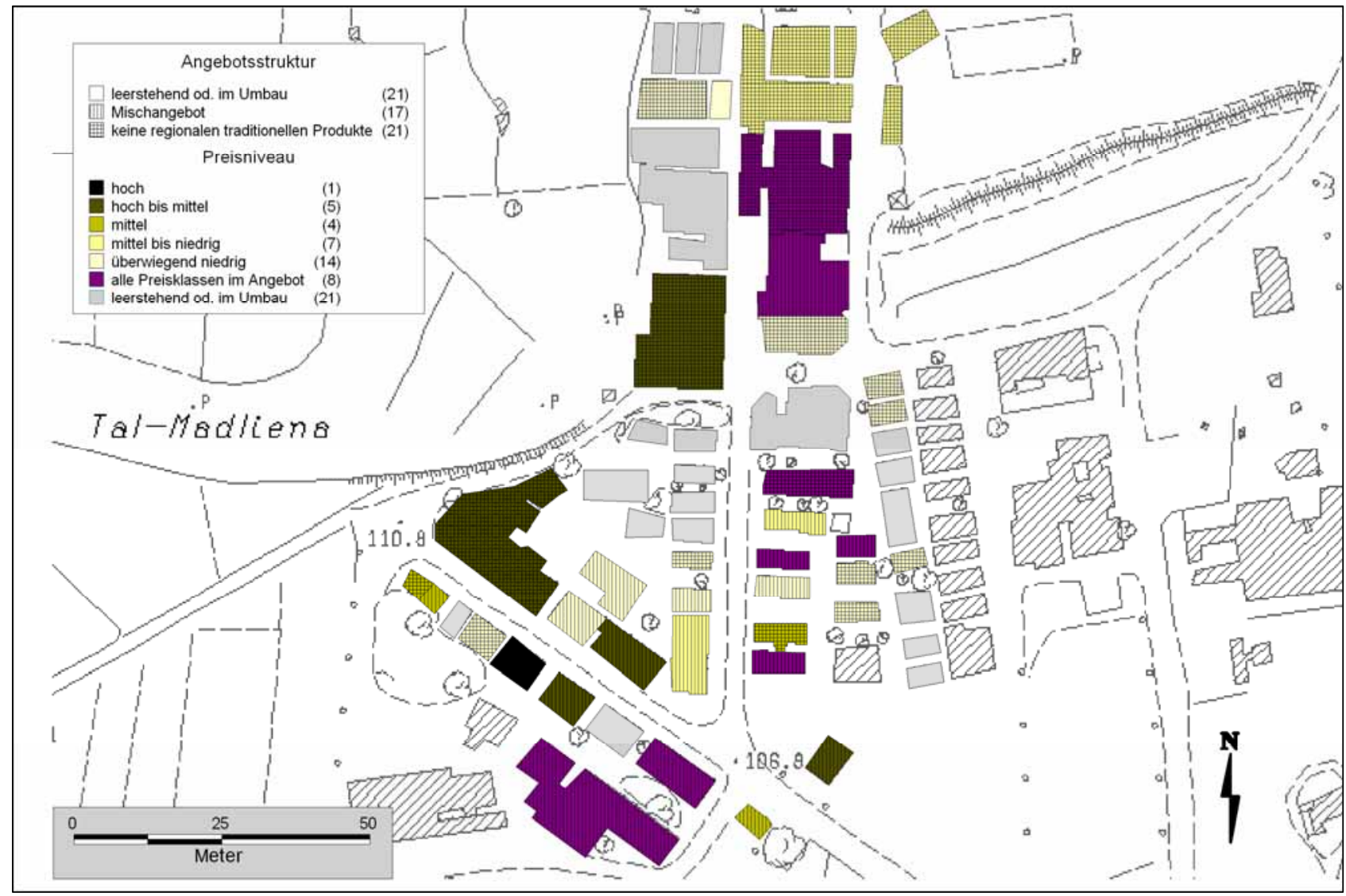

Karte 9: Geschäftskartierung von Ta'Qali nach Angebotsstruktur und Preisniveau. Entwurf \& Grafik: ASCHEMEIER

(Bitte beachten: Eine Vergrößerung dieser Darstellung liegt als Ausfaltkarte im Anhang bei!) 


\subsubsection{Zur Methodik}

Obige Kartierung ist das Ergebnis einer Geschäftsbegehung in deren Verlauf ein Schlüssel für die beiden untersuchten Parameter angewendet wurde. Dieser Schlüssel lautet wie folgt:

\section{a) Angebotsstruktur}

I - Keine regionalen traditionellen Produkte im Angebot

II - Überwiegend oder ausschließlich regionale traditionelle Produkte im Angebot

III - Mischangebot aus ca. 50\% regionalen traditionellen Produkten und ca. 50\% anderen Waren

IV - Laden befindet sich im Umbau oder ist geschlossen

b) Preisniveau

I - Alle Preisklassen sind vertreten

II - Überwiegend niedriges Preisniveau

III - Niedriges bis mittleres Preisniveau

IV - Mittleres bis hohes Preisniveau

V - Überwiegend hohes Preisniveau

$\mathrm{VI}$ - Laden befindet sich im Umbau oder ist geschlossen

Das Preisniveau b) musste in sieben Kategorien aufgeteilt werden, da es Läden mit weit gestreuten Sortimenten gibt (s. u.). So ist es in Ta'Qali keine Seltenheit, dass hochpreisige Waren wie mundgeblasenes Glas oder Silver Filigree unter einem Dach mit niedrigpreisigen Produkten wie T-Shirts oder den klassischen Billigsouvenirs angeboten werden.

\subsubsection{Auswertung}

\section{Angebotsstruktur}

Die o. a. Darstellung zeigt, dass regionale traditionelle Produkte überraschenderweise in Ta'Qali nur spärlich vertreten sind. Kein Laden in Ta'Qali ist ausschließlich auf regionale traditionelle Produkte Maltas spezialisiert. 17 Unternehmen führen ein Mischsortiment, in denen sich regionale traditionelle Produkte und Souvenirs, etc. in etwa die Waage halten. 21 Geschäfte führen gar keine Güter, die nach der Definition dieser Arbeit zu der Kategorie „regional traditionell“ gezählt werden könnten. Weitere 21 Gebäude befanden sich derzeit im Umbaustadium oder waren geschlossen.

Als Fazit kann festgehalten werden: Nur drei der vier vorher festgelegten Kategorien zur Angebotsstruktur konnten kartiert werden. Dies zeigt, dass in rund zwei Drittel der Geschäfte von Ta'Qali keine regionalen traditionellen Produkte zu finden sind. Das erstaunt um so mehr, weil die Regierung Ta'Qali und Ta'Dbiegi einst zum Zweck der Konzentration des regionalen Handwerks ins Leben gerufen hatte.

\section{Preisniveau}

Diese Entwicklung spiegelt sich auch in dem angebotenen Preisniveau wider. Ein großer Anteil der Geschäfte in Ta'Qali (14 Unternehmen) führen ausschließlich Waren des untersten Preisniveaus. 7 Läden führen Güter der mittleren bis unteren Preisklassen. Ein Mischangebot aller möglichen Preiskategorien von „ganz teuer" bis "ganz billig" haben 8 Läden im Angebot. 4 Geschäfte bieten ihrer Kundschaft Gegenstände einer mittleren Preisklasse, und 5 Läden konnten von der Preisgestaltung mit "mittel bis hoch“ veranschlagt werden. Nur ein Unternehmen führt ausschließlich Waren der höheren Preisklassen.

Als Fazit kann festgehalten werden, dass rund die Hälfte aller Geschäfte in Ta'Qali ein Produktsortiment im 
mittleren bis billigen Preisniveau unterhalten. Dabei handelt es sich überwiegend um Souvenirs wie T-Shirts, Mützen, Kaffeetassen, Schlüsselanhänger, Spielzeugautos und -Boote, etc. Aber die Kartierung zeigt auch, dass in vielen Geschäften der mittleren bis unteren Preisstufe ein Mischangebot besteht, in dem billige Verteter der "Gattung" der regionalen traditionellen Produkte enthalten sind. Das können die billigen Sorten von maltesischem Wein, Nougat, Gebäck, etc. sein. Es gibt in Malta aber auch billige Kunsthandwerksprodukte, die für etwa $1 \mathrm{Lm}$ zum Verkauf stehen.

\subsection{Kartierung Nr. 2}

Um die einzelnen Produktsortimente genauer zu untersuchen, wurde im Rahmen einer zweiten Kartierung (Karte 10) hinterfragt, aus welchen Produkten sich die einzelnen Ladensortimente zusammensetzen. Im Rahmen dieser Geschäftsbegehung wurden 19 Produktkategorien festgestellt (s. Tab. 3).

Tab. 3: Erhebungsschlüssel zur Kartierung der Produktkategorien in Ta'Qali

\begin{tabular}{|c|l|}
\hline Schlüssel-Nr. & Produktkategorie \\
\hline $\mathbf{1}$ & Glas \\
\hline $\mathbf{2}$ & Steinwaren \\
\hline $\mathbf{3}$ & Keramik \& Töpferei \\
\hline $\mathbf{4}$ & Spitze \& Häkelarbeiten \\
\hline $\mathbf{5}$ & Metallverarbeitung \\
\hline $\mathbf{6}$ & Lederwaren \\
\hline $\mathbf{7}$ & „Billigsouvenirs“, Postkarten \& Sonnenbrillen \\
\hline $\mathbf{8}$ & Silver \& Gold Filigree \\
\hline $\mathbf{9}$ & Schmuck \& Armbanduhren \\
\hline $\mathbf{1 0}$ & Tischlerei, Möbel, Holzwaren \\
\hline $\mathbf{1 1}$ & Tabakwaren \\
\hline $\mathbf{1 2}$ & Kacheln \& Fliesen \\
\hline 13 & Web- \& Strickarbeiten/Wollwaren \\
\hline 14 & Bücher \& Videos \\
\hline 15 & Lebensmittel \\
\hline 16 & Snack Bar od. Café \\
\hline 17 & Messing-Türklopfer \\
\hline 18 & Textilien (T-Shirts, Mützen, etc.) \\
\hline 19 & Rattan-Möbel \& Korbwaren \\
\hline Eigene Darstellung \\
\hline
\end{tabular}

In jedem Geschäft wurden anhand des Schlüssels Erhebungen vorgenommen. In der Auswertung zeigt sich, dass die Spannbreite des Produktsortiments in Ta'Qali von Geschäft zu Geschäft in starkem Maße schwankt: Einerseits gibt es Handwerksläden, die nur eine einzige Produktkategorie im Angebot haben, also als Spezialisten bzw. Fachgeschäfte bezeichnet werden können. Andererseits gibt es auch Verkäufer, die über zehn verschiedene Produktkategorien in ihrem Sortiment führen.

Im Fall der letztgenannten Geschäfte muss unterschieden werden in diejenigen, die ein Schwerpunktangebot aufweisen (z. B. Glas oder Keramik), zusätzlich aber zahlreiche Souvenirs führen und in diejenigen, in denen kein Schwerpunktangebot zu erkennen ist. Jene Läden also, die alle Produkte etwa gleich häufig führen. Zusätzlich gibt es noch die Kategorie des „Mischangebots“. Dies sind Geschäfte, die mehrere Produkte gleich häufig führen. In Ta'Qali zeigte sich, dass Geschäfte ohne Schwerpunktangebot den Charakter von „Ramsch-Läden“ aufweisen. Diese Geschäfte fungieren als negativer Imagefaktor, der Ta'Qali mittlerweile den Ruf beschert hat, zunehmend ein Standort für minderwertige und billige Produktqualität zu sein, obwohl die angesprochenen „Ramsch-Läden“ insgesamt in der Minderheit sind.

Auf der folgenden Seite ist eine detaillierte Produktauflistung für jedes Geschäft in Ta'Qali aufgeführt (vgl. Tab. 4). 
Tab. 4: Die Geschäfte in Ta'Qali und ihr Produktsortiment

\begin{tabular}{|c|c|c|}
\hline & Name des Geschäfts & Produktkategorien (vgl. Tab. 3) \\
\hline 1 & Super Gold Jewellers/Anthony's Filigree & $1,2,8,9$ \\
\hline 2 & Francis Gold \& Silver Jewellery \& Filigree Works & $1,8,9$ \\
\hline 3 & Bristow Pottery & $1,3,7,12,14,15,16,17$ \\
\hline 4 & Potterware & $1,2,3,4,6,13,14,15,17,18$ \\
\hline 5 & Double C Creations Soft Toys & Leerstehend \\
\hline 6 & ALF. Ciantar Filigree \& Jewellery Works & $1,8,9$ \\
\hline 7 & Mario Filigree \& Jewellery Works & $7,8,9$ \\
\hline 8 & The Wool Lace Leather Centre & $4,6,7,13,14,17,18$ \\
\hline 9 & Formosa Cane & 19 \\
\hline 10 & Ta'Qali Cafeteria \& Restaurant & 16 \\
\hline 11 & Debbie's Leather Goods \& Wool Souvenirs & Leerstehend \\
\hline 12 & Peter the Potter & $2,3,7,14$ \\
\hline 13 & Heritage Homes of Malta & 2 \\
\hline 14 & Dragon Forge & 5,17 \\
\hline 15 & Denis Jewellery & $1,8,9$ \\
\hline 16 & Glass Creations & $1,4,7$ \\
\hline 17 & Sculptures & $2,4,7,10,12,13,14,15,18$ \\
\hline 18 & Crafts Village Restaurant & 16 \\
\hline 19 & Leerstehend & \\
\hline 20 & Leerstehend & \\
\hline 21 & Leerstehend & \\
\hline 22 & Polybag Leathercrafts & $4,6,7,10,13,14,15,17,18$ \\
\hline 23 & Malta Pipes & $1,2,6,7,10,11,12,13,14,17,18$ \\
\hline 24 & J.C.F. Jewellers & 8,9 \\
\hline 25 & Leerstehend & \\
\hline 26 & Decorart & $1,2,3,4,5,7,8,9,13,14,15,17,18$ \\
\hline 27 & Leerstehend & \\
\hline 28 & Malta Stone - Strata Designs Ltd. & $2,7,9,10$ \\
\hline 29 & Leerstehend & \\
\hline 30 & Gaetano Camillieri Chairs \& Tables & 10 \\
\hline 31 & Abgerissen & Im Wiederaufbau \\
\hline 32 & Master Plaster & 2 \\
\hline 33 & Hut 122 & Im Umbau \\
\hline 34 & Phoenician Glassblowers & 1,7 \\
\hline 35 & The Carpenter & 10 \\
\hline 36 & Leerstehend & \\
\hline 37 & Leerstehend & \\
\hline 38 & Leerstehend & \\
\hline 39 & Tacsum Wood & 10 \\
\hline 40 & ALKA Ceramics & $1,2,3,9,12$ \\
\hline 41 & Emmanuel Galea Woodworks & 10 \\
\hline 42 & George Cassar Woodworks & 10 \\
\hline 43 & Scicluna Woodworks & 10 \\
\hline 44 & Woodworks & 10 \\
\hline 45 & Leerstehend & \\
\hline 46 & Leerstehend & \\
\hline 47 & Öffentliche Toilette & - \\
\hline 48 & Metcraft & 5,7 \\
\hline 49 & Polybag Leathercrafts (Werkstatt, vgl. Nr. 22) & - (vgl. Nr. 22) \\
\hline 50 & Leerstehend & \\
\hline 51 & Leerstehend & \\
\hline 52 & Famous for Cheapest in Prices & $1,2,4,6,7,10,12,13,14,15,17,18$ \\
\hline 53 & A. Inglott Jewellery & 7,9 \\
\hline 54 & Mdina Glass (nicht im kartierten Gebiet (s. o.)) & $1,7,9,14$ \\
\hline
\end{tabular}

Quelle: Eigene Darstellung auf der Grundlage eigener Erhebungen 
Werden die 19 festgestellten Produktkategorien klassifiziert, kann man noch deutlicher unterscheiden in ein Angebot mit eindeutiger Schwerpunktsetzung (z. B. auf Glas oder Keramik oder Wolle, etc.), ein Angebot ohne eindeutige Schwerpunktsetzung (alle Produktkategorien in etwa gleich häufig im Angebot) und ein Mischangebot aus beiden Bereichen (s. o.). Aus dieser Klassifizierung ergibt sich die folgende Darstellung:

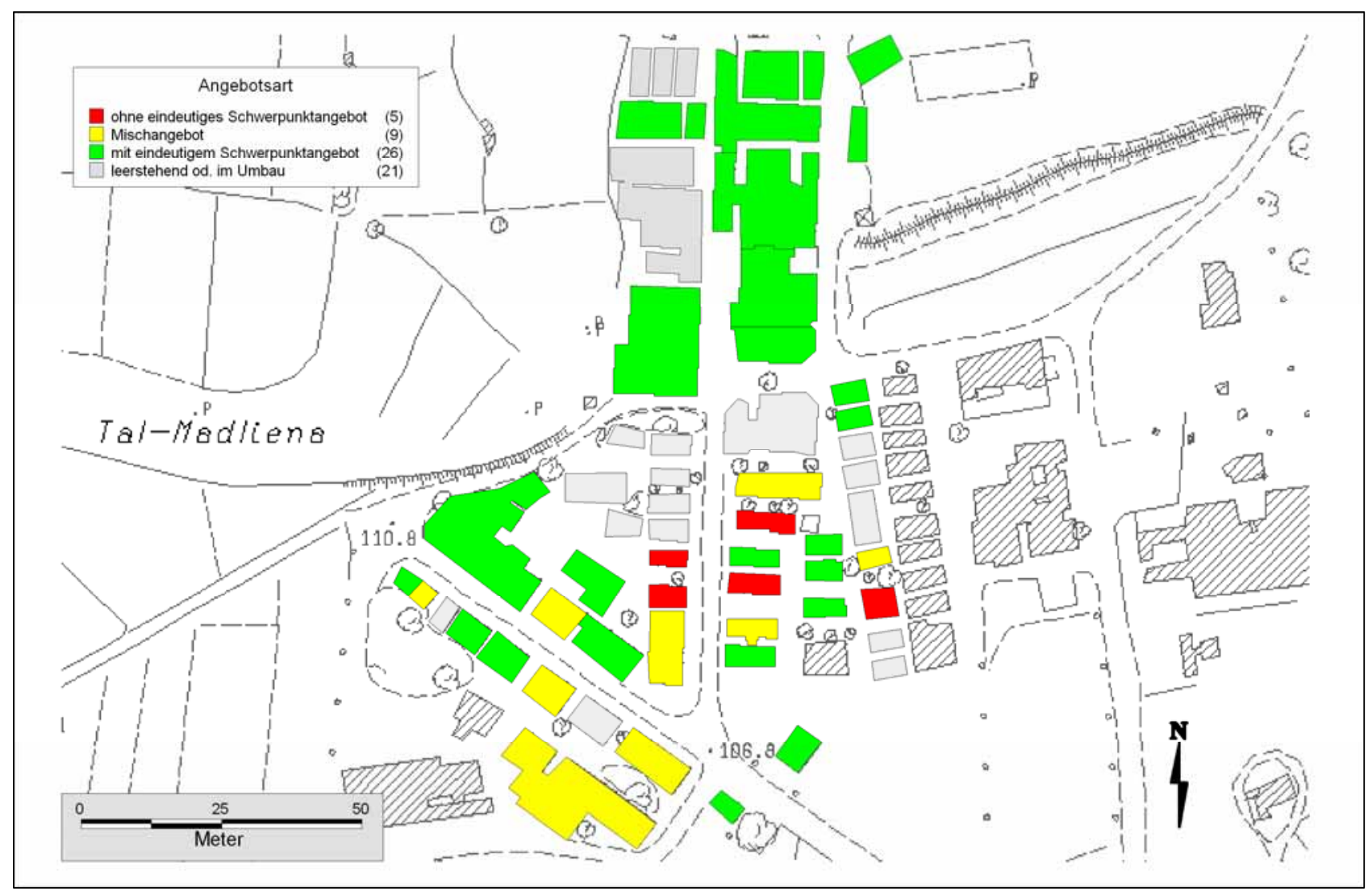

Karte 10: Geschäftskartierung in Ta'Qali nach der Angebotsart. Entwurf \& Grafik: AsCHEMEIER

Auffällig hierbei ist, dass sich die Läden mit Mischangebot sowie die Geschäfte mit einem Warenangebot ohne eindeutigen Schwerpunkt fast ausnahmslos im Bereich des Haupteingangs des Crafts Village im Südosten befinden. Je weiter man in die Handwerksansiedlung vordringt, desto mehr spezialisierte Geschäfte findet man.

Diese Feststellung ist deshalb aufschlussreich, weil der Haupteingangsbezirk die Zone ist, in der sich die meisten Touristen aufhalten. Dort haben sich überwiegend Läden mit einem breit gestreuten Produktsortiment angesiedelt. Der Anteil von „Billigsouvenirs“ ist hoch. Je weiter man nach Westen oder Norden vorstößt, desto spezialisierter wird das Angebot. Dies fällt insbesondere im Norden des Crafts Village auf, wo sich nahezu sämtliche Tischlereibetriebe angesiedelt haben, deren Angebot, bestehend aus Möbeln sowie Haus- und Wohnungstüren, ausschließlich für die einheimische Bevölkerung Maltas interessant ist.

Die Anzahl von 26 Läden mit eindeutigem Schwerpunktangebot im Verhältnis zu 5 Läden ohne Schwerpunktangebot und 9 Geschäften mit einem Mischangebot in dem etwa alle Waren gleich häufig vertreten sind, ist ein guter Wert. Dies zeigt, dass Ta'Qali nach der geplanten Umgestaltung wieder ein qualitativ hochwertiger Standort werden könnte. Es ist jedoch auch eine gegenteilige Entwicklung denkbar, wenngleich unwahrscheinlich. 


\section{Marketingkonzept für regionale traditionelle maltesische Produkte}

Die bisherigen Ausführungen haben ergeben, dass größere Schwächen der regionalen traditionellen Produkte Maltas offenbar im Bereich ihrer Vermarktung und ihres Images liegen. Maltesische Produkte weisen im Allgemeinen hohe Qualität auf, und sie sind in den allermeisten Fällen durchaus eigenständig. D. h. sie heben sich in mehr als nur in Details von Produkten aus anderen Mittelmeeranrainerstaaten ab und besitzen klare Alleinstellungsmerkmale.

Die Frage ist nun, wie das Marketing für regionale traditionelle Produkte in Malta verbessert werden kann, sodass auch bei einem möglichen Rückgang oder einer Stagnation der touristischen Ankünfte die bisherige vorteilhafte Marktstellung erhalten oder möglichst ausgebaut werden kann.

Der Autor schlägt diesbezüglich die Entwicklung eines Marketingkonzepts vor, das im Wesentlichen auf der Entwicklung einer Corporate Identity der wichtigsten regionalen traditionellen Produkte Maltas beruht. Hinzu kommt eine zentralisierte Vermarktung, bei der u.U. auch die Regierung Maltas gefragt ist.

Die Einzelpunkte des vom Autor erarbeiteten Marketingkonzepts, die im Folgenden erläutert werden, lauten:

- Zertifizierung und Labelisierung

- Corporate Identity und (zumindest teilweise) Corporate Design

- Ausschließlich regionale traditionelle Produkte dürfen aufgenommen werden

- Qualitätssicherung und Qualitätsprüfung => Schaffung von Richtlinien

- Enge Zusammenarbeit aller Hersteller

- Bereitstellung von Sekundärinformationen (z. B. in Form von Broschüren oder Büchern)

- Zentrale Verkaufsstellen in den größeren Städten und einheitliche Produktpräsentation

- Umfassende Plakat- und Fernsehwerbung

- U.U. Subventionierung durch Regierung und EU

- Ausnutzung von Online-Business-Lösungen

\subsection{Zertifizierung und Labelisierung}

Der bereits begonnene Prozess, Produkte über Standards und Labels auszuzeichnen, muss weiter geführt werden. Das Endresultat sollte eine lückenlose Erfassung aller regionalen traditionellen Produkte Maltas durch festgelegte Herstellungsstandards durch die MSA sein. Nur wer seine Waren nach den Richtlinien der MSA herstellt, kann das Prädikat „original maltesisch“ erwerben.

Das bisher bestehende Label des Malta Crafts Councils "Genuinely made in Malta" ist bisher zu wenig aussagekräftig. Es gibt zwar an, dass das Produkt in Malta hergestellt wurde. Unter diese Auszeichnung fallen allerdings auch solche Waren, deren Rohstoffe importiert wurden, wie z. B. die einige Liköre der größeren Hersteller oder das maltesische Glas.

Die MSA-Standards, die detailgenau festlegen, welcher Herstellungsprozess als "traditionell“ bzw. „authentisch“ bezeichnet werden kann, wird vom Autor positiv beurteilt. Anhand des MSA-Labels ist z. B. festzustellen, inwieweit Kunsthandwerk teilweise oder vollständig in Handarbeit hergestellt wurde und ob traditionelle Formen und Methoden (beim Handwerk) oder Rohstoffe (beim Lebensmittel) verwendet wurden. 
Problematisch ist jedoch der Freiwilligkeitsstatus des MSA-Labels, das in Zusammenhang mit der Erfüllung festgelegter Standards vergeben wird. Kein Handwerker ist derzeit gesetzlich gebunden, seine Ware am MSA-Standard zu messen. Beinhaltet ein Herstellungsprozess z. B. maschinelle Massenverarbeitung, womit das Produkt nicht mehr dem MSA-Standard genügen würde, kann der Hersteller auch ohne das MSA-Label sein Produkt als „traditionell maltesisch“ vermarkten, ohne dass es dagegen eine rechtliche Handhabe gäbe.

Es wird betont, dass die Qualitätssicherung, wie sie von den bereits bestehenden MSA-Standards festgesetzt wird, als prinzipiell geeignet für das vorgestellte Marketingkonzept erscheint. Jedoch muss, damit das Konzept in vollem Umfang wirksam werden kann, der MSA-Standard von der Regierung als gesetzlich verpflichtender Maßstab festgelegt werden. Das heißt in der Anwendung: Alle Hersteller von Produkten, für die es einen MSA-Standard gibt, müssen ihre Ware an diesem Maßstab messen lassen. Kann das Produkt den Standard erfüllen, bekommt es ein MSA-Label, kann der Standard nicht erfüllt werden, darf der Hersteller sein Gut weiter vermarkten, jedoch ohne MSA-Qualitätszertifizierung.

\subsection{Corporate Identity und (zumindest teilweise) Corporate Design}

Bisher herrscht auf dem maltesischen Markt ein reger Konkurrenzkampf der Hersteller. Einige der Firmen werben auch um touristische Kundschaft (z. B. die Anbieter von Wein, Likören, Keramik, Silberfiligranarbeiten, Olivenöl, etc.), andere wiederum bemühen sich hauptsächlich um maltesische Kunden (z. B. die Anbieter von Gebäck, Käse, Tomatenprodukten, etc.).

Für ausländische Konsumenten ist es teilweise sehr schwierig, maltesische regionale traditionelle Produkte im Laden zu identifizieren bzw. sie überhaupt zu finden. Unscheinbare Güter, wie z. B. die von der EU mit einer D.O.P.-Kennzeichnung geschützte Tomatenpaste Kunserva (vgl. Kap. 14.1), gehen fast nie in den Verkauf an Touristen. Große Vermarktungspotenziale werden auf diese Weise verschenkt.

Daher wird eine einheitliche Kennzeichnung maltesischer Produkte vorgeschlagen. Dabei ist vorstellbar, dass der Hersteller das äußere Erscheinungsbild seines Produktes nur marginal ändert. Unter voller Beibehaltung des individuellen Produktauftretens könnte ein Sticker in Form eines Malteserkreuzes unerfahrenen Käufern zu erkennen geben, dass es sich um ein regionales traditionelles maltesisches Produkt handelt.

Das Konzept sieht in diesem Fall die Schaffung einer interkooperativen Produktreihe vor: Hat der Konsument ein Produkt mit dem erwähnten Sticker gekauft, wird er andere Produkte der Produktreihe sofort erkennen und kann sein Konsumverhalten entsprechend ausrichten.

Ein gutes Beispiel dafür, wie ein solcher Sticker aussehen könnte, ist die Kunserva-Verpackung des Herstellers „Vera“ (s. Foto 29). Auf der Dosenmitte prangt ein grünes Malteserkreuz auf rotem Grund. In diesem Fall trägt das Label zusätzlich die Aufschrift „b'tadam malti“, was übersetzt „aus maltesischen Tomaten" bedeutet. Ebenso gut wäre aber auch ein Schriftzug im Sinne von „regional traditional product of Malta“ bzw. „regional traditional product of Gozo" auf der Verpackung denkbar. 


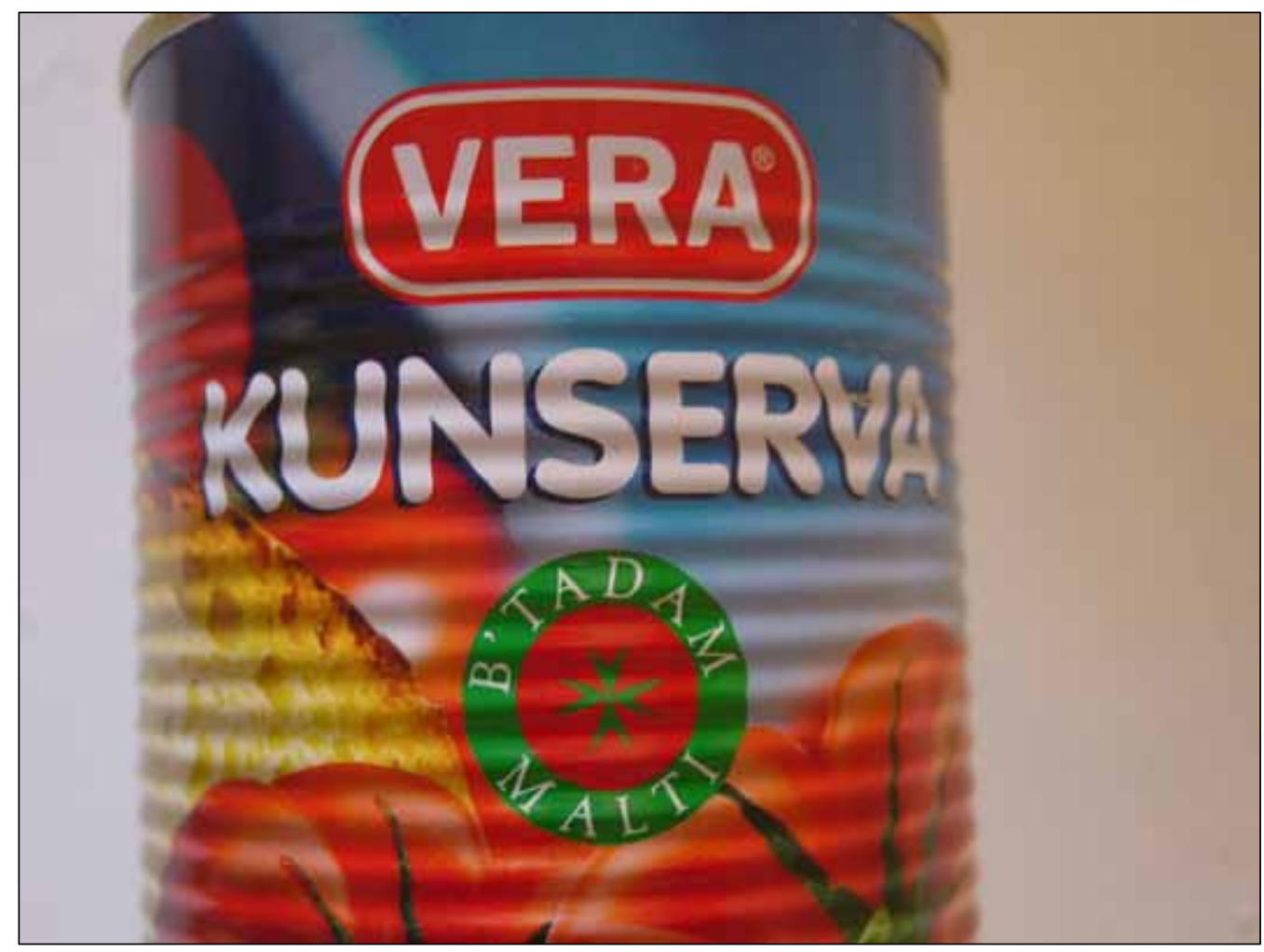

Foto 29: Beschriftung der Kunserva-Dose der Marke „Vera“. In der Dosenmitte ist ein Malteserkreuz zu sehen. Foto: AsCHEMEIER

\subsection{Ausschließlich regionale traditionelle Produkte dürfen aufgenommen werden}

Die Grundbedingung für den Erfolg des an dieser Stelle vorgestellten Marketingkonzepts ist die ausschließliche Beschränkung auf regionale traditionelle Produkte im Sortiment der vorgeschlagenen Produktreihe. Nur auf diese Weise wird die Qualität innerhalb des Sortiments gesichert. Gleichzeitig suggeriert diese Maßnahme nach außen den Charakter einer hochwertigen und exklusiven Produktreihe, was u.U. die Möglichkeit einer Preiserhöhung im Angebot für Touristen zugunsten der Hersteller und Händler ermöglicht.

Es sollte keinesfalls wieder der Fehler begangen werden, auch Produkte, die nur teilweise in Malta erzeugt wurden oder die ihre Rohstoffe importieren, ranggleich mit regionalen traditionellen Gütern zu zertifizieren, so wie es derzeit durch das Malta Crafts Council geschieht.

\subsection{Qualitätssicherung und Qualitätsprüfung - Schaffung von Richtlinien}

Die auf diese Weise erreichte Qualität innerhalb der Produktreihe muss fortlaufend kontrolliert und bestätigt werden. Ähnlich wie in einigen Bereichen des Lebensmittelhandels müssen unabhängige Kontrolleinrichtungen geschaffen werden, die exklusiv die Produkte mit der vorgeschlagenen Kennzeichnung überwachen.

Dies trägt nicht nur zum Positiv-Image der Produktreihe bei, sondern schafft auch Sicherheit für die Konsumenten, die umso lieber zu den „vertrauenswürdigen“ maltesischen Produkten greifen werden.

Ferner müssen Qualitätsrichtlinien erarbeitet werden, die qualitative Toleranzen festlegen. D. h., dem Hersteller wird ein Rahmen vorgegeben, in dem sich die Qualität seiner Produkte maximal bewegen darf. Es muss in dieser Hinsicht auch der Punkt erkennbar sein, an dem ein Produkt qualitativ nicht oder nicht mehr für die angestrebte Produktreihe tragbar ist. 
Diese Qualitätsrichtlinien können viele verschiedene Indikatoren umfassen, die von der grundsätzlichen chemischen/biologischen Unbedenklichkeit eines Produkts bis hin zu Werten wie „Haltbarkeit“", "Geschmack“ oder bei Handwerksprodukten „Detailgenauigkeit“ und „Fertigungstoleranzen von Handarbeit" reichen. Auch in diesem Sektor sind die bestehenden MSA-Standards ein Positiv-Beispiel, da sie bereits solche "Grenzwerte“ ausweisen.

Der Verfasser ist der Überzeugung, dass auf diese Weise neben Corporate Identity und Corporate Design auch eine „Corporate Quality“ der vorgeschlagenen Produktreihe erzielt werden kann.

\subsection{Enge Zusammenarbeit aller Hersteller}

Es ist bedauerlich sagen zu müssen, dass vor allem in diesem Punkt die größten Probleme für das vorgeschlagene Konzept zu erwarten sind. Die Erfahrung hat gezeigt, dass maltesische Hersteller in starkem Maße den Konkurrenzkampf suchen, wenn es sich dabei um Fabrikanten von Produkten ähnlicher Natur handelt.

Folgendes Beispiel möge zur Verdeutlichung des Sachverhalts dienen: Seit den 1990er-Jahren wird maltesischer Wein nach Deutschland exportiert. Dabei findet exklusiv der Wein der Firma Marsovin Berücksichtigung. Gleichzeitig wird durch denselben Importeur Likör der Firma Master Wine („Zeppi's“) nach Deutschland eingeführt. Auf die Frage, warum der Importeur nicht auch den Wein der Marke Master Wine importiere, bekam der Autor die Antwort, dass sonst Marsovin einen anderen Importeur suchen werde. Im Klartext bedeutet das: Marsovin möchte nicht durch denselben Importeur vertreten werden wie Master Wine.

Bedenkt man die angespannte Lage für Weinhersteller auf dem maltesischen Binnenmarkt, die durch die explosionsartige Vermehrung von Weinimportfirmen nach dem EU-Beitritt zusätzliche Brisanz bekommen hat, ist diese Haltung verständlich. Bedenkt man jedoch, dass deutsche Konsumenten bei maltesischem Wein vermutlich keine Markenunterschiede machen und nach Geschmack und Qualität einkaufen, wirkt die Firmenpolitik des erwähnten Weinproduzenten übertrieben. Im Gegenteil gilt hier die alte Regel: Konkurrenz belebt das Geschäft. Je mehr maltesische Weine im europäischen Ausland auftauchen und etabliert werden, desto höher ist die Wahrscheinlichkeit, dass der Gesamtabsatz aller Hersteller steigt.

So wie im Exportgeschäft verhält sich die Lage auch im Bereich des Angebots für Touristen: Für einen touristischen Käufer ist der Unterschied ob er maltesischen Wein in Malta oder in seinem Heimatland kauft nicht groß: Unerfahrene Reisende kennen die einzelnen Marken zunächst nicht und werden durch stetiges Ausprobieren Favoriten finden.

Die Ausführungen zeigen, dass es durchaus vorstellbar ist, dass auch Konkurrenzprodukte von Waren gleicher Art in ein und derselben Produktreihe erfolgreich koexistieren können, sofern sie die für alle gültigen Qualitätsrichtlinien erfüllen. Es ist jedoch zu erwarten, dass das übertriebene Konkurrenzgebahren der maltesischen Hersteller, das in vielen wissenschaftlichen Arbeiten beschrieben ist ${ }^{589}$ und schon zu vielen Problemen im Bereich der statistischen Datenerhebung geführt hat, auch in diesem Fall die größte Hürde sein wird.

Für das in dieser Arbeit vorgestellte Konzept ist es unabdingbar, dass eine Einigung unter den Herstellern erreicht wird, sodass die Produktreihe auch von der Basis her Unterstützung findet. Zudem garantieren eine hohe Zahl von beteiligten Herstellern auch eine große Vielzahl an Produkten, eine große Qualitätsbandbreite und eine kooperativ wirksame Motivation, die Produktreihe als „Ganzes“ zu unterstützen. 


\subsection{Bereitstellung von Sekundärinformationen}

Es gibt eine Reihe von maltesischen Autoren ${ }^{590}$, die sich in ihren Büchern in der Vergangenheit mit dem Ursprung und der Ausprägung der maltesischen Folklore beschäftigt haben, zu der - wie zum Beginn der vorliegenden Arbeit bewiesen wurde - auch der Bereich der regionalen traditionellen Produkte gehört. Unterstützend zu der Ausweisung einer Reihe von regionalen traditionellen Produkten, könnten diese Autorinnen und Autoren ein Buch herausgeben, das einerseits eine "Produktkunde“ für Konsumenten sein soll, andererseits aber auch ein zusätzliches Angebot im Sinne eines „Souvenirs“ für Malta-Touristen.

Der Autor ist sich darüber im Klaren, dass dies ein aufwändiger Schritt wäre, der jedoch durch das gut ausgebaute Verlagssystem Maltas mit einer langen Veröffentlichungstradition im Bereich der Landesfolklore nicht allzu problematisch erscheint.

Vorläufig genügen auch Informationsbroschüren, die jedem Produkt beigelegt werden können bzw. sollten. Festlands-Europäer sind sicherlich neugierig auf ein so ungewöhnliches Produkt, wie die maltesische Bohnenpaste Bigilla. Das merkwürdige Aussehen der Speise und ihre fehlende Produktkennzeichung verhindern aber u.U. den Kauf, weil ein Käufer sich nicht vorstellen kann, wie das Produkt schmeckt und woraus es hergestellt wurde. Eine Informationsbroschüre dient der Information, schafft Neugier auf und Vertrauen in das Produkt und bietet zusätzlich noch anregenden „Unterricht“ in maltesischer Folklore.

\subsection{Zentrale Verkaufsstellen und einheitliche Produktpräsentation}

In Valletta und Sliema gibt es mit den Shops des Malta Crafts Councils bereits Läden, in denen maltesische Produkte zentral angeboten werden. Darunter sind jedoch auch Waren, die nicht der in dieser Arbeit ausgewiesenen Definition des Begriffes „regionales traditionelles Produkt" genügen würden.

Ladenbesitzer sollten ein eigenes Regal haben, in dem nur Produkte der erwähnten qualitativ hochwertigen Produktreihe - möglichst mit Werbeflächen im Corporate Design - verkauft werden. Diese Praxis ist bereits von weltweit renommierten Konzernen erfolgreich erprobt worden. So verkauft z.B. das CD-Label Naxos seine Produkte weltweit ausschließlich in solchen Läden, die sich damit einverstanden erklären, bei der Produktpräsentation einen einheitlich gestalteten Präsentationsständer exklusiv für die Produkte der Firma Naxos einzusetzen. Ein ähnliches Vorgehen wäre im inländischen Verkauf von den Gütern der vorgeschlagenen Produktreihe zu empfehlen.

\subsection{Plakat- und Fernsehwerbung}

In Malta ist es üblich, auch für verhältnismäßig preisgünstige Produkte Fernsehwerbung zu schalten. Exklusivere Waren, wie z. B. Wein und Liköre, profitieren verstärkt von Print- und Plakatwerbung.

Soll die angesprochene Produktreihe in Malta auch unter der einheimischen Bevölkerung Akzeptanz finden und erfolgreich sein, so muss in der Werbung derselbe Aufwand erkennbar sein, wie für andere Produkte, die auch die großen internationalen Marken umfassen.

Für solche Produkte, die international vermarktet werden sollen, muss die Werbung auch in den Zielgruppenländern präsent sein. Eine Werbeoffensive für maltesische Produkte würde demnach z.B. für Großbritannien und Deutschland in Frage kommen, die die wichtigsten Quellnationen für touristische Reisen nach Malta und Gozo darstellen. Es ist anzunehmen, dass in diesen Ländern ein höheres Kaufinteresse zu erwarten ist, da Teile der Bevölkerung im Urlaub bereits mit maltesischen Produkten in Kontakt gekommen sind. 


\subsection{U.U. Subventionierung durch Regierung und EU}

Das hier vorgestellte Marketingkonzept trägt positiv zur Gesamtwirtschaft der Republik Malta bei. Des Weiteren wird auch das Image der Agrarprodukte dieser Region gefördert, die von der EU als landwirtschaftliches Ungunstgebiet ausgewiesen wurde. Dies könnte zur dringend notwendigen Wiederbelebung der maltesischen Landwirtschaft beitragen, die in den letzten Jahren nur noch marginale Anteile am BSP erwirtschaftet hat ${ }^{591}$ (vgl. zu Thema Subventionierung auch Kap. 23).

Es erscheint daher sinnvoll, eine Förderung durch die maltesische Regierung und möglicherweise auch die Europäische Union für das vorgeschlagene Konzept einzurichten, um die Realisierung in der Umsetzung sicherzustellen. Dies kann zwar auf unterschiedliche Art und Weise geschehen, in der Anfangsphase zählt jedoch vor allem Geld, das bei der Umsetzung des vorgeschlagegen Konzepts in die Praxis benötigt wird.

Maltesische Regierung und EU können auch durch die Bereitstellung von Prüfungsinstituten und Labors zum Gelingen des Marketingkonzepts beitragen.

\subsection{Ausnutzung von Online-Business-Lösungen}

Online-Business ist eine Möglichkeit für kleine und große Firmen, ihr Produktangebot auf globaler Ebene anzubieten ${ }^{592}$. Der Investitionsaufwand ist vergleichsweise gering, während der potenzielle Nutzen je nach Einsatz dieses Marketinginstruments sehr hoch sein kann.

Während auf Sizilien rund 20 Shops existieren, die regionale traditionelle Produkte im Internet anbieten, gibt es auf den Maltesischen Inseln nur einen einzigen Internetshop mit regionalem traditionellem Angebot ${ }^{593}$.

Der Autor ist der Überzeugung, dass bei einem touristisch in hohem Maße frequentierten Inselstaat wie Malta die Erfolgsaussichten für eine gut präsentierte und beworbene Online-Businessplattform exzeptionell gut sind. Nach Statistiken der MTA sucht ein Großteil der internationalen Malta-Reisenden vor allem im Internet nach weiterführenden Informationen über die Inselgruppe: „Thirty-six per cent of travellers to Malta and Gozo use the internet to find information about the islands (...). "(DEBATTISTA in THE TIMES OF MALTA (Beilage: i-tech), Ausgabe vom 22.07.2004, S. 2). Legt man diese Zahlen zugrunde, informieren sich jährlich ca. 432.000 Menschen im Internet über die Maltesischen Inseln.

Folgt man darüber hinaus den Untersuchungen von z. B. LITTRELL ET AL (1993), MACCANNELL (1999) oder MARKWICK (2001a), ist wahrscheinlich, dass Urlauber Produkte, die sie im Urlaub schätzen gelernt haben, auch in ihrem Heimatland regelmäßig konsumieren würden, wenn eine unaufwändige Gelegenheit zum Kauf bestünde. Auch die touristische Befragung im Rahmen der vorliegenden Dissertation legt dies nahe ${ }^{594}$.

Die Ausweitung der o. a. Vorschläge auf das Online-Business wird daher empfohlen. Eine zentralisierte Vermarktung $-\mathrm{d}$. h. in diesem Fall: Alle Produkte der vorgeschlagenen Produktreihe müssten über eine einzige Internetplattform zu beziehen sein - wird nahe gelegt, da auf diese Weise der subjektive Aufwand des Kunden beim Online-Einkauf minimiert wird.

591 Vgl. Pollacco (2003) sowie Aschemeier \& CYFFKA (2004b)

592 Vgl. AsCHEMEIER (2005) sowie MüLleR (2002)

$593 \mathrm{Vgl}$. ASCHEMEIER (2005)

594 Vgl. Kap. 7 


\section{Projekte zur Förderung der Vermarktung von regionalen traditionellen Produkten}

Maltesische Produkte verkaufen sich, ebenso wie Waren überall auf der Welt, nicht von selbst. Die Vermarktung und Werbung gestaltet sich in ihrem Fall vielmehr besonders schwierig. Der Grund liegt auf der Hand: Malta liegt als Ursprungsgebiet fernab von den Trend setzenden Metropolen Europas und dem Rest der Welt. Zwar stammen viele regionale traditionelle Produkte aus peripheren Regionen - z. B. aus der französischen Auvergne, dem schweizerischen Emmental, dem deutschen Erzgebirge, etc. - jedoch dürfte das „Erfolgsrezept“ dieser Produkte u.a. darin liegen, dass sie von großen Städten aus ohne besondere Mühe erreichbar sind. Dies garantiert einen kostengünstigen Überlandtransport mit kurzen Transportwegen. Hat sich dann erst einmal die herausragende Qualität eines regionalen traditionellen Produkts herumgesprochen, können auch weitere Transportwege etabliert werden, die über den überregionalen Massenabsatz der Ware gerechtfertigt sind.

$\mathrm{Zu}$ dem im vorangegangenen Kapitel vorgestellten Marketingkonzept für maltesische Produkte werden deshalb im Folgenden mögliche Projekte vorgeschlagen, die einen zusätzlichen positiven Effekt auf die Vermarktung regionaler traditioneller Produkte der Maltesischen Inseln erwarten lassen.

Im Fall der maltesischen Produkte ist bei einer Markteinführung außerhalb der Ursprungsregion ein umständlicher Transportweg zu Wasser oder in der Luft vonnöten. Die Etablierung der maltesischen Güter außerhalb Maltas ist daher schwierig und läuft bisher vor allem über die Mund-zu-Mund-Propaganda von Urlaubern, die selbst vor Ort auf den Maltesischen Inseln mit regionalen traditionellen Produkten in Kontakt gekommen sind.

Wenn man sich also mit Projekten zur Förderung regionaler traditioneller Produkte beschäftigt, so muss zunächst vornehmlich die Vermarktung im Ursprungsland Malta gefördert werden, weil aus Sicht des Verfassers nur solche Produkte der maltesischen Inselgruppe Chancen im Export haben, die vorher bei der touristischen Vermarktung vor Ort etabliert werden konnten.

Dabei wurde bisher von maltesischer Seite weitgehend außer Acht gelassen, dass Vermarktung und Werbung nicht nur über die bekannten „klassischen“ Kanäle funktionieren, sondern auch das subjektive Reiseerlebnis des Einzelnen teilweise als „Produktwerbung“ im weiteren Sinne arrangiert werden kann. So können beispielsweise über Museumsbesuche (s. u.) auch solche Informationen vermittelt werden, die die Aufmerksamkeit auf die regionalen traditionellen Produkte des Landes lenken.

Zur Optimierung des Produktmarketings sollte das Ausschöpfen aller möglichen staatlichen und „überstaatlichen“ (EU-Ebene, UNESCO, etc.) Fördermöglichkeiten und politischen Regulationsmittel eine Selbstverständlichkeit sein (vgl. Kap. 22). Erzeuger bestimmter regionaler Produkte werden in vielen Ländern Europas sowie auch in einigen Fällen von der EU selbst finanziell und/oder politisch unterstützt. In Malta ist diese Politik bisher noch nicht hinreichend etabliert. Das Problem dabei liegt sicherlich in der "Selbstverständlichkeit" begründet, die regionale traditionelle Produkte in Maltas Alltag auszeichnen. Dass diese „Selbstverständlichkeit“ auf europäischer Ebene eine „Außerordentlichkeit“ darstellt, ist in Malta bislang noch nicht in vollem Umfang erkannt worden.

Allein die Tatsache, dass bestimmte charakteristische regionale traditionelle Produkte ausschließlich in Malta erzeugt werden - sie sozusagen „endemisch“ auf den Maltesischen Archipel begrenzt auftreten macht sie zu Raritäten auf europäischer und erst recht auf globaler Ebene. Wenn man z.B. bedenkt, dass von der maltesischen Rebe Gennaru nur 1450 Rebstöcke weltweit existieren ${ }^{595}$ (alle in Gozo und Malta), dann besitzen die Trauben dieser Rebe zweifellos den Charakter eines raren Agrarprodukts. 
Im Folgenden werden in kurzer Form denkbare Projekte skizziert, deren praktische Umsetzung nach Meinung des Autors vorteilhaft auf die Vermarktung maltesischer Produkte wirken kann.

\section{Freilichtmuseum Agrarwirtschaft}

Vier Landnutzungsanalysen ${ }^{596}$ in dieser Dissertationsschrift haben gezeigt, dass sich die Landwirtschaft der Maltesischen Inseln in unterschiedlichen Anbauzonen manifestiert. Dabei wurde insbesondere im Rahmen der Teilerhebung 2 (II-Hofra) deutlich, dass noch Bereiche existieren, deren Bewirtschaftung und Ertrag nach wie vor hauptsächlich der Eigenversorgung dienen. Auf diesen Flächen haben sich Arbeitstechniken erhalten, die seit Jahrhunderten nur geringfügig verändert wurden. Auch AzZOPARDI weist in seiner "New Geography of the Maltese Islands" (2002) darauf hin, dass im kleinbäuerlichen maltesischen Anbau selbst im 21. Jh. stellenweise noch das Pflügen der Felder mit Ochsen praktiziert wird, Esel als Lastenträger eingesetzt werden und manche Feldfrüchte im Anbau persistent sind, die sich im professionellen, marktwirtschaftlichen Bereich längst nicht mehr rentieren. Dabei ist die Quote aufgegebener Ländereien in diesen ursprünglich und ländlich gebliebenen Arealen sehr gering. Das Alter der bewirtschaftenden Landwirte ist jedoch sehr hoch ${ }^{597}$.

Diese, aus ökonomischer Sicht zunächst wenig verlockende Ausgangsperspektive, könnte im Rahmen der Landschaftsinterpretation ${ }^{598}$ für den Tourismus effektiv genutzt werden.

Der Autor schlägt die Einrichtung eines „Freilichtmuseums Agrarwirtschaft" vor, in welchem die Besucher über das traditionelle maltesische Landleben mit all seinen Besonderheiten und typischen Charakteristika aufgeklärt werden. Anhand einer Art geführtem Lehrpfad ${ }^{599}$ könnten im Rahmen einer Kurzwanderung Stationen wie

1. das typische maltesische Farmhaus,

2. die traditionelle Pflugmethode,

3. der traditionelle Weinbau,

4. Aufschichtung und Erhaltung von Rubble Walls,

5. die Rubble Wall als Lebensraum,

6. Hangterrassen - gefährdete Kulturlandschaftsrelikte

7. die regionalen traditionellen Produkte der maltesischen Agrarwirtschaft und ihre Herstellung, etc.

erörtert werden. Während der kartierte Bereich II-Hofra für ein solches Vorhaben nur eingeschränkt zu empfehlen wäre ${ }^{600}$, könnte im Bereich Zurrieq/Siǵg̉iewi ein Freilichtmuseum erfolgreich etabliert werden.

Das ein solches Vorhaben durchaus keine Utopie ist, zeigt die erfolgreiche Etablierung des museal aufbereiteten „The Limestone Heritage"-Steinbruchs in Siǵgiewi: eine Ausstellung - halb Freilicht, halb in einem modernen Gebäudekomplex -, die die Tradition des Bausteinabbaus auf Malta thematisiert.

\footnotetext{
596 Vgl. Kap. 17

597 Der Autor weist darauf hin, dass die kleinbäuerlichen Bereiche traditionell vor allem im Norden und Westen Maltas verbreitet sind (vgl. auch BOWEN JONES ET AL. (1961)). ASCHEMEIER \& CYFFKA bewiesen anhand von Daten des NSO, dass vor allem diese Teile der Insel eine starke Überalterung der Landwirte aufweisen (vgl. AsCHEMEIER \& CYFFKA (2004b, S. 109). 
Hinsichtlich eines hypothetischen Freilichtmuseums zum Thema der ruralen Traditionen des Archipels sollte am Ende des Museumsrundgangs explizit auf die regionalen traditionellen Produkte hingewiesen werden, die ihren Ursprung in der maltesischen Landwirtschaft haben. Ein Museumsshop könnte die entsprechenden Waren in Verbindung mit der musealen Aufbereitung vermarkten.

Diese Form der Vermarktung wäre nach Ansicht des Verfassers besonders vielversprechend, da sich bereits in Untersuchungen von LITRELL ET AL. (1993) herausgestellt hat, dass Touristen beim Souvenirkauf Wert auf die Authentizität der Produkte legen. Die Authentizität messen die Reisenden dabei aber vor allem an der Herkunft der Produkte aus landestypischen Traditionen ${ }^{601}$.

Der Autor hat 2005 im Rahmen der Erstdefinition des Terms "regionales traditionelles Produkt“ darauf hingewiesen, dass Vermarktungsmethoden, die Tradition, Ursprung und Geschichte eines regionalen traditionellen Produkts thematisieren, besonders Erfolg versprechend $s^{1} d^{602}$. In diesem Zusammenhang kann die Verbindung von Vermarktung und musealer Information über die Herkunft und Entstehung regionaler traditioneller Produkte in verstärktem Maße nahe gelegt werden.

Darüber hinaus ist die maltesische Landwirtschaft reich an Besonderheiten und Alleinstellungsmerkmalen, was u.a. BOWEN JONES ET AL. (1962), LUKE (1968), BRADFORD (1971), AsCHEMEIER \& CYFFKA (2004b), BLOUET (2004) sowie CYFFKA (2005) explizit hervorhoben. Die „urig“ anmutende Agrarwirtschaft der Inselgruppe ist deshalb fast immer auch Bestandteil der Reiseliteratur zum Thema Malta. Dies beweist, dass das touristische Potenzial von Maltas Landwirtschaft nicht zu unterschätzen ist.

\section{Ausschöpfung von Fördermitteln und Schutzkategorien durch die EU}

Malta empfängt hohe Summen Fördermittel von Seiten der EU ${ }^{603}$. Vor allem im Bereich der Agrarwirtschaft sind nach dem EU-Beitritt Maltas Projekte mit großem Finanzbedarf initiiert worden ${ }^{604}$.

Es ist jedoch bisher kein Antrag auf Förderung des Schutzes der regionalen traditionellen Produkte eingereicht worden. Ein solcher Antrag könnte im Sinne des Schutzes von Kulturgut gestellt werden. Sowohl die EU als auch die UNESCO fühlen sich der Erhaltung und dem Schutz von gefährdeten Kulturgütern ihrer Mitgliedsstaaten verpflichtet. So erklären sich z. B. Projekte, die das Aussterben von bestimmten Dialekten und Sprachen verhindern, bzw. aussterbende Sprachen dokumentieren sollen. U.a. ist in diesem Zusammenhang auch die Aufnahme von Malti als offizielle EU-Sprache zu verstehen.

Eine ähnliche Vorgehensweise empfiehlt der Autor hinsichtlich der regionalen traditionellen Produkte: Auch in diesem Sektor gibt es gefährdete Sparten (in Malta sind das u.a. z.B. Töpferei \& Keramik, OlivenölHerstellung, Klöppelei, etc.). Das Ursprungs- und Verbreitungsgebiet ist ebenso klein, wie das der maltesischen Sprache. Aktiver Kulturschutz mit EU-Mitteln müsste somit auch die regionalen traditionellen Kunsthandwerker und Lebensmittelerzeuger umfassen.

Ein weiterer Pluspunkt für das regionale Produktmarketing ist die Einstufung bestimmter Waren als geschützte EU-Herkunftsbezeichnung (z. B. D.O.P.-Klassifizierung). Eine D.O.P.-Klassifizierung besteht in Malta bereits für die Tomatenpaste „Kunserva“.

Durch die geschützte Herkunftsbezeichnung der EU ist sichergestellt, dass Produkte, die namentlich als „Kunserva“ ausgewiesen sind, ausschließlich aus Malta stammen dürfen.

Diese Maßnahme gewährleistet, dass einzelne regionale traditionelle Produkte nicht in anderen Ländern, artifiziell eingeführt und imitiert werden und dann statt des Originalprodukts $-u$. U. zum billigeren Preis - den Weltmarkt oder gar den maltesischen Binnenmarkt beliefern. Inwiefern ein solches Szenario wahrscheinlich ist, ist jedoch von Produkt zu Produkt unterschiedlich zu beurteilen.

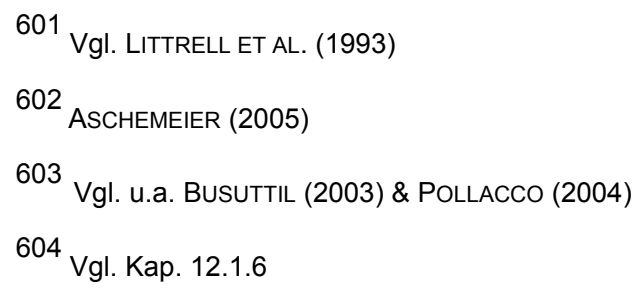


Im Fall der Limonade „Kinnie“ ist z. B. durchaus eine Gewinn versprechende Etablierung des Produkts auf europäischer Ebene denkbar, wie dem Autor auch der Kinnie-Importeur für Deutschland im persönlichen Interview bestätigte. Hier wäre eine geschützte Herkunftsbezeichung durch die EU durchaus wünschenswert, könnte sich aber aufgrund des Produktcharakters von Kinnie als alkoholfreier Longdrink in der „Tradition“ von Fanta, Coca Cola, etc. schwierig gestalten.

Des Weiteren hat sich gezeigt, dass die Labels der EU für geschützte Herkunftsbezeichungen von den Verbrauchern z. T. als "Gütesiegel“ missverstanden werden. So kann sich z. B. jeder ParmesankäseHersteller, der bei der entsprechenden Kontrollstelle für die Region Parma gelistet ist, mit dem D.O.P.-Label der EU schmücken. Dabei spielt es keine Rolle ob sein Käse qualitativ hochwertig ist oder nicht.

Viele Verbraucher sehen nur das Label der EU und sind nicht informiert, was die geschützte Herkunftsbezeichung bedeutet. Daher halten sie es für ein Gütesiegel. Dieses weit verbreitete Missverständnis könnte für Maltas regionale traditionelle Produkte von Vorteil sein. Deswegen sollte von Hersteller- sowie von Regierungsseite versucht werden, so viele regionale traditionelle Produkte wie möglich für die EU-Herkunftsschutzklassen-Beantragung anzumelden. Je mehr Produkte dabei erfolgreich sind, desto höher wird das nationale und internationale Ansehen maltesischer Produkte allgemein steigen.

\section{Thematische Verknüpfung der Folkloremuseen und des Crafts Villages Ta'Dbieǵi auf Gozo}

Im Allgemeinen lässt sich sagen, dass viele Vernetzungsmöglichkeiten, die im Endeffekt positiv auf die Vermarktungswege für regionale traditionelle Produkte wirken könnten, in Malta und Gozo bislang nicht genutzt werden.

Eine nahe liegende und Erfolg versprechende Vernetzungsmöglichkeit liegt z.B. in der thematischen Verknüpfung der Folkloremuseen in Gharb und Victoria auf Gozo mit dem gozitanischen Handwerksdorf Ta'Dbiegii. Da Ta'Dbieġi in unmittelbarer Nähe des Ortes Gharb gelegen ist, wäre das private Folkloremuseum dieses Dorfes - wohl eines der beeindruckendsten Museen der Maltesischen Inseln geradezu prädestiniert.

Die Verknüpfung soll in solch einer Weise erfolgen, dass beide Parteien, Museum und Crafts Village, davon profitieren. Ein Vermarktungskonzept auf Basis einer thematischen Vernetzung von Museen und Crafts Village könnte demnach schematisch aussehen wie folgt (vgl. Abb. 25).

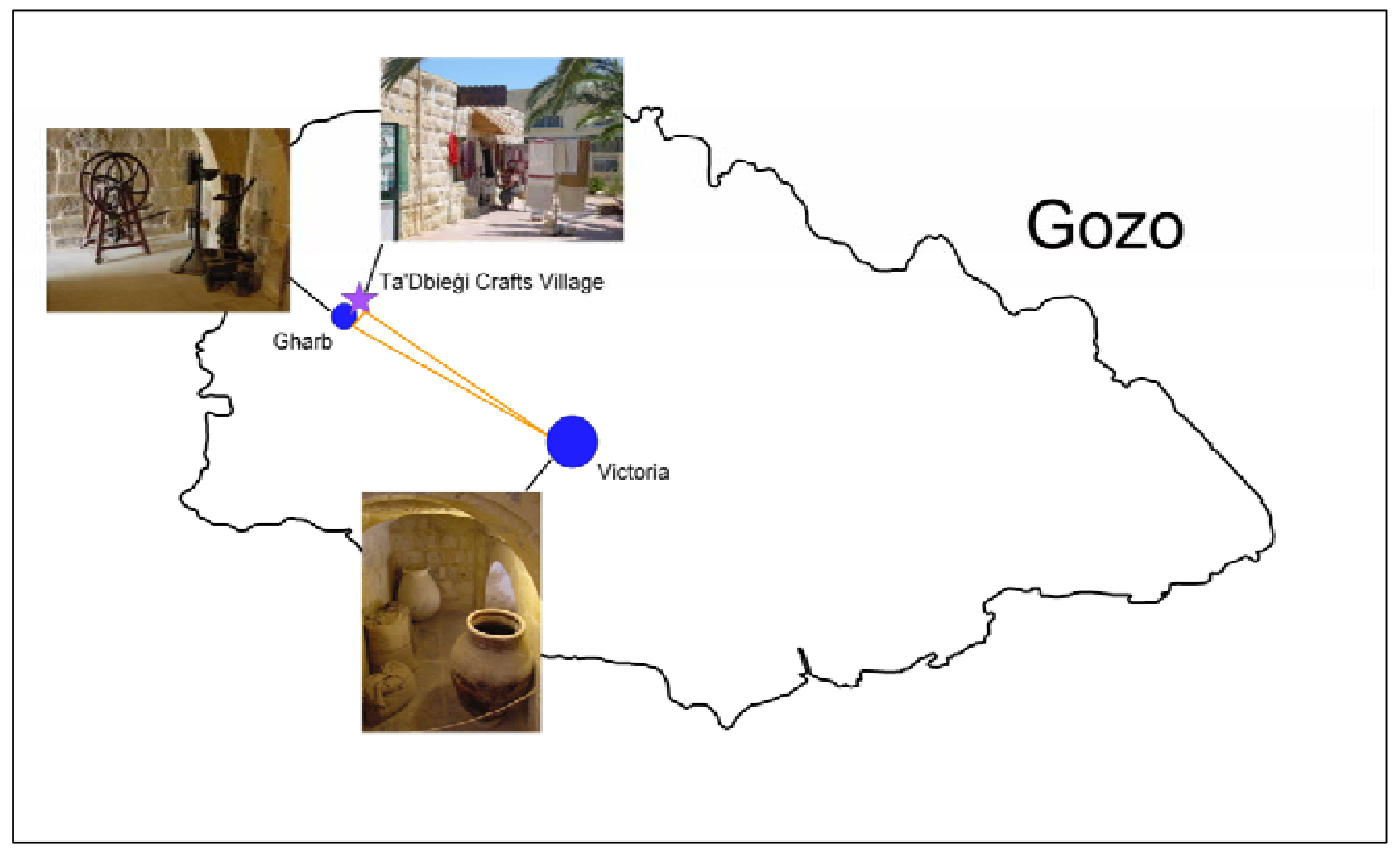

Abb 25: Vernetzungsmöglichkeiten der Folkloremuseen Gozos mit dem Crafts Village Ta'Dbieġi. Entwurf, Fotos und Grafik: ASCHEMEIER 
Die Abbildung veranschaulicht die Möglichkeit der Vernetzung (oranges Dreieck) zwischen den beiden Folkoremuseen in Gharb und Victoria mit dem Crafts Village Ta'Dbiegi auf halber Strecke zwischen Gћarb und San Lawrenz.

In den Folkloremuseen wird antikes Kunsthandwerk sowie die Werkzeuge, die zu seiner Fertigung notwendig waren, ausgestellt. Im Crafts Village wird aktuell Kunsthandwerk hergestellt, wobei sich an der Art und Weise wie dies geschieht häufig seit hunderten von Jahren nicht viel verändert hat. Als Beispiel möchte der Autor die Töpferei und die Klöppelei anführen. Antike Tongefäße sind im Folkloremuseum von Victoria ausgestellt (vgl. Abb. 25), im Folkloremuseum von Gharb sind u. a. Gerätschaften zum Spinnen von Baumwolle und für die Klöppelei ausgestellt (vgl. ebenda). Im Crafts Village von Ta'Dbiegi wird heute noch nach traditioneller Art und Weise getöpfert und geklöppelt (vgl. ebenda).

Die Möglichkeit der thematischen und kommerziellen Verknüpfung der drei genannten Standorte liegt demnach auf der Hand: Touristische Führungen, die die Folkloremuseen behandeln, sollten nach dem Besuch im Museum ebenfalls das Crafts Village ansteuern. Nachdem die Reisenden sich im Museum über das antike Kunsthandwerk Gozos informiert haben, können sie in Ta'Dbiegi nun u. a. Produkte, die nach antiken Vorbildern gearbeitet wurden, erwerben. Des Weiteren sollten sich die drei genannten Einrichtungen in einem Kooperationsverbund zusammenschließen und gegenseitig füreinander werben. D. h. im Crafts Village von Ta'Dbieġi müsste auf die Folkloremuseen von Victoria und Gharb hingewiesen werden, während in den genannten Museen auf die Möglichkeit des Besuchs von Ta'Dbieǵi hingewiesen werden sollte. U. U. ist auch ein Museumsshop an beiden Museumsstandorten denkbar, in dem Produkte, die in Ta'Dbiegi gefertigt wurden, angeboten werden. Ein Museumsshop existiert bereits im Folkoremuseum von Gharb. Im Zuge der vorgeschlagenen Standortvernetzung müsste jedoch das Angebot umgestaltet und erweitert werden.

Die Standortvernetzung würde zudem mehr Publikumsaufmerksamkeit auf das gozitanische Crafts Village lenken, welches z. Zt. darunter leidet, dass nur wenige Reiseveranstalter Ta'Dbieg̉i auf ihren Bustouren rund um Gozo berücksichtigen ${ }^{605}$. Das Folkloremuseum von Gharb ist im Rahmen eines ca. 15-minütigen Spaziergangs von Ta'Dbieg̉i aus zu erreichen. Die topographischen Bedingungen für eine Vernetzung wären optimal.

Leider kann man das von der ökonomischen Ausgangslage nicht behaupten: Das Folkloremuseum in Victoria ist eine staatliche Einrichtung, das Folkloremuseum von Gharb ist in privater Hand. Die Läden in Ta'Dbieġi befinden sich zwar alle im selben abgegrenzten „Crafts Village“, sind jedoch darüber hinaus nicht verbandsmäßig organisiert.

Das Problem wäre demnach, einen Organisator für den Vernetzungsvorgang zu finden und alle Parteien an einen Tisch zu bringen. Dies erscheint durchaus schwierig, wenn man die bisher eher reservierte Haltung der drei Parteien zueinander in Betracht zieht. Als vermittelndes Medium schlägt der Autor die Malta Tourism Authority (MTA) in Verbindung mit der University of Malta vor. Die University of Malta könnte im Rahmen eines studienbegleitenden Projekts ein detailliertes Vernetzungskonzept für die drei genannten Standorte erarbeiten. Dieses könnte mithilfe der MTA in die Tat umgesetzt werden. Das brächte nicht nur positive Publicity in der Presse, sondern stellt auch sicher, dass das - vornehmlich für Touristen angelegte Netzwerk im Tourismusprogramm der MTA Berücksichtigung findet und somit auch tatsächlich an die Reisenden herangetragen wird. Die Planungsautorität der Malta Environment \& Planning Authority (MEPA) muss bei dem Vorgang nicht zwangsläufig beteiligt werden, da im Rahmen des Vernetzungskonzepts keine baulichen Umgestaltungen notwendig sind. 


\section{Schaffung eines (Kunst-)Handwerksmuseums in Malta}

Auf den Maltesischen Inseln gibt es zwei Folkloremuseen. Beide sind in Gozo beheimatet, vermutlich deshalb, weil Maltas kleinere Nachbarinsel auf den ersten Blick ruraler und "folkloristischer" wirkt als die Hauptinsel mit ihrem quirligen Verkehrschaos.

Von Seiten der Folkloremuseen ist bereits viel zur musealen Aufbereitung des Kunsthandwerks getan worden. Dabei ist vor allem das private Folkloremuseum in Gharb lobend zu erwähnen, das mit fachlich fundierten Begleittexten und einem hervorragend informierenden Museumsführer des renommierten maltesischen Schriftstellers GUIDO LANFRANCO ein nahezu optimales Beispiel unter den Museen der Inselgruppe darstellt.

Auf der Hauptinsel Malta gibt es bislang keine museale Anlaufstelle für Interessierte, die sich näher über das Kunsthandwerk des Inselstaats informieren möchten. Anstatt ein weiteres Folkloremuseum ins Leben zu rufen, schlägt der Autor vor, ein spezielles Kunsthandwerksmuseum einzurichten. Aus marktwirtschaftlicher Persprektive wäre als Standort das Crafts Village Ta'Qali möglich. Jedoch sieht der Autor hierbei das Problem, dass ansässige Handwerksbetriebe im Vergleich zu den Handwerkern in den anderen Städten man denke z.B. an die hochqualitativen Kunsthandwerksbetriebe in Lija und Mdina - übervorteilt würden, was sie durch ihren Sitz am Standort Ta'Qali ohnedem schon sind ${ }^{606}$.

Ähnlich wie beim Folkloremuseum in Gharb wäre es u.U. sinnvoll, ein altes maltesisches Gebäude als stilvolle Kulisse für das Museum zu wählen. Vorstellbar wäre diesbezüglich z.B. einer der zahlreichen Wachtürme der Johanniter, die um die gesamte Insel herum verteilt auftreten. Vorstellbar für ein solches Projekt wäre konkret z.B. der Wignacourt Tower, der mitten im touristischen Zentrum St. Paul's Bay gelegen ist, sich in gutem, begehbaren Zustand befindet, jedoch bisher nur einer eingeschränkten touristischen Nutzung unterliegt. 


\section{Zusammenfassung}

Im Verlauf der vorliegenden Dissertation wurden die unter Kap.1.2 als Zielsetzung definierten Punkte bearbeitet.

Dabei wurden zunächst die naturräumlichen Gegebenheiten der Maltesischen Inseln sowie deren historische Entwicklung sowie die Geschichte des EU-Beitritts - ergänzt um ein Kapitel zu Maltas gesamtwirtschaftlicher Ausgangslage zum Zeitpunkt seiner EU-Angliederung - beleuchtet. Eine sozialgeographische Analyse von Maltas Volkskultur, bzw. Folkore, wurde notwendig, da sich im Rahmen einer touristischen Befragung zeigte, dass das Bild, welches die Befragten mit "typisch maltesischen Produkten“ verbanden, in der Endauswertung ein ambivalentes Ergebnis darstellte.

Ausgehend von diesem Standpunkt wurde untersucht, inwiefern Maltas regionale Produkte Bestandteil der Folklore der Inselgruppe sind. Um eine begriffliche Verwirrung zwischen Ausdrücken wie z. B. „authentisch“, „regional“, „traditionell“, „ursprünglich“ im Hinblick auf die in der vorliegenden Abhandlung untersuchten Produkte auszuschließen, wurde der Terminus technicus „regionales traditionelles Produkt" eingeführt und definiert.

Unter Zuhilfenahme dieses neuen Begriffs konnte die Analyse von Maltas Agrarprodukten stattfinden, die zunächst mit einem agrarhistorischen Kapitel eingeleitet wurde, auf das die Auflistung und Analyse der agrarischen Nutzpflanzen der Maltesischen Inseln nach der Methode von BOWEN JONES, DEWDNEY \& FISHER (1961) erfolgte.

Im Zentrum der Arbeit steht ein umfassendes Kapitel mit der Darstellung, Beschreibung und Untersuchung von Maltas regionalen traditionellen Produkten aus den Bereichen Lebensmittel und Kunsthandwerk. Es wurde diesbezüglich auch hinterfragt, welche maltesischen Waren nicht zu den regionalen traditionellen Produkten gezählt werden können.

Im Folgenden wurden die bisher geläufigen Anbau- und Marketingmechanismen der Inselgruppe analysiert. Dies begann mit einer Folge von agrargeographischen Landnutzungsanalysen um zu beleuchten, welche Anbauzonen Maltas und Gozos für den Fortbestand von regionalen traditionellen Agrarprodukten besonders wichtig sind. Danach wurde das Augenmerk auf die verschiedenen Herstellergruppen für regionale traditionelle Produkte gelegt. Aus der Analyse ihrer bisherigen Distributions- und Herstellungsweisen ergab sich die Hervorhebung zweier ausgewählter Vermarktungskonzepte sowie Vorschläge für die Ausweisung von zwei besonders entwicklungsfähigen Produkten: Artischocken und Olivenöl.

Es musste dann noch untersucht werden, inwiefern die sog. Handicraft Villages Ta'Qali auf Malta und Ta'Dbieg̉i auf Gozo Zentren der Vermarktung regionaler traditioneller Produkte darstellen oder nicht.

Zum Schluss der Arbeit wurde ein schematisches Marketingkonzept für die regionalen traditionellen Produkte der Maltesischen Inseln erarbeitet. Darüber hinaus wurden mögliche Projekte zur Förderung der Vermarktung erarbeitet.

Das Gesamtergebnis ist eine umfassende strukturelle Analyse des ausgedehnten Untersuchungsbereichs der regionalen traditionellen Produkte der Maltesischen Inseln. 


\section{Literatur}

ASCHEMEIER, Rainer:

- Neue Chancen und Entwicklungsmöglichkeiten für das touristische Marketing kleiner ländlicher Fremdenverkehrsorte. 140 S.. Unveröffentlichte Diplomarbeit. 2002.

- The Role of „Regional Traditional Products“ in the Tourism Economy of the Small Island State of Malta. In: WAIBEL, Michael; THIMM, Tatjana \& KREISEL, Werner (Hrsg.): Fragile Inselwelten - Tourismus, Umwelt und indigene Kulturen. = Pazifik Forum, Bd. 9. 2005. S. 233-254.

ASCHEMEIER, Rainer \& CYFFKA, Bernd:

- $\quad$ Malta: Neuling in der EU. In: Geographische Rundschau, 56, H. 12. (2004a). S. 62-65.

- Die Landwirtschaft Maltas an der Schwelle zum EU-Beitritt. In: Europa Regional, H. 2/2004. (2004b). S. 107-115.

ATTARD, Joseph:

- Malta - A History of Two Millennia. Valletta. 2002.

- The Battle of Malta. London. 1980.

AzZOPARDI, Aldo E.: Malta und seine Inseln. Terni. 1994.

AZZOPARDI, Anton: A New Geography of the Maltese Islands. 2. erweiterte Auflage. Valletta. 2002.

AzzoPARDI, Consiglia: Gozo Lace - An Introduction to Lace Making in the Maltese Islands. Victoria (Gozo). 1991.

BAIER-ALLEN, Susanne: Zypern - Ein geteilter Kleinstaat vor dem EU-Beitritt. In: KIRT, Romain \& WASCHKUHN, Arno (Hrsg.): Kleinstaaten-Kontinent Europa. Baden-Baden. 2001. S. 269-282.

BAILLIE, Sascha: The Position of Small States in the EU. In: GoETSCHEL, Laurent (Hrsg.): Small States inside and outside the European Union - Interests and Policies. Boston, Dordrecht, London. 1999. S. 193-205.

BECKER, Christoph; STEINECKE, Albrecht; HOPFINGER, Hans (Hrsg.): Geographie der Freizeit und des Tourismus: Bilanz und Ausblick. München, Wien. 2003.

BENDIX, Jörg; BENDIX, Astrid; REUDENBACH, Christoph: Umweltprobleme im Maltesischen Archipel. In: Geographische Rundschau, 54, H. 4. 2002. S. 12-19.

BesentHAL, Petra: Der Tourismus in Malta und seine Auswirkungen. Weiden. 1994.

BESTLER, Anita: Malta Reviews - kommentierte Bibliographie zur Malta-Forschung. = Augsburger Beiträge aus Kommunikationswissenschaft und Soziologie (ABAKUS), Bd. 16. 2., überarbeitete Auflage. Augsburg. 1994.

BESTLER, Anita \& WASCHKUHN, Arno: Das politische System Maltas. In: ISMAYR, Wolfgang (Hrsg.): Die politischen Systeme Westeuropas. 3. aktualisierte und überarbeitete Auflage. Opladen. 2003. S. 731739.

BEZZINA, Joseph:

- Vierzig Legenden von Gozo. Geschichten aus vergangener Zeit. Valletta. 1992.

- Gozo - a historical glimpse. Valletta. 1998. = Gaulitana, Bd. 6.

BHUGLAH, Assad: A Case for Special Treatment: Small Island Developing States in International Trade Negotiations. In: BRIGUGLIO, Lino \& CORDINA, Gordon (Hrsg.): Competitiveness Strategies for Small States. Blata I-Bajda. 2004. S. 338-348.

BIAGINI, Emilio: Le Isole Maltesi. Genua. 1974.

BIEGER, Thomas \& LAESSER, Christian: Tourismustrends - Eine aktuelle Bestandesaufnahme. (http://verdi.unisg.ch/org/idt/main.nsf/0/C1E221A15D731FCEC1256D3D002B31A3/\$file/TourismustrendsBieger-Laesser.pdf). 2003.

BLACK, Annabel: Negotiating the Tourist Gaze. The Example of Malta. In: BoISSEVAIN, Jeremy (Hrsg.): Coping With Tourists: European reactions to mass tourism. Providence, Oxford. 1996. S. 112-142. 
BLAKE, Adam; SINCLAIR, M. Thea \& SUgIYARTO, Guntur: Tourism and EU Accession in Malta and Cyprus. Unveröffentlichter Beitrag zur Konferenz „Tourism Modelling and Competitiveness: implications for policy and strategic planning“, 31.10.-01.11.2003, Paphos (Zypern).

BLOUET, Brian W.:

- The Story of Malta. 3. new revised edition. Valletta. 2004.

- $\quad$ The Story of Malta. 2. revised edition. Valletta. 1987.

- Rural Settlement in Malta. In: Geography, 56. 1971.

BLÜTHGEN, Joachim \& WEISCHET Wolfgang: Allgemeine Klimageographie. 3. Auflage. Berlin, New York. 1980. = Lehrbuch der Allgemeinen Geographie, Bd. 2.

BoISSEVAIN, Jeremy (Hrsg.): Coping With Tourists: European reactions to mass tourism. Providence, Oxford. 1996.

BONANNO, Anthony: Archaeology. In: MINISTRY OF YOUTH AND THE ARTS (Hrsg.) (1994): Malta - Culture and Identity. Marsa. 1994. S. 81-103.

BORG', Geoffrey: On Viticulture and Vintners: The Future of Vine Growing and Wine Making in Malta. In: Options Méditerranéennes, Ser. B, no. 7. Cachan Cedex. 1993. S. 105-111.

BORǴ, Paul P.:

- Snajja' u Xoghol il-Maltin. Volume 2. San Ǵwann. 2001. 373 S..

- Snajja' u Xoghol il-Maltin. Volume 1. San Ǵwann. 2000. 595 S..

BOWEN JONES, H.: Agriculture in Malta. A survey of land-use, part I. Durham. 1955.

BOWEN JONES, H.; DEWDNEY, J.C.; FISHER, W.B. (Hrsg.): Malta - Background for Development. Durham. 1961. = Research Papers of the Department of Geography of the University of Durham, Bd. 5.

BRADFORD, Ernle: Mediterranean - Portrait of a Sea. London. 1971.

BRAMWELL, Bill: Maltese Responses to Tourism. In: Annals of Tourism Research, 30, H. 3. 2003. S. 581-605.

BRAUN, O.L.: (Urlaubs-)Reisemotive. In: HAHN, Heinz \& KAGELMANN, H. Jürgen (Hrsg.) (1993):

Tourismuspsychologie und Tourismussoziologie. Ein Handbuch zur Tourismuswissenschaft. München. 1993.S. 199-207.

BRIGUGLIO, Lino:

- Sustainable Tourism in Small Island States: issues and policies. London. 1996.

- Sustainable Tourism in Small Island States: case studies. London. 1996.

- The Economy. In: MINISTRY OF YOUTH AND THE ARTS (Hrsg.): Malta - Culture and Identity. Marsa (Malta). 1994. S. 233-251.

BRIGUGLIO, Lino \& CORDINA, Gordon:

- Competitiveness Strategies for Small States. Blata I-Bajda. 2004a.

- Malta's Competitiveness Standing and the EU Lisbon Agenda. In: BRIGUgLIO, Lino \& CORDINA, Gordon (Hrsg.): Competitiveness Strategies for Small States. Blata I-Bajda. 2004b. S. 58-74.

- Malta's Competitiveness: Analysis and Prescriptions. In: Grip, H. 2/2004. Birkirkara. (2004c). S. 45.

BRIGUglIO, Lino; BARE Tendai R.W.; CORDINA, Gordon; ENDELEY, Rose Marie-Rita: Maximising Malta's Competitiveness Potential - A Consultative Process towards the Development of a National Competitiveness Strategy. Blata I-Bajda. 2004.

BMZ (Bundesministerium für wirtschaftliche Zusammenarbeit und Entwicklung) (Hrsg.): Erfolgsbedingungen der Euro-Mediterranen Assoziierungspolitik. Stellungnahme des wissenschaftlichen Beirats beim BMZ. Bonn. 2001.

BusutTIL, Salvino: Agriculture in Malta: A Historical Note. In: Options Méditerranéennes, Ser. B, no. 7. Cachan Cedex. 1993. S. 9-26.

BUSUTTIL, Simon \& DEMICOLI, Edward: Lenghty talks on Malta's Agriculture draw to a close. $=$ Aǵgiornat, $\mathrm{Nr}$. 16, Januar 2003. Valletta. 2003.

CALLEYA, Stephen C.: Looking Ahead - Malta's Foreign Policy Agenda. Luqa. 2002.

CAMILLIERI, Mariella: From the Vine to the Table - A Marketing Perspective. A Study of how the Wine Industry has grown with particular reference to the effects it is leaving on the Maltese population. Msida. 1994. (Unveröffentlichte Bachelorarbeit). 
CARABOtT, Romina: The Promotion of Traditional Maltese Textile Crafts. Msida. 1998. (Unveröffentlichte Bachelorarbeit).

CASSAR, Carmel: A Concise History of Malta. Msida. 2000.

CASSAR PULLICINo, Gużè:

- Studies in Maltese Folklore. Msida. 1992.

- Folklore. In: MINISTRY OF YOUTH AND THE ARTS (Hrsg.): Malta - Culture and Identity. Marsa. 1994. S. 181204.

CHARLTON, William A. \& BEELEY, Brian W.:

- Agriculture, Land Use and Resource Transformation in Malta. In: Tijdschrift voor Economische en Sociale Geografie, 84, H. 5. 1993. S. 325-331

- Land-use change in Malta. In: Land Use Policy. 4, H. 2. 1987. S. 96 -101.

CHURCHILL, Winston: Something to astonish you! (Protokoll einer mündlichen Rede vom 19.09.1946, gehalten in Zürich).

COHEN, E.: Authenticity and commoditization in tourism. In: Annals of Tourism Research, 15, H. 1. 1988. S. 371-386.

CORDINA, Elaine: Lenghty talks on Malta's Environment draw to a close. = Agggornat, Nr. 14, November 2002. Valletta. 2002.

CREMONA, Matty: A Year in the Country - Life and Food in Rural Malta. Valletta. 2003.

CYFFKA, Bernd: Land-Cover Changes by Land-Use changes on the Islands of Elba and Malta, Mediterranean Sea. In: MILANOVA, Elena, HIMIYAMA, Yukio \& BIĈ́ik, Ivan (Hrsg.): Understanding Land-Use and LandCover Change in Global and Regional Context. Enfield, Plymouth. 2005. S. 187-205.

DARMANIN, Francis: Die maltesische Küche. Sliema. 1991.

DEGUARA, Lina: Viticulture in Malta. Msida. 1979. (Unveröffentlichte Bachelorarbeit).

DORSON, Richard Mercer (Hrsg.): Folklore and Folklife. An Introduction. Chicago, London. 1972.

DREYER, Axel: Kulturtourismus. München, Wien. 2000.

EISENSTEIN, Bernd \& ROSINSKI, André: Ökonomische Effekte des Tourismus. In: BECKER, Christoph;

STEINECKE, Albrecht; HOPFINGER, Hans (Hrsg.): Geographie der Freizeit und des Tourismus: Bilanz und Ausblick. München, Wien. 2003. S. 805-814.

EUROPÄISCHE GEMEINSCHAFTEN, Kommission der...:

- Umfassender Monitoring-Bericht über die Vorbereitungen Maltas auf die Mitgliedschaft. Brüssel. 2003a.

- Bericht zur Aktualisierung der Stellungnahme der Kommission zum Beitrittsantrag von Malta. Brüssel. 2003b.

- Malta - Agriculture and Enlargement. Brüssel. 2002a.

- Agricultural Situation in the Candidate Countries. Brüssel. 2002b.

- Regelmäßiger Bericht 2001 über die Fortschritte Maltas auf dem Wege zum Beitritt. Brüssel. 2001.

EVANS, John D.: Malta. = Alte Kulturen und Völker, Bd. 3. Köln. 1963.

FARRUGIA, Victor:

- Malta. In: MYRTA, A.; DI TERLIZZI, B.; SAVINO, V (Hrsg.): Production and exchange of virus-free plant propagating material in the Mediterranean region $=$ Options Méditerranéennes: Séries B. Etudes et Recherches; Nr. 35. 2001. S. 103-124.

- Different Crop Types grown in Malta. (Unveröffentlichtes Manuskript).

FRENDO, Henry: National Identity. In: MINISTRY OF YOUTH AND THE ARTS (Hrsg.): Malta - Culture And Identity. Marsa. 1994. S. 1-25. 
Galea de GIovanNI, Martin: Agro Biodiversity Campaign - Paper on Maltese Agro-Biodiversity. Malta. (Ohne Jahresangabe).

GATT, Marica; MERCIECA, Vanessa \& FARRUGIA, Victor: Malta. In: Options Mediterranéennes, Séries B, H.19. 1998. S. $59-60$.

GEE, Kira; REEH, Tobias \& KREISEL, Werner (Hrsg).: Regionale Identität, Tourismus und Landschaftsinterpretation: Eine natürliche Symbiose? = ZELTForum - Göttinger Schriften zu Landschaftsinterpretation und Tourismus, Bd. 1. 2000.

GEORGES, Robert A. \& OWEN JONES, Michael: Folkloristics - An Introduction. Indianapolis, Bloomington. 1995.

GESER, Hans (2001): Was ist eigentlich ein Kleinstaat? In: KIRT, Romain \& WASCHKUHN, Arno (Hrsg.):

Kleinstaaten-Kontinent Europa. Baden-Baden. 2001. S. 89-100.

GOMME, George Laurence: The Handbook of Folklore. London. 1890.

GOWER, Jackie \& REDMOND, John: Enlarging the European Union: The Way Forward. Aldershot, Burlington, Singapur, Sydney. 2000.

GRECH, Jesmond: British Heritage in Malta. Luqa. 2003.

GRIENBERGER, Regine: Die Herkunftsangabe als Marketinginstrument. Fallstudien aus Italien und Spanien. Mainz. 2000.

HALTER, Liv: Kulturanleitung für Artischocken - Praxisempfehlungen für den Anbau. (http://www.weihenstephan.de/gb/main/Sop2.pdf)

HAUSMANN, Friederike: Kleine Geschichte Italiens von 1943 bis heute. Aktualisierte und erweiterte Neuausgabe. Berlin. 1997.

HENNIG, Christoph: Reiselust. Touristen, Tourismus und Urlaubskultur. Frankfurt/Main, Leipzig. 1999. (Taschenbuchausgabe der Erstausgabe von 1997 mit unverändertem Inhalt, jedoch durch das Taschenbuchformat bedingter Änderung der Seitenzahlen.)

HILLER, Dietmar: Wirtschaftsstandort Malta. Eine Bilanz deutscher Unternehmen. = BESTLER, Anita (Hrsg.): Mediterrane Studien, Bd. 1. Bruchköbel. 1995.

HUGHES, Quentin: Malta - „Fortress“. München. 1972.

HYDE, Herbert P.T.: Malta. Unbesiegte Insel im Abwehrkampf der Jahrhunderte. In: Die Erde, Bd. 5=84. 1953. S. 224-240.

ILG, Bertha:

- Maltesische Märchen und Schwänke: Aus dem Volksmunde gesammelt. Teil 1. = Beiträge zur Volkskunde, Bd. 2. Leipzig. 1906a.

- Maltesische Märchen und Schwänke: Aus dem Volksmunde gesammelt. Teil 2. = Beiträge zur Volkskunde, Bd. 3. Leipzig. 1906b.

KARABDIC, Zurah: Individuum, Tourismus und Internet in der entwickelten Industriegesellschaft: Eine theoretische Studie mit empirischer Untersuchung. Osnabrück. 2002.

KASCHUBA, Wolfgang: Einführung in die Europäische Ethnologie. München. 2. Aufl.. 2003.

KIRT, Romain \& WASCHKUHN, Arno:

- Kleinstaaten-Kontinent Europa. Baden-Baden. 2001a.

- Was ist und zu welchem Zweck betreibt man Kleinstaaten-Forschung? Ein Plädoyer für die wissenschaftliche Beschäftigung mit kleinen Nationen. In: KIRT, Romain \& WASCHKUHN, Arno (Hrsg.): Kleinstaaten-Kontinent Europa. Baden-Baden. 2001b. S. 23-48.

KONKEN, Michael: Stadtmarketing. Grundlagen für Städte und Gemeinden. Limburgerhof. 2000.

KRAPPE, Alexander Haggerty: The Science of Folk-Lore. London. 1930.

KREISEL, Werner: Trends in der Entwicklung von Freizeit und Tourismus. In: BECKER, Christoph (Hrsg.):

Geographie der Freizeit und des Tourismus. München. 2003. S. 74-85. 
KREISEL, Werner; HOPPE, Michael; REEH, Tobias: Mega-Trends im Tourismus: Auswirkungen auf Natur und Umwelt. Berlin. 2000. (Forschungsbericht des Umweltbundesamts, Nr. 28999 110).

KRETSCHMER, Ingrid: Malta: Junge Inselrepublik vor neuer Zukunft. In: Mitteilungen der Österreichischen Geographischen Gesellschaft, Bd. 122/2. 1980. S. 221-255.

KROEBER-RIEHL, Werner \& WEINBERG, Peter: Konsumentenverhalten. München. 2003.

KULINAT, Klaus: Tourismusnachfrage: Motive und Theorien. In: BECKER, Christoph; STEINECKE, Albrecht; HOPFINGER, Hans (Hrsg.): Geographie der Freizeit und des Tourismus: Bilanz und Ausblick. München, Wien. 2003. S. 97-111.

LANFRANCO, Guido:

- Gharb Folklore Museum. Ghajnsielem (Gozo). 2003.

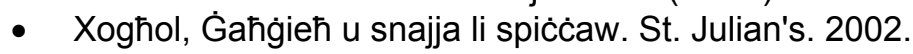

LESER, Hartmut (Hrsg.) et al.: Diercke Wörterbuch Allgemeine Geographie. Braunschweig, München. 1997.

LIPPERT, Barbara: Auf dem Weg in eine größere Union. In: BUNDESZENTRALE FÜR POLITISCHE BILDUNG (Hrsg.): Informationen zur Politischen Bildung, H. 279: Europäische Union. Bonn. 2003.

LITTRELL, Mary Ann; ANDERSON, Luella F. \& BROWN, Pamela J.: What makes a craft souvenir authentic? In: Annals of Tourism Research, 20, H. 1. 1993. S. 197-215.

LOCATELLI, Davide: The Roman Villa at San Pawl Milqi: history and perspectives of an archaoelogical site. In: Treasures of Malta, Nr. VII. Valletta. 2001. S. 73-77.

LUKE, Sir Harry: Malta - An Account and an Appreciation. $2^{\text {nd }}$ revised and enlarged edition. London. 1968.

MacCANNELL, Dean: The Tourist. A New Theory of the Leisure Class. $3^{\text {rd }}$ edition. Berkeley, Los Angeles, London. 1999.

MALTA CRAFTS COUNCIL:

- Crafts - A Directory of Craftsmen \& Entrepeneurs registered with the Malta Crafts Council. Valletta. 2001.

- Malta Crafts Council - For the Commercial Development and the Creation of New Jobs in the Crafts Sector. (Informationsbroschüre).

MARAUHN, Thilo (Hrsg.): Staatliche Förderung für regionale Produkte: Protektionismus oder Umwelt- und Verbraucherschutz? Tübingen. 2004.

MARKWICK, Marion:

- Tourism and the development of handicraft production in the Maltese Islands. In: Tourism Geographies, H. 3/2001. (2001a). S. 29-51.

- Postcards from Malta - Image, Consumption, Context. In: Annals of Tourism Research, 28, H. 2. 2001b. S. 417-438.

- Malta's Tourism Industry since 1985: Diversification, Cultural Tourism and Sustainability. In: Scottish Geographical Journal, 115, H. 3. 1999. S. 227-247.

- The Population of the Maltese Islands. In: Geography, 82, H. 2. 1997. S. 179-182.

MAULL, Otto: Politische Geographie. Berlin. 1956.

MELI, Anthony: Overview of Agricultural Land Use in Malta. In: Options Mediterranéennes, Séries B, H. 7. 1993. S. 71-75.

METWALLY, Mokhtar M.: Structure and performance of the Maltese economy. Valletta. 1977.

METzGer, Philipp: Local rural development in the hinterland of the Algarve, Portugal. In: GEE, Kira; REEH, Tobias \& KREISEL, Werner: Regionale Identität, Tourismus und Landschaftsinterpretation: Eine natürliche Smbiose? = ZELTForum - Göttinger Schriften zu Landschaftsinterpretation und Tourismus, Bd. 1. 2004. S. 83-90.

MEYER, F.: Euro-Mediterrane Partnerschaft oder Konfrontation? Die EU und die südlichen Mittelmeeranrainerstaaten. In: Geographische Rundschau. 53, H. 6. 2001. S. 32-38. 
MIFSUD-CHIRCOP, Ǵorǵ (2003a):

- II-Folklor Malti, Vol. I. Valletta. 2003a. = Kullana Kulturali, Bd. 56.

- II-Folklor Malti, Vol. II. Valletta 2003b. = Kullana Kulturali, Bd. 57.

MINISTRY OF YOUTH AND THE ARTS (Hrsg.): Malta - Culture and Identity. Marsa. 1994.

MiTCHELL, Jon. P.: Ambivalent Europeans. Ritual, Memory and the Public Sphere in Malta. London, New York. 2002.

MIzzI, Leonard: A General Overview of Agro-Industry. In: BusUTTIL, S.; LERIN, F. \& MIzzI, L. (Hrsg.): Malta: Food, Agriculture, Fisheries and the Environment. = Options Méditerranéennes, Séries B. Etudes et Recherches; Nr. 7. Montpellier. 1993. S. 93-104.

Monge-RoffaRELlo, Luca; SWIDINSKY, Michael; VANZETTI, David: Small Island Developing States and Agricultural Trade Liberalisation. In: BRIGUGLIO, Lino \& CORDINA, Gordon (Hrsg.): Competitiveness Strategies for Small States. Blata I-Bajda. 2004. S. 307-337.

MSA (Malta Standards Authority):

- Hand-made Maltese Lace - Characteristics and Identification Parameters. Valletta. 2003a.

- Traditional Hand-made Maltese Filigree: General Characteristics and Identification Parameters. Valletta. 2003.

MTA (Malta Tourism Authority): Strategic Plan 2002-2004. Valletta (Malta).2002.

MÜLLER, Klaus: Globalisierung. Frankfurt am Main, Köln. 2002.

NABU (Naturschutzbund Deutschland e.V.) \& DLV (Deutscher Verband für Landschaftspflege) (Hrsg.): Regionale Produktion und Vermarktung - Ziele, Rahmenbedingungen, Forderungen. Berlin, Bonn. 2002.

NEHRING, Bodo: Die Maltesischen Inseln. Tübingen. 1966.

NSO (National Statistics Office):

- News Release no. 95/2001. Tourism. Valletta. 2001.

- Agriculture and Fisheries 2000. Valletta. 2002a.

- Information Society. Valletta. 2002b.

- Census of Agriculture 2001. Valletta. 2003a.

- News Release no. 106/2003: Tourism. Valletta. 2003b.

- Malta in Figures 2002. Valletta. 2003c.

- Tourstat Survey Results on Inbound Tourism. Valletta. 2003d.

- News Release no. 41/2004: Tourism Statistics. Valletta. 2004a.

- Agriculture and Fisheries 2001. Valletta. 2004b.

- Shipping and Aviation Statistics 2002. Valletta. 2004c.

- Agriculture \& Fisheries 2002. Valletta. 2004d.

- News Release No. 14/2005: Tourism Statistics. Valletta. 2005.

O'SuILleabHaIN, Sean: A Handbook Of Irish Folklore. Hatboro. 1963. (Reprint der ersten Auflage von 1942).

OECKL, Walter: Kolonialherrschaft contra Selbstbestimmung - Das Streben nach politischer Emanzipation: Malta. Augsburg. 1980.

OPASCHOWSKI, Horst W.:

- Umwelt, Mobilität und Tourismus. Hamburg.1998.

- Einführung in die Freizeitwissenschaft. 3. aktualisierte und erweiterte Aufl. Opladen. 1997.

- Tourismusforschung. Opladen. 1989. 
PACE, Roderick:

- Microstate Security in the Global System - EU-Malta Relations. Valletta. 2001.

- Small States and the Internal Balance of the European Union. In: GoWER, Jackie \& REDMOND, John (2000): Enlarging the European Union: The Way Forward. Aldershot, Burlington, Singapore, Sydney. 2000. S. $107-122$.

- Malta, the Enlargement of the European Union and the Situation in the Mediterranean Region. In: Streit Kultur - Magazin für Politik und Kultur in Europa. H. 1/99. Bonn. 1999. S. 19-20.

PAGENSTECHER, Cord: Neue Ansätze für die Tourismusgeschichte - ein Literaturbericht. In: Archiv für Sozialgeschichte, H. 38. 1998. S. 591-619.

PfaffenBaCh, Carmella \& REUBER, Paul: Methoden der empirischen Humangeographie. Braunschweig. 2005.

PIROTTA, Fabio: Negotiations closed in crucial free trade area. = Aǵġornat, Nr. 1, Juli 2001. Valletta. 2001.

POLLACCO, Christopher:

- European Integration - The Maltese Experience. Luqa. 2004.

- An Outline of the Socio-Economic Development in Post-War Malta. Msida. 2003.

- Malta-EEC Relations. Valletta. 1992.

POLLACCO, John: In the National Interest - Towards a sustainable tourism industry in Malta. Valletta. 2003.

Pro Tour (Hrsg.): Mit der Natur gewinnen... Traditionen, Trends und Potentiale im Tourismus. Düren. 1995.

REEH, Tobias \& KREISEL, Werner: Motive, Merkmale und Marketing im Inseltourismus: Ein Überblick. In: WAIBEL, Michael; THIMM, Tatjana \& KREISEL, Werner (Hrsg.) (2005): Fragile Inselwelten - Tourismus, Umwelt und indigene Kulturen. = Pazifik Forum, Bd. 9. 2005. S. 7-26.

REEH, Tobias: Der Wunsch nach Urlaubsreisen in Abhängigkeit von Lebenszufriedenheit und Sensation Seeking - Entwicklung und Anwendung eines Modells der Urlaubsreisemotivation. Göttingen. 2005.

REIMANN, Horst:

- Research on Malta - A German Perspective. = Augsburger Beiträge aus Kommunikationswissenschaft und Soziologie (ABAKUS), Bd. 13. Augsburg. 1991.

- Malta - ein Sonderfall? Zur Problematik von Modernisierungstheorien. In: MACKENSEN, Rainer \& SAGEBIEL, Felizitas (Hrsg.): Soziologische Analysen. Referate beim 19. Deutschen Soziologentag. Berlin. 1979. S. 59-68.

ROBERTS, Warren E.: Folk Crafts. In: DORSON, Richard M. (Hrsg.): Folklore and Folklife. An Introduction. Chicago, London. 1972. S. 233-252.

RossI, Enzo: Malta on the brink: from western democracy to Libyan satellite. = European Security Studies, H. 5. London. 1986.

ROTHER, Klaus:

- Der Mittelmeerraum. Stuttgart. 1993.

- Die mediterranen Subtropen. Braunschweig. 1984.

SANT CASSIA, Paul: Tradition, tourism and memory in Malta. In: The Journal of The Royal Anthropological Institute, 5, H. 6. 1999. S. 247-264.

SCHEMBRI, Patrick J.: Natural Heritage. In: MINISTRY OF YOUTH AND THE ARTS (Hrsg.): Malta - Culture and Identity. Marsa. 1994.

SCHIFFARTH, B.: Vogeljagd in Malta. In: Deutsch-Maltesische Gesellschaft: Deutsch-Maltesischer Rundbrief $4 / 2002$.

SCHÖNFELDER, Ingrid \& SCHÖNFELDER, Peter: Die Kosmos-Mittelmeerflora. 3. Auflage. Stuttgart. 1999.

SCHRUTKA-RECHTENSTAMM, Adelheid: „Die Gäste fühlen sich wohl bei uns“ - Begegnungen durch Tourismus. In: PÖTTLER, Burkhart (Hrsg.): Tourismus und Regionalkultur - Referate der Österreichischen Volkskundetagung 1992 in Salzburg. Wien. 1994.

SCHWIND, Dieter: Allgemeine Staatengeographie. = Lehrbuch der Allgemeinen Geographie, Bd. 8. Berlin . New York. 1972. 
SEILER, Markus-René: Kleinststaaten im Europarat - Fallstudien zu Island, Liechtenstein, Luxemburg, Malta und San Marino. Bamberg. 1995.

SHORT, David: Traditional-style farming and values for sustainable development in Malta. In: Tijdschrift voor Economische en Sociale Geografie, 88, H.1. 1997. S. 41-52.

SICK, Wolf-Dieter: Agrargeographie. Braunschweig. 1983.

STEINECKE, Albrecht: Tourismus und neue Konsumkultur: Orientierungen - Schauplätze - Werthaltungen. In: STEINECKE, Albrecht (Hrsg.): Erlebnis- und Konsumwelten. München; Wien. 2000. S. 11-27.

STEINMÜLLER, Karlheinz: Trends und Konsumverhalten - Ein Blick in die Kristallkugel: Was bringt uns die Zukunft? Unveröffentlichte Präsentationsfolien zu einem Vortrag im Rahmen der Agendakonferenz „Verbraucherschutz und Gesundheit" der Agenda 21 NRW. Düsseldorf, 15.03.2002.

STUMmE, Hans:

- Maltesische Studien: Eine Sammlung prosaischer und poetischer Texte in maltesischer Sprache. $=$ Leipziger Semitistische Studien, Bd. 1., H. 4. Leipzig. 1904a.

- Maltesische Märchen: Gedichte und Rätsel in deutscher Übersetzung = Leipziger Semitistische Studien, Bd. 1, H. 5. Leipzig. 1904b.

SUlTANA, Joseph: National Tree. In: BEZZINA, Joseph: Gozo - a historical glimpse. = Gaulitana, Bd. 6 . Valletta. 1988.

TEUMA, Eugene Paul: San Pawl II-Baћar. St. Paul's Bay. 2003.

THIMM, T.: Kulturwandel und Tourismus in Französisch-Polynesien. = KREISEL, Werner \& WAIBEL, Michael (Hrsg.): Pazifik Forum, Bd. 7. Göttingen. 2002.

TRUMP, David H.: Malta: An Archaeological Guide. Valletta. 1997.

TURNER, Victor: Vom Ritual zum Theater. Vom Ernst des menschlichen Spiels. Frankfurt/Main. 1995.

USHER, John: Malta and the EU Three Issues. Msida. 1995.

VITAL, David: The Inequality of States - A Study of the Small Power in International Relations. Oxford. 1964.

VOTH, Andreas: Aufwertung regionaltypischer Produkte in Europa durch geographische Herkunftsbezeichnungen. In: Europa Regional, 11, H. 1. 2003. S. 2-11.

XUEREB, Paul (Hrsg.): Malta and the EU: Together in Change? Msida. 2001.

YODER, Don: Folk Cookery. In: DORSON, Richard M. (Hrsg.): Folklore and Folklife. An Introduction. Chicago, London. 1972.

ZARB, Tarcisio: Folklore of an Island - Maltese Threshold Customs. San Ǵwann. 1998.

ZECH, Wolfgang \& HINTERMAIER-ERHARD, Gerd: Böden der Welt. Ein Bildatlas. Heidelberg, Berlin. 2002.

ZIMBARDO, Philip G. \& GERRIG, Richard J.: Psychologie. 7., neu übersetzte und bearbeitete Auflage. Berlin, Heidelberg, New York. 1999. 


\section{Tageszeitungen und Wochenzeitschriften:}

\section{DER SPIEGEL}

Nr. 40/2002: KOCH, Dirk: Aufgeblähte Verträge. Die EU schlägt zehn Staaten zur Aufnahme in die Union vor. S. 51.

Nr. 28/2004: SCHNEIDER, Peter: Die deutsche Lähmung. Über Fußball, Politik und das Versagen der Intellektuellen in der aktuellen Reformdiskussion. S. 132-134.

\section{FRANKFURTER ALLGEMEINE ZEITUNG}

13.01.2003: Reichlich Wohlstand - und doch wird vieles sich ändern müssen. S. 14.

\section{MALTA TODAY}

Onlineausgabe, 16.02.2001: BRINCAT, Nadine: Haphazard Ta'Qali development to be checked.

Onlineausgabe, 28.10.2001: BUGEJA, Zilla: This Week - Sammy Cremona.

Onlineausgabe, 23.02.2003: BALZAN, Saviour: Malta as Gateway to the South for EU investors.

09.05.2004: SCHEMBRI, Karl: Gonzi says EP election is „PN's gift to the people“. S. 4.

09.05.2004: (keine Autorenangabe): Farsons to launch Celebration Brew today at Malta Fireworks Festival. S. 20.

\section{NEUE WESTFÄLISCHE}

29.04.2004: Gute Noten auch ohne Dolmetscher. S. 3.

03.05.2004: Euphorie von Riga bis Nikosia. S. 3.

\section{TASTE MAGAZINE}

Nr. 1/2004: GATT, Beatrice: This is the Season for Almonds. S. 41-42.

Nr. 3/2004: (keine Autorenangabe): Happy $200^{\text {th }}$ Birthday, Maltese Potato. S. 16-17.

\section{THE MALTA INDEPENDENT / THE MALTA INDEPENDENT ON SUNDAY}

04.05.2004: BRINCAT, Leo: Making a reality of the rhetoric. S. 6.

04.05.2004: (keine Autorenangabe): A missed chance for the MLP. S.7.

05.05.2004: (keine Autorenangabe): VAT on an additional 400 items - MLP. S. 8.

05.05.2004: (keine Autorenangabe): MTA tourism awareness campaign on the buses. S. 16.

\section{THE TIMES OF MALTA / THE SUNDAY TIMES / THE GOZO TIMES}

08.08.2000: FENECH, Natalino: Scheme to register tenants of government agicultural land. S. 17.

06.05.2004: ZAMMIT, Rosanne: Sant calls for the creation of a price watch unit. S. 44.

06.05.2004: (keine Autorenangabe): When you come to Malta, you think about fun. Business Section, S. 3. 25.05.2004: (keine Autorenangabe): Malta Tram promotion in Zurich. S. 6.

28.05.2004: TESTA, Michael: Potato exports yield Lm 1.3m. S. 16.

05.07.2004: (keine Autorenangabe): Malta's strong point: adaptability. S. 2.

05.07.2004: (keine Autorenangabe): Prickly pear extract prevents hangovers. S. 1.

05.07.2004: CINI, George: New Property found in prickly pear skin. S. 7.

05.07.2004: SPITERI, Lino: 100 days of introspection. S. 48.

12.07.2004: The Gozo Times: Meet... Joe Grima. S. 8.

13.07.2004: MASSA, Ariadne: Thousands of tons of tomatoes start being picked. S. 5.

13.07.2004: ZAMMIT TABONA, Kenneth: Take a walk on the wild side. S. 8.

18.07.2004: BARTOLO, Michael: Malta and the European Union: Now we know what we are receiving, do we know what we can give? S. 14.

22.07.2004: CINI, George: Campaign to claw back public land for walks draws support. S. 6 .

22.07.2004: DEBATTISTA, Martin: Internet could be attracting a third of travellers to our country. Itech, S. 1.

\section{TRUE}

09.05.2004: (keine Autorenangabe): „All the best..... S. 22-23. 


\section{Internet:}

http://www.aboutmalta.com/gozo/folklore1.html - Gozitan Folklore. Von ATTARD, Anton F.. Stand:

13.11.2003.

http://www.stern.de/wirtschaft/unternehmen/meldungen/index.html?id=521446 - ITB 2004 in Berlin öffnet inre Tore. Stand: 12.03.2004

http://www.maltatoday.com.mt - Onlineausgaben der Wochenzeitung Malta Today

http://mfea.gov.mt/commerce/maltacrafts_02.htm - Homepage des Malta Crafts Council

http://www.internationalspecialreports.com/archives/00/malta2000/20.html - Malta-Reportagensammlung der Washington Times. Stand: 23.09.2004

http://www.merkur-online.de/nachrichten/wirtschaft/aktuell/art279,335462.html - Malta lockt ausländische Hersteller. Stand: 08.09.2005 


\section{Anhang}

\section{Georg-August-Universität Göttingen - Malta Opinion Poll}

My name is... I am conducting a survey on behalf of the Geographical Institute of the University of Goettingen in Germany.

The survey deals with questions about typically Maltese products. It takes approx. $5 \mathrm{~min}$. to answer the questionnaire. Your data are treated anonymous and the survey does not have a commercial background of any kind.

Would you agree to help with your answers?

1. Location and date of the interview:

2. How long is your stay in Malta altogether?
a $\square<7$ days
c $\square$ 14-20 days
e $\square>30$ days
b $\square 7-13$ days
d $\square 21-30$ days
f $\square$ no answer

3. With whom did you travel?
$A \square$ noone $>$ single
C $\square$ Travel's group
E $\square$ family
B $\square$ partner
D $\square$ Group of friends
F $\square$ no answer

4. Do you know typical Maltese products? (If yes:) Which Maltese products do you know?

5. In which of the following procuct categories would you expect to find typical Maltese products?
A $\square$ lace
D $\square$ pottery \& ceramics
$\mathrm{G} \square$ jewellery
E $\square$ leather goods
H $\square$ fishery products
$\mathrm{J} \square$ refreshment beverages
B $\square$ textiles \& clothes
F $\square$ paintings \& arts
K $\square$ pastry
$\square$ no answer

6. Did you already buy Maltese products during your stay?
a $\square$ yes
b $\square$ no
c $\square$ I don't know
d $\square$ no answer

7. Which products did you buy?

8. Do you feel well informed about typical Maltese products?

$\begin{array}{ccc}\text { a } \square \text { yes } & \text { b } \square \text { no } & \text { c } \square \text { I don't know } \quad \text { d } \square \text { no answer }\end{array}$

9. Would you welcome the possibility to buy Maltese products also in your home country?

\begin{tabular}{|c|c|c|c|c|}
\hline a $\square$ yes & b $\square$ no & c $\square$ not sure & d $\square$ no answe & \\
\hline \multicolumn{5}{|l|}{ 10. Age } \\
\hline $\begin{array}{l}\text { a } \square<15 y \\
\text { b } \square 15-20 y\end{array}$ & $\begin{array}{l}\text { c } \square 21-30 \mathrm{y} \\
\text { d } \square 31-40 \mathrm{y}\end{array}$ & $\begin{array}{l}\text { e } \square 41-50 \mathrm{y} \\
\text { f } \square 51-60 \mathrm{y}\end{array}$ & $\begin{array}{l}\text { g } \square 61-70 \mathrm{y} \\
\text { h } \square 71-80 \mathrm{y}\end{array}$ & $\begin{array}{l}\mathrm{i} \quad \square>80 \mathrm{y} \\
\mathrm{j} \quad \square \text { no answer }\end{array}$ \\
\hline
\end{tabular}

11. Sex
a $\square$ male
b $\square$ female

12. Homeland

Englischsprachige Fassung des im Rahmen der Datenerhebung verwendeten Fragebogens. Eigener Entwurf 


\section{$\underline{\text { Lebenslauf }}$}

\section{Rainer Aschemeier}

Geburtsdatum: 04.01.1976

Geburtsort: Helmarshausen

Familienstand: Ledig

1982-1986 Städtische Grundschule Beverungen

1986-1995 Städtisches Gymnasium Beverungen

1995 Zeugnis der Allgemeinen Hochschulreife (Abitur)

1995-1996 Grundwehrdienst i. d. General Weber-Kaserne Höxter

1996-2002 Geographiestudium am Geographischen Institut der Georg-AugustUniversität Göttingen, Abschluss mit Diplom

2003-2005 Promotion am Geographischen Institut der Georg-August-Universität Göttingen

$2005 \quad$ Erlangung des Doktorgrades der mathematisch-naturwissenschaftlichen Fakultäten 


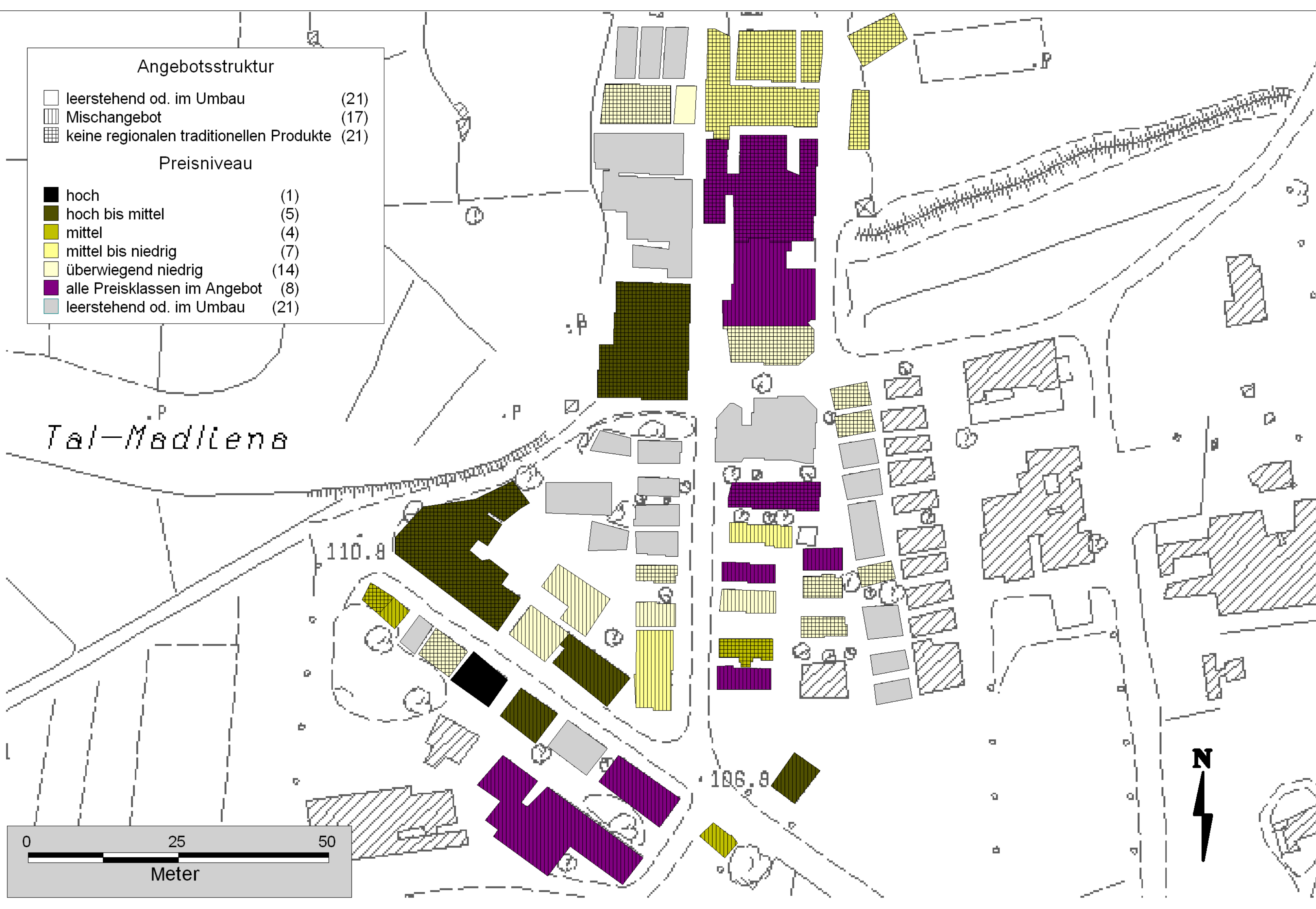




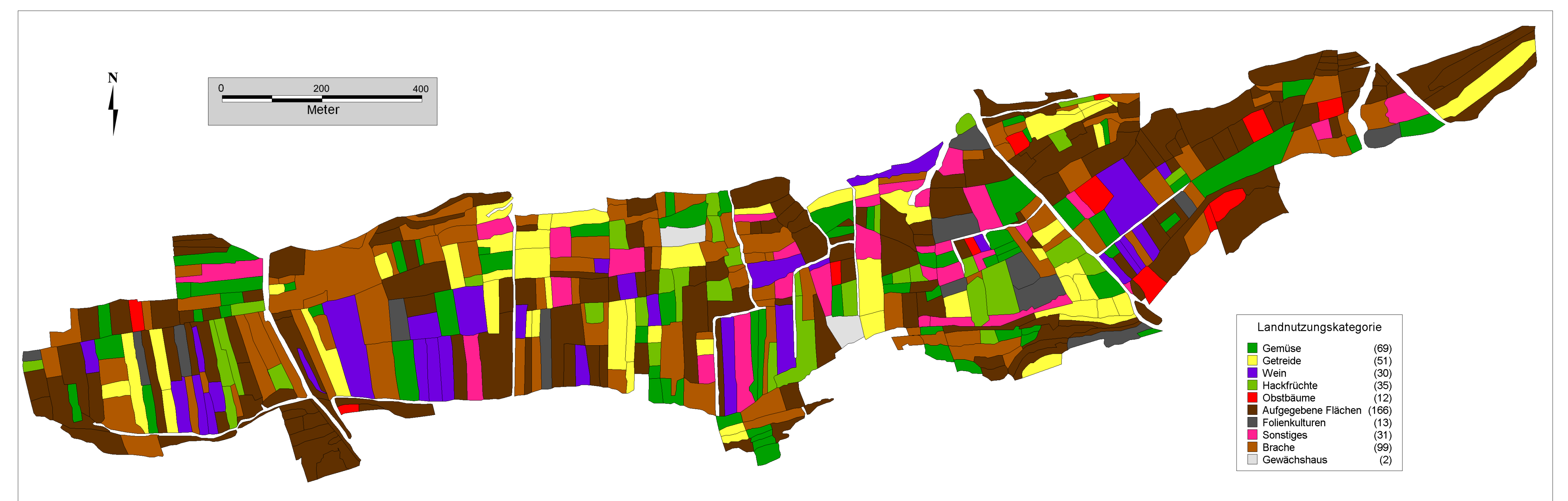

Karte I 


\begin{tabular}{|c|c|}
\hline \multicolumn{2}{|l|}{ Landnutzungskategorie } \\
\hline Gemüse & (40) \\
\hline Getreide & (2) \\
\hline Wein & (2) \\
\hline Hackfrüchte & (21) \\
\hline Obstbäume & (2) \\
\hline Aufgegebene Flächen (2 & (29) \\
\hline Folienkultur & (6) \\
\hline Sonstiges & (6) \\
\hline Brache & 44) \\
\hline Gewächshaus & \\
\hline
\end{tabular}

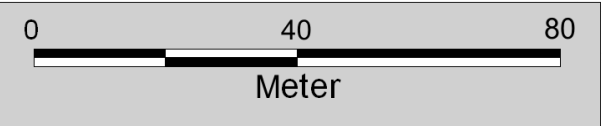

Karte II 


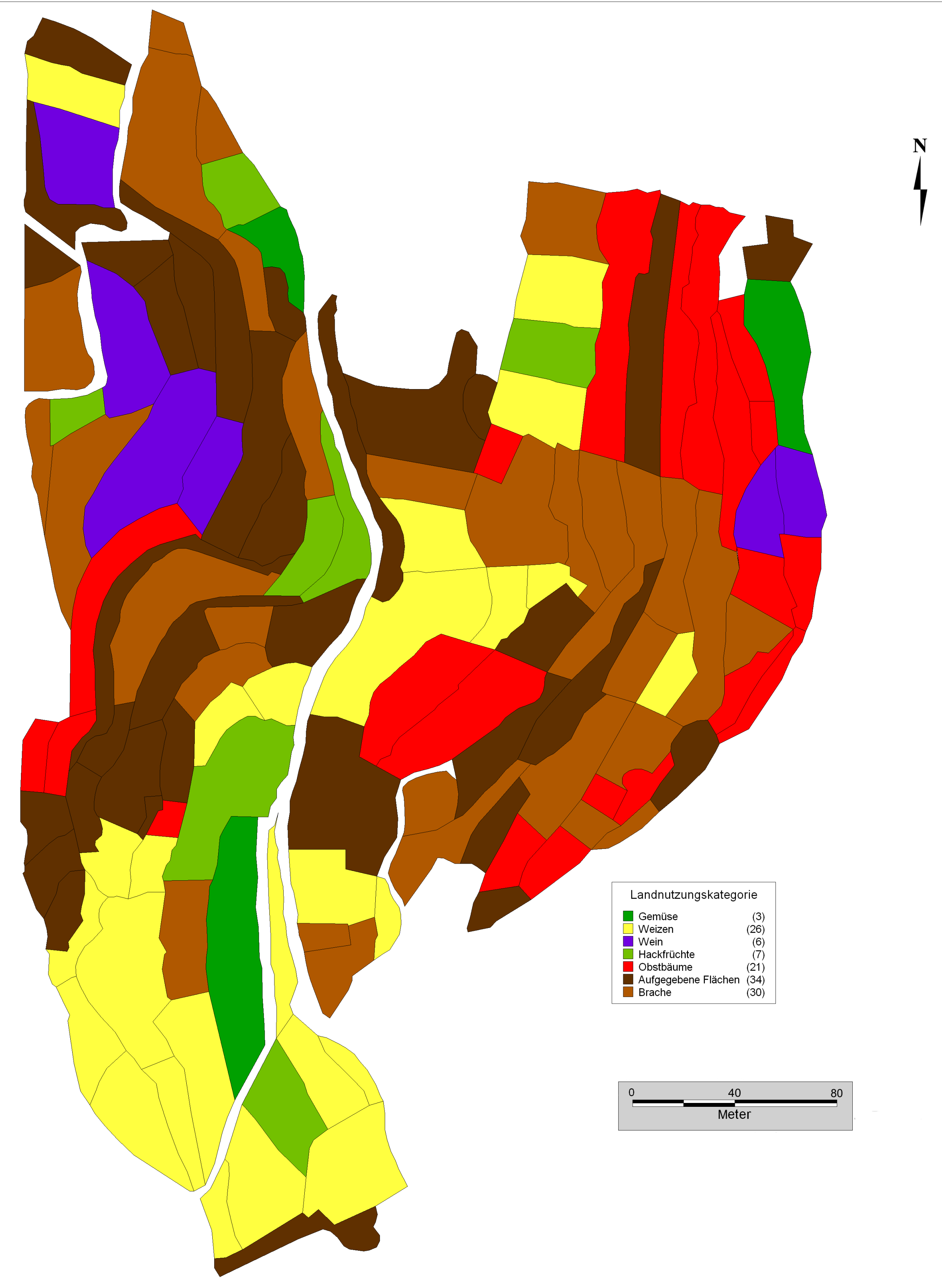

Karte III 


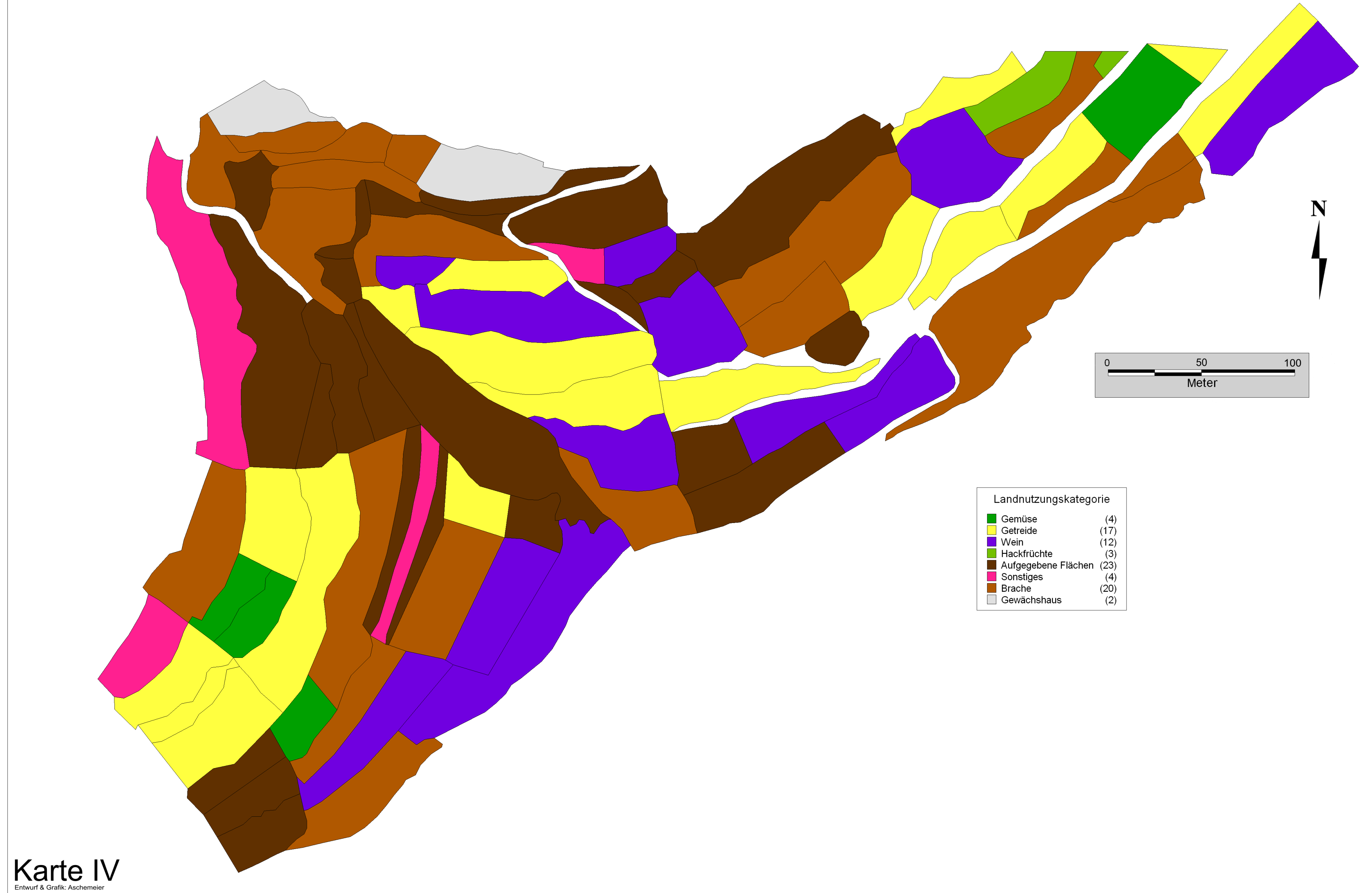

